\title{
Catalytic Asymmetric Three-Component Reaction of 2- Alkynylbenzaldehydes, Amines, and Dimethylphosphonate
}

Liangliang Zou, Jiaying Huang, Na Liao, Yan Liu, Qixiang Guo,* and Yungui Peng* Key Laboratory of Applied Chemistry of Chongqing Municipality, School of Chemistry and Chemical Engineering, Southwest University, Chongqing 400715, China

*E-mail address: qxguo@swu.edu.cn; *E-mail address: pengyungui@hotmail.com, pyg@swu.edu.cn

\section{Table of Contents}

1. General information.

2. Synthesis of chiral phosphoric acid 2

3. General procedure for the preparation of 2-alkynylbenzaldehyde derivatives

4. Optimization of the reaction conditions

5. General procedure for the catalytic asymmetric three-component reaction

6. Characterization of products

7. Amplification and Derivatizations of products 4 .

8. References 38

9. The spectra of ${ }^{1} \mathrm{H} N M R,{ }^{13} \mathrm{C} N M R$ and $\mathrm{HPLC}$. 40 


\section{General information}

Unless stated otherwise, all reactions were carried out under argon atmosphere. All the reactions that need to be heated, the oil bath is used as a heating source. All solvents were purified by standard procedures and distilled prior to use. Reagents obtained from commercial source were used without further purification. Petroleum ether and ethyl acetate for flash column chromatography was distilled before use. All reactions were monitored by TLC with silica gel coated plates. Flash column chromatography was performed on silica gel (300-400 mesh). NMR spectra were recorded on Bruker Avance $600 \mathrm{MHz}$ instruments. Chemical shifts $(\delta)$ are given in ppm relative to TMS, coupling constants $(J)$ in $\mathrm{Hz} .{ }^{1} \mathrm{H}$ NMR chemical shifts are reported in ppm relative to tetramethylsilane (TMS) with the solvent signal as the internal standard $\left(\mathrm{CDCl}_{3}\right.$ at $\left.7.26 \mathrm{ppm}\right) .{ }^{13} \mathrm{C} \mathrm{NMR}$ chemical shifts are reported in ppm from tetramethylsilane (TMS) with the solvent resonance as the internal standard $\left(\mathrm{CDCl}_{3}\right.$ at 77.00 ppm). Melting points were determined on an X-6 digital melting-point apparatus and were uncorrected. Optical rotations were measured on a Perkin Elmer 341 Polarimeter at $\lambda=589 \mathrm{~nm}$. IR spectra were recorded using a Perkin Elmer Spectrum 1 machine. Absorption maxima (vmax) are reported in wavenumbers $\left(\mathrm{cm}^{-1}\right)$. Analytical high performance liquid chromatography (HPLC) was carried out on WATERS 510 instrument (2487 Dual $\lambda$ Absorbance Detector and 515 HPLC Pump) using chiral column (Chiralpak IC, IF columns were purchased from Daicel Chemical Industries). ESI HRMS (Bio TOF Q) spectra were recorded on P-SIMS-Gly of Bruker Daltonics Inc. $3 \AA$ MS, $4 \AA$ MS and $5 \AA$ MS were purchased from J\&K Chemicals and used as received (500 mesh powder).

\section{Synthesis of chiral phosphoric acid}

\subsection{The compound $B$ was prepared according to the literature ${ }^{1}$}

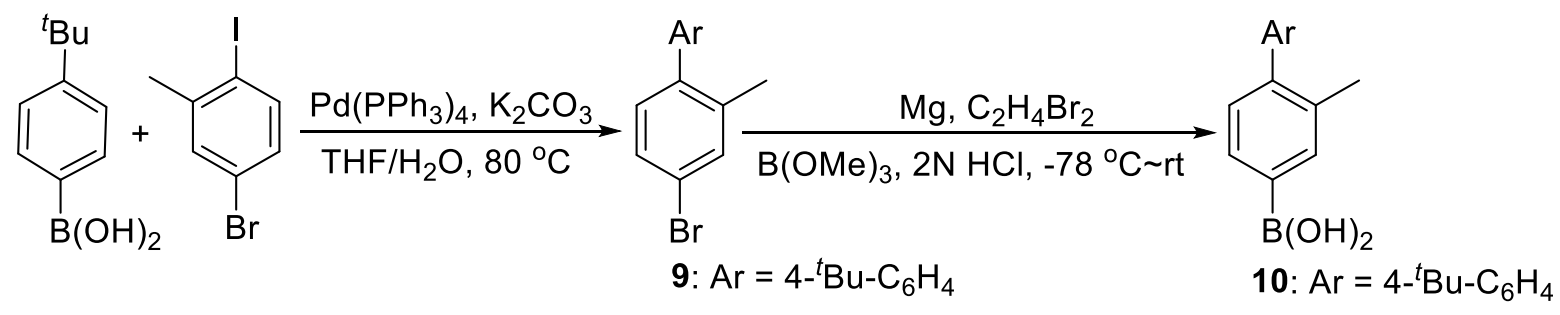

\subsection{The chiral phosphoric acid Id was prepared according to the literature ${ }^{2}$}




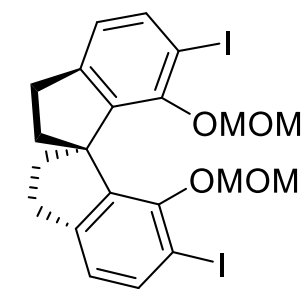

(S)-11

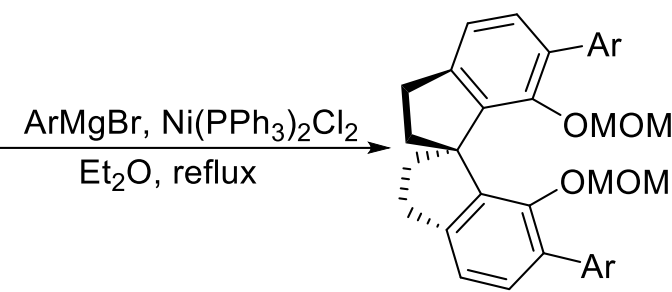

12

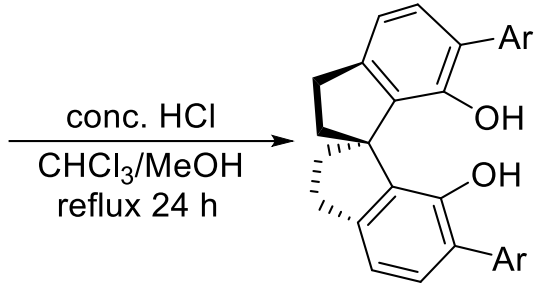

13

1) $\mathrm{POCl}_{3}$, Pyridine $110^{\circ} \mathrm{C}, 5$ days

2) $\mathrm{H}_{2} \mathrm{O}, 6 \mathrm{~N} \mathrm{HCl}$ $110^{\circ} \mathrm{C}, 2$ days

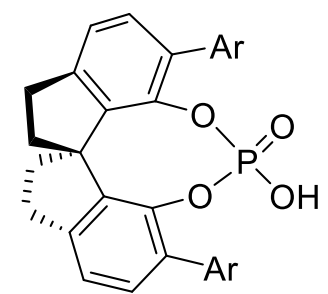

Id: $\mathrm{Ar}=2,4,6-\left({ }^{(} \mathrm{Pr}\right)_{3} \mathrm{C}_{6} \mathrm{H}_{2}$

\section{(R)-6,6'-Bis(2,4,6-triisopropylphenyl)-1,1'-spirobiindanyl-7,7'-diylhydrogenphosphate}

(ld)

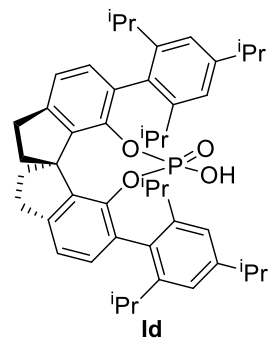

The product was prepared according to the literature, ${ }^{2}$ The residue was purified by silica gel column chromatography (ethyl acetate/petroleum ether $=1 / 15$ to $1 / 10)$ as white solid $(33.9 \mathrm{mg}, 62 \%$ yield $) . \mathrm{mp}: 154.2-156.8^{\circ} \mathrm{C},[\alpha]$ $\underset{\mathrm{D}}{25}=+200.6^{\circ}\left(c=1.0, \mathrm{CHCl}_{3}\right) ;{ }^{1} \mathrm{H}$ NMR $\left(600 \mathrm{MHz}, \mathrm{CDCl}_{3}\right) \delta 7.14(\mathrm{~d}, J=7.5$ $\mathrm{Hz}, 2 \mathrm{H}), 7.09$ (d, $J=7.6 \mathrm{~Hz}, 2 \mathrm{H}), 7.03(\mathrm{~d}, J=1.8 \mathrm{~Hz}, 2 \mathrm{H}), 6.96(\mathrm{~d}, J=1.8$ $\mathrm{Hz}, 2 \mathrm{H}), 3.76-3.51$ (m, 3H), 3.15 (ddd, $J=17.0,11.1,6.4 \mathrm{~Hz}, 2 \mathrm{H}), 2.97-$ $2.79(\mathrm{~m}, 6 \mathrm{H}), 2.65(\mathrm{p}, J=6.8 \mathrm{~Hz}, 2 \mathrm{H}), 2.31(\mathrm{dd}, J=12.1,6.4 \mathrm{~Hz}, 2 \mathrm{H}), 2.09(\mathrm{td}, J=11.6,8.5$ $\mathrm{Hz}, 2 \mathrm{H}), 1.26(\mathrm{dd}, J=6.9,1.9 \mathrm{~Hz}, 12 \mathrm{H}), 1.20(\mathrm{t}, J=6.5 \mathrm{~Hz}, 12 \mathrm{H}), 1.11(\mathrm{~d}, J=6.7 \mathrm{~Hz}, 6 \mathrm{H})$, $0.85(\mathrm{~d}, J=6.8 \mathrm{~Hz}, 6 \mathrm{H}) .{ }^{13} \mathrm{C} \mathrm{NMR}\left(151 \mathrm{MHz}, \mathrm{CDCl}_{3}\right) \delta 148.0,148.0,147.4,144.7,139.6$, 132.0 , 131.9 , 131.5 , 121.8 , 121.3 , 120.3 , 77.1 , 76.9 , 60.3 , 38.4 , 34.1 , 30.7 , 30.7 , 30.0 , 26.9 , 25.0 , 24.0 , 24.0 , 23.6 , 23.4 . ${ }^{31} \mathrm{P}$ NMR $\left(243 \mathrm{MHz}, \mathrm{CDCl}_{3}\right) \delta-10.58$. HRMS (ESI) m/z: $[\mathrm{M}+\mathrm{H}]^{+}$Calcd for $\mathrm{C}_{47} \mathrm{H}_{58} \mathrm{O}_{4} \mathrm{P} 717.4078$; Found 717.4066.

\subsection{Synthesis of chiral phosphoric acid Ii}

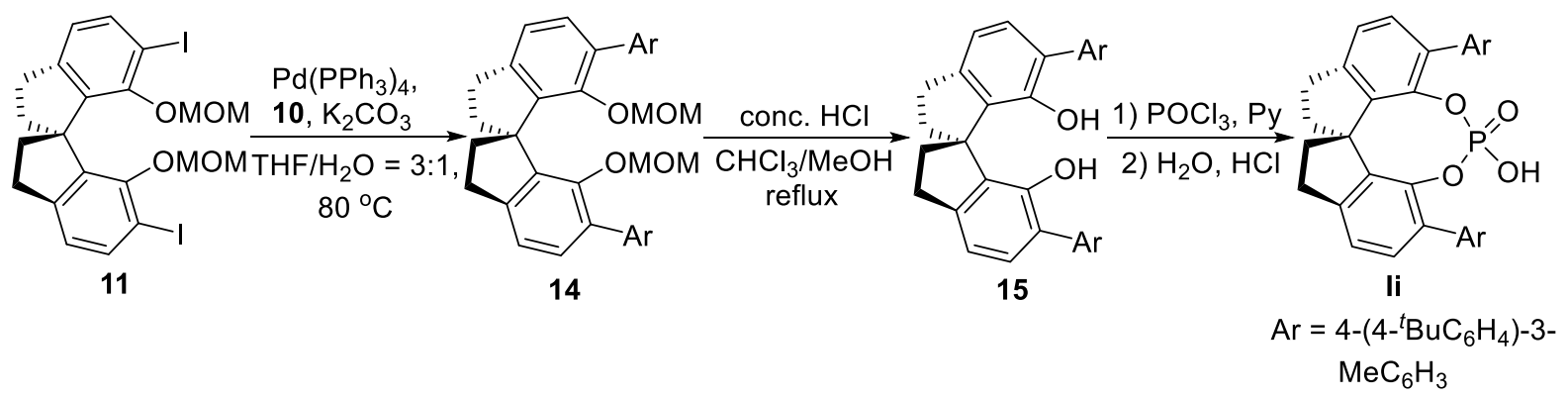

2.2.1 Synthesis of the compound $\mathbf{1 4}$ 
Under $\mathrm{N}_{2}$ atmosphere, $\mathrm{K}_{2} \mathrm{CO}_{3}(2.8 \mathrm{~g}, 20.4 \mathrm{mmol})$ was added to the solution of $(\boldsymbol{S})-\mathbf{1 1}^{3}(2.0 \mathrm{~g}$, $3.4 \mathrm{mmol})$, arylboronic acid $10(3.6 \mathrm{~g}, 13.6 \mathrm{mmol})$ and $\mathrm{Pd}\left(\mathrm{PPh}_{3}\right)_{4}(393 \mathrm{mg}, 0.34 \mathrm{mmol})$ in $\mathrm{THF}$ (45 mL) and $\mathrm{H}_{2} \mathrm{O}(15 \mathrm{~mL})$. The mixture was heated at reflux for $16 \mathrm{~h}$ and then cooled to ambient temperature, diluted with EA, washed with brine, dried over $\mathrm{Na}_{2} \mathrm{SO}_{4}$. The solvent was removed under reduced pressure and the residue was purified by column chromatography (ethyl acetate/petroleum ether, $\mathrm{v} / \mathrm{v}=1 / 20)$ to give $\mathbf{1 4}(2.4 \mathrm{~g}, 90 \%$ yield $)$ as a white solid.

\subsubsection{Synthesis of the compound $\mathbf{1 5}$}

To a solution of compound $14(500 \mathrm{mg}, 0.64 \mathrm{mmol})$ in $10 \mathrm{~mL} \mathrm{CHCl}_{3}$ and $15 \mathrm{~mL} \mathrm{MeOH}$, conc. $\mathrm{HCl}(10.0 \mathrm{~mL})$ was added and the mixture was heated at reflux for $3 \mathrm{~h}$. After cooled to ambient temperature, the mixture was poured into water, extracted by $\mathrm{CH}_{2} \mathrm{Cl}_{2}$ and the combined organic phase was washed with saturated brine, dried over $\mathrm{Na}_{2} \mathrm{SO}_{4}$, concentrated under reduce pressure and the residue was purified by chromatography (ethyl acetate/petroleum ether: $\mathrm{v} / \mathrm{v}=$ 1:10) to give 15 (0.42 $\mathrm{mg}, 94 \%$ yield) as a white solid.

\subsubsection{Synthesis of chiral phosphoric acid Ii}

$\mathrm{POCl}_{3}(280 \mu \mathrm{L}, 3.01 \mathrm{mmol})$ was added dropwise to the solution of compound 15 (300 $\mathrm{mg}$, $0.43 \mathrm{mmol})$ in pyridine $(6 \mathrm{~mL})$ at $0{ }^{\circ} \mathrm{C}$, and the resulted mixture was heated to $70{ }^{\circ} \mathrm{C}$ and stirred for $10 \mathrm{~h}$. After cooling to $0{ }^{\circ} \mathrm{C}, 6 \mathrm{~mL}$ of water was carefully added, and the resulted suspension was stirred at $110^{\circ} \mathrm{C}$ for an additional $4 \mathrm{~h}$. Dichloromethane was added and the pyridine was removed by reverse extraction with $6 \mathrm{~N} \mathrm{HCl}$. The organic layer was dried over $\mathrm{Na}_{2} \mathrm{SO}_{4}$ and concentrated under reduce pressure. The residue was purified by silica gel column chromatography (ethyl acetate/petroleum ether $=1 / 5$ to $1 / 1)$ to give the product $(270 \mathrm{mg}, 83 \%$ yield) as a white solid. mp: $341.2-342.5^{\circ} \mathrm{C}$ (decomposition), $[\alpha]_{\mathrm{D}}^{25}=+487.9^{\circ}\left(c=1.0, \mathrm{CHCl}_{3}\right)$; ${ }^{1} \mathrm{H}$ NMR (600 MHz, $\left.\mathrm{CDCl}_{3}\right) \delta 7.32(\mathrm{~d}, J=7.6 \mathrm{~Hz}, 2 \mathrm{H}), 7.23-7.16(\mathrm{~m}, 8 \mathrm{H}), 7.13(\mathrm{dd}, J=7.9$, $1.9 \mathrm{~Hz}, 2 \mathrm{H}), 6.99$ (dd, $J=14.8,8.0 \mathrm{~Hz}, 6 \mathrm{H}), 3.14$ (ddd, $J=17.0,11.5,6.5 \mathrm{~Hz}, 2 \mathrm{H}), 2.93(\mathrm{dd}, J$ $=15.9,7.8 \mathrm{~Hz}, 2 \mathrm{H}), 2.39-2.31(\mathrm{~m}, 2 \mathrm{H}), 2.17(\mathrm{td}, J=11.6,8.0 \mathrm{~Hz}, 2 \mathrm{H}), 1.99(\mathrm{~s}, 6 \mathrm{H}), 1.32(\mathrm{~s}$, $18 \mathrm{H}) .{ }^{13} \mathrm{C} \mathrm{NMR}\left(151 \mathrm{MHz}, \mathrm{CDCl}_{3}\right) \delta 148.8,144.9,142.5,140.5,139.1,136.2,135.2,134.8$, 131.0, 130.0, 129.4, 128.8, 126.7, 124.5, 122.5, 60.0, 38.5, 34.4, 31.5, 30.5, 20.5. ${ }^{31} \mathrm{P}$ NMR $\left(243 \mathrm{MHz}, \mathrm{CDCl}_{3}\right) \delta$-10.18. HRMS (ESI) $\mathrm{m} / \mathrm{z}$ : $[\mathrm{M}+\mathrm{H}]^{+}$Calcd for $\mathrm{C}_{51} \mathrm{H}_{52} \mathrm{O}_{4} \mathrm{P} 759.3598$; Found 759.3609 .

2.4 The chiral phosphoric acid Ij-Il, IIb, IIIc was prepared reference to the above method (R)-1,10-bis(4'-(tert-butyl)-2,6-dimethyl-[1,1'-biphenyl]-4-yl)-12-hydroxy-4,5,6,7-tetra hydrodiindeno[7,1-de:1',7'-fg][1,3,2] dioxaphosphocine 12-oxide (lj) 


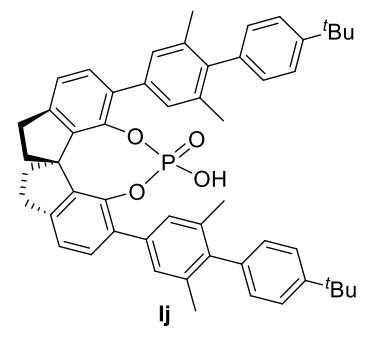

Following the general procedure from Ii, the residue was purified by silica gel column chromatography (ethyl acetate/petroleum ether $=1 / 1$ ) as eluent to afford the product as white solid (41.3 $\mathrm{mg}, 76 \%$ yield). $\mathrm{mp}$ : 242.6-247.1 ${ }^{\circ} \mathrm{C},[\alpha]_{\mathrm{D}}^{25}=-354.1^{\circ}\left(c=1.0, \mathrm{CHCl}_{3}\right) ;{ }^{1} \mathrm{H} \mathrm{NMR}(600 \mathrm{MHz}$, $\left.\mathrm{CDCl}_{3}\right) \delta 7.38(\mathrm{dt}, J=7.8,1.7 \mathrm{~Hz}, 2 \mathrm{H}), 7.29-7.26(\mathrm{~m}, 4 \mathrm{H}), 7.19(\mathrm{~d}, J=$ $3.3 \mathrm{~Hz}, 4 \mathrm{H}), 7.15$ (d, $J=7.7 \mathrm{~Hz}, 2 \mathrm{H}), 7.01-6.96$ (m, 4H), 3.09 (ddd, $J=16.8,11.1,6.5 \mathrm{~Hz}, 2 \mathrm{H}$ ), $2.79(\mathrm{dd}, J=15.9,7.8 \mathrm{~Hz}, 2 \mathrm{H}), 2.30(\mathrm{dd}, J=12.1,6.4 \mathrm{~Hz}, 2 \mathrm{H}), 2.16(\mathrm{td}, J=11.6,8.2 \mathrm{~Hz}, 2 \mathrm{H})$, $1.74(\mathrm{~s}, 12 \mathrm{H}), 1.30(\mathrm{~s}, 18 \mathrm{H}) .{ }^{13} \mathrm{C} \mathrm{NMR}\left(151 \mathrm{MHz}, \mathrm{CDCl}_{3}\right) \delta 148.8,145.0,142.6,142.5$, $140.6,140.4,137.9,136.2$, 136.1 , 134.8 , 130.2 , 128.8 , 128.3 , $124.8,122.5$, 67.1 , 60.1 , 38.5 , 34.4 , 31.4 , 30.7 , 30.2 , 20.7 . ${ }^{31} \mathrm{P}$ NMR (243 MHz, $\left.\mathrm{CDCl}_{3}\right) \delta-12.19$. HRMS (ESI) m/z: $[\mathrm{M}+\mathrm{H}]^{+}$Calcd for $\mathrm{C}_{53} \mathrm{H}_{54} \mathrm{O}_{4} \mathrm{P} 785.3765$; Found 785.3749.

(R)-1,10-bis(4'-(tert-butyl)-2-ethyl-[1,1'-biphenyl]-4-yl)-12-hydroxy-4,5,6,7-tetrahydrodi indeno[7,1-de:1',7'-fg][1,3,2] dioxaphosphocine 12-oxide (lk)

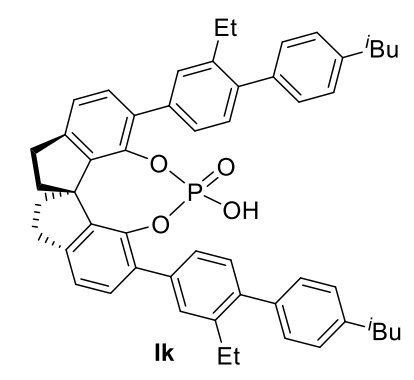

Following the general procedure from Ii, the residue was purified by silica gel column chromatography (ethyl acetate/petroleum ether $=1 / 1$ ) as eluent to afford the product as white solid (44.0 $\mathrm{mg}, 81 \%$ yield). mp: $306.1-308.5{ }^{\circ} \mathrm{C}$ (decomposition), $[\alpha]_{\mathrm{D}}^{25}=-394.5^{\circ}(c=1.0$, $\left.\mathrm{CHCl}_{3}\right) ;{ }^{1} \mathrm{H} \mathrm{NMR}\left(600 \mathrm{MHz}, \mathrm{CDCl}_{3}\right) \delta 7.31(\mathrm{~d}, J=7.6 \mathrm{~Hz}, 2 \mathrm{H}), 7.25$ $7.20(\mathrm{~m}, 6 \mathrm{H}), 7.19-7.13(\mathrm{~m}, 4 \mathrm{H}), 7.07$ (d, $J=8.2 \mathrm{~Hz}, 4 \mathrm{H}), 6.93$ (d, $J$ $=7.9 \mathrm{~Hz}, 2 \mathrm{H}), 3.12(\mathrm{ddd}, J=16.9,11.2,6.4 \mathrm{~Hz}, 2 \mathrm{H}), 2.88(\mathrm{dd}, J=15.9,7.8 \mathrm{~Hz}, 2 \mathrm{H}), 2.39-2.27$ (m, 6H), 2.19-2.11 (m, 2H), $1.31(\mathrm{~s}, 18 \mathrm{H}), 0.85(\mathrm{t}, J=7.5 \mathrm{~Hz}, 6 \mathrm{H}) .{ }^{13} \mathrm{C} \mathrm{NMR}\left(151 \mathrm{MHz}, \mathrm{CDCl}_{3}\right)$ $\delta 148.9,144.9$, $142.5,142.4,141.4,140.6,140.6,140.1,139.0,136.5,134.8,130.2$, $130.1,129.7,129.5,128.9,126.5,124.5,122.4,122.4,60.0$, 38.6 , 34.4 , 31.4 , 30.4 , 26.0, $19.1,15.6 .{ }^{31} \mathrm{P}$ NMR $\left(243 \mathrm{MHz}, \mathrm{CDCl}_{3}\right) \delta-10.15$. HRMS (ESI) m/z: $[\mathrm{M}+\mathrm{H}]^{+} \mathrm{Calcd}$ for $\mathrm{C}_{53} \mathrm{H}_{54} \mathrm{O}_{4} \mathrm{P} 785.3765$; Found 785.3755.

\section{(R)-1,10-bis(4'-(tert-butyl)-2-isopropyl-[1,1'-biphenyl]-4-yl)-12-hydroxy-4,5,6,7-tetra} hydrodiindeno[7,1-de:1',7'-fg][1,3,2] dioxaphosphocine 12-oxide (II)

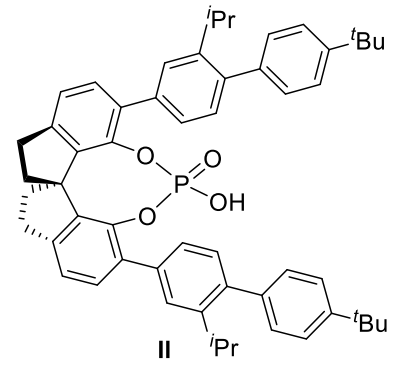

Following the general procedure from Ii, the residue was purified by silica gel column chromatography (ethyl acetate/petroleum ether $=$ $1 / 1$ ) as eluent to afford the product as white solid (39.5 $\mathrm{mg}, 73 \%$ yield). mp: $213.3-220.8^{\circ} \mathrm{C},[\alpha]_{\mathrm{D}}^{25}=-395.4^{\circ}\left(c=1.0, \mathrm{CHCl}_{3}\right) ;{ }^{1} \mathrm{H} \mathrm{NMR}(600$ $\left.\mathrm{MHz} \mathrm{CDCl}_{3}\right) \delta 7.33(\mathrm{~d}, J=4.8 \mathrm{~Hz}, 2 \mathrm{H}), 7.30(\mathrm{~d}, J=7.3 \mathrm{~Hz}, 2 \mathrm{H})$, $7.24(\mathrm{~d}, J=7.9 \mathrm{~Hz}, 4 \mathrm{H}), 7.16(\mathrm{~d}, J=7.0 \mathrm{~Hz}, 4 \mathrm{H}), 7.06(\mathrm{~d}, J=7.3 \mathrm{~Hz}$, 
4H), $6.83(\mathrm{~d}, J=7.4 \mathrm{~Hz}, 2 \mathrm{H}), 3.16-3.04(\mathrm{~m}, 2 \mathrm{H}), 2.88$ (ddt, $J=33.8,13.8,6.2 \mathrm{~Hz}, 4 \mathrm{H}), 2.27$ $(\mathrm{dd}, J=12.7,6.3 \mathrm{~Hz}, 2 \mathrm{H}), 2.12(\mathrm{q}, J=10.1,9.0 \mathrm{~Hz}, 2 \mathrm{H}), 1.31(\mathrm{~d}, J=4.0 \mathrm{~Hz}, 18 \mathrm{H}), 1.05(\mathrm{~d}, J$ $=6.6 \mathrm{~Hz}, 6 \mathrm{H}), 0.88(\mathrm{~d}, J=6.9 \mathrm{~Hz}, 6 \mathrm{H}) .{ }^{13} \mathrm{C} \mathrm{NMR}\left(151 \mathrm{MHz}, \mathrm{CDCl}_{3}\right) \delta 148.9,146.1,144.9$, $139.5,139.0,136.8,134.8,130.3,129.9,129.1,126.5,124.5,122.4,76.9$, 60.1 , 38.6 , $34.4,31.5,30.3,29.3,24.3,24.2 .{ }^{31} \mathrm{P}$ NMR $\left(243 \mathrm{MHz}, \mathrm{CDCl}_{3}\right) \delta-10.67$. HRMS (ESI) m/z: $[\mathrm{M}+\mathrm{H}]^{+}$Calcd for $\mathrm{C}_{55} \mathrm{H}_{58} \mathrm{O}_{4} \mathrm{P}$ 813.4078; Found 813.4078.

(4R)-2,6-bis(4'-(tert-butyl)-2-ethyl-[1,1'-biphenyl]-4-yl)-4-hydroxydinaphtho[2,1-d:1',2'f][1,3,2]dioxaphosphepine 4-oxide (llb)

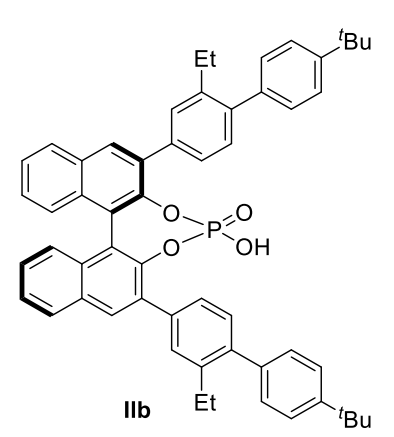

Following the general procedure from Ii, the residue was purified by silica gel column chromatography $\left(\mathrm{CH}_{3} \mathrm{OH} / \mathrm{CH}_{2} \mathrm{Cl}_{2}=1 / 20\right)$ as eluent to afford the product as white solid (46.0 $\mathrm{mg}, 85 \%$ yield). mp: 212.9 $217.3{ }^{\circ} \mathrm{C},[\alpha]_{\mathrm{D}}^{25}=-249.3^{\circ}\left(c=1.0, \mathrm{CHCl}_{3}\right) ;{ }^{1} \mathrm{H} \mathrm{NMR}\left(600 \mathrm{MHz}, \mathrm{CDCl}_{3}\right)$ $\delta 8.06(\mathrm{~d}, J=1.9 \mathrm{~Hz}, 2 \mathrm{H}), 7.98(\mathrm{~d}, J=8.2 \mathrm{~Hz}, 2 \mathrm{H}), 7.52-7.47(\mathrm{~m}, 4 \mathrm{H})$, $7.37(\mathrm{dt}, J=8.0,2.0 \mathrm{~Hz}, 2 \mathrm{H}), 7.32-7.25(\mathrm{~m}, 4 \mathrm{H}), 7.14-7.10(\mathrm{~m}, 4 \mathrm{H})$, $7.07(\mathrm{td}, J=8.3,7.8,2.1 \mathrm{~Hz}, 6 \mathrm{H}), 2.48-2.42(\mathrm{~m}, 4 \mathrm{H}), 1.24(\mathrm{~d}, J=1.8$ $\mathrm{Hz}, 18 \mathrm{H}), 0.91(\mathrm{td}, J=7.5,1.7 \mathrm{~Hz}, 6 \mathrm{H}) .{ }^{13} \mathrm{C} \mathrm{NMR}\left(151 \mathrm{MHz}, \mathrm{CDCl}_{3}\right) \delta 149.2,141.6,140.9$, 138.6 , 135.7 , 134.3 , 131.9 , 131.6 , 131.3 , 130.0 , 129.9 , 128.9 , 128.4 , 127.2 , 127.0 , 126.4 , $125.9,124.6,34.4,31.4,26.0,15.5 .{ }^{31} \mathrm{P} \mathrm{NMR}\left(243 \mathrm{MHz}, \mathrm{CDCl}_{3}\right) \delta 2.89 . \mathrm{HRMS}(\mathrm{ESI}) \mathrm{m} / \mathrm{z}$ : $[\mathrm{M}+\mathrm{H}]^{+}$Calcd for $\mathrm{C}_{56} \mathrm{H}_{52} \mathrm{O}_{4} \mathrm{P}$ 819.3609; Found 819.3601.

(4R)-2,6-bis(4'-(tert-butyl)-2-ethyl-[1,1'-biphenyl]-4-yl)-4-hydroxy-8,9,10,11,12,13,14,15octahydrodinaphtho[2,1-d:1',2'-f][1,3,2]dioxaphosphepine 4-oxide (Illc)

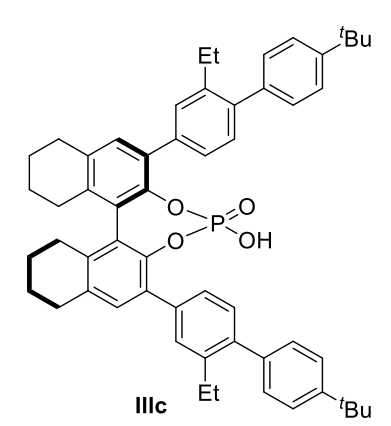

Following the general procedure from Ii, the residue was purified by silica gel column chromatography $\left(\mathrm{CH}_{3} \mathrm{OH} / \mathrm{CH}_{2} \mathrm{Cl}_{2}=1 / 20\right)$ as eluent to afford the product as yellow solid (43.7 $\mathrm{mg}, 81 \%$ yield). mp: $216.2-$ $220.0^{\circ} \mathrm{C},[\alpha]_{\mathrm{D}}^{25}=-237.9^{\circ}\left(c=1.0, \mathrm{CHCl}_{3}\right) ;{ }^{1} \mathrm{H} \mathrm{NMR}\left(600 \mathrm{MHz}, \mathrm{CDCl}_{3}\right)$ $\delta 7.39(\mathrm{~d}, J=2.0 \mathrm{~Hz}, 2 \mathrm{H}), 7.32-7.28(\mathrm{~m}, 2 \mathrm{H}), 7.27-7.24(\mathrm{~m}, 4 \mathrm{H}), 7.20$ (s, 2H), 7.15-7.11 (m, 4H), 7.05-7.00 (m, 2H), 2.87 (tq, $J=16.3,8.3$, $6.6 \mathrm{~Hz}, 4 \mathrm{H}), 2.67$ (ddd, $J=16.9,8.1,4.1 \mathrm{~Hz}, 2 \mathrm{H}), 2.51-2.42(\mathrm{~m}, 4 \mathrm{H})$, $2.29(\mathrm{dt}, J=16.8,5.4 \mathrm{~Hz}, 2 \mathrm{H}), 1.81$ (tq, $J=12.4,6.0 \mathrm{~Hz}, 6 \mathrm{H}), 1.59$ (ddt, $J=13.0,8.1,5.3 \mathrm{~Hz}$, $2 \mathrm{H}), 1.31(\mathrm{t}, J=1.1 \mathrm{~Hz}, 18 \mathrm{H}), 0.93(\mathrm{t}, J=7.5 \mathrm{~Hz}, 6 \mathrm{H}) .{ }^{13} \mathrm{C} \mathrm{NMR}\left(151 \mathrm{MHz}, \mathrm{CDCl}_{3}\right) \delta 149.1$, $141.4,140.3$, 138.8 , 137.2 , 135.9 , 135.1 , 131.1 , 129.8 , 129.6 , 129.0 , 127.1 , 126.6 , 124.6, $34.4,31.4,29.3,27.8,26.0,22.7,22.7,15.5 .{ }^{31} \mathrm{P} \mathrm{NMR}\left(243 \mathrm{MHz}, \mathrm{CDCl}_{3}\right) \delta 0.32 . \mathrm{HRMS}$ (ESI) m/z: $[\mathrm{M}+\mathrm{H}]^{+}$Calcd for $\mathrm{C}_{56} \mathrm{H}_{60} \mathrm{O}_{4} \mathrm{P}$ 827.4235; Found 827.4218. 


\section{General procedure for the preparation of 2-alkynylbenzaldehyde derivatives $^{4}$}

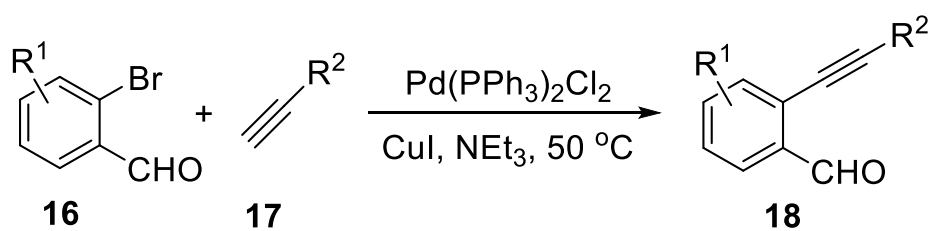

Under Ar atmosphere, the appropriate acetylene 17 (1.2 equiv) was added to the solution of the corresponding 2-bromobenzaldehyde 16 (1.0 equiv), $\mathrm{PdCl}_{2}\left(\mathrm{PPh}_{3}\right)_{2}(2 \mathrm{~mol} \%)$, and $\mathrm{CuI}(1$ mol \%) in $\operatorname{NEt}_{3}(0.25 \mathrm{M})$. The resulted mixture was heated at $50{ }^{\circ} \mathrm{C}$ for $12 \mathrm{~h}$ under $\mathrm{Ar}$ atmosphere. After the reaction was completed, quenched with distilled water and extracted with $\mathrm{CH}_{2} \mathrm{Cl}_{2}$ (three times). Combined organic layer and washed with brine, dried over $\mathrm{Na}_{2} \mathrm{SO}_{4}$, concentrated under reduce pressure. The residue was purified by column chromatography on silica gel (ethyl acetate/petroleum ether $=1 / 10$ ) as eluent to afford the desired product $18 .{ }^{4}$

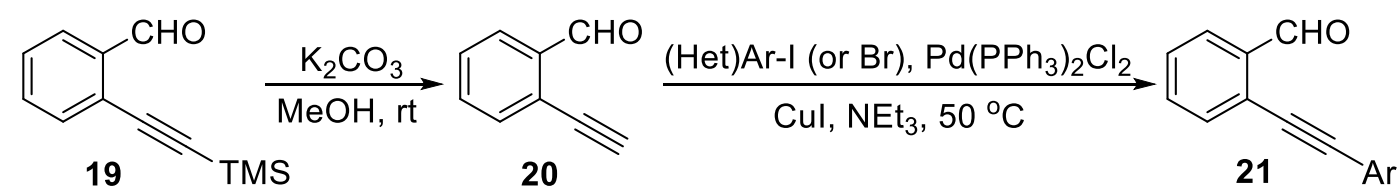

Compound 19 (202.3 mg, $1.0 \mathrm{mmol})$ was dissolved in $\mathrm{MeOH}(14 \mathrm{~mL}, 0.07 \mathrm{M})$ and treated with $\mathrm{K}_{2} \mathrm{CO}_{3}$ (276.4 mg, 2 equiv), stirred at $\mathrm{rt}$ for $1 \mathrm{~h}$, the reaction mixture was diluted with water and extracted with $\mathrm{CH}_{2} \mathrm{Cl}_{2}$ (three times). Combined organic layer and dried over $\mathrm{Na}_{2} \mathrm{SO}_{4}$, concentrated under reduce pressure and the residue was purified by column chromatography on silica gel (ethyl acetate/petroleum ether $=1 / 10)$ as eluent to afford the desired product $\mathbf{2 0}(108.0$ $\mathrm{mg}, 83 \%)$ as white solid.

Aryl halide (3.6 mmol, 1.2 equiv) was added to the solution of compound $\mathbf{2 0}(390 \mathrm{mg}, 3.0$ mmol), $\mathrm{PdCl}_{2}\left(\mathrm{PPh}_{3}\right)_{2}(42.0 \mathrm{mg}, 0.06 \mathrm{mmol}, 2 \mathrm{~mol} \%$ ) and $\mathrm{CuI}$ (5.7 mg, $0.03 \mathrm{mmol}, 1 \mathrm{~mol} \%$ ) in $\mathrm{NEt}_{3}(12 \mathrm{ml}, 0.25 \mathrm{M})$, stirred at $50{ }^{\circ} \mathrm{C}$ for $8 \mathrm{~h}$ under $\mathrm{Ar}$ atmosphere, the reaction mixture was quenched with distilled water and extracted with $\mathrm{CH}_{2} \mathrm{Cl}_{2}$ (three times). Combined organic layer and washed with brine, dried over $\mathrm{Na}_{2} \mathrm{SO}_{4}$, concentrated under reduce pressure and the residue was purified by column chromatography on silica gel (ethyl acetate/petroleum ether $=1 / 10$ ) as eluent to afford the desired product $\mathbf{2 1} .^{4}$

\section{Optimization of the reaction conditions}

Table S1: Screened phosphoric acids derived from 1,10-binaphthol (BINOL)- and octahydrodinaphthol (H8-BINOL) in the model reaction ${ }^{a}$ 


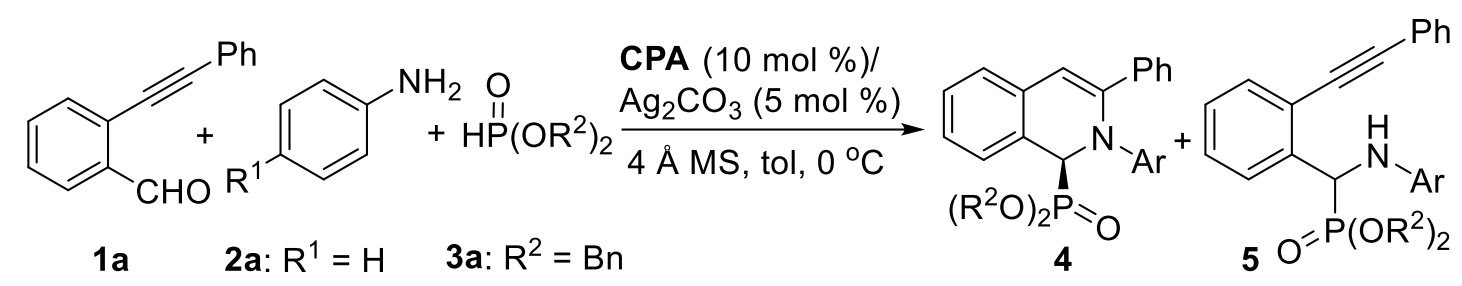<smiles></smiles>

Ila: $\mathrm{Ar}=2,4,6-\left({ }^{i} \mathrm{Pr}\right)_{3} \mathrm{C}_{6} \mathrm{H}_{2}$

Illb: $\mathrm{Ar}=3-\mathrm{Et}-4-\left(4-{ }^{\mathrm{t}} \mathrm{BuC}_{6} \mathrm{H}_{4}\right)-\mathrm{C}_{6} \mathrm{H}_{3}$

Ilc: $\mathrm{Ar}=2,6-\left({ }^{i} \mathrm{Pr}\right)_{2}-4-\left(4-{ }^{\mathrm{t}} \mathrm{BuC}_{6} \mathrm{H}_{4}\right)-\mathrm{C}_{6} \mathrm{H}_{2}$<smiles>O=P(O)(O)Oc1c(Br)cc2c(c1-c1c(Br)cc3c(c1Br)CCCC3)CCCC2</smiles>

IIIa: $\mathrm{Ar}=4-\mathrm{ClC}_{6} \mathrm{H}_{4}$

IIIb: $\mathrm{Ar}=$ 2-naphthyl

IIIc: $\mathrm{Ar}=3-\mathrm{Et}-4-\left(4-{ }^{\mathrm{t}} \mathrm{BuC}_{6} \mathrm{H}_{4}\right)-\mathrm{C}_{6} \mathrm{H}_{3}$

\begin{tabular}{cccccc}
\hline entry & CPA & $\mathrm{R}^{2}$ & yield of $\mathbf{4}(\%)^{b}$ & $e e(\%)^{c}$ & yield of $\mathbf{5}(\%)^{b}$ \\
\hline 1 & IIa & Bn & 4aaa, 62 & 43 & - \\
2 & IIb & Bn & 4aaa, 53 & 18 & - \\
3 & IIc & Bn & 4aaa, 45 & 26 & - \\
4 & IIIa & Bn & 4aaa, 35 & 10 & trace \\
5 & IIIb & Bn & 4aaa, 33 & 0 & trace \\
6 & IIIc & Bn & 4aaa, 50 & 24 & trace \\
\hline
\end{tabular}

${ }^{a}$ Unless otherwise specified, the reaction was performed with 1a $(0.12 \mathrm{mmol}), 2 \mathrm{a}(0.11 \mathrm{mmol}), 3 \mathbf{a}(0.1 \mathrm{mmol}), 4 \AA \mathrm{MS}(100$ $\mathrm{mg}$ ), $\mathrm{CPA}(10 \mathrm{~mol} \%)$, and $\mathrm{Ag}_{2} \mathrm{CO}_{3}(5 \mathrm{~mol} \%)$ in $1.0 \mathrm{~mL}$ of toluene at $0{ }^{\circ} \mathrm{C}$ for 3 days under argon. ${ }^{b}$ Isolated yield. ${ }^{c}$ Determined by chiral HPLC.

Table S2: Optimization reaction temperature ${ }^{a}$<smiles>O=Cc1ccccc1C#CP</smiles>

1a
$\mathrm{Ph}$

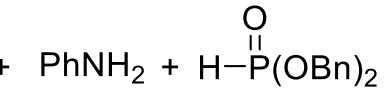

2a

3a

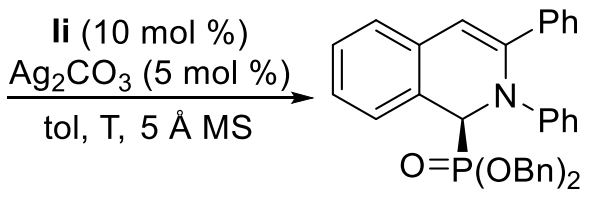

4aaa

\begin{tabular}{ccccc}
\hline entry & $\mathrm{T}\left({ }^{\circ} \mathrm{C}\right)$ & time $(\mathrm{h})$ & yield $(\%)^{b}$ & $e e(\%)^{c}$ \\
\hline 1 & 0 & 24 & 96 & 62 \\
2 & -5 & 48 & 92 & 66 \\
$\mathbf{3}$ & $\mathbf{- 1 0}$ & $\mathbf{7 2}$ & $\mathbf{9 0}$ & $\mathbf{7 0}$ \\
4 & -20 & 96 & 40 & 74 \\
\hline
\end{tabular}


${ }^{a}$ Unless otherwise specified, the reaction was carried out with 1a $(0.12 \mathrm{mmol}), \mathbf{2 a}(0.11 \mathrm{mmol}), \mathbf{3 a}(0.1 \mathrm{mmol}), 5 \AA \mathrm{MS}(100$ $\mathrm{mg}$ ), Ii (10 mol \%), and $\mathrm{Ag}_{2} \mathrm{CO}_{3}(5 \mathrm{~mol} \%)$ in $1.0 \mathrm{~mL}$ of toluene under argon. ${ }^{b}$ Isolated yield. ${ }^{c}$ Determined by chiral HPLC.

Table S3: The effect of molecular sieve on the reaction ${ }^{a}$

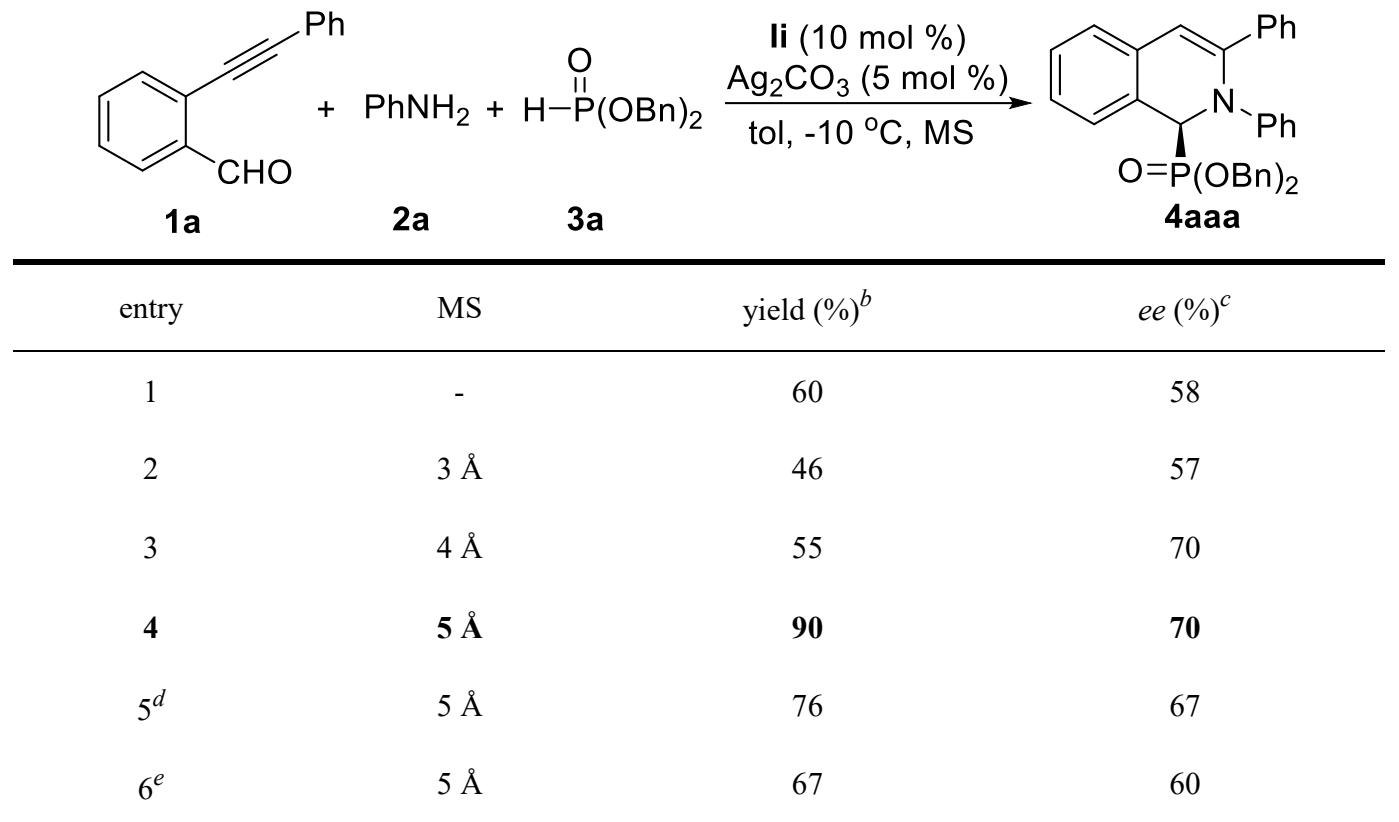

${ }^{a}$ Unless otherwise specified, the reaction was carried out with 1a $(0.12 \mathrm{mmol}), \mathbf{2 a}(0.11 \mathrm{mmol}), \mathbf{3 a}(0.1 \mathrm{mmol}), \quad \mathrm{MS}(100 \mathrm{mg})$, Ii (10 mol \%), and $\mathrm{Ag}_{2} \mathrm{CO}_{3}(5 \mathrm{~mol} \%)$ in the $1.0 \mathrm{~mL}$ of toluene at $-10{ }^{\circ} \mathrm{C}$ for $3 \mathrm{~d}$ under argon. ${ }^{b}$ Isolated yield. ${ }^{c}$ Determined by chiral HPLC. ${ }^{d} 5 \AA \mathrm{MS}(50 \mathrm{mg}) .{ }^{e} 5 \AA \mathrm{MS}(20 \mathrm{mg})$.

Table S4: The effect of counter ion on the reaction ${ }^{a}$

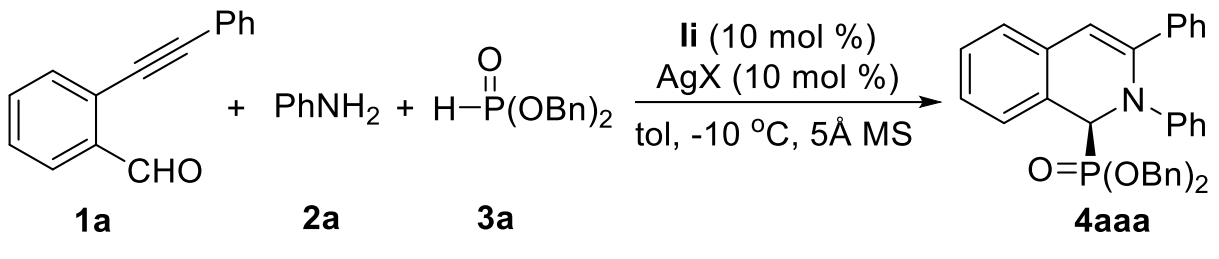

\begin{tabular}{cccc}
\hline entry & $\mathrm{X}$ & yield $(\%)^{b}$ & $e e(\%)^{c}$ \\
\hline 1 & $\mathrm{OAc}^{-}$ & 81 & 57 \\
2 & $\mathrm{CF}_{3} \mathrm{CO}_{2}^{-}$ & 79 & 59 \\
3 & $\mathrm{OTf}$ & 81 & 67 \\
4 & $\mathrm{BF}_{4}^{-}$ & 40 & 34 \\
5 & $\mathrm{ClO}_{4}^{-}$ & 65 & 64 \\
$\mathbf{6}$ & $\mathbf{1 / 2 \mathbf { C O } ^ { - }}$ & $\mathbf{9 0}$ & $\mathbf{7 0}$ \\
\hline
\end{tabular}

${ }^{a}$ Unless otherwise specified, the reaction was carried out with 1a $(0.12 \mathrm{mmol}), \mathbf{2 a}(0.11 \mathrm{mmol}), 3 \mathbf{3}(0.1 \mathrm{mmol}), 5 \AA \mathrm{MS}$ (100 $\mathrm{mg}$ ), Ii (10 mol \%), and $\mathrm{AgX}(10 \mathrm{~mol} \%)$ in the $1.0 \mathrm{~mL}$ of toluene at $-10{ }^{\circ} \mathrm{C}$ for $3 \mathrm{~d}$ under argon. ${ }^{b}$ Isolated yield. ${ }^{c}$ Determined by chiral HPLC. 
Table S5: The effect of the ratio of chiral phosphoric acid Ii to $\mathrm{Ag}_{2} \mathrm{CO}_{3}$ on the reaction ${ }^{a}$

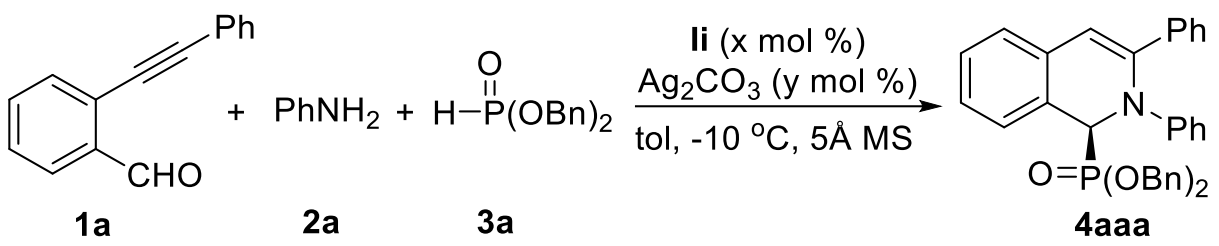

\begin{tabular}{ccccc}
\hline entry & $\mathrm{x}$ & $\mathrm{y}$ & yield $(\%)^{b}$ & $e e(\%)^{c}$ \\
\hline 1 & 5 & 5 & 83 & 52 \\
2 & 10 & 5 & 90 & 70 \\
3 & 11 & 5 & 90 & 70 \\
4 & 20 & 5 & 91 & 76 \\
5 & 5 & 2.5 & 75 & 57 \\
6 & 20 & 10 & 90 & 75 \\
\hline
\end{tabular}

${ }^{a}$ Unless otherwise specified, the reaction was carried out with 1a $(0.12 \mathrm{mmol}), \mathbf{2 a}(0.11 \mathrm{mmol}), \mathbf{3 a}(0.1 \mathrm{mmol}), 5 \AA \mathrm{MS}(100$ $\mathrm{mg}$ ), $\mathbf{I i}\left(\mathrm{x} \mathrm{mol} \mathrm{\% )}\right.$, and $\mathrm{Ag}_{2} \mathrm{CO}_{3}\left(\mathrm{y} \mathrm{mol} \mathrm{\% )}\right.$ in the $1.0 \mathrm{~mL}$ of toluene at $-10{ }^{\circ} \mathrm{C}$ for $3 \mathrm{~d}$ under argon. ${ }^{b}$ Isolated yield. ${ }^{c}$ Determined by chiral HPLC.

Table S6: The effect of solvent on the reaction ${ }^{a}$

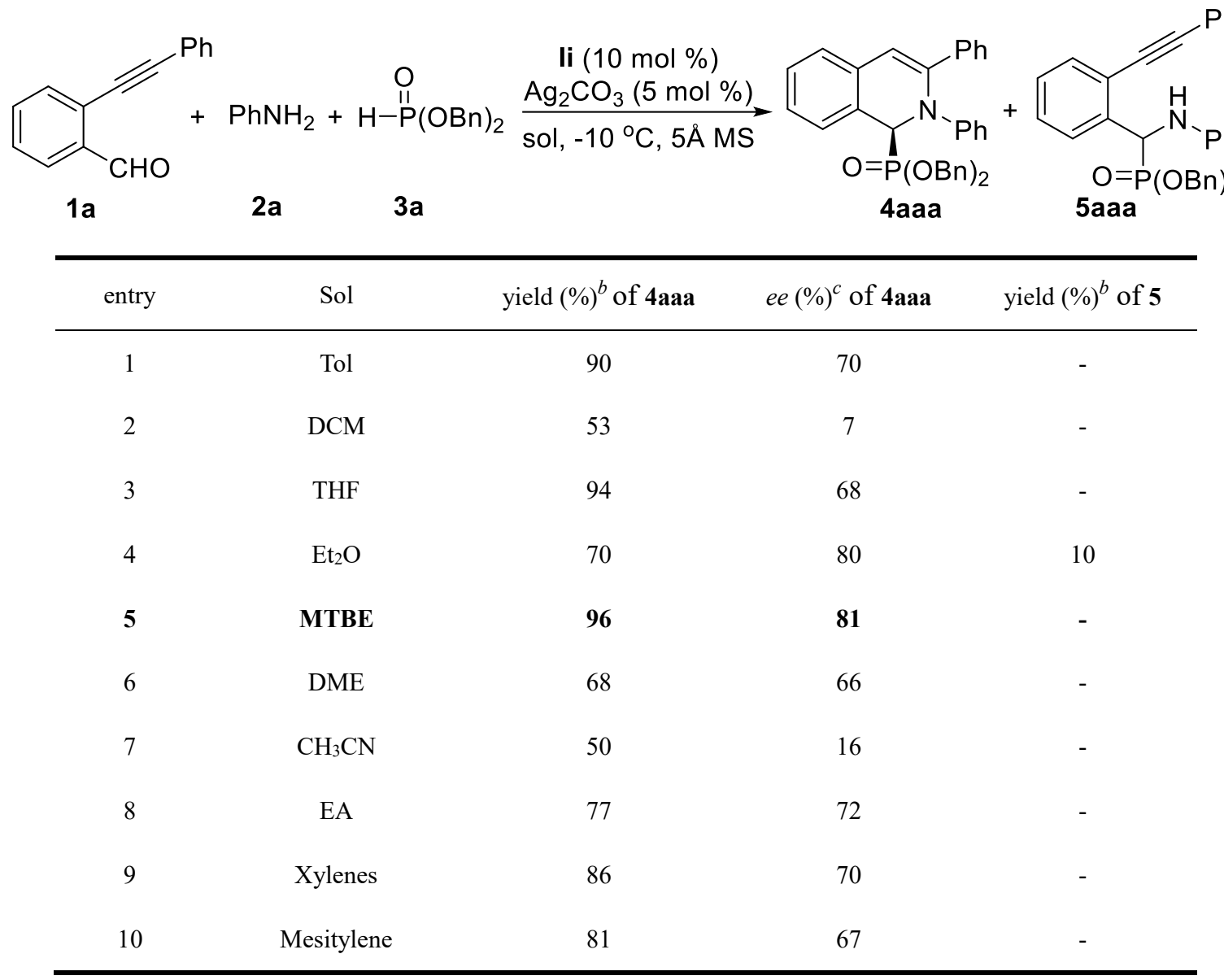




$11 \quad \mathrm{PhCl} \quad 78 \quad 50$

${ }^{a}$ Unless otherwise specified, the reaction was carried out with 1a $(0.12 \mathrm{mmol}), \mathbf{2 a}(0.11 \mathrm{mmol}), \mathbf{3 a}(0.1 \mathrm{mmol}), 5 \AA \mathrm{MS}(100$ $\mathrm{mg})$, Ii (10 mol \%), and $\mathrm{Ag}_{2} \mathrm{CO}_{3}(5 \mathrm{~mol} \%)$ in the $1.0 \mathrm{~mL}$ of solvent at $-10{ }^{\circ} \mathrm{C}$ for $3 \mathrm{~d}$ under argon. ${ }^{b}$ Isolated yield. ${ }^{c}$ Determined by chiral HPLC.

\section{General procedure for the catalytic asymmetric three-component reaction}<smiles>O=Cc1cc[R]c(C#CF)c1</smiles>

1<smiles>CC(=O)OP=C(C)C</smiles>

2

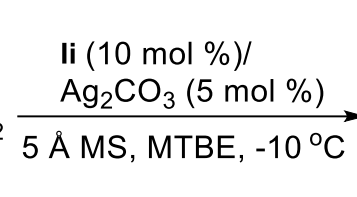<smiles>[R]C1=Cc2c([R])cccc2C([PH](C)=O)N1c1ccccc1</smiles>

4

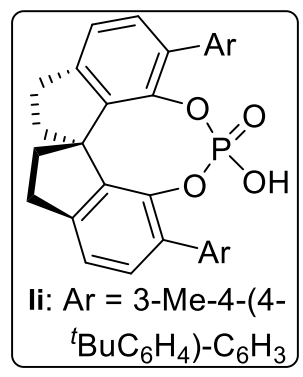

$\left.{ }^{t} \mathrm{BuC}_{6} \mathrm{H}_{4}\right)-\mathrm{C}_{6} \mathrm{H}_{3}$

To a reaction tube, $5 \AA$ MS (100 mg) powder was added and dried with flame for $10 \mathrm{~min}$ under vacuum, then charged with Ar atmosphere and cooled down to rt. Chiral phosphoric acid Ii (0.01 mmol, 0.1 equiv.), $\mathrm{Ag}_{2} \mathrm{CO}_{3}(0.005 \mathrm{mmol}, 0.05$ equiv.) and anhydrous MTBE $(1.0 \mathrm{~mL})$ were added subsequently. The resulted solution was stirred for $2.0 \mathrm{~h}$ at $30^{\circ} \mathrm{C}$ in dark and then the corresponding 2-alkynylbenzaldehyde derivatives 1 ( $0.12 \mathrm{mmol}, 1.2$ equiv. $)$ and amine 2 ( $0.11 \mathrm{mmol}, 1.1$ equiv.) were added, the mixture reacted at $30{ }^{\circ} \mathrm{C}$ for $0.5 \mathrm{~h}$. Then the reaction mixture was cooled down to $-10^{\circ} \mathrm{C}$, the methyl phosphonate $\mathbf{3 b}(0.1 \mathrm{mmol}, 1.0$ equiv. $)$ was added and the reaction mixture was stirred at $-10{ }^{\circ} \mathrm{C}$ for $4 \mathrm{~d}$, the solution was directly purified by column chromatography on silica gel $(\mathrm{PE} / \mathrm{EA}=2 / 1$ to $1 / 1)$ to give the corresponding $1,2-$ dihydroisoquinolin -1-ylphosphonate 4.

\section{Characterization of products}

\section{Dibenzyl (S)-(2, 3-diphenyl-1, 2-dihydroisoquinolin-1-yl)phosphonate (4aaa)}<smiles>CC(C)(C)CO[PH](=O)C1c2ccccc2C=C(c2ccccc2)N1c1ccccc1</smiles>

4aaa

Following the general procedure, reaction were performed with 1a $(0.12$ mmol), 2a (0.11 mmol), 3a (0.1 mmol), $5 \AA$ MS (100 mg), Ii (10 mol \%), and $\mathrm{Ag}_{2} \mathrm{CO}_{3}(5 \mathrm{~mol} \%)$ in the $1.0 \mathrm{~mL}$ of MTBE at $-10{ }^{\circ} \mathrm{C}$ for 3 days under argon, followed by column chromatography $(\mathrm{PE} / \mathrm{EA}=3: 1)$ to afford 4aaa as yellow oil, $52.1 \mathrm{mg}(96 \%$ yield, $81 \% e e) ;[\alpha]_{\mathrm{D}}^{25}=+341.9^{\circ}(c=1.0$, EA); HPLC condition: Chiralpak IC, $254 \mathrm{~nm}, 0.5 \mathrm{~mL} / \mathrm{min}$, hexane $/{ }^{i} \mathrm{PrOH}=90 / 10, t_{\text {major }}=25.814 \mathrm{~min}, t_{\text {minor }}=23.861 \mathrm{~min} ;{ }^{1} \mathrm{H}$ NMR $\left(600 \mathrm{MHz}, \mathrm{CDCl}_{3}\right) \delta 7.53(\mathrm{dd}, J=7.4,2.2 \mathrm{~Hz}, 2 \mathrm{H}), 7.25(\mathrm{ddd}, J=12.7,6.3,3.8 \mathrm{~Hz}, 7 \mathrm{H})$, 7.19 (ddd, $J=15.8,6.9,2.9 \mathrm{~Hz}, 7 \mathrm{H}), 7.13(\mathrm{t}, J=7.4 \mathrm{~Hz}, 1 \mathrm{H}), 7.10-7.02(\mathrm{~m}, 5 \mathrm{H}), 6.83$ (tt, $J=$ 6.0, $2.8 \mathrm{~Hz}, 1 \mathrm{H}), 6.43(\mathrm{~s}, 1 \mathrm{H}), 5.51(\mathrm{~d}, J=18.5 \mathrm{~Hz}, 1 \mathrm{H}), 4.95(\mathrm{dd}, J=11.7,7.1 \mathrm{~Hz}, 1 \mathrm{H}), 4.92-$ $4.81(\mathrm{~m}, 3 \mathrm{H}) .{ }^{13} \mathrm{C} \mathrm{NMR}\left(151 \mathrm{MHz}, \mathrm{CDCl}_{3}\right) \delta 147.6,142.1,137.2,128.5,128.4,128.4,128.3$, 
$128.2,128.1,128.0,127.9,127.7,127.3,127.3,126.7,126.7,125.4,124.5,124.5,122.7,122.7$, 122.4, 112.5, 68.1, 68.1, 68.0, 68.0, 65.0, 64.0. ${ }^{31} \mathrm{P}$ NMR (243 MHz, $\left.\mathrm{CDCl}_{3}\right) \delta 21.57 . \mathrm{HRMS}$ (ESI) $\mathrm{m} / \mathrm{z}:[\mathrm{M}+\mathrm{H}]^{+}$Calcd for $\mathrm{C}_{35} \mathrm{H}_{31} \mathrm{NO}_{3} \mathrm{P}$ 544.2036; Found 544.2036.

\section{Dimethyl (S)-(2,3-diphenyl-1, 2-dihydroisoquinolin-1-yl)phosphonate (4aab)}<smiles>COP(=O)(O)C1c2ccccc2C=C(c2ccccc2)N1c1ccccc1</smiles>

$4 \mathrm{aab}$

Following the general procedure, reaction were performed with $1 \mathbf{a}(0.12$

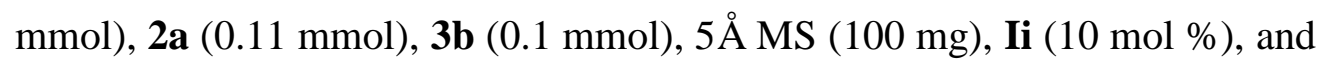
$\mathrm{Ag}_{2} \mathrm{CO}_{3}(5 \mathrm{~mol} \%)$ in the $1.0 \mathrm{~mL}$ of MTBE at $-10{ }^{\circ} \mathrm{C}$ for 3 days under argon, followed by column chromatography $(\mathrm{PE} / \mathrm{EA}=1: 1)$ to afford 4aab as white solid, $34.0 \mathrm{mg}$ (87\% yield, 91\% ee ); mp: $145.0-147.2^{\circ} \mathrm{C},[\alpha]_{\mathrm{D}}^{25}=+256.3^{\circ}(c=1.0$, EA); HPLC condition: Chiralpak IC, $254 \mathrm{~nm}, 0.5 \mathrm{~mL} / \mathrm{min}$, hexane $/{ }^{i} \mathrm{PrOH}=80 / 20, t_{\text {major }}=22.698 \mathrm{~min}, t_{\text {minor }}$ $=21.621 \mathrm{~min} ;{ }^{1} \mathrm{H}$ NMR $\left(600 \mathrm{MHz}, \mathrm{CDCl}_{3}\right) \delta$ 7.59-7.56 (m, 2H), 7.27-7.15 (m, 7H), 7.13-7.05 $(\mathrm{m}, 5 \mathrm{H}), 6.86(\mathrm{td}, J=7.0,1.7 \mathrm{~Hz}, 1 \mathrm{H}), 6.53(\mathrm{~s}, 1 \mathrm{H}), 5.48(\mathrm{~d}, J=18.6 \mathrm{~Hz}, 1 \mathrm{H}), 3.68(\mathrm{~d}, J=10.3$ $\mathrm{Hz}, 3 \mathrm{H}), 3.61(\mathrm{~d}, J=10.4 \mathrm{~Hz}, 3 \mathrm{H}) .{ }^{13} \mathrm{C} \mathrm{NMR}\left(151 \mathrm{MHz}, \mathrm{CDCl}_{3}\right) \delta 147.6,142.2,137.2,128.6$, 128.4, 128.4, 128.3, 128.0, 127.7, 127.2, 127.2, 126.7, 126.7, 125.4, 124.4, 124.4, 122.8, 122.8, 122.5, 112.0, 64.6, 63.5, 53.3, 53.3, 53.2. ${ }^{31} \mathrm{P}$ NMR (243 MHz, $\left.\mathrm{CDCl}_{3}\right) \delta$ 23.13. HRMS (ESI) $\mathrm{m} / \mathrm{z}:[\mathrm{M}+\mathrm{H}]^{+}$Calcd for $\mathrm{C}_{23} \mathrm{H}_{23} \mathrm{NO}_{3} \mathrm{P}$ 392.1410; Found 392.1417. IR $\left(\mathrm{KBr}, \mathrm{cm}^{-1}\right)$ 3397, 3051, 2948, 2848, 1595, 1493, 1452, 1382, 1142, 1055, 1024, 823, 765, 697, 524.

\section{X-ray Crystallography of 4aab}

The product of $\mathbf{4 a a b}$ was dissolved in $\mathrm{EA} / \mathrm{PE}=3: 1$ solution $(4 \mathrm{~mL})$, and colorless crystals were isolated when this solution was kept at room temperature for two days.

Single-crystal X-ray diffraction measurement was carried out on an Agilent SuperNova EosS2 diffractometer using graphite monochromated $\mathrm{Cu} \mathrm{K \alpha}$ radiation $(\lambda=1.54184 \AA)$. The crystal was kept at 194 (30) K during data collection. The structure was solved by the ShelXT ${ }^{5}$ structure solution program in Olex $2^{6}$ and refined using Full-matrix Least Squares based on $F^{2}$ with program SHELXL-2014 5 within Olex2. Crystal data and experimental data for 4aab are summarized in Table S7. 


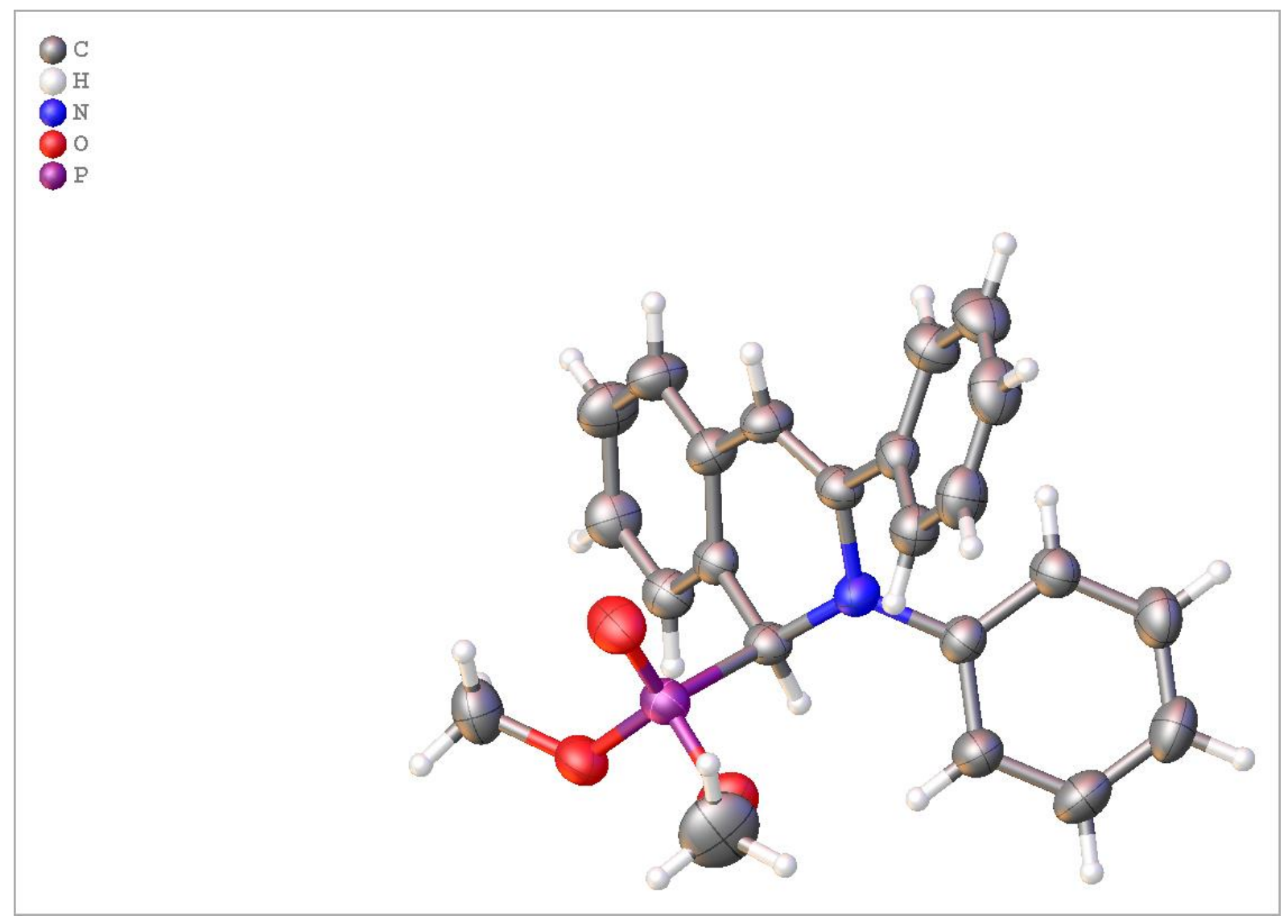

Figure S1. Molecular structure of 4aab (thermal ellipsoids drawn at the $35 \%$ probability level).

\begin{tabular}{ll}
\hline \multicolumn{2}{l}{ Table S7 Crystal data and structure refinement for 4aab. } \\
\hline Empirical formula & $\mathrm{C}_{23} \mathrm{H}_{22} \mathrm{NO}_{3} \mathrm{P}$ \\
Formula weight & 391.38 \\
Temperature/K & $194(30)$ \\
Crystal system & orthorhombic \\
Space group & $\mathrm{P} 2{ }_{1} 2_{1} 2_{1}$ \\
a/A & $8.63587(7)$ \\
$\mathrm{b} / \AA$ & $11.13974(8)$ \\
c/A & $20.37028(18)$ \\
$\alpha /{ }^{\circ}$ & 90 \\
$\beta /{ }^{\circ}$ & 90 \\
$\gamma /{ }^{\circ}$ & 90 \\
Volume $/ \AA^{3}$ & $1959.65(3)$ \\
$\mathrm{Z}$ & 4 \\
$\rho_{\text {calcg/cm }}{ }^{3}$ & 1.327 \\
$\mu / \mathrm{mm}^{-1}$ & 1.438 \\
$\mathrm{~F}(000)$ & 824.0 \\
$\mathrm{Crystal} \mathrm{size} / \mathrm{mm}^{3}$ & $0.2 \times 0.15 \times 0.15$ \\
\hline
\end{tabular}




\begin{tabular}{ll}
\hline Radiation & $\mathrm{CuK \alpha}(\lambda=1.54184)$ \\
$2 \Theta$ range for data collection ${ }^{\circ}$ & 8.682 to 143.724 \\
Index ranges & $-10 \leq \mathrm{h} \leq 9,-13 \leq \mathrm{k} \leq 11,-24 \leq 1 \leq 24$ \\
Reflections collected & 22091 \\
Independent reflections & $3822\left[\mathrm{R}_{\text {int }}=0.0300, \mathrm{R}_{\text {sigma }}=0.0164\right]$ \\
Data/restraints/parameters & $3822 / 0 / 255$ \\
Goodness-of-fit on $\mathrm{F}^{2}$ & 1.046 \\
Final R indexes [I $>=2 \sigma(\mathrm{I})]$ & $\mathrm{R}_{1}=0.0273, \mathrm{wR}_{2}=0.0755$ \\
Final R indexes [all data] & $\mathrm{R}_{1}=0.0280, \mathrm{wR}_{2}=0.0762$ \\
Largest diff. peak/hole / e $\AA^{-3}$ & $0.12 /-0.21$ \\
Flack parameter & $0.003(7)$ \\
\hline
\end{tabular}

Diethyl (S)-(2,3-diphenyl-1, 2-dihydroisoquinolin-1-yl)phosphonate (4aac)

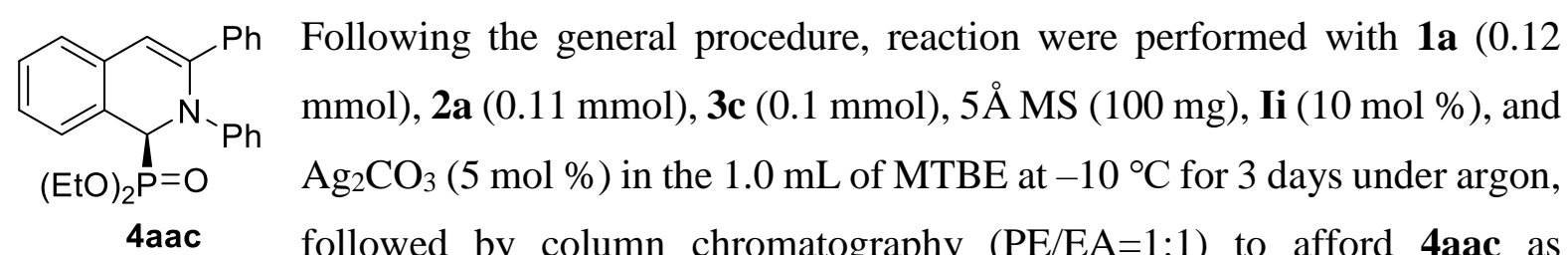
colorless oil, $24.8 \mathrm{mg}(59 \%$ yield, $90 \% e e) ;[\alpha]_{\mathrm{D}}^{25}=+940.4^{\circ}(c=1.0$, EA); HPLC condition: Chiralpak IC, $254 \mathrm{~nm}, 0.5 \mathrm{~mL} / \mathrm{min}$, hexane $/{ }^{i} \mathrm{PrOH}=80 / 20, t_{\text {major }}=15.356 \mathrm{~min}, t_{\text {minor }}=14.505$ min; ${ }^{1} \mathrm{H}$ NMR (600 MHz, $\left.\mathrm{CDCl}_{3}\right) \delta$ 7.62-7.53 (m, 2H), 7.28-7.05 (m, 11H), $6.85(\mathrm{td}, J=6.2$, $3.3 \mathrm{~Hz}, 1 \mathrm{H}), 6.50$ (s, 1H), 5.45 (d, $J=18.7 \mathrm{~Hz}, 1 \mathrm{H}), 4.13-4.06(\mathrm{~m}, 1 \mathrm{H}), 4.05-3.99(\mathrm{~m}, 1 \mathrm{H})$, 3.98-3.90 (m, 2H), $1.21(\mathrm{dt}, J=16.8,7.1 \mathrm{~Hz}, 6 \mathrm{H}) .{ }^{13} \mathrm{C} \mathrm{NMR}\left(151 \mathrm{MHz}, \mathrm{CDCl}_{3}\right) \delta 142.1,137.4$, $128.5,128.3,128.3,128.0,127.7,127.3,127.2$, 126.6, 126.6, 124.3, 124.3, 122.7, 122.7, 122.3, $112.3,76.9,64.7,63.7,62.8,62.7,62.6,62.5,16.5,16.5,16.5,16.4 .{ }^{31} \mathrm{P} \mathrm{NMR}\left(243 \mathrm{MHz}, \mathrm{CDCl}_{3}\right)$ $\delta$ 20.71. HRMS (ESI) m/z: [M+H] ${ }^{+}$Calcd for $\mathrm{C}_{25} \mathrm{H}_{27} \mathrm{NO}_{3} \mathrm{P} 420.1723$; Found 420.1717 .

\section{Diisopropyl (S)-(2,3-diphenyl-1, 2-dihydroisoquinolin-1-yl)phosphonate (4aad)}<smiles>O=P(O)(OC(F)(F)F)C1c2ccccc2C=C(c2ccccc2)N1c1ccccc1</smiles>

oil, $7.2 \mathrm{mg}(16 \%$ yield, $71 \% e e) ;[\alpha]_{\mathrm{D}}^{25}=+666.7^{\circ}(c=1.0$, EA); HPLC condition: Chiralpak IC, $254 \mathrm{~nm}, 0.5 \mathrm{~mL} / \mathrm{min}$, hexane $/{ }^{i} \mathrm{PrOH}=80 / 20, t_{\text {major }}=11.067 \mathrm{~min}, t_{\text {minor }}=11.889 \mathrm{~min} ;{ }^{1} \mathrm{H} \mathrm{NMR}$ $\left(600 \mathrm{MHz}, \mathrm{CDCl}_{3}\right) \delta 7.59(\mathrm{~d}, J=7.6 \mathrm{~Hz}, 2 \mathrm{H}), 7.27-7.05(\mathrm{~m}, 11 \mathrm{H}), 6.84(\mathrm{q}, J=4.4 \mathrm{~Hz}, 1 \mathrm{H})$, 6.49 (s, 1H), 5.39 (d, $J=18.9 \mathrm{~Hz}, 1 \mathrm{H}), 4.63$ (h, $J=6.3 \mathrm{~Hz}, 1 \mathrm{H}), 4.54$ (h, $J=6.3 \mathrm{~Hz}, 1 \mathrm{H}), 1.27$ $(\mathrm{dd}, J=23.4,6.2 \mathrm{~Hz}, 7 \mathrm{H}), 1.17(\mathrm{dd}, J=9.0,6.2 \mathrm{~Hz}, 6 \mathrm{H}) .{ }^{13} \mathrm{C} \mathrm{NMR}\left(151 \mathrm{MHz}, \mathrm{CDCl}_{3}\right) \delta 141.9$, 
137.6, 128.4, 128.2, 128.1, 128.1, 127.8, 127.6, 127.4, 127.4, 126.4, 126.4, 124.3, 124.3, 122.5, $122.5,122.1,112.8,71.5,71.5,71.3,71.2,65.1,64.0,24.4,24.4,24.3,24.3,23.9,23.9,23.8$, 23.8. ${ }^{31} \mathrm{P}$ NMR $\left(243 \mathrm{MHz}, \mathrm{CDCl}_{3}\right) \delta 18.97$. HRMS (ESI) m/z: $[\mathrm{M}+\mathrm{H}]^{+}$Calcd for $\mathrm{C}_{27} \mathrm{H}_{31} \mathrm{NO}_{3} \mathrm{P}$ 448.2036; Found 448.2035.

\section{Dimethyl (S)-(2-(4-methoxyphenyl)-3-phenyl-1, 2-dihydroisoquinolin-1-yl)phosphonate (4abb)}

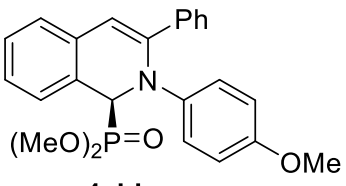

$4 \mathrm{abb}$

Following the general procedure, reaction were performed with $\mathbf{1 a}(0.12$ mmol), $2 \mathbf{b}$ (0.11 mmol), 3b (0.1 mmol), $5 \AA$ MS (100 mg), Ii (10 mol \%), and $\mathrm{Ag}_{2} \mathrm{CO}_{3}\left(5 \mathrm{~mol} \%\right.$ ) in the $1.0 \mathrm{~mL}$ of MTBE at $-10{ }^{\circ} \mathrm{C}$ for 3 days under argon, followed by column chromatography(PE/EA=1:1) to afford 4abb as yellow oil, $38.8 \mathrm{mg}(92 \%$ yield, $92 \% e e) ;[\alpha]_{\mathrm{D}}^{25}=+571.0^{\circ}(c=1.0, \mathrm{EA})$; HPLC condition: Chiralpak IC, $254 \mathrm{~nm}, 0.5 \mathrm{~mL} / \mathrm{min}$, hexane $/{ }^{i} \mathrm{PrOH}=80 / 20, t_{\text {major }}=26.412 \mathrm{~min}, t_{\text {minor }}$ $=30.860 \mathrm{~min} ;{ }^{1} \mathrm{H}$ NMR $\left(600 \mathrm{MHz}, \mathrm{CDCl}_{3}\right) \delta 7.62-7.52(\mathrm{~m}, 2 \mathrm{H}), 7.24(\mathrm{dd}, J=13.1,6.2 \mathrm{~Hz}$, $3 \mathrm{H}), 7.21-7.13(\mathrm{~m}, 3 \mathrm{H}), 7.09$ (d, $J=7.6 \mathrm{~Hz}, 1 \mathrm{H}), 7.06-7.00(\mathrm{~m}, 2 \mathrm{H}), 6.69-6.58(\mathrm{~m}, 2 \mathrm{H}), 6.47$ (s, 1H), $5.36(\mathrm{~d}, J=18.8 \mathrm{~Hz}, 1 \mathrm{H}), 3.71-3.58(\mathrm{~m}, 9 \mathrm{H}) .{ }^{13} \mathrm{C} \mathrm{NMR}\left(151 \mathrm{MHz}, \mathrm{CDCl}_{3}\right) \delta 155.5$, 142. 7, 141.4, 137.3, 133.2, 128.4, 128.3, 128.3, 128.0, 127.8, 127.3, 127.2, 126.6, 126.6, 124.6, 124.6, 124.2, 124.2, 113.9, 110.8, 65.3, 64.2, 55.3, 53.4, 53.3, 53.2, 53.2. ${ }^{31} \mathrm{P}$ NMR (243 MHz, $\left.\mathrm{CDCl}_{3}\right) \delta$ 23.10. HRMS (ESI) m/z: [M+H] $]^{+}$Calcd for $\mathrm{C}_{24} \mathrm{H}_{25} \mathrm{NO}_{4} \mathrm{P} 422.1516$; Found 422.1509 . IR $\left(\mathrm{KBr}, \mathrm{cm}^{-1}\right)$ 3435, 3046, 2950, 2834, 1606, 1510, 1451, 1385, 1255, 1048, 1021, 837, 765, $695,525$.

\section{Dimethyl (S)-(2-(4-isopropoxyphenyl)-3-phenyl-1, 2-dihydroisoquinolin-1-yl) phosphonate (4acb)}

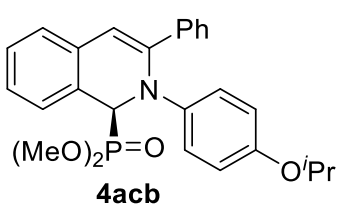

Following the general procedure, reaction were performed with 1a (0.12 mmol), 2c (0.11 mmol), $3 \mathbf{b}(0.1 \mathrm{mmol}), 5 \AA \mathrm{MS}(100 \mathrm{mg})$, Ii (10 mol \%), and $\mathrm{Ag}_{2} \mathrm{CO}_{3}(5 \mathrm{~mol} \%)$ in the $1.0 \mathrm{~mL}$ of $\mathrm{MTBE}$ at $-10{ }^{\circ} \mathrm{C}$ for 3 days under argon, followed by column chromatography(PE/EA=1:1) to afford 4acb as yellow solid, $40.9 \mathrm{mg}$ (91\% yield, 94\% ee); $\mathrm{mp}: 137.1-138.5{ }^{\circ} \mathrm{C},[\alpha]_{\mathrm{D}}^{25}=$ $+773.0^{\circ}(c=1.0, \mathrm{EA})$; HPLC condition: Chiralpak IC, $254 \mathrm{~nm}, 0.5 \mathrm{~mL} / \mathrm{min}$, hexane/ ${ }^{i} \mathrm{PrOH}=$ $80 / 20, t_{\text {major }}=20.480 \mathrm{~min}, t_{\text {minor }}=22.374 \mathrm{~min} ;{ }^{1} \mathrm{H}$ NMR $\left(600 \mathrm{MHz}, \mathrm{CDCl}_{3}\right) \delta 7.63-7.52(\mathrm{~m}$, 2H), 7.28-7.21 (m, 3H), 7.17 (ddd, $J=21.4,11.1,6.1 \mathrm{~Hz}, 3 \mathrm{H}), 7.09$ (d, $J=7.6 \mathrm{~Hz}, 1 \mathrm{H}), 7.04$ $6.97(\mathrm{~m}, 2 \mathrm{H}), 6.67-6.55(\mathrm{~m}, 2 \mathrm{H}), 6.46(\mathrm{~s}, 1 \mathrm{H}), 5.36(\mathrm{~d}, J=18.9 \mathrm{~Hz}, 1 \mathrm{H}), 4.34$ (hept, $J=6.0 \mathrm{~Hz}$, $1 \mathrm{H}), 3.68(\mathrm{~d}, J=10.3 \mathrm{~Hz}, 3 \mathrm{H}), 3.61(\mathrm{~d}, J=10.4 \mathrm{~Hz}, 3 \mathrm{H}), 1.22(\mathrm{dd}, J=6.1,2.3 \mathrm{~Hz}, 6 \mathrm{H}) .{ }^{13} \mathrm{C}$ $\operatorname{NMR}\left(151 \mathrm{MHz}, \mathrm{CDCl}_{3}\right) \delta 153.8,142.7,137.4,128.3,128.3,128.2,128.0,127.8,127.3,127.2$, 
$126.5,126.5,124.8,124.6,124.6,124.2,124.1,116.0,110.8,70.1,64.7,53.3,53.3,53.2,53.1$, 22.1, 22.0. ${ }^{31} \mathrm{P}$ NMR (243 MHz, $\left.\mathrm{CDCl}_{3}\right) \delta$ 23.12. HRMS (ESI) $\mathrm{m} / \mathrm{z}:[\mathrm{M}+\mathrm{H}]^{+}$Calcd for $\mathrm{C}_{26} \mathrm{H}_{29} \mathrm{NO}_{4} \mathrm{P}$ 450.1829; Found 450.1824. IR $\left(\mathrm{KBr}, \mathrm{cm}^{-1}\right)$ 3055, 2975, 2952, 2850, 1609, 1562 , $1505,1452,1382,1238,1181,1055,1030,951,831,766,697,550,527$.

\section{Dimethyl (S)-(3-(4-fluorophenyl)-2-(4-isopropoxyphenyl)-1, 2-dihydroisoquinolin-1-yl) phosphonate (4bcb)}

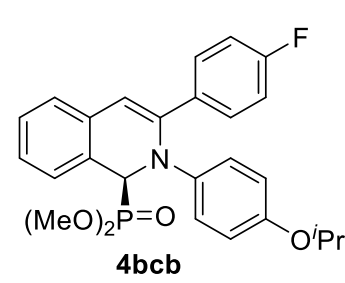

Following the general procedure, reaction were performed with $\mathbf{1 b}(0.12$ mmol), 2c (0.11 mmol), 3b (0.1 mmol), $5 \AA$ MS (100 mg), Ii (10 mol \%), and $\mathrm{Ag}_{2} \mathrm{CO}_{3}(5 \mathrm{~mol} \%)$ in the $1.0 \mathrm{~mL}$ of MTBE at $-10{ }^{\circ} \mathrm{C}$ for 3 days under argon, followed by column chromatography $(\mathrm{PE} / \mathrm{EA}=1: 1)$ to afford 4bcb as yellow oil, $45.8 \mathrm{mg}(98 \%$ yield, $94 \% e e)$; $[\alpha]_{\mathrm{D}}^{25}=+647.9^{\circ}$ ( $c=1.0$, EA); HPLC condition: Chiralpak IC, $254 \mathrm{~nm}, 0.5 \mathrm{~mL} / \mathrm{min}$, hexane $/{ }^{i} \mathrm{PrOH}=80 / 20$, $t_{\text {major }}=42.739 \mathrm{~min}, t_{\text {minor }}=41.094 \mathrm{~min} ;{ }^{1} \mathrm{H} \mathrm{NMR}\left(600 \mathrm{MHz}, \mathrm{CDCl}_{3}\right) \delta 7.54(\mathrm{dd}, J=8.5,5.3 \mathrm{~Hz}$, 2H), 7.29-7.22 (m, 1H), 7.17 (dd, $J=19.3,7.6 \mathrm{~Hz}, 2 \mathrm{H}), 7.08$ (d, $J=7.6 \mathrm{~Hz}, 1 \mathrm{H}), 6.99$ (d, $J=$ $8.4 \mathrm{~Hz}, 2 \mathrm{H}), 6.92(\mathrm{t}, J=8.5 \mathrm{~Hz}, 2 \mathrm{H}), 6.63(\mathrm{~d}, J=8.7 \mathrm{~Hz}, 2 \mathrm{H}), 6.41(\mathrm{~s}, 1 \mathrm{H}), 5.34(\mathrm{~d}, J=18.9$ Hz, 1H), 4.36 (p, $J=6.1 \mathrm{~Hz}, 1 \mathrm{H}), 3.65$ (dd, $J=19.3,10.4 \mathrm{~Hz}, 6 \mathrm{H}), 1.23$ (dd, $J=6.1,2.8 \mathrm{~Hz}$, $6 \mathrm{H}) .{ }^{13} \mathrm{C} \mathrm{NMR}\left(151 \mathrm{MHz}, \mathrm{CDCl}_{3}\right) \delta 162.5,153.9,141.7,140.9,133.4,133.1,129.5,128.4$, 127.2, 126.6, 124.7, 124.6, 124.2, 116.1, 115.3, 110.6, 70.1, 64.7, 53.3, 53.1, 22.0. ${ }^{31} \mathrm{P}$ NMR $\left(243 \mathrm{MHz}, \mathrm{CDCl}_{3}\right) \delta$ 23.20. HRMS (ESI) $\mathrm{m} / \mathrm{z}$ : $[\mathrm{M}+\mathrm{H}]^{+}$Calcd for $\mathrm{C}_{26} \mathrm{H}_{28} \mathrm{FNO}_{4} \mathrm{P} 468.1734$; Found 468.1744 .

\section{Dimethyl (S)-(3-(4-chlorophenyl)-2-(4-isopropoxyphenyl)-1, 2-dihydroisoquinolin-1-yl) phosphonate (4ccb)}

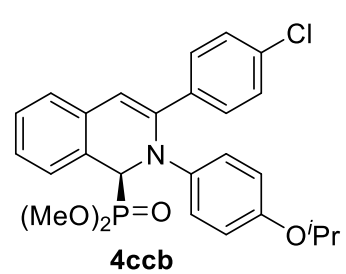

Following the general procedure, reaction were performed with 1c $(0.12$ mmol), 2c (0.11 mmol), 3b (0.1 mmol), $5 \AA$ MS (100 mg), Ii (10 mol \%), and $\mathrm{Ag}_{2} \mathrm{CO}_{3}(5 \mathrm{~mol} \%)$ in the $1.0 \mathrm{~mL}$ of MTBE at $-10{ }^{\circ} \mathrm{C}$ for 3 days under argon, followed by column chromatography(PE/EA=1:1) to afford 4 ccb as yellow oil, $41.6 \mathrm{mg}(86 \%$ yield, $92 \% e e) ;[\alpha]_{\mathrm{D}}^{25}=+679.4^{\circ}(c=$ 1.0, EA); HPLC condition: Chiralpak IC, $254 \mathrm{~nm}, 0.5 \mathrm{~mL} / \mathrm{min}$, hexane $/{ }^{i} \mathrm{PrOH}=80 / 20, t_{\text {major }}=$ $18.000 \mathrm{~min}, t_{\text {minor }}=19.774 \mathrm{~min} ;{ }^{1} \mathrm{H} \mathrm{NMR}\left(600 \mathrm{MHz}, \mathrm{CDCl}_{3}\right) \delta 7.50(\mathrm{~d}, J=8.4 \mathrm{~Hz}, 2 \mathrm{H}), 7.28-$ $7.23(\mathrm{~m}, 1 \mathrm{H}), 7.19(\mathrm{dd}, J=8.6,6.7 \mathrm{~Hz}, 3 \mathrm{H}), 7.16(\mathrm{~d}, J=7.4 \mathrm{~Hz}, 1 \mathrm{H}), 7.08(\mathrm{~d}, J=7.6 \mathrm{~Hz}, 1 \mathrm{H})$, 7.03-6.92 (m, 2H), 6.68-6.57 (m, 2H), $6.45(\mathrm{~s}, 1 \mathrm{H}), 5.34$ (d, $J=18.9 \mathrm{~Hz}, 1 \mathrm{H}), 4.36$ (hept, $J=$ $6.1 \mathrm{~Hz}, 1 \mathrm{H}), 3.64(\mathrm{dd}, J=18.9,10.5 \mathrm{~Hz}, 6 \mathrm{H}), 1.23(\mathrm{dd}, J=6.1,3.4 \mathrm{~Hz}, 6 \mathrm{H}) .{ }^{13} \mathrm{C}$ NMR $(151$ $\left.\mathrm{MHz}, \mathrm{CDCl}_{3}\right) \delta 154.0,141.5,140.8,135.9,133.7,129.0,128.5,128.4,127.2,126.8,124.6$, 
124.3, 116.1, 111.2, 70.1, 64.6, 53.2, 22.0. ${ }^{31} \mathrm{P} \mathrm{NMR}\left(243 \mathrm{MHz}, \mathrm{CDCl}_{3}\right) \delta 23.09$. HRMS (ESI) m/z: $[\mathrm{M}+\mathrm{H}]^{+}$Calcd for $\mathrm{C}_{26} \mathrm{H}_{28} \mathrm{ClNO}_{4} \mathrm{P}$ 484.1439; Found 484.1440.

\section{Dimethyl (S)-(3-(4-bromophenyl)-2-(4-isopropoxyphenyl)-1, 2-dihydroisoquinolin-1-yl) phosphonate (4dcb)}

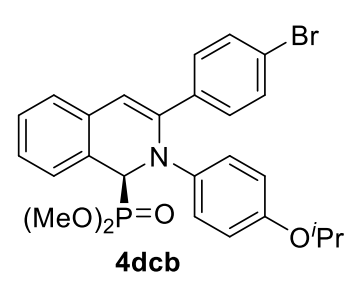

Following the general procedure, reaction were performed with $\mathbf{1 d}(0.12$ mmol), $2 \mathbf{c}(0.11 \mathrm{mmol}), \mathbf{3 b}(0.1 \mathrm{mmol}), 5 \AA \mathrm{MS}(100 \mathrm{mg}), \mathbf{I i}(10 \mathrm{~mol} \%)$, and $\mathrm{Ag}_{2} \mathrm{CO}_{3}(5 \mathrm{~mol} \%)$ in the $1.0 \mathrm{~mL}$ of MTBE at $-10{ }^{\circ} \mathrm{C}$ for 3 days under argon, followed by column chromatography(PE/EA=1:1) to afford $\mathbf{4 d c b}$ as yellow solid, $41.6 \mathrm{mg}$ ( $81 \%$ yield, $91 \% e e)$; $\mathrm{mp}: 51.6-54.0{ }^{\circ} \mathrm{C},[\alpha]_{\mathrm{D}}^{25}=$ $+679.4^{\circ}\left(c=1.0\right.$, EA); HPLC condition: Chiralpak IC, $254 \mathrm{~nm}, 0.5 \mathrm{~mL} / \mathrm{min}$, hexane/ ${ }^{i} \mathrm{PrOH}=$ $80 / 20, t_{\text {major }}=18.504 \mathrm{~min}, t_{\text {minor }}=21.002 \mathrm{~min} ;{ }^{1} \mathrm{H} \mathrm{NMR}\left(600 \mathrm{MHz}, \mathrm{CDCl}_{3}\right) \delta 7.44(\mathrm{~d}, J=8.5$ $\mathrm{Hz}, 2 \mathrm{H}), 7.35$ (d, $J=8.4 \mathrm{~Hz}, 2 \mathrm{H}), 7.28-7.22(\mathrm{~m}, 1 \mathrm{H}), 7.18(\mathrm{dd}, J=15.7,7.6 \mathrm{~Hz}, 2 \mathrm{H}), 7.08$ (d, $J=7.5 \mathrm{~Hz}, 1 \mathrm{H}), 7.02-6.94(\mathrm{~m}, 2 \mathrm{H}), 6.68-6.58(\mathrm{~m}, 2 \mathrm{H}), 6.45(\mathrm{~s}, 1 \mathrm{H}), 5.33(\mathrm{~d}, J=18.9 \mathrm{~Hz}, 1 \mathrm{H})$, 4.36 (hept, $J=6.1 \mathrm{~Hz}, 1 \mathrm{H}), 3.64(\mathrm{dd}, J=19.8,10.5 \mathrm{~Hz}, 6 \mathrm{H}), 1.24(\mathrm{dd}, J=6.1,3.8 \mathrm{~Hz}, 6 \mathrm{H}) .{ }^{13} \mathrm{C}$ NMR $\left(151 \mathrm{MHz}, \mathrm{CDCl}_{3}\right) \delta 154.0,141.6,140.8,136.4,132.9,131.5,129.3,128.4,127.2,126.8$, 124.8, 124.6, 124.3, 122.0, 116.1, 111.2, 70.1, 64.6, 53.2, 22.0. ${ }^{31} \mathrm{P}$ NMR (243 MHz, $\left.\mathrm{CDCl}_{3}\right) \delta$ 23.07. HRMS (ESI) m/z: $[\mathrm{M}+\mathrm{H}]^{+}$Calcd for $\mathrm{C}_{26} \mathrm{H}_{28} \mathrm{BrNO}_{4} \mathrm{P}$ 528.0934; Found 528.0936.

\section{Dimethyl (S)-(2-(4-isopropoxyphenyl)-3-(p-tolyl)-1, 2-dihydroisoquinolin-1-yl)} phosphonate (4ecb)

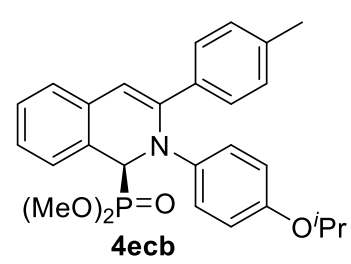

Following the general procedure, reaction were performed with $1 \mathbf{e}(0.12$ mmol), 2c (0.11 mmol), 3b (0.1 mmol), $5 \AA$ MS (100 mg), Ii (10 mol \%), and $\mathrm{Ag}_{2} \mathrm{CO}_{3}(5 \mathrm{~mol} \%)$ in the $1.0 \mathrm{~mL}$ of MTBE at $-10{ }^{\circ} \mathrm{C}$ for 3 days under argon, followed by column chromatography(PE/EA=1:1) to afford 4ecb as yellow oil, $45.8 \mathrm{mg}(99 \%$ yield, $92 \% e e) ;[\alpha]_{\mathrm{D}}^{25}=+728.9^{\circ}$ ( $c=1.0$, EA); HPLC condition: Chiralpak IC, $254 \mathrm{~nm}, 0.5 \mathrm{~mL} / \mathrm{min}$, hexane $/{ }^{i} \mathrm{PrOH}=80 / 20$, $t_{\text {major }}=24.017 \mathrm{~min}, t_{\text {minor }}=22.547 \mathrm{~min} ;{ }^{1} \mathrm{H} \mathrm{NMR}\left(600 \mathrm{MHz}, \mathrm{CDCl}_{3}\right) \delta 7.46(\mathrm{~d}, J=7.9 \mathrm{~Hz}, 2 \mathrm{H})$, $7.23(\mathrm{dt}, J=7.5,1.7 \mathrm{~Hz}, 1 \mathrm{H}), 7.18(\mathrm{~d}, J=7.5 \mathrm{~Hz}, 1 \mathrm{H}), 7.14(\mathrm{t}, J=7.4 \mathrm{~Hz}, 1 \mathrm{H}), 7.08$ (d, $J=7.6$ $\mathrm{Hz}, 1 \mathrm{H}), 7.03$ (d, J=7.9 Hz, 2H), 6.66-6.57 (m, 2H), $6.43(\mathrm{~s}, 1 \mathrm{H}), 5.35$ (d, $J=18.9 \mathrm{~Hz}, 1 \mathrm{H})$, 4.35 (hept, $J=5.9 \mathrm{~Hz}, 1 \mathrm{H}), 3.64(\mathrm{dd}, J=44.7,10.5 \mathrm{~Hz}, 6 \mathrm{H}), 2.27$ (s, 3H), 1.22 (dd, $J=6.0,3.5$ $\mathrm{Hz}, 6 \mathrm{H}) .{ }^{13} \mathrm{C} \mathrm{NMR}\left(151 \mathrm{MHz}, \mathrm{CDCl}_{3}\right) \delta 153.7,142.7,141.3,137.9,134.5,133.3,129.0,128.3$, $127.7,127.2$, 126.4, 124.7, 124.6, 124.0, 116.0, 110.2, 70.1, 64.8, 53.3, 53.2, 22.1, 22.0, 21.2. ${ }^{31} \mathrm{P}$ NMR $\left(243 \mathrm{MHz}, \mathrm{CDCl}_{3}\right) \delta$ 23.18. HRMS (ESI) m/z: $[\mathrm{M}+\mathrm{H}]^{+}$Calcd for $\mathrm{C}_{27} \mathrm{H}_{31} \mathrm{NO}_{4} \mathrm{P}$ 464.1985; Found 464.1990. IR (KBr, $\left.\mathrm{cm}^{-1}\right)$ 3453, 3022, 2975, 2851, 1610, 1562, 1505, 1426, 
$1237,1181,1055,1029,951,831,810,757,664,559,531$.

\section{Dimethyl (S)-(3-(4-ethylphenyl)-2-(4-isopropoxyphenyl)-1, 2-dihydroisoquinolin-1-yl) phosphonate (4fcb)}

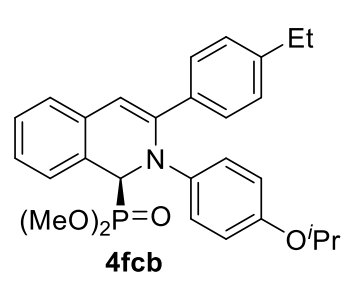

Following the general procedure, reaction were performed with $\mathbf{1 f}(0.12$ mmol), $2 \mathbf{c}(0.11 \mathrm{mmol}), \mathbf{3 b}(0.1 \mathrm{mmol}), 5 \AA \mathrm{MS}$ (100 mg), Ii (10 mol \%), and $\mathrm{Ag}_{2} \mathrm{CO}_{3}(5 \mathrm{~mol} \%)$ in the $1.0 \mathrm{~mL}$ of MTBE at $-10{ }^{\circ} \mathrm{C}$ for 3 days under argon, followed by column chromatography(PE/EA=1:1) to afford 4fcb as yellow oil, $45.8 \mathrm{mg}(94 \%$ yield, $90 \%$ ee $) ;[\alpha]_{\mathrm{D}}^{25}=+353.0^{\circ}(c=$ 1.0, EA); HPLC condition: Chiralpak IF, $254 \mathrm{~nm}, 0.5 \mathrm{~mL} / \mathrm{min}$, hexane $/{ }^{i} \mathrm{PrOH}=60 / 40, t_{\text {major }}=$ $13.810 \mathrm{~min}, t_{\text {minor }}=11.631 \mathrm{~min} ;{ }^{1} \mathrm{H} \mathrm{NMR}\left(600 \mathrm{MHz}, \mathrm{CDCl}_{3}\right) \delta 7.51-7.43(\mathrm{~m}, 2 \mathrm{H}), 7.23(\mathrm{dt}, J$ $=7.5,1.7 \mathrm{~Hz}, 1 \mathrm{H}), 7.20-7.16(\mathrm{~m}, 1 \mathrm{H}), 7.14(\mathrm{t}, J=7.4 \mathrm{~Hz}, 1 \mathrm{H}), 7.10-7.04(\mathrm{~m}, 3 \mathrm{H}), 7.03-6.98$ $(\mathrm{m}, 2 \mathrm{H}), 6.68-6.58(\mathrm{~m}, 2 \mathrm{H}), 6.44(\mathrm{~s}, 1 \mathrm{H}), 5.36(\mathrm{~d}, J=18.9 \mathrm{~Hz}, 1 \mathrm{H}), 4.35$ (hept, $J=6.0 \mathrm{~Hz}, 1 \mathrm{H})$, $3.68(\mathrm{~d}, J=10.5 \mathrm{~Hz}, 3 \mathrm{H}), 3.60(\mathrm{~d}, J=10.5 \mathrm{~Hz}, 3 \mathrm{H}), 2.57$ (q, $J=7.6 \mathrm{~Hz}, 2 \mathrm{H}), 1.22(\mathrm{~d}, J=6.1$ $\mathrm{Hz}, 6 \mathrm{H}), 1.18(\mathrm{t}, J=7.6 \mathrm{~Hz}, 3 \mathrm{H}) .{ }^{13} \mathrm{C} \mathrm{NMR}\left(151 \mathrm{MHz}, \mathrm{CDCl}_{3}\right) \delta 153.7,144.1,142.8,134.7$, $128.3,127.8,127.2$, 126.3, 124.7, 124.6, 124.0, 116.0, 110.2, 70.1, 64.8, 53.2, 28.5, 22.0, 15.2. ${ }^{31} \mathrm{P}$ NMR $\left(243 \mathrm{MHz}, \mathrm{CDCl}_{3}\right) \delta$ 23.18. HRMS (ESI) $\mathrm{m} / \mathrm{z}$ : $[\mathrm{M}+\mathrm{H}]^{+}$Calcd for $\mathrm{C}_{28} \mathrm{H}_{33} \mathrm{NO}_{4} \mathrm{P}$ 478.2142; Found 478.2151. IR (KBr, cm $\left.{ }^{-1}\right)$ 3463, 3021, 2970, 2851, 1736, 1611, 1562, 1505, $1451,1381,1237,1182,1118,1056,1029,951,833,756,555,521$.

\section{Dimethyl (S)-(2-(4-isopropoxyphenyl)-3-(4-methoxyphenyl)-1, 2-dihydroisoquinolin-1- yl) phosphonate (4gcb)}

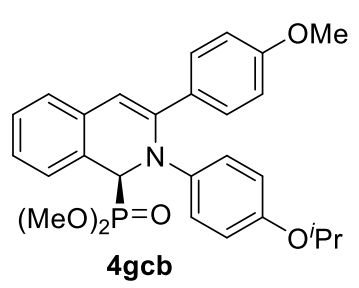

Following the general procedure, reaction were performed with $\mathbf{1 g}(0.12$ mmol), 2c (0.11 mmol), 3b (0.1 mmol), $5 \AA$ MS (100 mg), Ii (10 mol \%), and $\mathrm{Ag}_{2} \mathrm{CO}_{3}(5 \mathrm{~mol} \%)$ in the $1.0 \mathrm{~mL}$ of MTBE at $-10{ }^{\circ} \mathrm{C}$ for 3 days under argon, followed by column chromatography(PE/EA=1:1) to

afford 4gcb as yellow solid, $47.5 \mathrm{mg}$ (99\% yield, 90\% ee); $\mathrm{mp}: 50.1-$ $51.6^{\circ} \mathrm{C},[\alpha]_{\mathrm{D}}^{25}=+767.7^{\circ}(c=1.0, \mathrm{EA})$; HPLC condition: Chiralpak IF, $254 \mathrm{~nm}, 0.5 \mathrm{~mL} / \mathrm{min}$, hexane $/{ }^{i} \mathrm{PrOH}=60 / 40, t_{\text {major }}=25.278 \mathrm{~min}, t_{\text {minor }}=14.156 \mathrm{~min} ;{ }^{1} \mathrm{H} \mathrm{NMR}\left(600 \mathrm{MHz}, \mathrm{CDCl}_{3}\right) \delta$ 7.53-7.46 (m, 2H), 7.27-7.20 (m, 1H), 7.19-7.15 (m, 1H), 7.13 (t, $J=7.4 \mathrm{~Hz}, 1 \mathrm{H}), 7.07$ (d, $J$ $=8.1 \mathrm{~Hz}, 1 \mathrm{H}), 7.03-6.98(\mathrm{~m}, 2 \mathrm{H}), 6.81-6.70(\mathrm{~m}, 2 \mathrm{H}), 6.67-6.56(\mathrm{~m}, 2 \mathrm{H}), 6.38(\mathrm{~s}, 1 \mathrm{H}), 5.35(\mathrm{~d}$, $J=18.9 \mathrm{~Hz}, 1 \mathrm{H}), 4.35$ (hept, $J=6.0 \mathrm{~Hz}, 1 \mathrm{H}), 3.74$ (s, 3H), 3.67 (d, $J=10.5 \mathrm{~Hz}, 3 \mathrm{H}), 3.61$ (d, $J=10.4 \mathrm{~Hz}, 3 \mathrm{H}), 1.23(\mathrm{dd}, J=6.1,3.4 \mathrm{~Hz}, 6 \mathrm{H}) .{ }^{13} \mathrm{C} \mathrm{NMR}\left(151 \mathrm{MHz}, \mathrm{CDCl}_{3}\right) \delta 159.5,153.7$, 142.4, 129.8, 129.1, 128.3, 127.2, 126.2, 124.6, 123.9, 116.0, 113.7., 109.5, 70.1, 64.8, 55.2, 53.2, 22.0. ${ }^{31} \mathrm{P}$ NMR $\left(243 \mathrm{MHz}, \mathrm{CDCl}_{3}\right) \delta$ 23.29. HRMS (ESI) $\mathrm{m} / \mathrm{z}$ : $[\mathrm{M}+\mathrm{H}]^{+}$Calcd for 
$\mathrm{C}_{27} \mathrm{H}_{31} \mathrm{NO}_{5} \mathrm{P}$ 480.1934; Found 480.1941. IR $\left(\mathrm{KBr}, \mathrm{cm}^{-1}\right)$ 3454, 3039, 2974, 2847, 1609, 1507 , $1451,1382,1249,1176,1114,1055,1029,950,834,756,540$.

Dimethyl (S)-(3-(4-cyanophenyl)-2-(4-isopropoxyphenyl)-1, 2-dihydroisoquinolin-1-yl) phosphonate (4hcb)

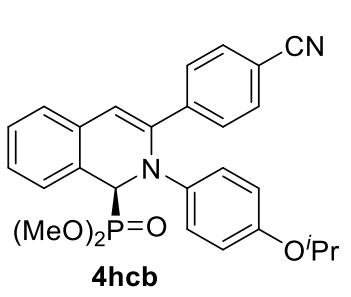

Following the general procedure, reaction were performed with $\mathbf{1 h}(0.12$ mmol), $2 \mathbf{c}(0.11 \mathrm{mmol}), \mathbf{3 b}(0.1 \mathrm{mmol}), 5 \AA \mathrm{MS}$ (100 mg), Ii (10 mol \%), and $\mathrm{Ag}_{2} \mathrm{CO}_{3}(5 \mathrm{~mol} \%)$ in the $1.0 \mathrm{~mL}$ of MTBE at $-10{ }^{\circ} \mathrm{C}$ for 3 days under argon, followed by column chromatography(PE/EA=1:3) to afford 4hcb as yellow oil, $24.6 \mathrm{mg}(52 \%$ yield, $92 \% e e) ;[\alpha]_{\mathrm{D}}^{25}=+512.9^{\circ}(c=$ 1.0, EA); HPLC condition: Chiralpak IC, $254 \mathrm{~nm}, 0.5 \mathrm{~mL} / \mathrm{min}$, hexane $/{ }^{i} \mathrm{PrOH}=50 / 50, t_{\text {major }}=$ $27.622 \mathrm{~min}, t_{\text {minor }}=36.281 \mathrm{~min} ;{ }^{1} \mathrm{H}$ NMR $\left(600 \mathrm{MHz} \mathrm{CDCl}_{3}\right) \delta 7.67(\mathrm{~d}, J=8.0 \mathrm{~Hz}, 2 \mathrm{H}), 7.52$ $(\mathrm{d}, J=8.1 \mathrm{~Hz}, 2 \mathrm{H}), 7.29$ (t, $J=7.5 \mathrm{~Hz}, 1 \mathrm{H}), 7.22(\mathrm{dd}, J=10.3,7.4 \mathrm{~Hz}, 2 \mathrm{H}), 7.10(\mathrm{~d}, J=7.5$ $\mathrm{Hz}, 1 \mathrm{H}), 6.97(\mathrm{~d}, J=8.8 \mathrm{~Hz}, 2 \mathrm{H}), 6.64(\mathrm{~d}, J=8.5 \mathrm{~Hz}, 2 \mathrm{H}), 6.56(\mathrm{~s}, 1 \mathrm{H}), 5.34(\mathrm{~d}, J=18.9 \mathrm{~Hz}$, $1 \mathrm{H}), 4.37(\mathrm{p}, J=6.1 \mathrm{~Hz}, 1 \mathrm{H}), 3.64(\mathrm{dd}, J=10.5,2.9 \mathrm{~Hz}, 6 \mathrm{H}), 1.24(\mathrm{dd}, J=6.1,2.5 \mathrm{~Hz}, 6 \mathrm{H})$. ${ }^{13} \mathrm{C} \mathrm{NMR}\left(151 \mathrm{MHz}, \mathrm{CDCl}_{3}\right) \delta 154.2,142.1,140.8,140.5,132.4,132.2,128.6,128.5,128.1$, 127.5, 127.3, 127.3, 124.8, 124.7, 124.4, 124.4, 118.8, 116.2, 113.3, 111.2, 70.2, 65.0, 63.9, 53.4, 53.3, 53.3, 53.2, 22.0, 22.0. ${ }^{31} \mathrm{P}$ NMR (243 MHz, $\left.\mathrm{CDCl}_{3}\right) \delta 22.84$. HRMS (ESI) m/z: $[\mathrm{M}+\mathrm{H}]^{+}$Calcd for $\mathrm{C}_{27} \mathrm{H}_{28} \mathrm{~N}_{2} \mathrm{O}_{4} \mathrm{P}$ 475.1781; Found 475.1781.

Dimethyl (S)-(3-(3-fluorophenyl)-2-(4-isopropoxyphenyl)-1, 2-dihydroisoquinolin-1-yl) phosphonate (4icb)

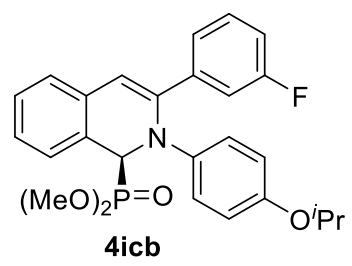

Following the general procedure, reaction were performed with $\mathbf{1 i}(0.12$ mmol), 2c (0.11 mmol), 3b (0.1 mmol), $5 \AA$ MS (100 mg), Ii (10 mol \%), and $\mathrm{Ag}_{2} \mathrm{CO}_{3}(5 \mathrm{~mol} \%)$ in the $1.0 \mathrm{~mL}$ of MTBE at $-10{ }^{\circ} \mathrm{C}$ for 3 days under argon, followed by column chromatography(PE/EA=1:1) to afford 4icb as yellow oil, $29.9 \mathrm{mg}(64 \%$ yield, 90\% ee $) ;[\alpha]_{\mathrm{D}}^{25}=+762.7^{\circ}(c=$ 1.0, EA); HPLC condition: Chiralpak IF, $254 \mathrm{~nm}, 0.5 \mathrm{~mL} / \mathrm{min}$, hexane $/{ }^{i} \mathrm{PrOH}=70 / 30, t_{\text {major }}=$ $22.112 \mathrm{~min}, t_{\text {minor }}=13.482 \mathrm{~min} ;{ }^{1} \mathrm{H}$ NMR $\left(600 \mathrm{MHz}, \mathrm{CDCl}_{3}\right) \delta 7.36(\mathrm{~d}, J=7.8 \mathrm{~Hz}, 1 \mathrm{H}), 7.30$ $7.23(\mathrm{~m}, 2 \mathrm{H}), 7.23-7.15(\mathrm{~m}, 3 \mathrm{H}), 7.09(\mathrm{~d}, J=7.6 \mathrm{~Hz}, 1 \mathrm{H}), 7.03-6.96(\mathrm{~m}, 2 \mathrm{H}), 6.87(\mathrm{td}, J=8.4$, $2.6 \mathrm{~Hz}, 1 \mathrm{H}), 6.67-6.60(\mathrm{~m}, 2 \mathrm{H}), 6.49(\mathrm{~s}, 1 \mathrm{H}), 5.34$ (d, $J=19.0 \mathrm{~Hz}, 1 \mathrm{H}), 4.36$ (hept, $J=6.1 \mathrm{~Hz}$, $1 \mathrm{H}), 3.68(\mathrm{~d}, J=10.5 \mathrm{~Hz}, 3 \mathrm{H}), 3.62(\mathrm{~d}, J=10.4 \mathrm{~Hz}, 3 \mathrm{H}), 1.23(\mathrm{dd}, J=6.1,2.0 \mathrm{~Hz}, 6 \mathrm{H}) .{ }^{13} \mathrm{C}$ NMR (151 MHz, $\left.\mathrm{CDCl}_{3}\right) \delta 162.0,154.0,141.5,140.9,132.8,129.7$ 128.4, 127.3, 126.9, 124.8, $124.5,124.4,123.4,116.2,114.8,114.5,111.7,70.2,64.6,53.3,22.0 .{ }^{31} \mathrm{P}$ NMR $(243 \mathrm{MHz}$, $\left.\mathrm{CDCl}_{3}\right) \delta$ 22.96. HRMS (ESI) m/z: [M+H] $]^{+}$Calcd for $\mathrm{C}_{26} \mathrm{H}_{28} \mathrm{FNO}_{4} \mathrm{P} 468.1734$; Found 468.1741. 


\section{Dimethyl (S)-(3-(3-bromophenyl)-2-(4-isopropoxyphenyl)-1, 2-dihydroisoquinolin-1-yl) phosphonate $(4 \mathrm{jcb})$}

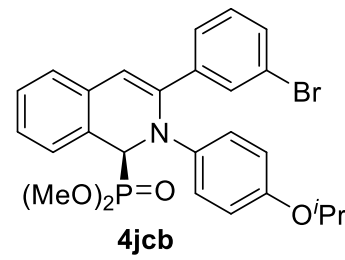

Following the general procedure, reaction were performed with $\mathbf{1 j}(0.12$ mmol), $2 \mathbf{c}(0.11 \mathrm{mmol}), 3 \mathbf{b}(0.1 \mathrm{mmol}), 5 \AA \mathrm{MS}$ (100 mg), Ii (10 mol \%), and $\mathrm{Ag}_{2} \mathrm{CO}_{3}(5 \mathrm{~mol} \%)$ in the $1.0 \mathrm{~mL}$ of MTBE at $-10{ }^{\circ} \mathrm{C}$ for 3 days under argon, followed by column chromatography $(\mathrm{PE} / \mathrm{EA}=1: 1)$ to afford 4 jcb as yellow oil, $38.0 \mathrm{mg}(72 \%$ yield, $93 \% e e) ;[\alpha]_{\mathrm{D}}^{25}=+643.5^{\circ}$ ( $c=1.0$, EA); HPLC condition: Chiralpak IC, $254 \mathrm{~nm}, 0.5 \mathrm{~mL} / \mathrm{min}$, hexane $/{ }^{i} \mathrm{PrOH}=80 / 20$, $t_{\text {major }}=19.812 \mathrm{~min}, t_{\text {minor }}=21.245 \mathrm{~min} ;{ }^{1} \mathrm{H} \mathrm{NMR}\left(600 \mathrm{MHz}, \mathrm{CDCl}_{3}\right) \delta 7.74(\mathrm{t}, J=1.8 \mathrm{~Hz}, 1 \mathrm{H})$, 7.46 (dt, $J=7.8,1.3 \mathrm{~Hz}, 1 \mathrm{H}), 7.34-7.29$ (m, 1H), 7.29-7.23 (m, 2H), 7.19 (dd, $J=16.3,8.1 \mathrm{~Hz}$, 2H), 7.12-7.04 (m, 2H), 7.03-6.96 (m, 2H), 6.68-6.60 (m, 2H), 6.47 (s, 1H), 5.33 (d, J = 19.0 $\mathrm{Hz}, 1 \mathrm{H}), 4.37$ (hept, $J=6.1 \mathrm{~Hz}, 1 \mathrm{H}), 3.68(\mathrm{~d}, J=10.5 \mathrm{~Hz}, 3 \mathrm{H}), 3.62(\mathrm{~d}, J=10.5 \mathrm{~Hz}, 3 \mathrm{H}), 1.24$ $(\mathrm{dd}, J=6.1,3.5 \mathrm{~Hz}, 6 \mathrm{H}) .{ }^{13} \mathrm{C} \mathrm{NMR}\left(151 \mathrm{MHz}, \mathrm{CDCl}_{3}\right) \delta 154.0,141.2,139.6,132.8,130.9$, 130.4, 129.8, 128.4, 128.4, 127.3, 127.2, 127.0, 126.6, 124.5, 124.5, 124.4, 124.4, 122.4, 116.2, $111.8,70.2,65.1,64.0,53.3,53.3,53.3,53.2,22.1,22.0 .{ }^{31} \mathrm{P}$ NMR $\left(243 \mathrm{MHz}, \mathrm{CDCl}_{3}\right) \delta 22.93$. HRMS (ESI) m/z: [M+H] ${ }^{+}$Calcd for $\mathrm{C}_{26} \mathrm{H}_{28} \mathrm{BrNO}_{4} \mathrm{P}$ 528.0934; Found 528.0917.

Dimethyl (S)-(2-(4-isopropoxyphenyl)-3-(m-tolyl)-1, 2-dihydroisoquinolin-1-yl)phosphon ate $(4 \mathrm{kcb})$

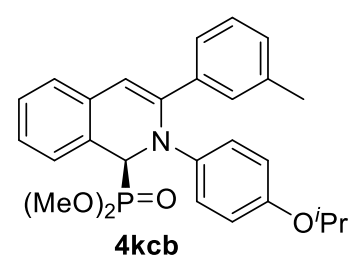

Following the general procedure, reaction were performed with $1 \mathbf{k}(0.12$ mmol), $2 \mathbf{c}(0.11 \mathrm{mmol}), \mathbf{3 b}(0.1 \mathrm{mmol}), 5 \AA \mathrm{MS}(100 \mathrm{mg}), \mathbf{I i}(10 \mathrm{~mol} \%)$, and $\mathrm{Ag}_{2} \mathrm{CO}_{3}(5 \mathrm{~mol} \%)$ in the $1.0 \mathrm{~mL}$ of MTBE at $-10{ }^{\circ} \mathrm{C}$ for 3 days under argon, followed by column chromatography(PE/EA=1:1) to afford 4kcb as yellow oil, $39.8 \mathrm{mg}(86 \%$ yield, $93 \% e e) ;[\alpha]_{\mathrm{D}}^{25}=+754.9^{\circ}$ ( $c=1.0$, EA); HPLC condition: Chiralpak IC, $254 \mathrm{~nm}, 0.5 \mathrm{~mL} / \mathrm{min}$, hexane $/{ }^{i} \mathrm{PrOH}=80 / 20$, $t_{\text {major }}=23.319 \mathrm{~min}, t_{\text {minor }}=19.239 \mathrm{~min} ;{ }^{1} \mathrm{H} \mathrm{NMR}\left(600 \mathrm{MHz}, \mathrm{CDCl}_{3}\right) \delta 7.40(\mathrm{~d}, J=2.1 \mathrm{~Hz}, 1 \mathrm{H})$, $7.35(\mathrm{~d}, J=7.8 \mathrm{~Hz}, 1 \mathrm{H}), 7.24(\mathrm{ddd}, J=7.5,5.8,2.7 \mathrm{~Hz}, 1 \mathrm{H}), 7.21-7.17(\mathrm{~m}, 1 \mathrm{H}), 7.14(\mathrm{t}, J=$ $7.6 \mathrm{~Hz}, 1 \mathrm{H}), 7.12-7.06(\mathrm{~m}, 2 \mathrm{H}), 7.03-6.97(\mathrm{~m}, 3 \mathrm{H}), 6.70-6.55(\mathrm{~m}, 2 \mathrm{H}), 6.45(\mathrm{~s}, 1 \mathrm{H}), 5.35$ (d, $J$ $=18.9 \mathrm{~Hz}, 1 \mathrm{H}), 4.35$ (hept, $J=6.1 \mathrm{~Hz}, 1 \mathrm{H}), 3.68(\mathrm{~d}, J=10.5 \mathrm{~Hz}, 3 \mathrm{H}), 3.61(\mathrm{~d}, J=10.5 \mathrm{~Hz}$, 3H), 2.29 (s, 3H), $1.22(\mathrm{dd}, J=6.1,4.1 \mathrm{~Hz}, 6 \mathrm{H}) .{ }^{13} \mathrm{C} \mathrm{NMR}\left(151 \mathrm{MHz}, \mathrm{CDCl}_{3}\right) \delta 153.7,142.9$, 137.7, 137.3, 133.3, 128.8, 128.3, 128.3, 128.3, 128.1, 127.3, 127.2, 126.4, 126.4, 125.2, 124.8, 124.6, 124.6, 124.1, 124.1, 116.1, 110.7, 77.2, 70.1, 64.8, 53.3, 53.3, 53.2, 53.1, 22.1, 22.0, 21.5 . ${ }^{31} \mathrm{P}$ NMR $\left(243 \mathrm{MHz}, \mathrm{CDCl}_{3}\right) \delta$ 23.12. HRMS (ESI) $\mathrm{m} / \mathrm{z}$ : $[\mathrm{M}+\mathrm{H}]^{+}$Calcd for $\mathrm{C}_{27} \mathrm{H}_{31} \mathrm{NO}_{4} \mathrm{P}$ 464.1985; Found 464.1986. 
Dimethyl (S)-(2-(4-isopropoxyphenyl)-3-(3-methoxyphenyl)-1, 2-dihydroisoquinolin-1-yl) phosphonate (4lcb)

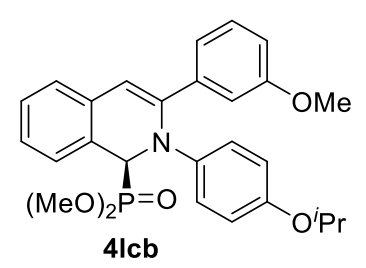

Following the general procedure, reaction were performed with $\mathbf{1 l}(0.12$ mmol), $2 \mathbf{c}(0.11 \mathrm{mmol}), 3 \mathbf{b}(0.1 \mathrm{mmol}), 5 \AA \mathrm{MS}$ (100 mg), Ii (10 mol \%), and $\mathrm{Ag}_{2} \mathrm{CO}_{3}(5 \mathrm{~mol} \%)$ in the $1.0 \mathrm{~mL}$ of MTBE at $-10{ }^{\circ} \mathrm{C}$ for 3 days under argon, followed by column chromatography $(\mathrm{PE} / \mathrm{EA}=1: 1)$ to afford 4lcb as yellow oil, $43.1 \mathrm{mg}(90 \%$ yield, $90 \% e e) ;[\alpha]_{\mathrm{D}}^{25}=+768.5^{\circ}$ ( $c=1.0$, EA); HPLC condition: Chiralpak IF, $254 \mathrm{~nm}, 0.5 \mathrm{~mL} / \mathrm{min}$, hexane $/{ }^{i} \mathrm{PrOH}=70 / 30$, $t_{\text {major }}=20.442 \mathrm{~min}, t_{\text {minor }}=13.051 \mathrm{~min} ;{ }^{1} \mathrm{H} \mathrm{NMR}\left(600 \mathrm{MHz}, \mathrm{CDCl}_{3}\right) \delta 7.28-7.23(\mathrm{~m}, 1 \mathrm{H}), 7.22-$ $7.12(\mathrm{~m}, 5 \mathrm{H}), 7.09$ (d, J=7.7 Hz, 1H), 7.05-6.99 (m, 2H), 5.36 (d, J=18.7 Hz, 1H), 4.36 (hept, $J=6.1 \mathrm{~Hz}, 1 \mathrm{H}), 3.72(\mathrm{~s}, 3 \mathrm{H}), 3.69(\mathrm{~d}, J=10.5 \mathrm{~Hz}, 3 \mathrm{H}), 3.61(\mathrm{~d}, J=10.4 \mathrm{~Hz}, 3 \mathrm{H}), 1.23(\mathrm{dd}, J$ $=6.1,3.9 \mathrm{~Hz}, 6 \mathrm{H}) .{ }^{13} \mathrm{C} \mathrm{NMR}\left(151 \mathrm{MHz}, \mathrm{CDCl}_{3}\right) \delta 159.5,153.8,142.5,138.8,129.2,128.3$, 128.3, 127.2, 127.2 126.6, 126.6, 124.4, 124.4, 124.2, 124.2, 120.4, 116.1, 114.0, 113.1, 110.9, 70.1, 65.1, 64.1, 55.1, 53.3, 53.3, 53.2, 53.1, 22.1, 22.0. ${ }^{31} \mathrm{P}$ NMR (243 MHz, $\left.\mathrm{CDCl}_{3}\right) \delta 23.22$. HRMS (ESI) m/z: $[\mathrm{M}+\mathrm{H}]^{+}$Calcd for $\mathrm{C}_{27} \mathrm{H}_{31} \mathrm{NO}_{5} \mathrm{P}$ 480.1934; Found 480.1941. IR $\left(\mathrm{KBr}, \mathrm{cm}^{-1}\right)$ 3453, 3061, 2975, 2849, 1602, 1505, 1454, 1380, 1232, 1178, 1117, 1053, 1031, 952, 839, 785, $673,556,515$.

Dimethyl (S)-(3-(3,5-dimethylphenyl)-2-(4-isopropoxyphenyl)-1, 2-dihydroisoquinolin-1yl)phosphonate $(4 \mathrm{mcb})$

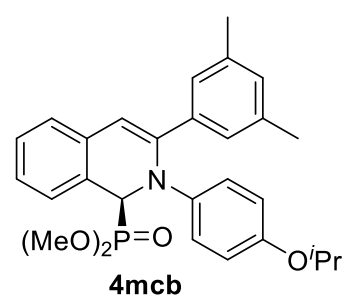

Following the general procedure, reaction were performed with $\mathbf{1 m}(0.12$ mmol), $2 \mathbf{c}(0.11 \mathrm{mmol}), \mathbf{3 b}(0.1 \mathrm{mmol}), 5 \AA \mathrm{MS}(100 \mathrm{mg}), \mathbf{I i}(10 \mathrm{~mol} \%)$, and $\mathrm{Ag}_{2} \mathrm{CO}_{3}(5 \mathrm{~mol} \%)$ in the $1.0 \mathrm{~mL}$ of MTBE at $-10{ }^{\circ} \mathrm{C}$ for 3 days under argon, followed by column chromatography(PE/EA=1:1) to afford

4mcb as yellow oil, $47.2 \mathrm{mg}(99 \%$ yield, $94 \% e e) ;[\alpha]_{\mathrm{D}}^{25}=+732.3^{\circ}(c=$ 1.0, EA); HPLC condition: Chiralpak IC, $254 \mathrm{~nm}, 0.5 \mathrm{~mL} / \mathrm{min}$, hexane $/{ }^{i} \mathrm{PrOH}=80 / 20, t_{\text {major }}=$ $18.358 \mathrm{~min}, t_{\mathrm{minor}}=17.000 \mathrm{~min} ;{ }^{1} \mathrm{H} \mathrm{NMR}\left(600 \mathrm{MHz}, \mathrm{CDCl}_{3}\right) \delta 7.25-7.22(\mathrm{~m}, 1 \mathrm{H}), 7.19-7.12$ (m, 5H), 7.07 (d, J=7.6 Hz, 1H), 7.04-6.99 (m, 2H), $6.82(\mathrm{~s}, 1 \mathrm{H}), 6.66-6.58(\mathrm{~m}, 2 \mathrm{H}), 6.43$ (s, $1 \mathrm{H}), 5.35(\mathrm{~d}, J=18.9 \mathrm{~Hz}, 1 \mathrm{H}), 4.35(\mathrm{~h}, J=6.0 \mathrm{~Hz}, 1 \mathrm{H}), 3.69(\mathrm{~d}, J=10.5 \mathrm{~Hz}, 3 \mathrm{H}), 3.60(\mathrm{~d}, J=$ $10.2 \mathrm{~Hz}, 3 \mathrm{H}), 2.23(\mathrm{~s}, 6 \mathrm{H}), 1.22(\mathrm{t}, J=5.9 \mathrm{~Hz}, 6 \mathrm{H}) .{ }^{13} \mathrm{C} \mathrm{NMR}\left(151 \mathrm{MHz}, \mathrm{CDCl}_{3}\right) \delta$ 153.7, 143.0, 137.5, 137.3, 133.4, 129.8, 129.0, 128.3, 128.3, 128.2, 127.2, 127.2, 126.3, 125.8, 124.7, 124.6, 124.6, 124.1, 124.0, 116.1, 110.5, 70.2, 64.8, 53.3, 53.3, 53.2, 53.1, 22.1, 22.0, 21.3. ${ }^{31} \mathrm{P}$ NMR $\left(243 \mathrm{MHz}, \mathrm{CDCl}_{3}\right) \delta$ 23.11. HRMS (ESI) m/z: [M+H] $]^{+}$Calcd for $\mathrm{C}_{28} \mathrm{H}_{33} \mathrm{NO}_{4} \mathrm{P} 478.2142$; Found 478.2131. IR ( $\left.\mathrm{KBr}, \mathrm{cm}^{-1}\right)$ 3453, 2952, 2922, 2852, 1599, 1563, 1504, 1453, 1378, 1227, 1182 , 
$1117,1055,1028,951,847,754,665,558,527$.

\section{Dimethyl (S)-(3-(2-chlorophenyl)-2-(4-isopropoxyphenyl)-1, 2-dihydroisoquinolin-1-yl) phosphonate (4ncb)}

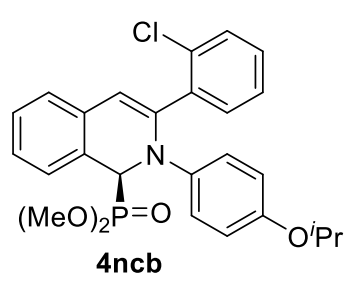

Following the general procedure, reaction were performed with 1 n $(0.12$ mmol), $2 \mathbf{c}(0.11 \mathrm{mmol}), \mathbf{3 b}(0.1 \mathrm{mmol}), 5 \AA \mathrm{MS}(100 \mathrm{mg}), \mathbf{I i}(10 \mathrm{~mol} \%)$, and $\mathrm{Ag}_{2} \mathrm{CO}_{3}(5 \mathrm{~mol} \%)$ in the $1.0 \mathrm{~mL}$ of MTBE at $-10{ }^{\circ} \mathrm{C}$ for 3 days under argon, followed by column chromatography(PE/EA=1:1) to afford 4ncb as yellow oil, $20.3 \mathrm{mg}$ (42\% yield, $27 \%$ ee $) ;[\alpha]_{\mathrm{D}}^{25}=+180.2^{\circ}(c=$ 1.0, EA); HPLC condition: Chiralpak IF, $254 \mathrm{~nm}, 0.5 \mathrm{~mL} / \mathrm{min}$, hexane $/{ }^{i} \mathrm{PrOH}=60 / 40, t_{\text {major }}=$ $12.447 \mathrm{~min}, t_{\text {minor }}=11.213 \mathrm{~min} ;{ }^{1} \mathrm{H}$ NMR $\left(600 \mathrm{MHz}, \mathrm{CDCl}_{3}\right) \delta 7.64-7.58(\mathrm{~m}, 1 \mathrm{H}), 7.35-7.30$ (m, 1H), 7.29-7.23 (m, 1H), $7.19(\mathrm{dd}, J=12.0,7.4 \mathrm{~Hz}, 2 \mathrm{H}), 7.08(\mathrm{tq}, J=7.8,3.4,2.6 \mathrm{~Hz}, 5 \mathrm{H})$, 6.68-6.58 (m, 2H), 6.41 (s, 1H), 5.37 (d, $J=17.9 \mathrm{~Hz}, 1 \mathrm{H}), 4.35$ (hept, $J=6.1 \mathrm{~Hz}, 1 \mathrm{H}), 3.67$ $(\mathrm{dd}, J=15.8,10.5 \mathrm{~Hz}, 6 \mathrm{H}), 1.22(\mathrm{dd}, J=6.1,3.3 \mathrm{~Hz}, 6 \mathrm{H}) .{ }^{13} \mathrm{C} \mathrm{NMR}\left(151 \mathrm{MHz}, \mathrm{CDCl}_{3}\right) \delta 154.0$, $138.5,135.8,133.2,132.7,132.1,130.2,128.8,128.4,128.38,127.2,127.1,126.8,126.7,124.5$, $124.4,124.3,124.2,116.0,114.0,70.1,65.0,64.0,53.2,53.2,53.1,53.0,22.1,22.0 .{ }^{31} \mathrm{P}$ NMR $\left(243 \mathrm{MHz}, \mathrm{CDCl}_{3}\right) \delta$ 23.47. HRMS (ESI) $\mathrm{m} / \mathrm{z}:[\mathrm{M}+\mathrm{H}]^{+}$Calcd for $\mathrm{C}_{26} \mathrm{H}_{28} \mathrm{ClNO}_{4} \mathrm{P}$ 484.1439; Found 484.1435.

Dimethyl (S)-(2-(4-isopropoxyphenyl)-3-(o-tolyl)-1, 2-dihydroisoquinolin-1-yl)phosphona te (4ocb)

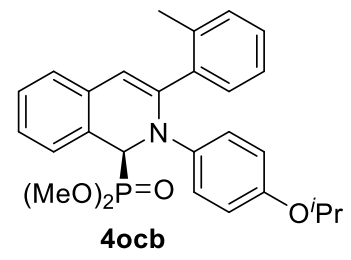

Following the general procedure, reaction were performed with $10(0.12$ mmol), 2c (0.11 mmol), 3b (0.1 mmol), $5 \AA$ MS (100 mg), Ii (10 mol \%), and $\mathrm{Ag}_{2} \mathrm{CO}_{3}(5 \mathrm{~mol} \%)$ in the $1.0 \mathrm{~mL}$ of MTBE at $-10{ }^{\circ} \mathrm{C}$ for 3 days under argon, followed by column chromatography(PE/EA=1:1) to afford 4ocb as yellow oil, $18.5 \mathrm{mg}(40 \%$ yield, $15 \% e e) ;[\alpha]_{\mathrm{D}}^{25}=+150.3^{\circ}$ ( $c=1.0$, EA); HPLC condition: Chiralpak IF, $254 \mathrm{~nm}, 0.5 \mathrm{~mL} / \mathrm{min}$, hexane $/{ }^{i} \mathrm{PrOH}=60 / 40$, $t_{\text {major }}=13.353 \mathrm{~min}, t_{\text {minor }}=11.801 \mathrm{~min} ;{ }^{1} \mathrm{H} \mathrm{NMR}\left(600 \mathrm{MHz}, \mathrm{CDCl}_{3}\right) \delta 7.52(\mathrm{dd}, J=7.2,2.3 \mathrm{~Hz}$, 1H), $7.24(\mathrm{~d}, J=9.2 \mathrm{~Hz}, 2 \mathrm{H}), 7.19-7.12(\mathrm{~m}, 2 \mathrm{H}), 7.11-7.04(\mathrm{~m}, 4 \mathrm{H}), 7.02(\mathrm{~d}, J=8.8 \mathrm{~Hz}, 2 \mathrm{H})$, $6.58(\mathrm{~d}, J=8.7 \mathrm{~Hz}, 2 \mathrm{H}), 6.09(\mathrm{~s}, 1 \mathrm{H}), 5.35$ (d, $J=17.8 \mathrm{~Hz}, 1 \mathrm{H}), 4.34$ (hept, $J=6.1 \mathrm{~Hz}, 1 \mathrm{H})$, $3.66(\mathrm{dd}, J=29.4,10.5 \mathrm{~Hz}, 6 \mathrm{H}), 2.54(\mathrm{~s}, 3 \mathrm{H}), 1.21(\mathrm{dd}, J=6.1,3.8 \mathrm{~Hz}, 6 \mathrm{H}) .{ }^{13} \mathrm{C}$ NMR $(151$ $\left.\mathrm{MHz} \mathrm{CDCl}_{3}\right) \delta 154.0,142.4,140.8,136.8,136.7,130.9,130.7,128.4,128.3,127.8,127.2$, 127.2, 126.2, 126.2, 125.6, 125.2, 125.2, 123.6, 123.6, 115.7, 111.3, 70.0, 65.3, 64.2, 53.1, 53.1, 53.1, 53.0, 22.1, 22.0, 20.7. ${ }^{31} \mathrm{P}$ NMR $\left(243 \mathrm{MHz}, \mathrm{CDCl}_{3}\right) \delta 23.09$. HRMS (ESI) m/z: $[\mathrm{M}+\mathrm{H}]^{+}$ Calcd for $\mathrm{C}_{27} \mathrm{H}_{31} \mathrm{NO}_{4} \mathrm{P}$ 464.1985; Found 464.1980. 
Dimethyl (S)-(2-(4-isopropoxyphenyl)-3-(thiophen-2-yl)-1, 2-dihydroisoquinolin-1-yl)pho sphonate (4pcb)

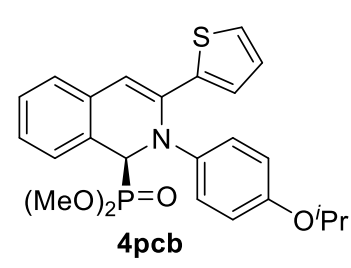

Following the general procedure, reaction were performed with $1 \mathbf{p}(0.12$ mmol), 2c (0.11 mmol), 3b (0.1 mmol), $5 \AA$ MS (100 mg), Ii (10 mol \%), and $\mathrm{Ag}_{2} \mathrm{CO}_{3}(5 \mathrm{~mol} \%)$ in the $1.0 \mathrm{~mL}$ of MTBE at $-10{ }^{\circ} \mathrm{C}$ for 3 days under argon, followed by column chromatography(PE/EA=1:1) to afford 4 pcb as yellow oil, $38.7 \mathrm{mg}(85 \%$ yield, $88 \% e e) ;[\alpha]_{\mathrm{D}}^{25}=+750.3^{\circ}$ ( $c=1.0$, EA); HPLC condition: Chiralpak IF, $254 \mathrm{~nm}, 0.5 \mathrm{~mL} / \mathrm{min}$, hexane $/{ }^{i} \mathrm{PrOH}=60 / 40$, $t_{\text {major }}=23.172 \mathrm{~min}, t_{\text {minor }}=13.707 \mathrm{~min} ;{ }^{1} \mathrm{H} \mathrm{NMR}\left(600 \mathrm{MHz}, \mathrm{CDCl}_{3}\right) \delta 7.27-7.22(\mathrm{~m}, 1 \mathrm{H}), 7.20$ $7.09(\mathrm{~m}, 4 \mathrm{H}), 7.07(\mathrm{dd}, J=8.9,3.4 \mathrm{~Hz}, 3 \mathrm{H}), 6.86(\mathrm{t}, J=4.4 \mathrm{~Hz}, 1 \mathrm{H}), 6.67(\mathrm{~d}, J=8.9 \mathrm{~Hz}, 2 \mathrm{H})$, $6.57(\mathrm{~s}, 1 \mathrm{H}), 5.25(\mathrm{~d}, J=19.9 \mathrm{~Hz}, 1 \mathrm{H}), 4.39$ (hept, $J=6.1 \mathrm{~Hz}, 1 \mathrm{H}), 3.72(\mathrm{~d}, J=10.5 \mathrm{~Hz}, 3 \mathrm{H})$, $3.65(\mathrm{~d}, J=10.4 \mathrm{~Hz}, 3 \mathrm{H}), 1.25(\mathrm{dd}, J=6.1,2.7 \mathrm{~Hz}, 6 \mathrm{H}) .{ }^{13} \mathrm{C} \mathrm{NMR}\left(151 \mathrm{MHz}, \mathrm{CDCl}_{3}\right) \delta 154.2$, 141.5, 141.5, 136.6, 128.4, 128.4, 127.4, 127.4, 127.2, 126.7, 126.7, 126.3, 125.5, 124.7, 124.6, 124.6, 124.3, 124.3, 116.1, 110.4, 70.1, 65.5, 64.4, 53.6, 53.6, 53.5, 53.4, 22.1, 22.0. ${ }^{31} \mathrm{P}$ NMR $\left(243 \mathrm{MHz}, \mathrm{CDCl}_{3}\right) \delta$ 22.45. HRMS (ESI) m/z: $[\mathrm{M}+\mathrm{H}]^{+}$Calcd for $\mathrm{C}_{24} \mathrm{H}_{27} \mathrm{NO}_{4} \mathrm{PS}$ 456.1393; Found 456.1392 .

Dimethyl (S)-(3-(furan-2-yl)-2-(4-isopropoxyphenyl)-1, 2-dihydroisoquinolin-1-yl) phosph onate $(4 \mathrm{qcb})$

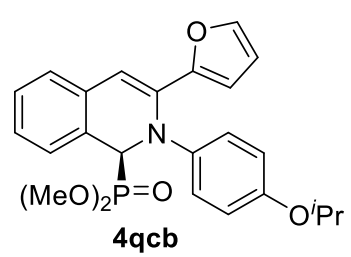

Following the general procedure, reaction were performed with $\mathbf{1 q}(0.12$ mmol), 2c (0.11 mmol), 3b (0.1 mmol), $5 \AA$ MS (100 mg), Ii (10 mol \%), and $\mathrm{Ag}_{2} \mathrm{CO}_{3}\left(5 \mathrm{~mol} \%\right.$ ) in the $1.0 \mathrm{~mL}$ of MTBE at $-10{ }^{\circ} \mathrm{C}$ for 3 days under argon, followed by column chromatography $(\mathrm{PE} / \mathrm{EA}=1: 1)$ to afford $4 \mathbf{q c b}$ as yellow oil, $19.7 \mathrm{mg}(54 \%$ yield, $88 \% e e) ;[\alpha]_{\mathrm{D}}^{25}=+375.0^{\circ}$ ( $c=1.0$, EA); HPLC condition: Chiralpak IF, $254 \mathrm{~nm}, 0.5 \mathrm{~mL} / \mathrm{min}$, hexane $/{ }^{i} \mathrm{PrOH}=60 / 40$, $t_{\text {major }}=18.224 \mathrm{~min}, t_{\text {minor }}=12.306 \mathrm{~min} ;{ }^{1} \mathrm{H} \mathrm{NMR}\left(600 \mathrm{MHz}, \mathrm{CDCl}_{3}\right) \delta 7.61(\mathrm{dt}, J=8.1,1.8 \mathrm{~Hz}$, 1H), $7.55(\mathrm{~d}, J=7.7 \mathrm{~Hz}, 1 \mathrm{H}), 7.46(\mathrm{~d}, J=1.8 \mathrm{~Hz}, 1 \mathrm{H}), 7.33$ (t, $J=7.6 \mathrm{~Hz}, 1 \mathrm{H}), 7.29-7.23$ (m, $2 \mathrm{H}), 6.71(\mathrm{~d}, J=3.4 \mathrm{~Hz}, 1 \mathrm{H}), 6.70-6.65(\mathrm{~m}, 2 \mathrm{H}), 6.60-6.53(\mathrm{~m}, 2 \mathrm{H}), 6.46(\mathrm{dd}, J=3.4,1.9 \mathrm{~Hz}$, 1H), 5.42 (d, $J=24.5 \mathrm{~Hz}, 1 \mathrm{H}), 4.29$ (hept, $J=6.1 \mathrm{~Hz}, 1 \mathrm{H}), 3.86$ (d, $J=10.7 \mathrm{~Hz}, 3 \mathrm{H}), 3.46$ (d, $J=10.5 \mathrm{~Hz}, 3 \mathrm{H}), 1.23(\mathrm{~d}, J=6.1 \mathrm{~Hz}, 6 \mathrm{H}) .{ }^{13} \mathrm{C} \mathrm{NMR}\left(151 \mathrm{MHz}, \mathrm{CDCl}_{3}\right) \delta 150.9,144.0,140.0$, 138.1, 136.8, 132.1, 129.4, 129.4, 127.8, 127.8, 127.6, 127.5, 117.7, 115.7, 115.0, 111.2, 91.0, 84.9, 70.9, 54.0, 54.0, 53.8, 53.7, 53.0, 22.2, 22.1. $\left.{ }^{31} \mathrm{P} \mathrm{NMR} \mathrm{(243} \mathrm{MHz,} \mathrm{CDCl}_{3}\right) \delta 24.94$. HRMS (ESI) $\mathrm{m} / \mathrm{z}:[\mathrm{M}+\mathrm{H}]^{+}$Calcd for $\mathrm{C}_{24} \mathrm{H}_{27} \mathrm{NO}_{5} \mathrm{P}$ 440.1621; Found 440.1626. 
Dimethyl (S)-(2-(4-isopropoxyphenyl)-3-(naphthalen-2-yl)-1, 2-dihydroisoquinolin-1-yl) phosphonate (4rcb)

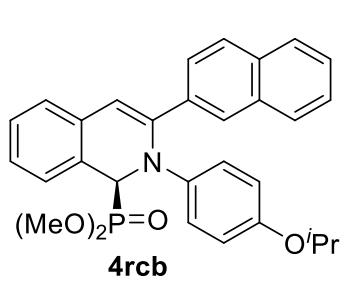

Following the general procedure, reaction were performed with $\mathbf{1 r}(0.12$ mmol), $2 \mathbf{c}(0.11 \mathrm{mmol}), \mathbf{3 b}(0.1 \mathrm{mmol}), 5 \AA \mathrm{MS}(100 \mathrm{mg}), \mathbf{I i}(10 \mathrm{~mol} \%)$, and $\mathrm{Ag}_{2} \mathrm{CO}_{3}(5 \mathrm{~mol} \%)$ in the $1.0 \mathrm{~mL}$ of MTBE at $-10{ }^{\circ} \mathrm{C}$ for 3 days under argon, followed by column chromatography $(\mathrm{PE} / \mathrm{EA}=1: 1)$ to afford 4rcb as yellow oil, $42.0 \mathrm{mg}(84 \%$ yield, $91 \% e e) ;[\alpha]_{\mathrm{D}}^{25}=+599.8^{\circ}(c=$ 1.0, EA); HPLC condition: Chiralpak IF, $254 \mathrm{~nm}, 0.5 \mathrm{~mL} / \mathrm{min}$, hexane $/{ }^{i} \mathrm{PrOH}=60 / 40, t_{\text {major }}=$ $18.532 \mathrm{~min}, t_{\text {minor }}=13.329 \mathrm{~min} ;{ }^{1} \mathrm{H}$ NMR $\left(600 \mathrm{MHz}, \mathrm{CDCl}_{3}\right) \delta 8.08(\mathrm{~s}, 1 \mathrm{H}), 7.80(\mathrm{dd}, J=7.3$, $2.0 \mathrm{~Hz}, 1 \mathrm{H}), 7.76-7.71(\mathrm{~m}, 1 \mathrm{H}), 7.70-7.64(\mathrm{~m}, 2 \mathrm{H}), 7.45-7.37(\mathrm{~m}, 2 \mathrm{H}), 7.30-7.22(\mathrm{~m}, 2 \mathrm{H})$, 7.20-7.15 (m, 1H), $7.11(\mathrm{~d}, J=7.6 \mathrm{~Hz}, 1 \mathrm{H}), 7.09-7.03$ (m, 2H), 6.64-6.54 (m, 3H), 5.41 (d, $J$ $=18.8 \mathrm{~Hz}, 1 \mathrm{H}), 4.30$ (hept, $J=6.1 \mathrm{~Hz}, 1 \mathrm{H}), 3.70(\mathrm{~d}, J=10.5 \mathrm{~Hz}, 3 \mathrm{H}), 3.63(\mathrm{~d}, J=10.4 \mathrm{~Hz}$, $3 \mathrm{H}), 1.18(\mathrm{~d}, J=6.1 \mathrm{~Hz}, 6 \mathrm{H}) .{ }^{13} \mathrm{C} \mathrm{NMR}\left(151 \mathrm{MHz}, \mathrm{CDCl}_{3}\right) \delta 153.8,142.6,135.0,133.3,133.2$, 128.4, 128.4, 128.3, 127.9, 127.6, 127.3, 127.3, 126.9, 126.7, 126.6, 126.1, 126.0, 125.8, 124.6, 124.6, 124.3, 124.2, 116.1, 111.4, 70.1, 65.3, 64.2, 53.4, 53.3, 53.21, 53.2, 22.1, 22.0. ${ }^{31} \mathrm{P}$ NMR $\left(243 \mathrm{MHz}, \mathrm{CDCl}_{3}\right) \delta$ 23.16. HRMS (ESI) m/z: [M+H] $]^{+}$Calcd for $\mathrm{C}_{30} \mathrm{H}_{31} \mathrm{NO}_{4} \mathrm{P} 500.1985$; Found 500.1996. IR ( $\left.\mathrm{KBr}, \mathrm{cm}^{-1}\right)$ 3435, 3054, 2974, 2849, 1734, 1603, 1562, 1504, 1383, 1235, 1183 , 1118, 1055, 1029, 952, 817, 530, 498, 478.

Dimethyl $(S, E)$-(2-(4-isopropoxyphenyl)-3-styryl-1, 2-dihydroisoquinolin-1-yl)phosphon ate $(4 \mathrm{scb})$

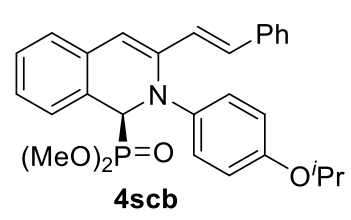

Following the general procedure, reaction were performed with $\mathbf{1 s}(0.12$ mmol), $2 \mathbf{c}(0.11 \mathrm{mmol}), \mathbf{3 b}(0.1 \mathrm{mmol}), 5 \AA \mathrm{MS}(100 \mathrm{mg}), \mathbf{I i}(10 \mathrm{~mol} \%)$, and $\mathrm{Ag}_{2} \mathrm{CO}_{3}(5 \mathrm{~mol} \%)$ in the $1.0 \mathrm{~mL}$ of MTBE at $-10{ }^{\circ} \mathrm{C}$ for 3 days under argon, followed by column chromatography $(\mathrm{PE} / \mathrm{EA}=1: 1)$ to afford 4scb as yellow oil, $26.6 \mathrm{mg}(56 \%$ yield, $60 \% e e) ;[\alpha]_{\mathrm{D}}^{25}=+368.2^{\circ}(c=1.0$, EA); HPLC condition: Chiralpak IC, $254 \mathrm{~nm}, 0.5 \mathrm{~mL} / \mathrm{min}$, hexane $/{ }^{i} \mathrm{PrOH}=80 / 20, t_{\text {major }}=23.053 \mathrm{~min}, t_{\text {minor }}$ $=27.951 \mathrm{~min} ;{ }^{1} \mathrm{H}$ NMR (600 MHz, $\left.\mathrm{CDCl}_{3}\right) \delta 7.31-7.21(\mathrm{~m}, 5 \mathrm{H}), 7.20-7.16(\mathrm{~m}, 1 \mathrm{H}), 7.16-7.09$ (m, 4H), 7.07-7.02 (m, 1H), $6.74(\mathrm{dd}, J=8.6,6.5 \mathrm{~Hz}, 2 \mathrm{H}), 6.70(\mathrm{~s}, 1 \mathrm{H}), 6.58(\mathrm{~d}, J=16.1 \mathrm{~Hz}$, $1 \mathrm{H}), 6.49$ (s, 1H), 5.21 (d, $J=19.9 \mathrm{~Hz}, 1 \mathrm{H}), 4.42$ (hept, $J=6.1 \mathrm{~Hz}, 1 \mathrm{H}), 3.71$ (d, $J=10.4 \mathrm{~Hz}$, $3 \mathrm{H}), 3.66(\mathrm{~d}, J=10.3 \mathrm{~Hz}, 3 \mathrm{H}), 1.29-1.25(\mathrm{~m}, 6 \mathrm{H}) .{ }^{13} \mathrm{C} \mathrm{NMR}\left(151 \mathrm{MHz}, \mathrm{CDCl}_{3}\right) \delta 154.2,140.5$, 137.0, 131.9, 128.5, 128.4, 128.3, 127.7, 127.3, 127.3, 126.8, 126.8, 126.6, 125.4, 124.4, 124.4, 124.3, 124.3, 116.4, 113.6, 70.3, 65.5, 64.4, 53.6, 53.6, 22.1, 22.0. ${ }^{31} \mathrm{P}$ NMR (243 MHz, $\left.\mathrm{CDCl}_{3}\right)$ $\delta$ 22.36. HRMS (ESI) m/z: $[\mathrm{M}+\mathrm{H}]^{+}$Calcd for $\mathrm{C}_{28} \mathrm{H}_{31} \mathrm{NO}_{4} \mathrm{P} 476.1985$; Found 476.1982. 


\section{Dimethyl (S)-(2-(4-isopropoxyphenyl)-3-methyl-1, 2-dihydroisoquinolin-1-yl) phosphonate (4tcb)}

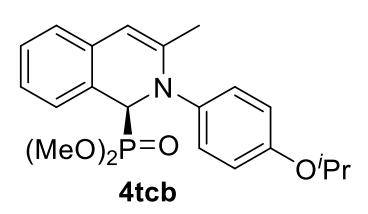

Following the general procedure, reaction were performed with $\mathbf{1 t}(0.12$ mmol), 2c (0.11 mmol), 3b (0.1 mmol), $5 \AA$ MS (100 mg), Ii (10 mol \%), and $\mathrm{Ag}_{2} \mathrm{CO}_{3}(5 \mathrm{~mol} \%)$ in the $1.0 \mathrm{~mL}$ of MTBE at $-10^{\circ} \mathrm{C}$ for 3 days under argon, followed by column chromatography $(\mathrm{PE} / \mathrm{EA}=1: 1)$ to afford 4tcb as yellow oil, $23.2 \mathrm{mg}(60 \%$ yield, $25 \% e e)$; $[\alpha]_{\mathrm{D}}^{25}=+44.8^{\circ}(c=1.0$, EA); HPLC condition: Chiralpak IF, $254 \mathrm{~nm}, 0.5 \mathrm{~mL} / \mathrm{min}$, hexane $/{ }^{i} \mathrm{PrOH}=80 / 20, t_{\text {major }}=16.308 \mathrm{~min}, t_{\text {minor }}$ $=17.366 \mathrm{~min} ;{ }^{1} \mathrm{H}$ NMR $\left(600 \mathrm{MHz}, \mathrm{CDCl}_{3}\right) \delta 7.22-7.13(\mathrm{~m}, 3 \mathrm{H}), 7.06(\mathrm{t}, J=7.4 \mathrm{~Hz}, 1 \mathrm{H}), 7.02-$ $6.94(\mathrm{~m}, 2 \mathrm{H}), 6.86-6.74(\mathrm{~m}, 2 \mathrm{H}), 5.89(\mathrm{~s}, 1 \mathrm{H}), 5.11$ (d, $J=17.4 \mathrm{~Hz}, 1 \mathrm{H}), 4.48$ (hept, $J=6.1 \mathrm{~Hz}$, $1 \mathrm{H}), 3.66(\mathrm{dd}, J=17.5,10.4 \mathrm{~Hz}, 6 \mathrm{H}), 1.91(\mathrm{~s}, 3 \mathrm{H}), 1.31(\mathrm{dd}, J=6.1,2.6 \mathrm{~Hz}, 6 \mathrm{H}) .{ }^{13} \mathrm{C} \mathrm{NMR}$ $\left(151 \mathrm{MHz}, \mathrm{CDCl}_{3}\right) \delta 155.0,140.3,139.8,133.5,128.2,128.2,126.9,126.8,126.3,126.3,125.4$, $125.4,122.9,122.8,122.8,116.2,107.8,70.2,65.4,64.3,53.3,53.3,53.3,53.3,22.1,20.1 .{ }^{31} \mathrm{P}$ NMR (243 MHz, $\left.\mathrm{CDCl}_{3}\right) \delta$ 22.84. HRMS (ESI) m/z: $[\mathrm{M}+\mathrm{H}]^{+}$Calcd for $\mathrm{C}_{21} \mathrm{H}_{27} \mathrm{NO}_{4} \mathrm{P} 388.1672$; Found 388.1678.

Dimethyl (S)-(3-hexyl-2-(4-isopropoxyphenyl)-1, 2-dihydroisoquinolin-1-yl)phosphonate (4ucb)

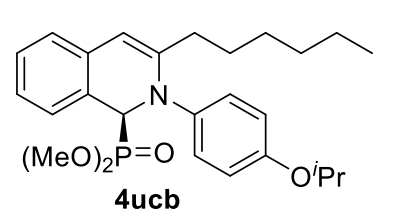

Following the general procedure, reaction were performed with $\mathbf{1 u}$ (0.12 mmol), $\mathbf{2 c}(0.11 \mathrm{mmol}), \mathbf{3 b}(0.1 \mathrm{mmol}), 5 \AA \mathrm{MS}(100 \mathrm{mg}), \mathbf{I i}(10$ $\mathrm{mol} \%$ ), and $\mathrm{Ag}_{2} \mathrm{CO}_{3}\left(5 \mathrm{~mol} \%\right.$ ) in the $1.0 \mathrm{~mL}$ of MTBE at $-10{ }^{\circ} \mathrm{C}$ for 3 days under argon, followed by column chromatography $(\mathrm{PE} / \mathrm{EA}=1: 1)$ to afford 4ucb as yellow oil, $35.2 \mathrm{mg}(77 \%$ yield, $41 \% e e)$; $[\alpha]_{\mathrm{D}}^{25}=+44.4^{\circ}(c=1.0$, EA); HPLC condition: Chiralpak IF, $254 \mathrm{~nm}, 0.5 \mathrm{~mL} / \mathrm{min}$, hexane $/{ }^{i} \mathrm{PrOH}=70 / 30, t_{\text {major }}=10.547 \mathrm{~min}, t_{\text {minor }}$ $=11.735 \mathrm{~min} ;{ }^{1} \mathrm{H} \mathrm{NMR}\left(600 \mathrm{MHz}, \mathrm{CDCl}_{3}\right) \delta 7.16(\mathrm{dd}, J=16.5,8.0 \mathrm{~Hz}, 3 \mathrm{H}), 7.06(\mathrm{t}, J=7.5$ $\mathrm{Hz}, 1 \mathrm{H}), 6.99$ (t, $J=7.0 \mathrm{~Hz}, 2 \mathrm{H}), 6.78$ (d, $J=8.4 \mathrm{~Hz}, 2 \mathrm{H}), 5.94(\mathrm{~s}, 1 \mathrm{H}), 5.08$ (d, $J=18.3 \mathrm{~Hz}$, 1H), 4.47 (hept, $J=6.0 \mathrm{~Hz}, 1 \mathrm{H}), 3.64$ (dd, $J=31.0,10.3 \mathrm{~Hz}, 6 \mathrm{H}), 2.26$ (ddd, $J=15.2,9.8,5.5$ $\mathrm{Hz}, 1 \mathrm{H}), 2.08(\mathrm{ddd}, J=15.5,9.8,6.5 \mathrm{~Hz}, 1 \mathrm{H}), 1.58-1.45(\mathrm{~m}, 2 \mathrm{H}), 1.31$ (dd, $J=6.2,3.0 \mathrm{~Hz}$, $6 \mathrm{H}), 1.27-1.16(\mathrm{~m}, 6 \mathrm{H}), 0.84(\mathrm{t}, J=7.0 \mathrm{~Hz}, 3 \mathrm{H}) .{ }^{13} \mathrm{C} \mathrm{NMR}\left(151 \mathrm{MHz}, \mathrm{CDCl}_{3}\right) \delta 154.9,145.1$, $145.1,140.3,133.5,128.2$, 128.2, 127.0, 127.0, 126.3, 126.3, 125.5, 125.5, 123.1, 123.1, 116.2, $107.4,70.2,65.4,64.4,53.3,53.3,53.0,53.0,33.3,31.6,29.0,28.1,22.5,22.1,22.1,14.0 .{ }^{31} \mathrm{P}$ NMR (243 MHz, $\left.\mathrm{CDCl}_{3}\right) \delta$ 22.87. HRMS (ESI) m/z: $[\mathrm{M}+\mathrm{H}]^{+}$Calcd for $\mathrm{C}_{26} \mathrm{H}_{37} \mathrm{NO}_{4} \mathrm{P} 458.2455$; Found 458.2461 
Dimethyl (S)-(3-cyclopropyl-2-(4-isopropoxyphenyl)-1, 2-dihydroisoquinolin-1-yl) phosphonate (4vcb)

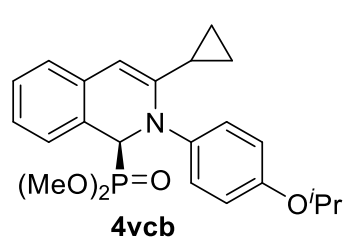

Following the general procedure, reaction were performed with $\mathbf{1 v}(0.12$ mmol), 2c (0.11 mmol), 3b (0.1 mmol), $5 \AA$ MS (100 mg), Ii (10 mol \%), and $\mathrm{Ag}_{2} \mathrm{CO}_{3}(5 \mathrm{~mol} \%)$ in the $1.0 \mathrm{~mL}$ of MTBE at $-10{ }^{\circ} \mathrm{C}$ for 3 days under argon, followed by column chromatography(PE/EA=1:1) to afford 4vcb as yellow oil, $28.1 \mathrm{mg}(68 \%$ yield, $44 \% e e) ;[\alpha]_{\mathrm{D}}^{25}=+345.7^{\circ}(c=$ 1.0, EA); HPLC condition: Chiralpak IF, $254 \mathrm{~nm}, 0.5 \mathrm{~mL} / \mathrm{min}$, hexane $/{ }^{i} \mathrm{PrOH}=60 / 40, t_{\text {major }}=$ $12.238 \mathrm{~min}, t_{\text {minor }}=12.928 \mathrm{~min} ;{ }^{1} \mathrm{H}$ NMR $\left(600 \mathrm{MHz}, \mathrm{CDCl}_{3}\right) \delta 7.28-7.23(\mathrm{~m}, 2 \mathrm{H}), 7.16(\mathrm{tt}, J=$ 7.5, 1.6 Hz, 1H), 7.04 (t, $J=7.4 \mathrm{~Hz}, 1 \mathrm{H}), 6.97$ (dd, $J=11.2,7.6 \mathrm{~Hz}, 2 \mathrm{H}), 6.87-6.73(\mathrm{~m}, 2 \mathrm{H})$, $5.72(\mathrm{~s}, 1 \mathrm{H}), 5.16(\mathrm{~d}, J=17.7 \mathrm{~Hz}, 1 \mathrm{H}), 4.48$ (hept, $J=6.1 \mathrm{~Hz}, 1 \mathrm{H}), 3.66(\mathrm{dd}, J=36.0,10.4 \mathrm{~Hz}$, $6 \mathrm{H}), 1.36(\mathrm{ddd}, J=13.3,8.2,5.3 \mathrm{~Hz}, 1 \mathrm{H}), 1.31(\mathrm{dd}, J=6.1,3.5 \mathrm{~Hz}, 6 \mathrm{H}), 0.86-0.78(\mathrm{~m}, 1 \mathrm{H})$, 0.75 (ddd, $J=11.2,8.3,3.7 \mathrm{~Hz}, 1 \mathrm{H}), 0.67$ (ddt, $J=8.4,6.0,2.7 \mathrm{~Hz}, 2 \mathrm{H}) .{ }^{13} \mathrm{C}$ NMR $(151 \mathrm{MHz}$, $\left.\mathrm{CDCl}_{3}\right) \delta 154.7,146.6,140.5,140.4,128.2,128.2,126.9,126.8,126.0,125.99,125.3,125.3$, 123.0, 123.0, 116.1, 102.5, 70.2, 65.5, 64.4, 53.4, 53.4, 53.3, 53.3, 22.1, 22.1, 14.1, 10.3, 7.6. ${ }^{31} \mathrm{P}$ NMR $\left(243 \mathrm{MHz}, \mathrm{CDCl}_{3}\right) \delta$ 22.81. HRMS (ESI) $\mathrm{m} / \mathrm{z}$ : $[\mathrm{M}+\mathrm{H}]^{+}$Calcd for $\mathrm{C}_{23} \mathrm{H}_{29} \mathrm{NO}_{4} \mathrm{P}$ 414.1829; Found 414.1830.

Dimethyl (S)-(3-(4-chlorophenyl)-2-phenyl-1， 2-dihydroisoquinolin-1-yl)phosphonate (4cab)

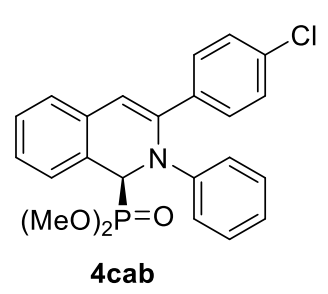

Following the general procedure, reaction were performed with 1c $(0.12$ mmol), $2 \mathbf{a}(0.11 \mathrm{mmol}), \mathbf{3 b}(0.1 \mathrm{mmol}), 5 \AA \mathrm{MS}(100 \mathrm{mg}), \mathbf{I i}(10 \mathrm{~mol} \%)$, and $\mathrm{Ag}_{2} \mathrm{CO}_{3}(5 \mathrm{~mol} \%)$ in the $1.0 \mathrm{~mL}$ of MTBE at $-10{ }^{\circ} \mathrm{C}$ for 3 days under argon, followed by column chromatography(PE/EA=1:1) to afford 4cab as yellow oil, $39.2 \mathrm{mg}$ (92\% yield, $89 \% e e) ;[\alpha]_{\mathrm{D}}^{25}=+542.4^{\circ}(c=1.0$,

$\left.{ }^{i} \mathrm{PrOH}\right)$; HPLC condition: Chiralpak IF, $254 \mathrm{~nm}, 0.5 \mathrm{~mL} / \mathrm{min}$, hexane $/{ }^{i} \mathrm{PrOH}=60 / 40, t_{\text {major }}=$ $18.040 \mathrm{~min}, t_{\text {minor }}=14.223 \mathrm{~min} ;{ }^{1} \mathrm{H} \mathrm{NMR}\left(600 \mathrm{MHz}, \mathrm{CDCl}_{3}\right) \delta 7.51(\mathrm{~d}, J=8.5 \mathrm{~Hz}, 2 \mathrm{H}), 7.29$ $7.24(\mathrm{~m}, 1 \mathrm{H}), 7.23-7.16(\mathrm{~m}, 4 \mathrm{H}), 7.14-7.08(\mathrm{~m}, 3 \mathrm{H}), 7.07-7.01(\mathrm{~m}, 2 \mathrm{H}), 6.89$ (t, J = 7.3 Hz, 1H), $6.52(\mathrm{~s}, 1 \mathrm{H}), 5.45(\mathrm{~d}, J=18.7 \mathrm{~Hz}, 1 \mathrm{H}), 3.64(\mathrm{dd}, J=18.4,10.5 \mathrm{~Hz}, 6 \mathrm{H}) .{ }^{13} \mathrm{C}$ NMR $(151$ $\left.\mathrm{MHz}, \mathrm{CDCl}_{3}\right) \delta 141.0,135.8,133.8,132.8,128.8,128.7,128.6,128.5,128.5,127.2,127.2$, 127.0, 127.0, 124.5, 124.5, 122.8, 122.8, 122.7, 112.4, 77.1, 64.5, 63.4, 53.3, 53.2, 53.2, 53.2. ${ }^{31} \mathrm{P}$ NMR $\left(243 \mathrm{MHz}, \mathrm{CDCl}_{3}\right) \delta$ 23.06. HRMS (ESI) m/z: $[\mathrm{M}+\mathrm{H}]^{+}$Calcd for $\mathrm{C}_{23} \mathrm{H}_{22} \mathrm{ClNO}_{3} \mathrm{P}$ 426.1020; Found 426.1019. IR (KBr, cm $\left.{ }^{-1}\right)$ 3396, 3068, 2951, 2849, 1592, 1488, 1375, 1255 , 1177, 1039, 1011, 828, 757, 528, 501. 


\section{Dimethyl (S)-(2-phenyl-3-(p-tolyl)-1, 2-dihydroisoquinolin-1-yl)phosphonate (4eab)}

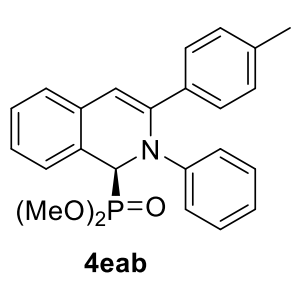

Following the general procedure, reaction were performed with 1e $(0.12$

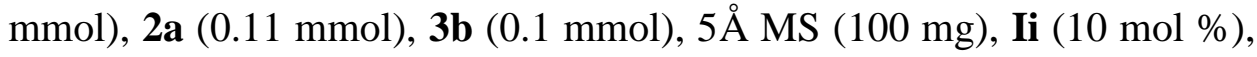
and $\mathrm{Ag}_{2} \mathrm{CO}_{3}(5 \mathrm{~mol} \%)$ in the $1.0 \mathrm{~mL}$ of $\mathrm{MTBE}$ at $-10{ }^{\circ} \mathrm{C}$ for 3 days under argon, followed by column chromatography $(\mathrm{PE} / \mathrm{EA}=1: 1)$ to afford 4eab as yellow oil, $35.7 \mathrm{mg}$ (88\% yield, $82 \% e e) ;[\alpha]_{\mathrm{D}}^{25}=+609.4^{\circ}\left(c=1.0,{ }^{i} \mathrm{PrOH}\right)$;

HPLC condition: Chiralpak IF, $254 \mathrm{~nm}, 0.5 \mathrm{~mL} / \mathrm{min}$, hexane $/{ }^{i} \mathrm{PrOH}=60 / 40, t_{\text {major }}=17.874$ $\min , t_{\text {minor }}=13.712 \mathrm{~min} ;{ }^{1} \mathrm{H}$ NMR $\left(600 \mathrm{MHz}, \mathrm{CDCl}_{3}\right) \delta 7.46(\mathrm{~d}, J=7.9 \mathrm{~Hz}, 2 \mathrm{H}), 7.28-7.22(\mathrm{~m}$, $1 \mathrm{H}), 7.19(\mathrm{~d}, J=7.6 \mathrm{~Hz}, 1 \mathrm{H}), 7.15(\mathrm{t}, J=7.4 \mathrm{~Hz}, 1 \mathrm{H}), 7.13-7.01(\mathrm{~m}, 7 \mathrm{H}), 6.92-6.80(\mathrm{~m}, 1 \mathrm{H})$, $6.50(\mathrm{~s}, 1 \mathrm{H}), 5.46(\mathrm{~d}, J=18.6 \mathrm{~Hz}, 1 \mathrm{H}), 3.74-3.53(\mathrm{~m}, 6 \mathrm{H}), 2.27(\mathrm{~s}, 3 \mathrm{H}) .{ }^{13} \mathrm{C} \mathrm{NMR}(151 \mathrm{MHz}$, $\left.\mathrm{CDCl}_{3}\right) \delta 147.7,142.2,138.0,134.4,133.2,129.1,128.6,128.4,128.4,127.6,127.2,127.2$, $126.5,126.5,124.3,124.2,122.8,122.8,122.5,111.4,64.6,63.6,53.3,53.3,53.2,53.2,21.2$. ${ }^{31} \mathrm{P}$ NMR $\left(243 \mathrm{MHz}, \mathrm{CDCl}_{3}\right) \delta$ 23.19. HRMS (ESI) $\mathrm{m} / \mathrm{z}$ : $[\mathrm{M}+\mathrm{H}]^{+}$Calcd for $\mathrm{C}_{24} \mathrm{H}_{25} \mathrm{NO}_{3} \mathrm{P}$ 406.1567; Found 406.1565. IR (KBr, cm $\left.{ }^{-1}\right)$ 3653, 3023, 2951, 2849, 1596, 1491, 1451, 1379, $1235,1145,1028,811,757,531$.

\section{Dimethyl (S)-(3-(4-methoxyphenyl)-2-phenyl-1, 2-dihydroisoquinolin-1-yl)phosphonate (4gab)}

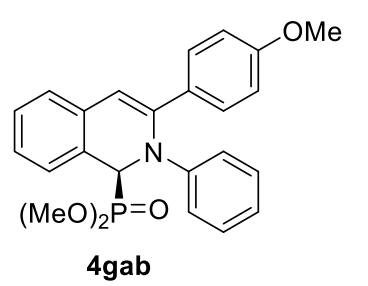

Following the general procedure, reaction were performed with $\mathbf{1 g}(0.12$ mmol), $2 \mathbf{a}(0.11 \mathrm{mmol}), \mathbf{3 b}(0.1 \mathrm{mmol}), 5 \AA \mathrm{MS}(100 \mathrm{mg}), \mathbf{I i}(10 \mathrm{~mol} \%)$, and $\mathrm{Ag}_{2} \mathrm{CO}_{3}(5 \mathrm{~mol} \%)$ in the $1.0 \mathrm{~mL}$ of MTBE at $-10{ }^{\circ} \mathrm{C}$ for 3 days under argon, followed by column chromatography(PE/EA=1:1) to afford 4gab as yellow oil, $40.0 \mathrm{mg}(95 \%$ yield, $90 \% e e) ;[\alpha]_{\mathrm{D}}^{25}=+637.1^{\circ}$ ( $\left.c=1.0,{ }^{i} \mathrm{PrOH}\right)$; HPLC condition: Chiralpak IC, $254 \mathrm{~nm}, 0.5 \mathrm{~mL} / \mathrm{min}$, hexane $/{ }^{i} \mathrm{PrOH}=80 / 20$, $t_{\text {major }}=29.193 \mathrm{~min}, t_{\text {minor }}=32.676 \mathrm{~min} ;{ }^{1} \mathrm{H} \mathrm{NMR}\left(600 \mathrm{MHz}, \mathrm{CDCl}_{3}\right) \delta 7.54-7.45(\mathrm{~m}, 2 \mathrm{H}), 7.27-$ $7.22(\mathrm{~m}, 1 \mathrm{H}), 7.18(\mathrm{~d}, J=7.5 \mathrm{~Hz}, 1 \mathrm{H}), 7.14(\mathrm{t}, J=7.4 \mathrm{~Hz}, 1 \mathrm{H}), 7.12-7.03(\mathrm{~m}, 5 \mathrm{H}), 6.86(\mathrm{t}, J=$ $7.2 \mathrm{~Hz}, 1 \mathrm{H}), 6.81-6.73(\mathrm{~m}, 2 \mathrm{H}), 6.45$ (s, 1H), 5.46 (d, J = 18.8 Hz, 1H), 3.74 (s, 3H), 3.64 (dd, $J=35.1,10.5 \mathrm{~Hz}, 6 \mathrm{H}) .{ }^{13} \mathrm{C} \mathrm{NMR}\left(151 \mathrm{MHz}, \mathrm{CDCl}_{3}\right) \delta 159.6,141.9,129.7,129.0,128.6,128.4$, 128.4, 127.2, 127.1, 126.4, 126.4, 125.3, 124.2, 124.1, 122.9, 122.9, 122.5, 113.8, 110.7, 64.7, 63.6, 55.2, 53.3, 53.3, 53.2, 53.2. ${ }^{31} \mathrm{P}$ NMR (243 $\left.\mathrm{MHz} \mathrm{CDCl}_{3}\right) \delta$ 23.29. HRMS (ESI) m/z: $[\mathrm{M}+\mathrm{H}]^{+}$Calcd for $\mathrm{C}_{24} \mathrm{H}_{25} \mathrm{NO}_{4} \mathrm{P}$ 422.1516; Found 422.1515. IR $\left(\mathrm{KBr}, \mathrm{cm}^{-1}\right) 3435,3010,2952$, 2847, 1598, 1510, 1491, 1380, 1250, 1176, 1054, 1030, 839, 732, 504. 


\section{Dimethyl (S)-(2-phenyl-3-(m-tolyl)-1, 2-dihydroisoquinolin-1-yl)phosphonate (4kab)}

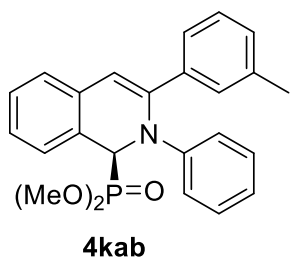

Following the general procedure, reaction were performed with $\mathbf{1 k}(0.12$ mmol), 2a (0.11 mmol), 3b (0.1 mmol), $5 \AA$ MS (100 mg), Ii (10 mol \%), and $\mathrm{Ag}_{2} \mathrm{CO}_{3}(5 \mathrm{~mol} \%)$ in the $1.0 \mathrm{~mL}$ of $\mathrm{MTBE}$ at $-10{ }^{\circ} \mathrm{C}$ for 3 days under argon, followed by column chromatography $(\mathrm{PE} / \mathrm{EA}=1: 1)$ to afford $\mathbf{4 k a b}$ as yellow oil, $33.6 \mathrm{mg}(83 \%$ yield, $90 \% e e) ;[\alpha]_{\mathrm{D}}^{25}=+698.2^{\circ}\left(c=1.0,{ }^{i} \mathrm{PrOH}\right)$;

$\min , t_{\text {minor }}=20.234 \mathrm{~min} ;{ }^{1} \mathrm{H}$ NMR $\left(600 \mathrm{MHz}, \mathrm{CDCl}_{3}\right) \delta 7.42(\mathrm{~s}, 1 \mathrm{H}), 7.34(\mathrm{~d}, J=7.8 \mathrm{~Hz}, 1 \mathrm{H})$, $7.25(\mathrm{qd}, J=4.8,3.8,1.6 \mathrm{~Hz}, 1 \mathrm{H}), 7.20(\mathrm{~d}, J=7.5 \mathrm{~Hz}, 1 \mathrm{H}), 7.15(\mathrm{t}, J=7.4 \mathrm{~Hz}, 1 \mathrm{H}), 7.13-7.04$ (m, 6H), $7.00(\mathrm{~d}, J=7.5 \mathrm{~Hz}, 1 \mathrm{H}), 6.90-6.81(\mathrm{~m}, 1 \mathrm{H}), 6.52(\mathrm{~s}, 1 \mathrm{H}), 5.47(\mathrm{~d}, J=18.7 \mathrm{~Hz}, 1 \mathrm{H})$, $3.65(\mathrm{dd}, J=46.4,10.5 \mathrm{~Hz}, 6 \mathrm{H}), 2.29(\mathrm{~s}, 3 \mathrm{H}) .{ }^{13} \mathrm{C} \mathrm{NMR}\left(151 \mathrm{MHz}, \mathrm{CDCl}_{3}\right) \delta 147.7,142.4$, 137.8, 137.2, 133.1, 128.9, 128.6, 128.4, 128.4, 128.2, 128.2, 127.2, 127.2, 126.6, 126.6, 125.4, 125.4, 125.1, 124.3, 124.3, 122.8, 122.7, 122.5, 111.9, 77.1, 64.6, 63.6, 53.3, 53.2, 53.2, 53.2, 21.5. ${ }^{31} \mathrm{P}$ NMR $\left(243 \mathrm{MHz}, \mathrm{CDCl}_{3}\right) \delta 23.13$. HRMS (ESI) m/z: $[\mathrm{M}+\mathrm{H}]^{+}$Calcd for $\mathrm{C}_{24} \mathrm{H}_{25} \mathrm{NO}_{3} \mathrm{P}$ 406.1567; Found 406.1565. IR (KBr, cm $\left.{ }^{-1}\right)$ 3309, 3058, 2950, 2847, 1595, 1562, 1451, 1382 , $1258,1187,1042,845,789,702,557,492$.

Dimethyl (S)-(3-(3-methoxyphenyl)-2-phenyl-1, 2-dihydroisoquinolin-1-yl)phosphonate (4lab)

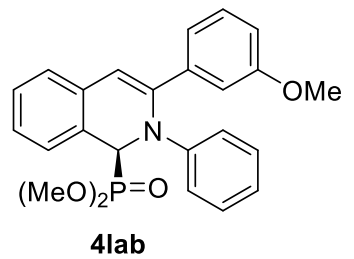

Following the general procedure, reaction were performed with $\mathbf{1 l}(0.12$ mmol), 2a (0.11 mmol), 3b (0.1 mmol), $5 \AA$ MS (100 mg), Ii (10 mol \%), and $\mathrm{Ag}_{2} \mathrm{CO}_{3}(5 \mathrm{~mol} \%)$ in the $1.0 \mathrm{~mL}$ of MTBE at $-10{ }^{\circ} \mathrm{C}$ for 3 days under argon, followed by column chromatography $(\mathrm{PE} / \mathrm{EA}=1: 1)$ to afford 4lab as yellow oil, $39.6 \mathrm{mg}(94 \%$ yield, $91 \% e e) ;[\alpha]_{\mathrm{D}}^{25}=+639.5^{\circ}$

( $\left.c=1.0,{ }^{i} \mathrm{PrOH}\right)$; HPLC condition: Chiralpak IC, $254 \mathrm{~nm}, 0.5 \mathrm{~mL} / \mathrm{min}$, hexane $/{ }^{i} \mathrm{PrOH}=80 / 20$, $t_{\text {major }}=28.421 \mathrm{~min}, t_{\text {minor }}=50.602 \mathrm{~min} ;{ }^{1} \mathrm{H} \mathrm{NMR}\left(600 \mathrm{MHz}, \mathrm{CDCl}_{3}\right) \delta 7.25(\mathrm{dt}, J=6.0,4.0 \mathrm{~Hz}$, $1 \mathrm{H}), 7.21(\mathrm{~d}, J=7.5 \mathrm{~Hz}, 1 \mathrm{H}), 7.19-7.13(\mathrm{~m}, 4 \mathrm{H}), 7.09$ (qd, $J=9.7,3.4 \mathrm{~Hz}, 5 \mathrm{H}), 6.87$ (td, $J=$ 6.9, $1.8 \mathrm{~Hz}, 1 \mathrm{H}), 6.74(\mathrm{dd}, J=8.0,2.6 \mathrm{~Hz}, 1 \mathrm{H}), 6.54(\mathrm{~s}, 1 \mathrm{H}), 5.47$ (d, $J=18.5 \mathrm{~Hz}, 1 \mathrm{H}), 3.71$ (s, $3 \mathrm{H}), 3.65(\mathrm{dd}, J=42.3,10.5 \mathrm{~Hz}, 6 \mathrm{H}) .{ }^{13} \mathrm{C} \mathrm{NMR}\left(151 \mathrm{MHz}, \mathrm{CDCl}_{3}\right) \delta$ 159.6, 142.0, 138.7, 133.0, 129.3, 128.6, 128.4, 128.4, 127.2, 127.1, 126.8, 126.8, 125.5, 124.4, 124.4, 122.6, 122.6, 122.6, 120.2, 114.1, 113.0, 112.1, 64.5, 63.4, 55.1, 53.3, 53.2, 53.2. ${ }^{31} \mathrm{P}$ NMR $\left(243 \mathrm{MHz}, \mathrm{CDCl}_{3}\right) \delta$ 23.21. HRMS (ESI) m/z: $[\mathrm{M}+\mathrm{H}]^{+}$Calcd for $\mathrm{C}_{24} \mathrm{H}_{25} \mathrm{NO}_{4} \mathrm{P}$ 422.1514; Found 422.1516. IR ( $\mathrm{KBr}$, $\mathrm{cm}^{-1}$ ) 3454, 3059, 2952, 2848, 1598, 1491, 1453, 1233, 1178, 1052, 1030, 818, 758, 702, 553, 506. 


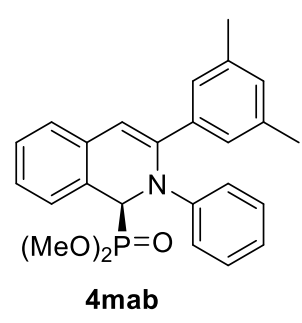

Following the general procedure, reaction were performed with $\mathbf{1 m}(0.12$ mmol), 2a (0.11 mmol), 3b (0.1 mmol), $5 \AA$ MS (100 mg), Ii (10 mol \%), and $\mathrm{Ag}_{2} \mathrm{CO}_{3}(5 \mathrm{~mol} \%)$ in the $1.0 \mathrm{~mL}$ of MTBE at $-10{ }^{\circ} \mathrm{C}$ for 3 days under argon, followed by column chromatography(PE/EA=1:1) to afford $\mathbf{4 m a b}$ as yellow oil, $40.2 \mathrm{mg}(96 \%$ yield, $88 \% e e) ;[\alpha]_{\mathrm{D}}^{25}=+622.7^{\circ}(c=1.0$,

$\left.{ }^{i} \mathrm{PrOH}\right)$; HPLC condition: Chiralpak IC, $254 \mathrm{~nm}, 0.5 \mathrm{~mL} / \mathrm{min}$, hexane $/{ }^{i} \mathrm{PrOH}=80 / 20, t_{\text {major }}=$ $18.246 \mathrm{~min}, t_{\text {minor }}=17.242 \mathrm{~min} ;{ }^{1} \mathrm{H}$ NMR $\left(600 \mathrm{MHz}, \mathrm{CDCl}_{3}\right) \delta 7.26-7.22(\mathrm{~m}, 1 \mathrm{H}), 7.19(\mathrm{~d}, J=$ $6.3 \mathrm{~Hz}, 3 \mathrm{H}), 7.14(\mathrm{t}, J=7.4 \mathrm{~Hz}, 1 \mathrm{H}), 7.12-7.04(\mathrm{~m}, 5 \mathrm{H}), 6.88-6.79(\mathrm{~m}, 2 \mathrm{H}), 6.50(\mathrm{~s}, 1 \mathrm{H}), 5.46$ $(\mathrm{d}, J=18.8 \mathrm{~Hz}, 1 \mathrm{H}), 3.64(\mathrm{dd}, J=53.9,10.5 \mathrm{~Hz}, 6 \mathrm{H}), 2.22(\mathrm{~s}, 6 \mathrm{H}) .{ }^{13} \mathrm{C} \mathrm{NMR}\left(151 \mathrm{MHz}, \mathrm{CDCl}_{3}\right)$ $\delta 142.5,137.6,137.2,133.2,129.9,128.6,128.4,128.4,127.2,127.2,126.6,126.5,125.6$, $125.4,124.3,124.3,122.8,122.7,122.5,111.8,64.7,63.6,53.3,53.2,21.3 .{ }^{31} \mathrm{P}$ NMR (243 $\left.\mathrm{MHz}, \mathrm{CDCl}_{3}\right) \delta$ 23.13. HRMS (ESI) $\mathrm{m} / \mathrm{z}$ : $[\mathrm{M}+\mathrm{H}]^{+}$Calcd for $\mathrm{C}_{25} \mathrm{H}_{27} \mathrm{NO}_{3} \mathrm{P}$ 420.1723; Found 420.1722. IR $\left(\mathrm{KBr}, \mathrm{cm}^{-1}\right)$ 3436, 3015, 2950, 2913, 2847, 1736, 1595, 1562, 1492, 1454, 1388, $1284,1238,1055,1030,852,819,756,702,558,537$.

\section{Dimethyl (S)-(2-(4-(benzyloxy)phenyl)-3-phenyl-1, 2-dihydroisoquinolin-1-yl) phosphonate (4adb)}

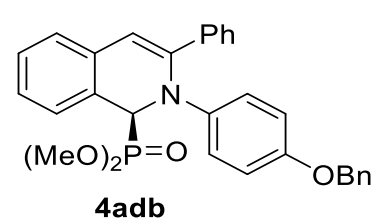

$4 a d b$
Following the general procedure, reaction were performed with 1a (0.12 mmol), 2d (0.11 mmol), 3b (0.1 mmol), $5 \AA$ MS (100 mg), Ii (10 $\mathrm{mol} \%$ ), and $\mathrm{Ag}_{2} \mathrm{CO}_{3}(5 \mathrm{~mol} \%)$ in the $1.0 \mathrm{~mL}$ of MTBE at $-10{ }^{\circ} \mathrm{C}$ for 3 days under argon, followed by column chromatography $(\mathrm{PE} / \mathrm{EA}=1: 1)$ to afford 4adb as yellow oil, $42.3 \mathrm{mg}(85 \%$ yield, 93\% ee $)$; $[\alpha]_{\mathrm{D}}^{25}=+594.8^{\circ}(c=1.0$, EA); HPLC condition: Chiralpak IC, $254 \mathrm{~nm}, 0.5 \mathrm{~mL} / \mathrm{min}$, hexane $/{ }^{i} \mathrm{PrOH}=80 / 20, t_{\text {major }}=27.097 \mathrm{~min}, t_{\text {minor }}$ $=31.878 \mathrm{~min} ;{ }^{1} \mathrm{H}$ NMR $\left(600 \mathrm{MHz}, \mathrm{CDCl}_{3}\right) \delta 7.61-7.53(\mathrm{~m}, 2 \mathrm{H}), 7.37-7.30(\mathrm{~m}, 4 \mathrm{H}), 7.26$ (ddd, $J=23.9,9.1,5.5 \mathrm{~Hz}, 4 \mathrm{H}), 7.17(\mathrm{dd}, J=26.0,7.3 \mathrm{~Hz}, 3 \mathrm{H}), 7.08(\mathrm{~d}, J=7.6 \mathrm{~Hz}, 1 \mathrm{H}), 7.06-7.00$ (m, 2H), 6.76-6.65 (m, 2H), $6.47(\mathrm{~s}, 1 \mathrm{H}), 5.36(\mathrm{~d}, J=18.8 \mathrm{~Hz}, 1 \mathrm{H}), 4.89$ (d, $J=3.5 \mathrm{~Hz}, 2 \mathrm{H})$, $3.65(\mathrm{dd}, J=38.5,10.4 \mathrm{~Hz}, 6 \mathrm{H}) .{ }^{13} \mathrm{C} \mathrm{NMR}\left(151 \mathrm{MHz}, \mathrm{CDCl}_{3}\right) \delta 154.7,142.6,137.3,137.0$, 128.5, 128.4, 128.4, 128.3, 128.0, 127.9, 127.8, 127.4, 127.3, 127.2, 126.6, 124.8, 124.6, 124.5, 124.2, 124.2, 115.0, 111.0, 70.2, 65.2, 64.1, 53.4, 53.3, 53.2, 53.2. ${ }^{31} \mathrm{P} \mathrm{NMR}\left(243 \mathrm{MHz}, \mathrm{CDCl}_{3}\right)$ $\delta$ 23.09. HRMS (ESI) m/z: $[\mathrm{M}+\mathrm{H}]^{+}$Calcd for $\mathrm{C}_{30} \mathrm{H}_{29} \mathrm{NO}_{4} \mathrm{P} 498.1829$; Found 498.1825. IR (KBr, $\mathrm{cm}^{-1}$ ) 3453, 3059, 2951, 2850, 1608, 1562, 1506, 1453, 1383, 1235, 1177, 1054, 1028, 830, $766,698,551,519$. 


\section{Dimethyl (S)-(2-(4-fluorophenyl)-3-phenyl-1, 2-dihydroisoquinolin-1-yl)phosphonate (4aeb)}

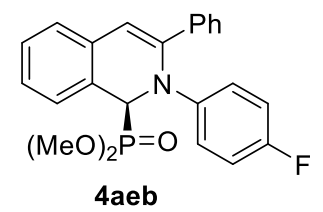

Following the general procedure, reaction were performed with 1a $(0.12$ mmol), 2e (0.11 mmol), 3b (0.1 mmol), $5 \AA$ MS (100 mg), Ii (10 mol \%), and $\mathrm{Ag}_{2} \mathrm{CO}_{3}(5 \mathrm{~mol} \%)$ in the $1.0 \mathrm{~mL}$ of MTBE at $-10{ }^{\circ} \mathrm{C}$ for 3 days under argon, followed by column chromatography(PE/EA=1:1) to afford 4aeb as yellow solid, $40.1 \mathrm{mg}$ (98\% yield, 89\% ee); mp: 146.2-148.0, $[\alpha]_{\mathrm{D}}^{25}=+771.8^{\circ}(c=1.0, \mathrm{EA})$; HPLC condition: Chiralpak IC, $254 \mathrm{~nm}, 0.5 \mathrm{~mL} / \mathrm{min}$, hexane $/{ }^{i} \mathrm{PrOH}=80 / 20, t_{\text {major }}=20.637$ min, $t_{\text {minor }}=19.611 \mathrm{~min} ;{ }^{1} \mathrm{H}$ NMR $\left(600 \mathrm{MHz}, \mathrm{CDCl}_{3}\right) \delta 7.59-7.51(\mathrm{~m}, 2 \mathrm{H}), 7.30-7.16(\mathrm{~m}, 6 \mathrm{H})$, $7.11(\mathrm{~d}, J=7.6 \mathrm{~Hz}, 1 \mathrm{H}), 7.08-7.03(\mathrm{~m}, 2 \mathrm{H}), 6.78(\mathrm{t}, J=8.7 \mathrm{~Hz}, 2 \mathrm{H}), 6.50(\mathrm{~s}, 1 \mathrm{H}), 5.37(\mathrm{~d}, J=$ $18.7 \mathrm{~Hz}, 1 \mathrm{H}), 3.65(\mathrm{dd}, J=29.5,10.4 \mathrm{~Hz}, 6 \mathrm{H}) .{ }^{13} \mathrm{C} \mathrm{NMR}\left(151 \mathrm{MHz}, \mathrm{CDCl}_{3}\right) \delta 159.5,142.2$, $137.0,132.9,128.5,128.5,128.4,128.2$, 127.8, 127.2 , 127.2 , 126.8, 126.8, 125.0, 124.5, 124.5, $124.5,124.5,124.4,124.4,115.4,115.2,111.7,65.0,63.9,53.4,53.4,53.2,53.2 .{ }^{31} \mathrm{P}$ NMR $\left(243 \mathrm{MHz}, \mathrm{CDCl}_{3}\right) \delta$ 22.84. HRMS (ESI) $\mathrm{m} / \mathrm{z}$ : $[\mathrm{M}+\mathrm{H}]^{+}$Calcd for $\mathrm{C}_{23} \mathrm{H}_{22} \mathrm{FNO}_{3} \mathrm{P}$ 410.1316; Found 410.1313.

\section{Dimethyl (S)-(3-phenyl-2-(m-tolyl)-1, 2-dihydroisoquinolin-1-yl)phosphonate (4afb)}

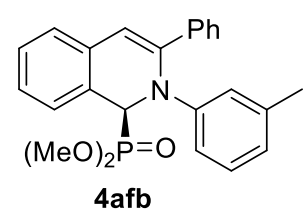

Following the general procedure, reaction were performed with $1 \mathbf{a}(0.12$ mmol), $2 \mathbf{f}(0.11 \mathrm{mmol}), \mathbf{3 b}(0.1 \mathrm{mmol}), 5 \AA \mathrm{MS}(100 \mathrm{mg}), \mathbf{I i}(10 \mathrm{~mol} \%)$, and $\mathrm{Ag}_{2} \mathrm{CO}_{3}\left(5 \mathrm{~mol} \%\right.$ ) in the $1.0 \mathrm{~mL}$ of MTBE at $-10{ }^{\circ} \mathrm{C}$ for 3 days under argon, followed by column chromatography(PE/EA=1:1) to afford $\mathbf{4 a f b}$ as yellow oil, $36.9 \mathrm{mg}(91 \%$ yield, $89 \% e e)$; $[\alpha]_{\mathrm{D}}^{25}=+781.3^{\circ}(c=1.0$, EA); HPLC condition: Chiralpak IC, $254 \mathrm{~nm}, 0.5 \mathrm{~mL} / \mathrm{min}$, hexane $/{ }^{i} \mathrm{PrOH}=80 / 20, t_{\text {major }}=22.311 \mathrm{~min}, t_{\text {minor }}=20.940$ min; ${ }^{1} \mathrm{H}$ NMR $\left(600 \mathrm{MHz}, \mathrm{CDCl}_{3}\right) \delta$ 7.54-7.47 (m, 2H), 7.20-7.07 (m, 6H), $7.04(\mathrm{~d}, J=7.6 \mathrm{~Hz}$, $1 \mathrm{H}), 6.92-6.81(\mathrm{~m}, 2 \mathrm{H}), 6.75(\mathrm{dd}, J=8.2,2.4 \mathrm{~Hz}, 1 \mathrm{H}), 6.60(\mathrm{~d}, J=7.5 \mathrm{~Hz}, 1 \mathrm{H}), 6.45(\mathrm{~s}, 1 \mathrm{H})$, $5.40(\mathrm{~d}, J=18.8 \mathrm{~Hz}, 1 \mathrm{H}), 3.61(\mathrm{~d}, J=10.5 \mathrm{~Hz}, 3 \mathrm{H}), 3.53(\mathrm{~d}, J=10.4 \mathrm{~Hz}, 3 \mathrm{H}), 2.11(\mathrm{~s}, 3 \mathrm{H}) .{ }^{13} \mathrm{C}$ NMR $\left(151 \mathrm{MHz}, \mathrm{CDCl}_{3}\right) \delta 141.3,137.4,136.3,127.4,127.4,127.3,127.3,127.0,126.6,126.2$, 126.2 , 125.7, 125.7, 124.4, 123.3, 123.3, 122.4, 122.3, 122.3, 119.1, 119.0, 110.9, 63.6, 62.5, 52.3, 52.3, 52.2, 52.2, 20.4. ${ }^{31} \mathrm{P}$ NMR (243 MHz, $\left.\mathrm{CDCl}_{3}\right) \delta 23.20$. HRMS (ESI) m/z: $[\mathrm{M}+\mathrm{H}]^{+}$ Calcd for $\mathrm{C}_{24} \mathrm{H}_{25} \mathrm{NO}_{3} \mathrm{P}$ 406.1567; Found 406.1569. IR (KBr, $\left.\mathrm{cm}^{-1}\right)$ 3396, 3060, 2949, 2848, 1600, 1562, 1489, 1452, 1378, 1252, 1179,1053, 1025, 824, 767, 697, 552, 525. 
Dimethyl (S)-(5-fluoro-2-(4-isopropoxyphenyl)-3-phenyl-1, 2-dihydroisoquinolin-1-yl)ph osphonate (4agb)

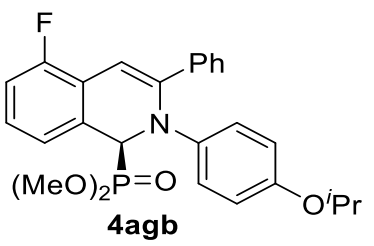

Following the general procedure, reaction were performed with $\mathbf{1}$ (0.12 mmol), $2 \mathbf{c}(0.11 \mathrm{mmol}), 3 \mathbf{b}(0.1 \mathrm{mmol}), 5 \AA \mathrm{MS}(100 \mathrm{mg}), \mathbf{I i}(10$ mol \%), and $\mathrm{Ag}_{2} \mathrm{CO}_{3}(5 \mathrm{~mol} \%)$ in the $1.0 \mathrm{~mL}$ of MTBE at $-10{ }^{\circ} \mathrm{C}$ for

3 days under argon, followed by column chromatography (PE/EA=1:1) to afford 4agb as yellow solid, $13.1 \mathrm{mg}$ (28\% yield, 84\% ee); mp: 60.5-61.7 ${ }^{\circ} \mathrm{C}$, $[\alpha]_{\mathrm{D}}^{25}=+341.4^{\circ}(c=1.0$, EA); HPLC condition: Chiralpak IC, $254 \mathrm{~nm}, 0.5 \mathrm{~mL} / \mathrm{min}$, hexane/ ${ }^{i} \mathrm{PrOH}=80 / 20, t_{\text {major }}=16.391 \mathrm{~min}, t_{\text {minor }}=14.450 \mathrm{~min} ;{ }^{1} \mathrm{H} \mathrm{NMR}\left(600 \mathrm{MHz}, \mathrm{CDCl}_{3}\right) \delta 7.64-$ $7.55(\mathrm{~m}, 2 \mathrm{H}), 7.24(\mathrm{t}, J=9.2 \mathrm{~Hz}, 2 \mathrm{H}), 7.19(\mathrm{t}, J=7.1 \mathrm{~Hz}, 1 \mathrm{H}), 7.10(\mathrm{td}, J=7.8,5.2 \mathrm{~Hz}, 1 \mathrm{H})$, 7.05-7.00 (m, 2H), $6.98(\mathrm{t}, J=9.1 \mathrm{~Hz}, 1 \mathrm{H}), 6.88(\mathrm{dd}, J=7.6,2.1 \mathrm{~Hz}, 1 \mathrm{H}), 6.62(\mathrm{dd}, J=6.6,2.2$ $\mathrm{Hz}, 3 \mathrm{H}), 5.38(\mathrm{~d}, J=19.0 \mathrm{~Hz}, 1 \mathrm{H}), 4.35(\mathrm{~h}, J=6.0 \mathrm{~Hz}, 1 \mathrm{H}), 3.70(\mathrm{~d}, J=10.5 \mathrm{~Hz}, 3 \mathrm{H}), 3.64(\mathrm{~d}$, $J=10.5 \mathrm{~Hz}, 3 \mathrm{H}), 1.22(\mathrm{dd}, J=6.1,1.6 \mathrm{~Hz}, 6 \mathrm{H}) .{ }^{13} \mathrm{C} \mathrm{NMR}\left(151 \mathrm{MHz}, \mathrm{CDCl}_{3}\right) \delta 154.0,143.4$, 140.9, 137.1, 128.3, 128.3, 128.0, 127.0, 124.7, 123.0-122.6 (m), 116.1, 114.7, 103.1, 70.1, 65.2-63.4 (m), 53.3, 22.0. ${ }^{31} \mathrm{P}$ NMR (243 MHz, $\left.\mathrm{CDCl}_{3}\right) \delta 22.64$. HRMS (ESI) m/z: $[\mathrm{M}+\mathrm{H}]^{+}$ Calcd for $\mathrm{C}_{26} \mathrm{H}_{28} \mathrm{FNO}_{4} \mathrm{P} 468.1734$; Found 468.1733 .

Dimethyl (S)-(6-fluoro-2-(4-isopropoxyphenyl)-3-phenyl-1, 2-dihydroisoquinolin-1-yl)ph osphonate (4ahb)

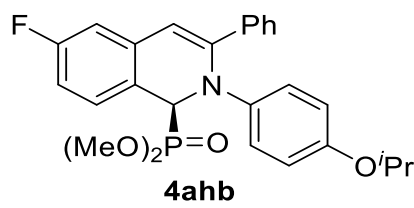

Following the general procedure, reaction were performed with $\mathbf{1}$ (0.12 mmol), 2c (0.11 mmol), 3b (0.1 mmol), $5 \AA$ MS (100 mg), Ii (10 mol \%), and $\mathrm{Ag}_{2} \mathrm{CO}_{3}(5 \mathrm{~mol} \%)$ in the $1.0 \mathrm{~mL}$ of MTBE at -

$10{ }^{\circ} \mathrm{C}$ for 3 days under argon, followed by column chromatography (PE/EA=1:1) to afford 4ahb as yellow solid, $44.4 \mathrm{mg}$ (95\% yield, 94\% ee); mp: 57.2-58.3 ${ }^{\circ} \mathrm{C}$, $[\alpha]_{\mathrm{D}}^{25}=+688.9^{\circ}(c=1.0$, EA); HPLC condition: Chiralpak IC, $254 \mathrm{~nm}, 0.5 \mathrm{~mL} / \mathrm{min}$, hexane/ ${ }^{i} \mathrm{PrOH}=84 / 16, t_{\text {major }}=22.221 \mathrm{~min}, t_{\text {minor }}=20.954 \mathrm{~min} ;{ }^{1} \mathrm{H} \mathrm{NMR}\left(600 \mathrm{MHz}, \mathrm{CDCl}_{3}\right) \delta 7.55(\mathrm{dt}$, $J=6.1,1.5 \mathrm{~Hz}, 2 \mathrm{H}), 7.25-7.17(\mathrm{~m}, 3 \mathrm{H}), 7.04(\mathrm{td}, J=5.7,2.8 \mathrm{~Hz}, 1 \mathrm{H}), 7.02-6.97(\mathrm{~m}, 2 \mathrm{H}), 6.88$ $(\mathrm{dd}, J=9.4,2.6 \mathrm{~Hz}, 1 \mathrm{H}), 6.84(\mathrm{td}, J=8.4,2.6 \mathrm{~Hz}, 1 \mathrm{H}), 6.66-6.58(\mathrm{~m}, 2 \mathrm{H}), 6.38(\mathrm{~s}, 1 \mathrm{H}), 5.33$ (d, $J=18.4 \mathrm{~Hz}, 1 \mathrm{H}), 4.35$ (hept, $J=6.1 \mathrm{~Hz}, 1 \mathrm{H}), 3.70(\mathrm{~d}, J=10.5 \mathrm{~Hz}, 3 \mathrm{H}), 3.64$ (d, $J=10.5$ $\mathrm{Hz}, 3 \mathrm{H}), 1.22(\mathrm{dd}, J=6.1,1.5 \mathrm{~Hz}, 6 \mathrm{H}) .{ }^{13} \mathrm{C} \mathrm{NMR}\left(151 \mathrm{MHz}, \mathrm{CDCl}_{3}\right) \delta 154.0,143.9,140.9$, 137.0, 128.6, 128.3, 128.3, 128.0, 124.8, 120.2, 116.0, 113.1, 110.6, 110.3, 109.7, 70.1, 64.2, 53.3, 22.0. ${ }^{31} \mathrm{P}$ NMR (243 MHz, $\left.\mathrm{CDCl}_{3}\right) \delta 22.83$ (d, $\left.J=4.7 \mathrm{~Hz}\right)$. HRMS (ESI) m/z: $[\mathrm{M}+\mathrm{H}]^{+}$ Calcd for $\mathrm{C}_{26} \mathrm{H}_{28} \mathrm{FNO}_{4} \mathrm{P}$ 468.1734; Found 468.1736. 


\section{Dimethyl (S)-(2-(4-isopropoxyphenyl)-6-methyl-3-phenyl-1, 2-dihydroisoquinolin-1-yl) phosphonate (4aib)}

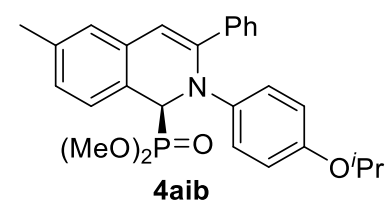

Following the general procedure, reaction were performed with 1 ( 0.12 mmol), $2 \mathbf{c}(0.11 \mathrm{mmol}), \mathbf{3 b}(0.1 \mathrm{mmol}), 5 \AA \mathrm{MS}(100 \mathrm{mg}), \mathbf{I i}(10 \mathrm{~mol} \%)$, and $\mathrm{Ag}_{2} \mathrm{CO}_{3}(5 \mathrm{~mol} \%)$ in the $1.0 \mathrm{~mL}$ of MTBE at $-10{ }^{\circ} \mathrm{C}$ for 3 days under argon, followed by column chromatography $(\mathrm{PE} / \mathrm{EA}=1: 1)$ to afford 4aib as yellow oil, $42.6 \mathrm{mg}(92 \%$ yield, $94 \% e e)$; $[\alpha]_{\mathrm{D}}^{25}=+597.6^{\circ}(c=1.0$, EA); HPLC condition: Chiralpak IC, $254 \mathrm{~nm}, 0.5 \mathrm{~mL} / \mathrm{min}$, hexane $/{ }^{i} \mathrm{PrOH}=86 / 14, t_{\text {major }}=36.207 \mathrm{~min}, t_{\text {minor }}$ $=34.811 \mathrm{~min} ;{ }^{1} \mathrm{H} \mathrm{NMR}\left(600 \mathrm{MHz}, \mathrm{CDCl}_{3}\right) \delta 7.56(\mathrm{~d}, J=7.6 \mathrm{~Hz}, 2 \mathrm{H}), 7.22(\mathrm{t}, J=7.5 \mathrm{~Hz}, 2 \mathrm{H})$, $7.17(\mathrm{t}, J=7.3 \mathrm{~Hz}, 1 \mathrm{H}), 7.06-6.91(\mathrm{~m}, 5 \mathrm{H}), 6.61(\mathrm{~d}, J=8.5 \mathrm{~Hz}, 2 \mathrm{H}), 6.42(\mathrm{~s}, 1 \mathrm{H}), 5.33(\mathrm{~d}, J=$ $18.5 \mathrm{~Hz}, 1 \mathrm{H}), 4.34$ (hept, $J=6.1 \mathrm{~Hz}, 1 \mathrm{H}), 3.65(\mathrm{dd}, J=34.0,10.5 \mathrm{~Hz}, 6 \mathrm{H}), 2.33(\mathrm{~d}, J=2.1 \mathrm{~Hz}$, $3 \mathrm{H}), 1.21(\mathrm{dd}, J=6.1,2.8 \mathrm{~Hz}, 6 \mathrm{H}) .{ }^{13} \mathrm{C} \mathrm{NMR}\left(151 \mathrm{MHz}, \mathrm{CDCl}_{3}\right) \delta 153.7,142.6,141.3,138.0$, 137.4, 128.3, 127.9, 127.8, 127.4, 127.4, 127.1, 127.1, 124.9, 124.8, 124.6, 124.5, 121.9, 116.0, $110.9,70.1,65.0,63.9,53.3,53.3,53.2,53.1,22.1,22.0,21.3 .{ }^{31} \mathrm{P}$ NMR $\left(243 \mathrm{MHz}, \mathrm{CDCl}_{3}\right) \delta$ 23.35. HRMS (ESI) m/z: $[\mathrm{M}+\mathrm{H}]^{+}$Calcd for $\mathrm{C}_{27} \mathrm{H}_{31} \mathrm{NO}_{4} \mathrm{P}$ 464.1985; Found 464.1988.

Dimethyl (S)-(2-(4-isopropoxyphenyl)-6-methoxy-3-phenyl-1, 2-dihydroisoquinolin-1-yl) Phosphonate (4ajb)

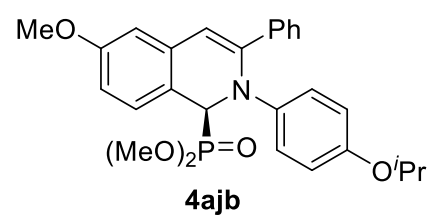

Following the general procedure, reaction were performed with $\mathbf{1}$ (0.12 mmol), $2 \mathbf{c}(0.11 \mathrm{mmol}), \mathbf{3 b}(0.1 \mathrm{mmol}), 5 \AA \mathrm{MS}(100 \mathrm{mg}), \mathbf{I i}$ (10 mol \%), and $\mathrm{Ag}_{2} \mathrm{CO}_{3}(5 \mathrm{~mol} \%)$ in the $1.0 \mathrm{~mL}$ of MTBE at $10{ }^{\circ} \mathrm{C}$ for 3 days under argon, followed by column chromatography(PE/EA=1:2) to afford 4ajb as yellow oil, $24.9 \mathrm{mg}(52 \%$ yield, 63\% ee $)$; $[\alpha]_{\mathrm{D}}^{25}$ $=+313.4^{\circ}\left(c=1.0\right.$, EA); HPLC condition: Chiralpak IC, $254 \mathrm{~nm}, 0.5 \mathrm{~mL} / \mathrm{min}$, hexane $/{ }^{i} \mathrm{PrOH}$ $=80 / 20, t_{\text {major }}=30.820 \mathrm{~min}, t_{\text {minor }}=29.187 \mathrm{~min} ;{ }^{1} \mathrm{H} \mathrm{NMR}\left(600 \mathrm{MHz}, \mathrm{CDCl}_{3}\right) \delta 7.56(\mathrm{dt}, J=$ 6.1, 1.4 Hz, 2H), 7.25-7.20 (m, 2H), 7.20-7.16 (m, 1H), 7.04-6.96 (m, 3H), 6.78-6.68 (m, 2H), 6.65-6.57 (m, 2H), $6.41(\mathrm{~s}, 1 \mathrm{H}), 5.31(\mathrm{~d}, J=17.9 \mathrm{~Hz}, 1 \mathrm{H}), 4.34$ (hept, $J=6.1 \mathrm{~Hz}, 1 \mathrm{H}), 3.81$ (s, $3 \mathrm{H}), 3.69(\mathrm{~d}, J=10.4 \mathrm{~Hz}, 3 \mathrm{H}), 3.62(\mathrm{~d}, J=10.4 \mathrm{~Hz}, 3 \mathrm{H}), 1.22(\mathrm{dd}, J=6.1,2.2 \mathrm{~Hz}, 6 \mathrm{H}) .{ }^{13} \mathrm{C}$ NMR (151 MHz, $\left.\mathrm{CDCl}_{3}\right) \delta 159.8,153.8,143.2,141.2,137.4,134.4,128.3,128.2,128.0,127.9$, 124.6, 117.1, 116.0, 112.4, 110.8, 109.1, 70.1 , 64.2, 55.2, 53.2, 22.0. ${ }^{31} \mathrm{P}$ NMR (243 MHz, $\left.\mathrm{CDCl}_{3}\right) \delta$ 23.31. HRMS (ESI) m/z: [M+H] ${ }^{+}$Calcd for $\mathrm{C}_{27} \mathrm{H}_{31} \mathrm{NO}_{5} \mathrm{P} 480.1934$; Found 480.1935. 


\section{Dimethyl (S)-(2-(4-isopropoxyphenyl)-7-methyl-3-phenyl-1, 2-dihydroisoquinolin-1-yl)}

phosphonate (4akb)

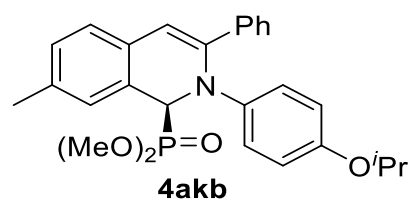

4 akb

Following the general procedure, reaction were performed with 1 (0.12 mmol), 2c (0.11 mmol), 3b (0.1 mmol), $5 \AA$ MS (100 mg), Ii (10 $\mathrm{mol} \%)$, and $\mathrm{Ag}_{2} \mathrm{CO}_{3}(5 \mathrm{~mol} \%)$ in the $1.0 \mathrm{~mL}$ of MTBE at $-10^{\circ} \mathrm{C}$ for 3 days under argon, followed by column chromatography $(\mathrm{PE} / \mathrm{EA}=1: 1)$ to afford 4akb as yellow oil, $39.9 \mathrm{mg}(86 \%$ yield, $90 \% e e) ;[\alpha]_{\mathrm{D}}^{25}=+756.4^{\circ}(c=$ 1.0, EA); HPLC condition: Chiralpak IF, $254 \mathrm{~nm}, 0.5 \mathrm{~mL} / \mathrm{min}$, hexane $/{ }^{i} \mathrm{PrOH}=70 / 30, t_{\text {major }}=$ $26.669 \mathrm{~min}, t_{\text {minor }}=13.045 \mathrm{~min} ;{ }^{1} \mathrm{H} \mathrm{NMR}\left(600 \mathrm{MHz}, \mathrm{CDCl}_{3}\right) \delta 7.61-7.51(\mathrm{~m}, 2 \mathrm{H}), 7.22(\mathrm{t}, J=$ $7.7 \mathrm{~Hz}, 2 \mathrm{H}), 7.19-7.14(\mathrm{~m}, 1 \mathrm{H}), 7.10$ (d, $J=7.7 \mathrm{~Hz}, 1 \mathrm{H}), 7.06$ (t, $J=4.8 \mathrm{~Hz}, 1 \mathrm{H}), 7.02-6.96$ (m, 2H), $6.91(\mathrm{~s}, 1 \mathrm{H}), 6.65-6.57(\mathrm{~m}, 2 \mathrm{H}), 6.47(\mathrm{~s}, 1 \mathrm{H}), 5.32$ (d, $J=19.0 \mathrm{~Hz}, 1 \mathrm{H}), 4.33$ (hept, $J$ $=6.1 \mathrm{~Hz}, 1 \mathrm{H}), 3.69(\mathrm{~d}, J=10.5 \mathrm{~Hz}, 3 \mathrm{H}), 3.61(\mathrm{~d}, J=10.4 \mathrm{~Hz}, 3 \mathrm{H}), 2.30(\mathrm{~s}, 3 \mathrm{H}), 1.21(\mathrm{dd}, J=$ 6.1, $2.7 \mathrm{~Hz}, 6 \mathrm{H}) .{ }^{13} \mathrm{C} \mathrm{NMR}\left(151 \mathrm{MHz}, \mathrm{CDCl}_{3}\right) \delta 153.6,141.8,137.4,130.5,129.1,129.1,128.2$, 127.9, 127.8, 127.8, 127.7, 124.9, 124.4, 124.4, 124.2, 124.1, 116.1, 111.0, 70.1, 65.2, 64.1, 53.3, 53.2, 22.1, 22.0, 21.3. ${ }^{31} \mathrm{P}$ NMR $\left(243 \mathrm{MHz}, \mathrm{CDCl}_{3}\right) \delta 23.42$. HRMS (ESI) m/z: $[\mathrm{M}+\mathrm{H}]^{+}$ Calcd for $\mathrm{C}_{27} \mathrm{H}_{31} \mathrm{NO}_{4} \mathrm{P} 464.1985$; Found 464.1985.

Dimethyl (S)-(2-(4-isopropoxyphenyl)-7-methoxy-3-phenyl-1, 2-dihydroisoquinolin-1-yl) phosphonate (4alb)

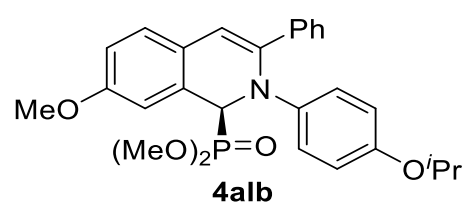

Following the general procedure, reaction were performed with 1 (0.12 mmol), 2c (0.11 mmol), 3b (0.1 mmol), $5 \AA$ MS (100 mg), Ii $(10 \mathrm{~mol} \%)$, and $\mathrm{Ag}_{2} \mathrm{CO}_{3}(5 \mathrm{~mol} \%)$ in the $1.0 \mathrm{~mL}$ of $\mathrm{MTBE}$ at

$-10{ }^{\circ} \mathrm{C}$ for 3 days under argon, followed by column chromatography(PE/EA=1:2) to afford 4alb as yellow oil, $39.8 \mathrm{mg}(83 \%$ yield, $92 \% e e) ;[\alpha]_{\mathrm{D}}^{25}$ $=+595.9^{\circ}(c=1.0$, EA); HPLC condition: Chiralpak IC, $254 \mathrm{~nm}, 0.5 \mathrm{~mL} / \mathrm{min}$, hexane $/ \mathrm{PrOH}$ $=80 / 20, t_{\text {major }}=47.393 \mathrm{~min}, t_{\text {minor }}=44.422 \mathrm{~min} ;{ }^{1} \mathrm{H} \mathrm{NMR}\left(600 \mathrm{MHz}, \mathrm{CDCl}_{3}\right) \delta 7.60-7.53(\mathrm{~m}$, $2 \mathrm{H}), 7.22(\mathrm{t}, J=7.6 \mathrm{~Hz}, 2 \mathrm{H}), 7.15(\mathrm{dd}, J=20.8,7.8 \mathrm{~Hz}, 2 \mathrm{H}), 7.04-6.94(\mathrm{~m}, 2 \mathrm{H}), 6.82(\mathrm{dt}, J=$ 8.5, 2.4 Hz, 1H), 6.67 (q, $J=2.6 \mathrm{~Hz}, 1 \mathrm{H}), 6.64-6.59$ (m, 2H), 6.47 (s, 1H), 5.32 (d, $J=19.4$ $\mathrm{Hz}, 1 \mathrm{H}), 4.34$ (hept, $J=6.0 \mathrm{~Hz}, 1 \mathrm{H}), 3.77(\mathrm{~s}, 3 \mathrm{H}), 3.70$ (d, $J=10.5 \mathrm{~Hz}, 3 \mathrm{H}), 3.63$ (d, $J=10.4$ $\mathrm{Hz}, 3 \mathrm{H}), 1.22(\mathrm{dd}, J=6.1,2.7 \mathrm{~Hz}, 6 \mathrm{H}) .{ }^{13} \mathrm{C} \mathrm{NMR}\left(151 \mathrm{MHz}, \mathrm{CDCl}_{3}\right) \delta 158.7,153.6,141.4$, 140.6, 137.4, 128.2, 127.6, 127.5, 125.4, 125.4, 124.2, 124.2, 116.1, 114.2, 114.2, 112.8, 112.8 , 110.8, 70.1, 65.3, 64.2, 55.4, 53.3, 53.3, 53.2, 53.2, 22.1, 22.0. ${ }^{31} \mathrm{P}$ NMR $\left(243 \mathrm{MHz}, \mathrm{CDCl}_{3}\right) \delta$ 23.18. HRMS (ESI) m/z: $[\mathrm{M}+\mathrm{H}]^{+}$Calcd for $\mathrm{C}_{27} \mathrm{H}_{31} \mathrm{NO}_{5} \mathrm{P} 480.1934$; Found 480.1938. 
Dimethyl (S)-(7-chloro-2-(4-isopropoxyphenyl)-3-phenyl-1, 2-dihydroisoquinolin-1-yl)ph osphonate (4amb)

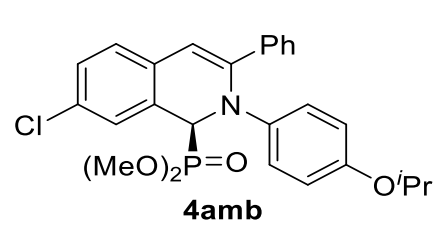

Following the general procedure, reaction were performed with $\mathbf{1}$ (0.12 mmol), 2c (0.11 mmol), 3b (0.1 mmol), $5 \AA$ MS (100 mg), Ii (10 mol \%), and $\mathrm{Ag}_{2} \mathrm{CO}_{3}(5 \mathrm{~mol} \%)$ in the $1.0 \mathrm{~mL}$ of MTBE at $10{ }^{\circ} \mathrm{C}$ for 3 days under argon, followed by column chromatography $(\mathrm{PE} / \mathrm{EA}=1: 1)$ to afford $\mathbf{4 a m b}$ as yellow solid, $45.0 \mathrm{mg}$ (93\% yield, 92\% ee); mp: 169.2$172.8^{\circ} \mathrm{C},[\alpha]_{\mathrm{D}}^{25}=+732.5^{\circ}(c=1.0$, EA); HPLC condition: Chiralpak IC, $254 \mathrm{~nm}, 0.5 \mathrm{~mL} / \mathrm{min}$, hexane $/{ }^{i} \mathrm{PrOH}=80 / 20, t_{\text {major }}=20.525 \mathrm{~min}, t_{\text {minor }}=18.349 \mathrm{~min} ;{ }^{1} \mathrm{H} \mathrm{NMR}\left(600 \mathrm{MHz}, \mathrm{CDCl}_{3}\right) \delta$ 7.61-7.52 (m, 2H), 7.25-7.16 (m, 4H), 7.12 (d, $J=8.2 \mathrm{~Hz}, 1 \mathrm{H}), 7.07$ (d, $J=2.2 \mathrm{~Hz}, 1 \mathrm{H}), 7.02-$ $6.96(\mathrm{~m}, 2 \mathrm{H}), 6.65-6.58(\mathrm{~m}, 2 \mathrm{H}), 6.43(\mathrm{~s}, 1 \mathrm{H}), 5.30$ (d, $J=19.3 \mathrm{~Hz}, 1 \mathrm{H}), 4.35$ (hept, $J=6.1 \mathrm{~Hz}$, $1 \mathrm{H}), 3.68(\mathrm{~d}, J=10.5 \mathrm{~Hz}, 6 \mathrm{H}), 1.22(\mathrm{dd}, J=6.1,1.6 \mathrm{~Hz}, 6 \mathrm{H}) .{ }^{13} \mathrm{C} \mathrm{NMR}\left(151 \mathrm{MHz}, \mathrm{CDCl}_{3}\right) \delta$ 154.0, 143.2, 140.9, 137.0, 131.8, 131.7, 128.4, 128.3, 128.2, 127.8, 127.1, 126.2, 125.2, 124.7, 116.1, 109.8, 70.1, 64.4, 53.3, 22.0. ${ }^{31} \mathrm{P}$ NMR (243 MHz, $\left.\mathrm{CDCl}_{3}\right) \delta 22.47 . \mathrm{HRMS}(\mathrm{ESI}) \mathrm{m} / \mathrm{z}$ : $[\mathrm{M}+\mathrm{H}]^{+}$Calcd for $\mathrm{C}_{26} \mathrm{H}_{28} \mathrm{ClNO}_{4} \mathrm{P}$ 484.1439; Found 484.1440. IR $\left(\mathrm{KBr}, \mathrm{cm}^{-1}\right) 3453,3057,2975$, 2851, 1731, 1609, 1554, 1504, 1448, 1381, 1238, 1180, 1119, 1055, 1030, 952, 833, 764, 699, $571,532$.

Dimethyl (S)-(6-(4-isopropoxyphenyl)-7-phenyl-5, 6-dihydro-[1， 3]dioxolo[4, 5g]isoquinolin-5-yl)phosphonate (4anb)

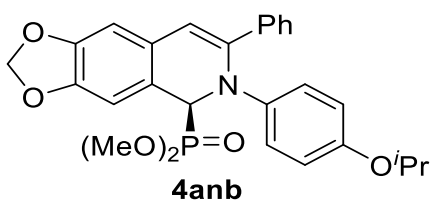

Following the general procedure, reaction were performed with $\mathbf{1}$ (0.12 mmol), 2c (0.11 mmol), 3b (0.1 mmol), $5 \AA$ MS (100 mg), Ii (10 mol \%), and $\mathrm{Ag}_{2} \mathrm{CO}_{3}(5 \mathrm{~mol} \%)$ in the $1.0 \mathrm{~mL}$ of MTBE at $10{ }^{\circ} \mathrm{C}$ for 3 days under argon, followed by column chromatography $(\mathrm{PE} / \mathrm{EA}=1: 5)$ to afford 4anb as yellow oil, $29.6 \mathrm{mg}(60 \%$ yield, $88 \% e e) ;[\alpha]_{\mathrm{D}}^{25}=+351.0^{\circ}(c=$ 1.0, EA); HPLC condition: Chiralpak IC, $254 \mathrm{~nm}, 0.5 \mathrm{~mL} / \mathrm{min}$, hexane $/{ }^{i} \mathrm{PrOH}=60 / 40, t_{\text {major }}=$ $29.955 \mathrm{~min}, t_{\text {minor }}=24.092 \mathrm{~min} ;{ }^{1} \mathrm{H} \mathrm{NMR}\left(600 \mathrm{MHz}, \mathrm{CDCl}_{3}\right) \delta 7.58-7.51(\mathrm{~m}, 2 \mathrm{H}), 7.22(\mathrm{t}, J=$ $7.6 \mathrm{~Hz}, 2 \mathrm{H}), 7.18-7.13(\mathrm{~m}, 1 \mathrm{H}), 7.02-6.95(\mathrm{~m}, 2 \mathrm{H}), 6.69$ (s, 1H), 6.65-6.56 (m, 3H), 6.38 (s, 1H), $5.92(\mathrm{q}, J=1.6 \mathrm{~Hz}, 2 \mathrm{H}), 5.25(\mathrm{~d}, J=18.7 \mathrm{~Hz}, 1 \mathrm{H}), 4.34$ (hept, $J=6.0 \mathrm{~Hz}, 1 \mathrm{H}), 3.68$ (dd, $J=24.7,10.5 \mathrm{~Hz}, 6 \mathrm{H}), 1.22(\mathrm{dd}, J=6.1,2.1 \mathrm{~Hz}, 6 \mathrm{H}) .{ }^{13} \mathrm{C} \mathrm{NMR}\left(151 \mathrm{MHz}, \mathrm{CDCl}_{3}\right) \delta 153.8$, 147.8, 146.5, 141.1, 137.2, 128.2, 127.8, 127.7, 127.6, 124.3, 117.9, 116.1, 111.0, 107.9, 104.7, 101.1, 70.1, 64.6, 53.2, 22.0. ${ }^{31} \mathrm{P}$ NMR (243 MHz, $\left.\mathrm{CDCl}_{3}\right) \delta 23.22$. HRMS (ESI) m/z: $[\mathrm{M}+\mathrm{H}]^{+}$ Calcd for $\mathrm{C}_{27} \mathrm{H}_{29} \mathrm{NO}_{6} \mathrm{P}$ 494.1727; Found 494.1734. 
Dimethyl (S)-(6-(4-isopropoxyphenyl)-7-phenyl-5, 6-dihydro-1, 6-naphthyridin-5-yl)phos phonate (4aob)

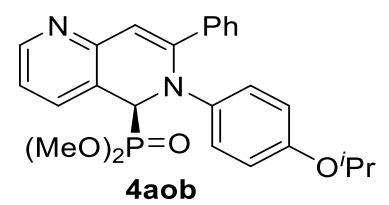

Following the general procedure, reaction were performed with 1 (0.12 mmol), $2 \mathbf{c}(0.11 \mathrm{mmol}), \mathbf{3 b}(0.1 \mathrm{mmol}), 5 \AA \mathrm{MS}(100 \mathrm{mg}), \mathbf{I i}$ (10 mol \%), and $\mathrm{Ag}_{2} \mathrm{CO}_{3}(5 \mathrm{~mol} \%)$ in the $1.0 \mathrm{~mL}$ of MTBE at $-10^{\circ} \mathrm{C}$ for 3 days under argon, followed by column chromatography $(\mathrm{PE} / \mathrm{EA}=1: 3)$ to afford 4aob as yellow solid, $23.4 \mathrm{mg}(52 \%$ yield, $87 \% e e)$; mp: $97.8-99.6^{\circ} \mathrm{C}$, $[\alpha]_{\mathrm{D}}^{25}=+891.7^{\circ}$ ( $c=1.0$, EA); HPLC condition: Chiralpak IF, $254 \mathrm{~nm}, 0.5 \mathrm{~mL} / \mathrm{min}$, hexane $/{ }^{i} \mathrm{PrOH}=70 / 30$, $t_{\text {major }}=18.304 \mathrm{~min}, t_{\text {minor }}=20.185 \mathrm{~min} ;{ }^{1} \mathrm{H} \mathrm{NMR}\left(600 \mathrm{MHz}, \mathrm{CDCl}_{3}\right) \delta 8.51-8.37(\mathrm{~m}, 1 \mathrm{H}), 7.59$ $(\mathrm{d}, J=7.4 \mathrm{~Hz}, 2 \mathrm{H}), 7.36(\mathrm{~d}, J=7.5 \mathrm{~Hz}, 1 \mathrm{H}), 7.25(\mathrm{td}, J=11.8,5.8 \mathrm{~Hz}, 4 \mathrm{H}), 7.13-6.99(\mathrm{~m}, 3 \mathrm{H})$, 6.69-6.56 (m, 3H), 5.36 (d, $J=19.0 \mathrm{~Hz}, 1 \mathrm{H}), 4.36$ (hept, $J=6.1 \mathrm{~Hz}, 1 \mathrm{H}), 3.75$ (d, $J=10.6 \mathrm{~Hz}$, $3 \mathrm{H}), 3.64(\mathrm{~d}, J=10.5 \mathrm{~Hz}, 3 \mathrm{H}), 1.23(\mathrm{~d}, J=6.1 \mathrm{~Hz}, 6 \mathrm{H}) .{ }^{13} \mathrm{C} \mathrm{NMR}\left(151 \mathrm{MHz}, \mathrm{CDCl}_{3}\right) \delta 154.3$, 152.2, 149.4, 149.3, 147.6, 140.7, 136.7, 134.6, 134.5, 128.7, 128.4, 128.2, 125.2, 125.2, 121.2, $121.1,120.0,116.0,110.9,70.1,65.3,64.2,53.6,53.5,53.3,53.2,22.0,21.9 .{ }^{31} \mathrm{P}$ NMR (243 $\left.\mathrm{MHz}, \mathrm{CDCl}_{3}\right) \delta$ 22.11. HRMS (ESI) m/z: $[\mathrm{M}+\mathrm{H}]^{+}$Calcd for $\mathrm{C}_{25} \mathrm{H}_{28} \mathrm{~N}_{2} \mathrm{O}_{4} \mathrm{P}$ 451.1781; Found 451.1781 .

\section{Dibenzyl ((phenylamino)(2-(phenylethynyl)phenyl)methyl)phosphonate (5aaa)}

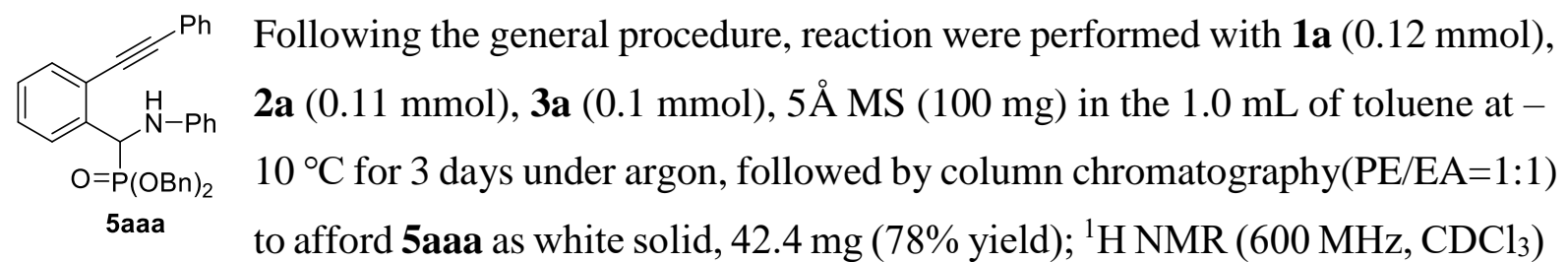
$\delta 7.62(\mathrm{dd}, J=7.9,2.6 \mathrm{~Hz}, 1 \mathrm{H}), 7.51$ (d, $J=7.8 \mathrm{~Hz}, 1 \mathrm{H}), 7.49-7.43$ (m, 2H), 7.38-7.27 (m, 5H), 7.26-7.13 (m, 9H), 7.12-7.03 (m, 4H), 6.67 (t, $J=7.3 \mathrm{~Hz}, 1 \mathrm{H}), 6.63(\mathrm{~d}, J=8.0 \mathrm{~Hz}, 2 \mathrm{H})$, $5.67(\mathrm{~d}, J=24.9 \mathrm{~Hz}, 1 \mathrm{H}), 5.13-5.07(\mathrm{~m}, 2 \mathrm{H}), 4.83(\mathrm{dd}, J=11.8,7.4 \mathrm{~Hz}, 1 \mathrm{H}), 4.52(\mathrm{dd}, J=11.9$, $8.0 \mathrm{~Hz}, 1 \mathrm{H}) .{ }^{13} \mathrm{C} \mathrm{NMR}\left(151 \mathrm{MHz}, \mathrm{CDCl}_{3}\right) \delta 146.0,138.0,136.1,136.0,132.2,131.6,129.2$, 129.0, 128.7, 128.6 , 128.5, 128.4, 128.3, 128.2, 127.9, 127.8, 127.6, 127.4, 123.4, 123.0, 118.5, 113.8, 95.3, 87.1, 68.6, 68.5, 53.5. ${ }^{31} \mathrm{P}$ NMR $\left(243 \mathrm{MHz}, \mathrm{CDCl}_{3}\right) \delta 23.51 . \mathrm{HRMS}(\mathrm{ESI}) \mathrm{m} / \mathrm{z}$ : $[\mathrm{M}+\mathrm{H}]^{+}$Calcd for $\mathrm{C}_{35} \mathrm{H}_{31} \mathrm{NO}_{3} \mathrm{P}$ 544.2036; Found 544.2036. 


\section{Amplification and Derivatizations of products 4}

\subsection{Amplification reaction}

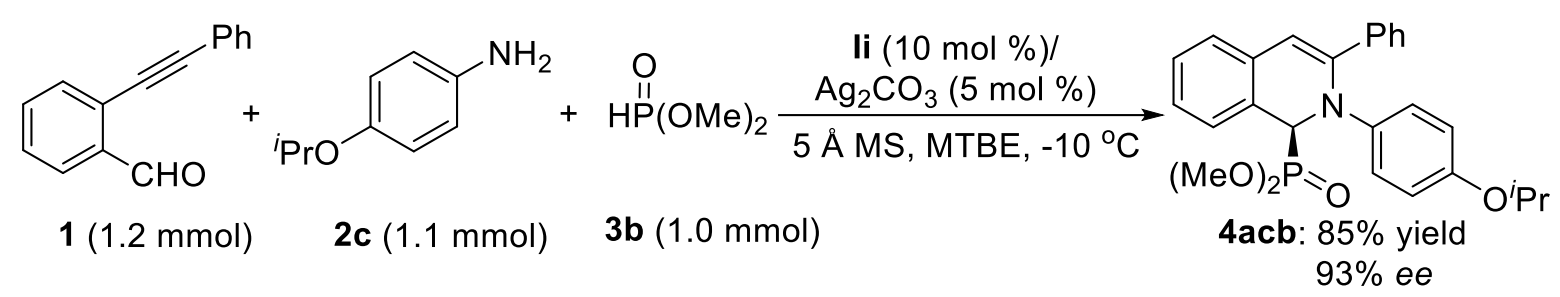

$5 \AA \mathrm{MS}(1.0 \mathrm{~g})$ powder was added to a $25 \mathrm{~mL}$ reaction tube and dried with flame for $10 \mathrm{~min}$ under vacuum, then charged with Ar atmosphere and cooled down to rt. Chiral phosphoric acid Ii ( $75.9 \mathrm{mg}, 0.1 \mathrm{mmol}, 0.1$ equiv.), $\mathrm{Ag}_{2} \mathrm{CO}_{3}(13.8 \mathrm{mg}, 0.05 \mathrm{mmol}, 0.05$ equiv.) and anhydrous $\operatorname{MTBE}(10.0 \mathrm{~mL})$ were added subsequently. The resulted solution was stirred for $2.0 \mathrm{~h}$ at $30^{\circ} \mathrm{C}$ in dark and then the corresponding 2-alkynylbenzaldehyde 1a (247.5 mg, $1.2 \mathrm{mmol}, 1.2$ equiv.) and amine $2 \mathrm{c}$ (166.3 mg, $1.1 \mathrm{mmol}, 1.1$ equiv.) were added, the mixture reacted at $30^{\circ} \mathrm{C}$ for 0.5 $\mathrm{h}$. Then the reaction mixture was cooled down to $-10^{\circ} \mathrm{C}$, the methyl phosphonate $3 \mathbf{b}(110.0 \mathrm{mg}$, $1.0 \mathrm{mmol}, 1.0$ equiv.) was added and the reaction mixture was stirred at $-10{ }^{\circ} \mathrm{C}$ for $3 \mathrm{~d}$, the solution was directly purified by column chromatography on silica gel $(\mathrm{PE} / \mathrm{EA}=2 / 1$ to $1 / 1)$ to give the corresponding 1,2-dihydroisoquinolin -1-ylphosphonate 4acb $(0.38 \mathrm{~g}, 85 \%$ yield, 93\% $e e)$.

\subsection{Derivatizations of Products ${ }^{7}$}

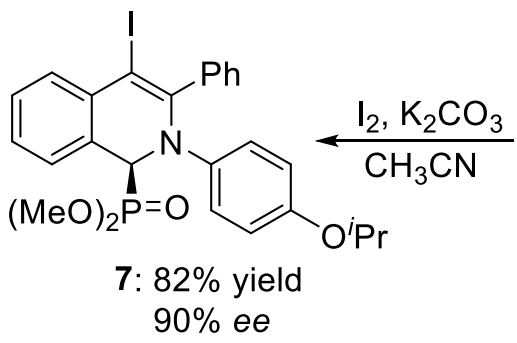

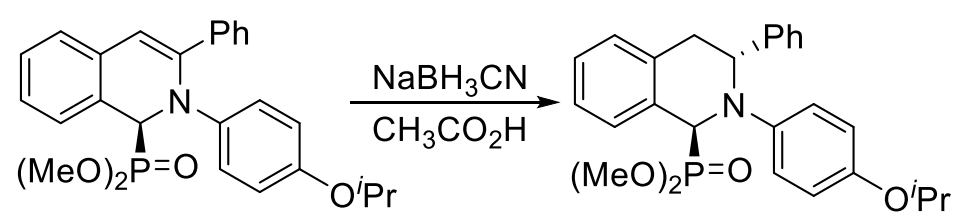

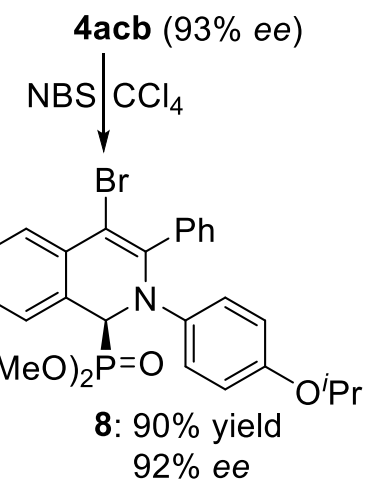


7.2.1 Synthesis of dimethyl ((1S, 3R)-2-(4-isopropoxyphenyl)-3-phenyl-1, 2, 3, 4tetrahydro-isoquinolin-1-yl)phosphonate 6

$\mathrm{NaBH}_{3} \mathrm{CN}$ (125.7 mg, $\left.2.0 \mathrm{mmol}\right)$ was added to the solution of dimethyl $(S)$-(2-(4isopropoxyphenyl)-3-phenyl-1, 2-dihydroisoquinolin- 1-yl)phosphonate 4acb (89.9 mg, 0.2 $\mathrm{mmol})$ in Acetic acid $(10.0 \mathrm{~mL})$ at $0{ }^{\circ} \mathrm{C}$. The reaction mixture was warmed to room temperature and stirred until the starting material was disappeared. The reaction solution was neutralized with saturated sodium bicarbonate and extracted with EA (three times), combined organic layers and washed with brine, dried over $\mathrm{Na}_{2} \mathrm{SO}_{4}$, and concentrated under reduce pressure and the residue was purified by column chromatography on silica gel to give 6 ( $85.7 \mathrm{mg}, 95 \%$ yield, $92 \% e e, d r>20: 1)$ as a yellow oil. $[\alpha]_{\mathrm{D}}^{25}=-8.2^{\circ}(c=1.0$, EA $)$; HPLC condition: Chiralpak IC, $254 \mathrm{~nm}, 0.5 \mathrm{~mL} / \mathrm{min}$, hexane $/{ }^{i} \mathrm{PrOH}=80 / 20, t_{\text {major }}=20.284 \mathrm{~min}, t_{\text {minor }}=16.754 \mathrm{~min} ;{ }^{1} \mathrm{H} \mathrm{NMR}$ $\left(600 \mathrm{MHz}, \mathrm{CDCl}_{3}\right) \delta$ 7.58-7.49 (m, 2H), 7.32-7.15 (m, 7H), 6.97-6.89 (m, 2H), 6.70-6.62 (m, $2 \mathrm{H}), 5.09(\mathrm{~d}, J=18.3 \mathrm{~Hz}, 1 \mathrm{H}), 4.40-4.28(\mathrm{~m}, 2 \mathrm{H}), 3.82(\mathrm{~d}, J=10.5 \mathrm{~Hz}, 3 \mathrm{H}), 3.73(\mathrm{dd}, J=15.1$, $12.4 \mathrm{~Hz}, 1 \mathrm{H}), 3.58(\mathrm{~d}, J=10.5 \mathrm{~Hz}, 3 \mathrm{H}), 2.92(\mathrm{dd}, J=15.1,3.9 \mathrm{~Hz}, 1 \mathrm{H}), 1.23(\mathrm{dd}, J=6.1,3.2$ $\mathrm{Hz}, 6 \mathrm{H}) .{ }^{13} \mathrm{C} \mathrm{NMR}\left(151 \mathrm{MHz}, \mathrm{CDCl}_{3}\right) \delta 152.2,144.7,131.6,128.5,128.1,128.06,128.0127 .9$, 127.2, 127.1, 127.07, 126.9, 126.51, 126.5, 120.8, 116.3, 70.3, 65.4, 64.2, 62.4, 53.7, 53.6, 53.25, 53.2, 39.3, 22.2, 22.1. ${ }^{31} \mathrm{P}$ NMR (243 MHz, $\left.\mathrm{CDCl}_{3}\right) \delta 24.70$. HRMS (ESI) m/z: $[\mathrm{M}+\mathrm{H}]^{+}$ Calcd for $\mathrm{C}_{26} \mathrm{H}_{31} \mathrm{NO}_{4} \mathrm{P}$ 452.1985; Found 452.1982.

\subsubsection{Synthesis of dimethyl (S)-(4-iodo-2-(4-isopropoxyphenyl)-3-phenyl-1,2- dihydroisoquino lin- 1-yl)phosphonate 7}

$\mathrm{K}_{2} \mathrm{CO}_{3}(34.6 \mathrm{mg}, 0.25 \mathrm{mmol})$ and $\mathrm{I}_{2}(63.5 \mathrm{mg}, 0.25 \mathrm{mmol})$ was successively added to the solution of dimethyl (S)-(2-(4-isopropoxyphenyl)-3-phenyl-1,2-dihydroisoquinolin-1-yl) phosphonate $4 \mathbf{a c b}(44.9 \mathrm{mg}, 0.10 \mathrm{mmol})$ in $\mathrm{CH}_{3} \mathrm{CN}(1.5 \mathrm{~mL})$ at $0{ }^{\circ} \mathrm{C}$. The reaction mixture was warmed to room temperature and stirred until the starting material was disappeared. The reaction was quenched with saturated sodium sulfite and extracted with EA (three times), combined organic layers and washed with brine, dried over $\mathrm{Na}_{2} \mathrm{SO}_{4}$, and concentrated under reduce pressure. The residue was purified by column chromatography on silica gel to give 7 (47.1 mg, 82\% yield, 90\% ee $)$ as a yellow solid. mp: $119.1-120.3^{\circ} \mathrm{C},[\alpha]_{\mathrm{D}}^{25}=+449.3^{\circ}(c=1.0$, EA); HPLC condition: Chiralpak IF, $254 \mathrm{~nm}, 0.5 \mathrm{~mL} / \mathrm{min}$, hexane $/{ }^{i} \mathrm{PrOH}=60 / 40, t_{\text {major }}=$ $11.386 \mathrm{~min}, t_{\text {minor }}=13.572 \mathrm{~min} ;{ }^{1} \mathrm{H} \mathrm{NMR}\left(600 \mathrm{MHz}, \mathrm{CDCl}_{3}\right) \delta 7.67-7.58(\mathrm{~m}, 3 \mathrm{H}), 7.35(\mathrm{tt}, J=$ 7.7, $1.7 \mathrm{~Hz}, 1 \mathrm{H}), 7.28-7.17(\mathrm{~m}, 5 \mathrm{H}), 6.99(\mathrm{dd}, J=7.6,5.0 \mathrm{~Hz}, 3 \mathrm{H}), 6.59-6.51(\mathrm{~m}, 2 \mathrm{H}), 5.28(\mathrm{~d}$, $J=18.1 \mathrm{~Hz}, 1 \mathrm{H}), 4.38-4.28(\mathrm{~m}, J=6.1 \mathrm{~Hz}, 1 \mathrm{H}), 3.79(\mathrm{~d}, J=10.7 \mathrm{~Hz}, 3 \mathrm{H}), 3.64(\mathrm{~d}, J=10.6$ $\mathrm{Hz}, 3 \mathrm{H}), 1.20(\mathrm{dd}, J=6.1,2.3 \mathrm{~Hz}, 6 \mathrm{H}) .{ }^{13} \mathrm{C} \mathrm{NMR}\left(151 \mathrm{MHz}, \mathrm{CDCl}_{3}\right) \delta 154.4,145.5,140.6$, 
$140.5,138.6,135.5,131.9,129.9,129.9,128.6,128.6,128.6,128.3,127.8,127.6,127.5,126.8$, 126.8, 126.6, 126.5, 125.5, 125.5, 116.0, 115.8, 70.0, 64.9, 63.8, 53.9, 53.8, 53.5, 53.5, 22.0, 22.0. ${ }^{31} \mathrm{P} \mathrm{NMR}\left(243 \mathrm{MHz}, \mathrm{CDCl}_{3}\right) \delta 23.43$. HRMS (ESI) $\mathrm{m} / \mathrm{z}$ : $[\mathrm{M}+\mathrm{H}]^{+}$Calcd for $\mathrm{C}_{26} \mathrm{H}_{28} \mathrm{INO}_{4} \mathrm{P}$ 576.0795; Found 576.0794.

\subsubsection{Synthesis of dimethyl $(S)$-(4-bromo-2-(4-isopropoxyphenyl)-3-phenyl-1,2-dihydro isoquinolin-1-yl)phosphonate 8}

NBS (23.1 mg, $0.13 \mathrm{mmol})$ was added to the solution of dimethyl (S)-(2-(4isopropoxyphenyl)-3-phenyl-1,2-dihydroisoquinolin-1-yl)phosphonate 4acb (44.9 mg, 0.10 $\mathrm{mmol})$ in $\mathrm{CCl}_{4}(1.5 \mathrm{~mL})$ at $\mathrm{rt}$. The reaction mixture was stirred until the starting material was disappeared and directly purified by column chromatography on silica gel (PE/EA $=3 / 1$ to $1 / 1$ ) to give 8 (47.5 mg, 90\% yield, $92 \% e e)$ as a yellow oil. $[\alpha]_{\mathrm{D}}^{25}=+714.8^{\circ}(c=1.0$, EA); HPLC condition: Chiralpak IF, $254 \mathrm{~nm}, 0.5 \mathrm{~mL} / \mathrm{min}$, hexane $/{ }^{i} \mathrm{PrOH}=60 / 40, t_{\text {major }}=10.847 \mathrm{~min}, t_{\text {minor }}$ $=12.440 \mathrm{~min} ;{ }^{1} \mathrm{H} \mathrm{NMR}\left(600 \mathrm{MHz}, \mathrm{CDCl}_{3}\right) \delta 7.71-7.63(\mathrm{~m}, 3 \mathrm{H}), 7.37(\mathrm{tt}, J=7.7,1.6 \mathrm{~Hz}, 1 \mathrm{H})$, 7.29-7.22 (m, 3H), 7.21-7.16 (m, 1H), $7.06(\mathrm{dt}, J=7.6,1.6 \mathrm{~Hz}, 1 \mathrm{H}), 7.03-6.97(\mathrm{~m}, 2 \mathrm{H}), 6.61-$ $6.54(\mathrm{~m}, 2 \mathrm{H}), 5.28(\mathrm{~d}, J=18.6 \mathrm{~Hz}, 1 \mathrm{H}), 4.32($ hept, $J=6.0 \mathrm{~Hz}, 1 \mathrm{H}), 3.77(\mathrm{~d}, J=10.6 \mathrm{~Hz}, 3 \mathrm{H})$, $3.63(\mathrm{~d}, J=10.5 \mathrm{~Hz}, 3 \mathrm{H}), 1.20(\mathrm{dd}, J=6.1,2.6 \mathrm{~Hz}, 6 \mathrm{H}) .{ }^{13} \mathrm{C} \mathrm{NMR}\left(151 \mathrm{MHz}, \mathrm{CDCl}_{3}\right) \delta 154.3$, 141.2 136.2, 131.6, 128.6, 128.5, 128.5, 127.8, 127.6, 126.7, 126.6, 125.8, 125.8, 125.2 125.2, 115.8, 70.0, 64.8, 63.7, 53.7, 53.7, 53.4, 53.4, 22.0, 22.0. ${ }^{31} \mathrm{P}$ NMR (243 MHz, $\left.\mathrm{CDCl}_{3}\right) \delta 23.14$. HRMS (ESI) m/z: [M+H] $]^{+}$Calcd for $\mathrm{C}_{26} \mathrm{H}_{28} \mathrm{BrNO}_{4} \mathrm{P}$ 528.0934; Found 528.0932.

\section{References}

[1] (a) Zheng, B.; Chen, H. H.; Zhu, L.; Hou, X. Q.; Wang, Y.; Lan, Y.; Peng, Y. G. Org. Lett. 2019, 21, 593. (b) Kazuteru, U.; Kensuke, T.; Kosuke, Y.; Takashi, S.; Hiroshi, S. Org. Lett. 2014, 16, 4662.

[2] (a) Čorić, I.; Müller, S.; List, B. J. Am. Chem. Soc. 2010, 132, 17370. (b) Wen, Y.; Wang, Z. B.; Sun, J. W. Angew. Chem. Int. Ed. 2016, 55, 6954.

[3] (a) Vladimir, B. B.; Arnold, L. R.; Lam, K. C. Tetrahedron: Asymmetry, 1999, 10, 125. (b) Zhang, J. H.; L J.; Cui X.; Yu K. B.; Zhu J.; Deng J. G.; Zhu S. F.; Wang L. X.; Zhou Q. L.; Chung L. W.; Ye T. Tetrahedron: Asymmetry, 2002, 13, 1363. (c) Xu, F.; Huang, D.; Han, C.; Shen, W.; Lin, X.; Wang, Y. J. Org. Chem. 2010, 75, 8677.

[4] Jong, H. P.; Sachin, V. B.; So, W. Y. Org. Lett. 2011, 13, 2228.

[5] Sheldrick, G. M. Acta Cryst. 2015, C71, 3. 
[6] Dolomanov, O.V.; Bourhis, L. J.; Gildea, R. J.; Howard, J. A. K.; Puschmann, H.; J. Appl. Cryst. 2009, 42, 339.

[7] (a) Shashikant, U. D.; Sanjay, B. Tetrahedron 2013, 9875. (b) Huang, J. R.; Qin, L.; Zhu, Y. Q.; Songa, Q.; Dong, L. Chem. Commun. 2015, 51, 2844. 


\section{The spectra of ${ }^{1} \mathrm{H}$ NMR, ${ }^{13} \mathrm{C}$ NMR and HPLC}

$600 \mathrm{MHz}, \mathrm{CDCl}_{3},{ }^{1} \mathrm{H} \mathrm{NMR}$

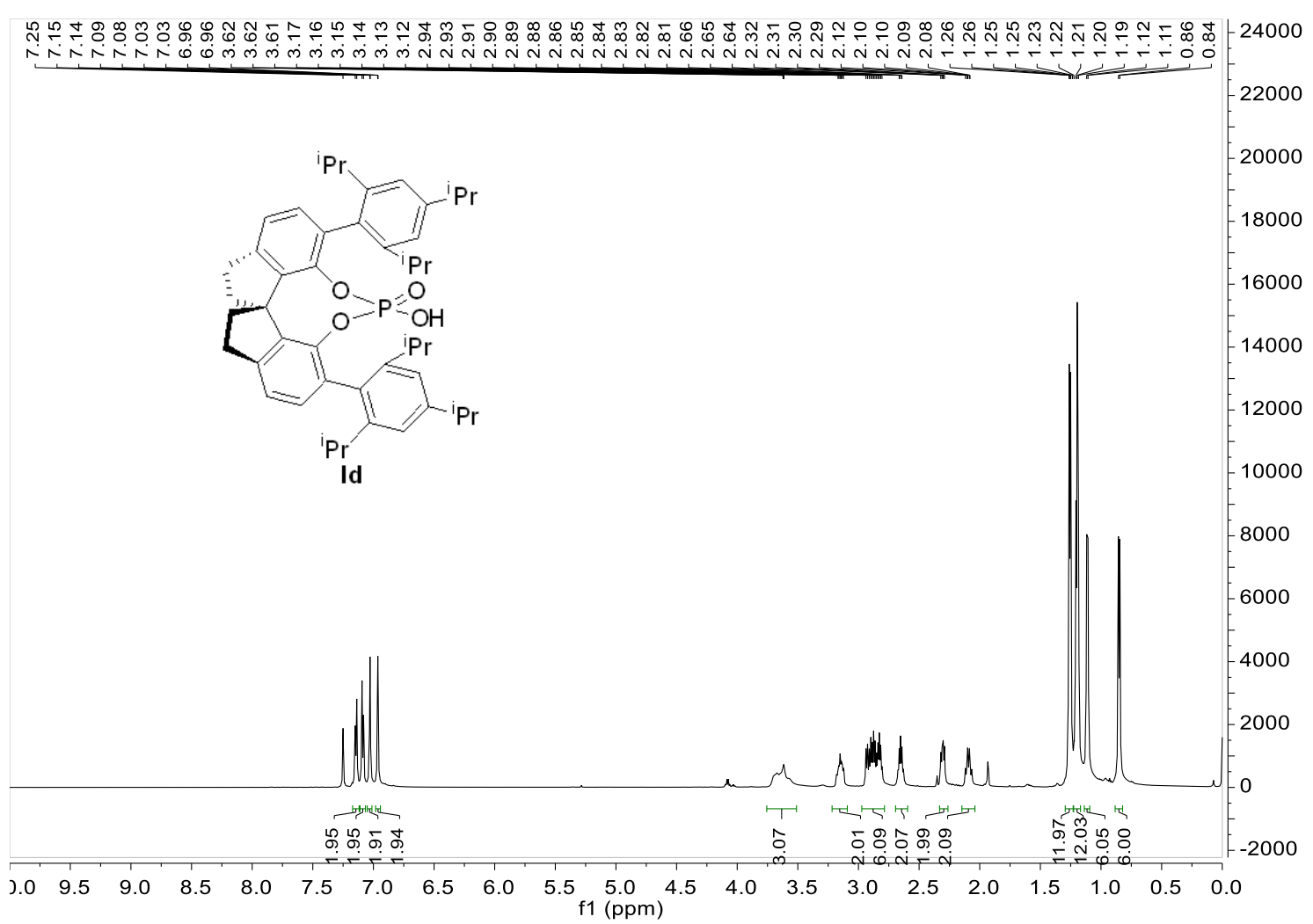

$151 \mathrm{MHz}, \mathrm{CDCl}_{3},{ }^{13} \mathrm{C} \mathrm{NMR}$

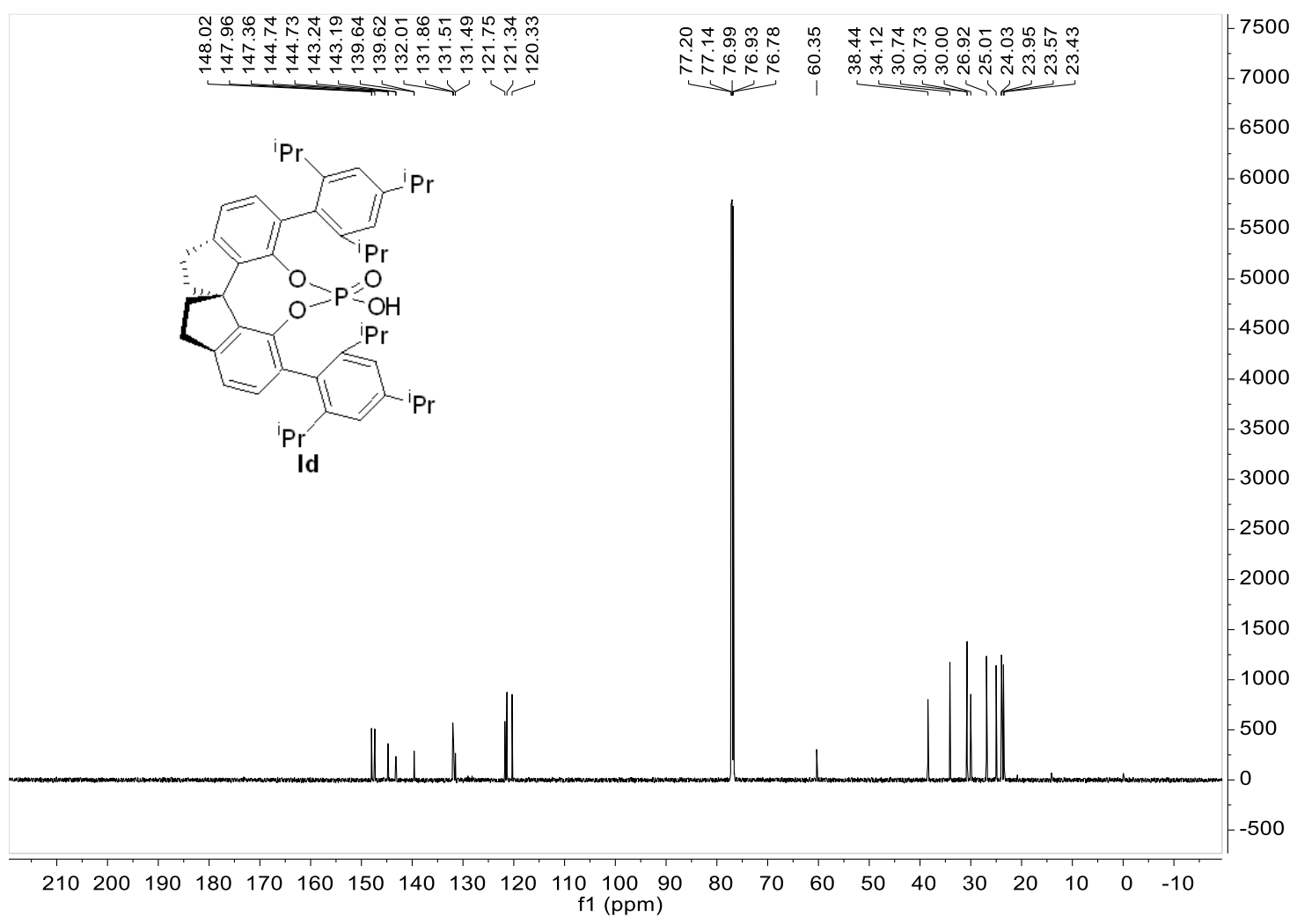


$600 \mathrm{MHz}, \mathrm{CDCl}_{3},{ }^{1} \mathrm{H} \mathrm{NMR}$

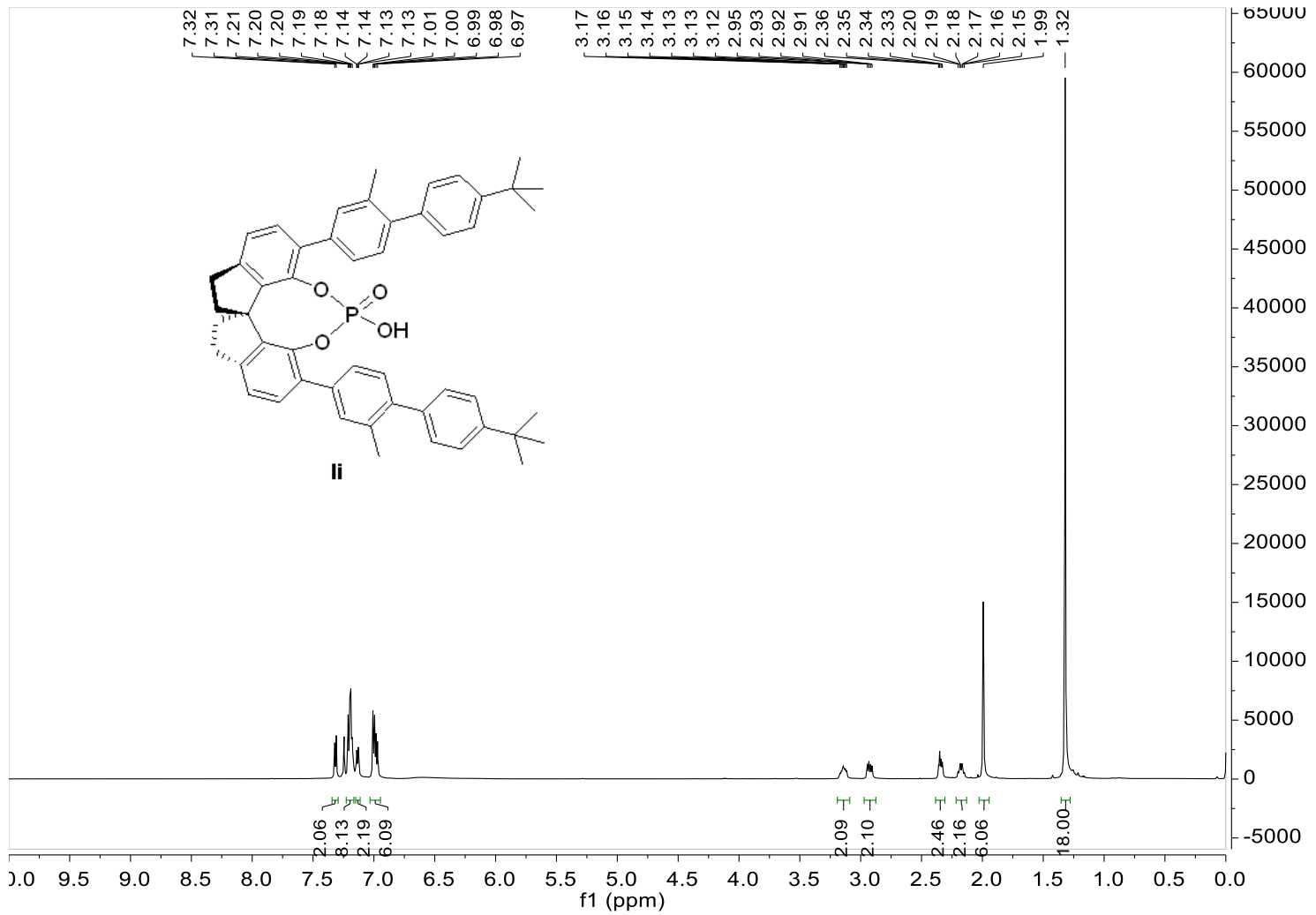

$151 \mathrm{MHz}, \mathrm{CDCl}_{3},{ }^{13} \mathrm{C} \mathrm{NMR}$

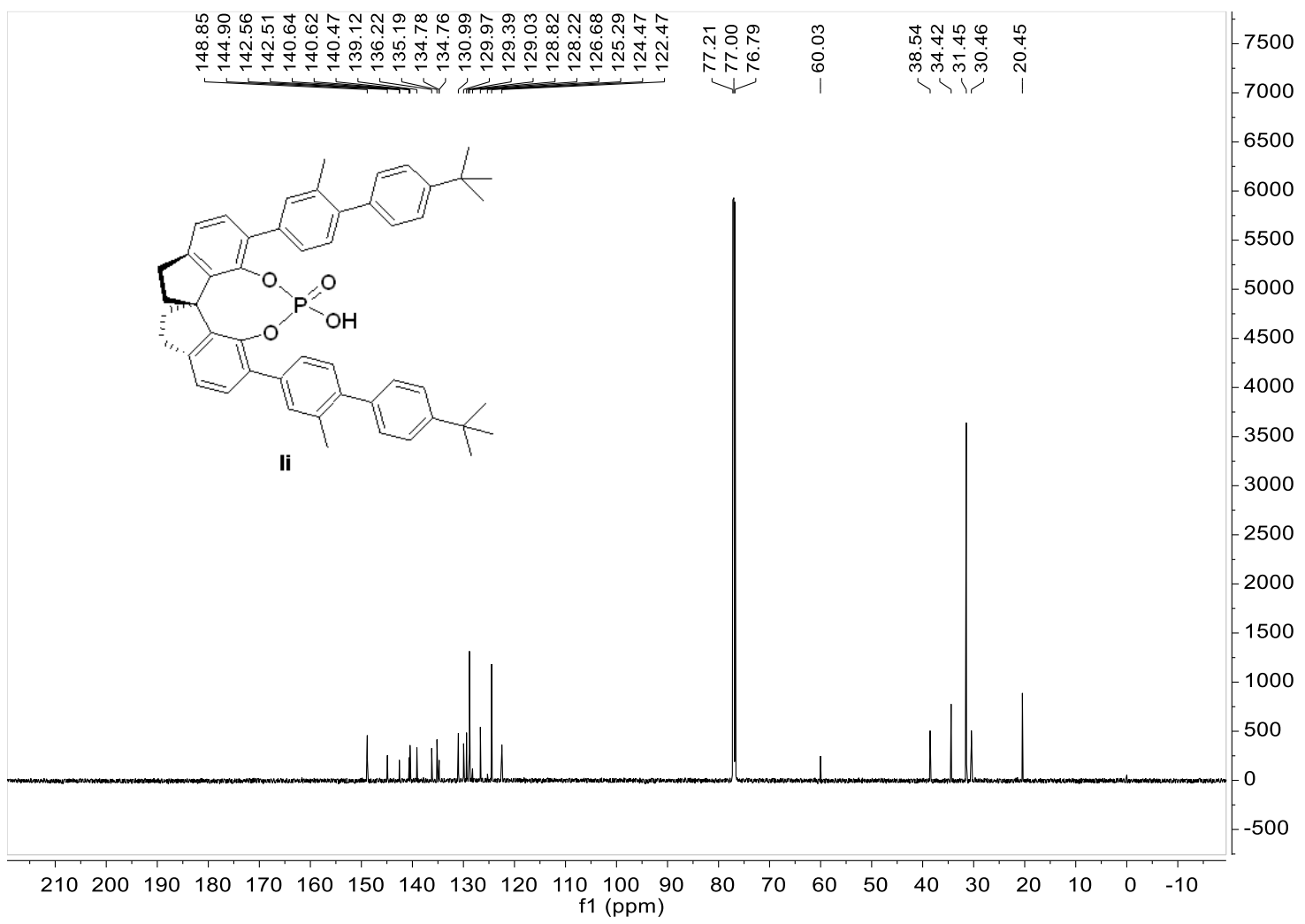


$600 \mathrm{MHz}, \mathrm{CDCl}_{3},{ }^{1} \mathrm{H} \mathrm{NMR}$

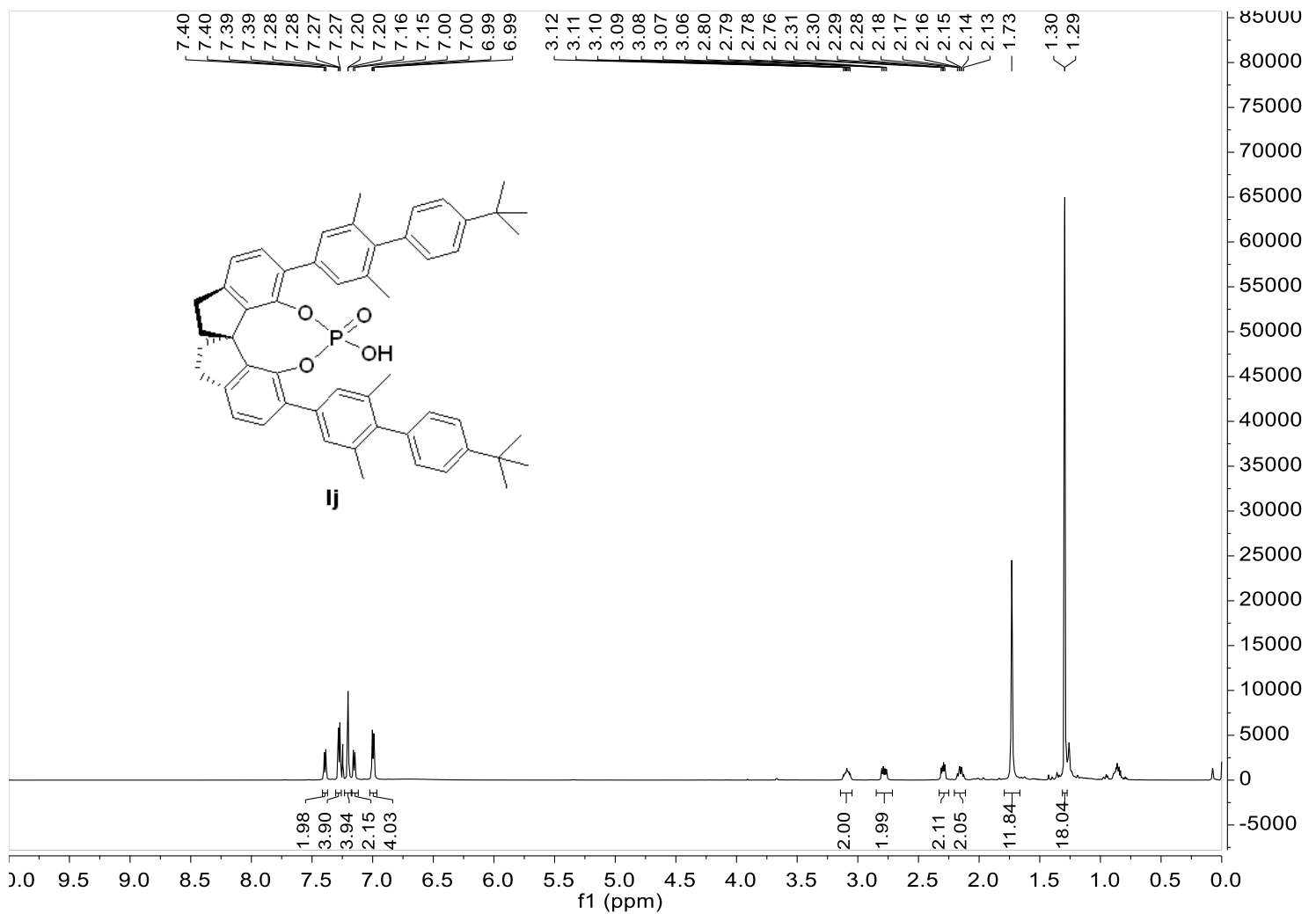

$151 \mathrm{MHz}, \mathrm{CDCl}_{3},{ }^{13} \mathrm{C} \mathrm{NMR}$

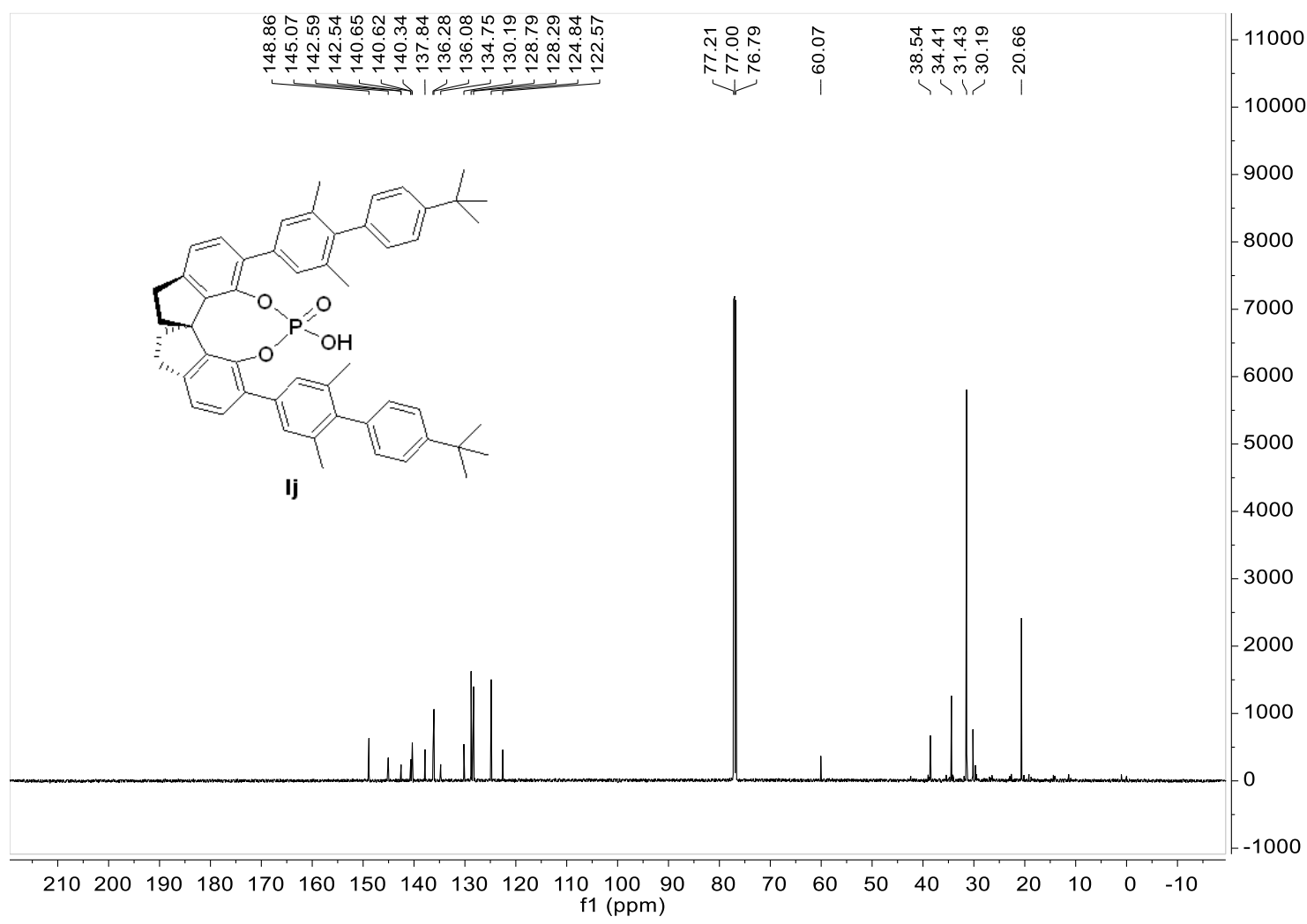


$600 \mathrm{MHz}, \mathrm{CDCl}_{3},{ }^{1} \mathrm{H} \mathrm{NMR}$

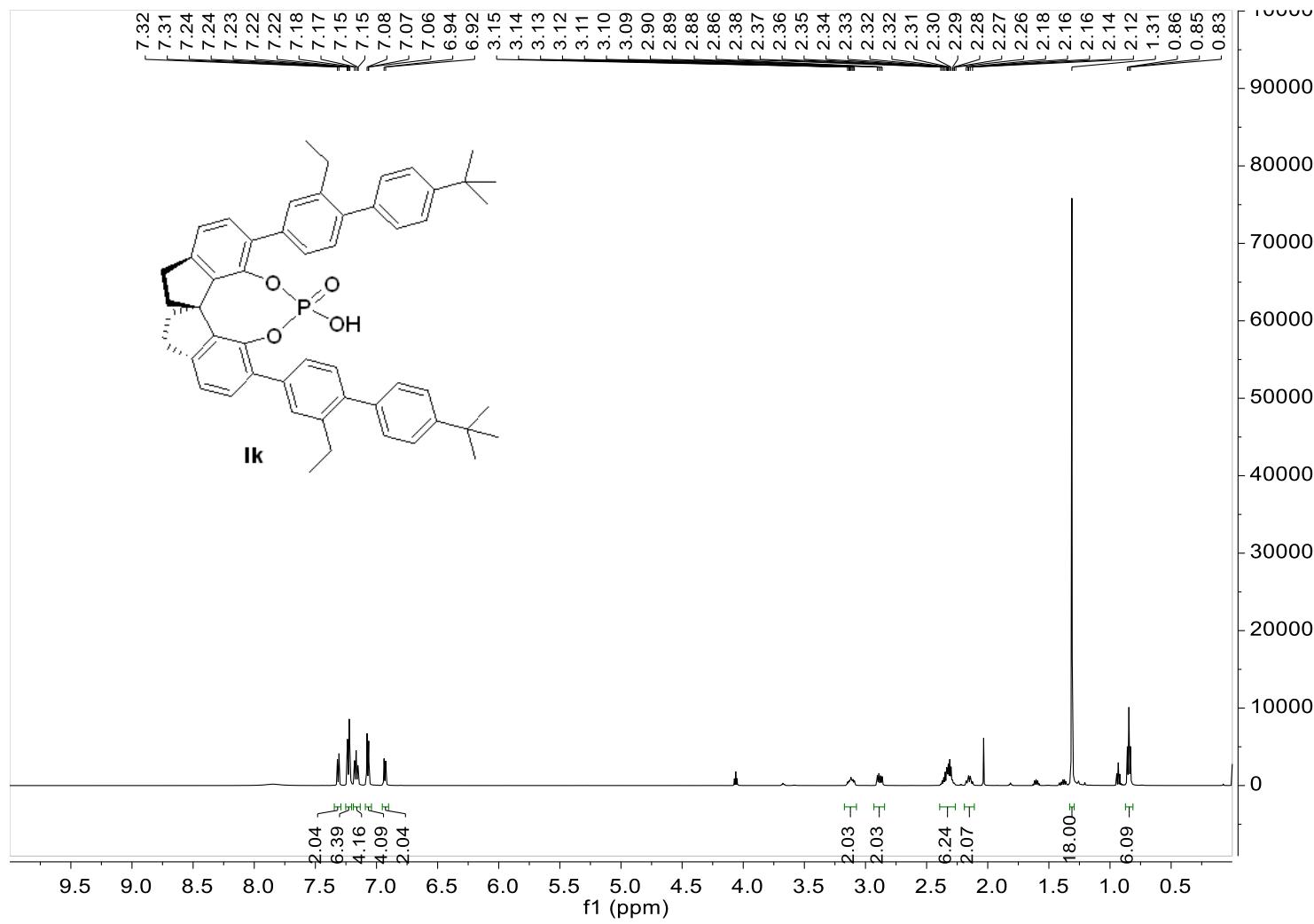

$151 \mathrm{MHz}, \mathrm{CDCl}_{3},{ }^{13} \mathrm{C} \mathrm{NMR}$

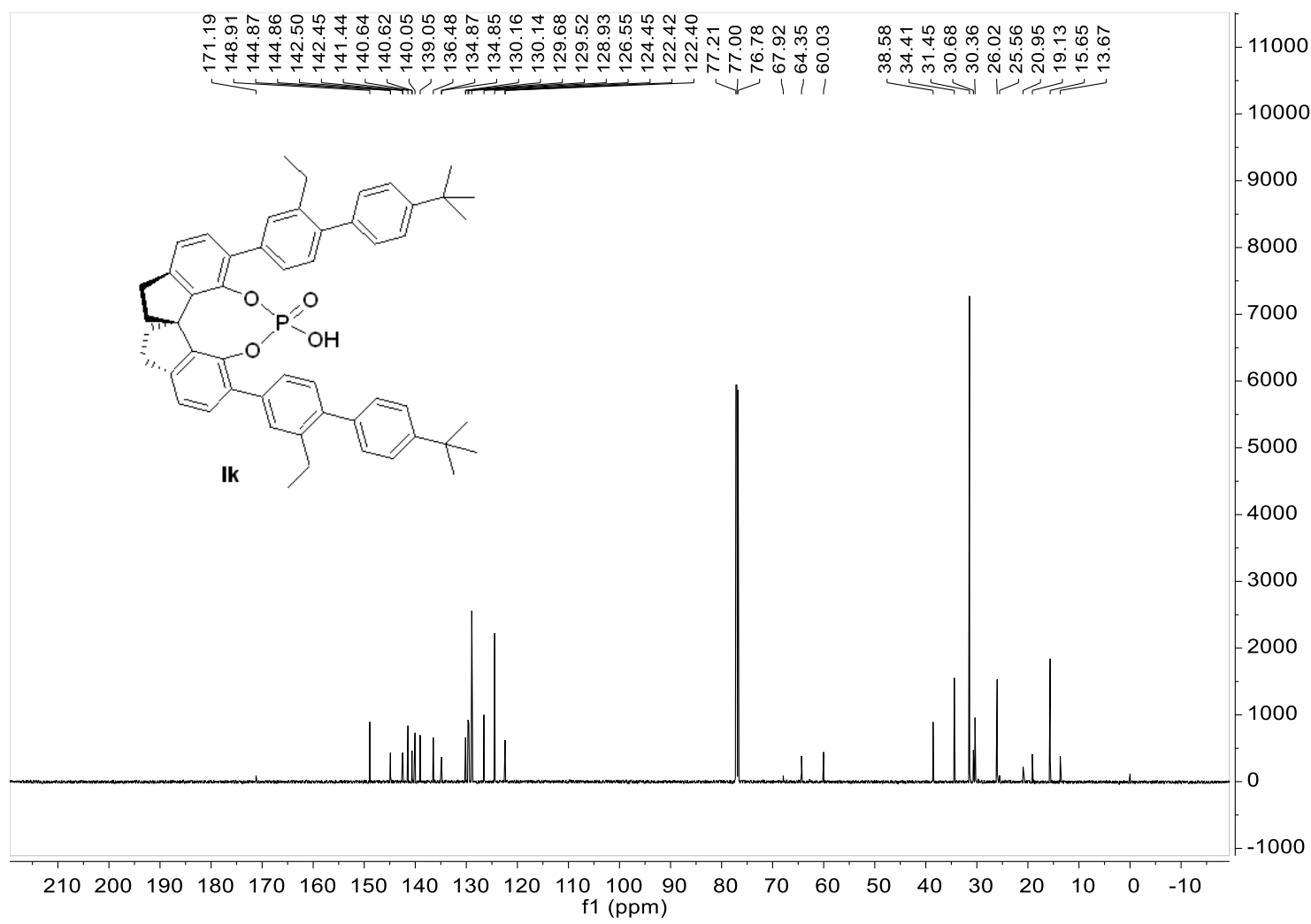


$600 \mathrm{MHz}, \mathrm{CDCl}_{3},{ }^{1} \mathrm{H} \mathrm{NMR}$

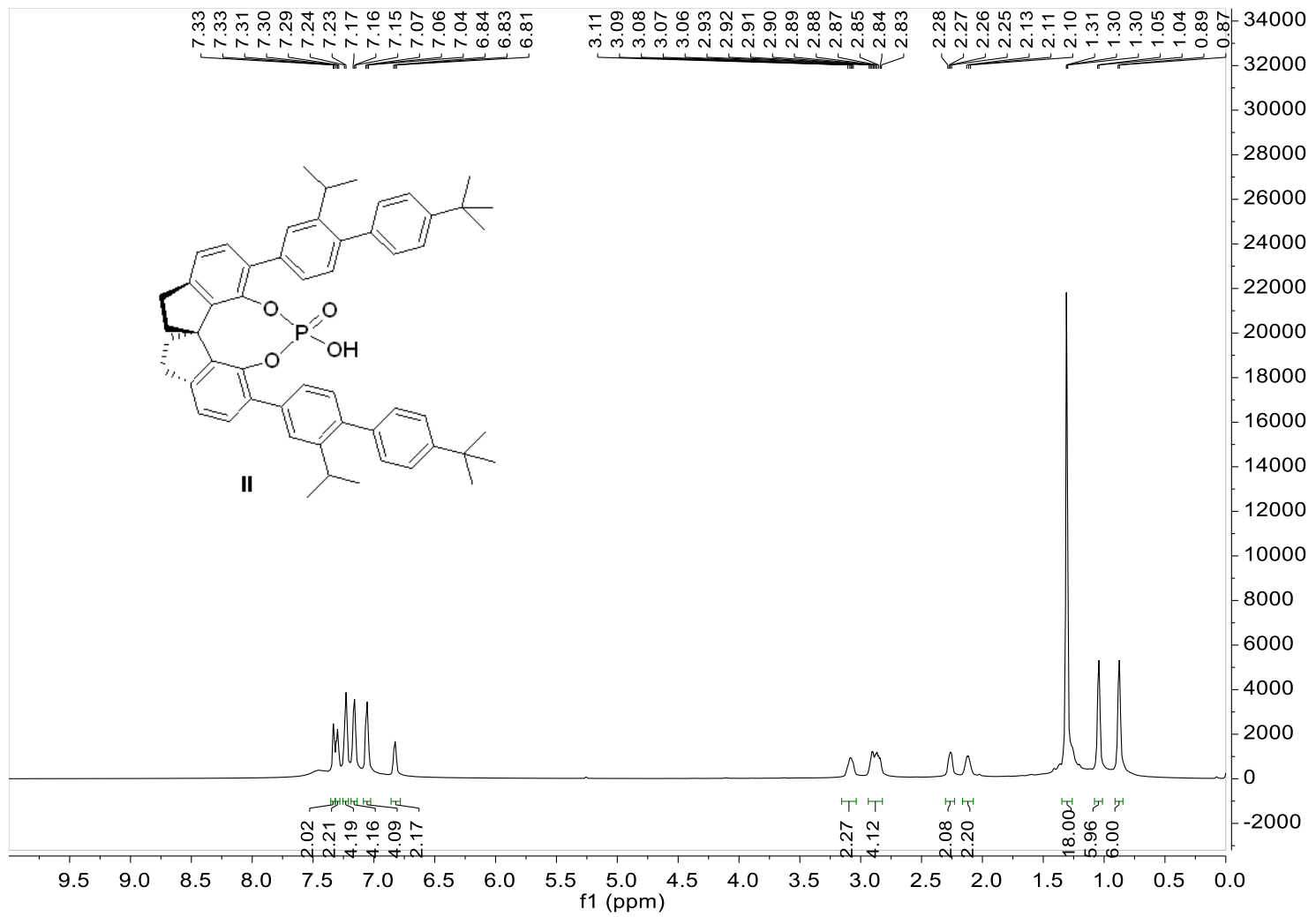

$151 \mathrm{MHz}, \mathrm{CDCl}_{3},{ }^{13} \mathrm{C} \mathrm{NMR}$

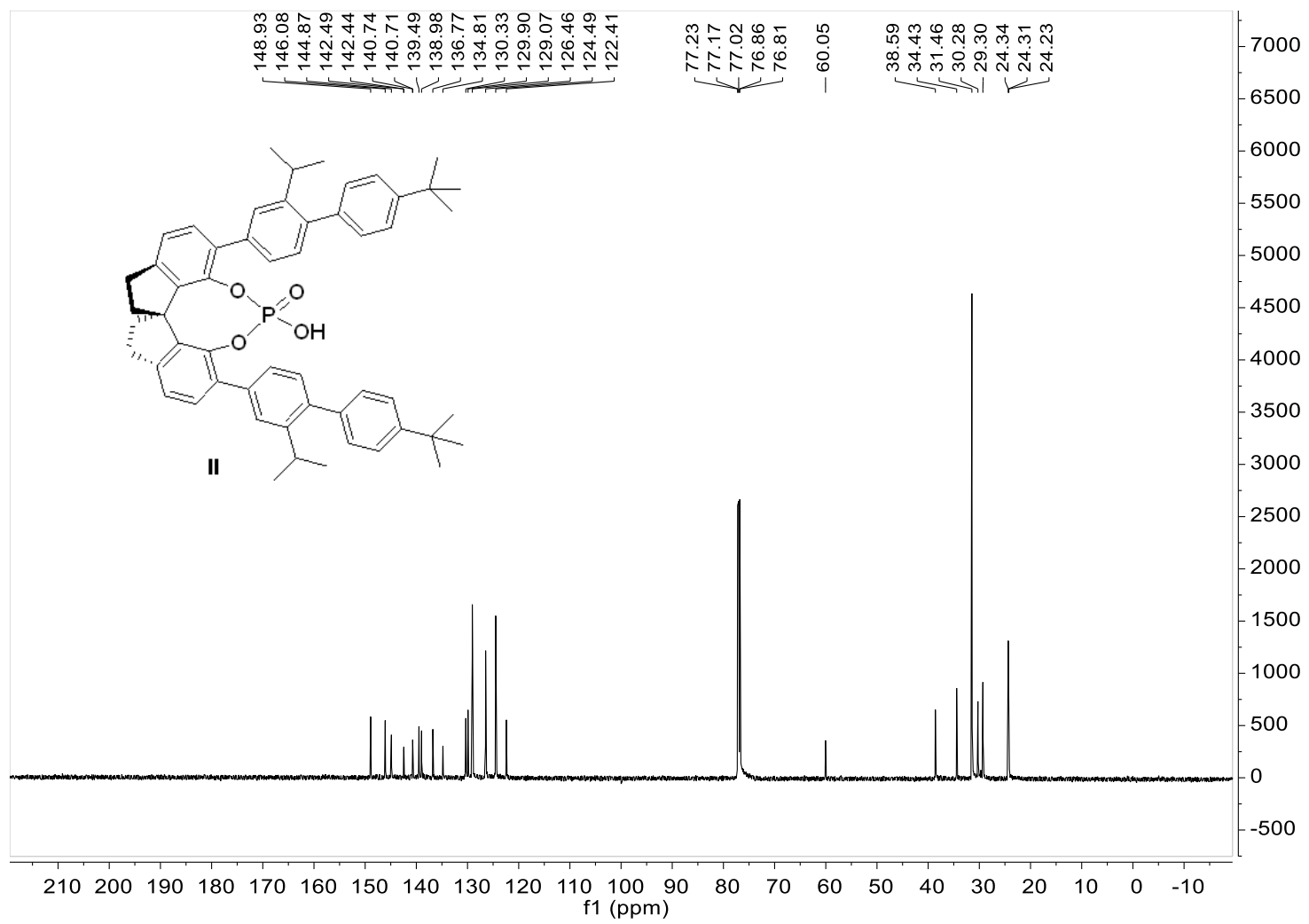


$600 \mathrm{MHz}, \mathrm{CDCl}_{3},{ }^{1} \mathrm{H} \mathrm{NMR}$

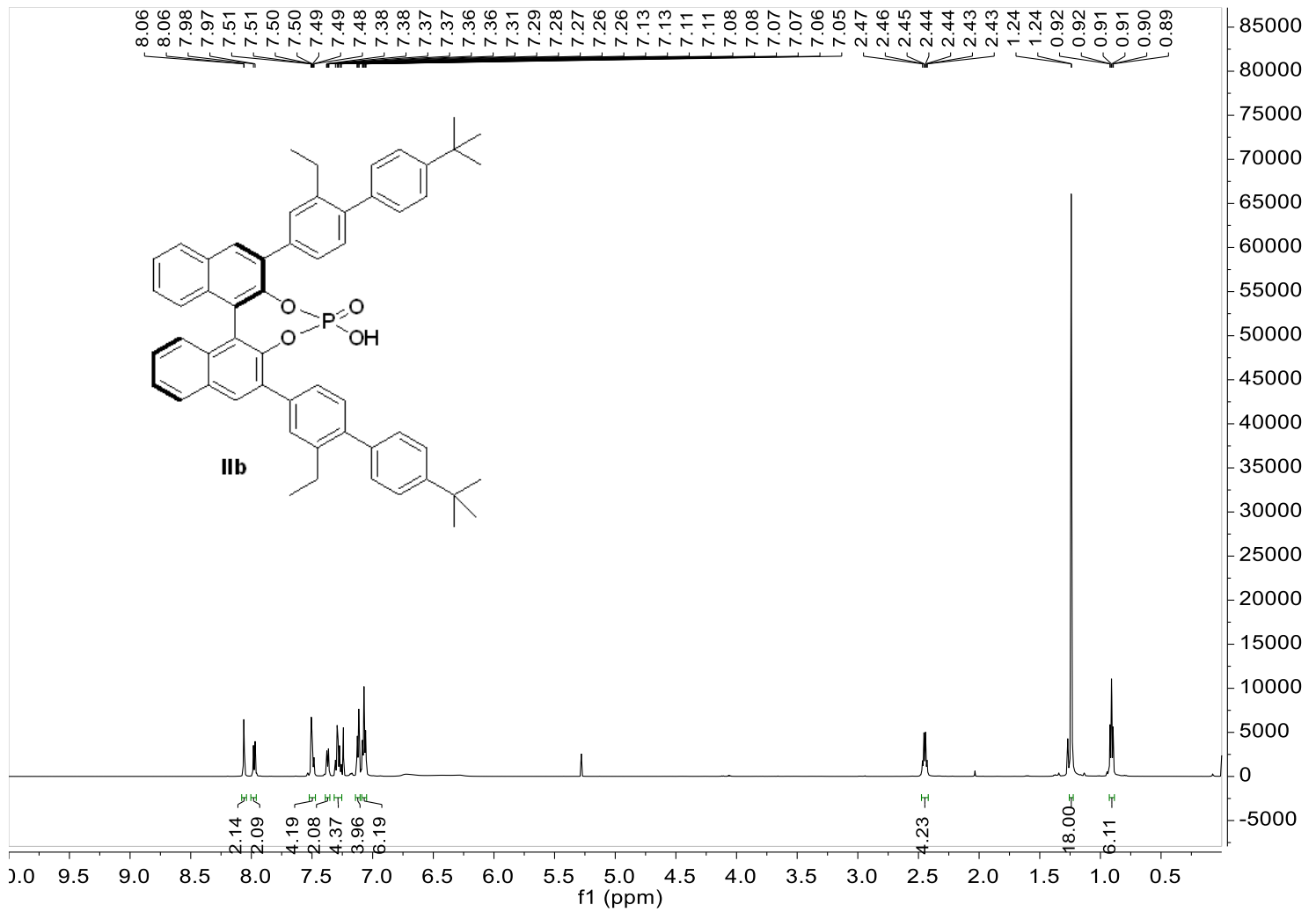

$151 \mathrm{MHz}, \mathrm{CDCl}_{3},{ }^{13} \mathrm{C} \mathrm{NMR}$

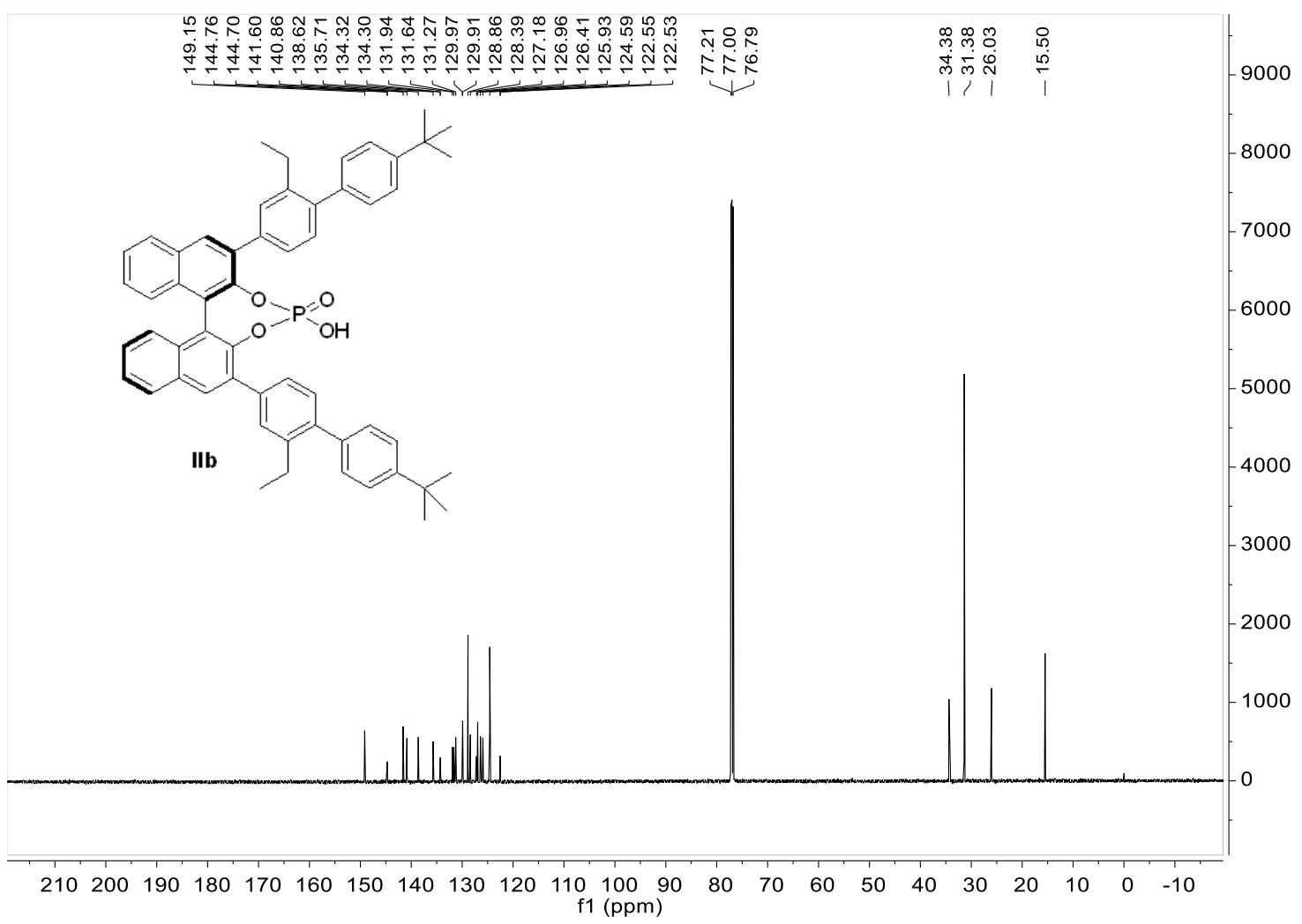



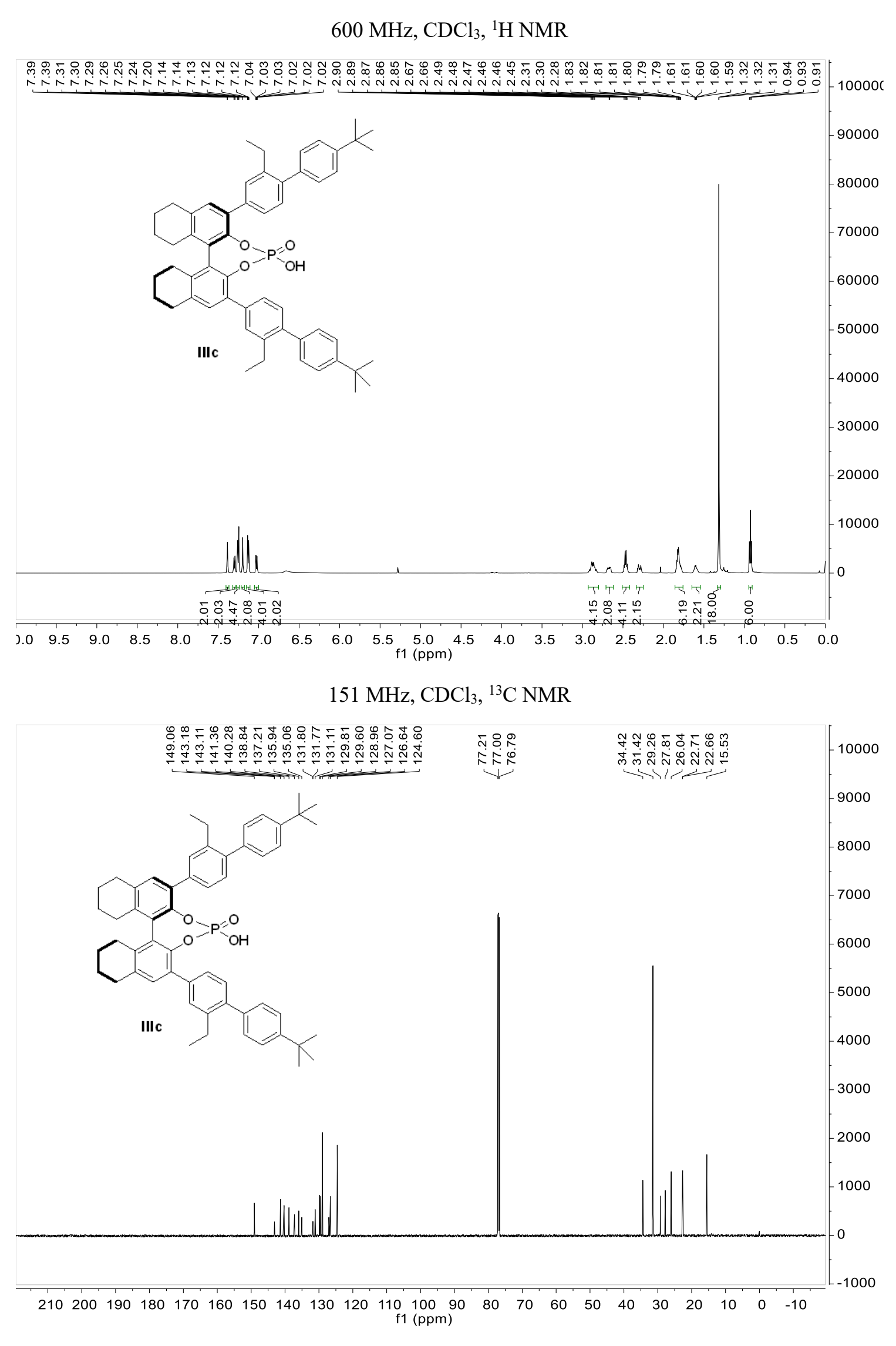
$600 \mathrm{MHz}, \mathrm{CDCl}_{3},{ }^{1} \mathrm{H} \mathrm{NMR}$

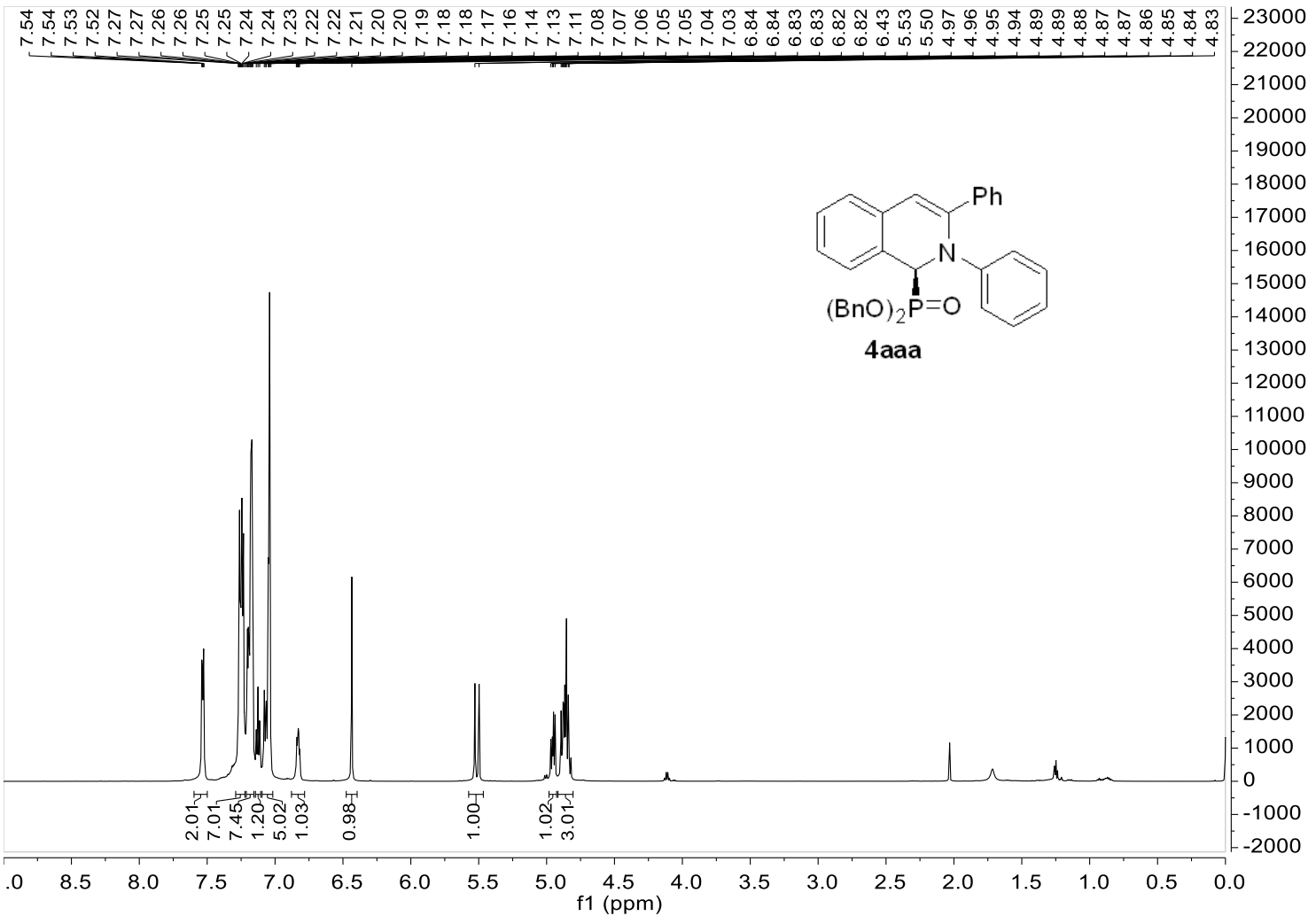

$151 \mathrm{MHz}, \mathrm{CDCl}_{3},{ }^{13} \mathrm{C} \mathrm{NMR}$

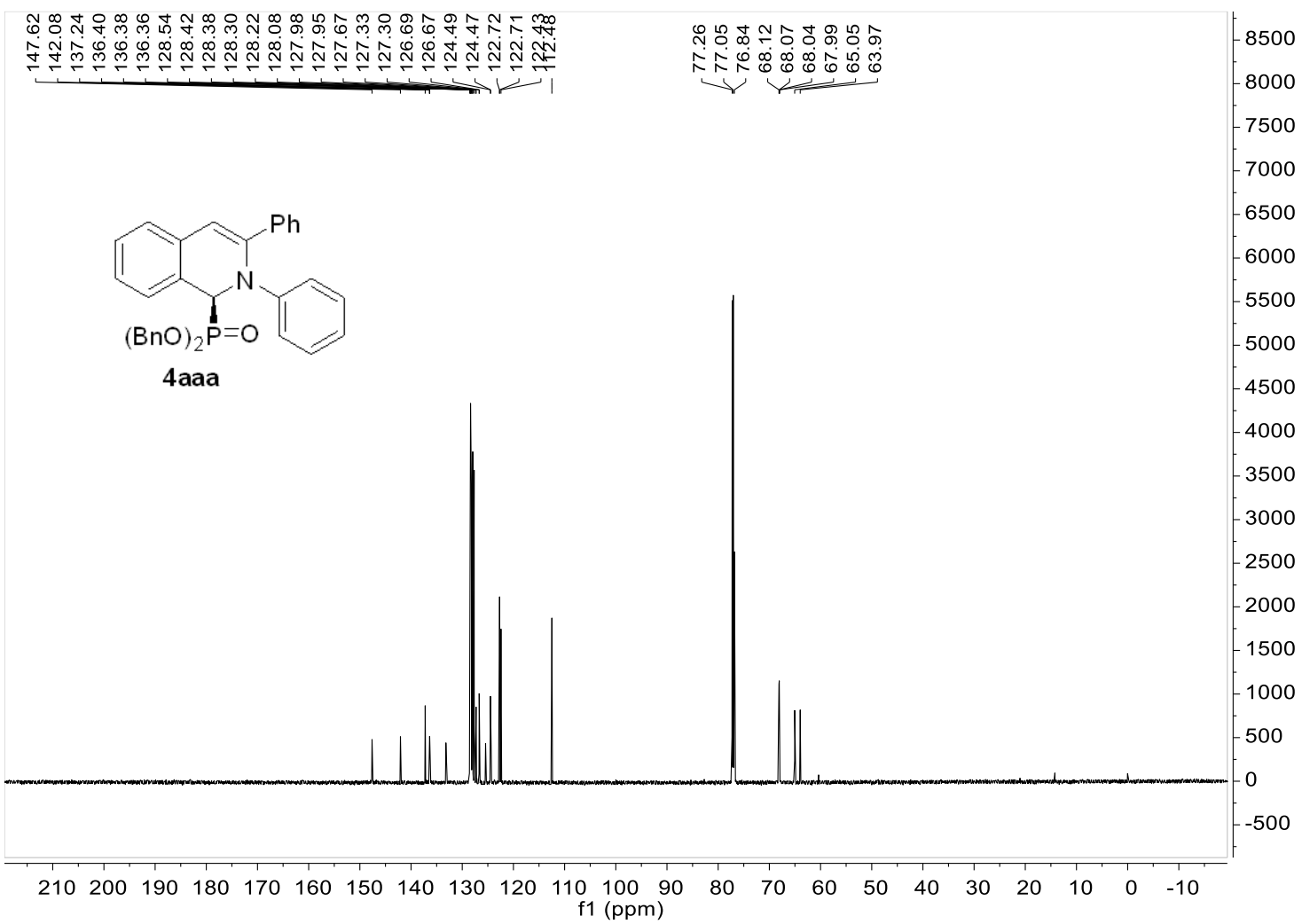


$600 \mathrm{MHz}, \mathrm{CDCl}_{3},{ }^{1} \mathrm{H}$ NMR

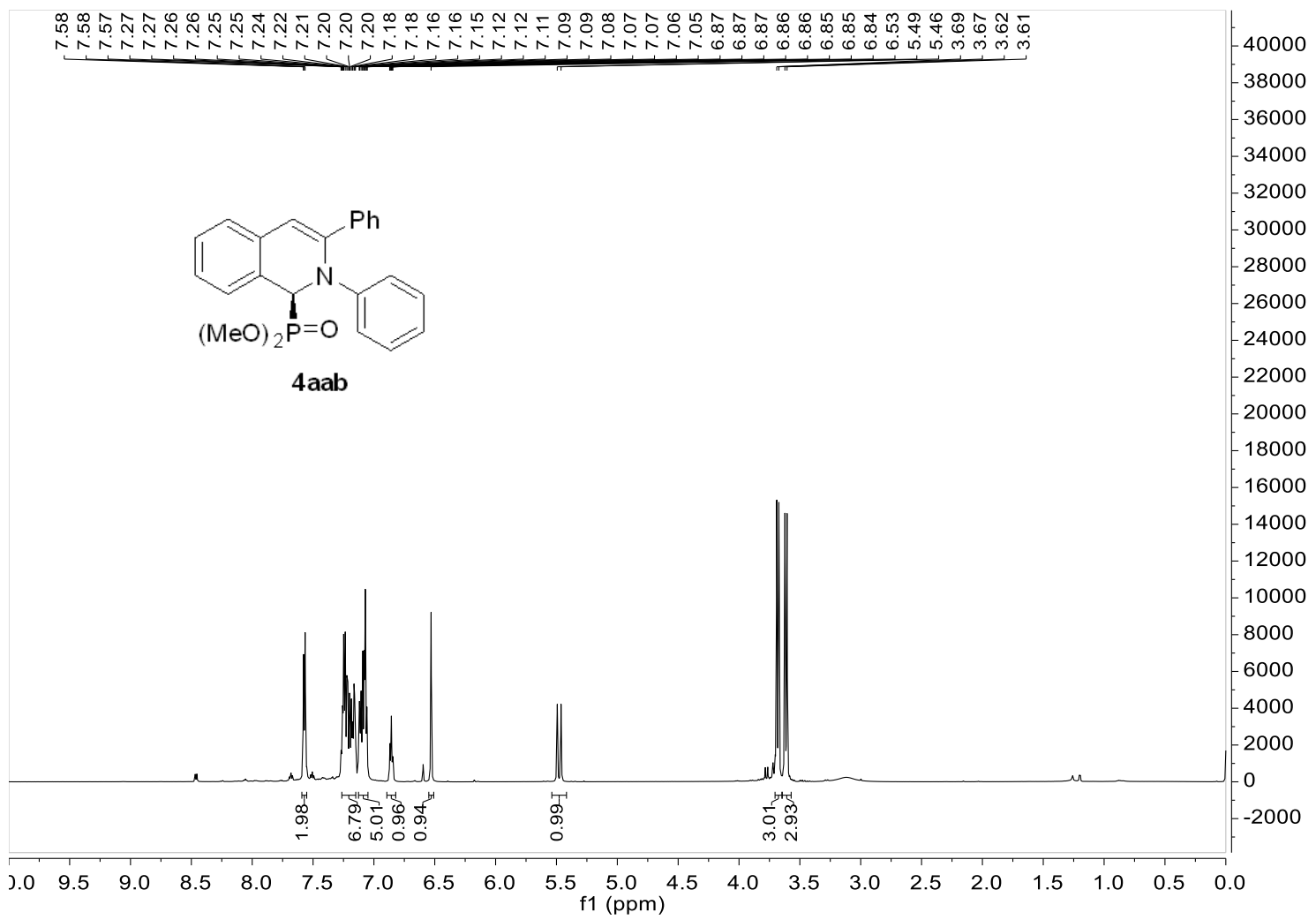

$151 \mathrm{MHz}, \mathrm{CDCl}_{3},{ }^{13} \mathrm{C} \mathrm{NMR}$

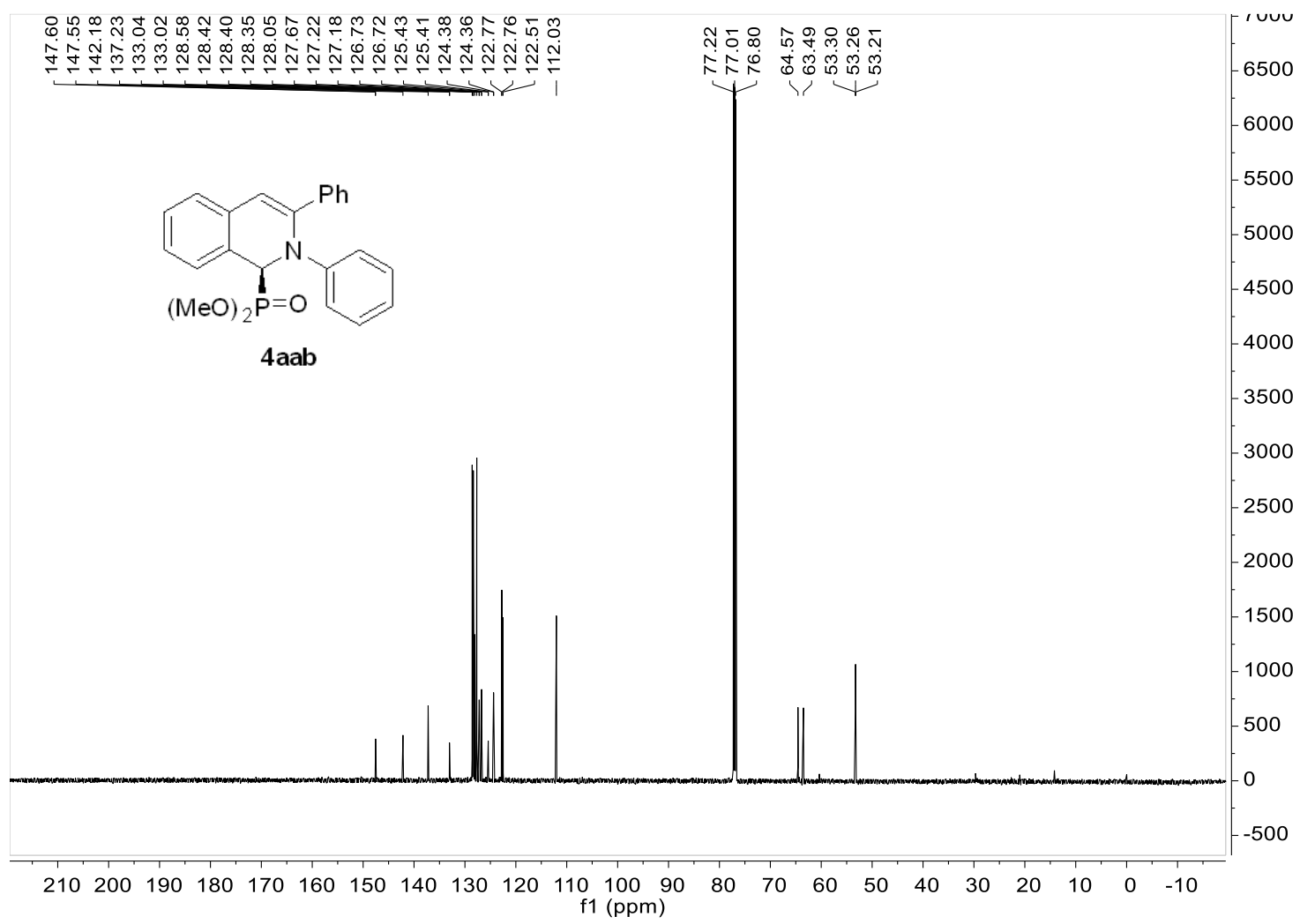


$600 \mathrm{MHz}, \mathrm{CDCl}_{3},{ }^{1} \mathrm{H} \mathrm{NMR}$

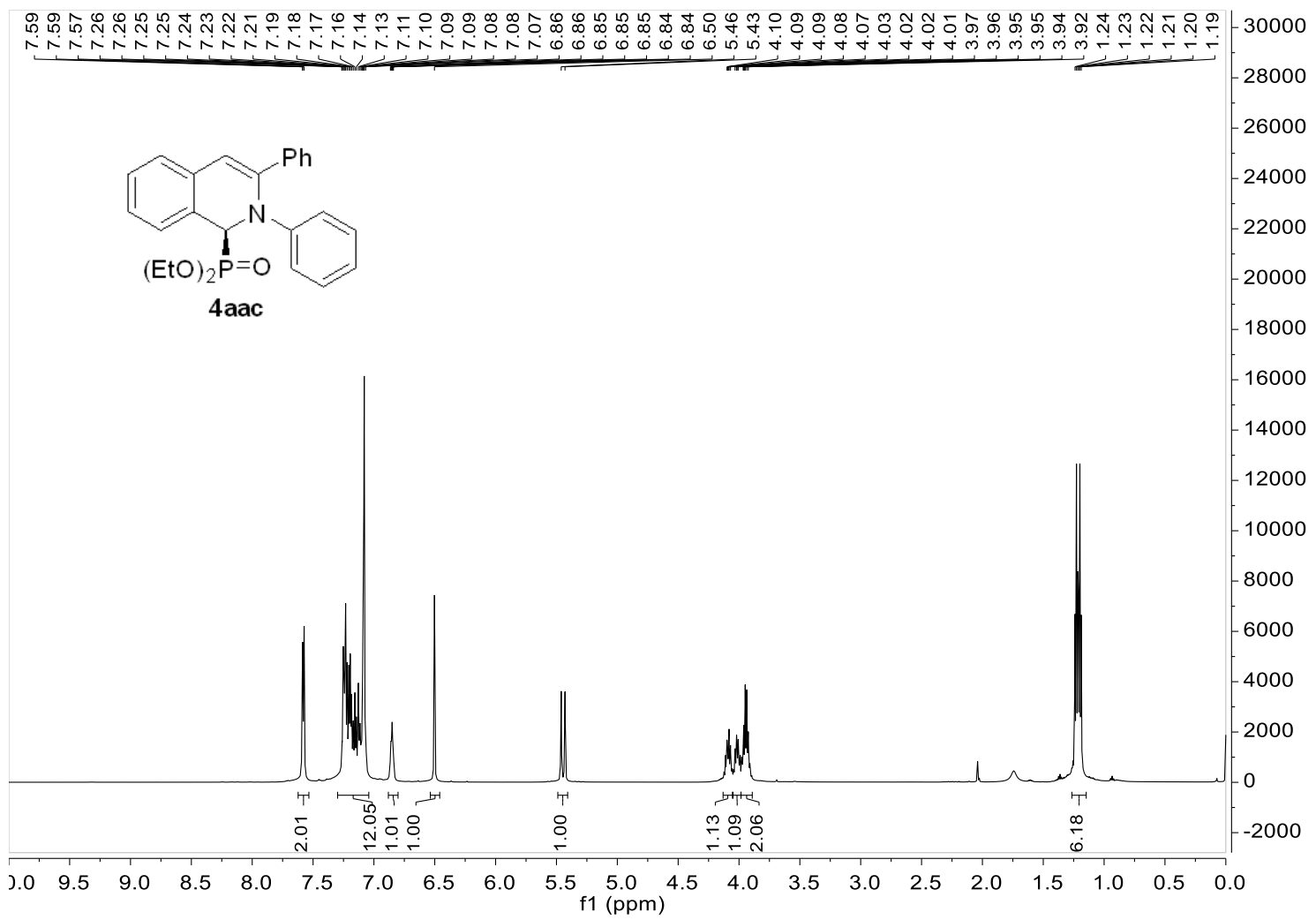

$151 \mathrm{MHz}, \mathrm{CDCl}_{3},{ }^{13} \mathrm{C} \mathrm{NMR}$

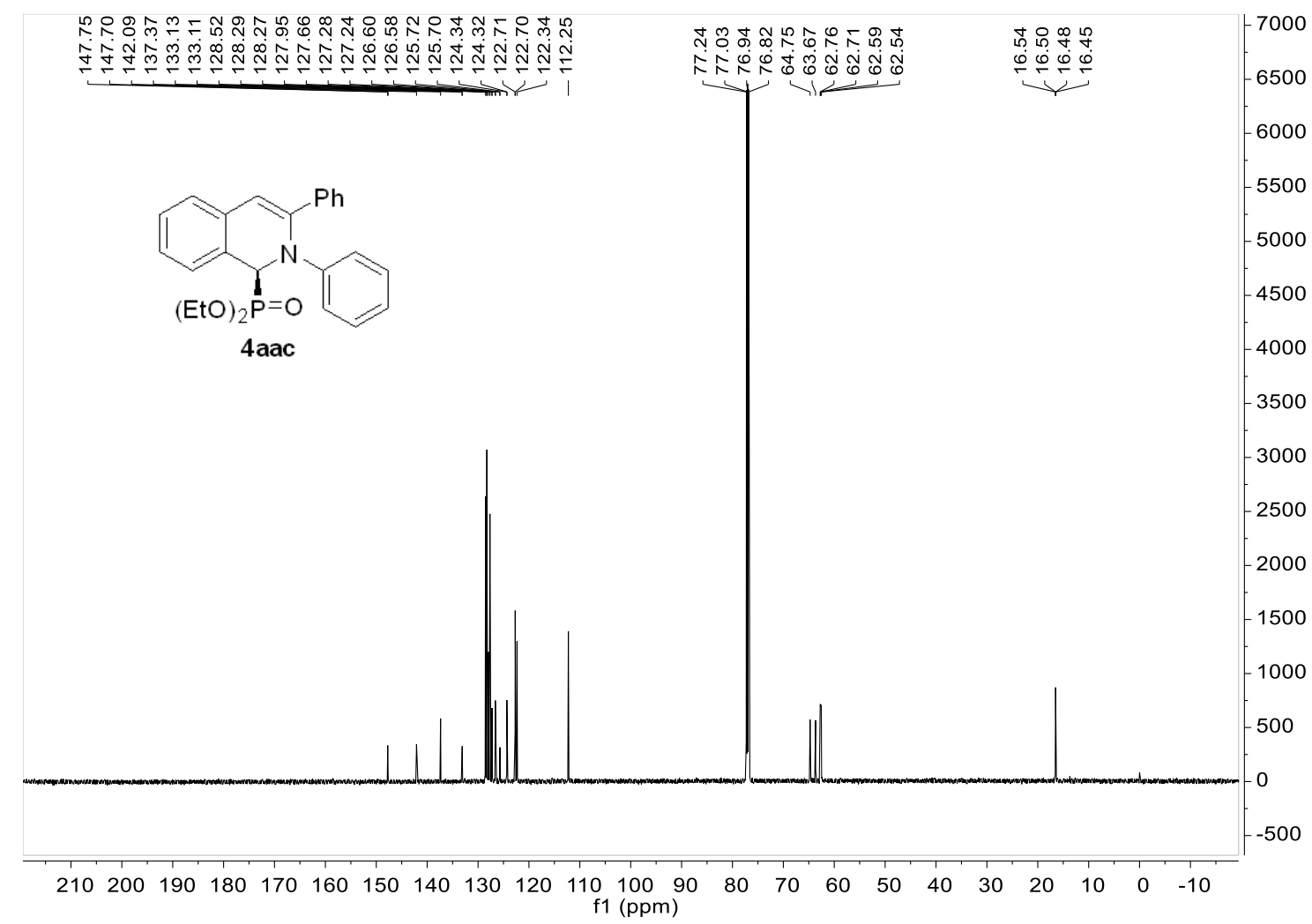


$600 \mathrm{MHz}, \mathrm{CDCl}_{3},{ }^{1} \mathrm{H} \mathrm{NMR}$

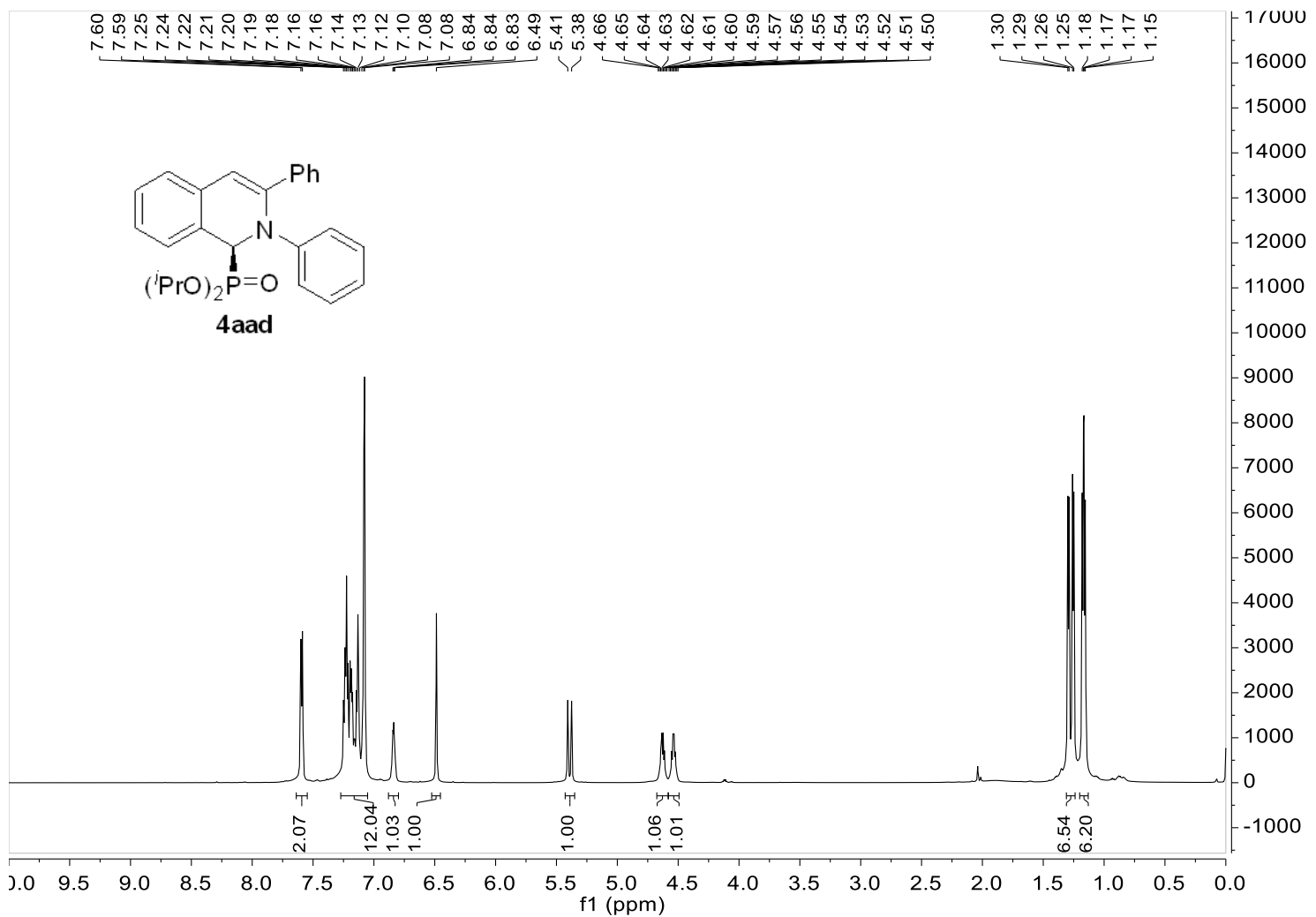

$151 \mathrm{MHz}, \mathrm{CDCl}_{3},{ }^{13} \mathrm{C} \mathrm{NMR}$

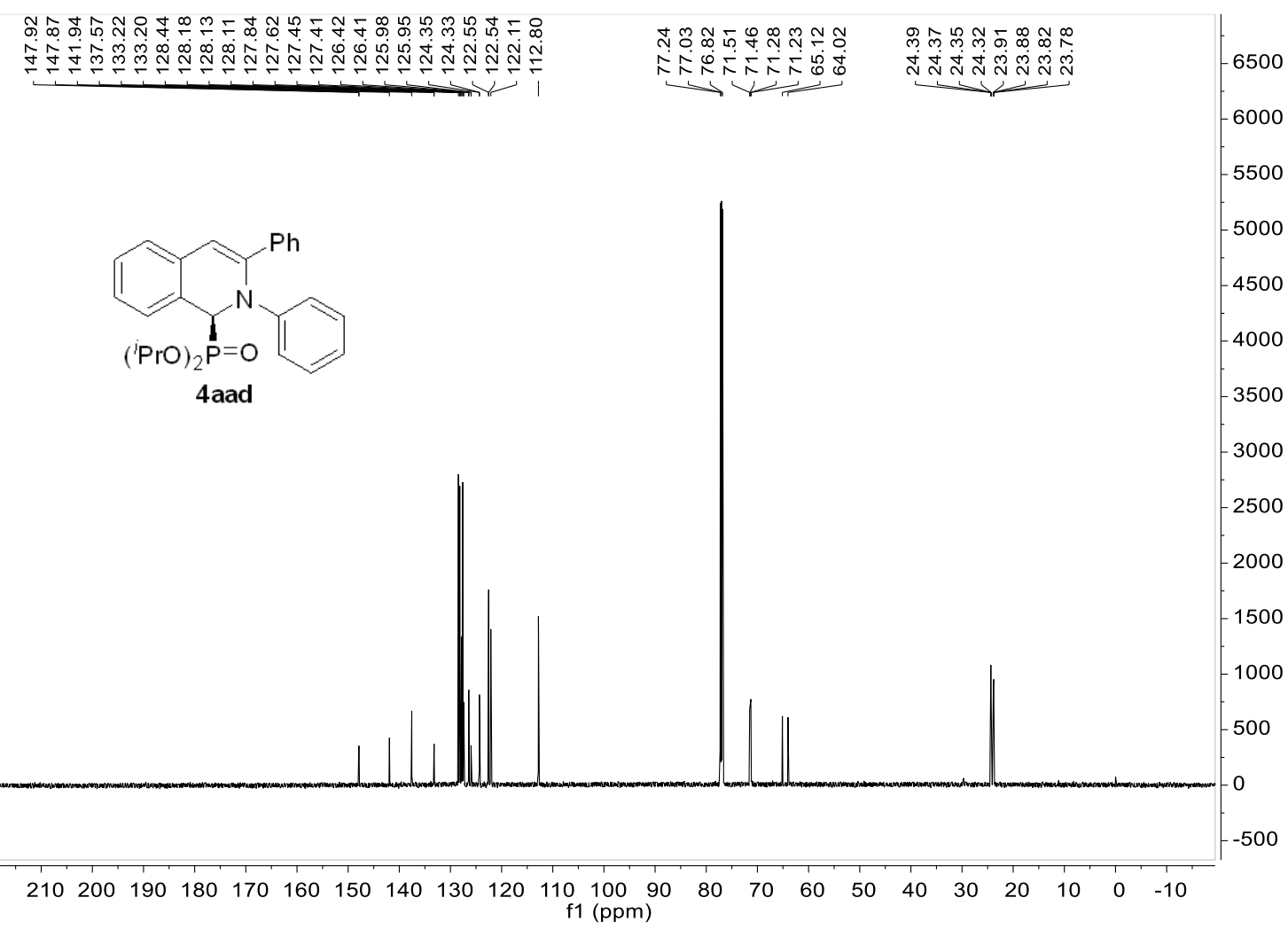


$600 \mathrm{MHz}, \mathrm{CDCl}_{3},{ }^{1} \mathrm{H} \mathrm{NMR}$

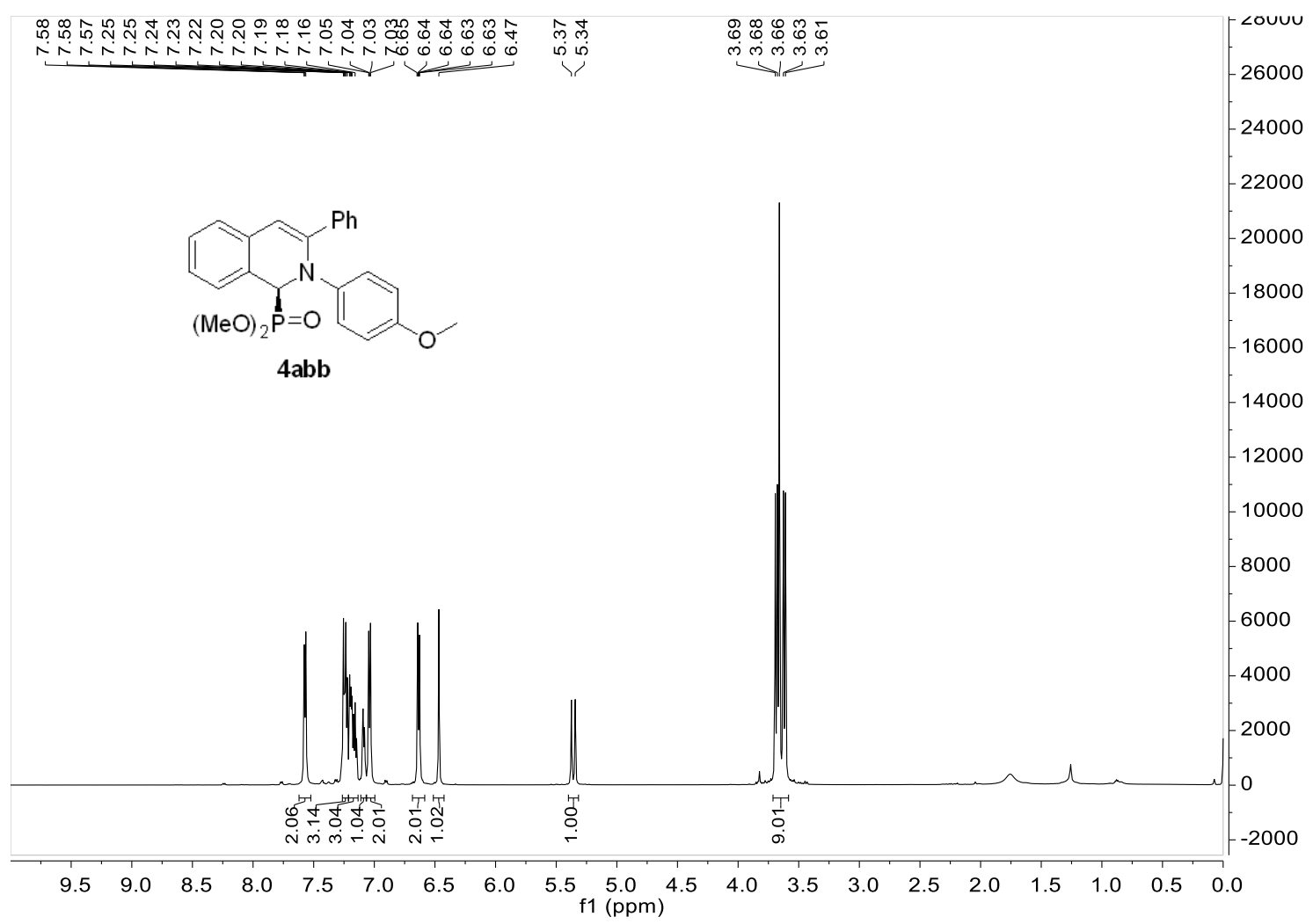

$151 \mathrm{MHz}, \mathrm{CDCl}_{3},{ }^{13} \mathrm{C} \mathrm{NMR}$

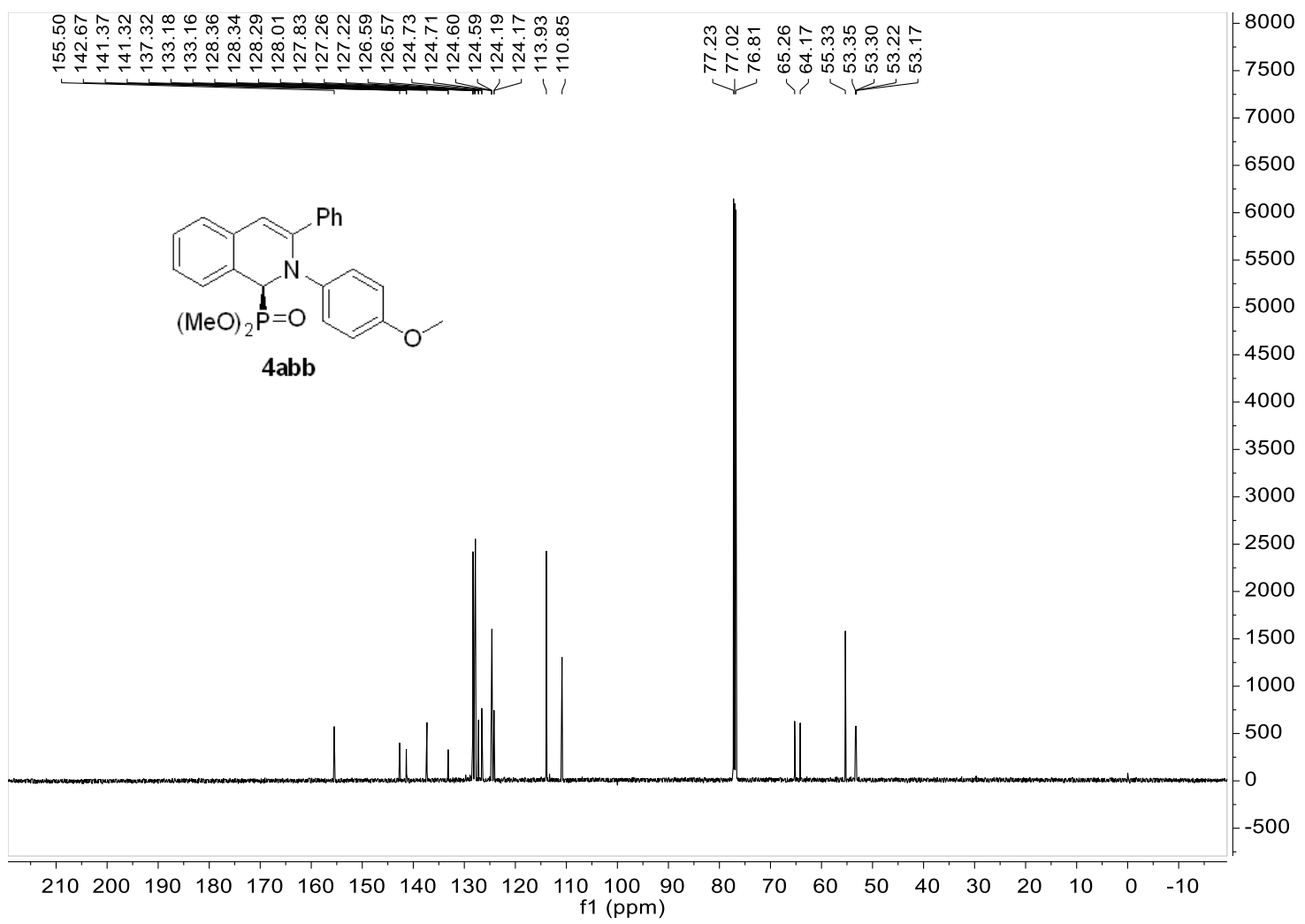


$600 \mathrm{MHz}, \mathrm{CDCl}_{3},{ }^{1} \mathrm{H}$ NMR

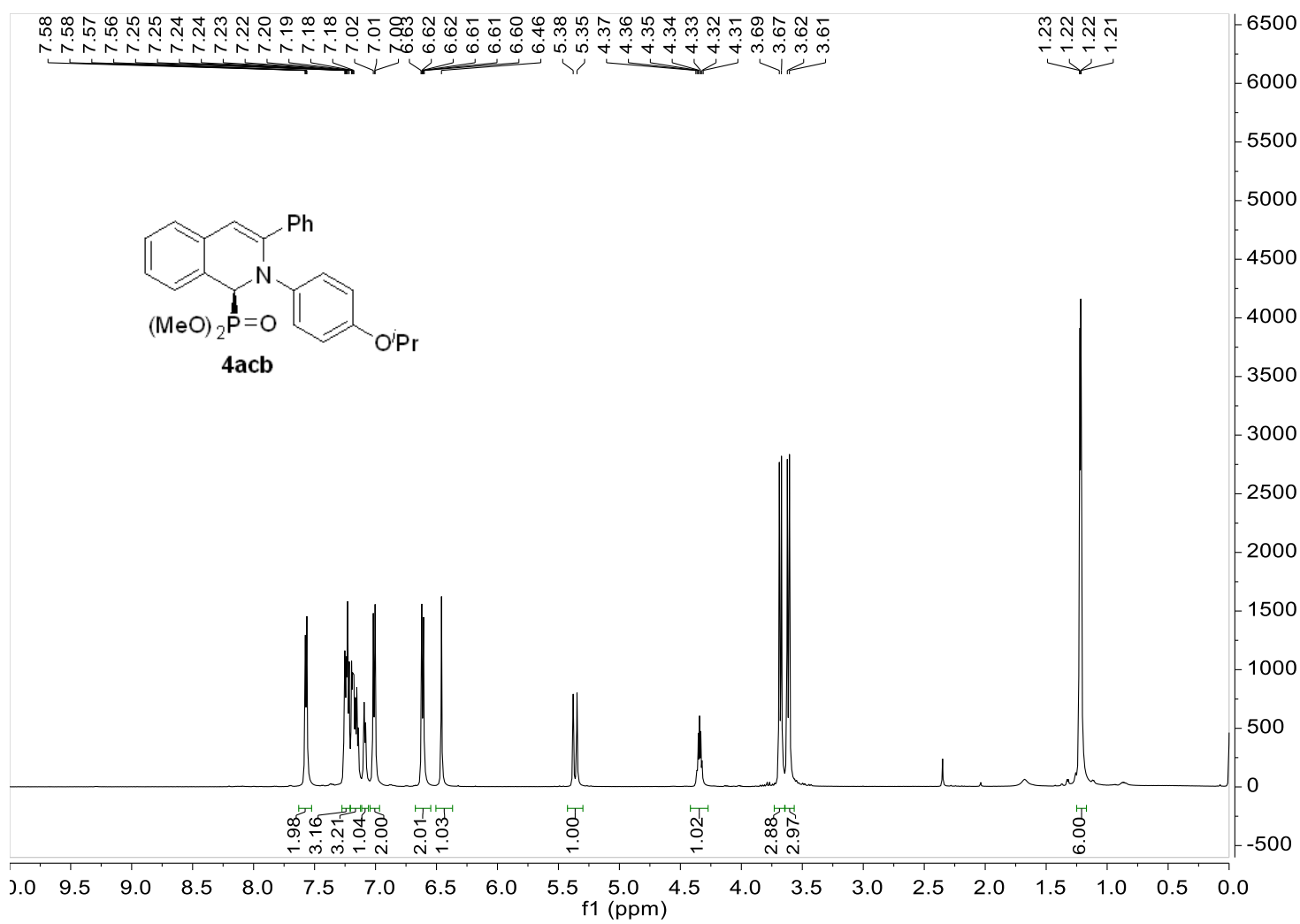

$151 \mathrm{MHz}, \mathrm{CDCl}_{3},{ }^{13} \mathrm{C} \mathrm{NMR}$

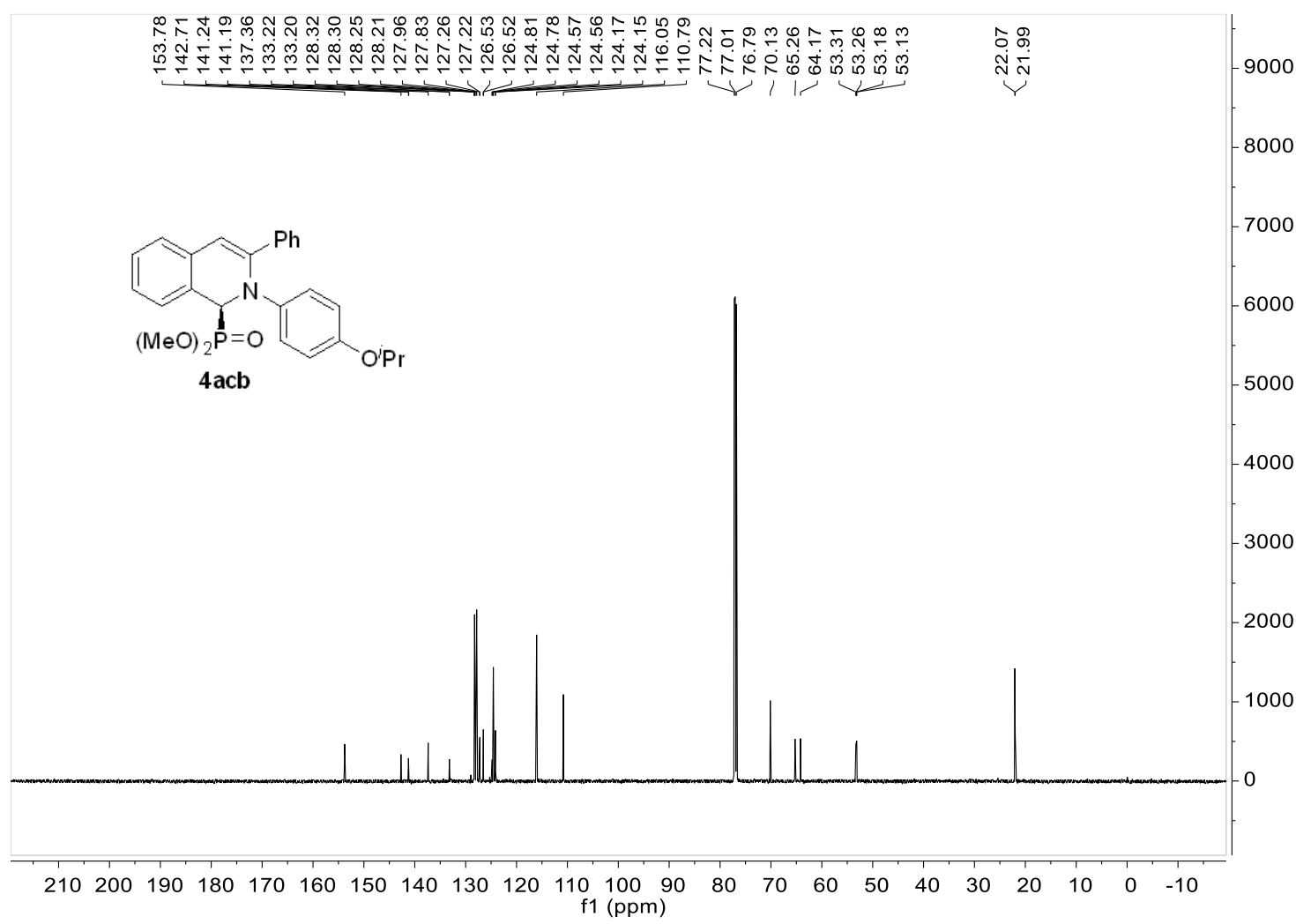


$600 \mathrm{MHz}, \mathrm{CDCl}_{3},{ }^{1} \mathrm{H} \mathrm{NMR}$

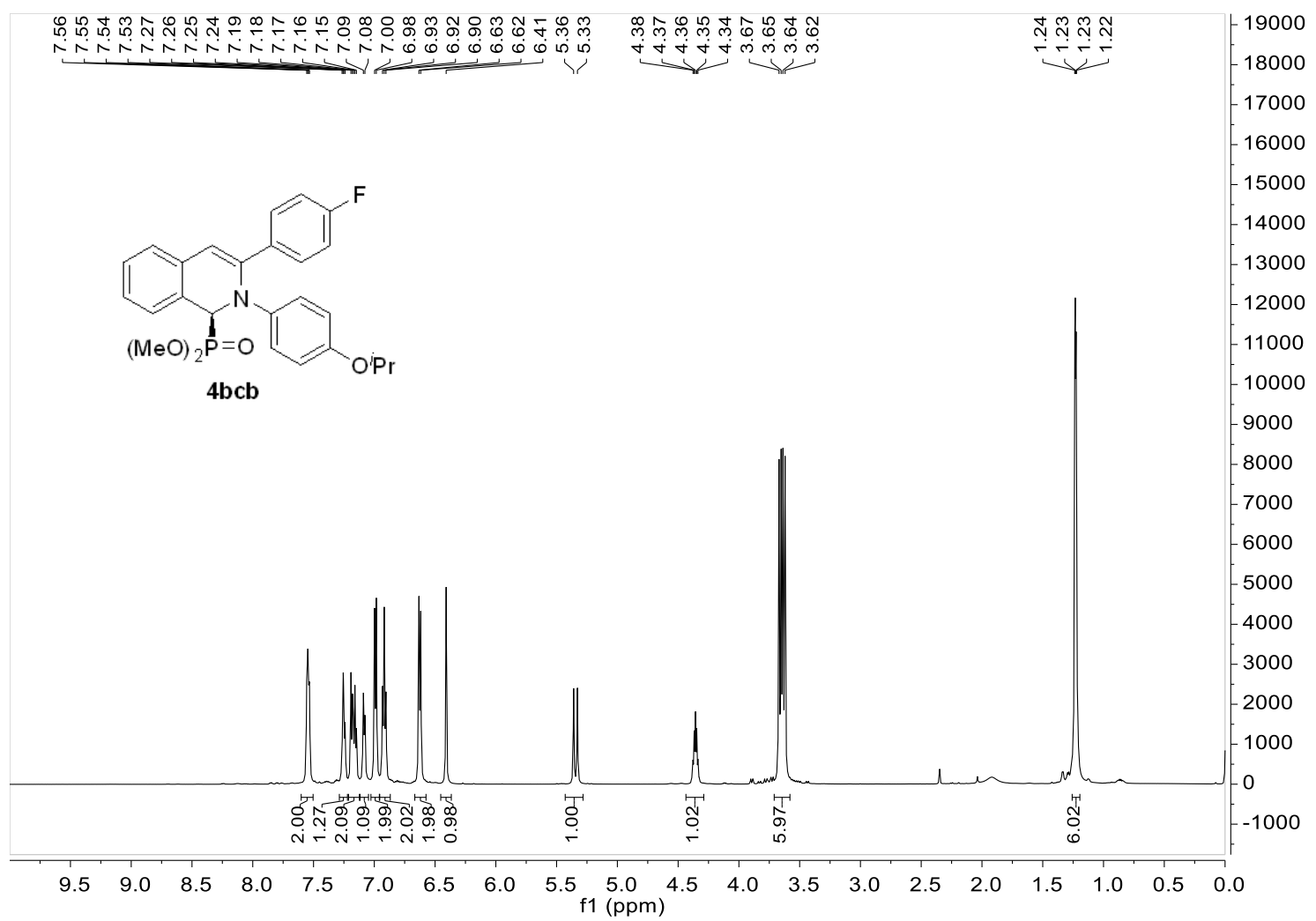

$151 \mathrm{MHz}, \mathrm{CDCl}_{3},{ }^{13} \mathrm{C} \mathrm{NMR}$

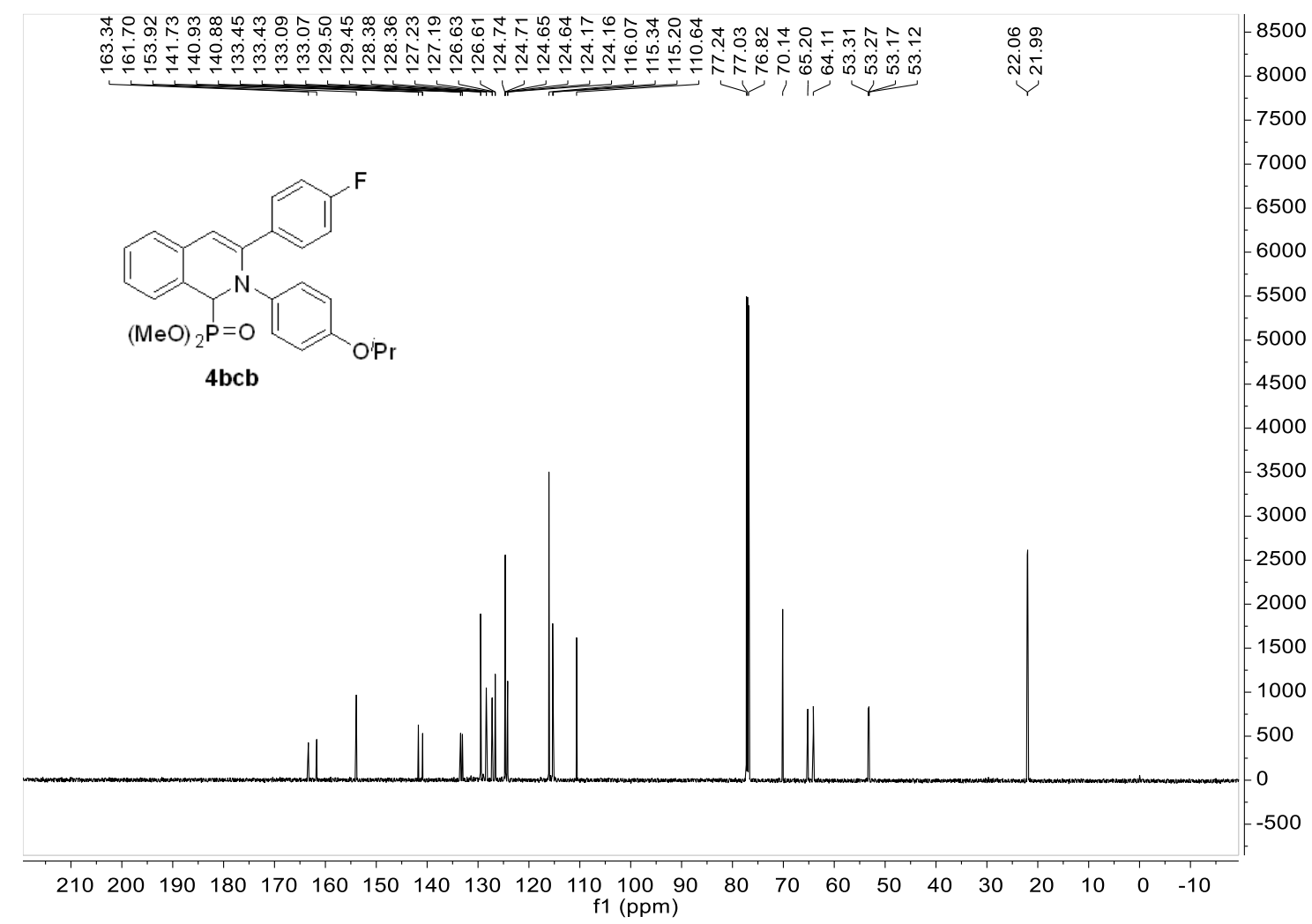


$600 \mathrm{MHz}, \mathrm{CDCl}_{3},{ }^{1} \mathrm{H} \mathrm{NMR}$

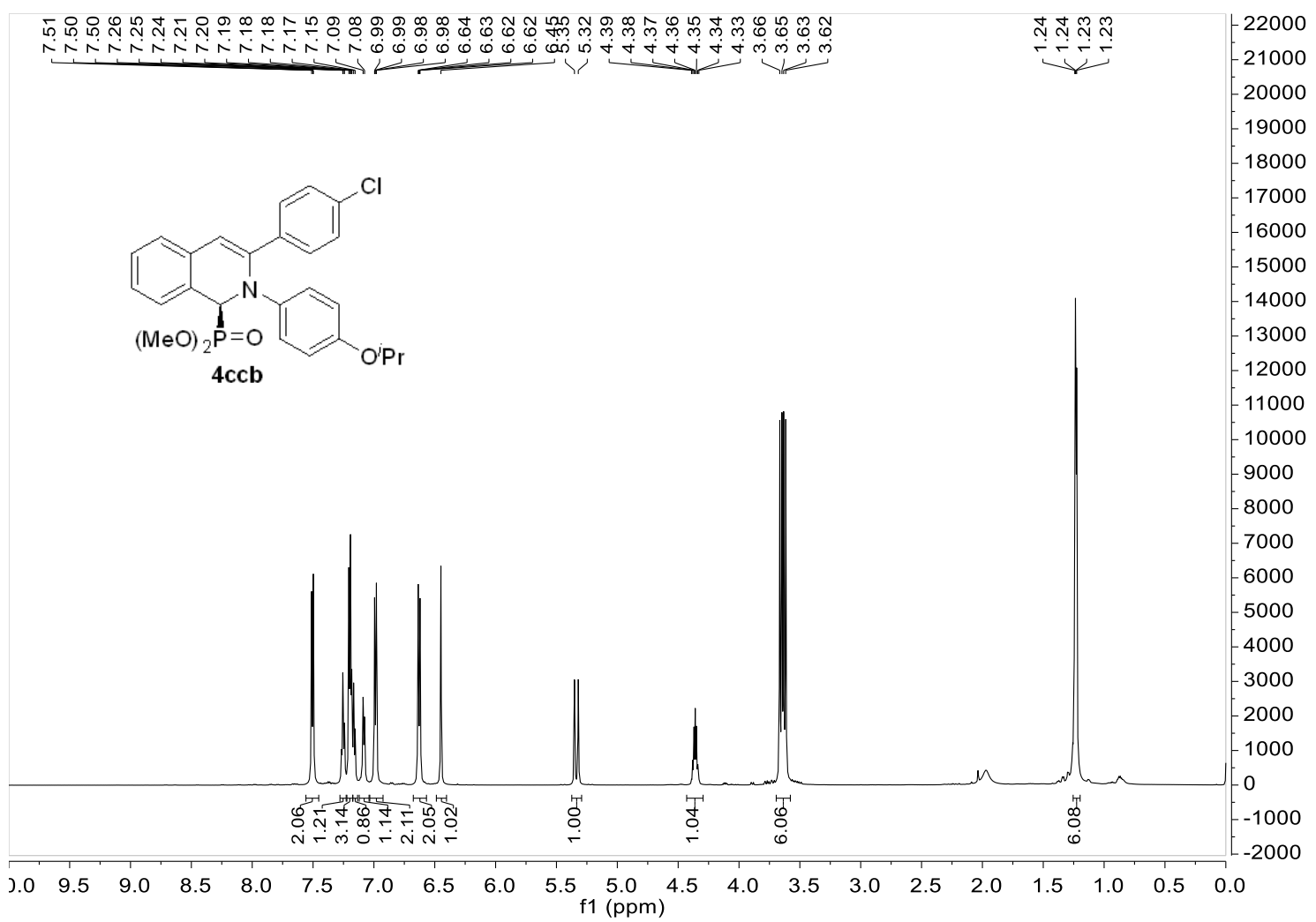

$151 \mathrm{MHz}, \mathrm{CDCl}_{3},{ }^{13} \mathrm{C} \mathrm{NMR}$

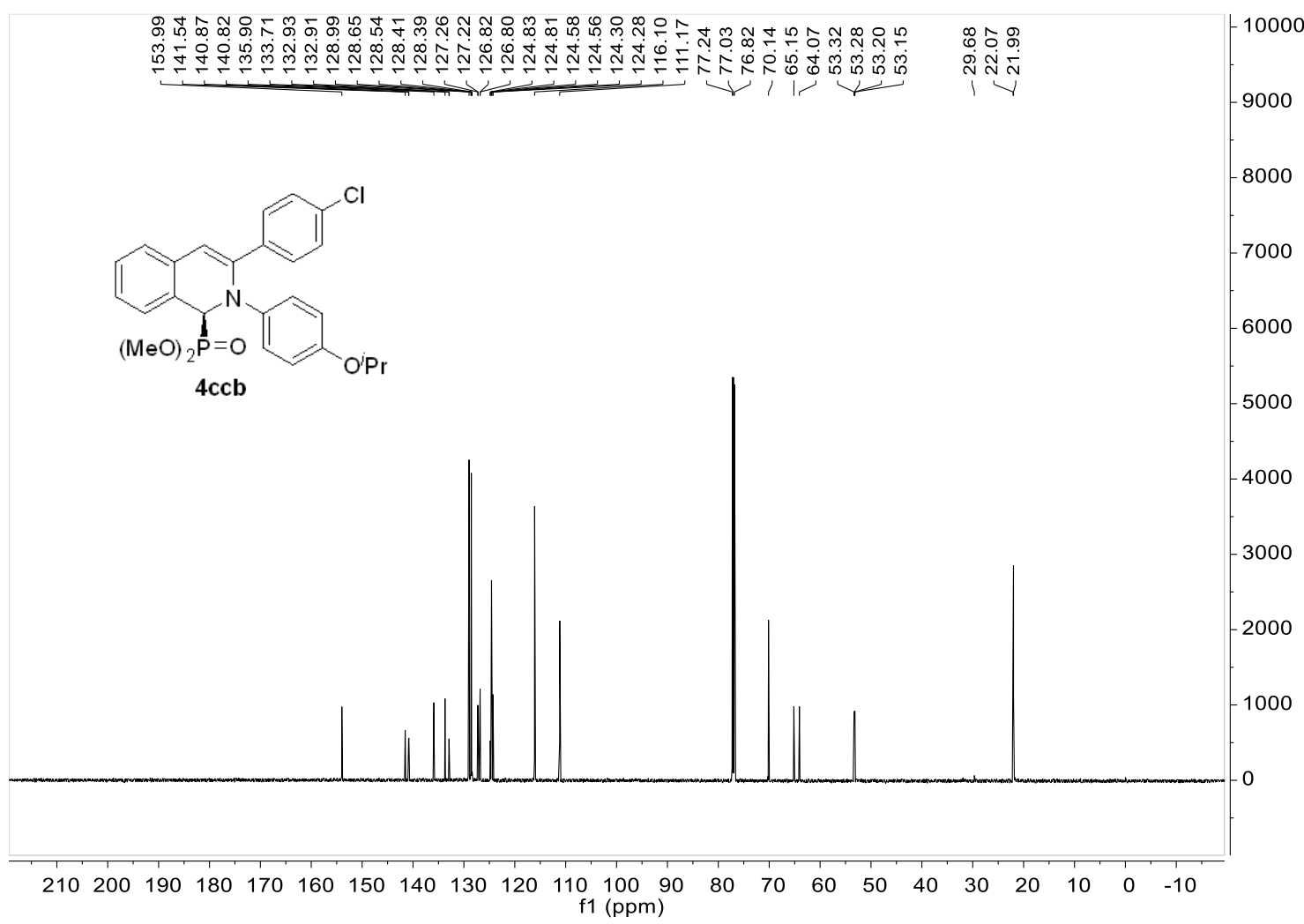


$600 \mathrm{MHz}, \mathrm{CDCl}_{3},{ }^{1} \mathrm{H} \mathrm{NMR}$

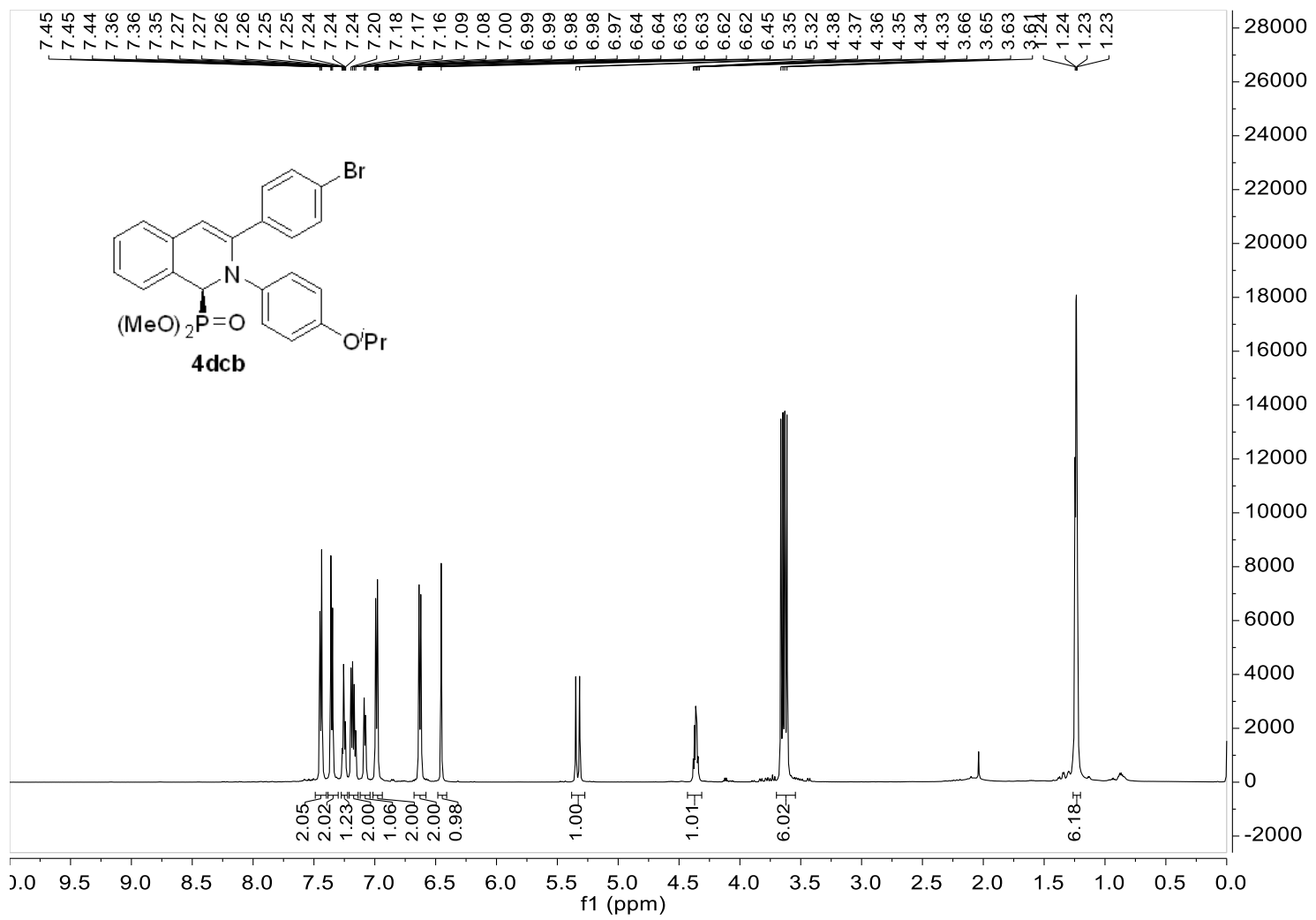

$151 \mathrm{MHz}, \mathrm{CDCl}_{3},{ }^{13} \mathrm{C} \mathrm{NMR}$

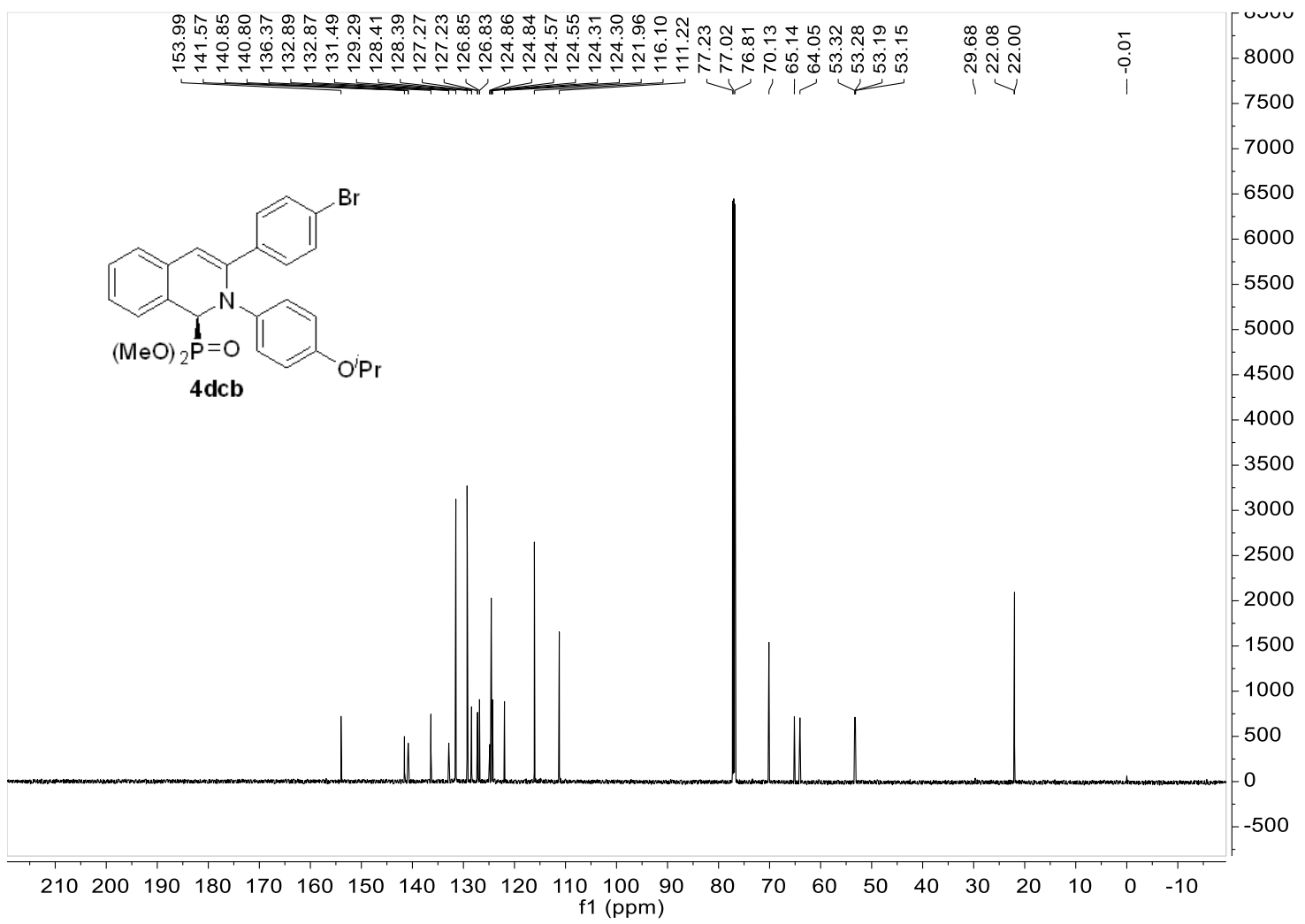


$600 \mathrm{MHz}, \mathrm{CDCl}_{3},{ }^{1} \mathrm{H} \mathrm{NMR}$

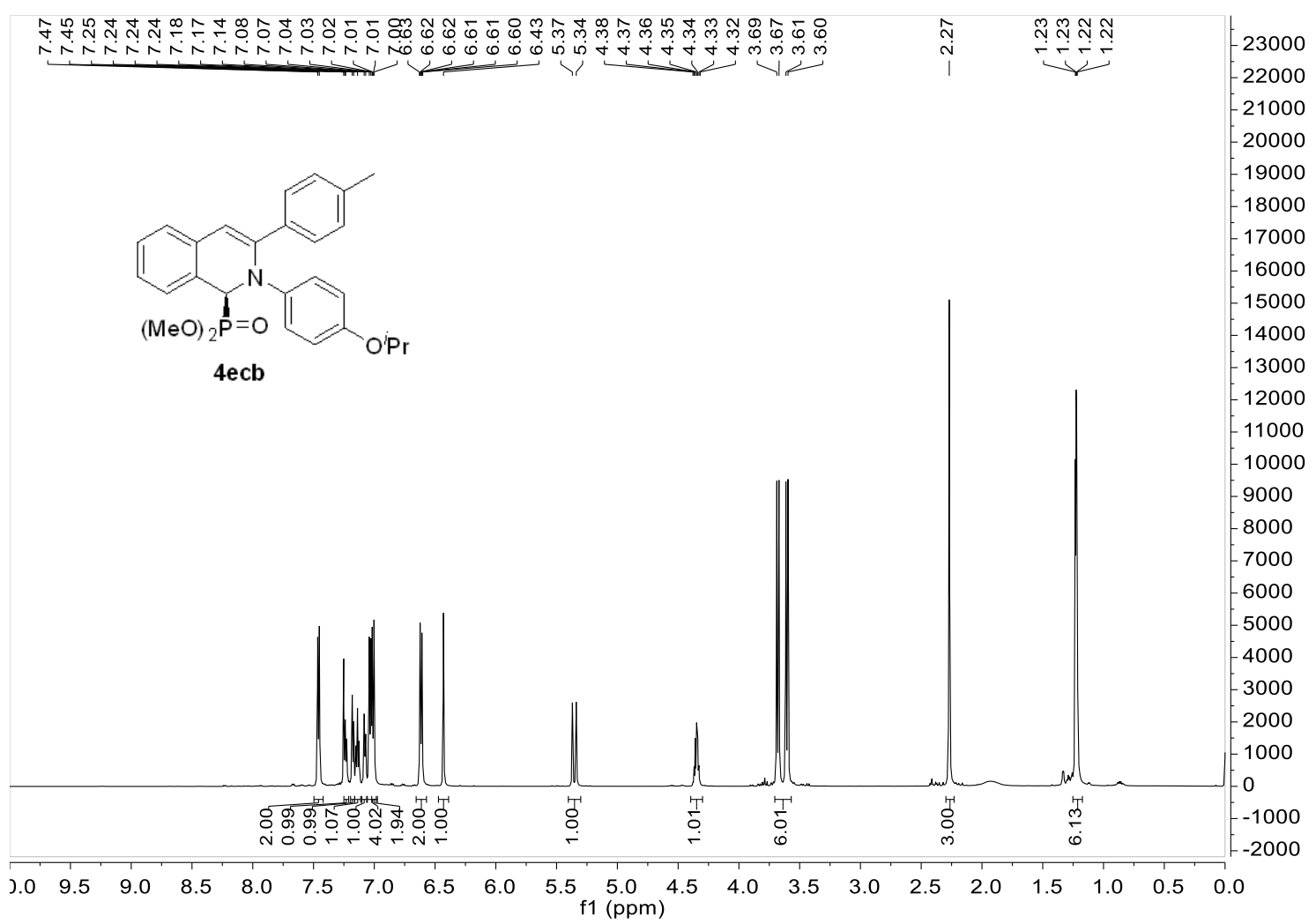

$151 \mathrm{MHz}, \mathrm{CDCl}_{3},{ }^{13} \mathrm{C} \mathrm{NMR}$

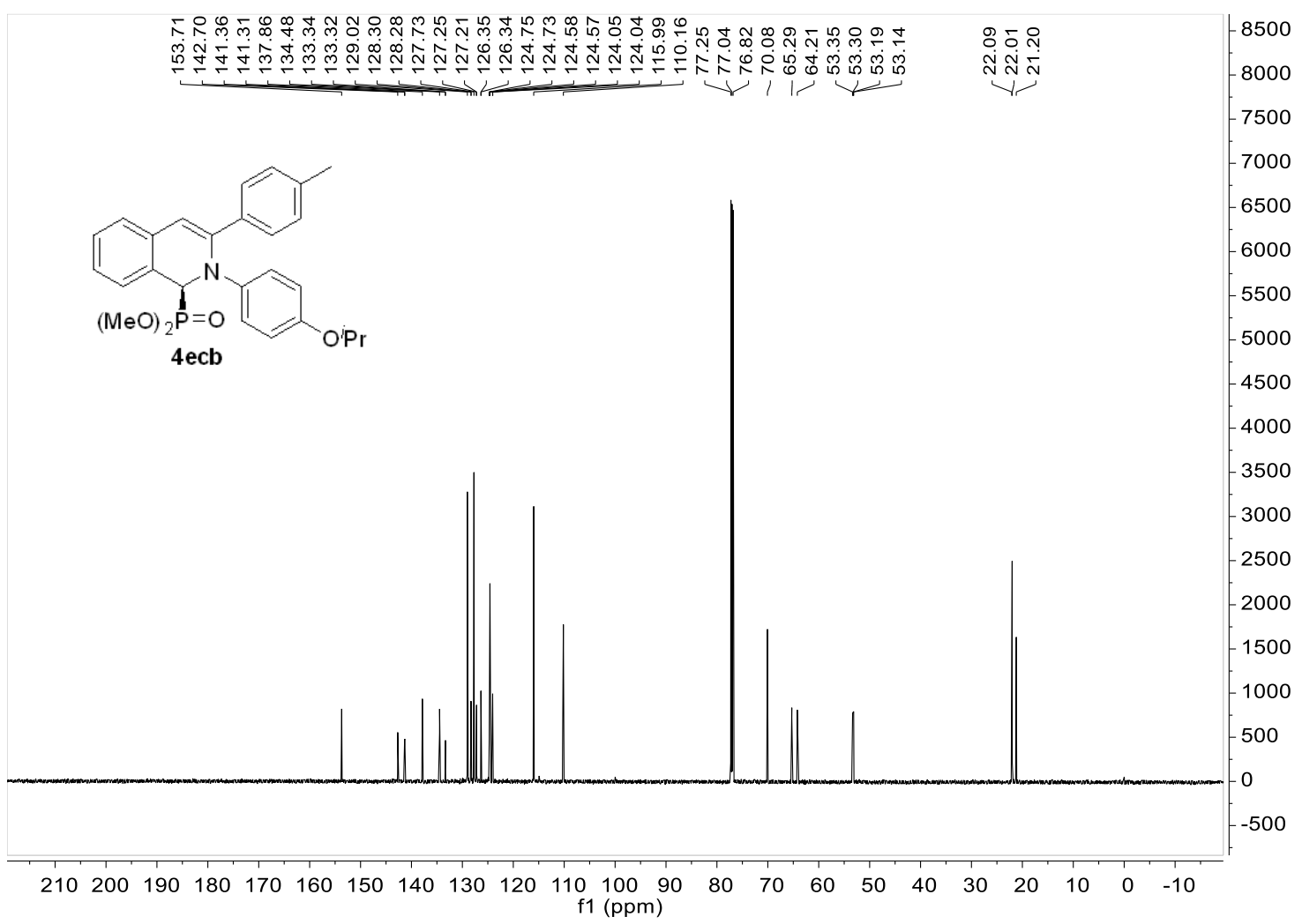


$600 \mathrm{MHz}, \mathrm{CDCl}_{3},{ }^{1} \mathrm{H} \mathrm{NMR}$

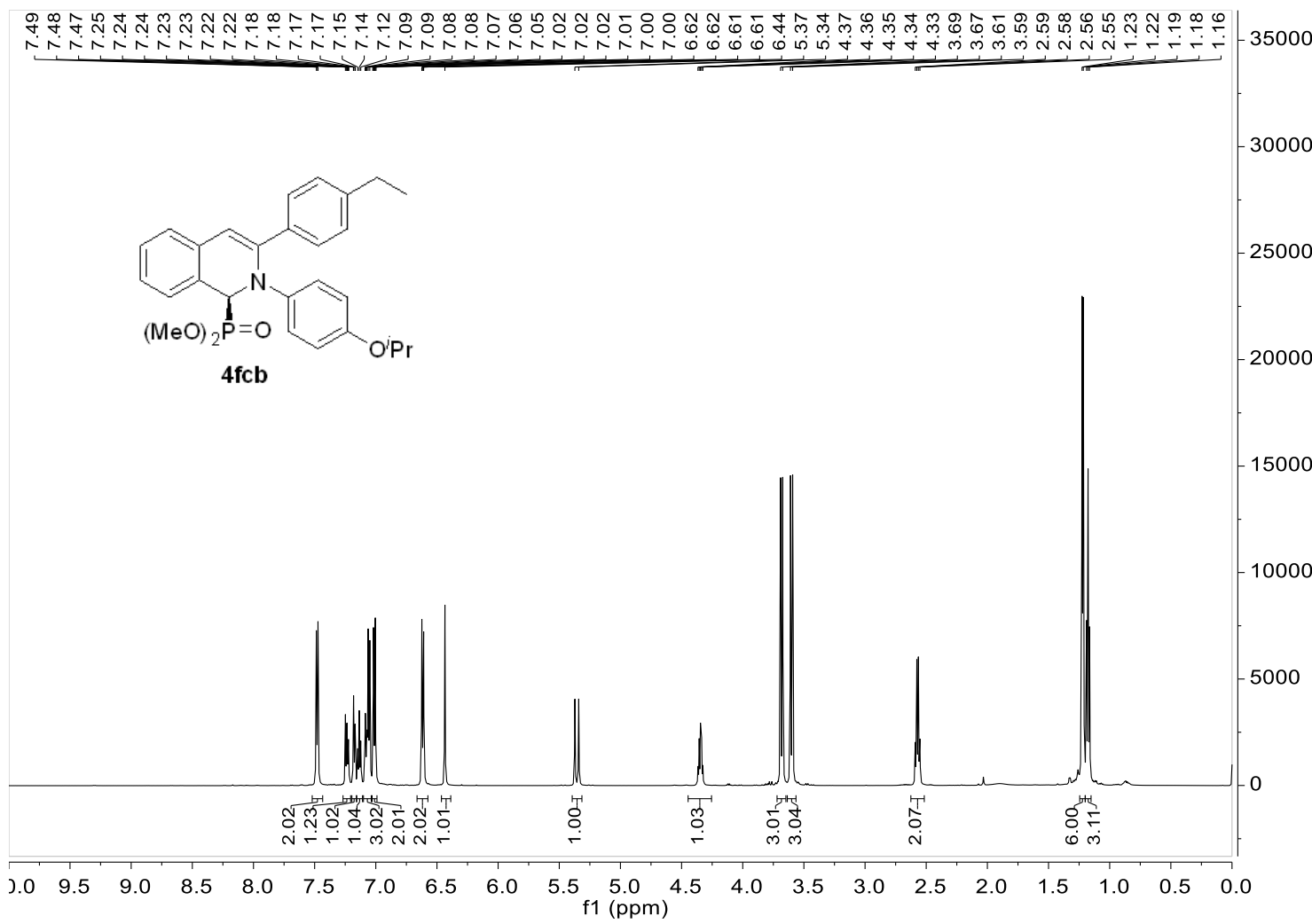

$151 \mathrm{MHz}, \mathrm{CDCl}_{3},{ }^{13} \mathrm{C} \mathrm{NMR}$

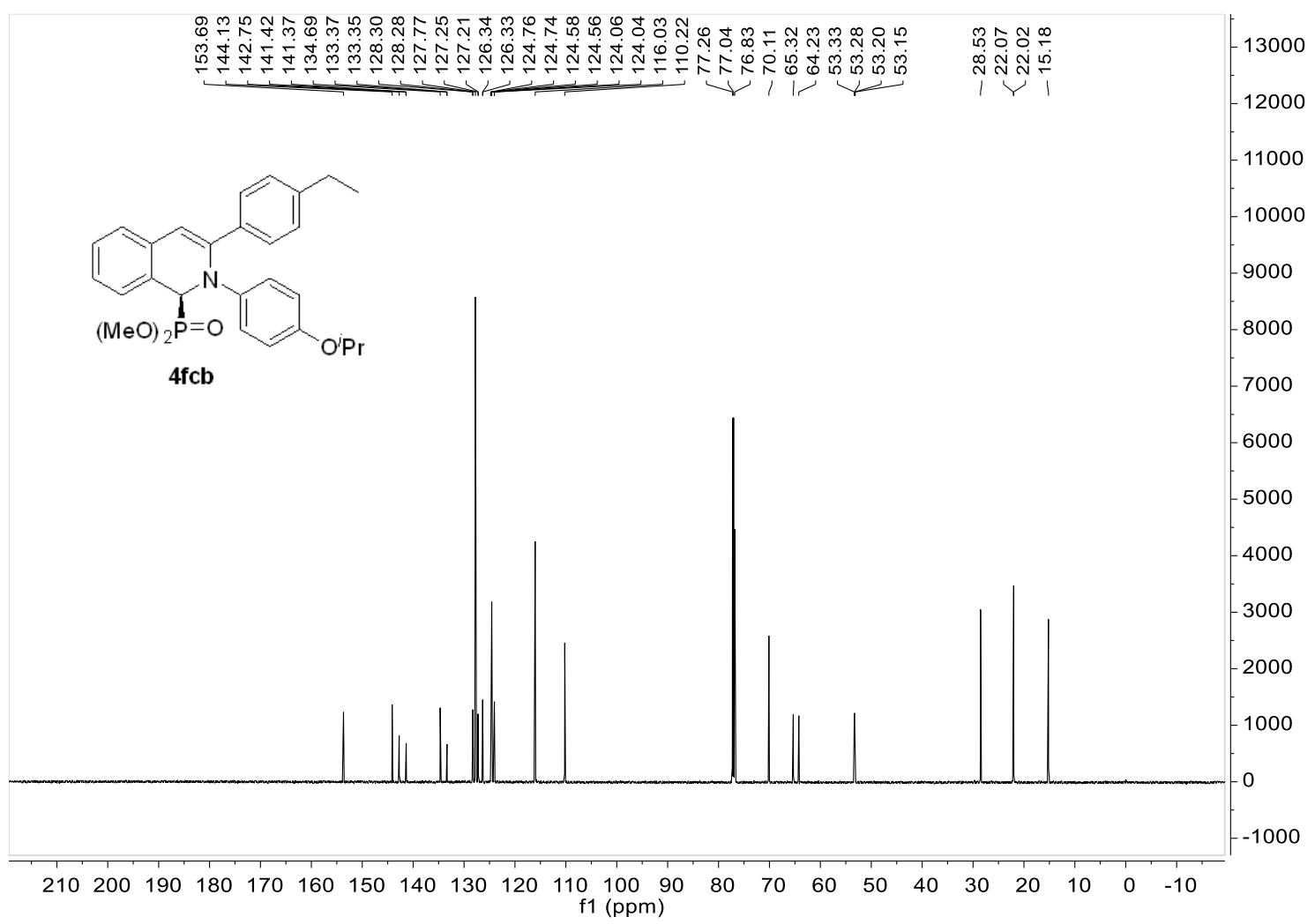


$600 \mathrm{MHz}, \mathrm{CDCl}_{3},{ }^{1} \mathrm{H} \mathrm{NMR}$

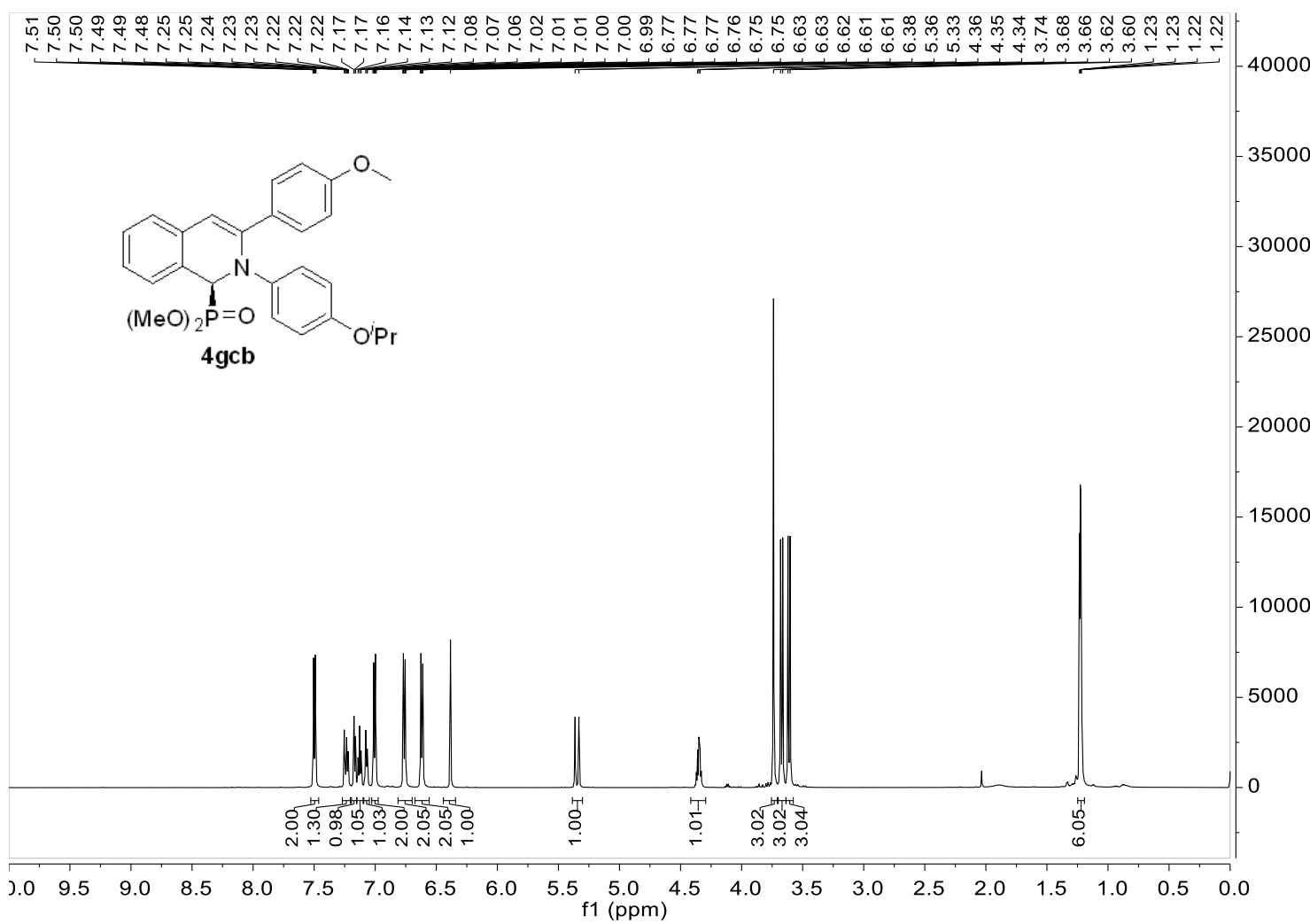

$151 \mathrm{MHz}, \mathrm{CDCl}_{3},{ }^{13} \mathrm{C} \mathrm{NMR}$

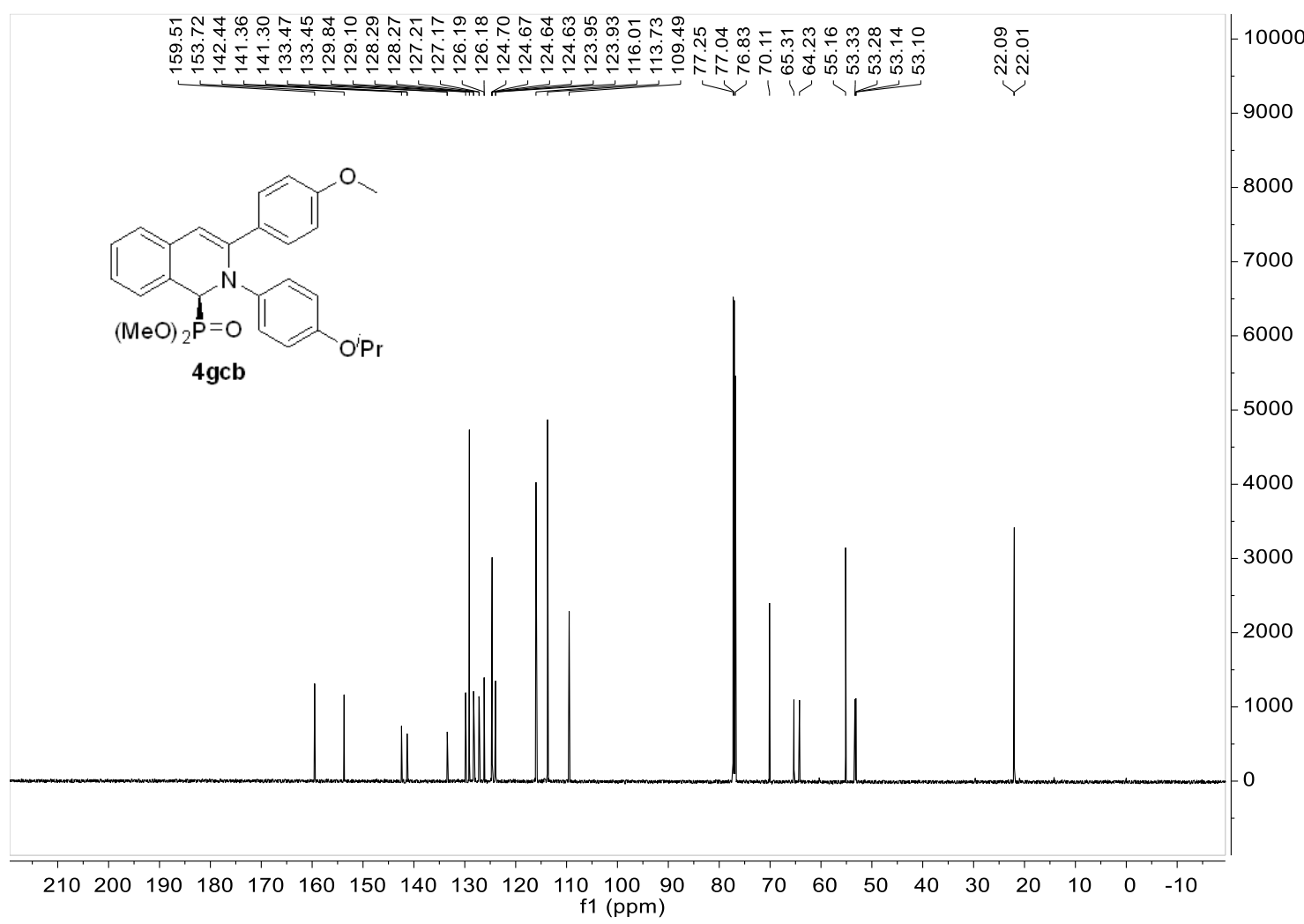


$600 \mathrm{MHz}, \mathrm{CDCl}_{3},{ }^{1} \mathrm{H} \mathrm{NMR}$

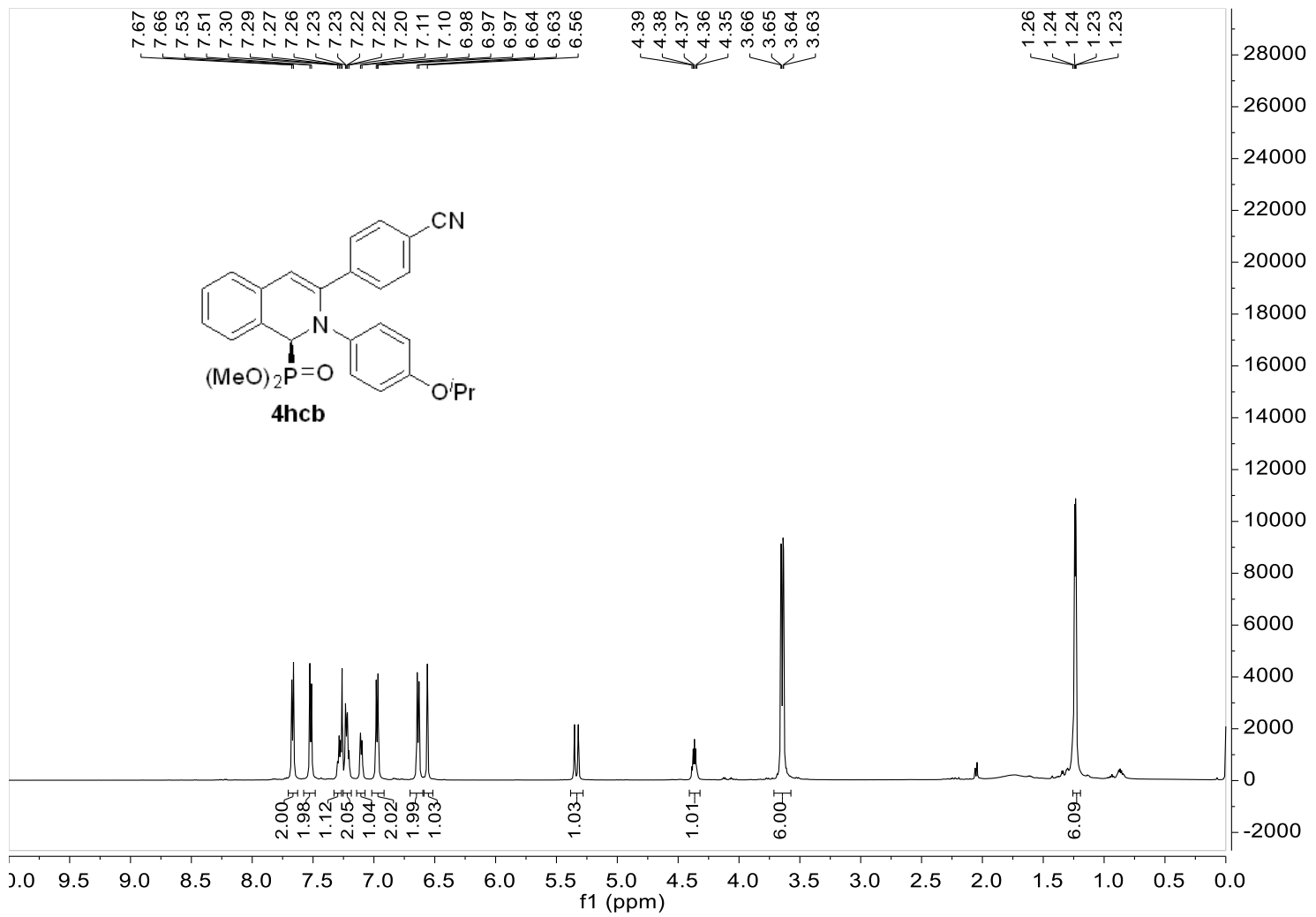

$151 \mathrm{MHz}, \mathrm{CDCl}_{3},{ }^{13} \mathrm{C} \mathrm{NMR}$

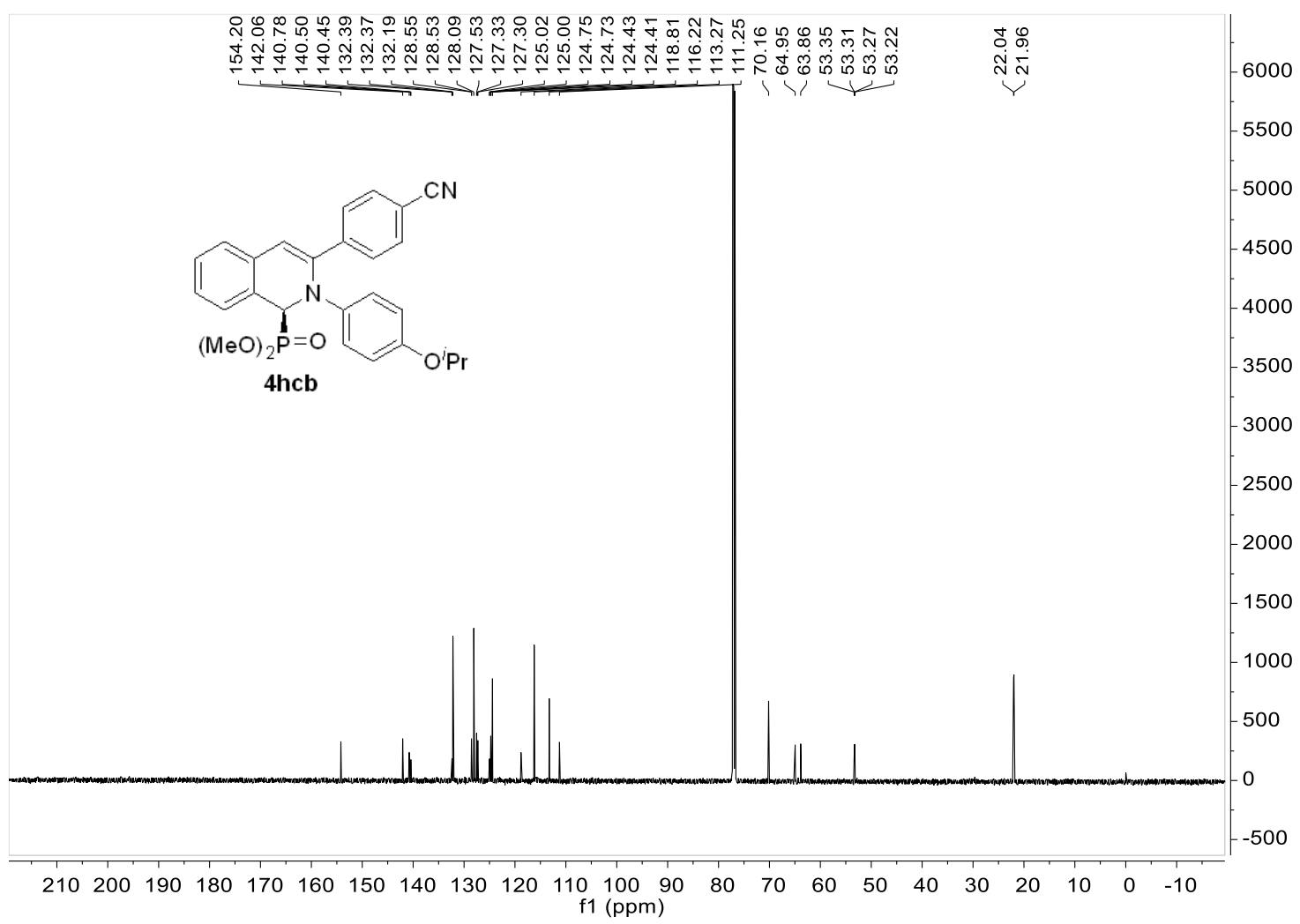


$600 \mathrm{MHz}, \mathrm{CDCl}_{3},{ }^{1} \mathrm{H} \mathrm{NMR}$

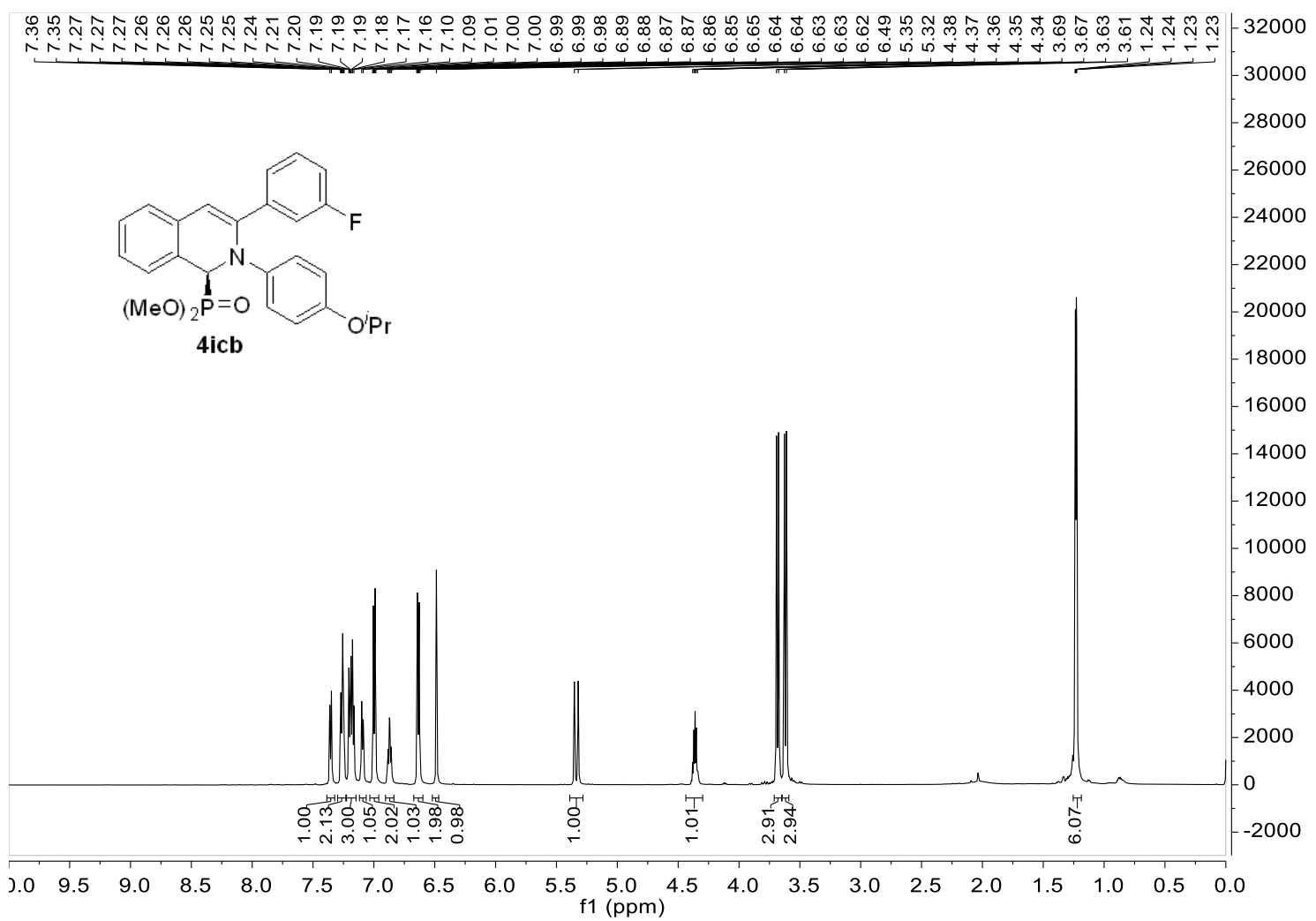

$151 \mathrm{MHz}, \mathrm{CDCl}_{3},{ }^{13} \mathrm{C} \mathrm{NMR}$

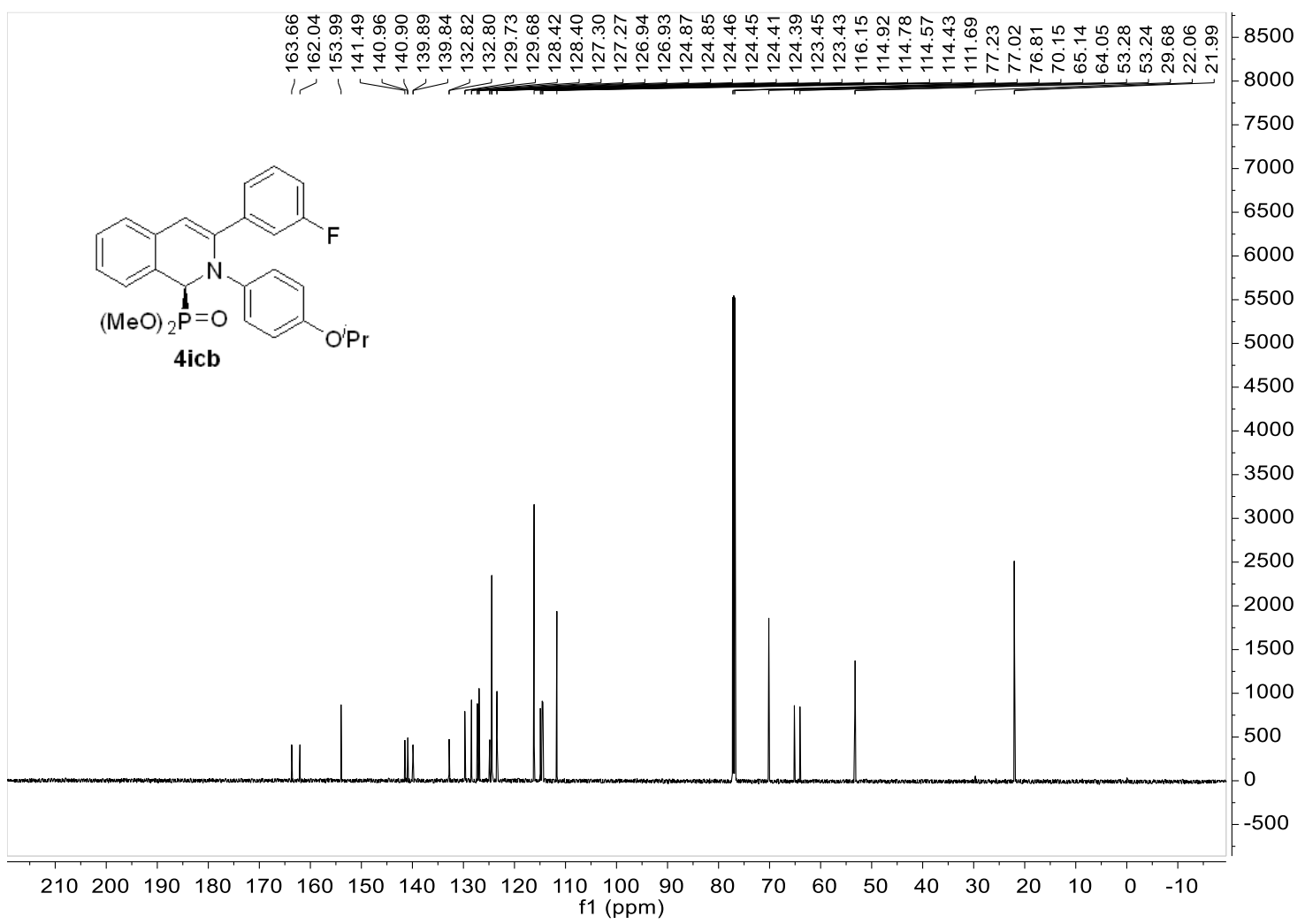


$600 \mathrm{MHz}, \mathrm{CDCl}_{3},{ }^{1} \mathrm{H} \mathrm{NMR}$

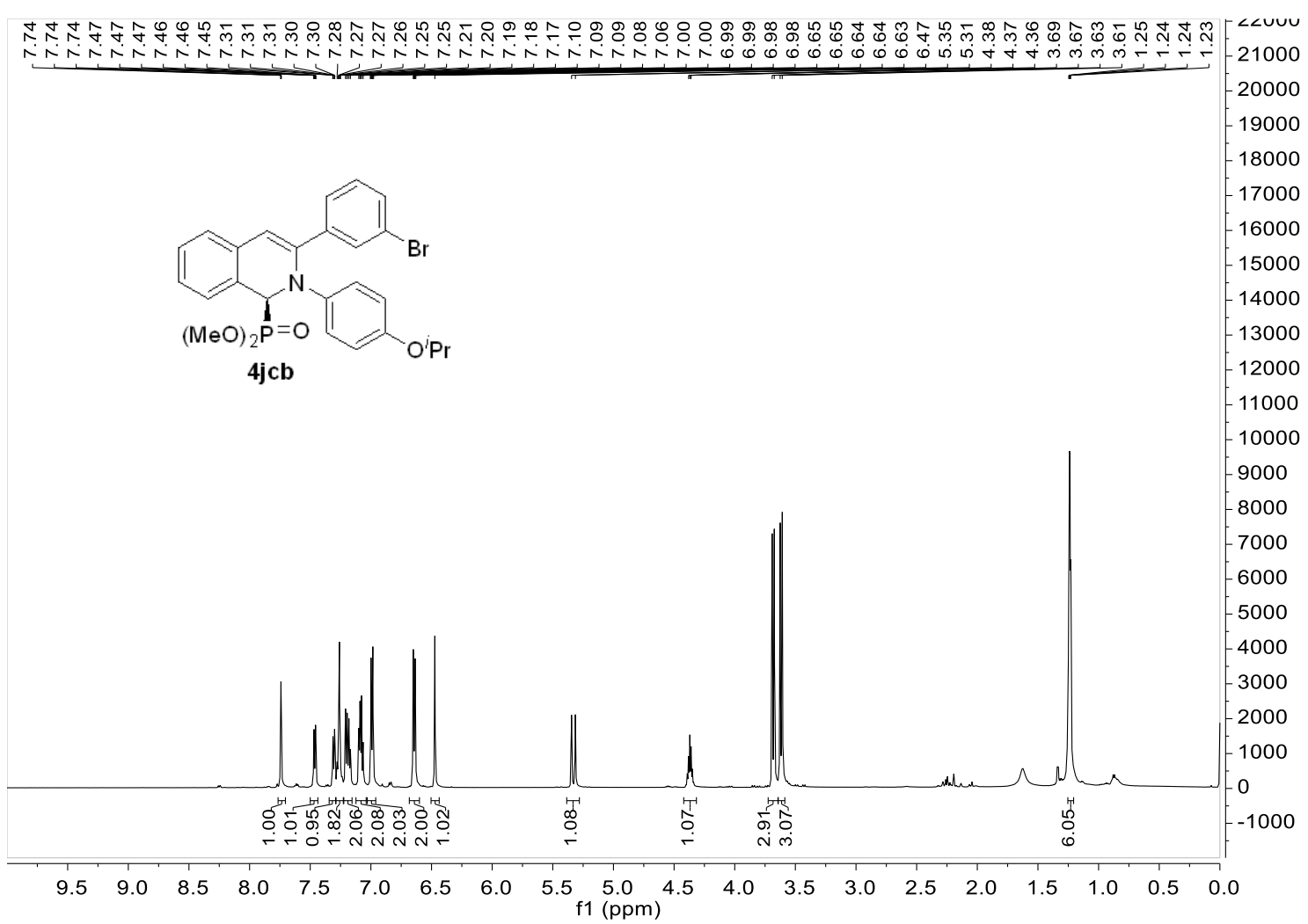

$151 \mathrm{MHz}, \mathrm{CDCl}_{3},{ }^{13} \mathrm{C} \mathrm{NMR}$

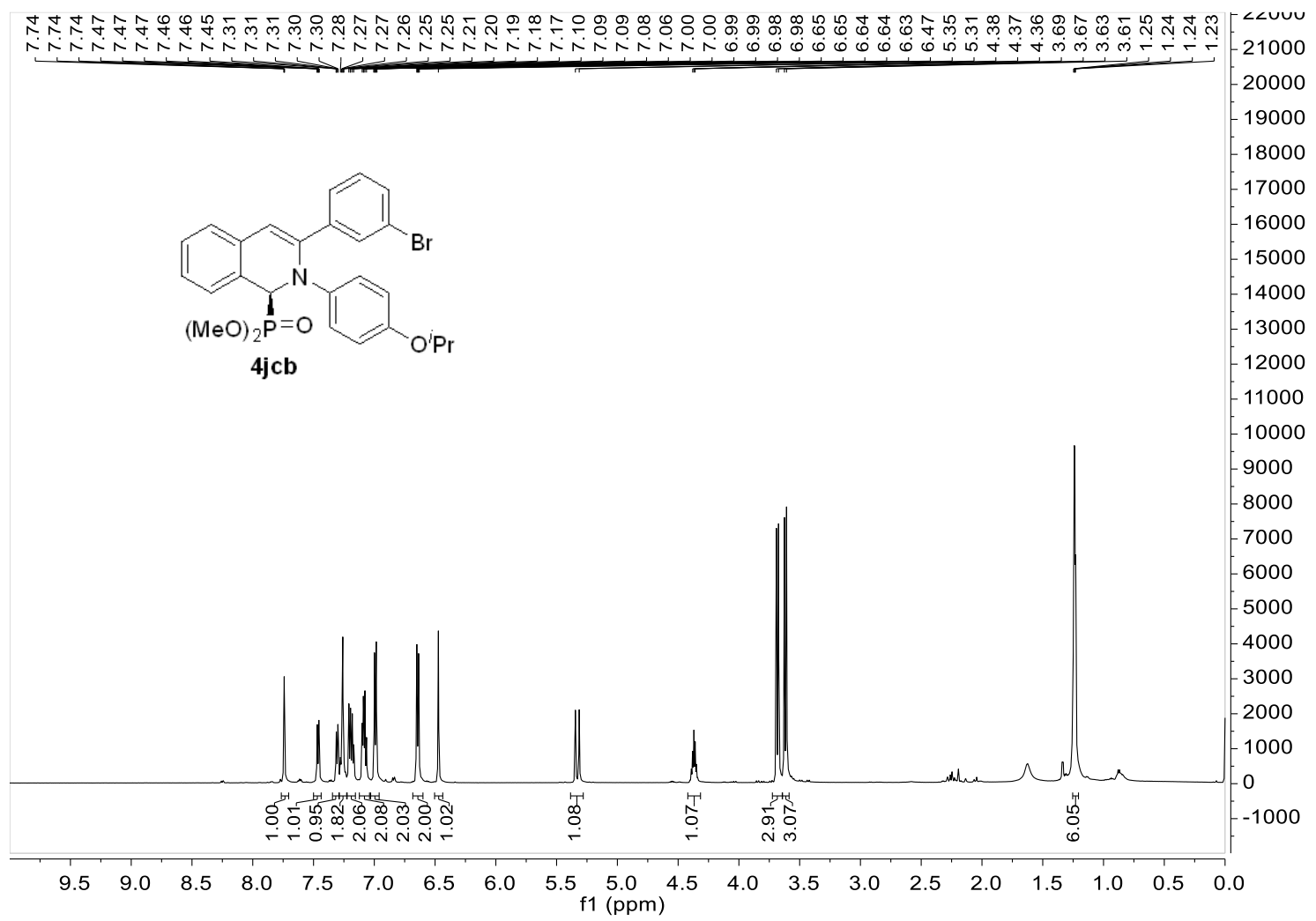


$600 \mathrm{MHz}, \mathrm{CDCl}_{3},{ }^{1} \mathrm{H} \mathrm{NMR}$

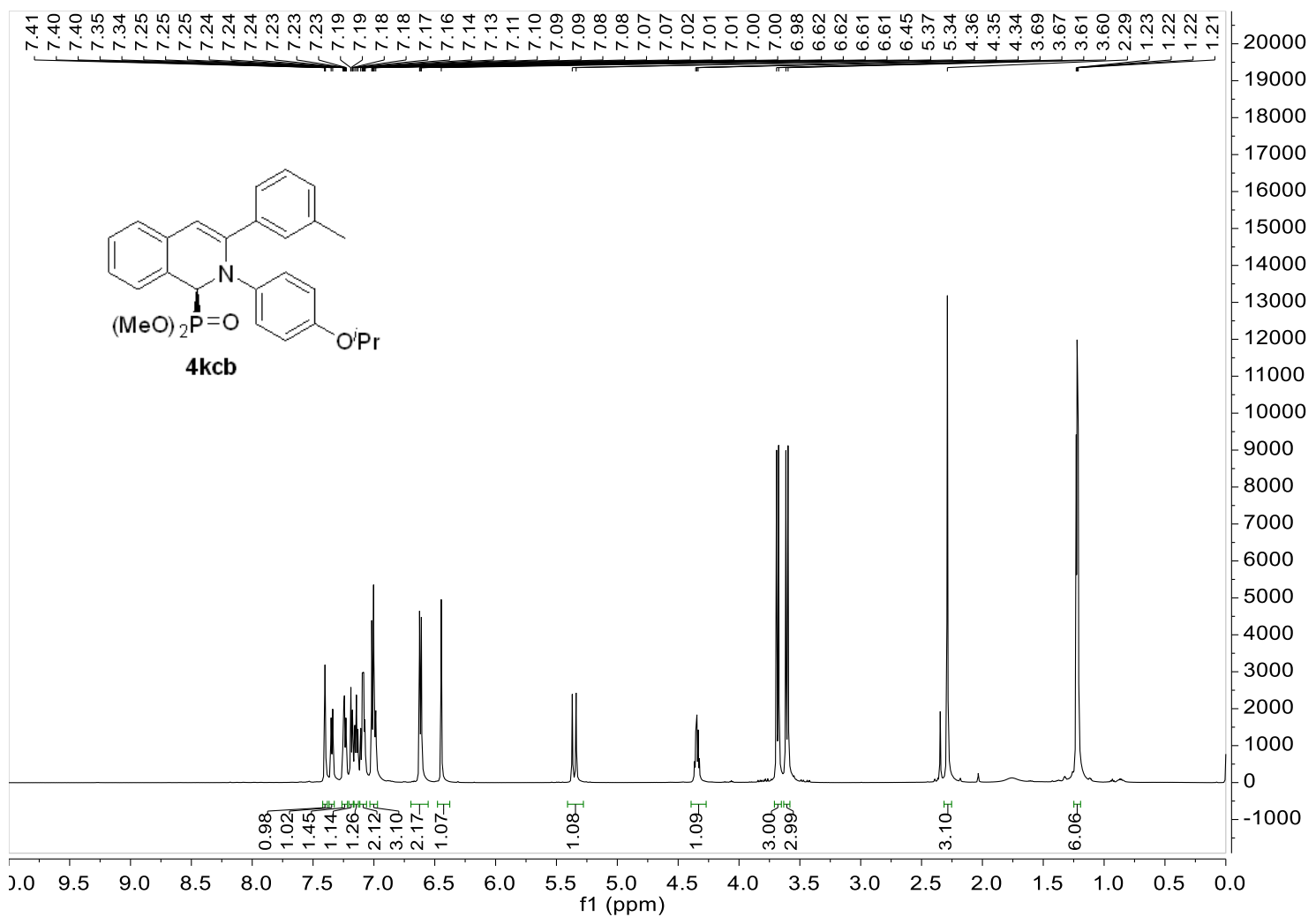

$151 \mathrm{MHz}, \mathrm{CDCl}_{3},{ }^{13} \mathrm{C} \mathrm{NMR}$

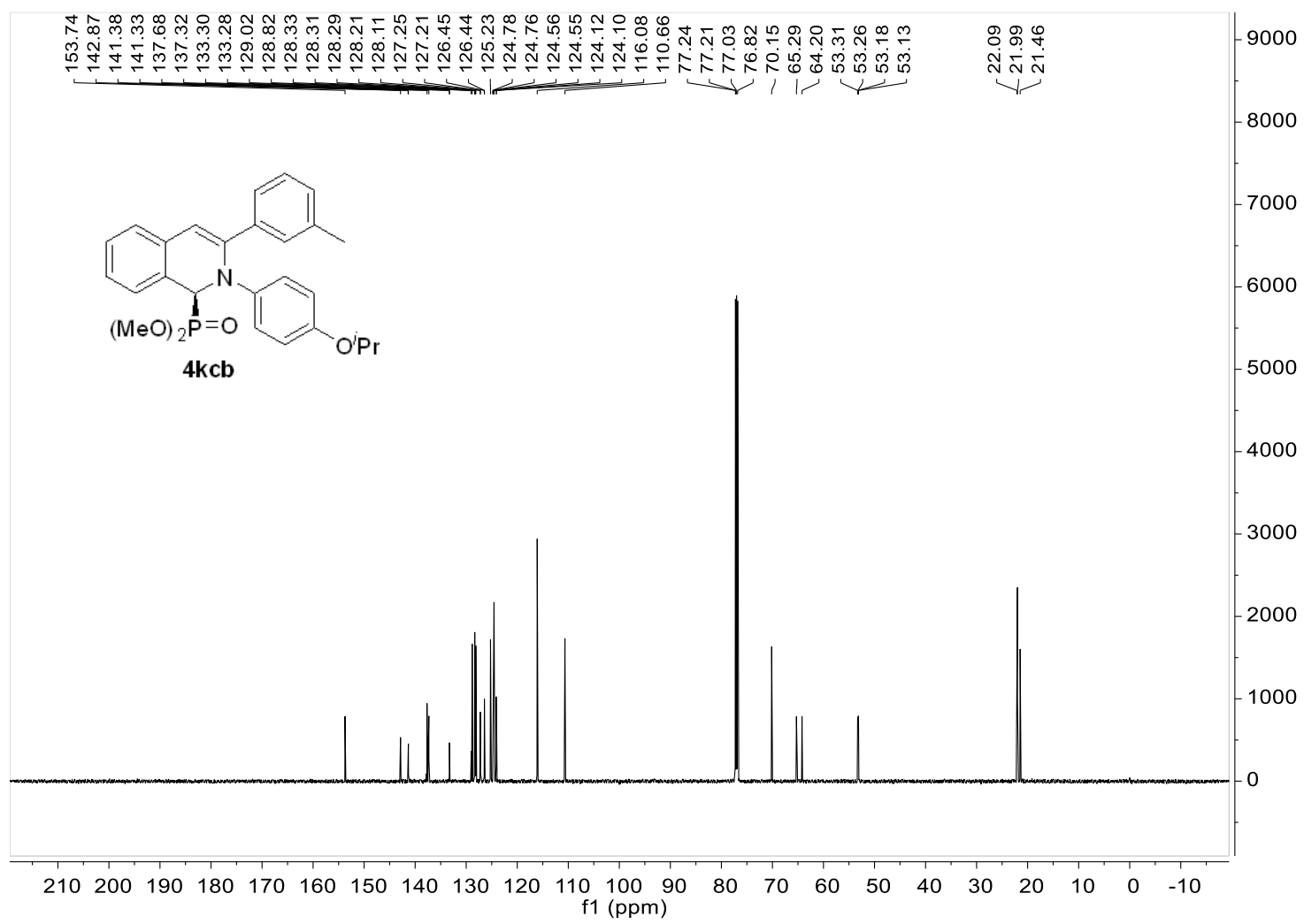


$600 \mathrm{MHz}, \mathrm{CDCl}_{3},{ }^{1} \mathrm{H} \mathrm{NMR}$

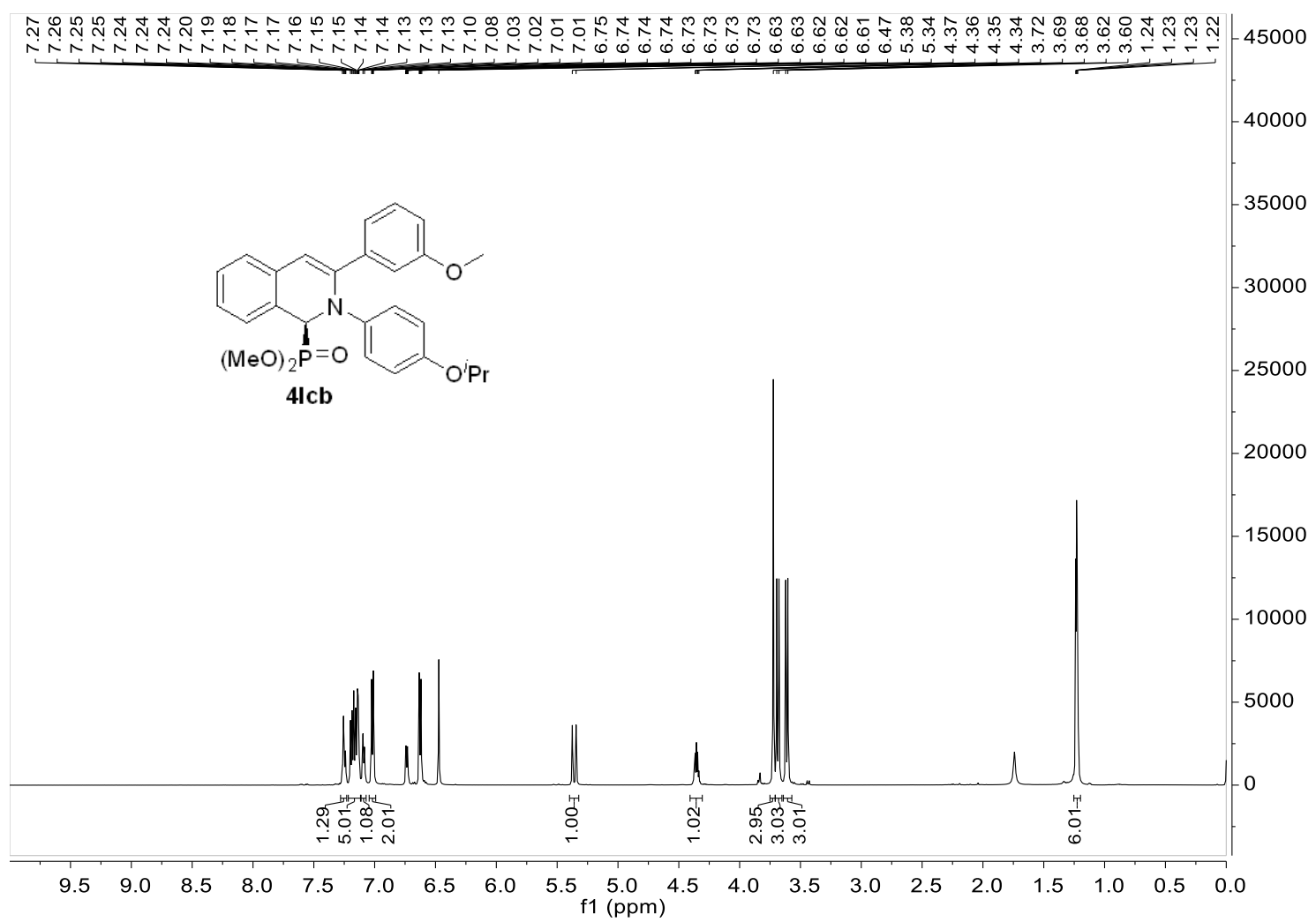

$151 \mathrm{MHz}, \mathrm{CDCl}_{3},{ }^{13} \mathrm{C} \mathrm{NMR}$

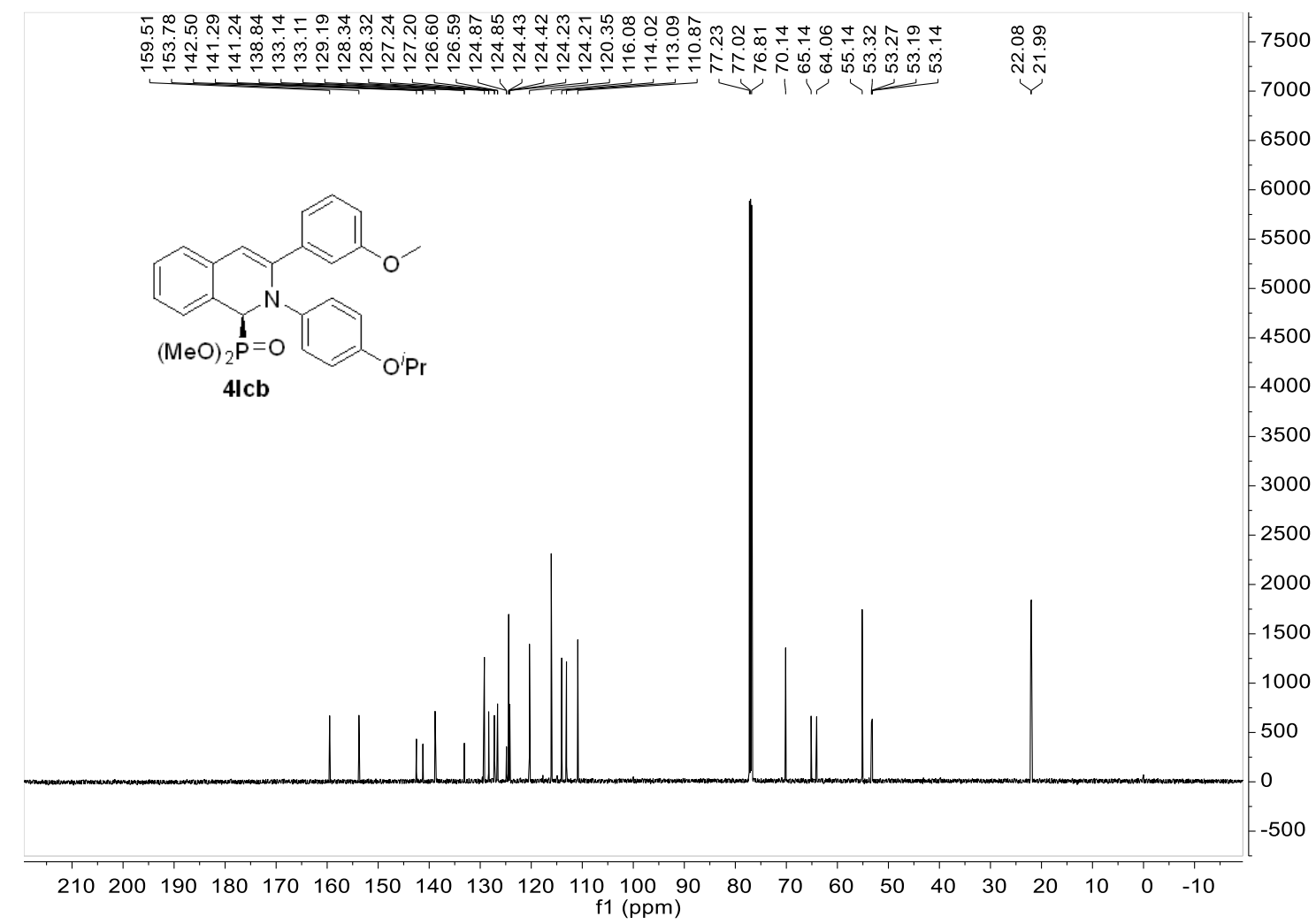


$600 \mathrm{MHz}, \mathrm{CDCl}_{3},{ }^{1} \mathrm{H} \mathrm{NMR}$

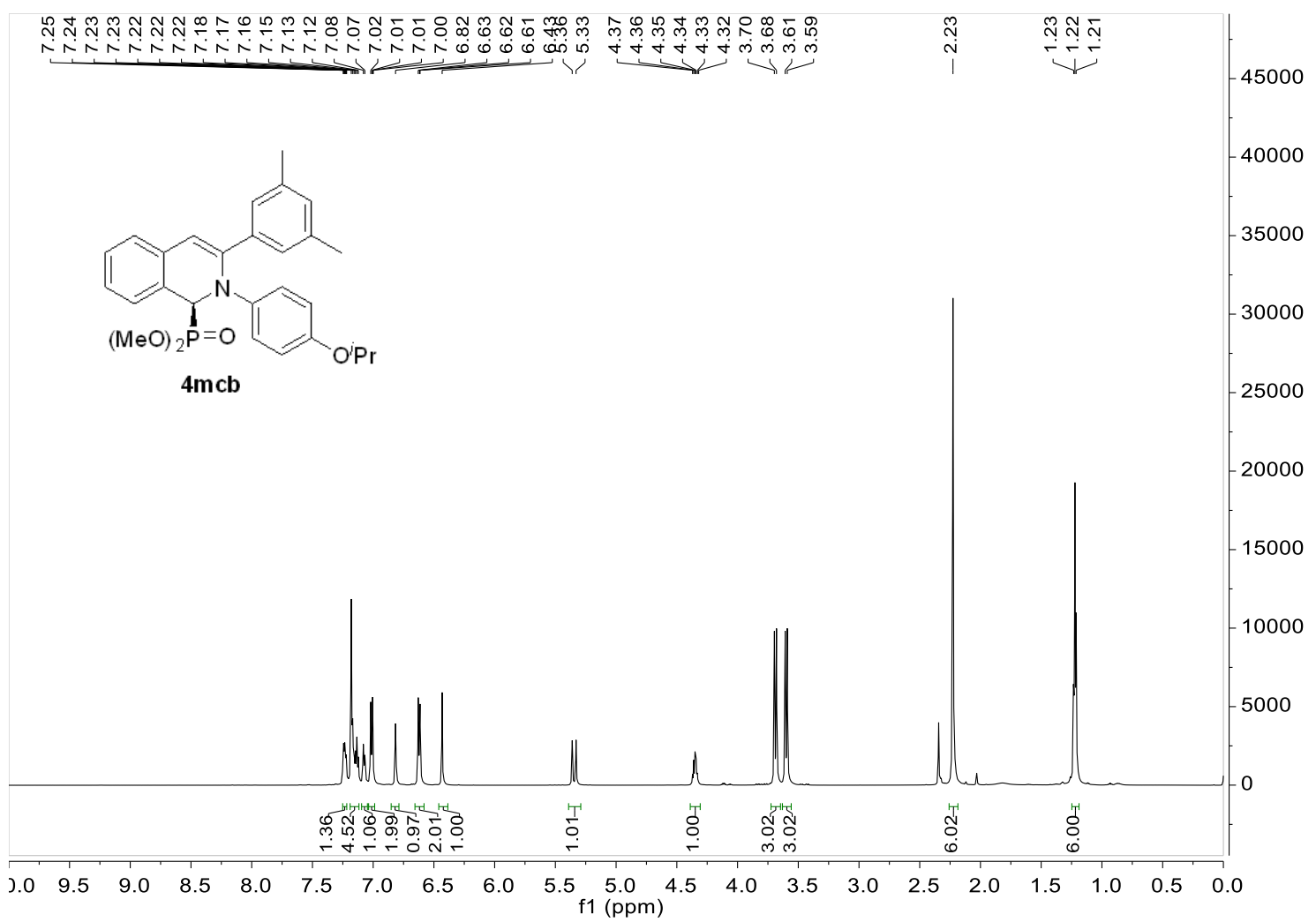

$151 \mathrm{MHz}, \mathrm{CDCl}_{3},{ }^{13} \mathrm{C} \mathrm{NMR}$

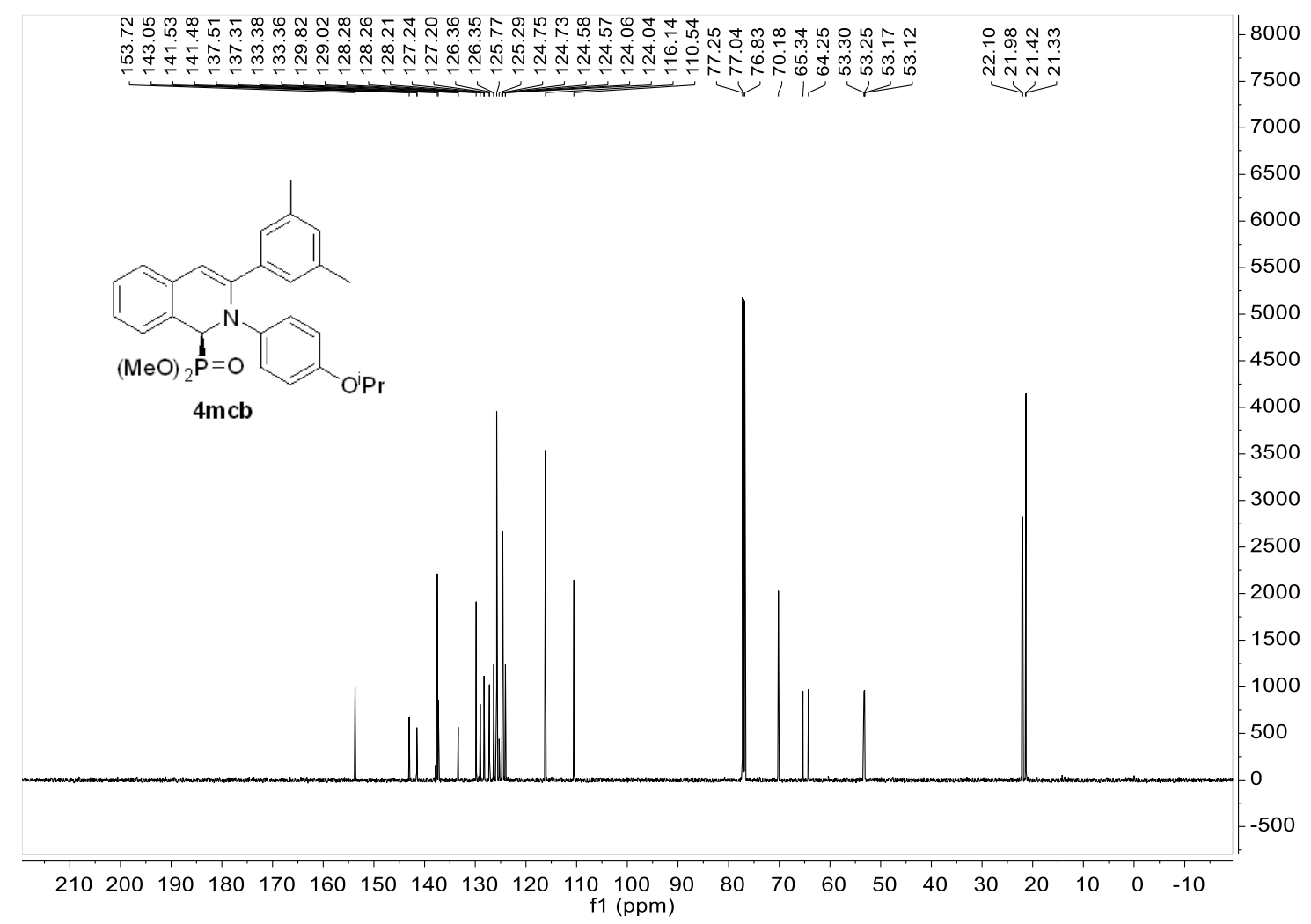


$600 \mathrm{MHz}, \mathrm{CDCl}_{3},{ }^{1} \mathrm{H} \mathrm{NMR}$

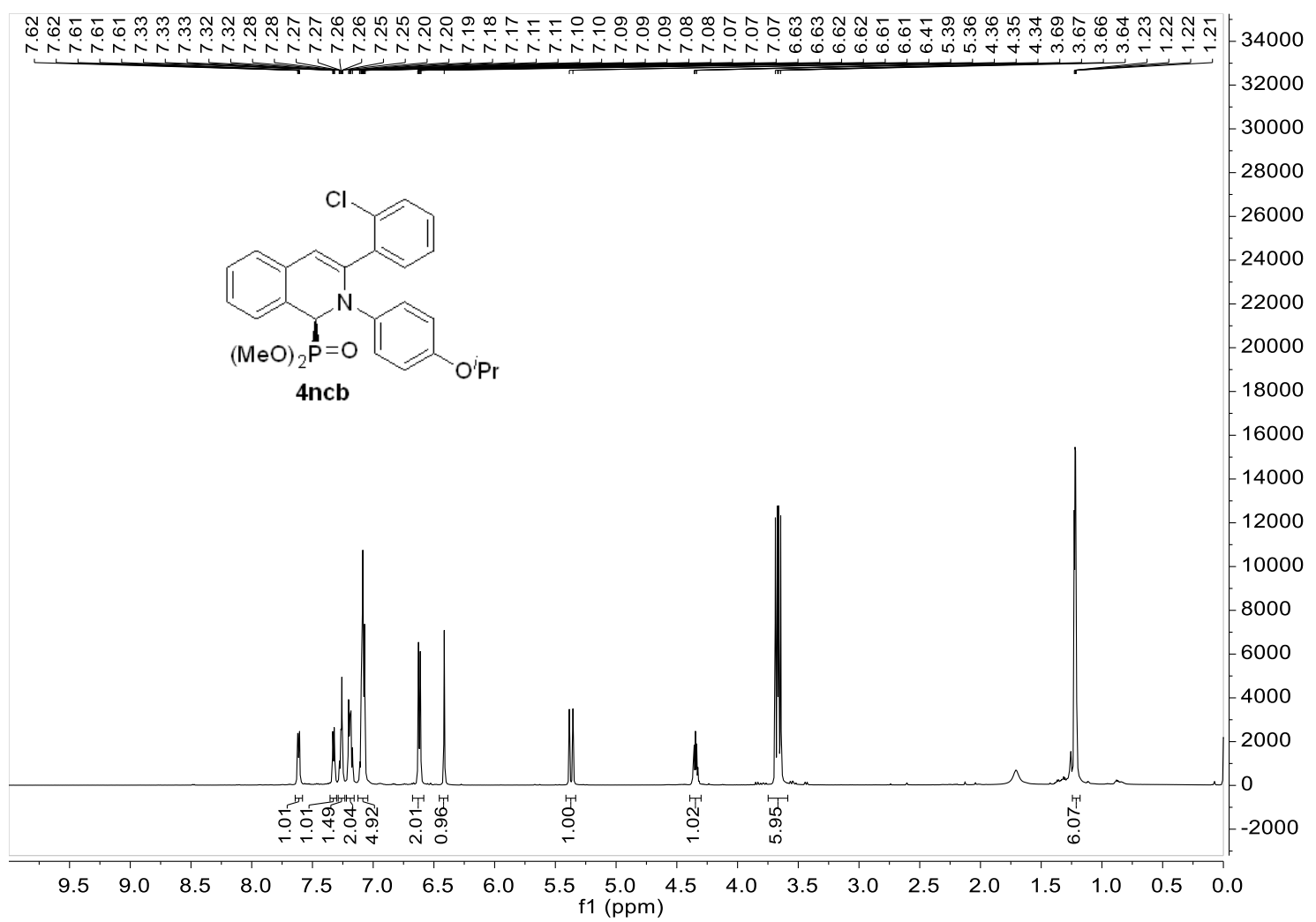

$151 \mathrm{MHz}, \mathrm{CDCl}_{3},{ }^{13} \mathrm{C} \mathrm{NMR}$

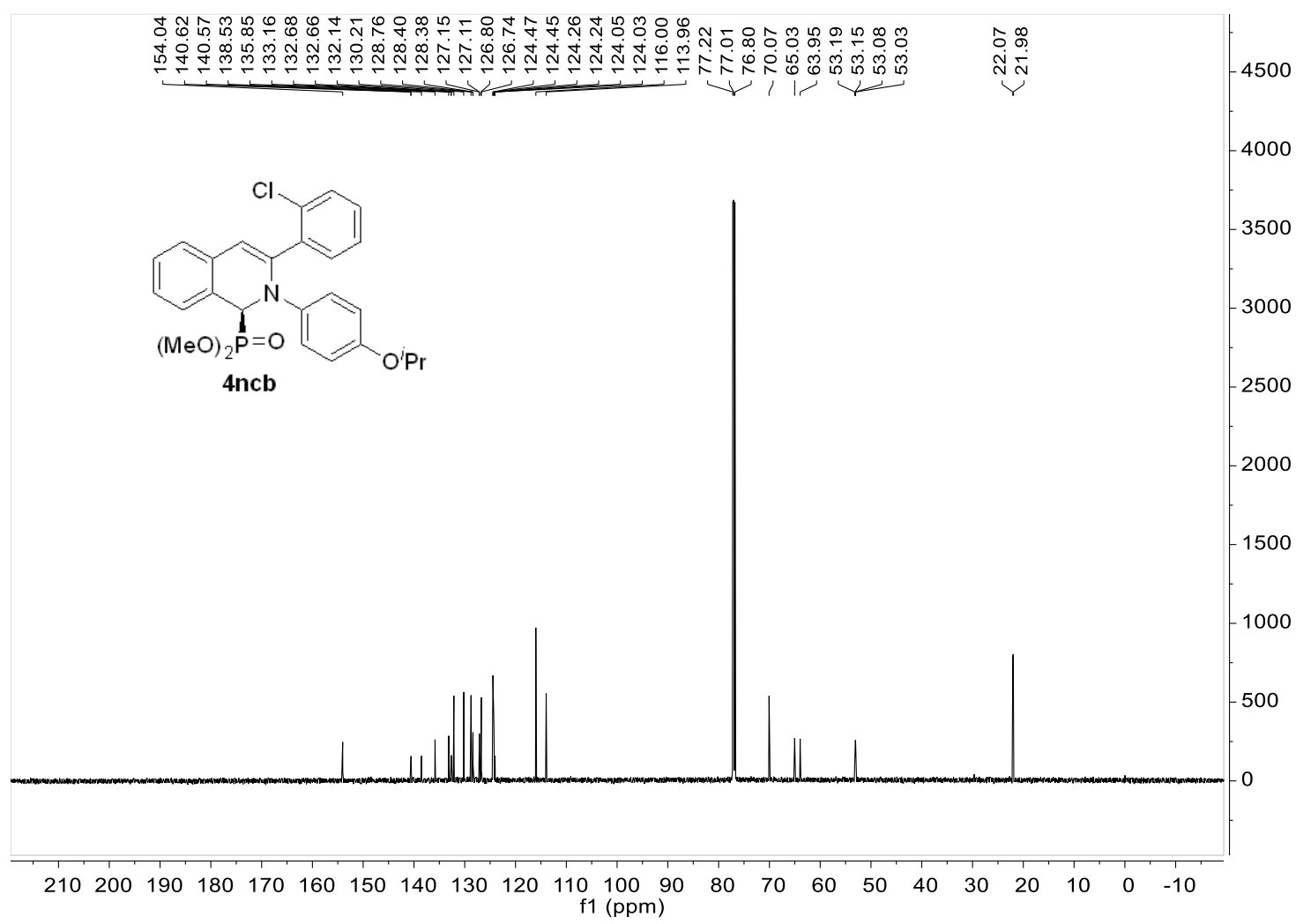


$600 \mathrm{MHz}, \mathrm{CDCl}_{3},{ }^{1} \mathrm{H} \mathrm{NMR}$

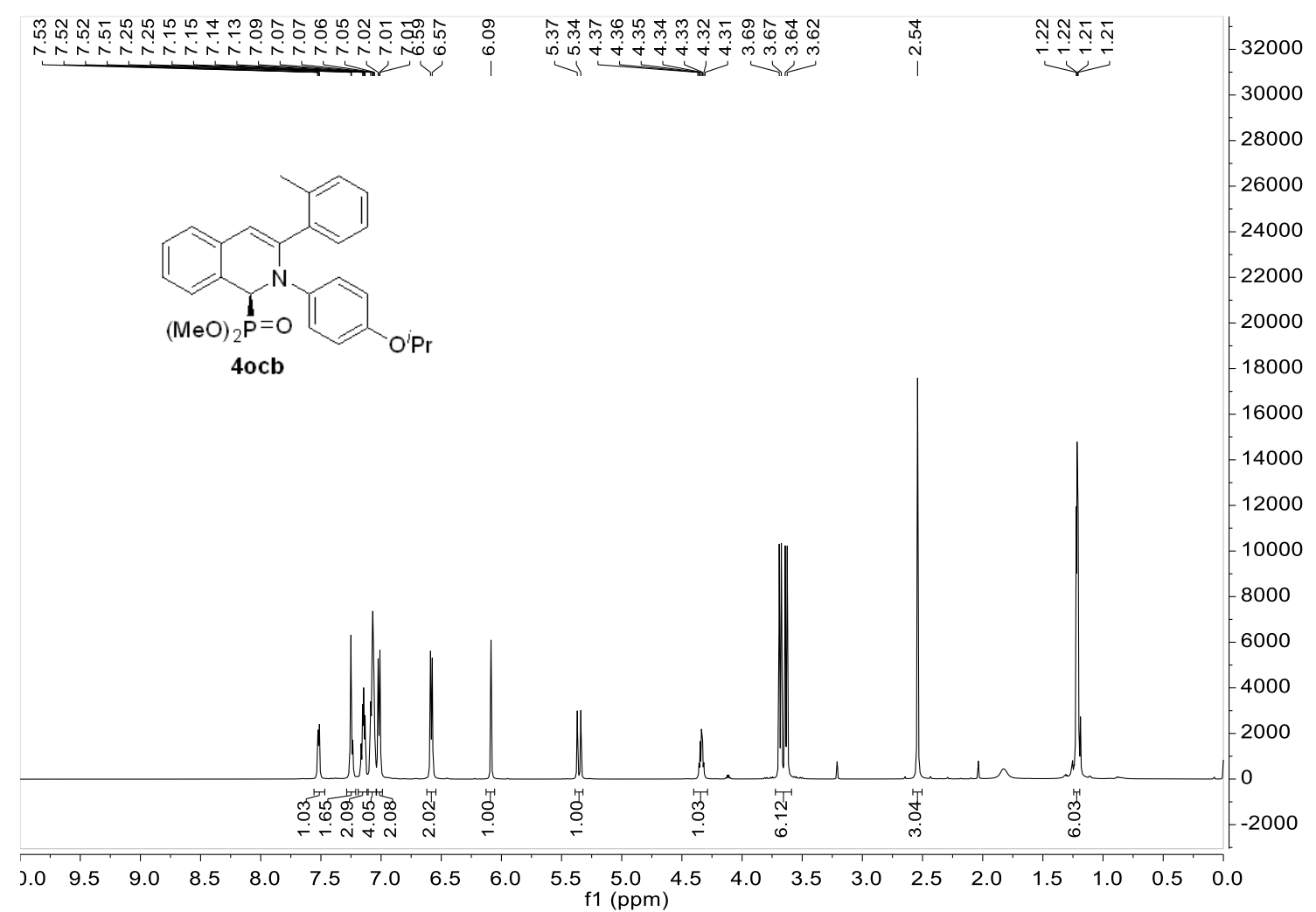

$151 \mathrm{MHz}, \mathrm{CDCl}_{3},{ }^{13} \mathrm{C} \mathrm{NMR}$

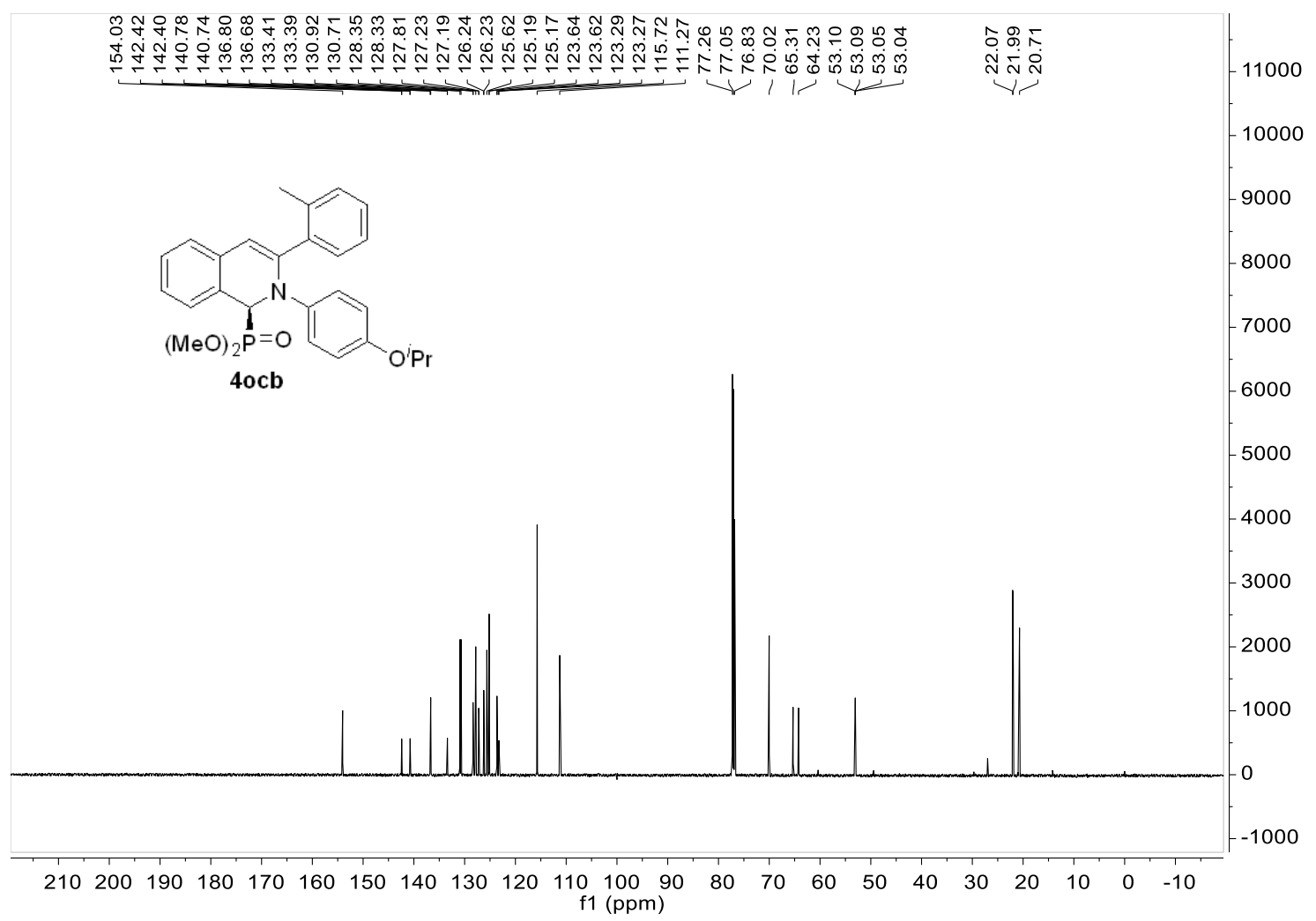


$600 \mathrm{MHz}, \mathrm{CDCl}_{3},{ }^{1} \mathrm{H} \mathrm{NMR}$

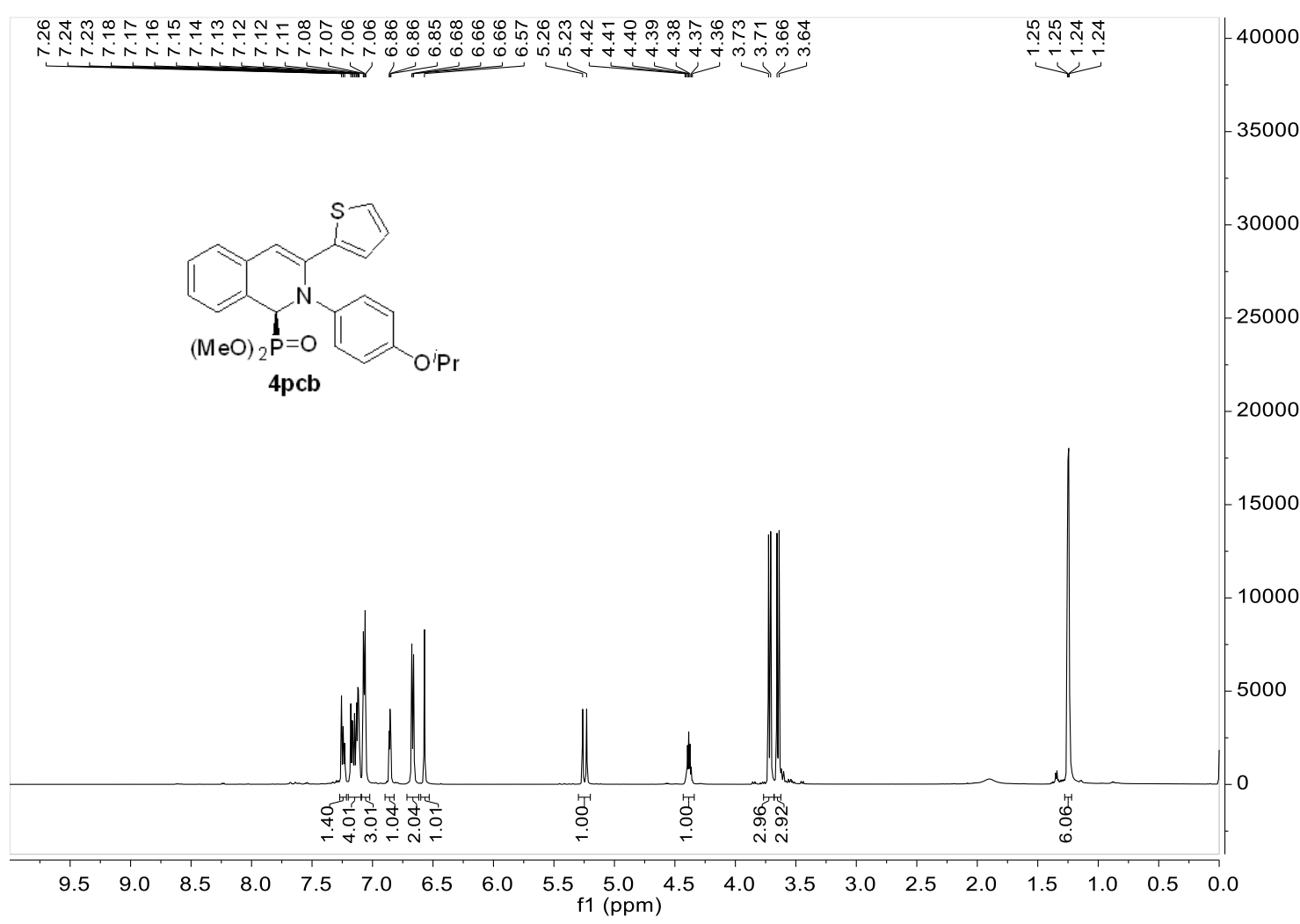

$151 \mathrm{MHz}, \mathrm{CDCl}_{3},{ }^{13} \mathrm{C} \mathrm{NMR}$

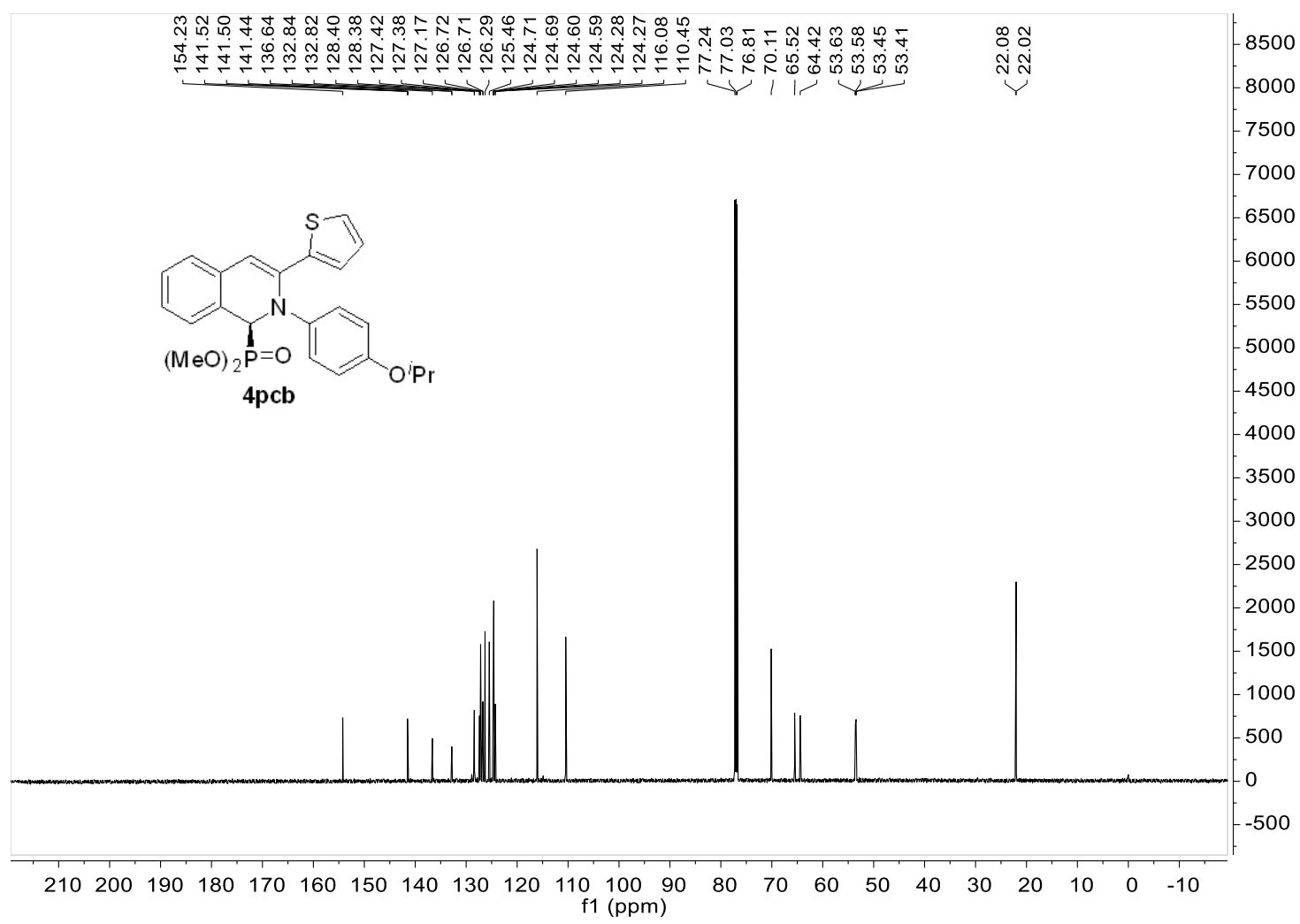


$600 \mathrm{MHz}, \mathrm{CDCl}_{3},{ }^{1} \mathrm{H} \mathrm{NMR}$

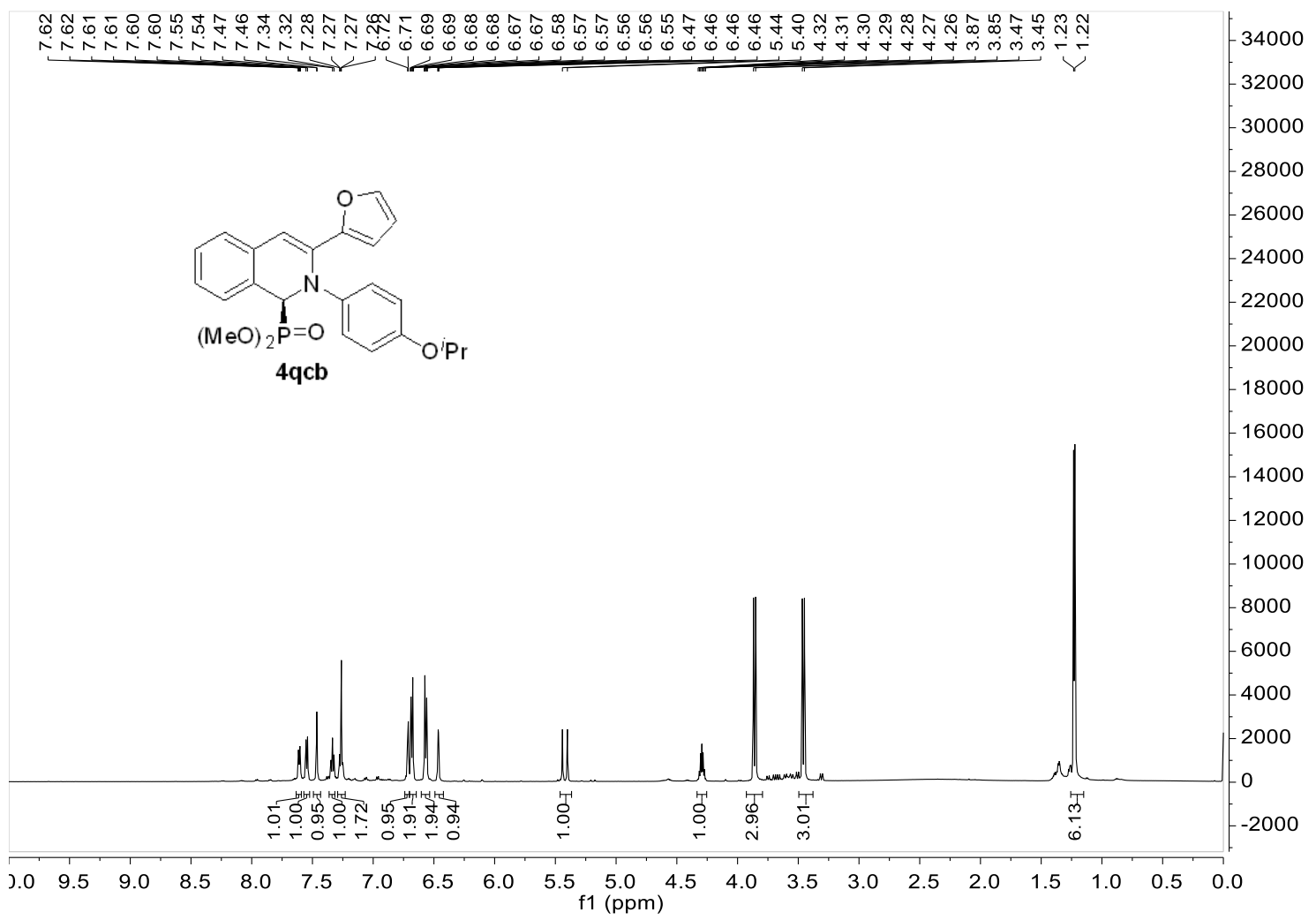

$151 \mathrm{MHz}, \mathrm{CDCl}_{3},{ }^{13} \mathrm{C} \mathrm{NMR}$

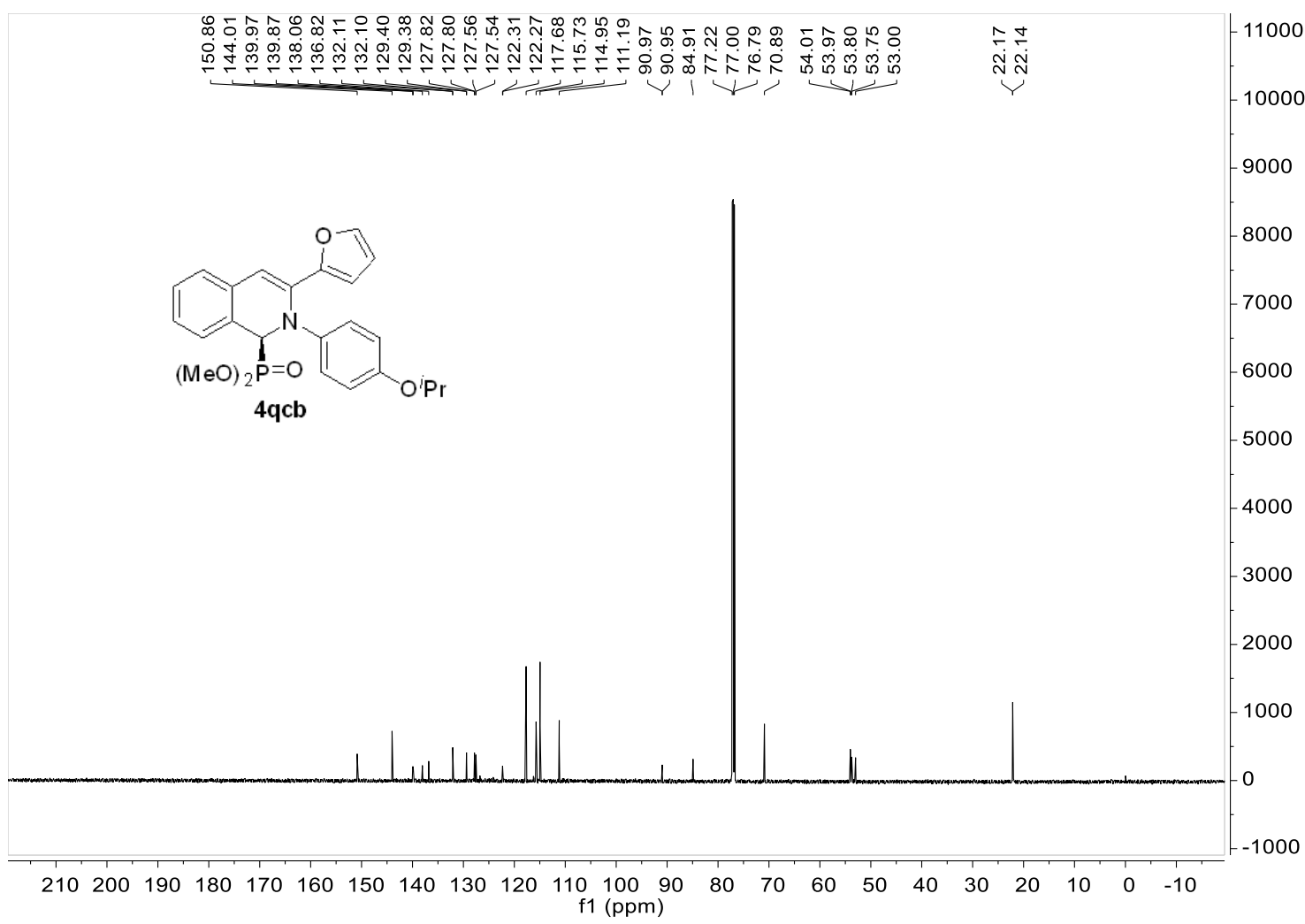


$600 \mathrm{MHz}, \mathrm{CDCl}_{3},{ }^{1} \mathrm{H} \mathrm{NMR}$

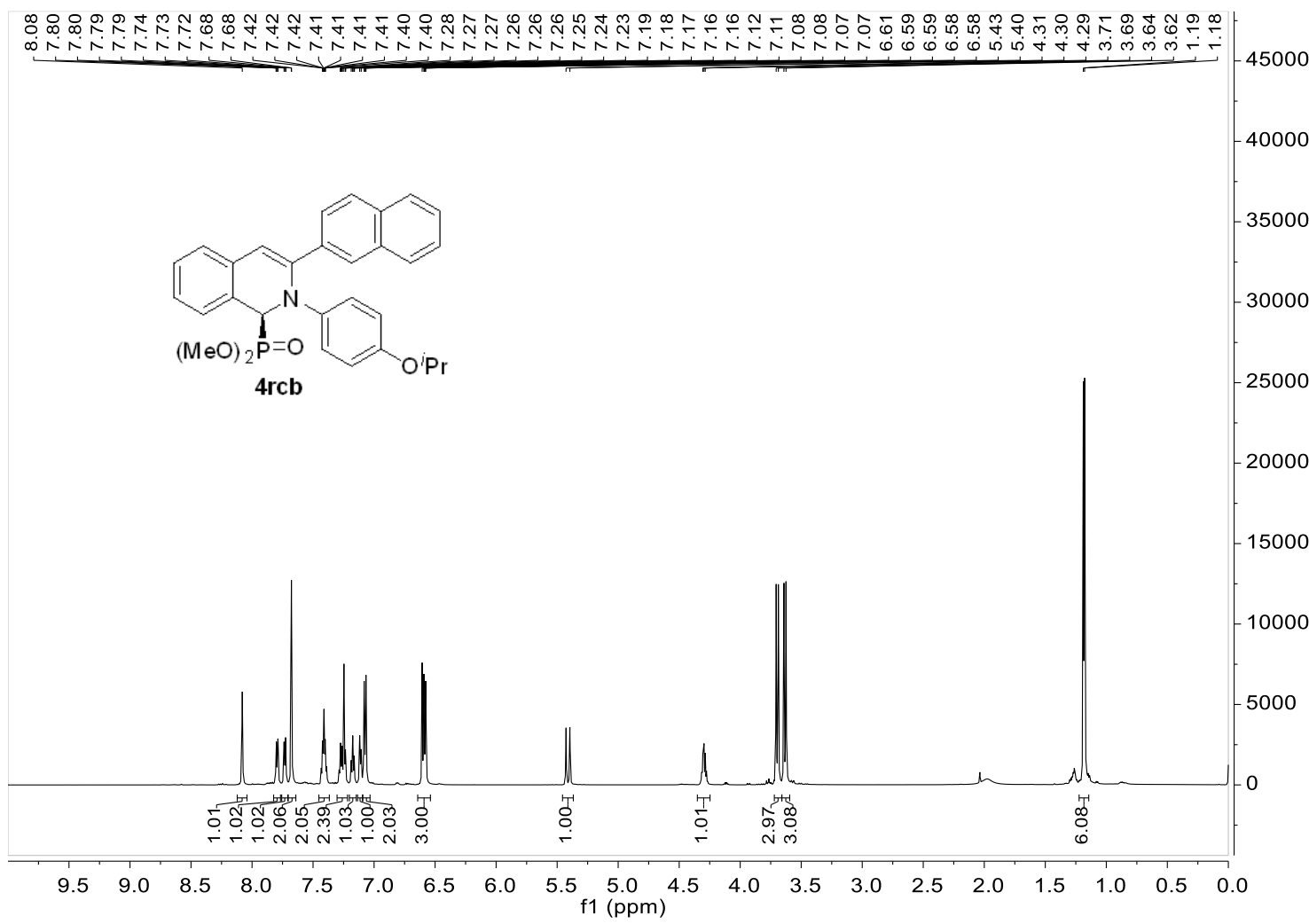

$151 \mathrm{MHz}, \mathrm{CDCl}_{3},{ }^{13} \mathrm{C} \mathrm{NMR}$

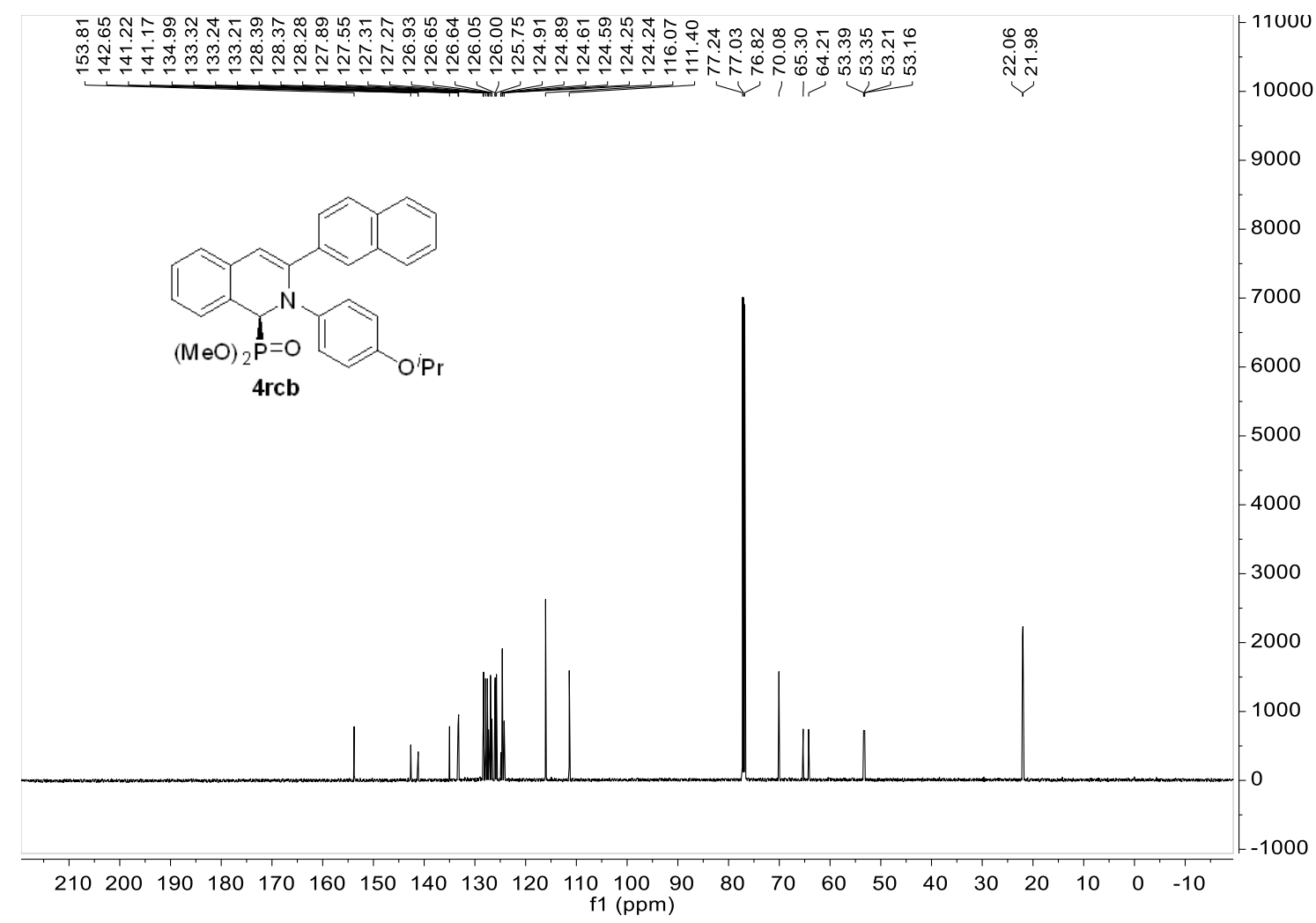


$600 \mathrm{MHz}, \mathrm{CDCl}_{3},{ }^{1} \mathrm{H} \mathrm{NMR}$

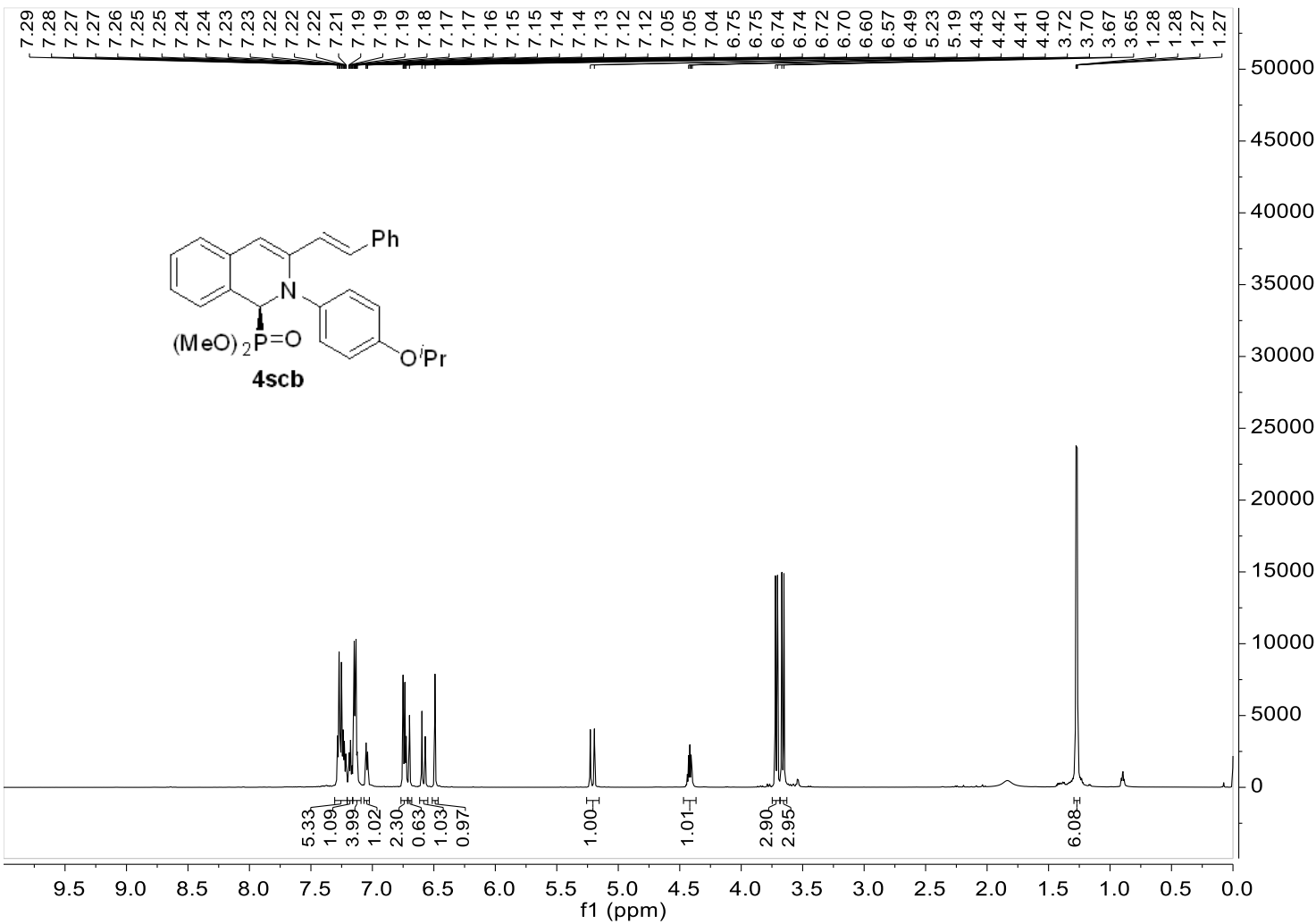

$151 \mathrm{MHz}, \mathrm{CDCl}_{3},{ }^{13} \mathrm{C} \mathrm{NMR}$

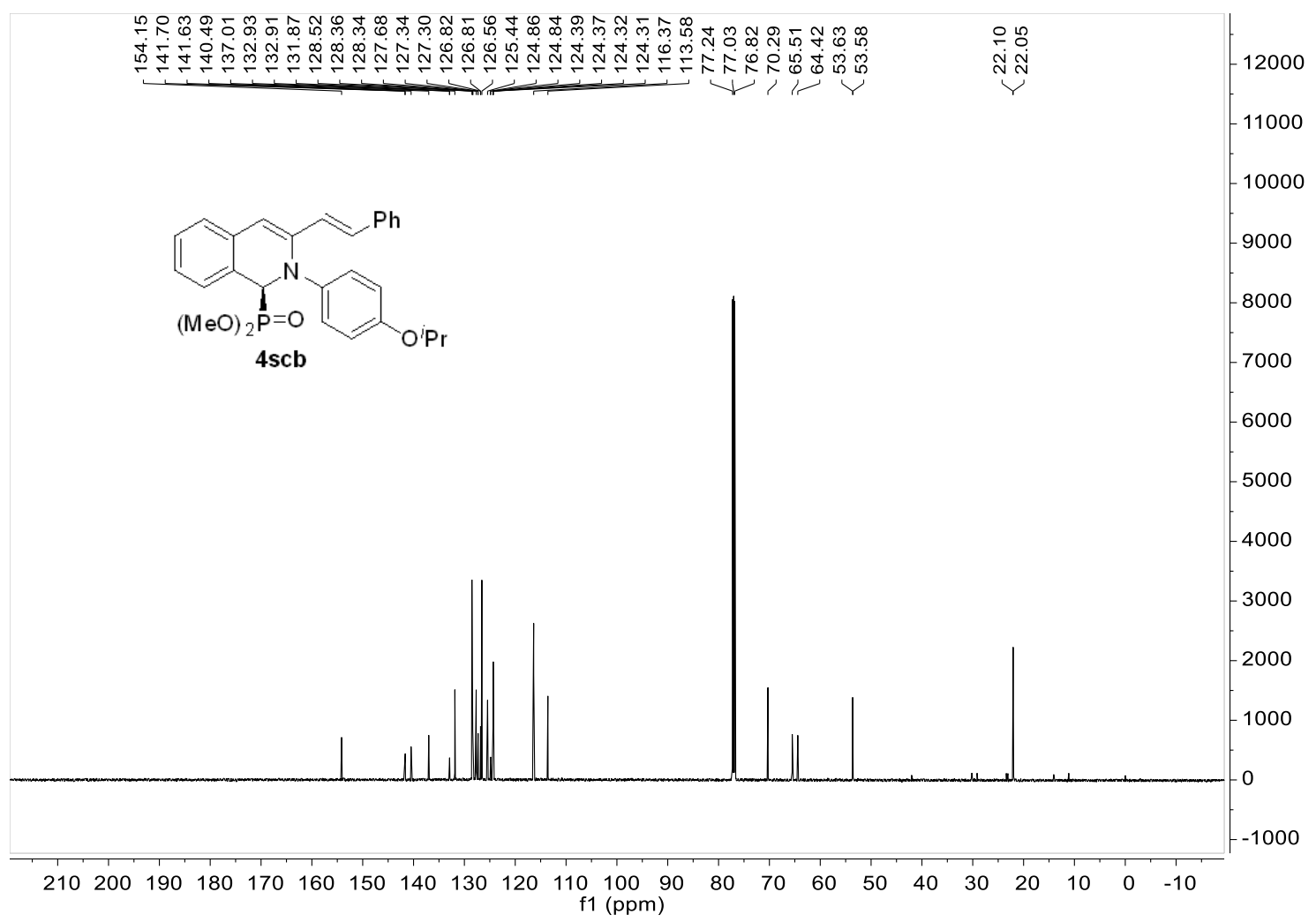


$600 \mathrm{MHz}, \mathrm{CDCl}_{3},{ }^{1} \mathrm{H} \mathrm{NMR}$

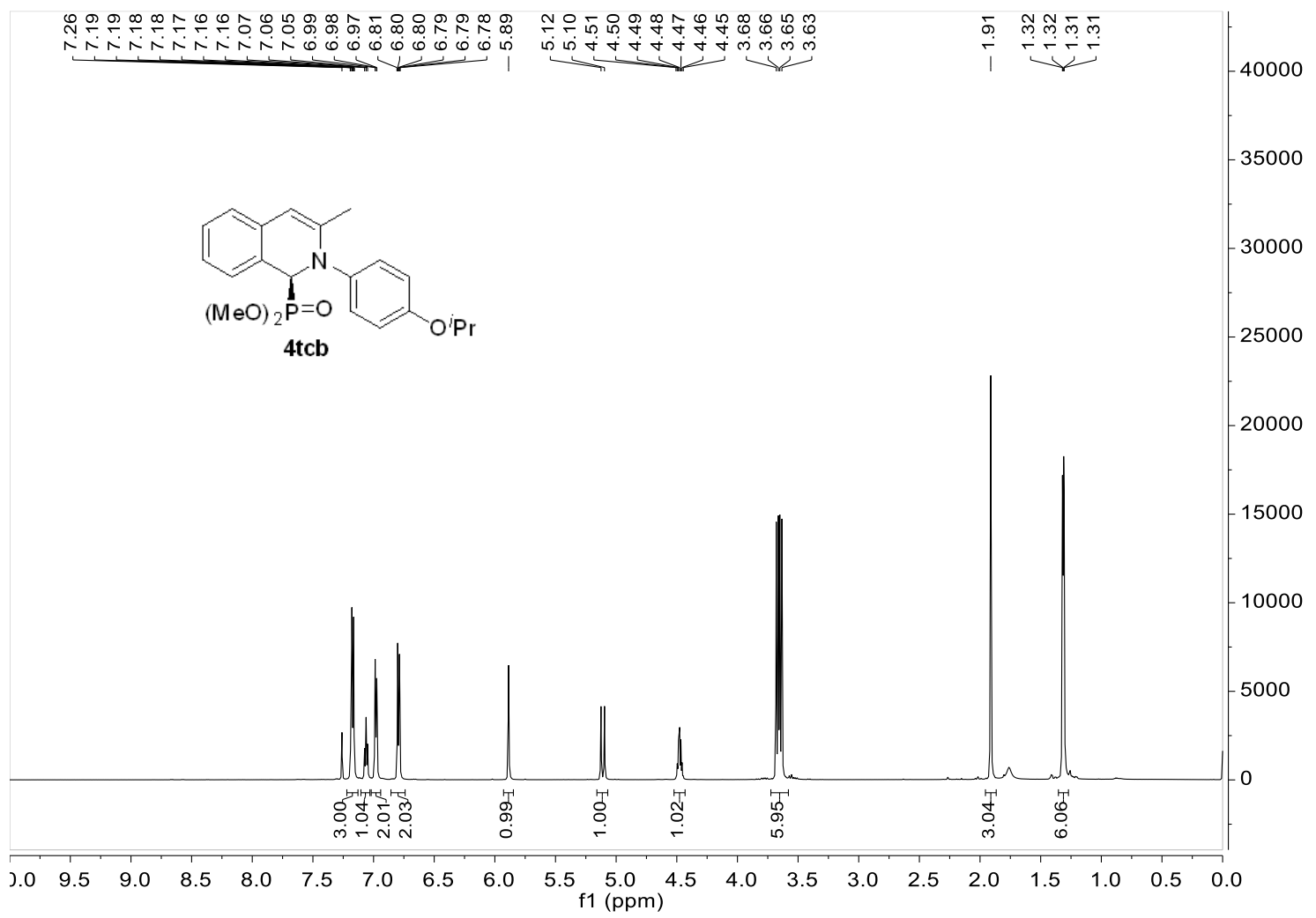

$151 \mathrm{MHz}, \mathrm{CDCl}_{3},{ }^{13} \mathrm{C} \mathrm{NMR}$

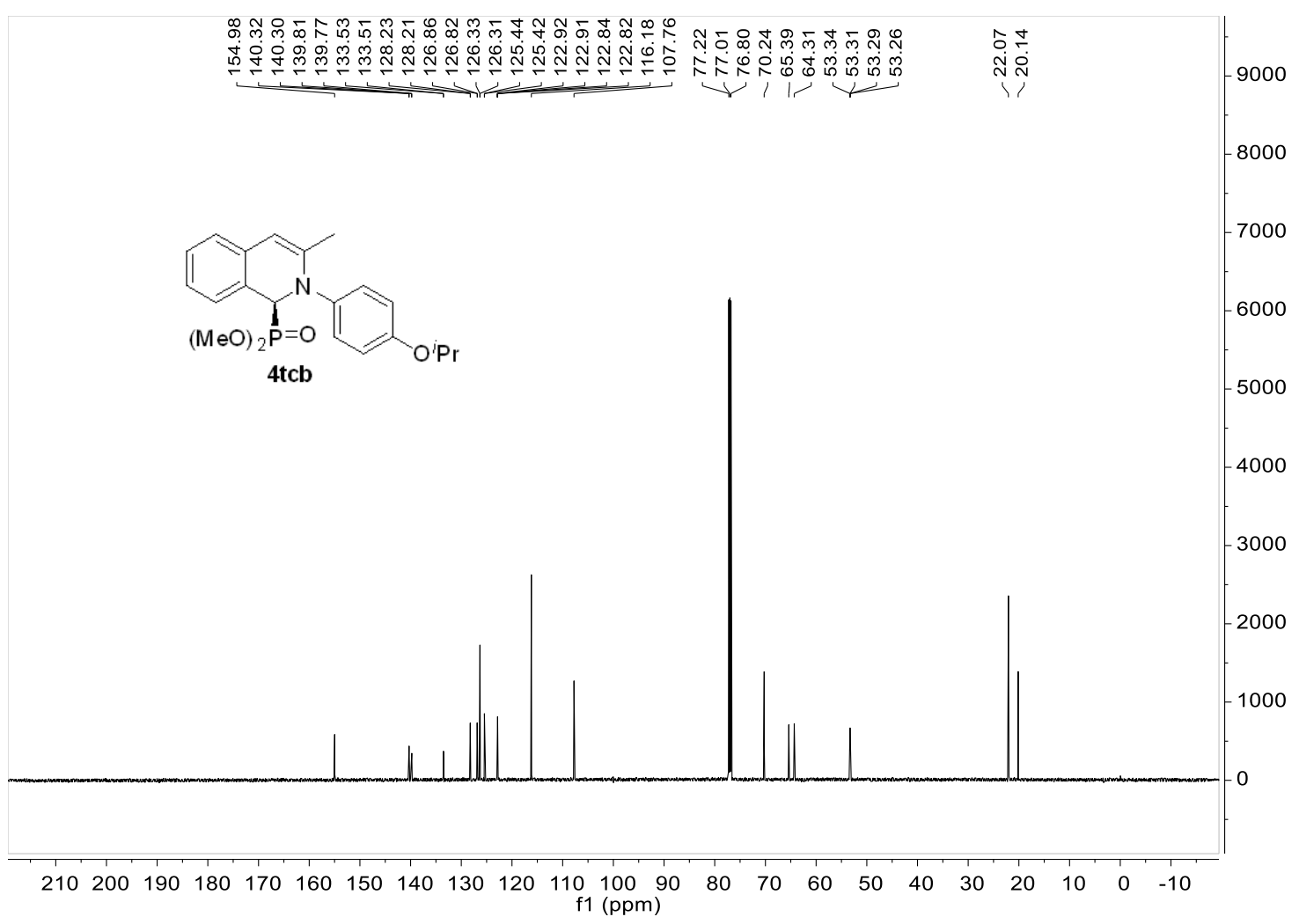


$600 \mathrm{MHz}, \mathrm{CDCl}_{3},{ }^{1} \mathrm{H} \mathrm{NMR}$

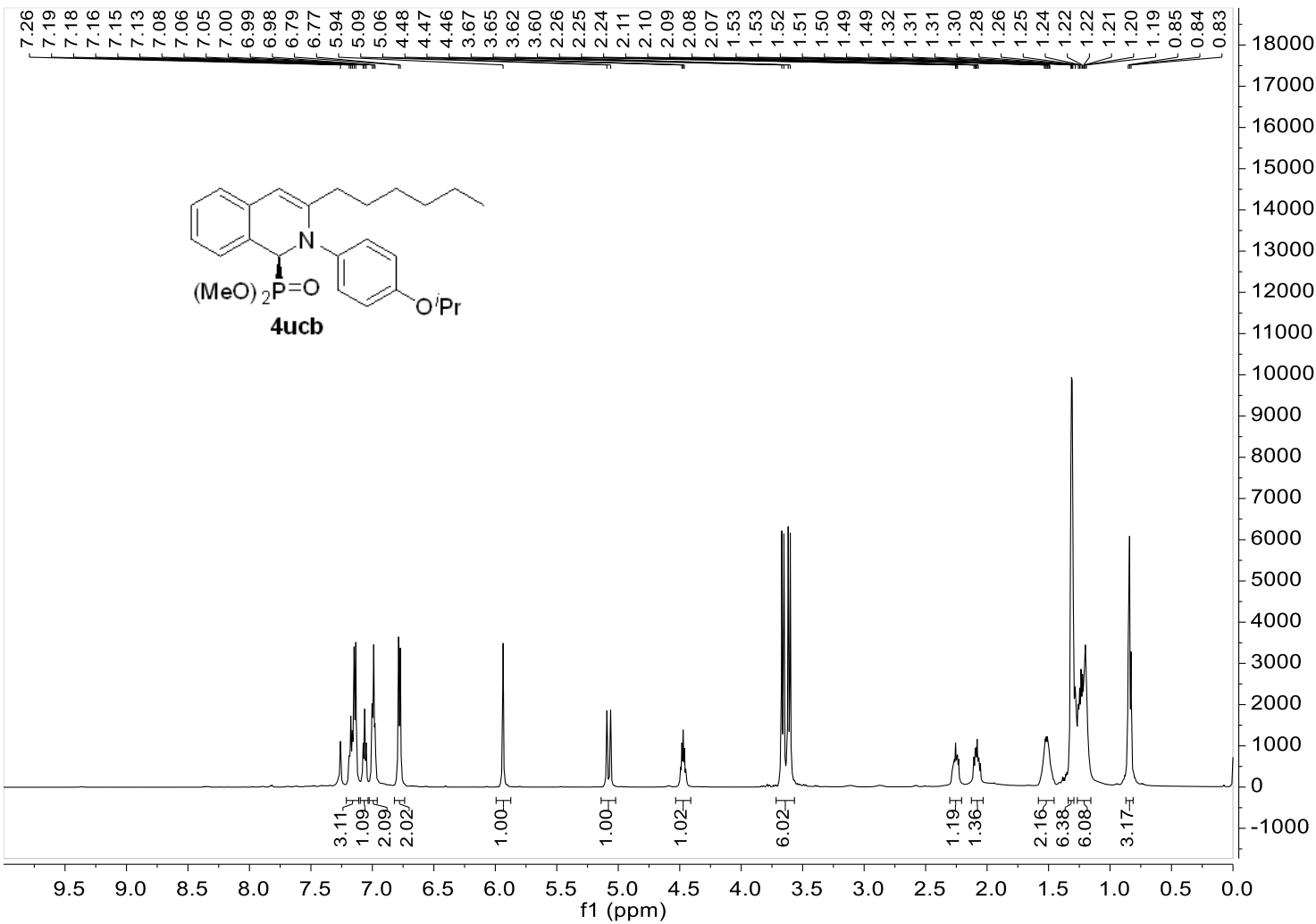

$151 \mathrm{MHz}, \mathrm{CDCl}_{3},{ }^{13} \mathrm{C} \mathrm{NMR}$

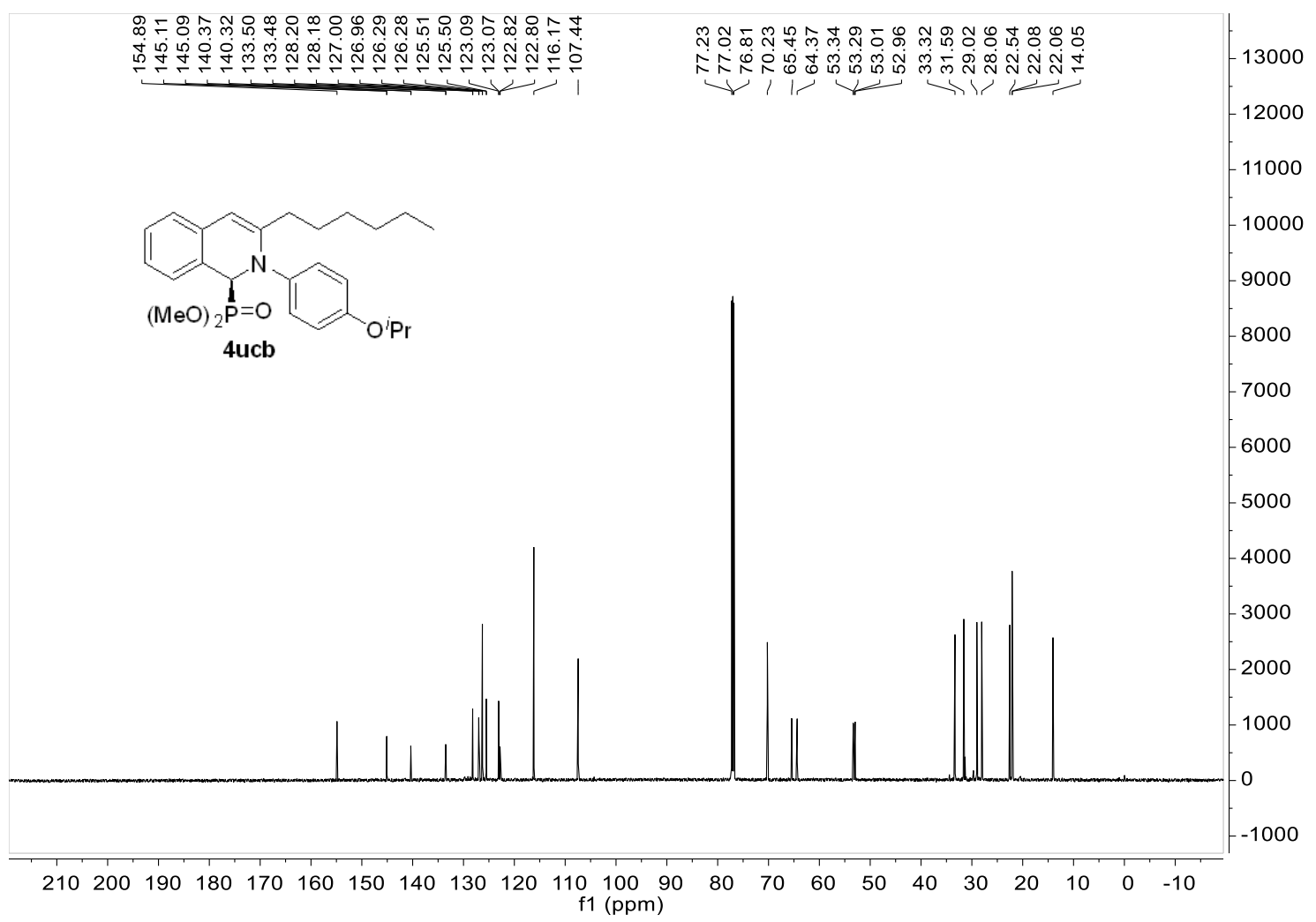


$600 \mathrm{MHz}, \mathrm{CDCl}_{3},{ }^{1} \mathrm{H} \mathrm{NMR}$

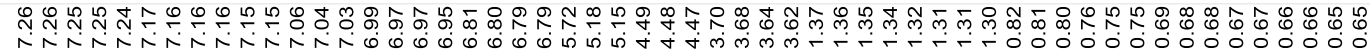

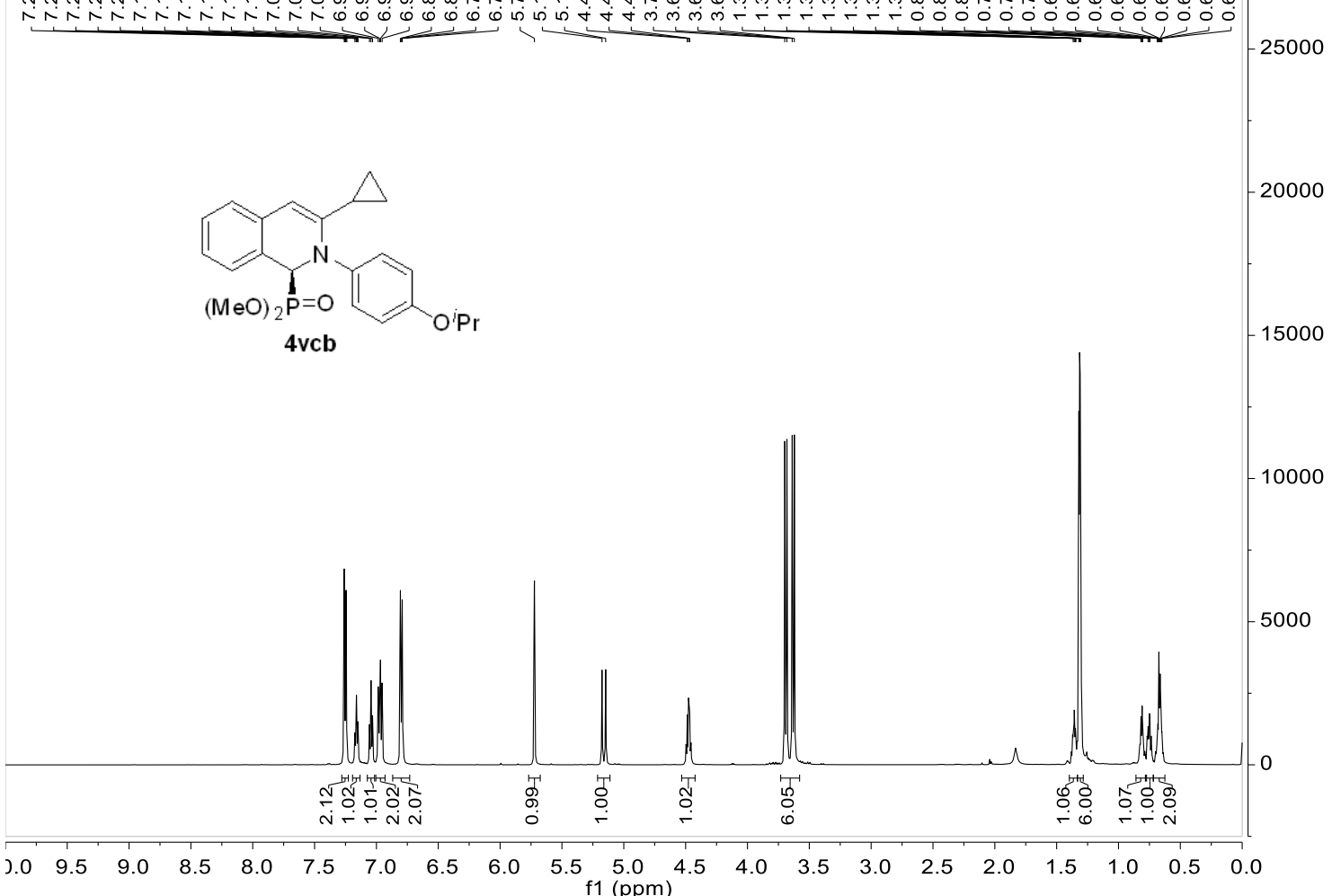

$151 \mathrm{MHz}, \mathrm{CDCl}_{3},{ }^{13} \mathrm{C} \mathrm{NMR}$

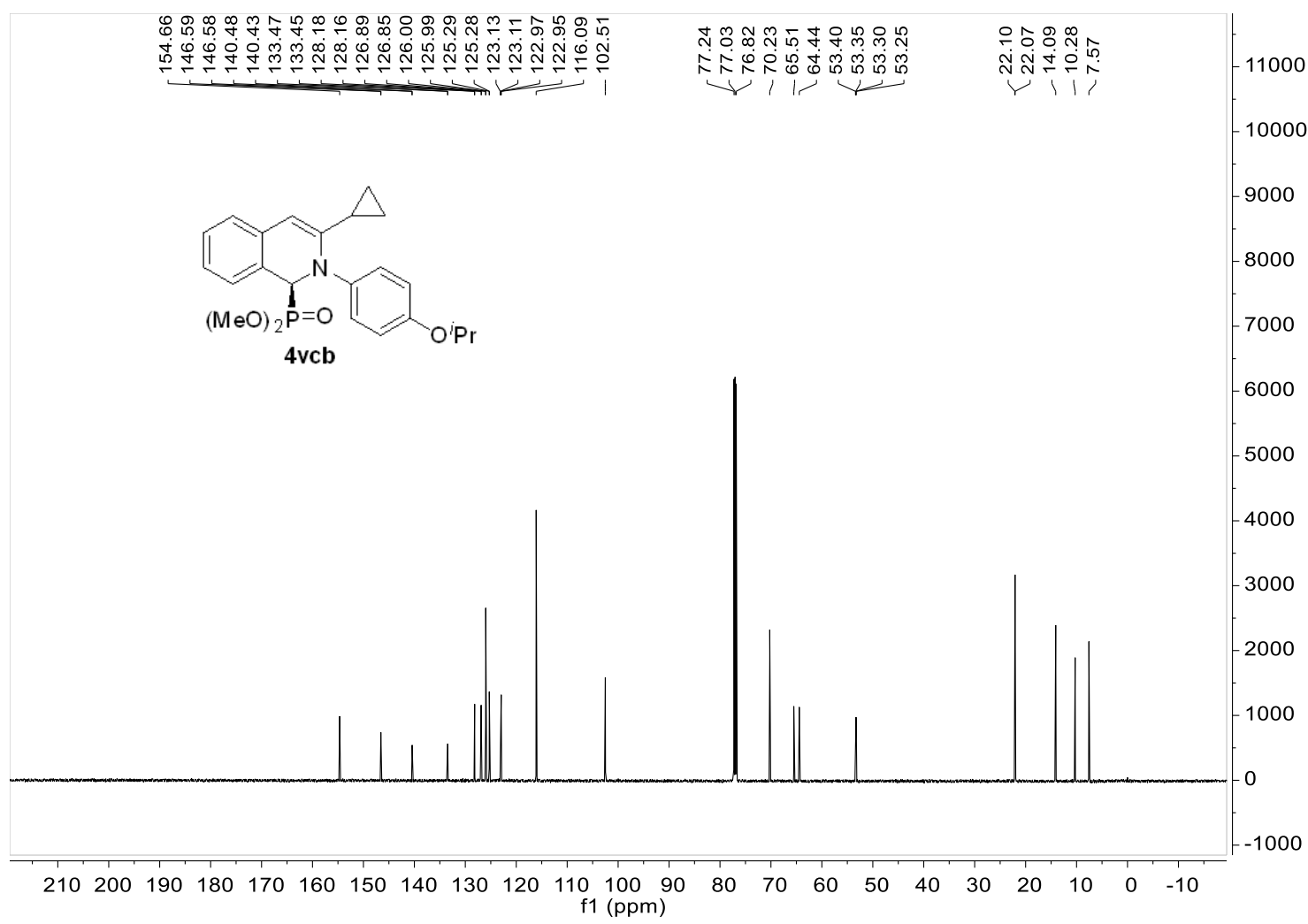


$600 \mathrm{MHz}, \mathrm{CDCl}_{3},{ }^{1} \mathrm{H} \mathrm{NMR}$

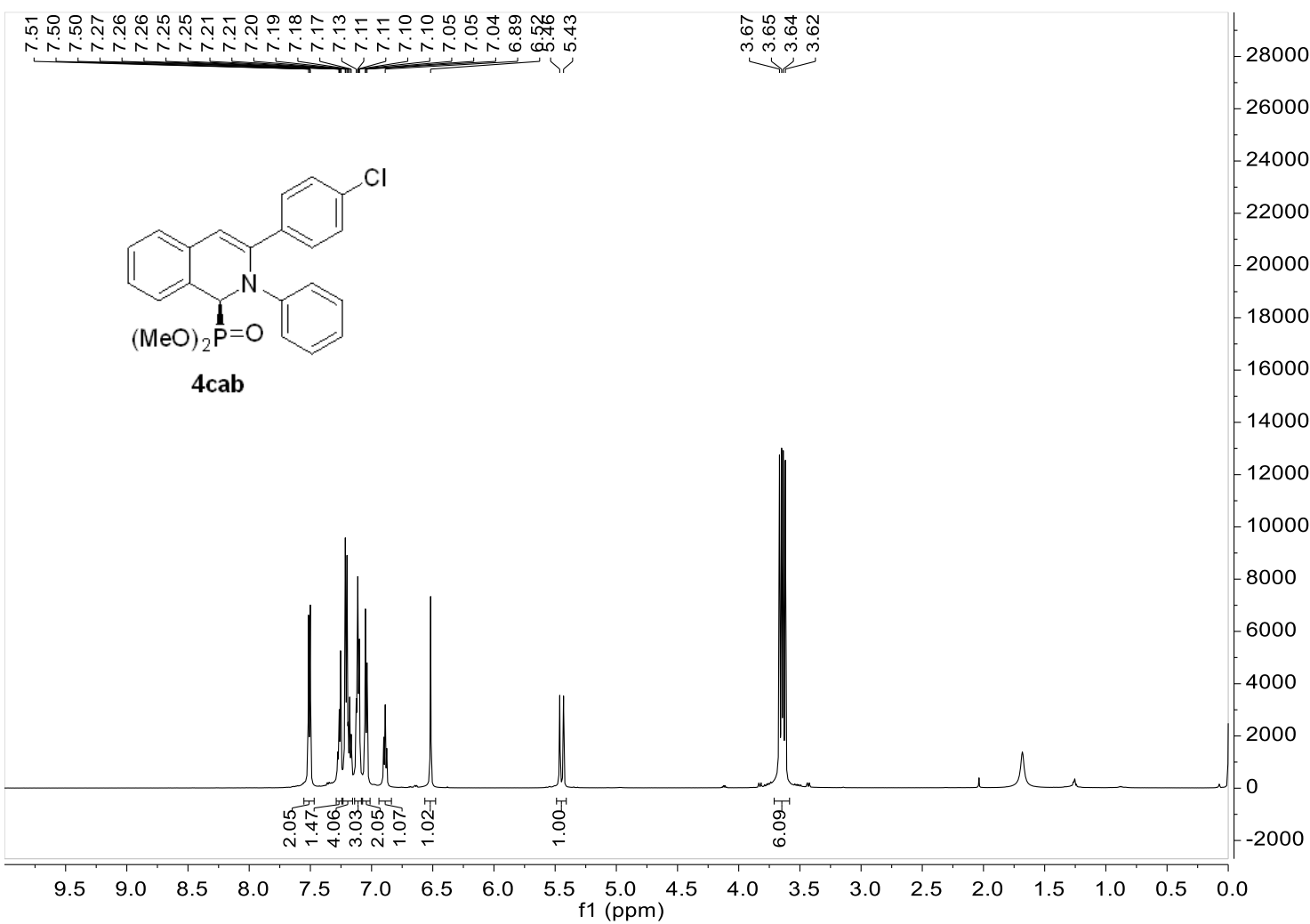

$151 \mathrm{MHz}, \mathrm{CDCl}_{3},{ }^{13} \mathrm{C} \mathrm{NMR}$

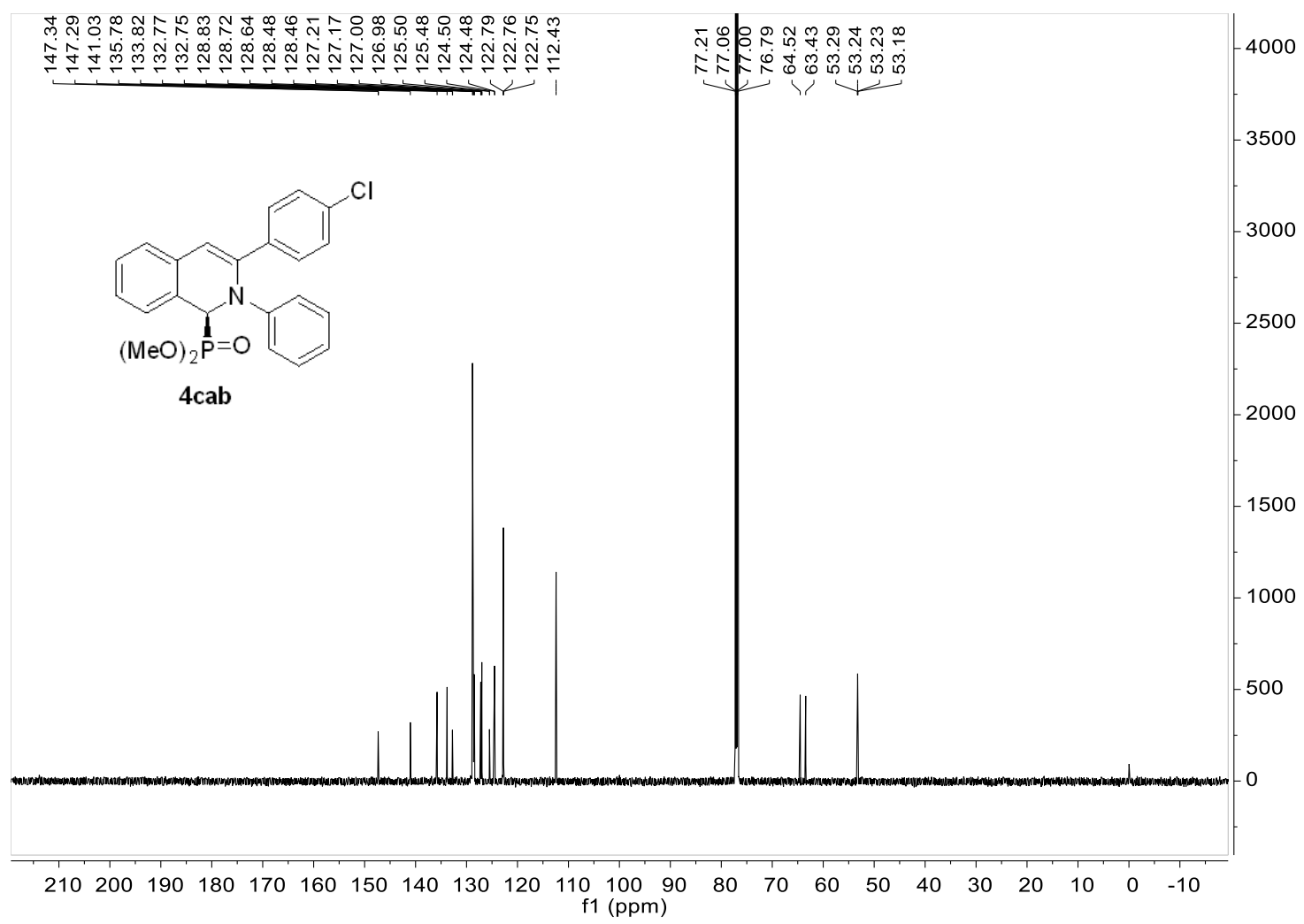


$600 \mathrm{MHz}, \mathrm{CDCl}_{3},{ }^{1} \mathrm{H} \mathrm{NMR}$

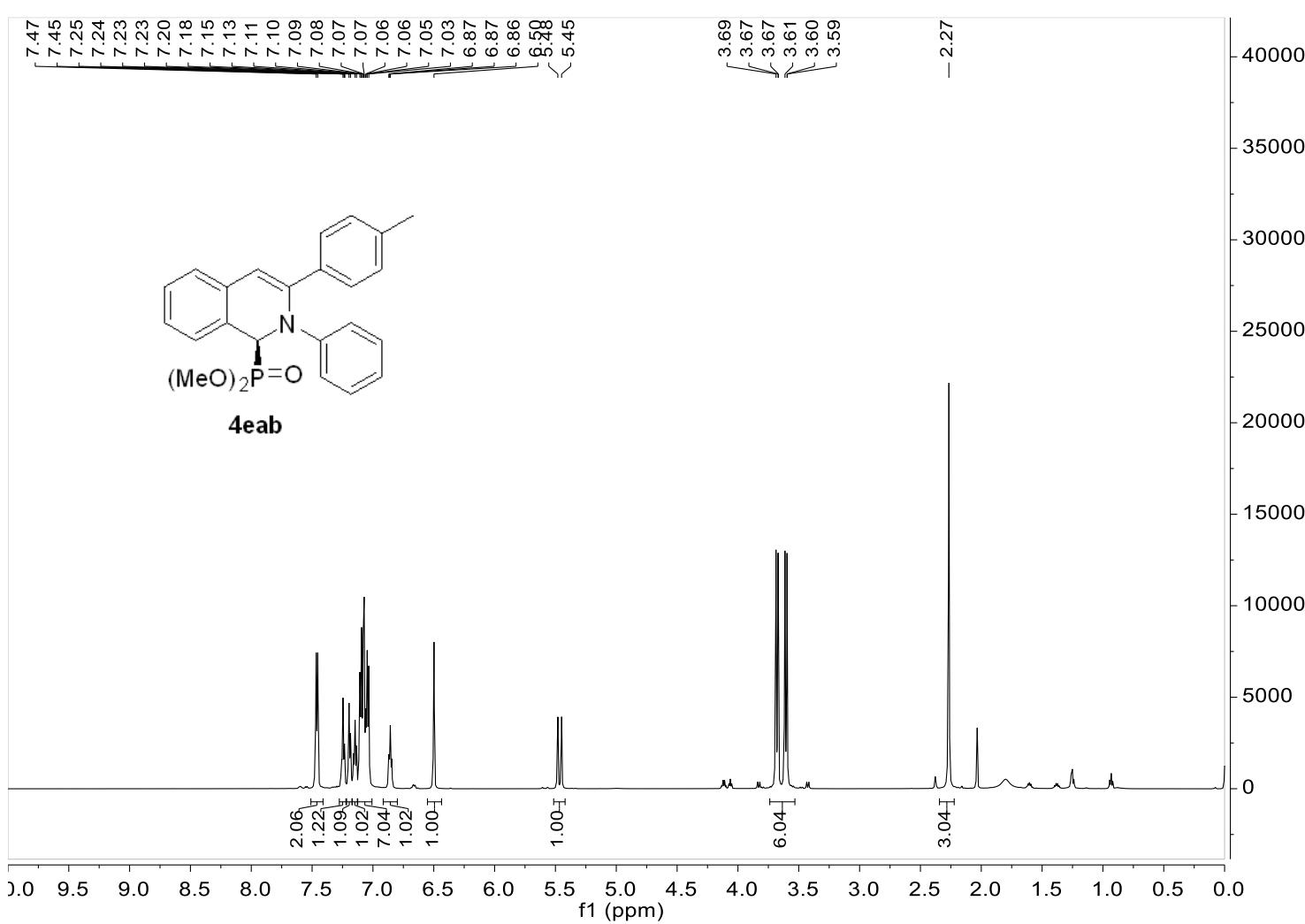

$151 \mathrm{MHz}, \mathrm{CDCl}_{3},{ }^{13} \mathrm{C} \mathrm{NMR}$

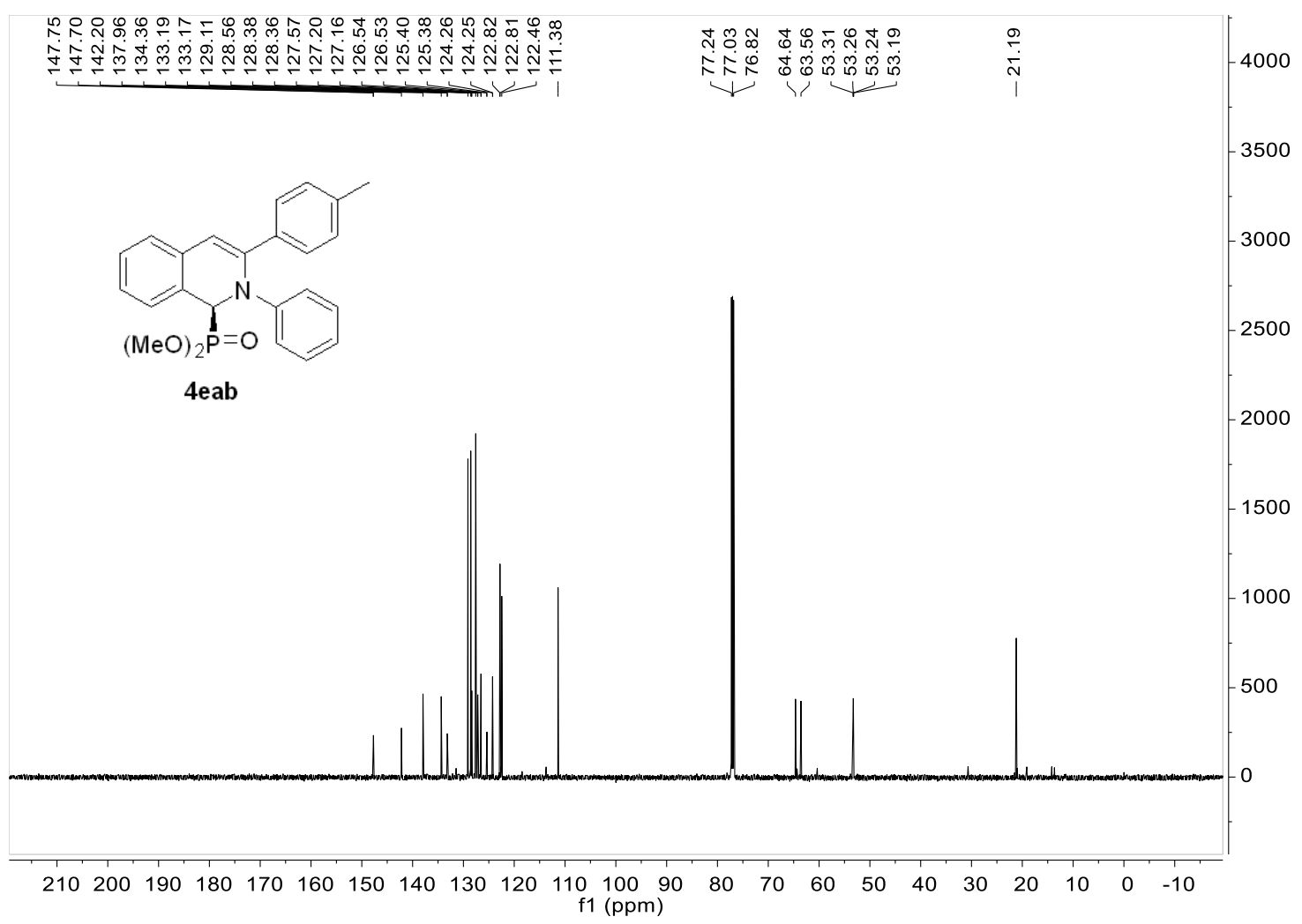


$600 \mathrm{MHz}, \mathrm{CDCl}_{3},{ }^{1} \mathrm{H} \mathrm{NMR}$

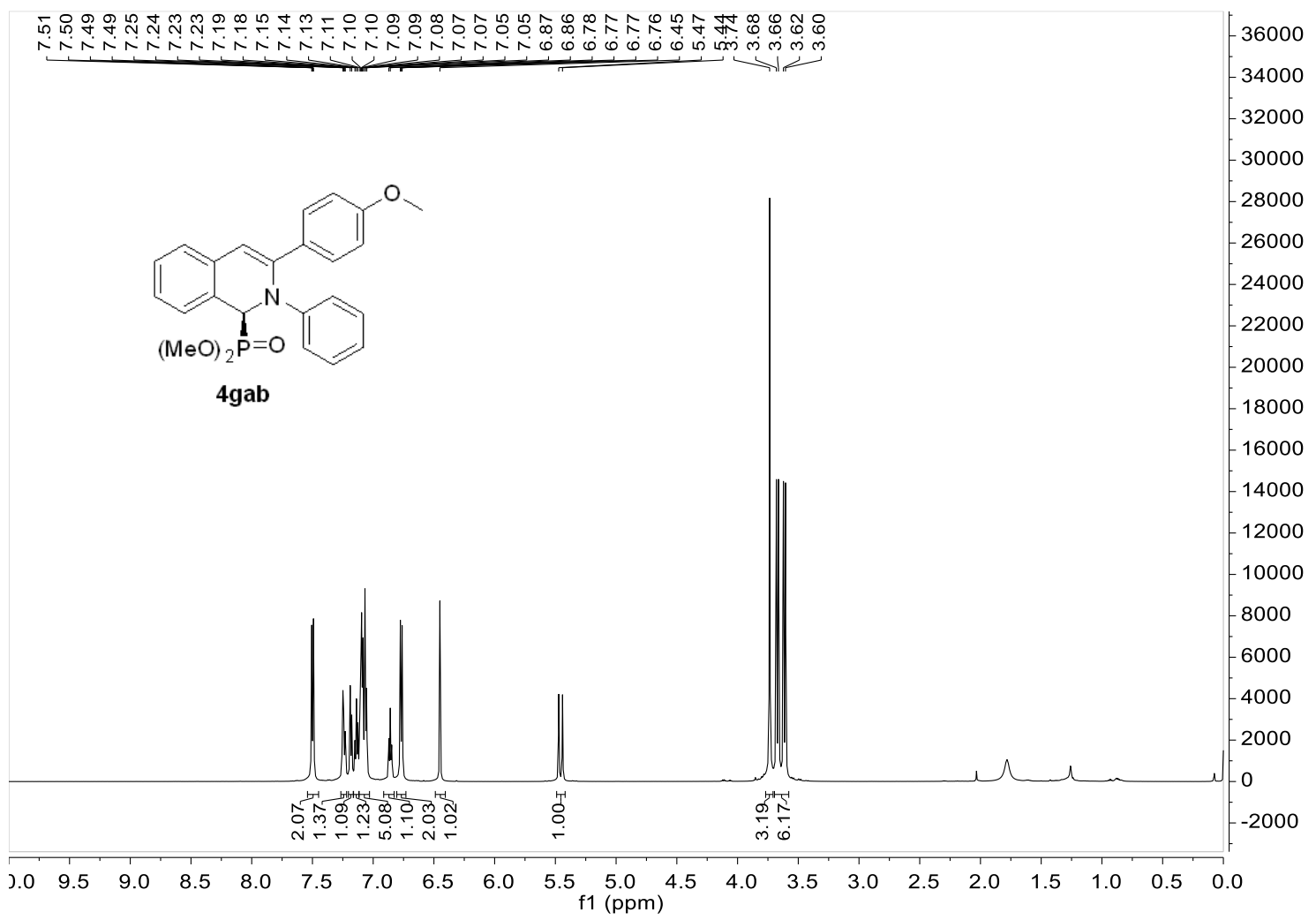

$151 \mathrm{MHz}, \mathrm{CDCl}_{3},{ }^{13} \mathrm{C} \mathrm{NMR}$

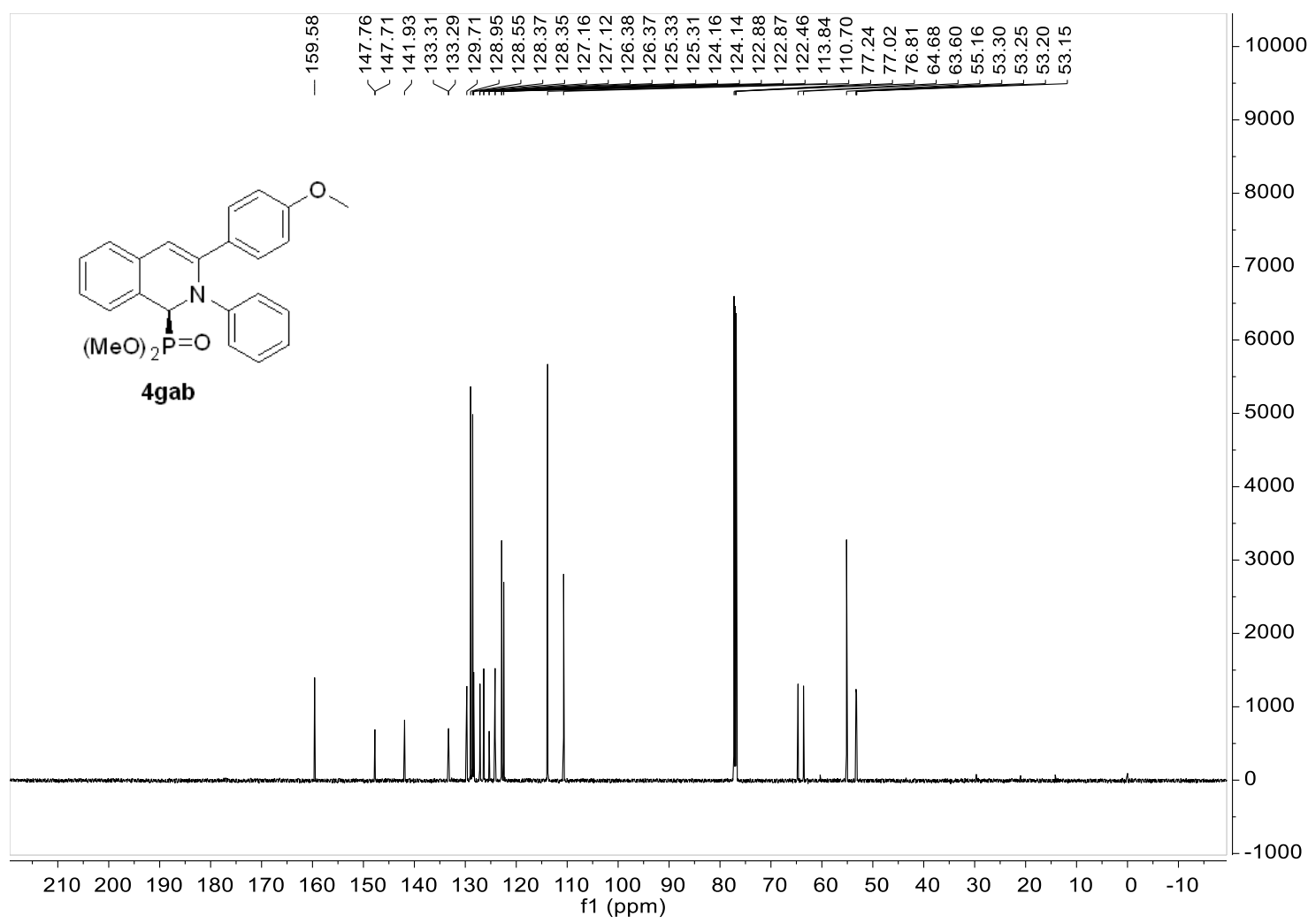


$600 \mathrm{MHz}, \mathrm{CDCl}_{3},{ }^{1} \mathrm{H} \mathrm{NMR}$

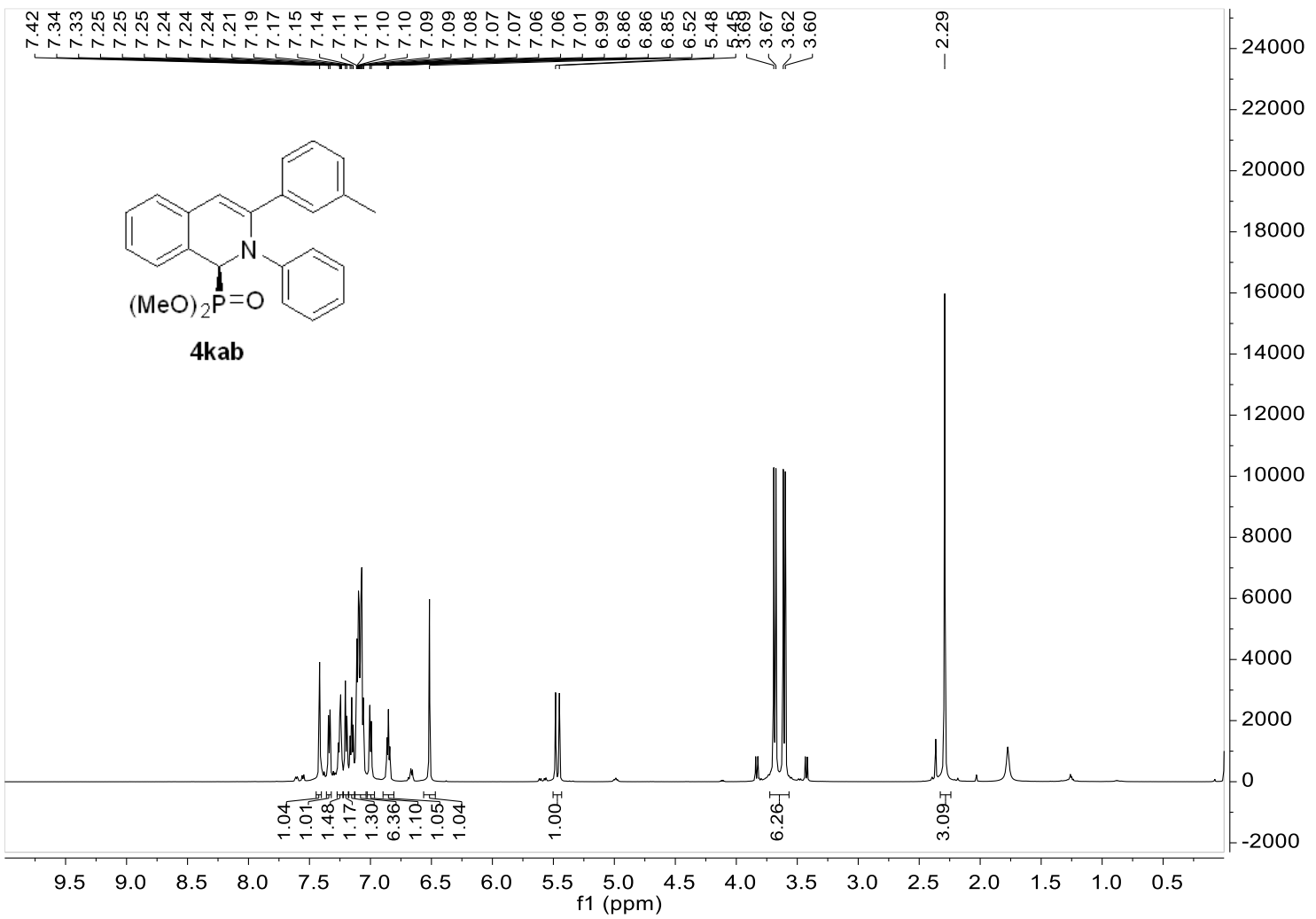

$151 \mathrm{MHz}, \mathrm{CDCl}_{3},{ }^{13} \mathrm{C} \mathrm{NMR}$

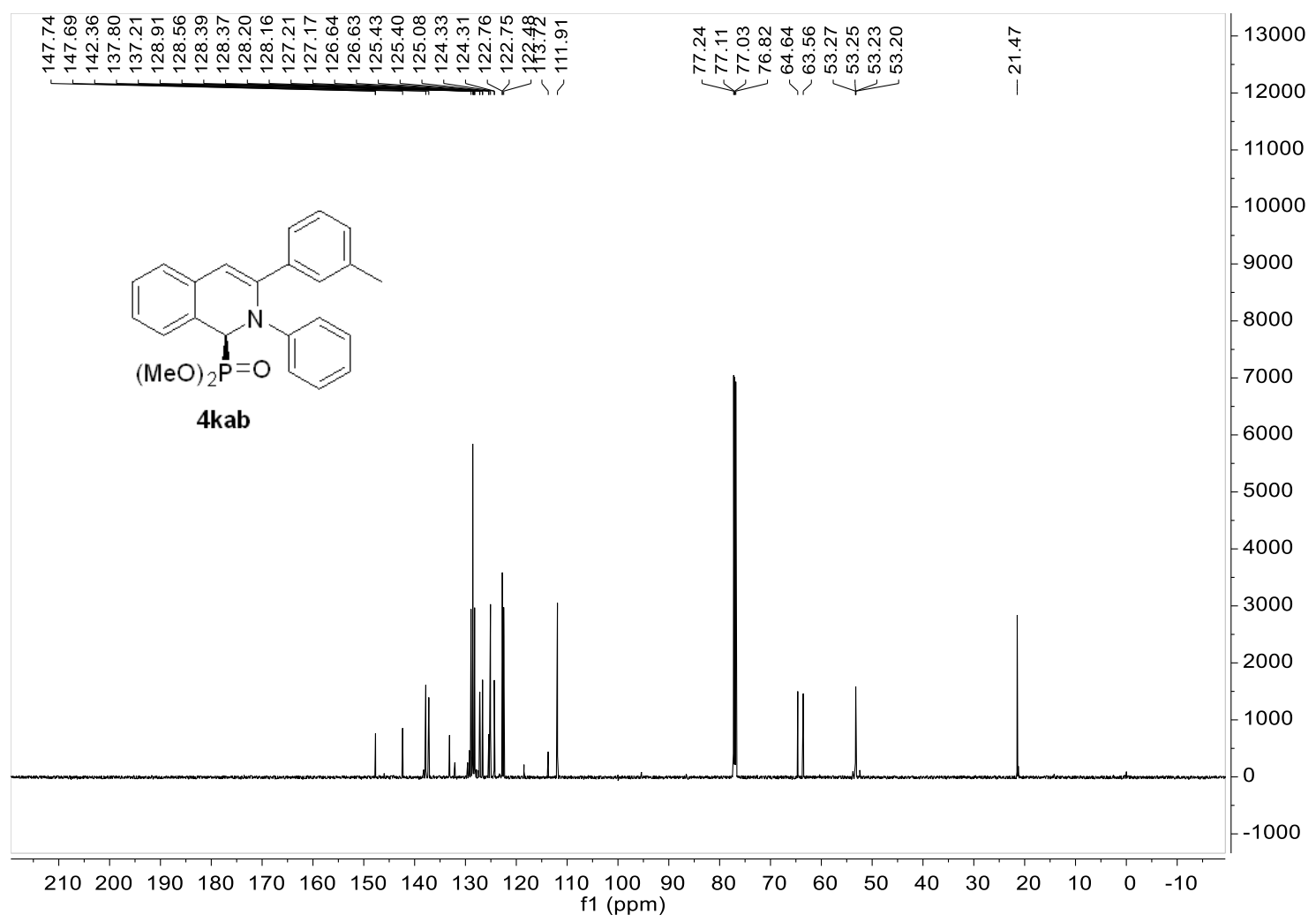


$600 \mathrm{MHz}, \mathrm{CDCl}_{3},{ }^{1} \mathrm{H} \mathrm{NMR}$

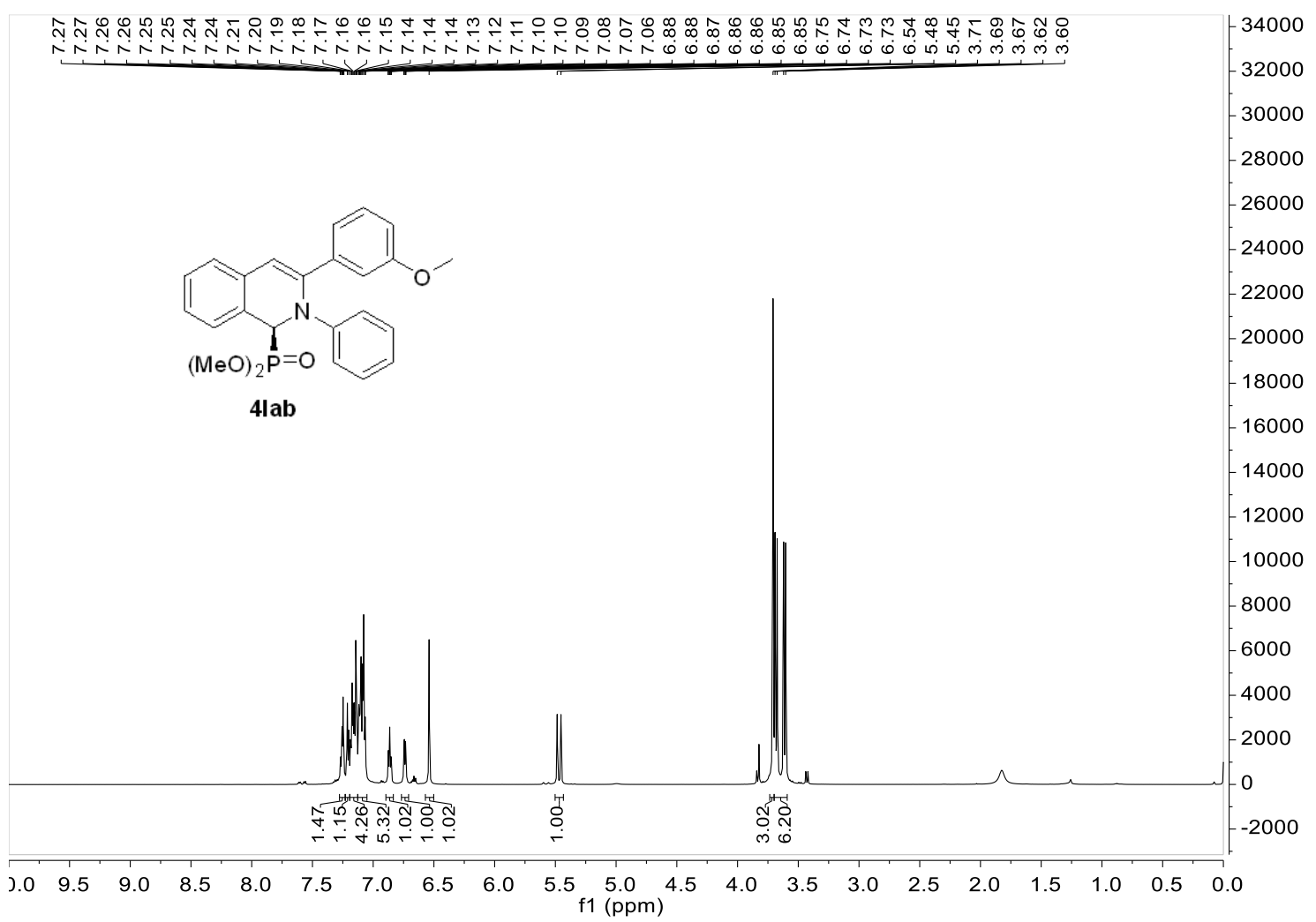

$151 \mathrm{MHz}, \mathrm{CDCl}_{3},{ }^{13} \mathrm{C} \mathrm{NMR}$

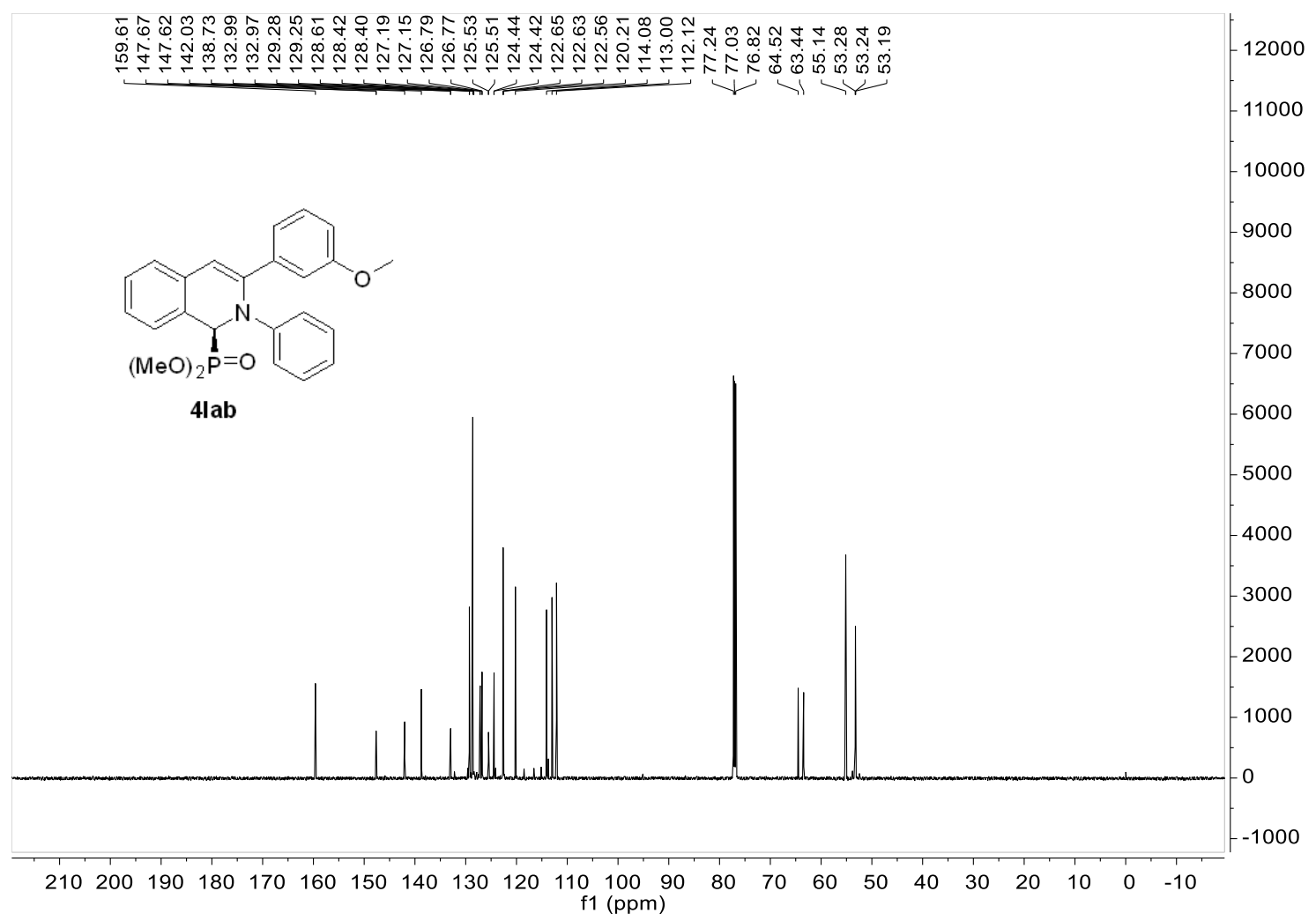


$600 \mathrm{MHz}, \mathrm{CDCl}_{3},{ }^{1} \mathrm{H} \mathrm{NMR}$

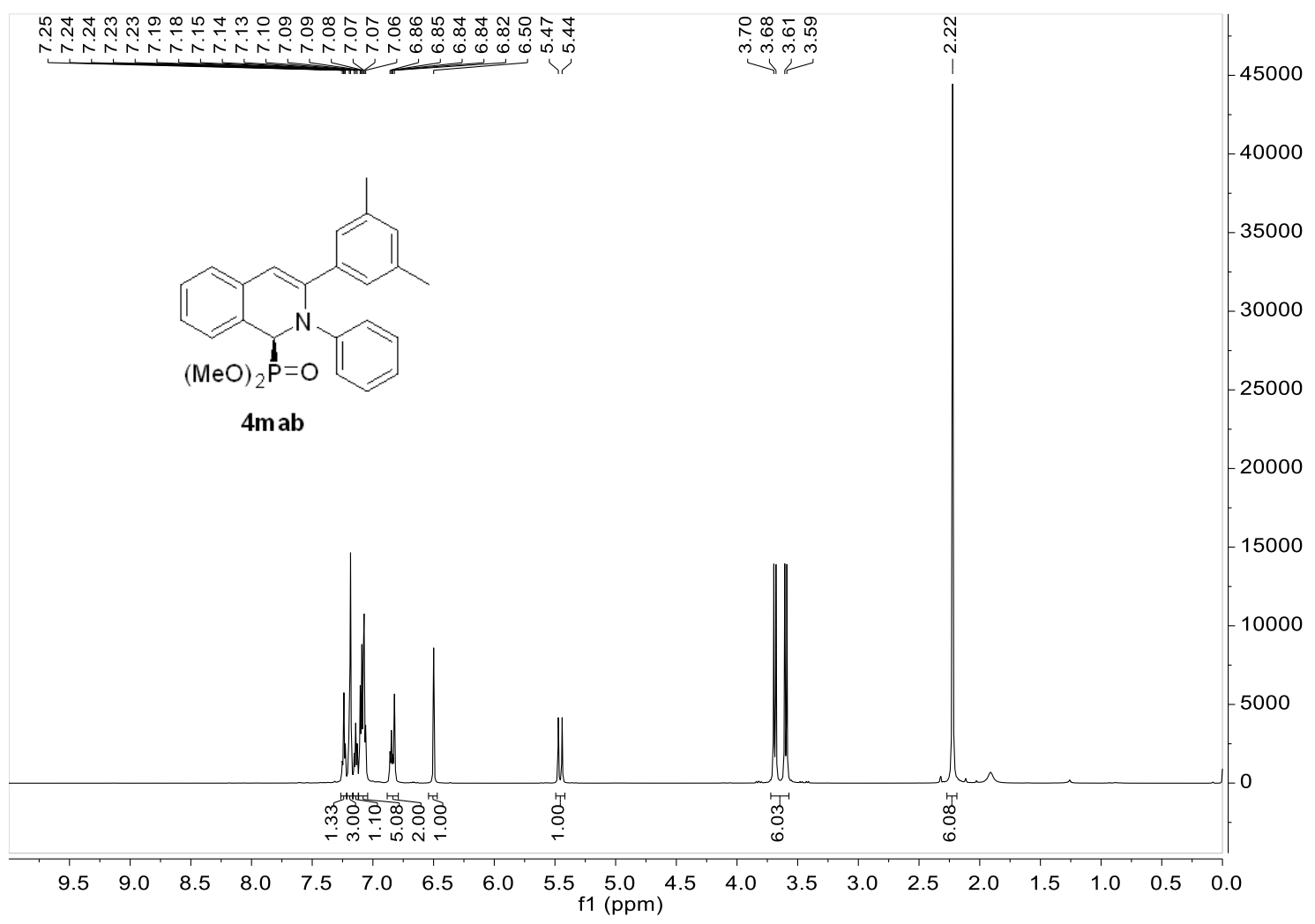

$151 \mathrm{MHz}, \mathrm{CDCl}_{3},{ }^{13} \mathrm{C} \mathrm{NMR}$

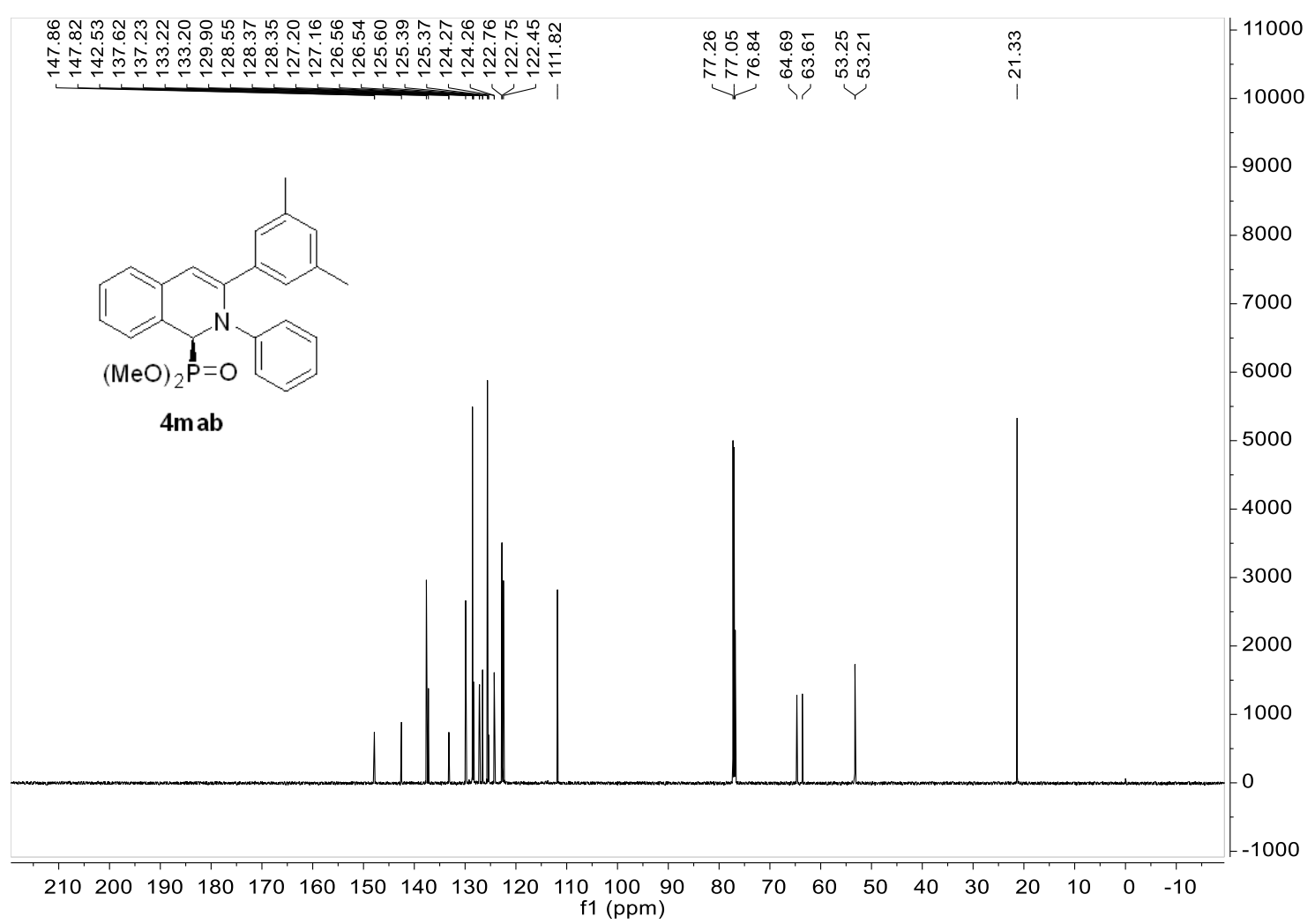


$600 \mathrm{MHz}, \mathrm{CDCl}_{3},{ }^{1} \mathrm{H} \mathrm{NMR}$

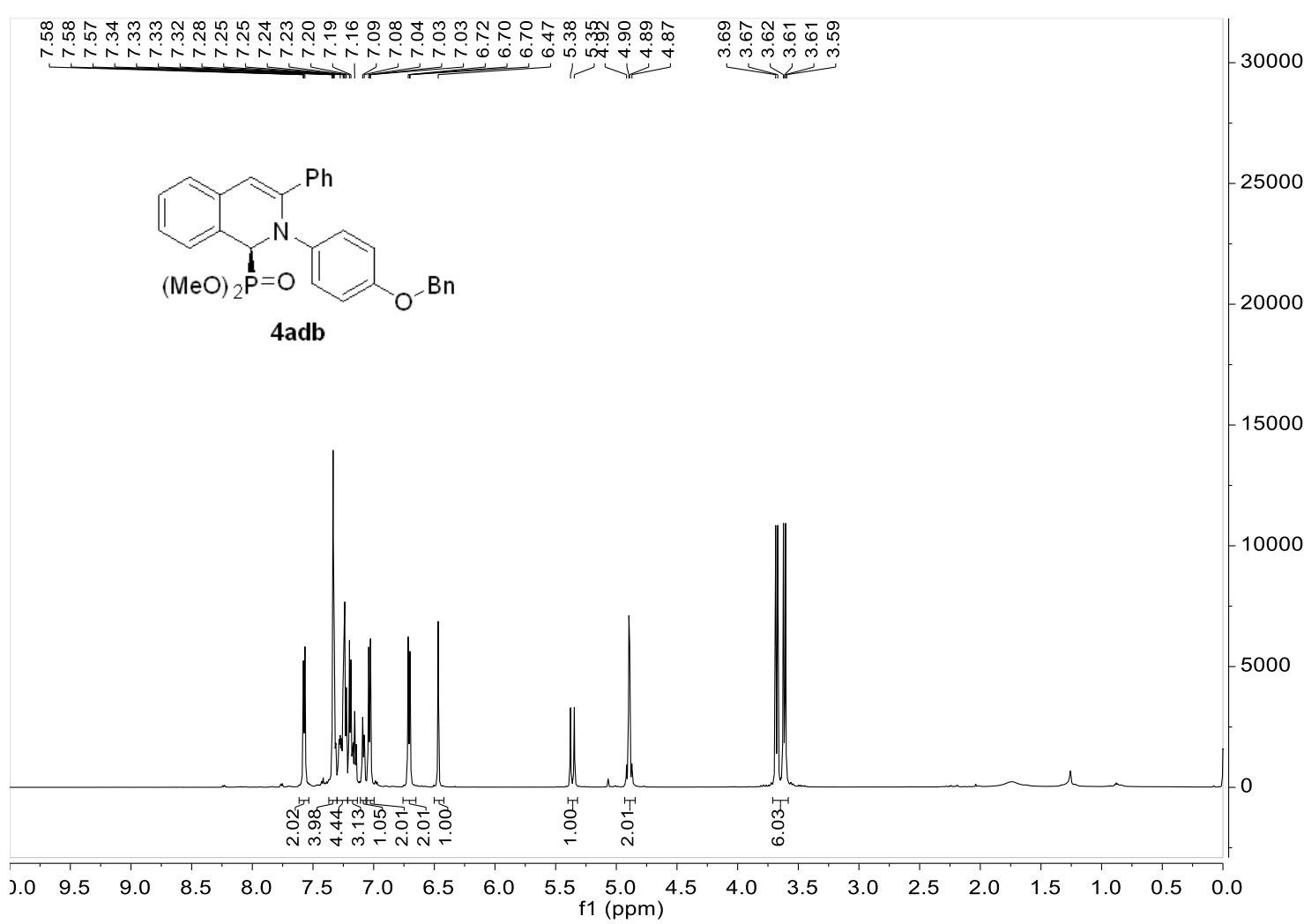

$151 \mathrm{MHz}, \mathrm{CDCl}_{3},{ }^{13} \mathrm{C} \mathrm{NMR}$

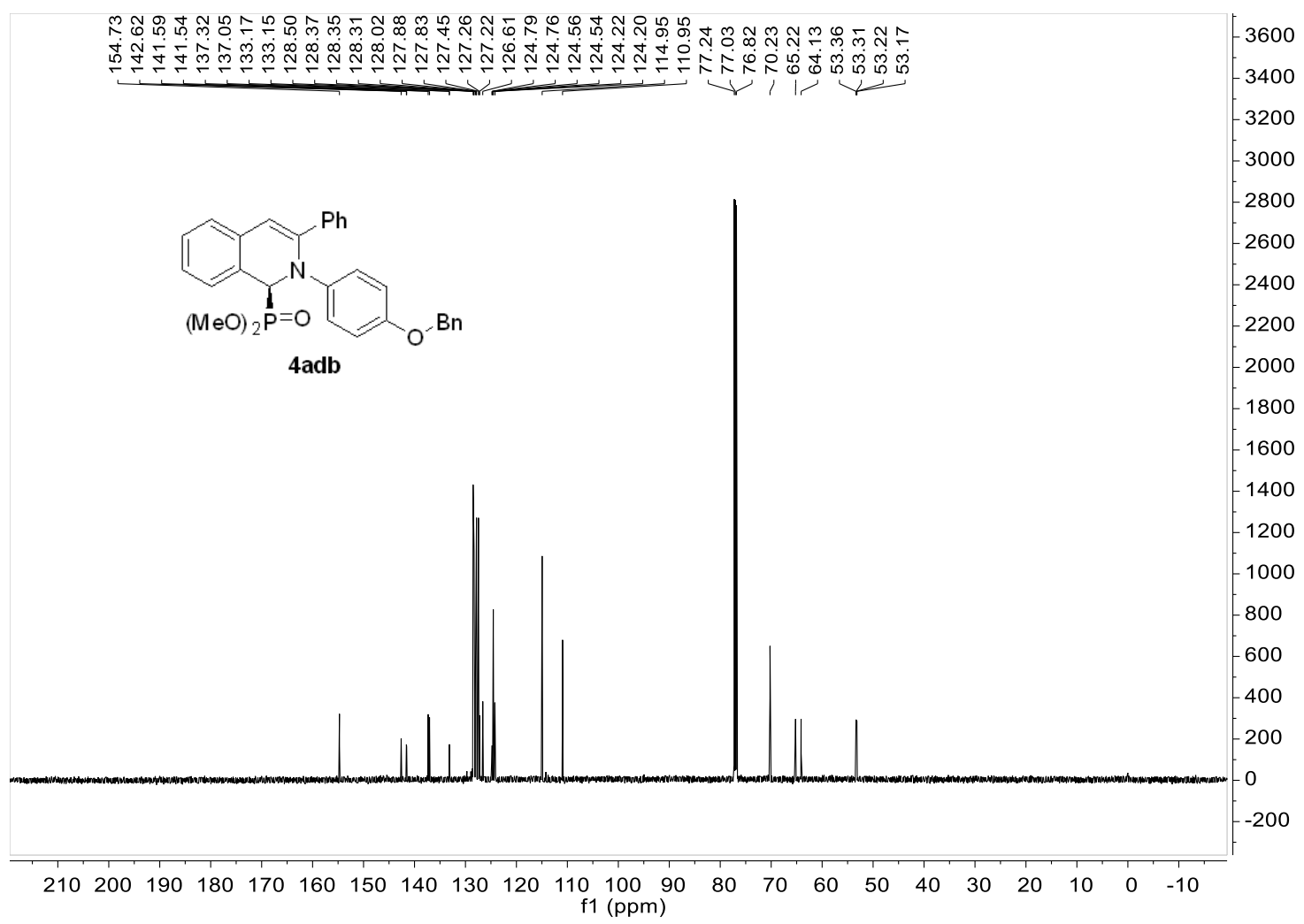


$600 \mathrm{MHz}, \mathrm{CDCl}_{3},{ }^{1} \mathrm{H}$ NMR

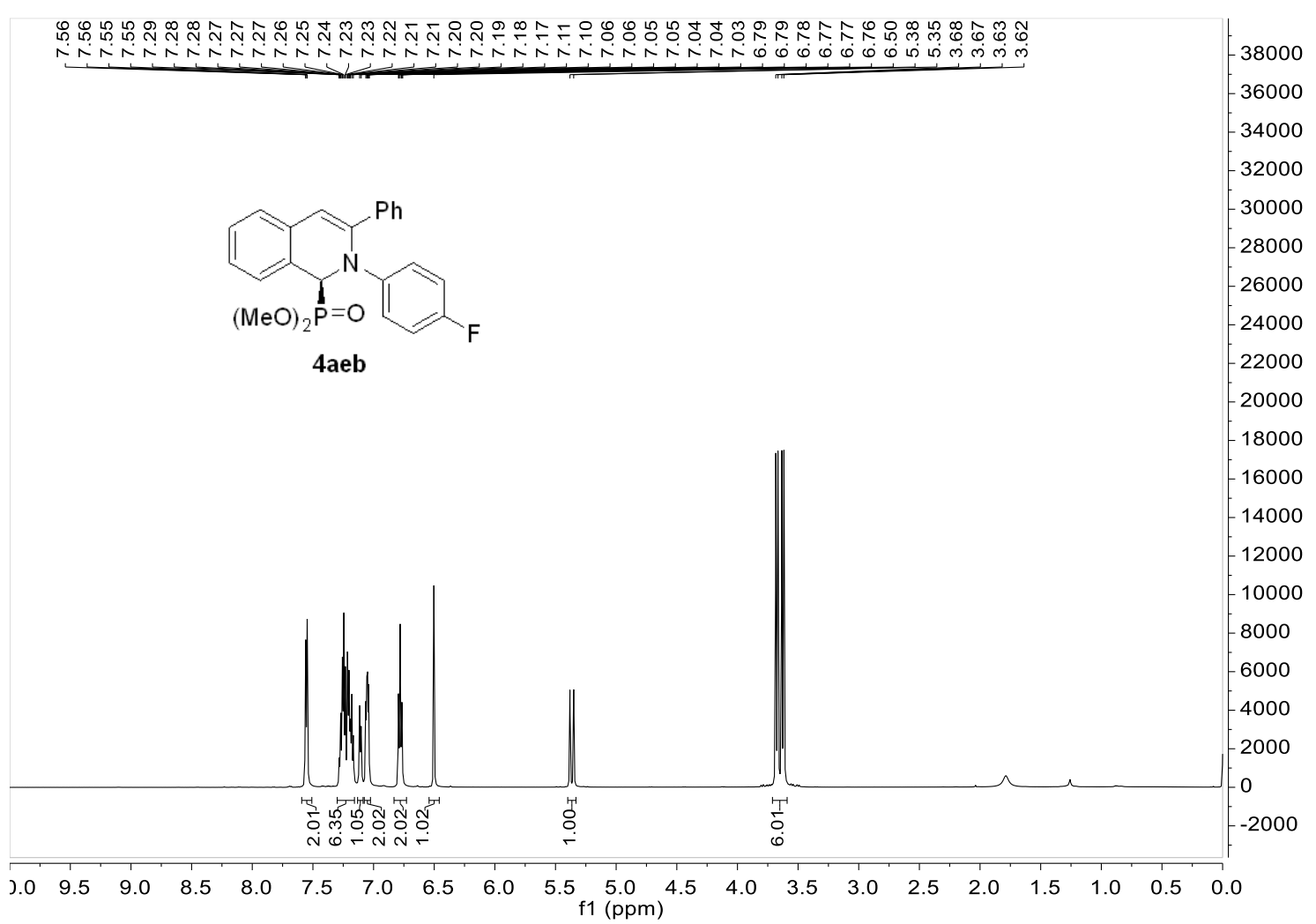

$151 \mathrm{MHz}, \mathrm{CDCl}_{3},{ }^{13} \mathrm{C} \mathrm{NMR}$

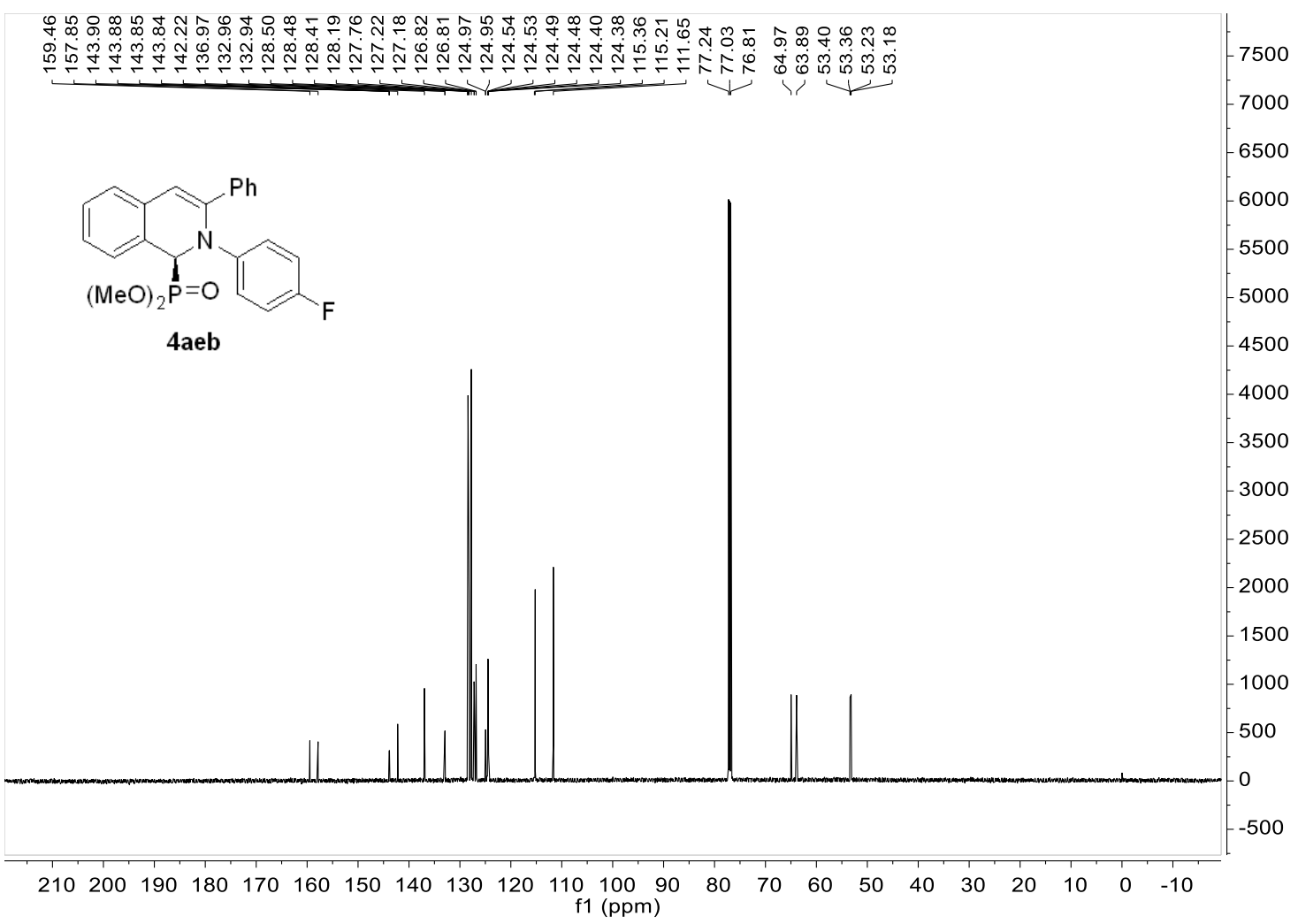


$600 \mathrm{MHz}, \mathrm{CDCl}_{3},{ }^{1} \mathrm{H} \mathrm{NMR}$

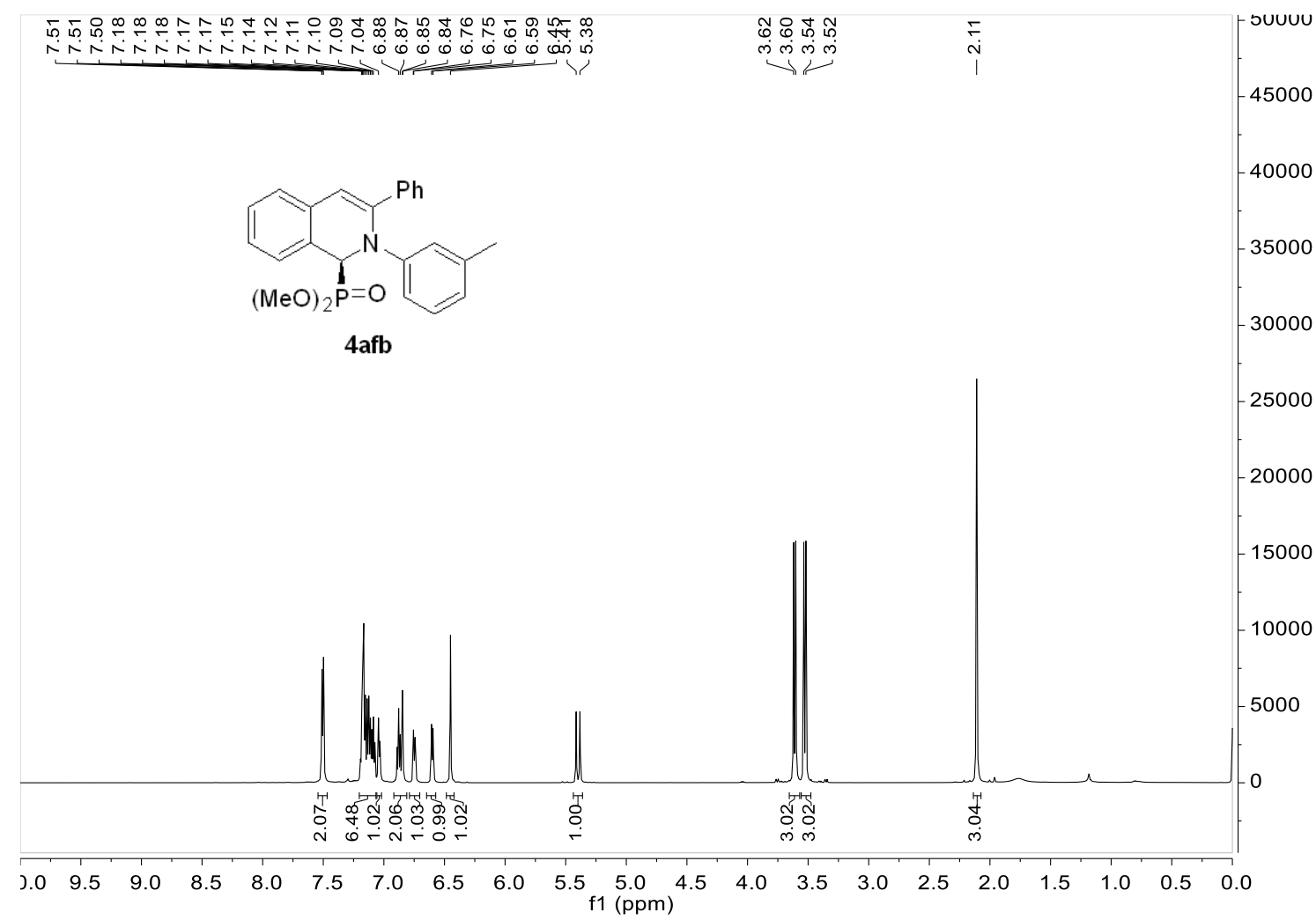

$151 \mathrm{MHz}, \mathrm{CDCl}_{3},{ }^{13} \mathrm{C} \mathrm{NMR}$

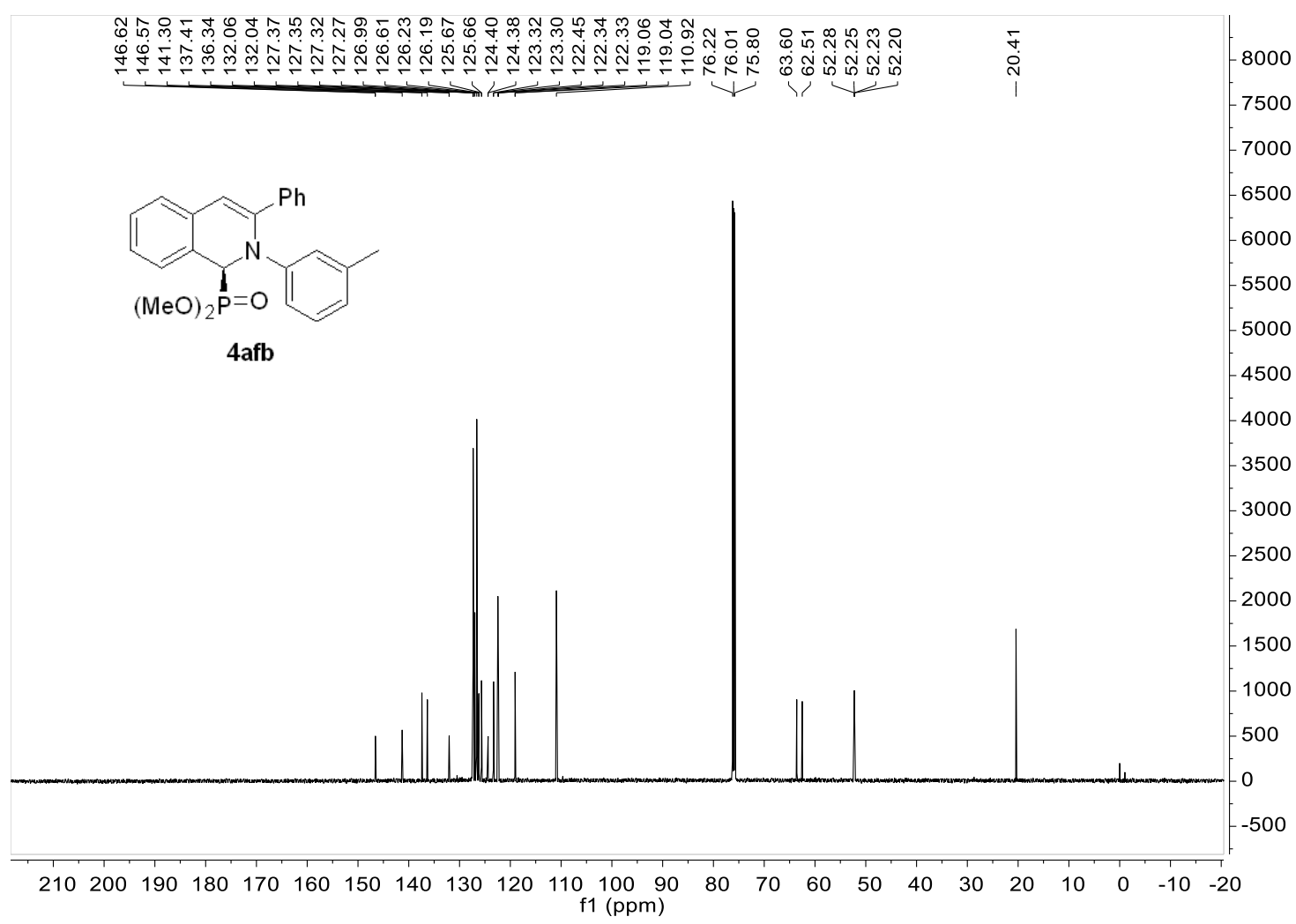


$600 \mathrm{MHz}, \mathrm{CDCl}_{3},{ }^{1} \mathrm{H} \mathrm{NMR}$

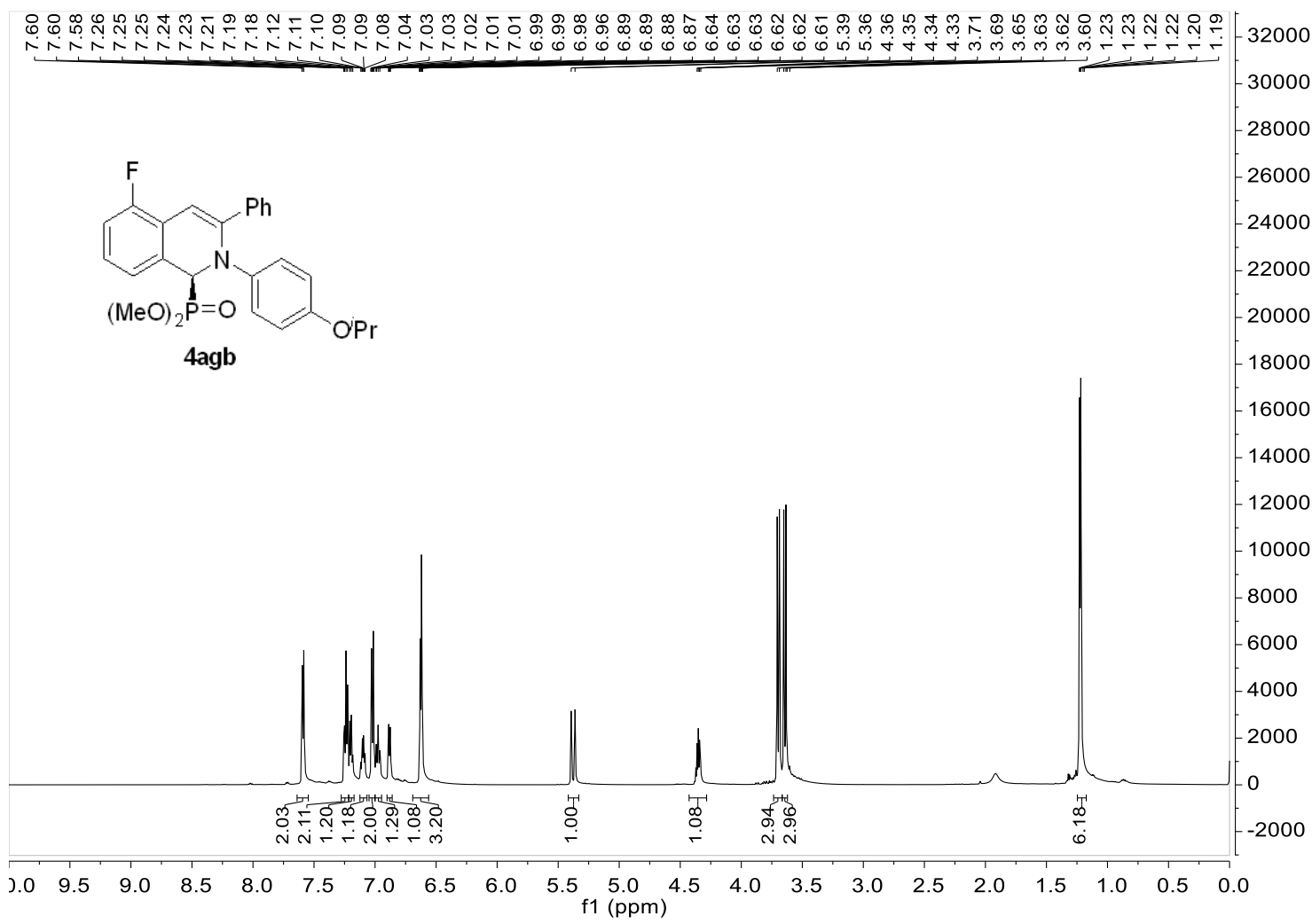

$151 \mathrm{MHz}, \mathrm{CDCl}_{3},{ }^{13} \mathrm{C} \mathrm{NMR}$

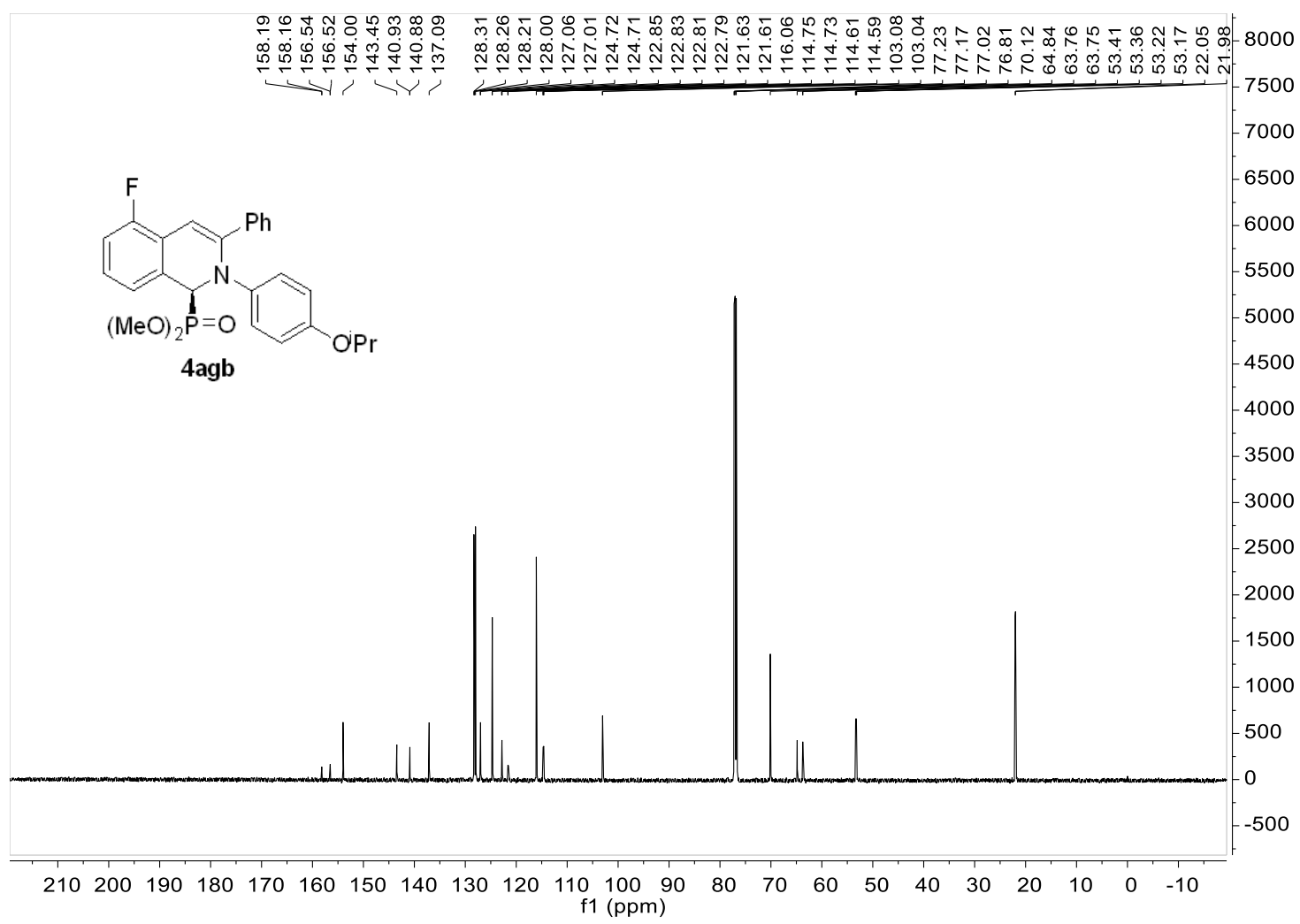


$600 \mathrm{MHz}, \mathrm{CDCl}_{3},{ }^{1} \mathrm{H} \mathrm{NMR}$

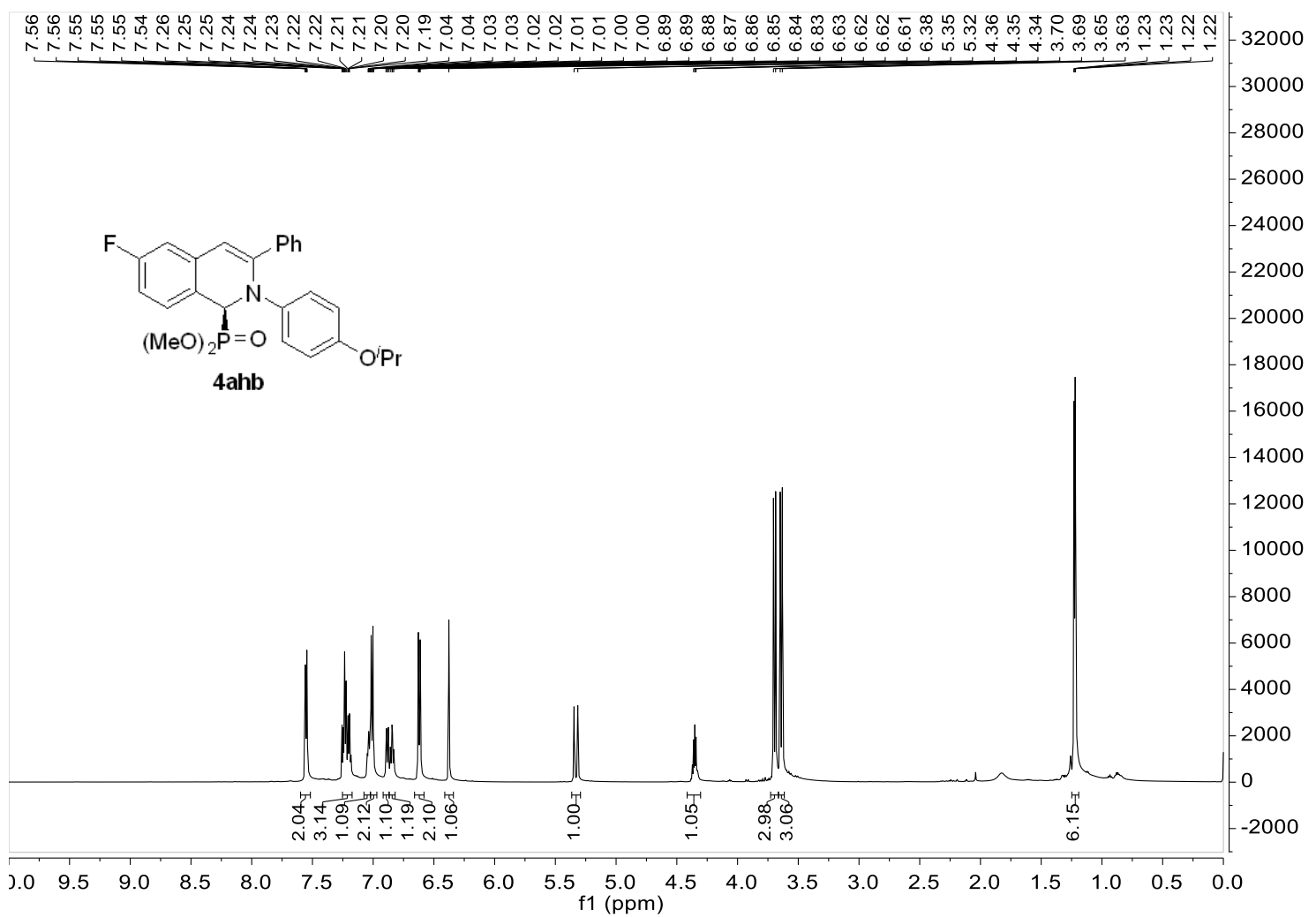

$151 \mathrm{MHz}, \mathrm{CDCl}_{3},{ }^{13} \mathrm{C} \mathrm{NMR}$

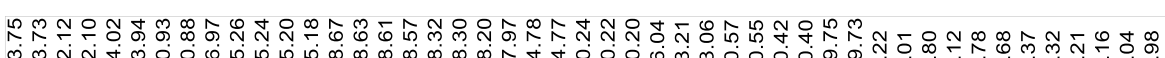

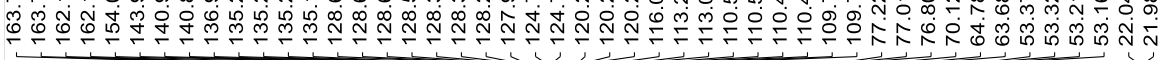

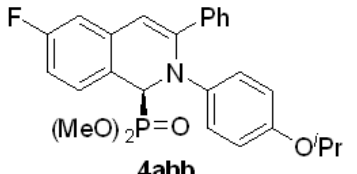
4ahb 
$600 \mathrm{MHz}, \mathrm{CDCl}_{3},{ }^{1} \mathrm{H} \mathrm{NMR}$

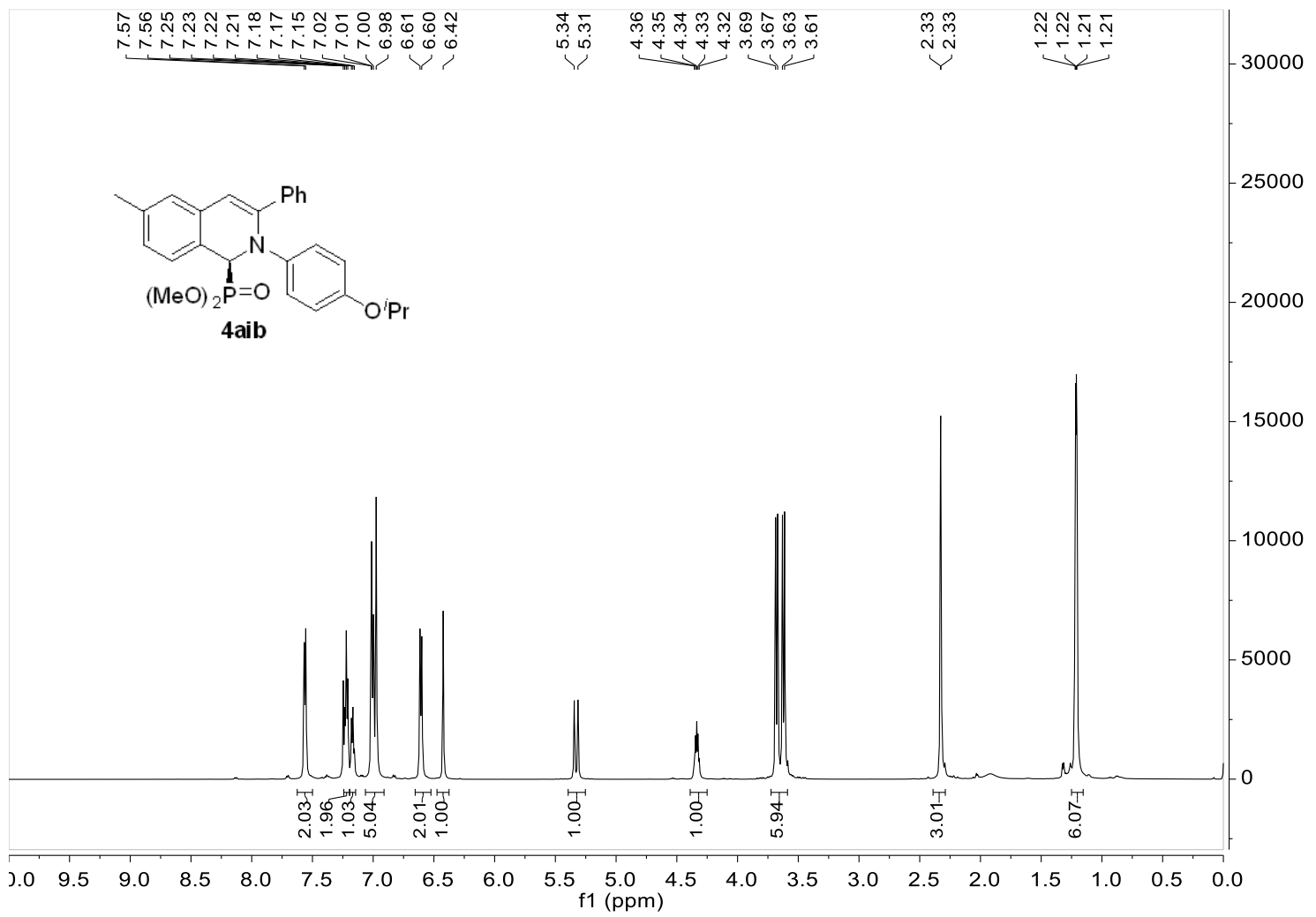

$151 \mathrm{MHz}, \mathrm{CDCl}_{3},{ }^{13} \mathrm{C} \mathrm{NMR}$

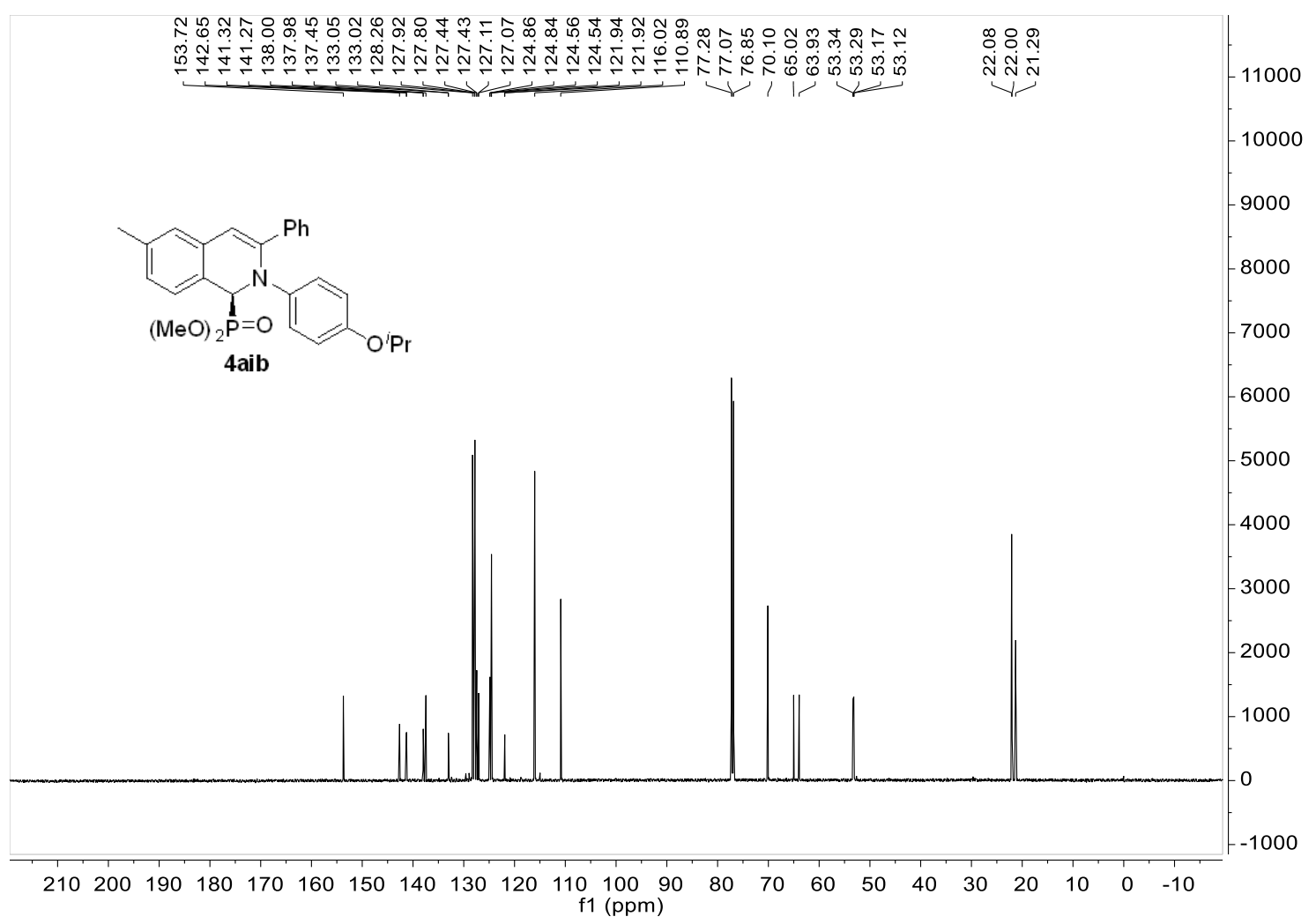


$600 \mathrm{MHz}, \mathrm{CDCl}_{3},{ }^{1} \mathrm{H} \mathrm{NMR}$

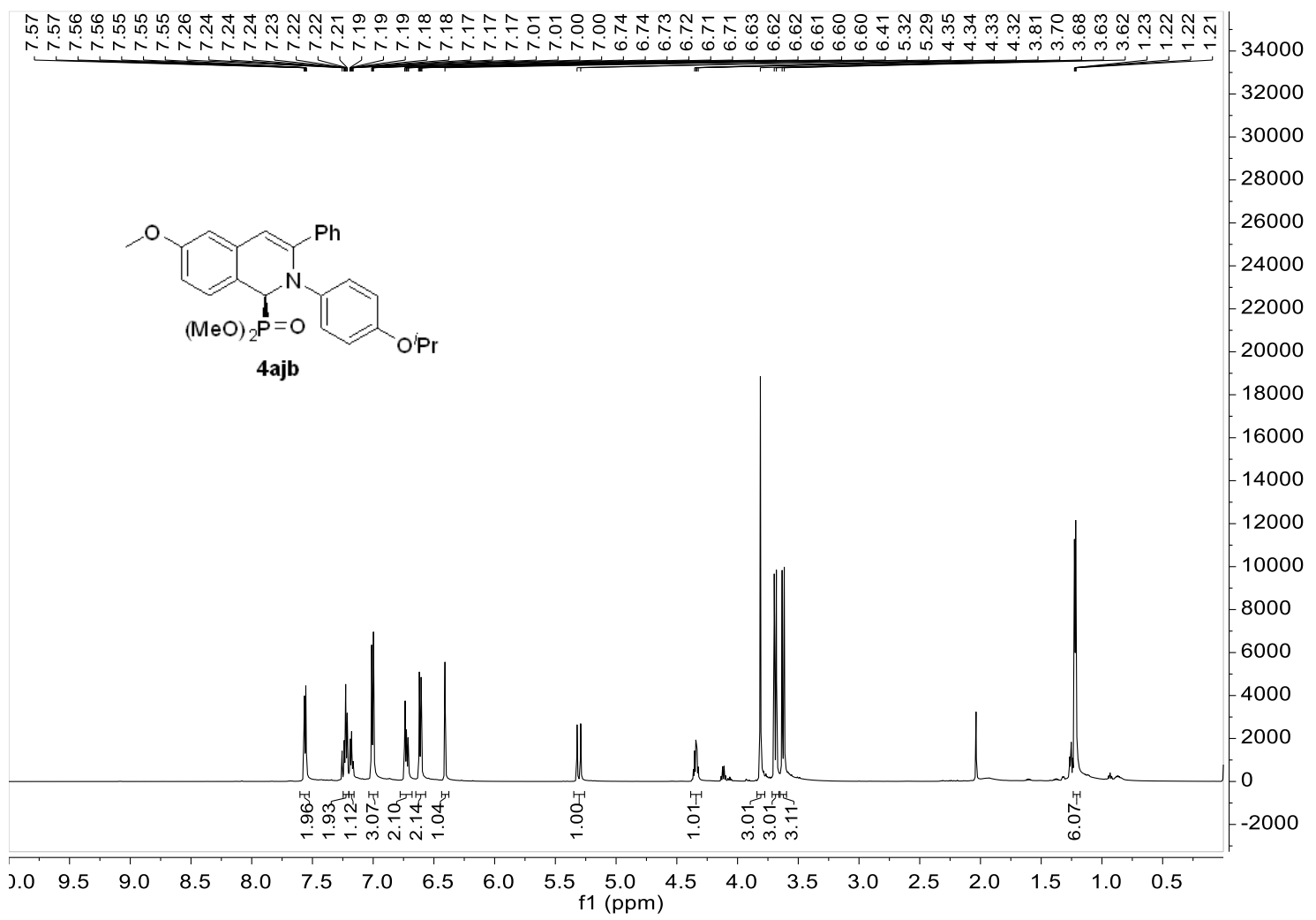

$151 \mathrm{MHz}, \mathrm{CDCl}_{3},{ }^{13} \mathrm{C} \mathrm{NMR}$

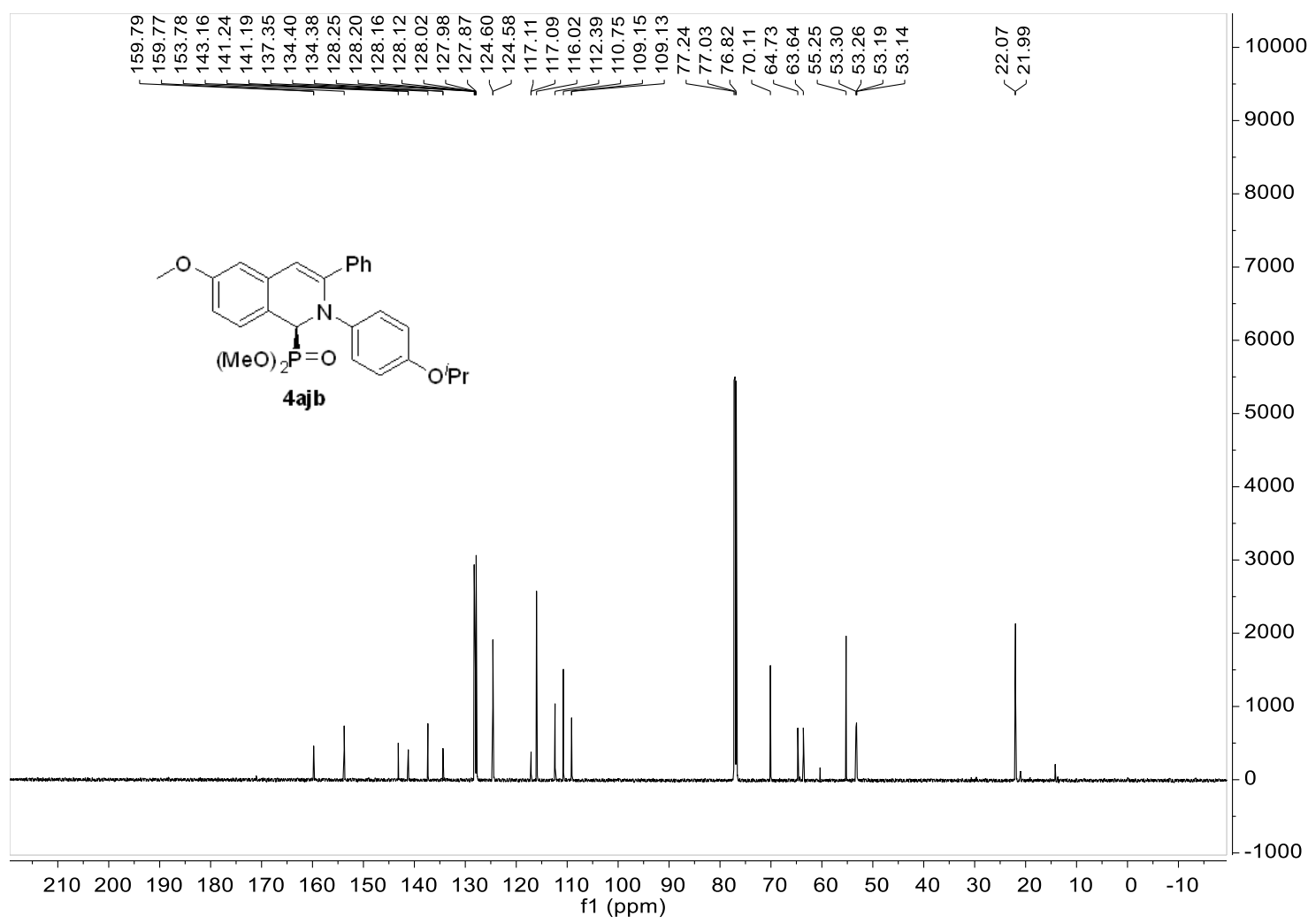


$600 \mathrm{MHz}, \mathrm{CDCl}_{3},{ }^{1} \mathrm{H} \mathrm{NMR}$

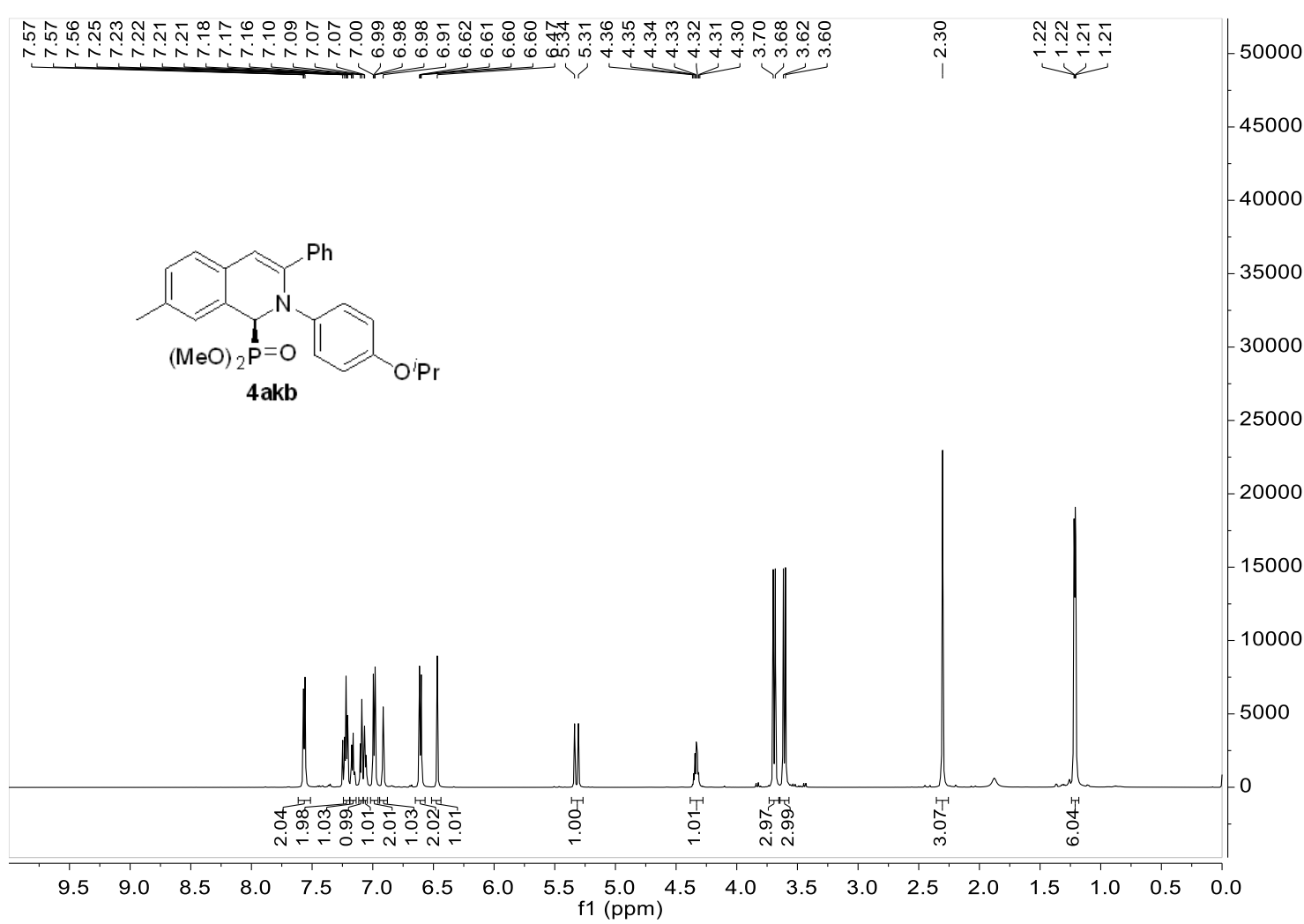

$151 \mathrm{MHz}, \mathrm{CDCl}_{3},{ }^{13} \mathrm{C} \mathrm{NMR}$

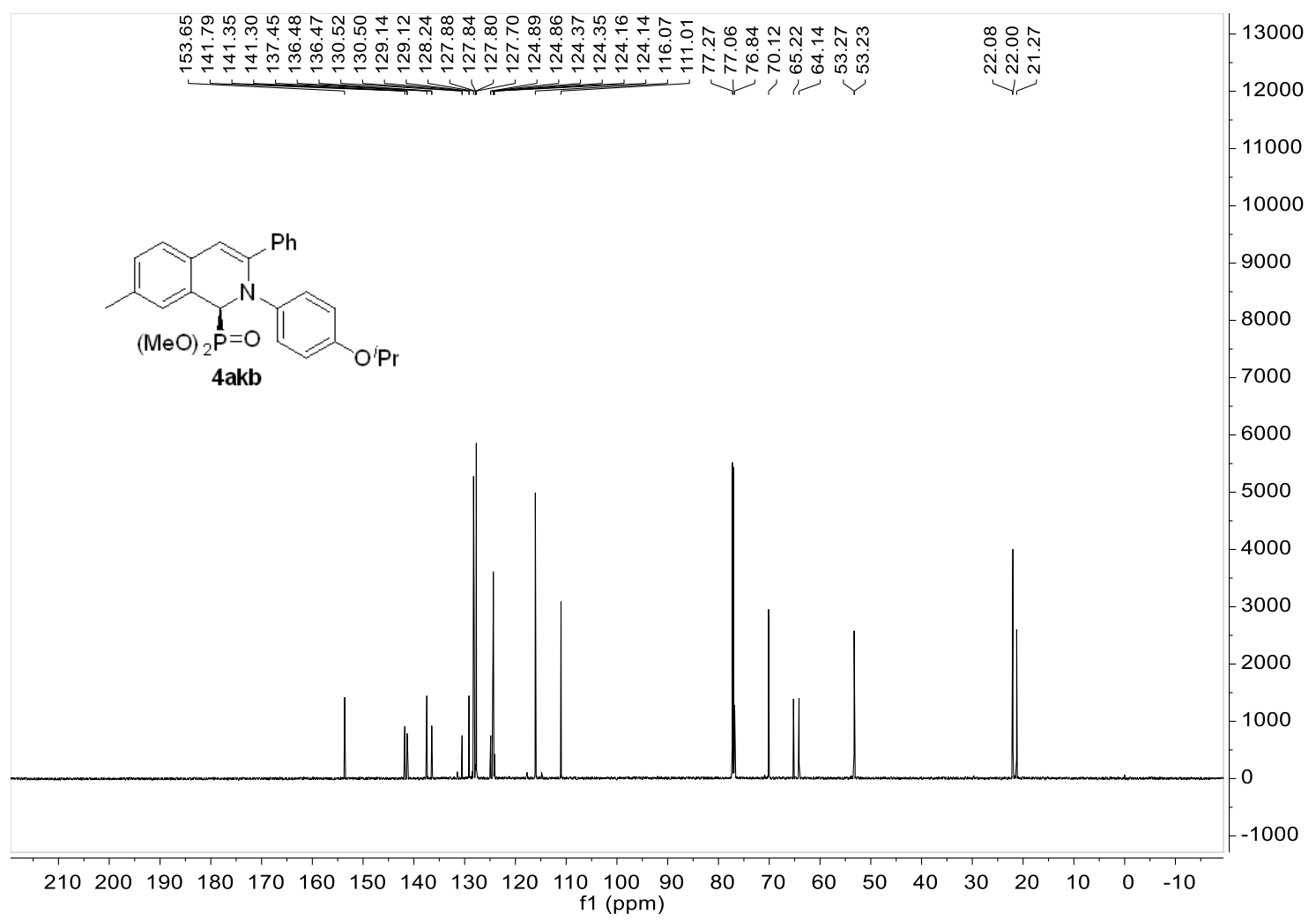


$600 \mathrm{MHz}, \mathrm{CDCl}_{3},{ }^{1} \mathrm{H}$ NMR

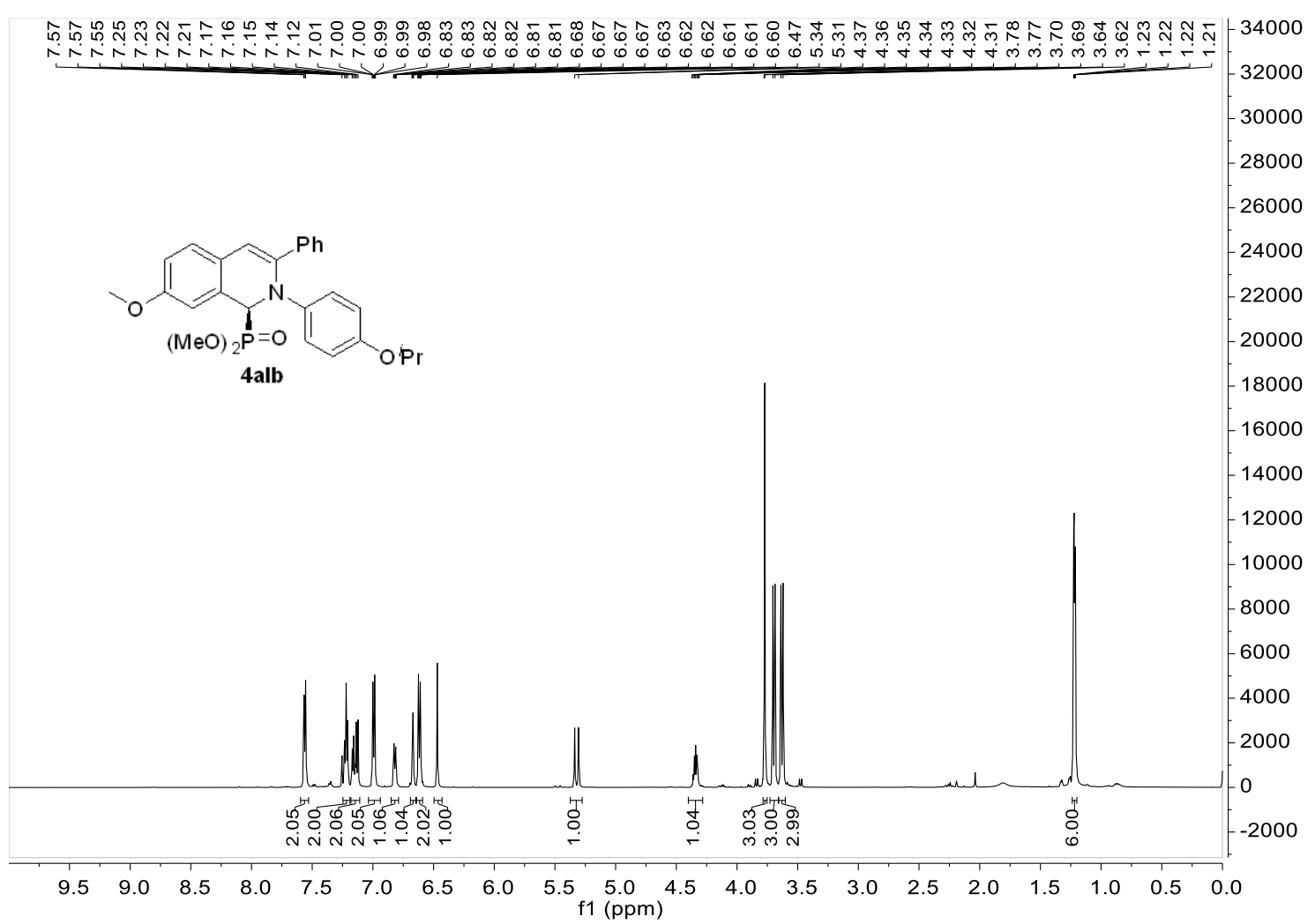

$151 \mathrm{MHz}, \mathrm{CDCl}_{3},{ }^{13} \mathrm{C} \mathrm{NMR}$

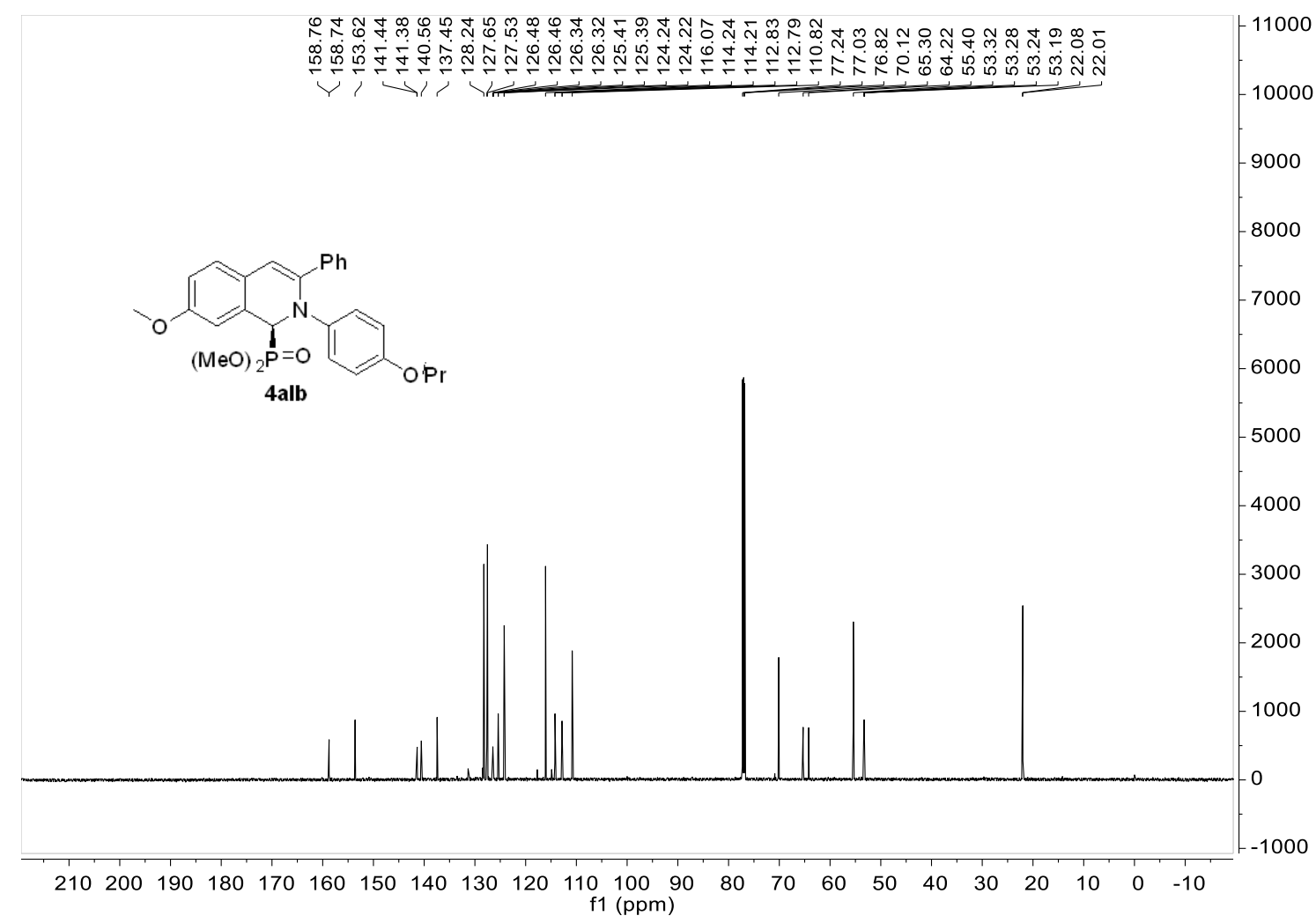


$600 \mathrm{MHz}, \mathrm{CDCl}_{3},{ }^{1} \mathrm{H} \mathrm{NMR}$

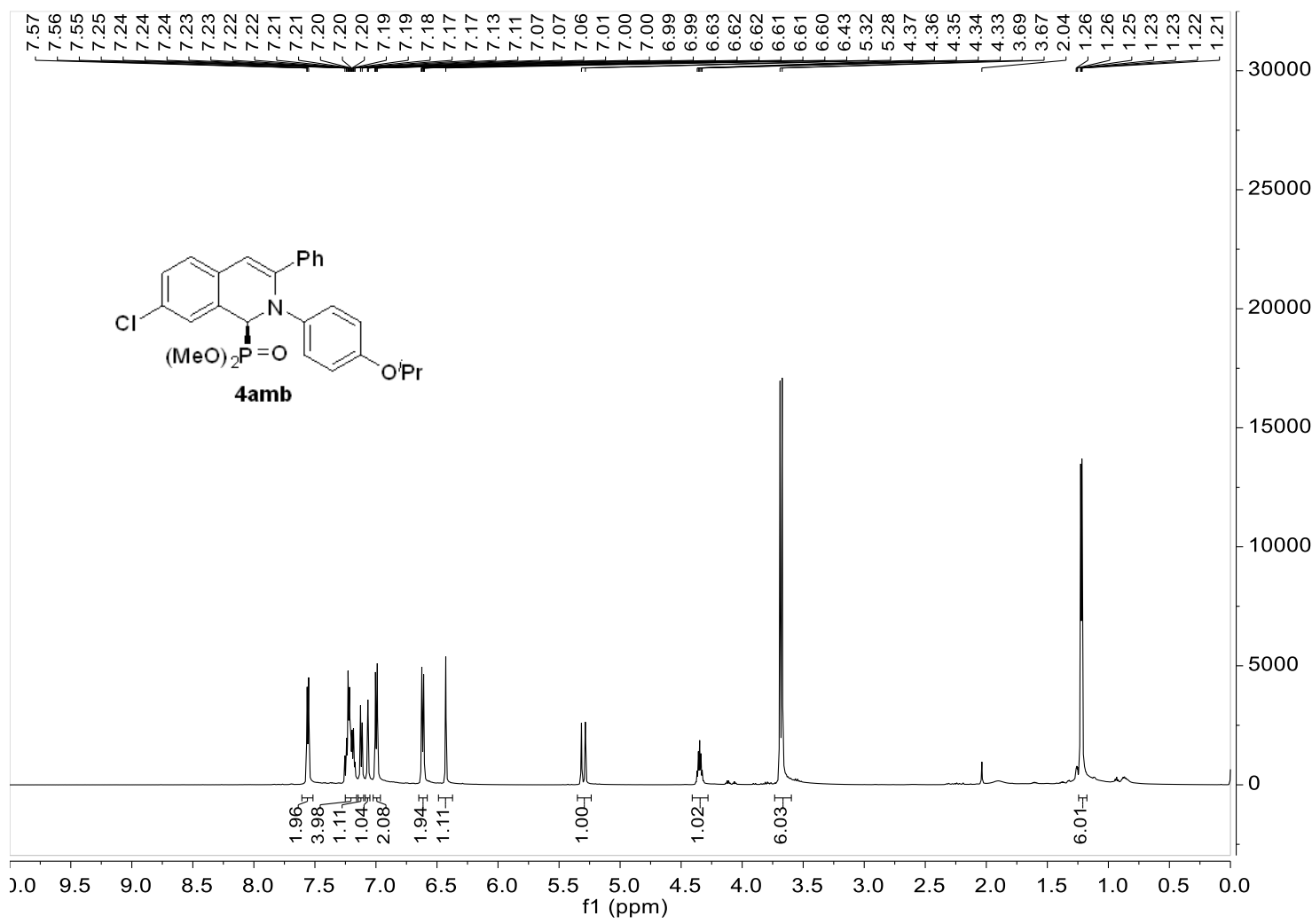

$151 \mathrm{MHz}, \mathrm{CDCl}_{3},{ }^{13} \mathrm{C} \mathrm{NMR}$

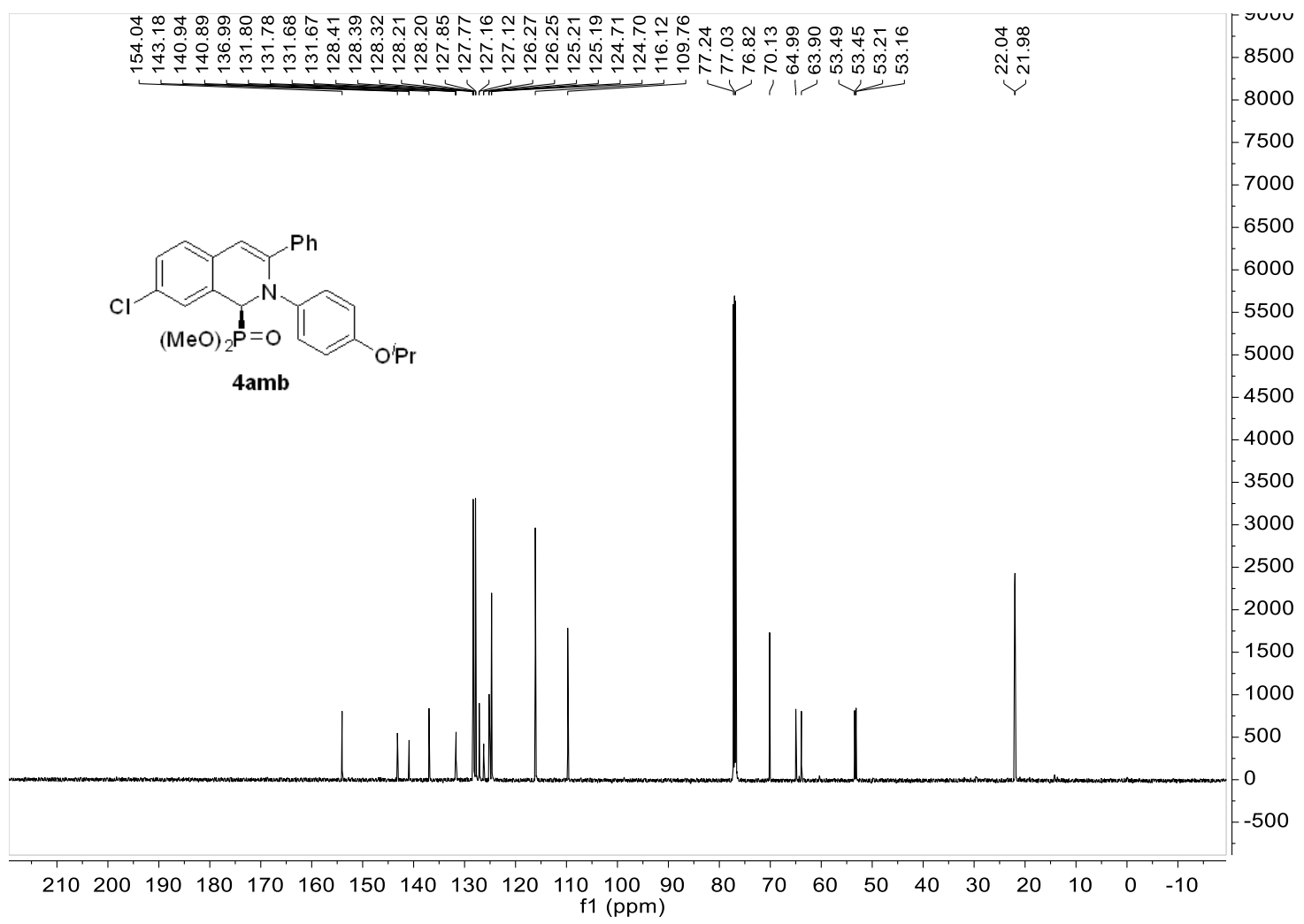


$600 \mathrm{MHz}, \mathrm{CDCl}_{3},{ }^{1} \mathrm{H} \mathrm{NMR}$

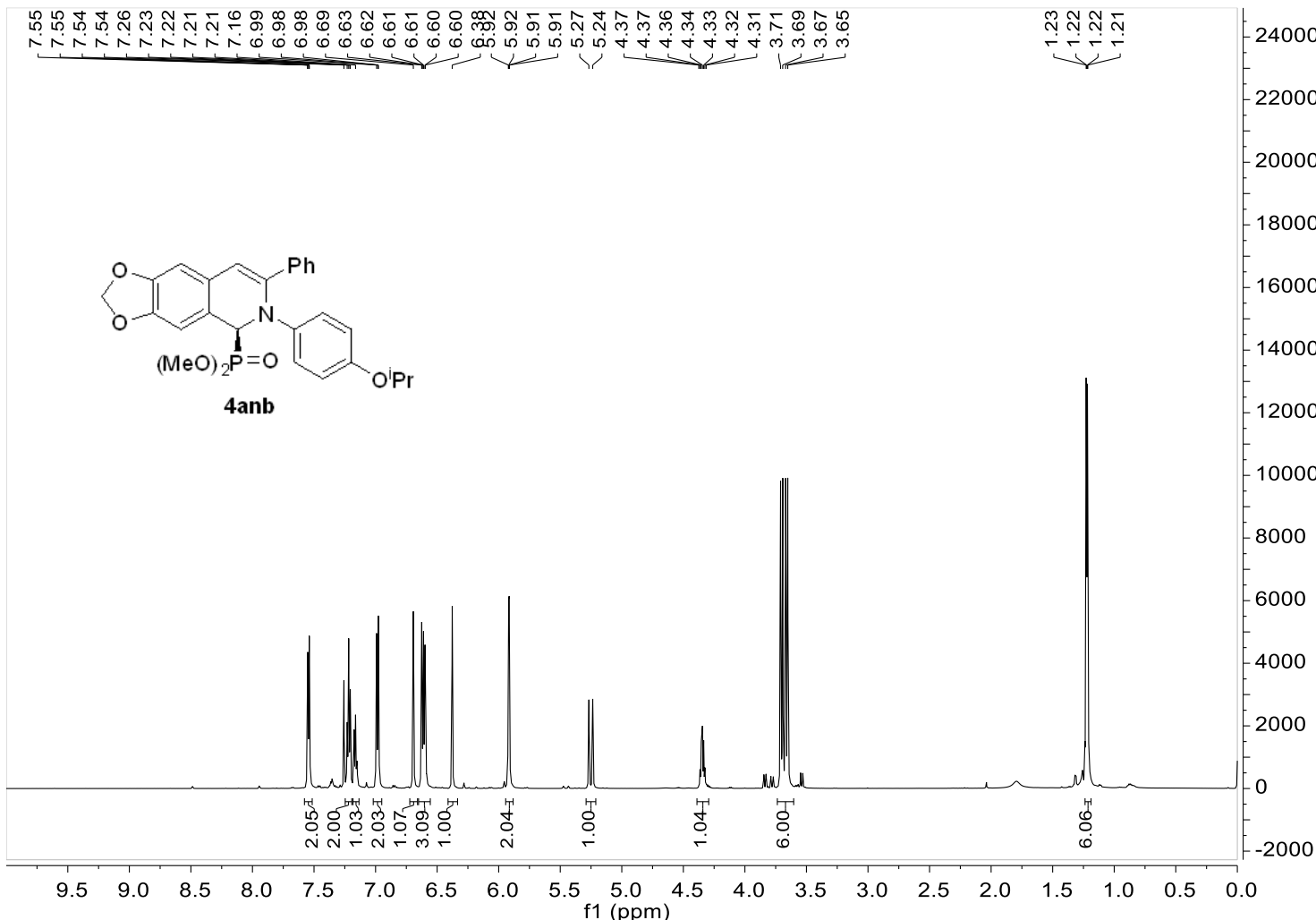

$151 \mathrm{MHz}, \mathrm{CDCl}_{3},{ }^{13} \mathrm{C} \mathrm{NMR}$

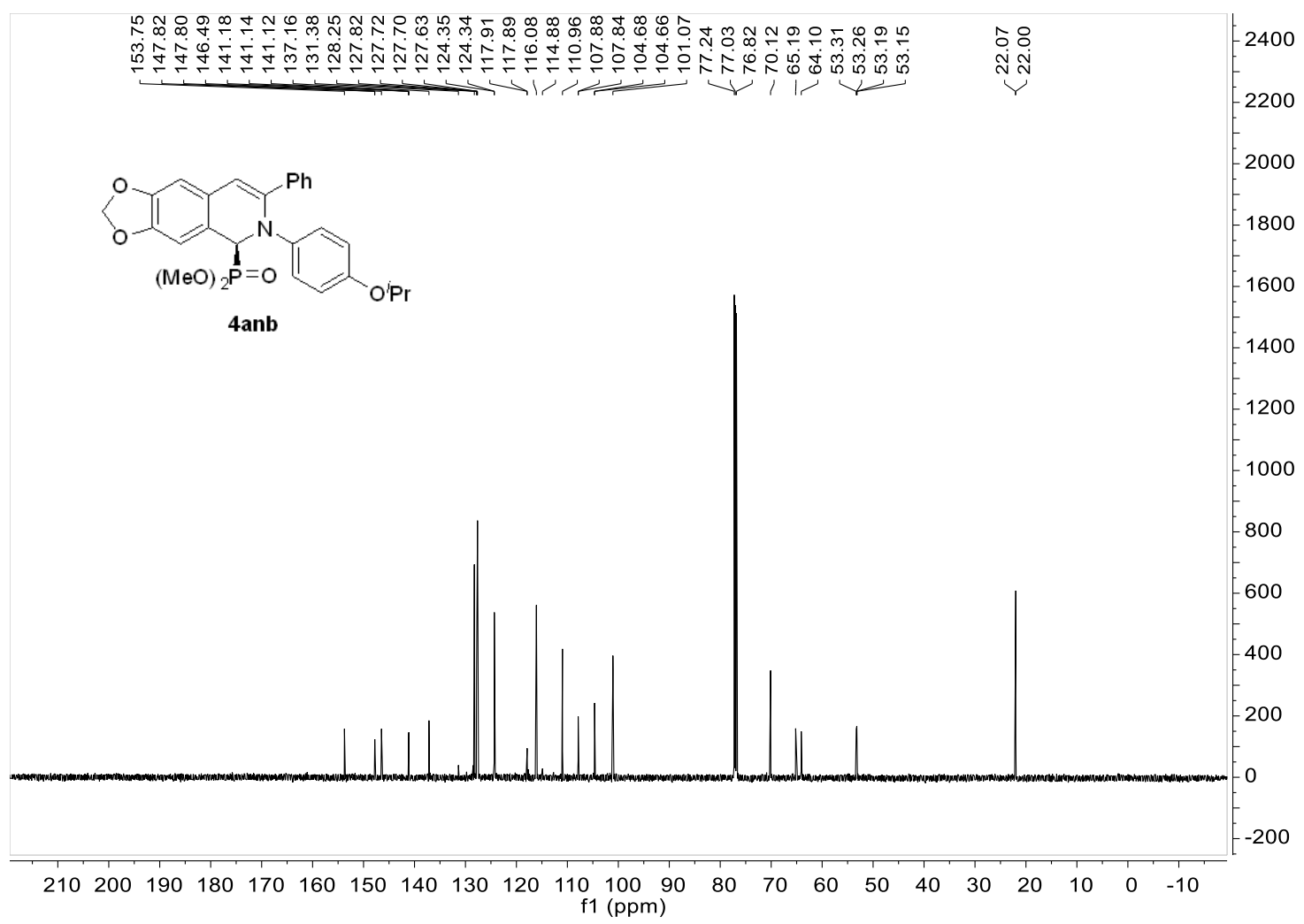


$600 \mathrm{MHz}, \mathrm{CDCl}_{3},{ }^{1} \mathrm{H} \mathrm{NMR}$

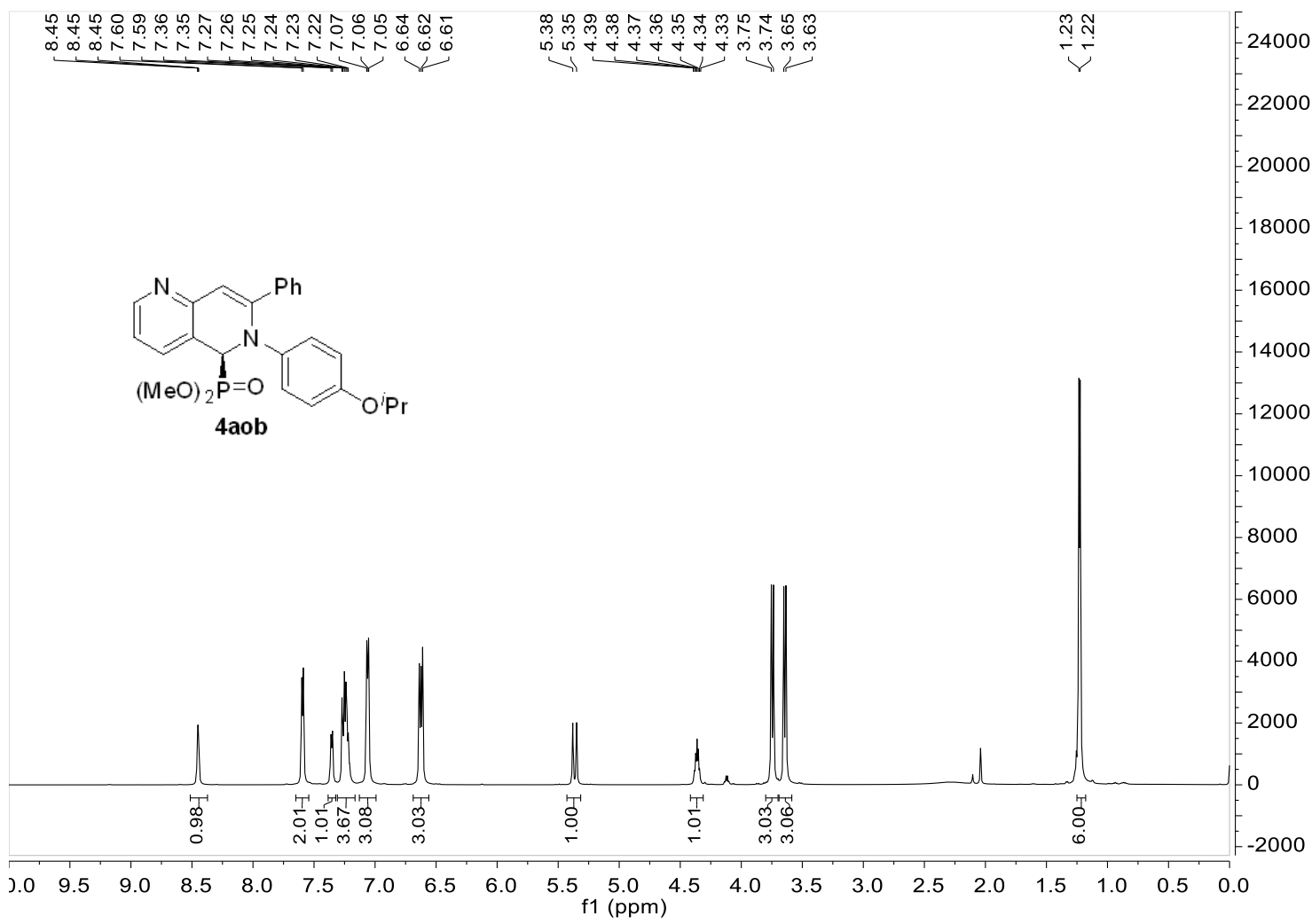

$151 \mathrm{MHz}, \mathrm{CDCl}_{3},{ }^{13} \mathrm{C} \mathrm{NMR}$

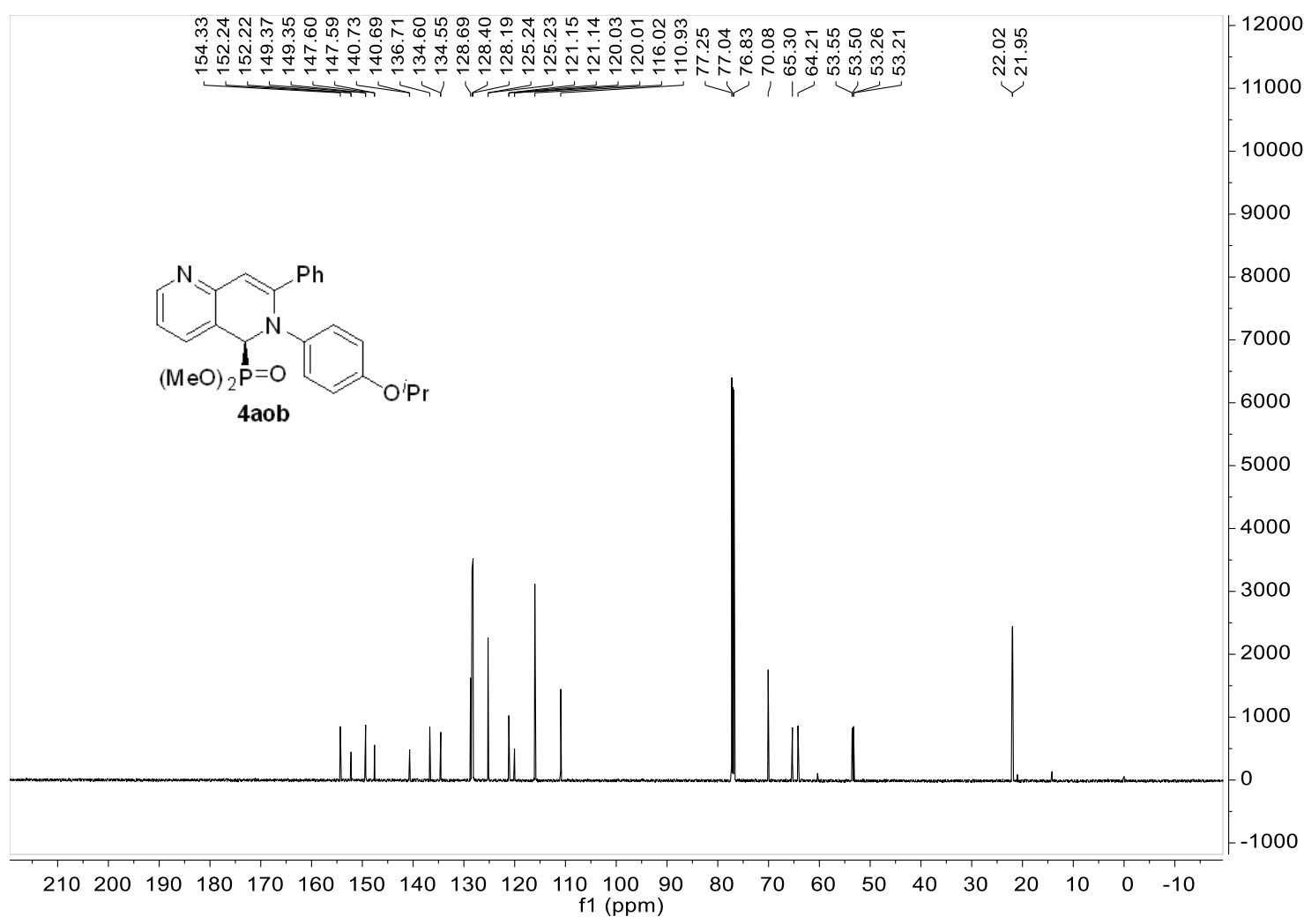


$600 \mathrm{MHz}, \mathrm{CDCl}_{3},{ }^{1} \mathrm{H} \mathrm{NMR}$

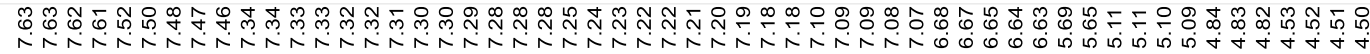

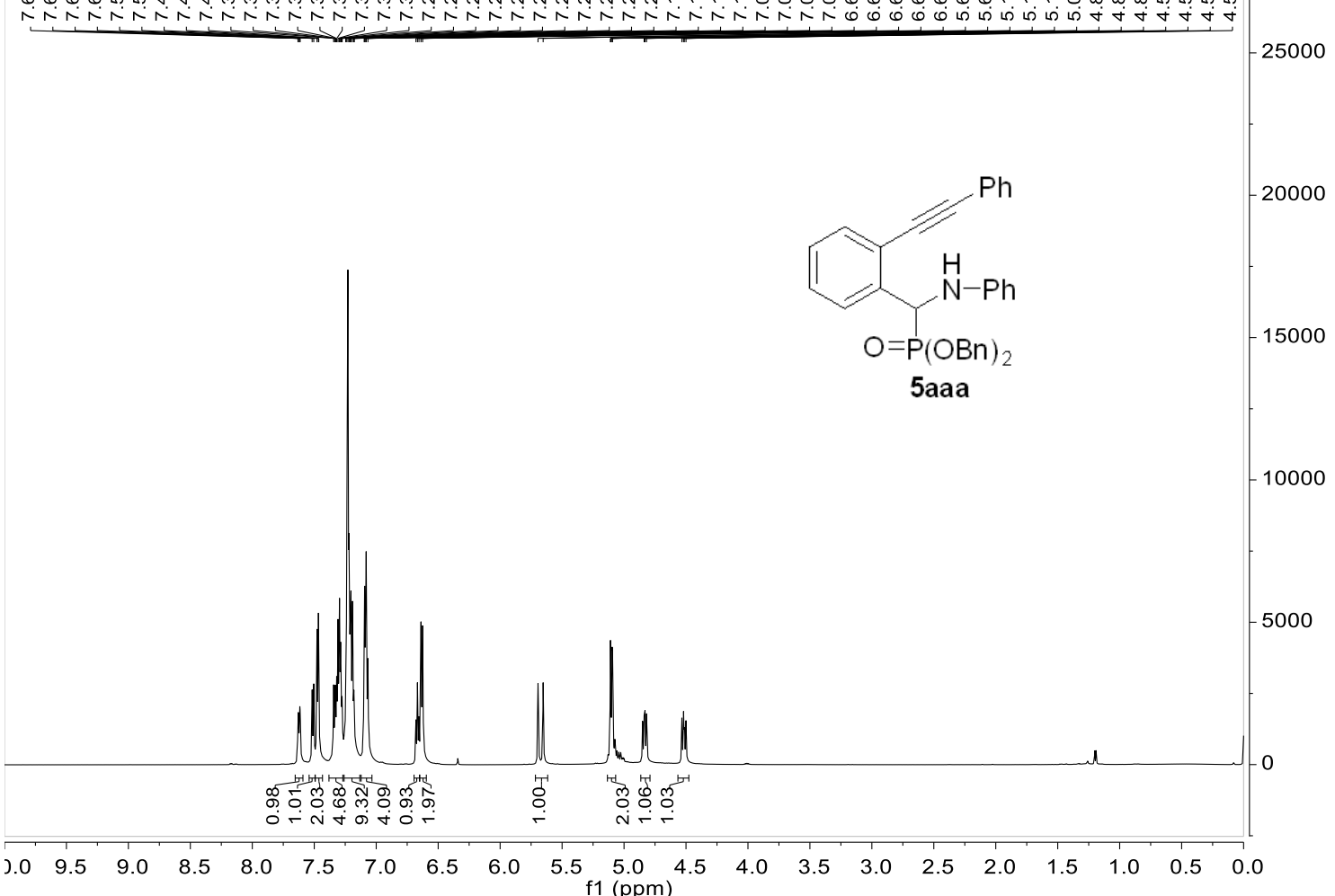

$151 \mathrm{MHz}, \mathrm{CDCl}_{3},{ }^{13} \mathrm{C} \mathrm{NMR}$

ธิ㇒木

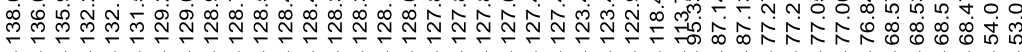

7000<smiles>O=P(O)(O)c1ccccc1NC(c1ccccc1C#Cc1ccccc1)P(=O)(O)c1ccccc1</smiles> 
$600 \mathrm{MHz}, \mathrm{CDCl}_{3},{ }^{1} \mathrm{H} \mathrm{NMR}$

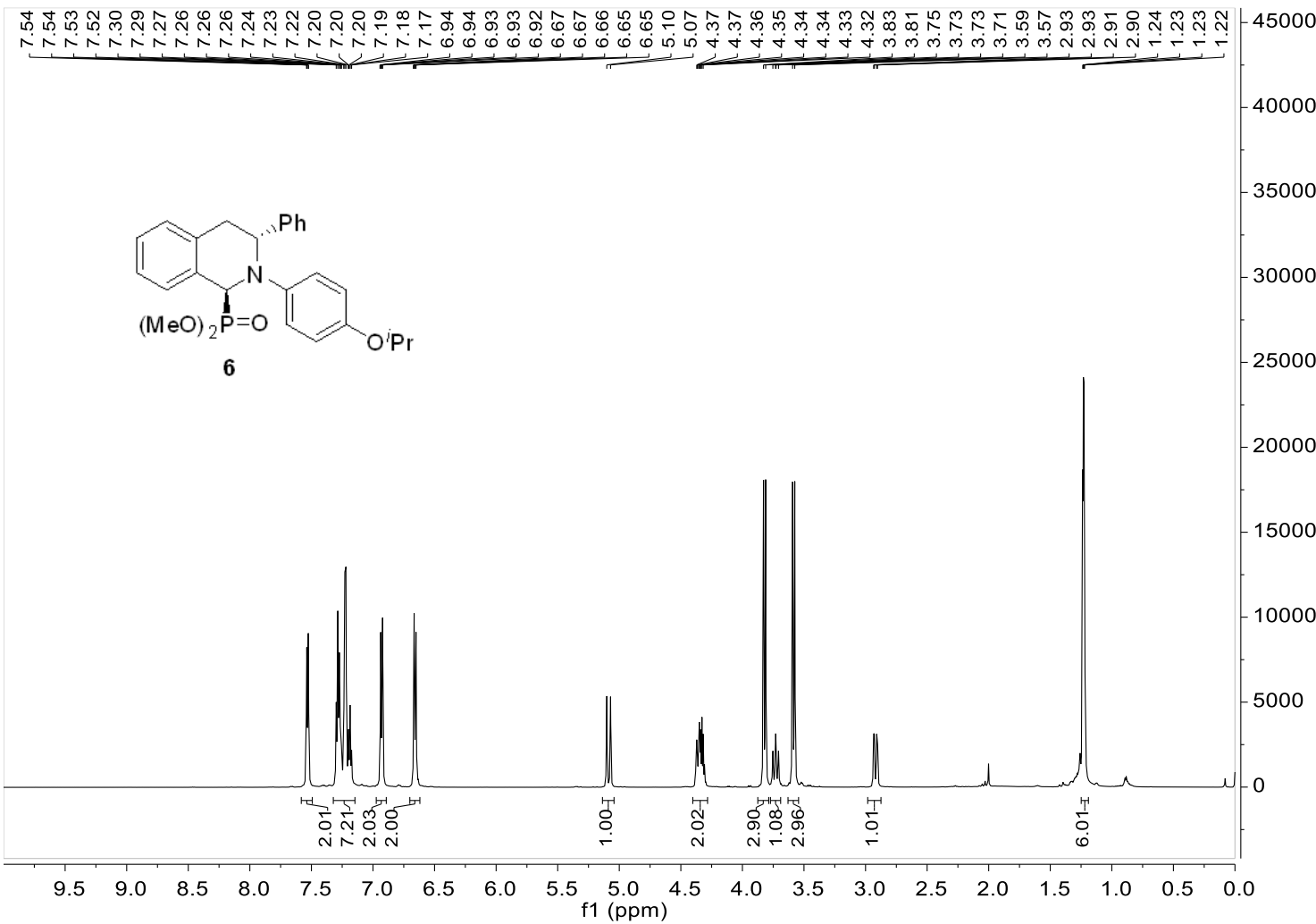

$151 \mathrm{MHz}, \mathrm{CDCl}_{3},{ }^{13} \mathrm{C} \mathrm{NMR}$

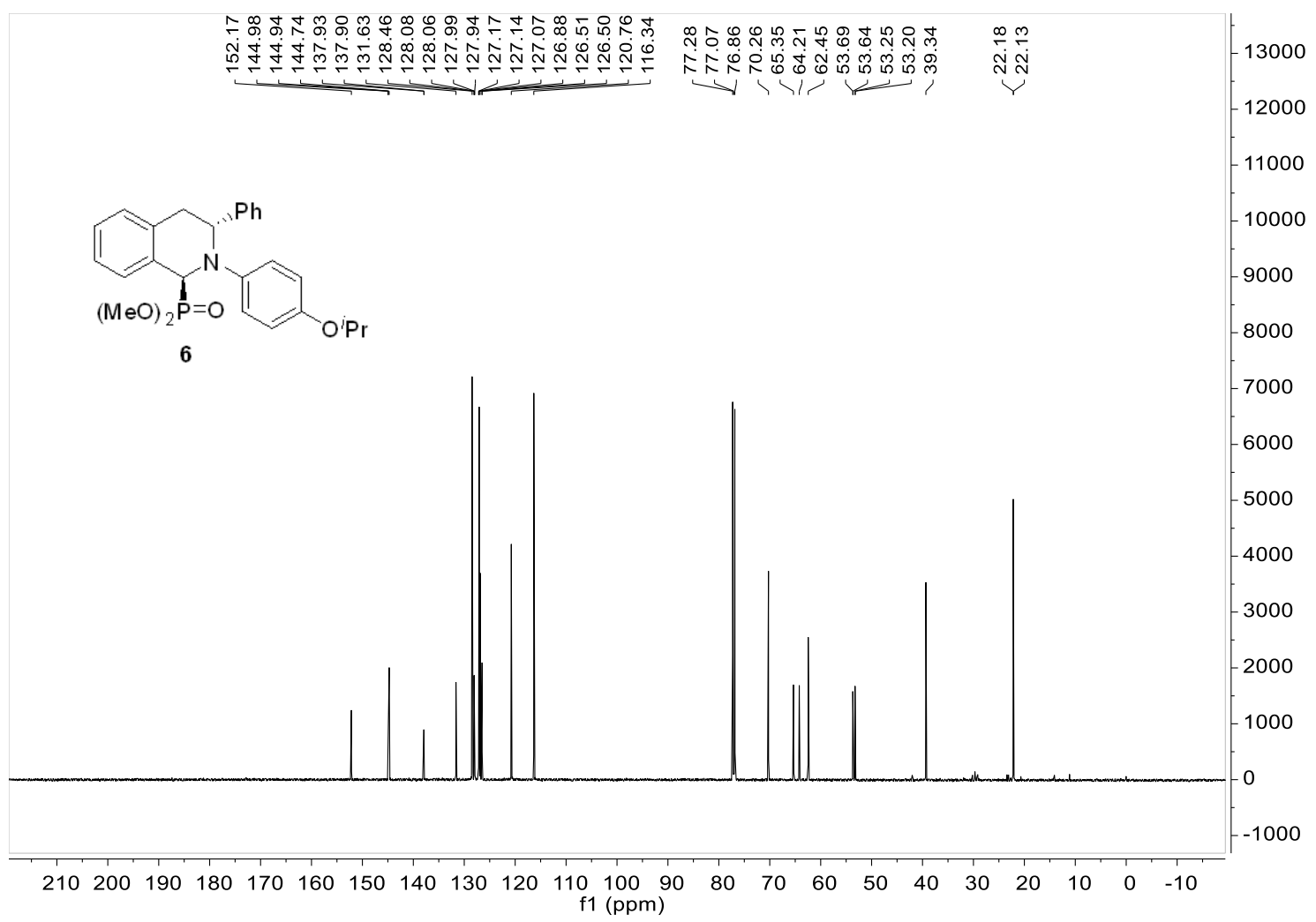



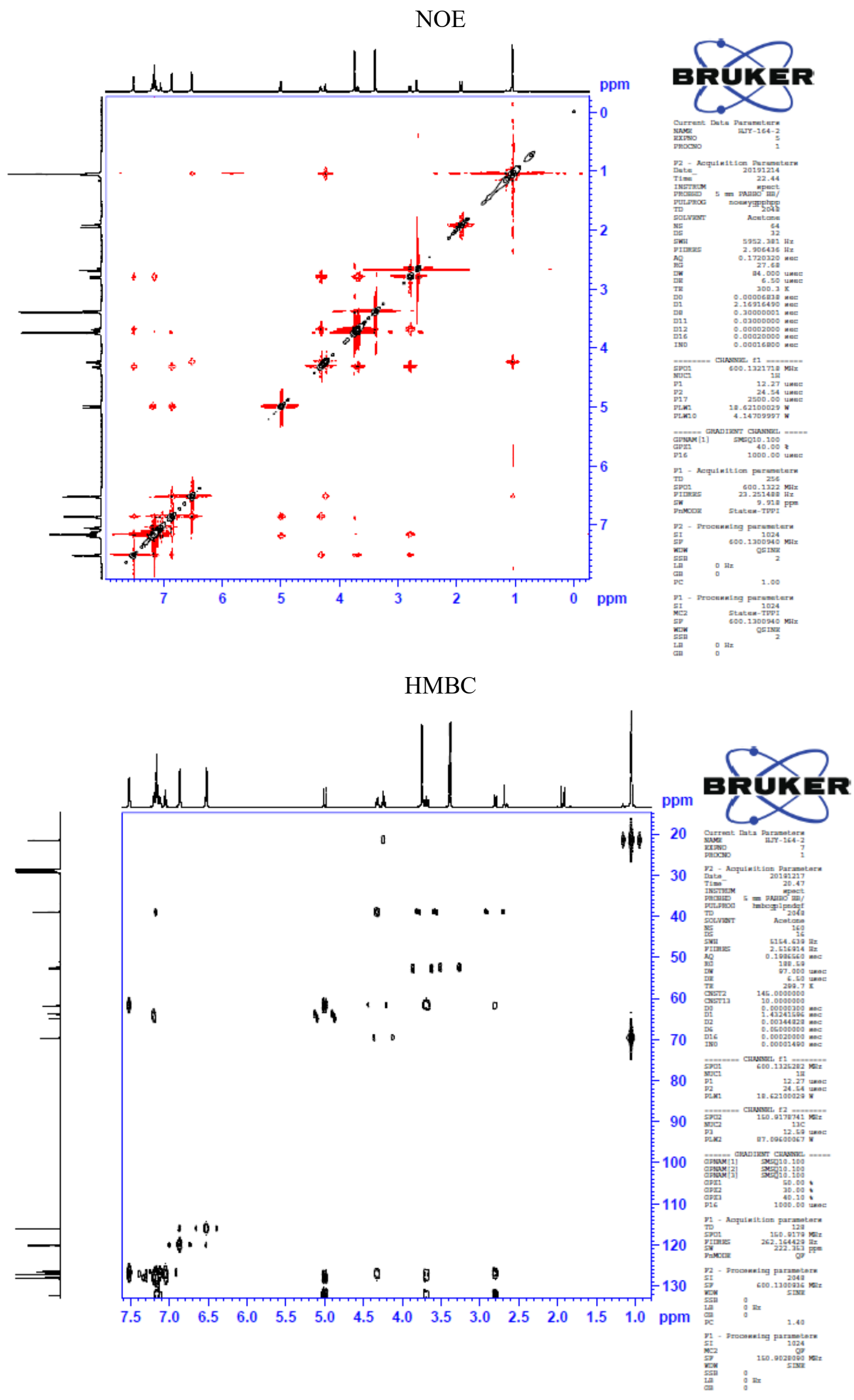


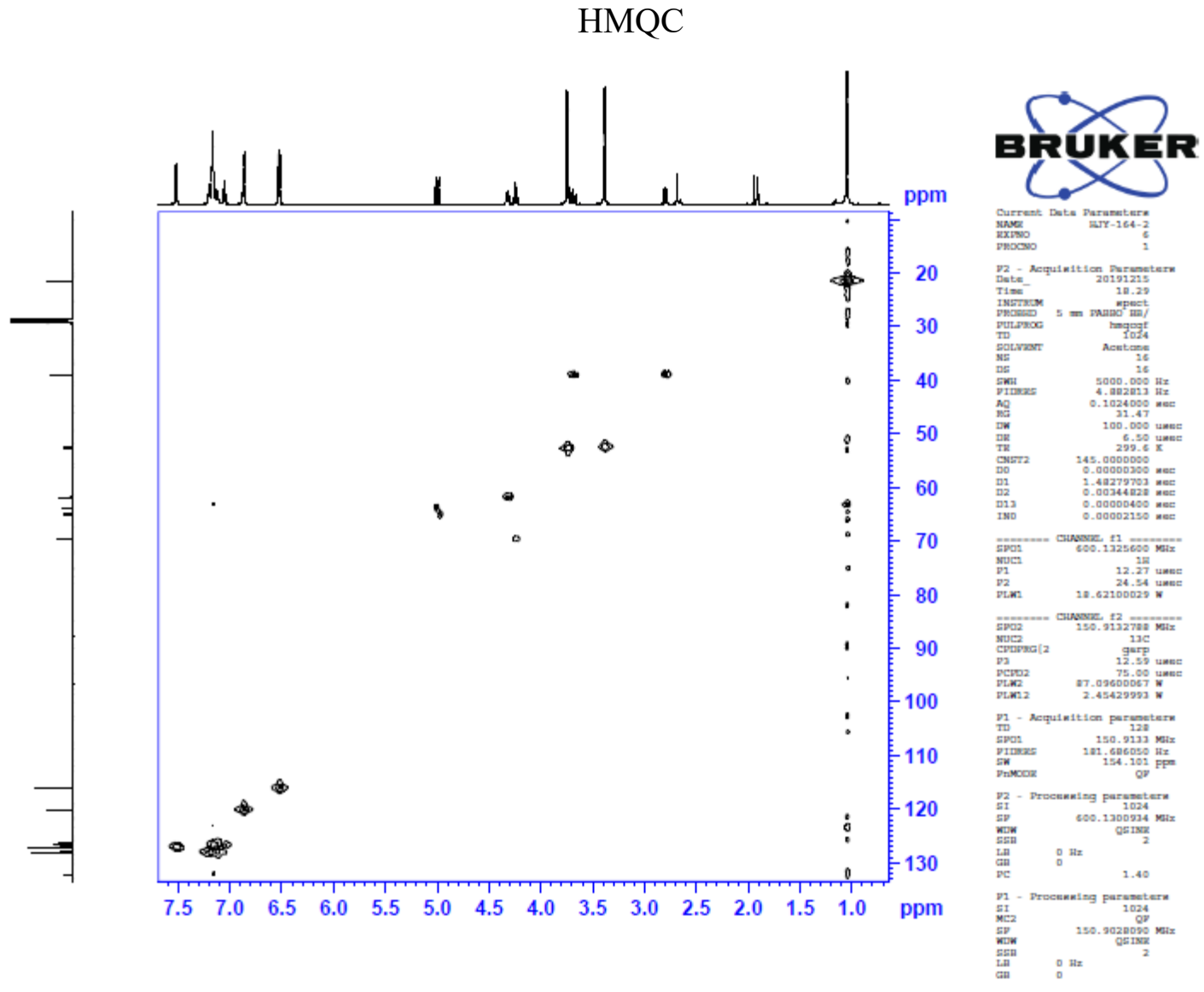

95 
$600 \mathrm{MHz}, \mathrm{CDCl}_{3},{ }^{1} \mathrm{H} \mathrm{NMR}$

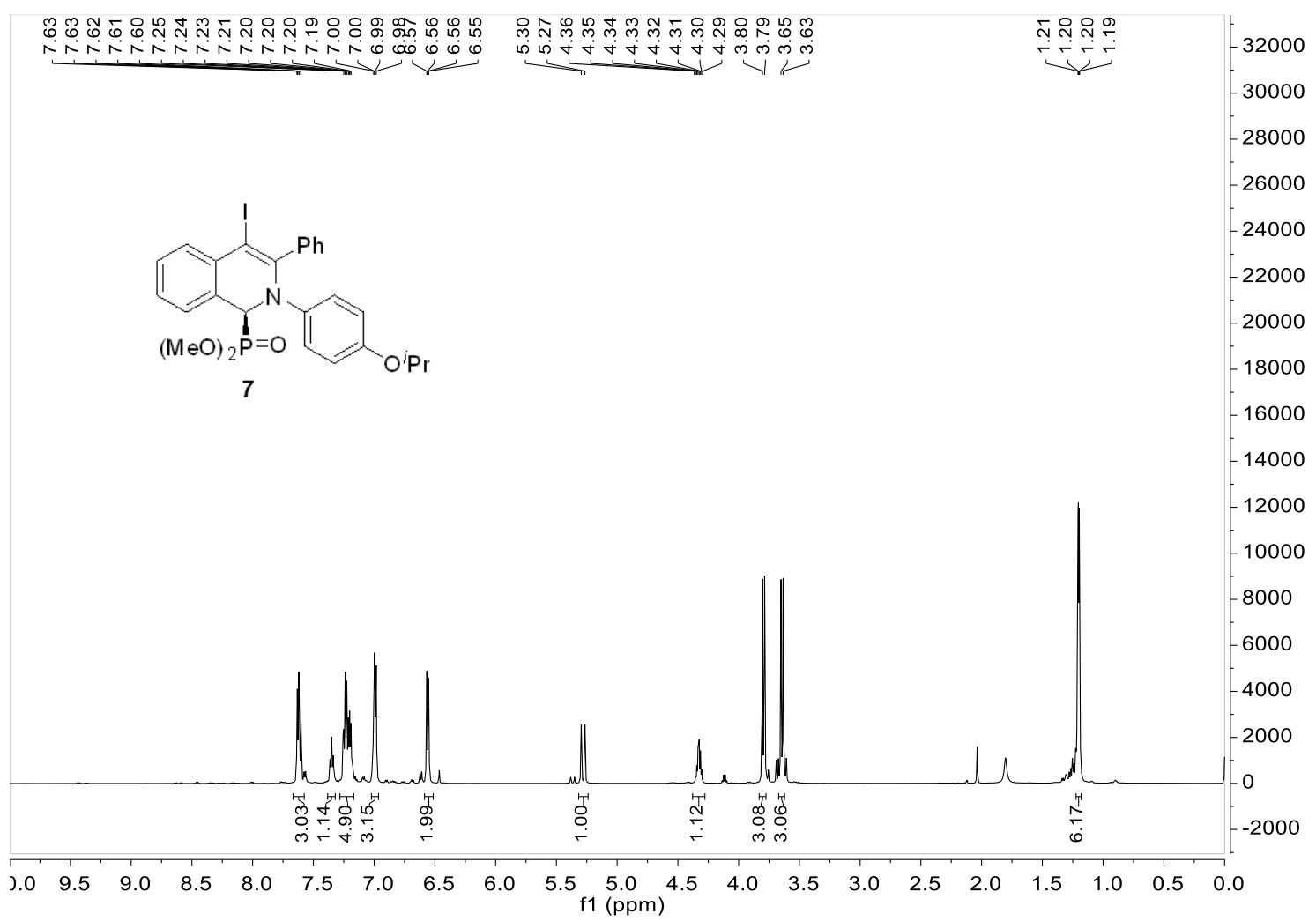

$151 \mathrm{MHz}, \mathrm{CDCl}_{3},{ }^{13} \mathrm{C} \mathrm{NMR}$

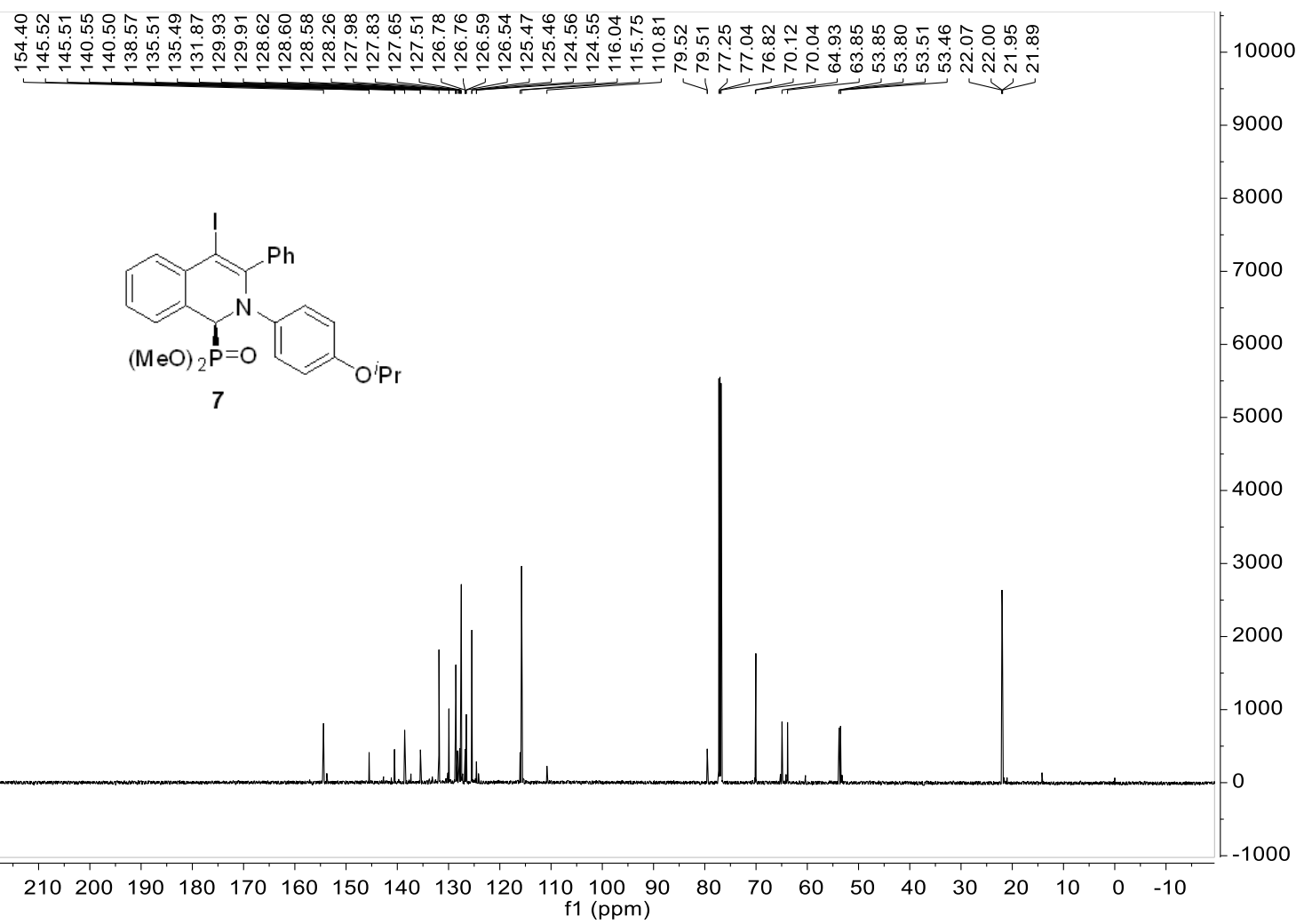


$600 \mathrm{MHz}, \mathrm{CDCl}_{3},{ }^{1} \mathrm{H} \mathrm{NMR}$

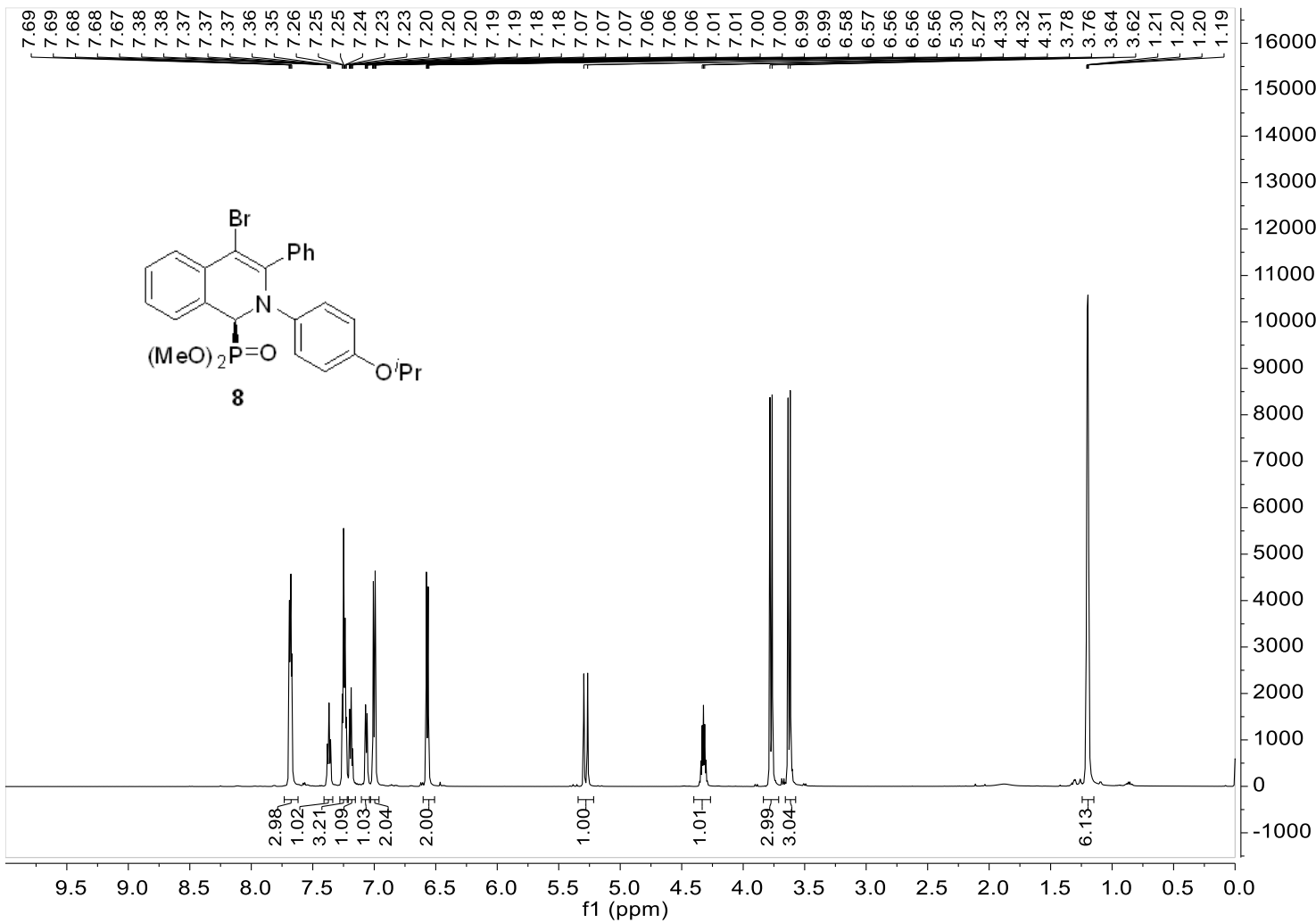

$151 \mathrm{MHz}, \mathrm{CDCl}_{3},{ }^{13} \mathrm{C} \mathrm{NMR}$

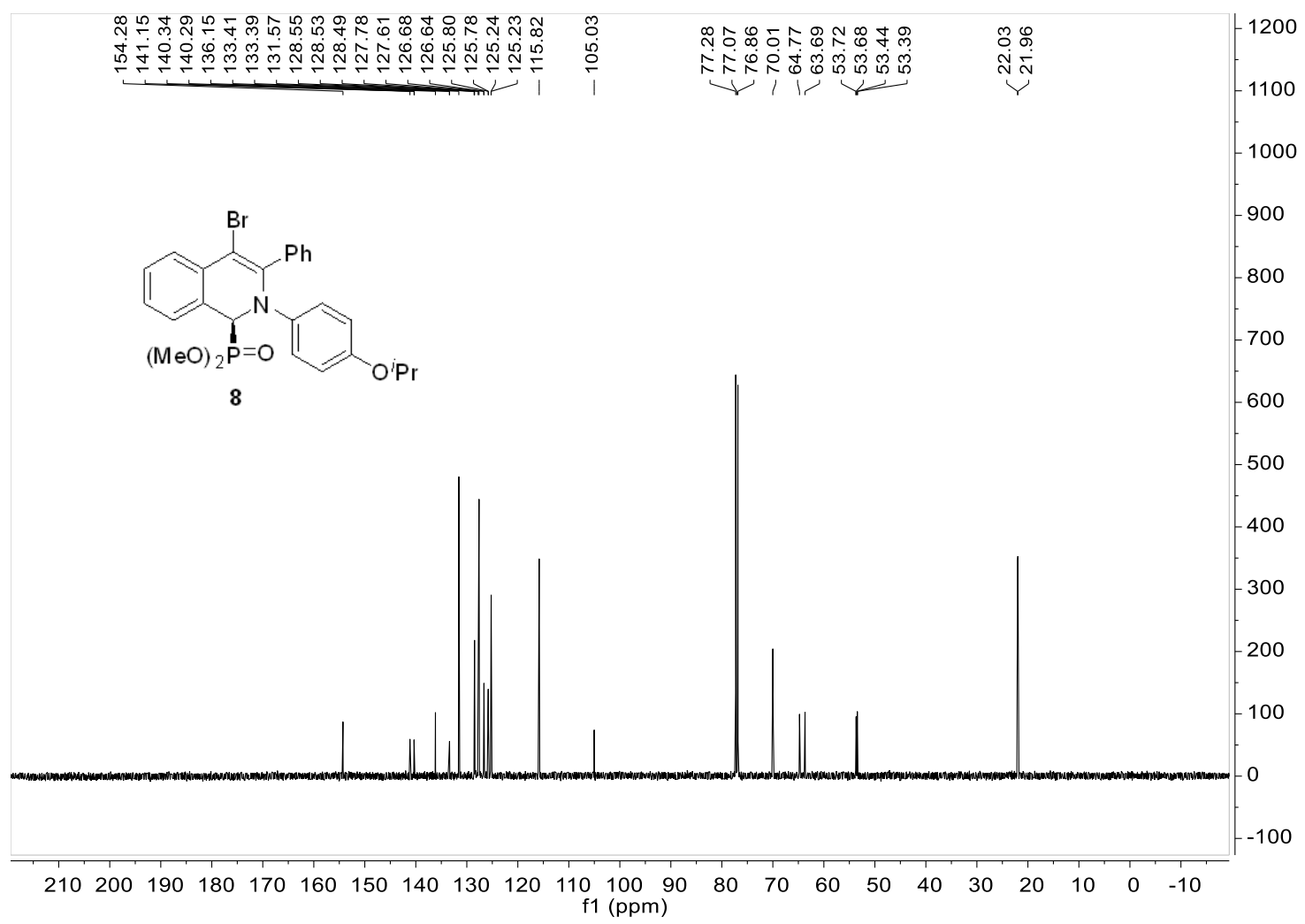




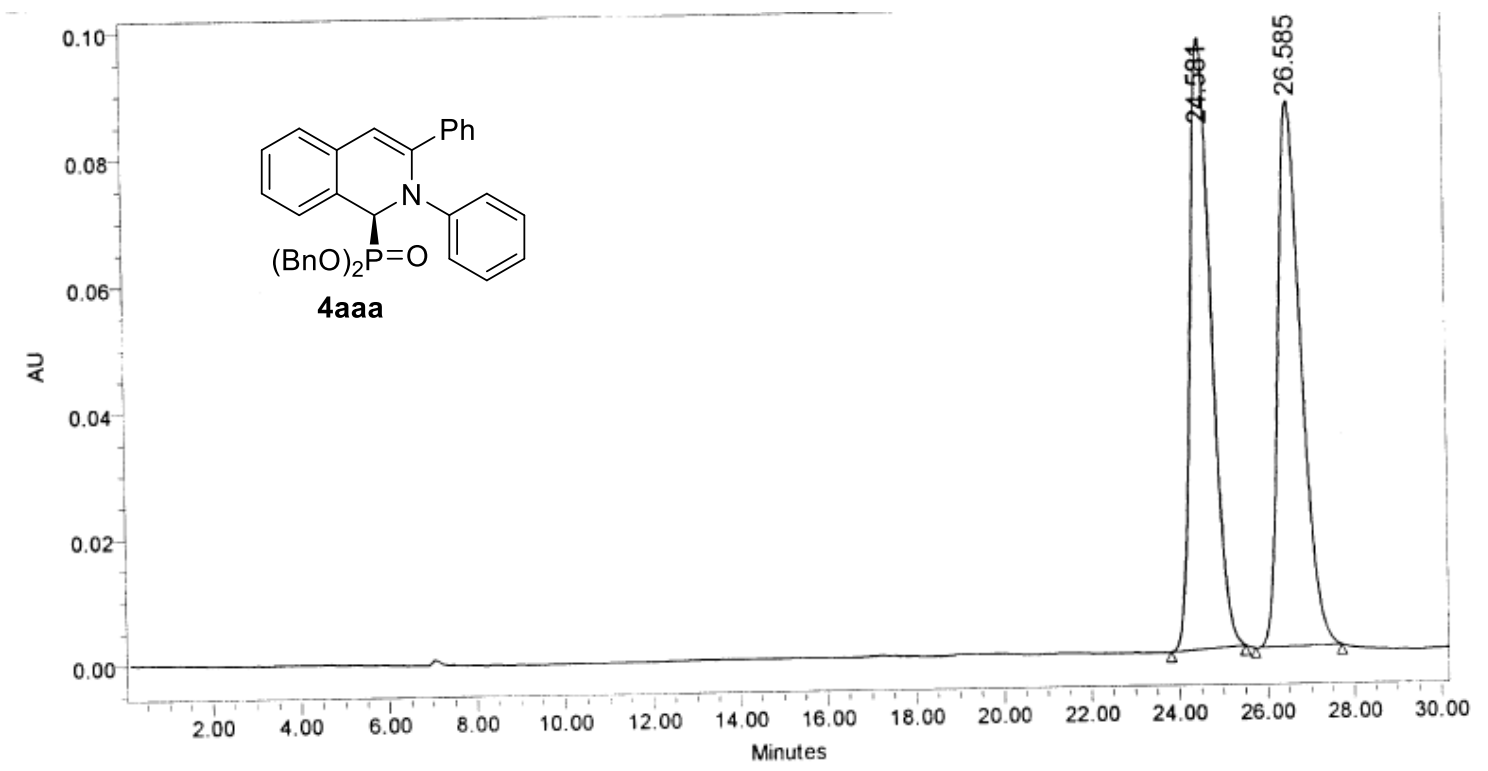

\begin{tabular}{|c|c|c|c|c|c|}
\hline & $\begin{array}{c}\mathrm{RT} \\
(\mathrm{min})\end{array}$ & $\begin{array}{c}\text { Area } \\
(\mu \mathrm{V} * \mathrm{sec})\end{array}$ & $\%$ Area & $\begin{array}{c}\text { Height } \\
(\mu \mathrm{V})\end{array}$ & $\begin{array}{c}\% \\
\text { Height }\end{array}$ \\
\hline 1 & 24.581 & 3208721 & 50.02 & 96770 & 52.83 \\
\hline 2 & 26.585 & 3206083 & 49.98 & 86410 & 47.17 \\
\hline
\end{tabular}

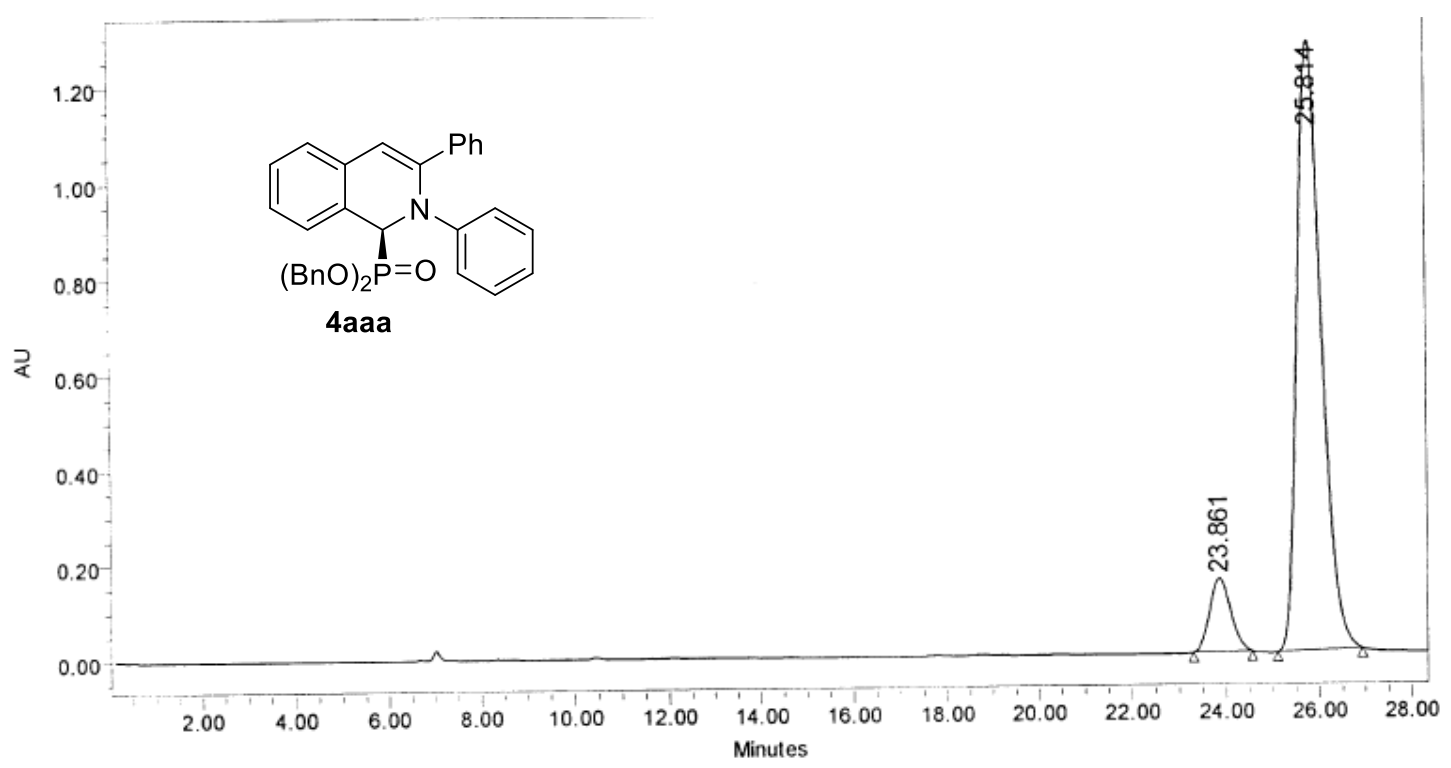

\begin{tabular}{|c|c|c|r|r|c|}
\hline & $\begin{array}{c}\mathrm{RT} \\
(\mathrm{min})\end{array}$ & $\begin{array}{c}\text { Area } \\
\left(\mu \mathrm{V}^{*} \mathrm{sec}\right)\end{array}$ & $\%$ Area & $\begin{array}{c}\text { Height } \\
(\mu \mathrm{V})\end{array}$ & $\begin{array}{c}\% \\
\text { Height }\end{array}$ \\
\hline 1 & 23.861 & 4815982 & 9.42 & 154994 & 10.83 \\
\hline 2 & 25.814 & 46335276 & 90.58 & 1276763 & 89.17 \\
\hline
\end{tabular}




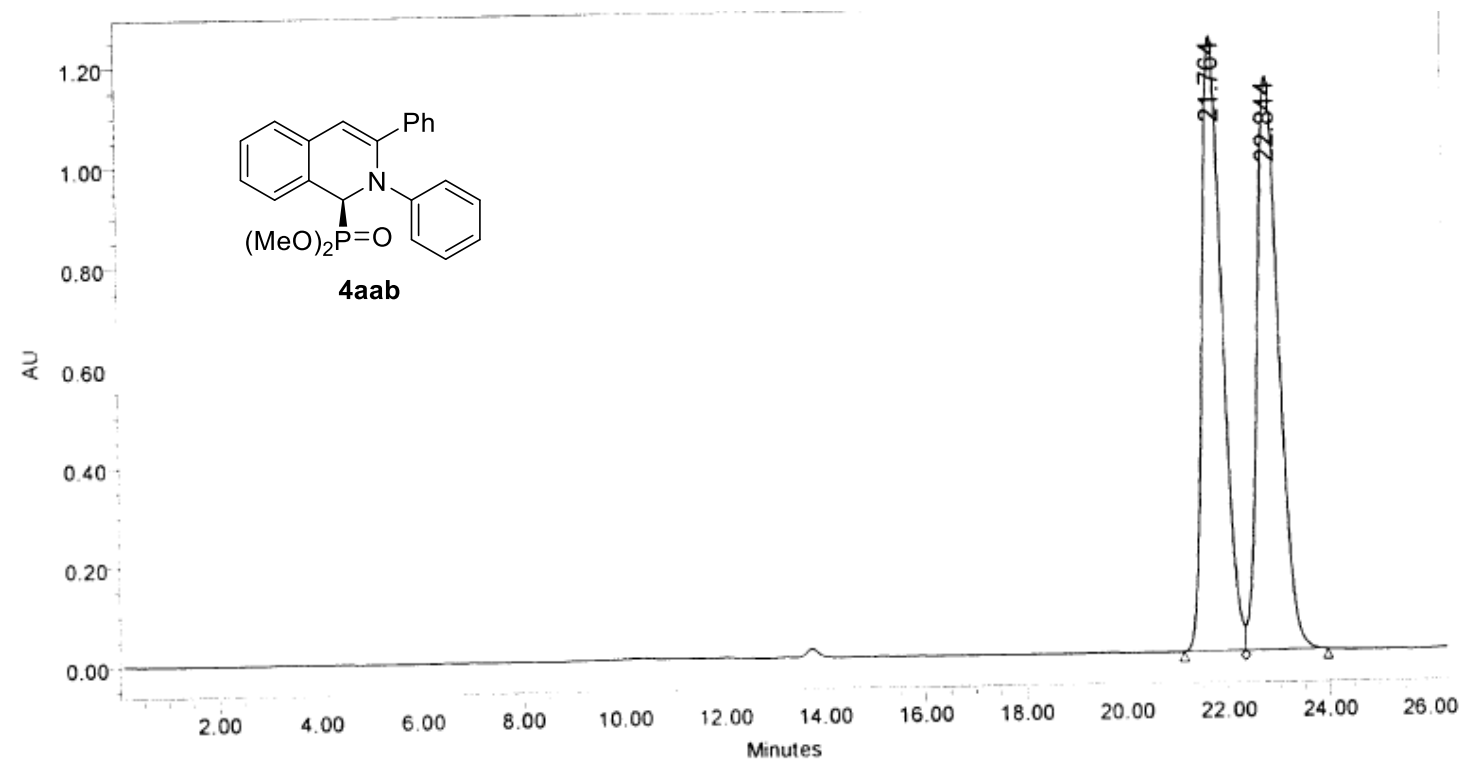

\begin{tabular}{|c|c|c|c|c|c|}
\hline & $\begin{array}{c}\mathrm{RT} \\
(\mathrm{min})\end{array}$ & $\begin{array}{c}\text { Area } \\
\left(\mu \mathrm{V}^{*} \mathrm{sec}\right)\end{array}$ & $\%$ Area & $\begin{array}{c}\text { Height } \\
(\mu \mathrm{V})\end{array}$ & $\begin{array}{c}\% \\
\text { Height }\end{array}$ \\
\hline 1 & 21.764 & 33419426 & 49.66 & 1232910 & 51.73 \\
\hline 2 & 22.844 & 33879803 & 50.34 & 1150232 & 48.27 \\
\hline
\end{tabular}

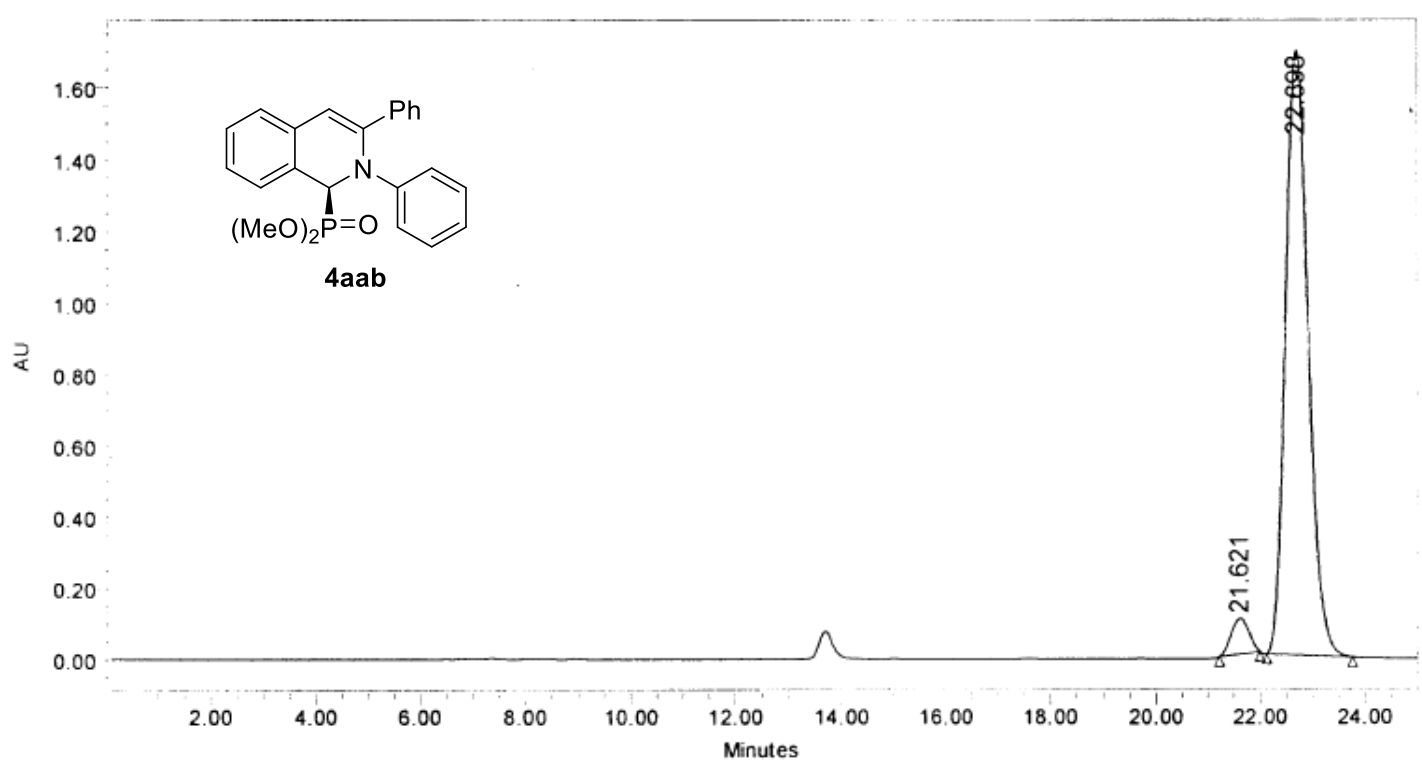

\begin{tabular}{|c|c|r|r|r|r|}
\hline & $\begin{array}{c}\mathrm{RT} \\
(\mathrm{min})\end{array}$ & $\begin{array}{c}\text { Area } \\
(\mu \mathrm{V} * \mathrm{sec})\end{array}$ & $\%$ Area & $\begin{array}{c}\text { Height } \\
(\mu \mathrm{V})\end{array}$ & $\begin{array}{c}\% \\
\text { Height }\end{array}$ \\
\hline 1 & 21.621 & 2371670 & 4.47 & 99883 & 5.57 \\
\hline 2 & 22.698 & 50744875 & 95.53 & 1692614 & 94.43 \\
\hline
\end{tabular}




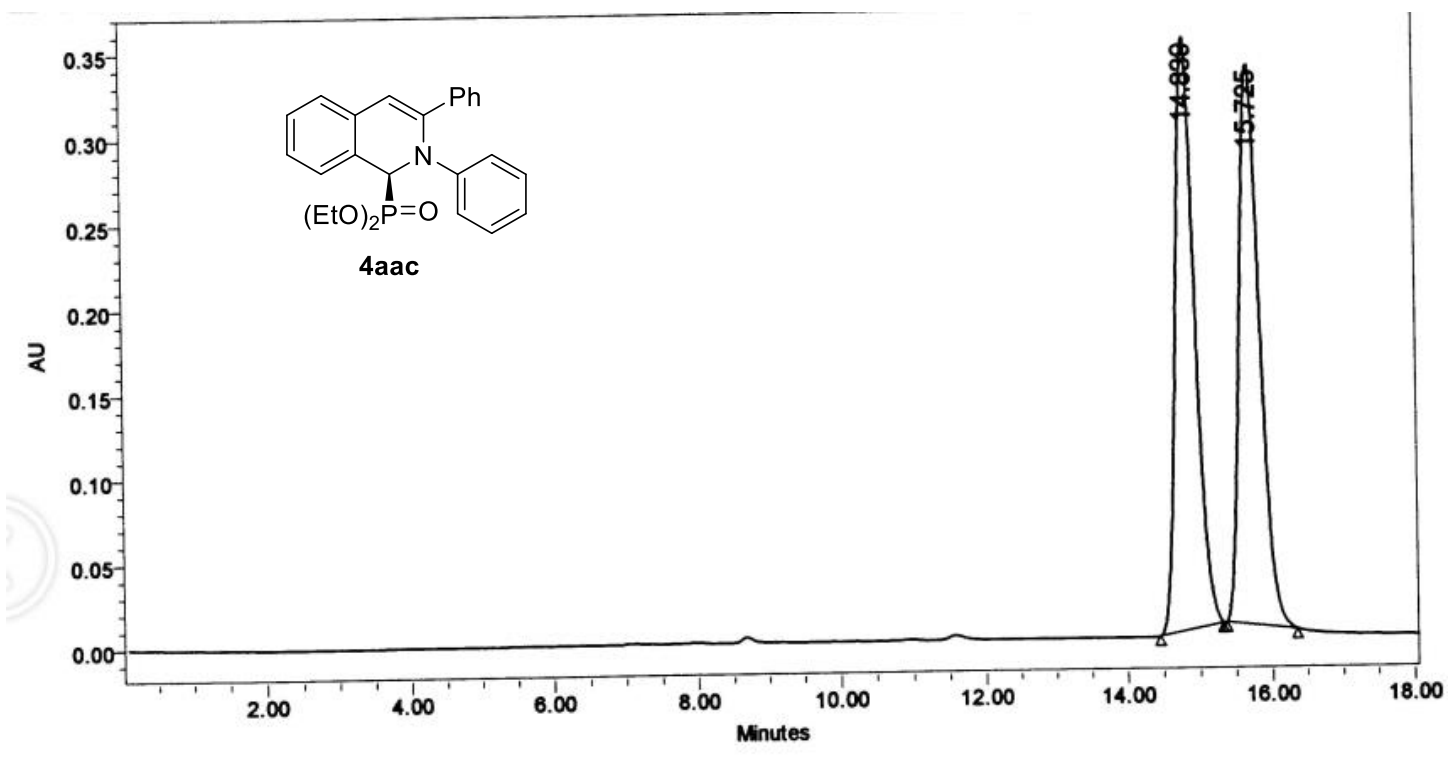

\begin{tabular}{|c|c|c|c|c|c|}
\hline & $\begin{array}{c}\text { RT } \\
(\mathrm{min})\end{array}$ & $\begin{array}{c}\text { Area } \\
(\mu \mathrm{N} * \mathrm{sec})\end{array}$ & $\%$ Area & $\begin{array}{c}\text { Height } \\
(\mu \mathrm{V})\end{array}$ & $\begin{array}{c}\% \\
\text { Height }\end{array}$ \\
\hline 1 & 14.839 & 6468646 & 49.89 & 349236 & 51.42 \\
\hline 2 & 15.725 & 6496661 & 50.11 & 329962 & 48.58 \\
\hline
\end{tabular}

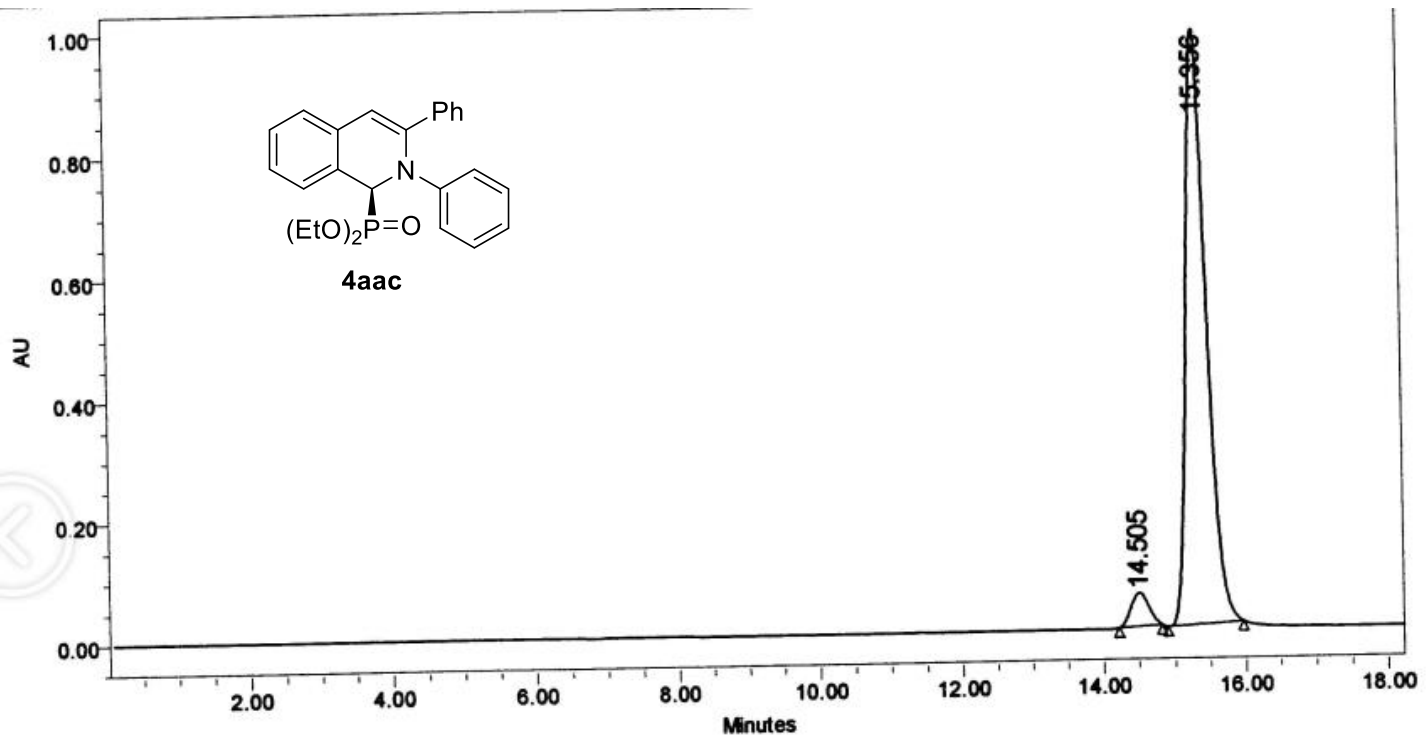

\begin{tabular}{|c|c|r|r|r|r|}
\hline & $\begin{array}{c}\text { RT } \\
(\mathrm{min})\end{array}$ & $\begin{array}{c}\text { Area } \\
(\mu \mathrm{N} * \mathrm{sec})\end{array}$ & \% Area & $\begin{array}{c}\text { Height } \\
(\mu \mathrm{V})\end{array}$ & $\begin{array}{c}\% \\
\text { Height }\end{array}$ \\
\hline 1 & 14.505 & 942762 & 4.61 & 55217 & 5.34 \\
\hline 2 & 15.356 & 19512197 & 95.39 & 978448 & 94.66 \\
\hline
\end{tabular}




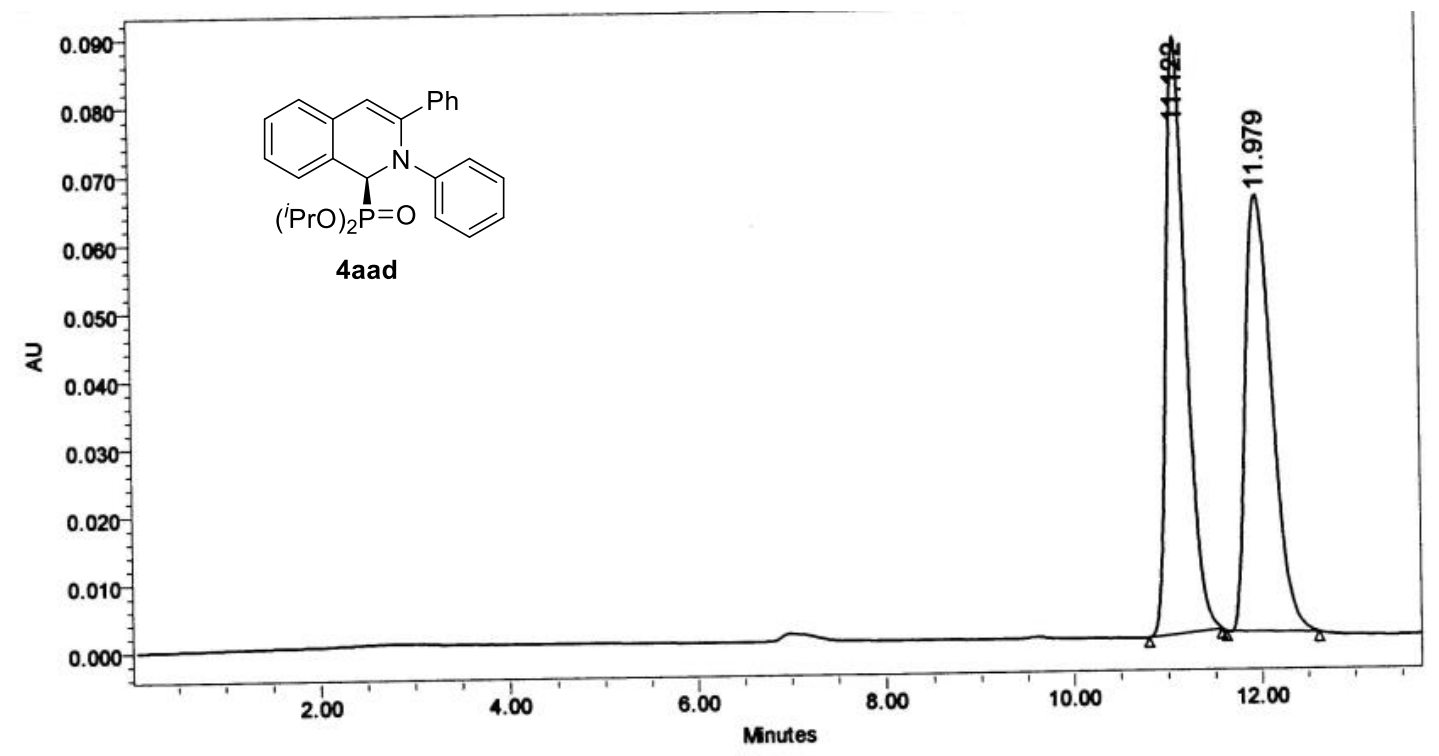

\begin{tabular}{|c|c|c|c|c|c|}
\hline & $\begin{array}{c}\text { RT } \\
(\mathrm{min})\end{array}$ & $\begin{array}{c}\text { Area } \\
(\mu \mathrm{N} * \mathrm{sec})\end{array}$ & \% Area & $\begin{array}{c}\text { Height } \\
(\mu \mathrm{N})\end{array}$ & $\begin{array}{c}\% \\
\text { Height }\end{array}$ \\
\hline 1 & 11.122 & 1276969 & 49.96 & 87887 & 57.74 \\
\hline 2 & 11.979 & 1279132 & 50.04 & 64322 & 42.26 \\
\hline
\end{tabular}

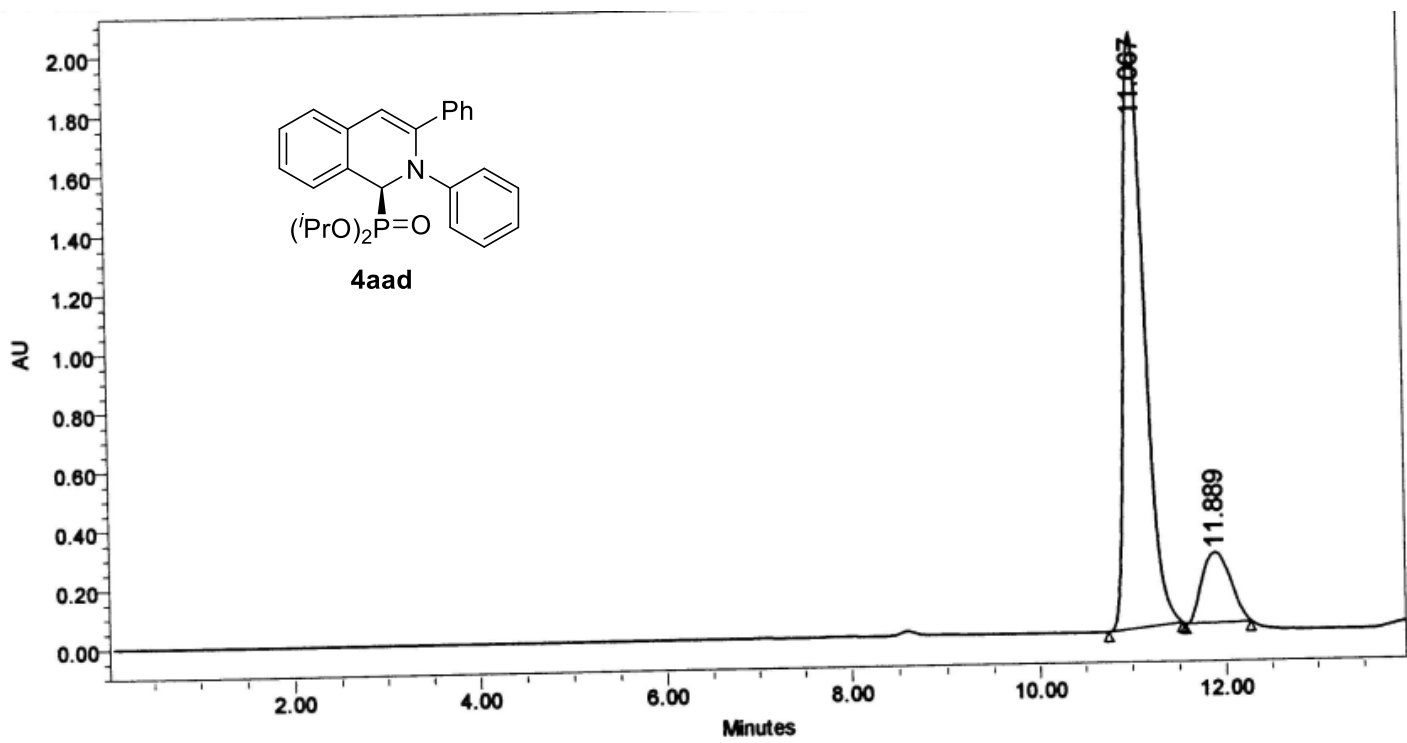

\begin{tabular}{|c|c|c|c|c|c|}
\hline & $\begin{array}{c}\text { RT } \\
(\min )\end{array}$ & $\begin{array}{c}\text { Area } \\
(\mu \mathrm{V} * \mathrm{sec})\end{array}$ & $\%$ Area & $\begin{array}{c}\text { Height } \\
(\mu \mathrm{V})\end{array}$ & $\begin{array}{c}\% \\
\text { Height }\end{array}$ \\
\hline 1 & 11.067 & 30570595 & 85.84 & 2014174 & 89.46 \\
\hline 2 & 11.889 & 5043721 & 14.16 & 237393 & 10.54 \\
\hline
\end{tabular}




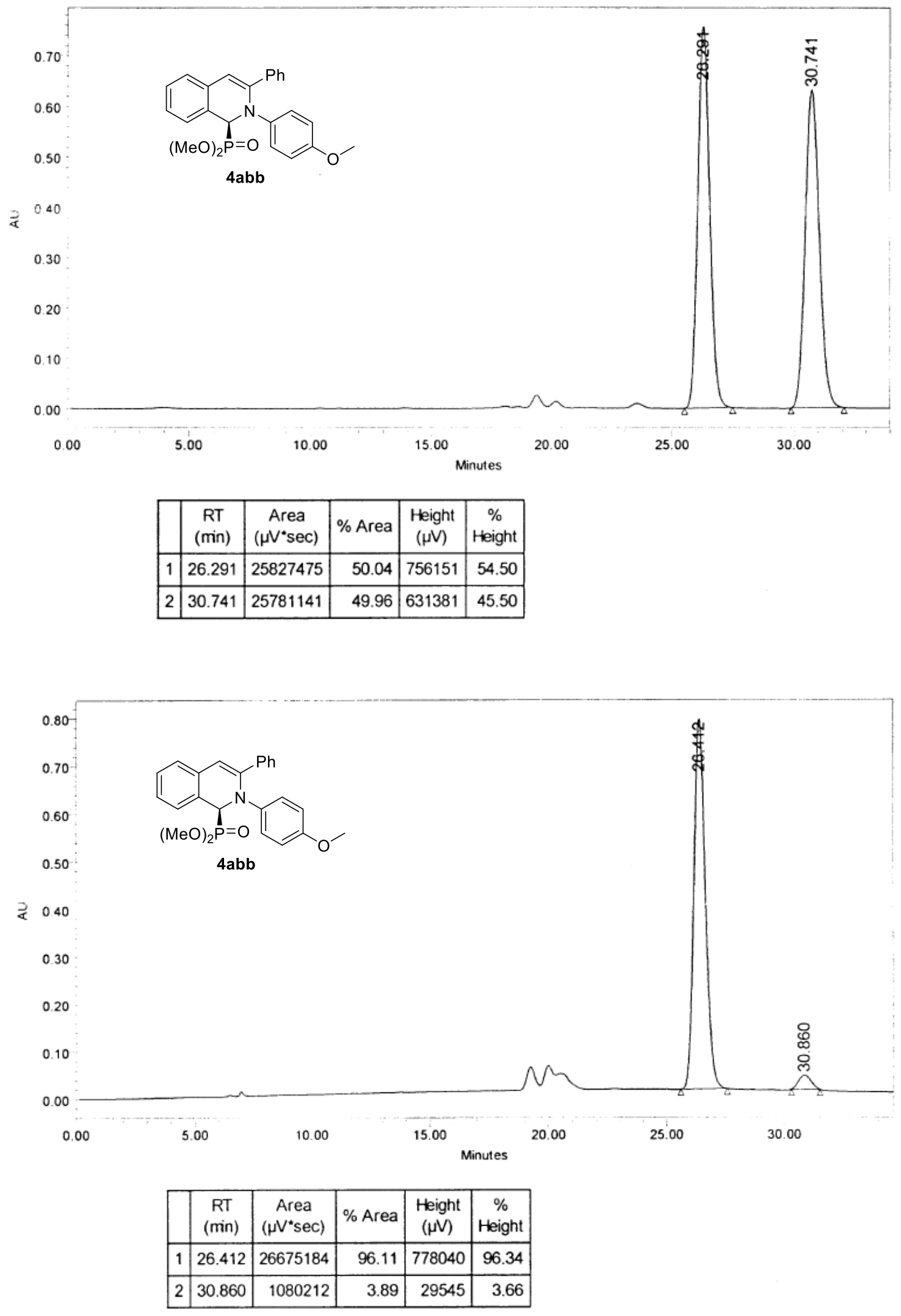




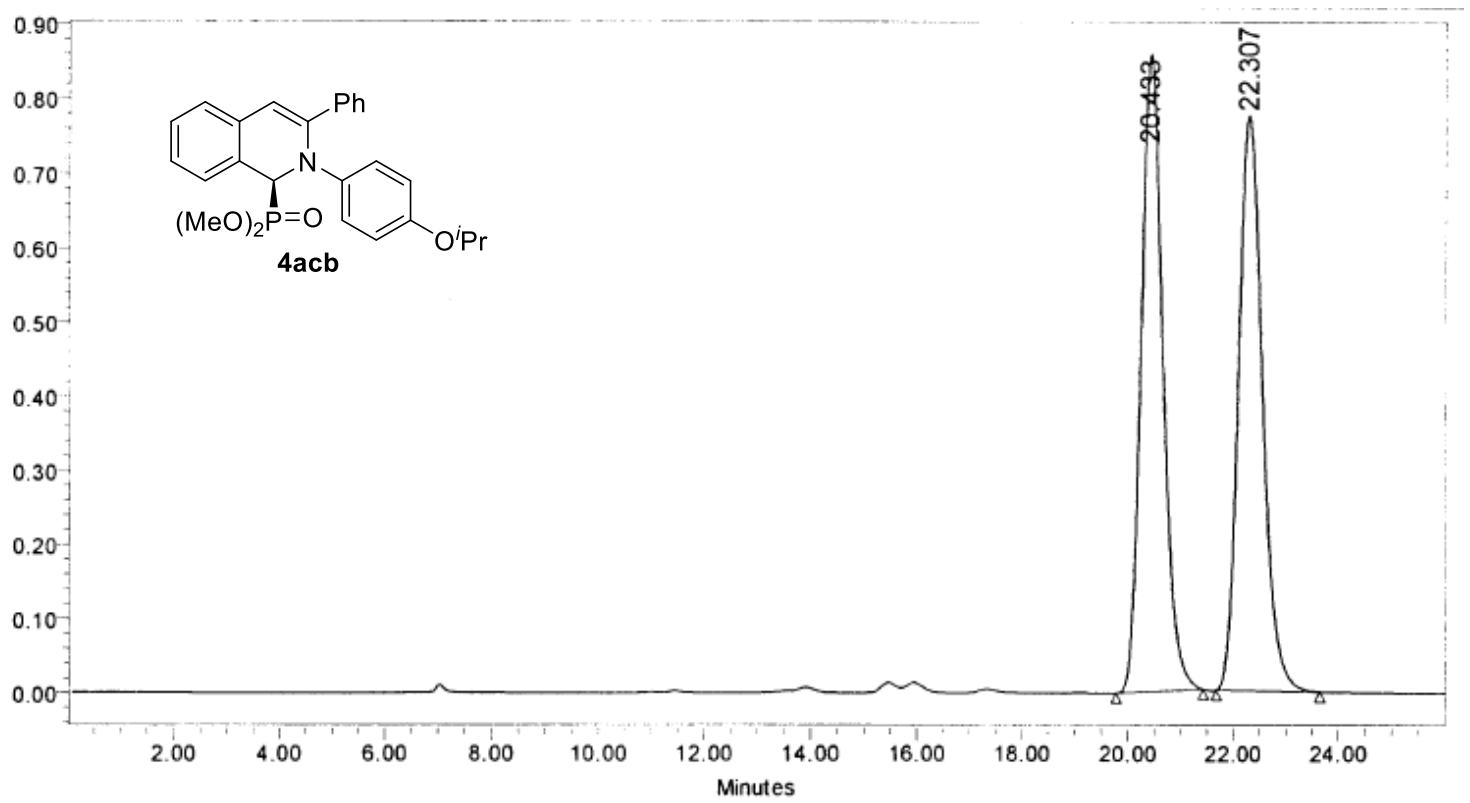

\begin{tabular}{|c|c|c|c|c|c|}
\hline & $\begin{array}{c}\text { RT } \\
(\mathrm{min})\end{array}$ & $\begin{array}{c}\text { Area } \\
(\mu \mathrm{V} * \mathrm{sec})\end{array}$ & $\%$ Area & $\begin{array}{c}\text { Height } \\
(\mu \mathrm{V})\end{array}$ & $\begin{array}{c}\% \\
\text { Height }\end{array}$ \\
\hline 1 & 20.433 & 24607610 & 50.00 & 858751 & 52.56 \\
\hline 2 & 22.307 & 24605688 & 50.00 & 775243 & 47.44 \\
\hline
\end{tabular}

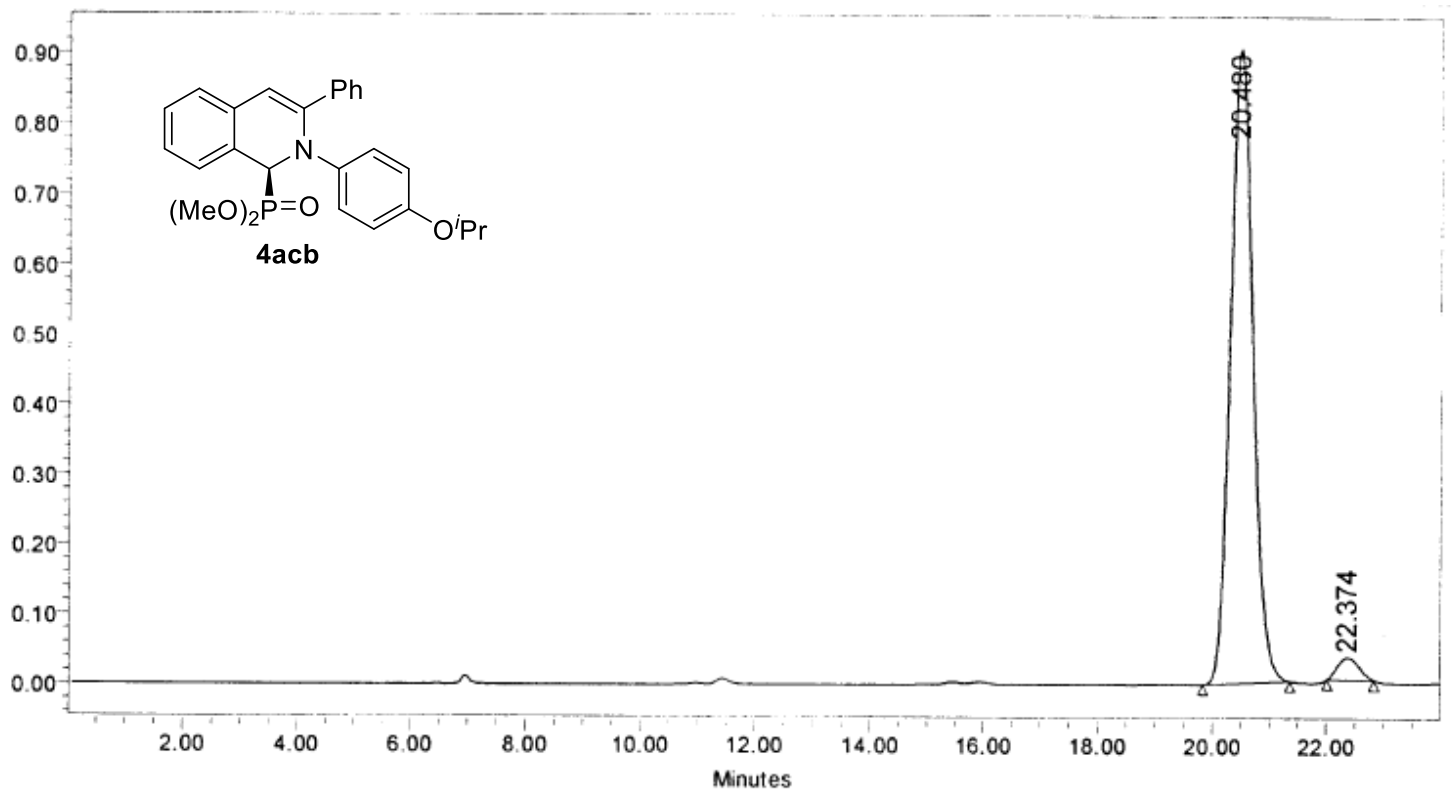

\begin{tabular}{|c|c|r|r|r|r|}
\hline & $\begin{array}{c}\text { RT } \\
(\mathrm{min})\end{array}$ & $\begin{array}{c}\text { Area } \\
(\mu \mathrm{V} * \mathrm{sec})\end{array}$ & $\%$ Area & $\begin{array}{c}\text { Height } \\
(\mu \mathrm{V})\end{array}$ & $\begin{array}{c}\% \\
\text { Height }\end{array}$ \\
\hline 1 & 20.480 & 24988692 & 96.81 & 905752 & 96.59 \\
\hline 2 & 22.374 & 824006 & 3.19 & 31976 & 3.41 \\
\hline
\end{tabular}




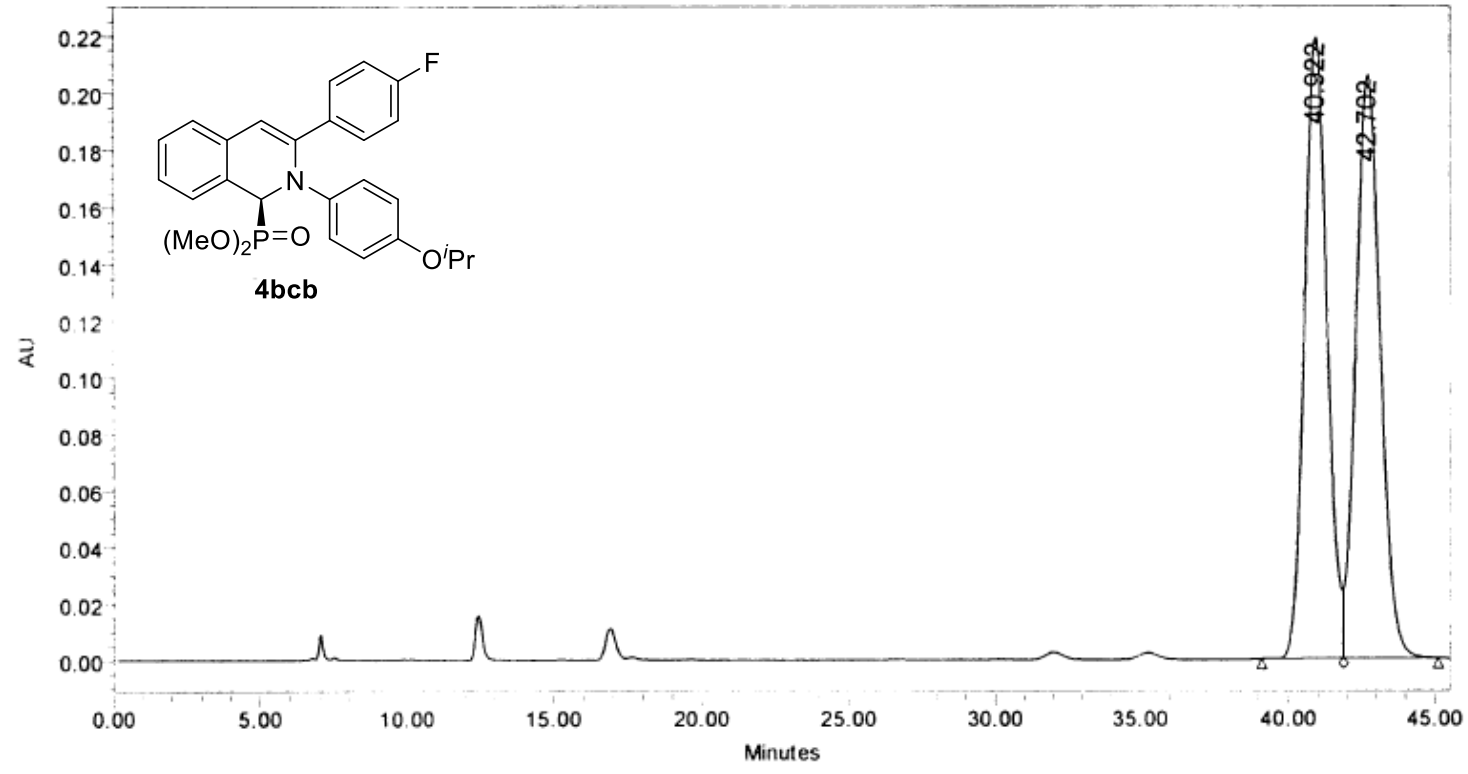

\begin{tabular}{|c|c|c|c|c|c|}
\hline & $\begin{array}{c}\text { RT } \\
(\mathrm{min})\end{array}$ & $\begin{array}{c}\text { Area } \\
(\mu \mathrm{V} * \mathrm{sec})\end{array}$ & $\%$ Area & $\begin{array}{c}\text { Height } \\
(\mu \mathrm{V})\end{array}$ & $\begin{array}{c}\% \\
\text { Height }\end{array}$ \\
\hline 1 & 40.922 & 11675959 & 49.43 & 218926 & 51.55 \\
\hline 2 & 42.702 & 11945440 & 50.57 & 205740 & 48.45 \\
\hline
\end{tabular}

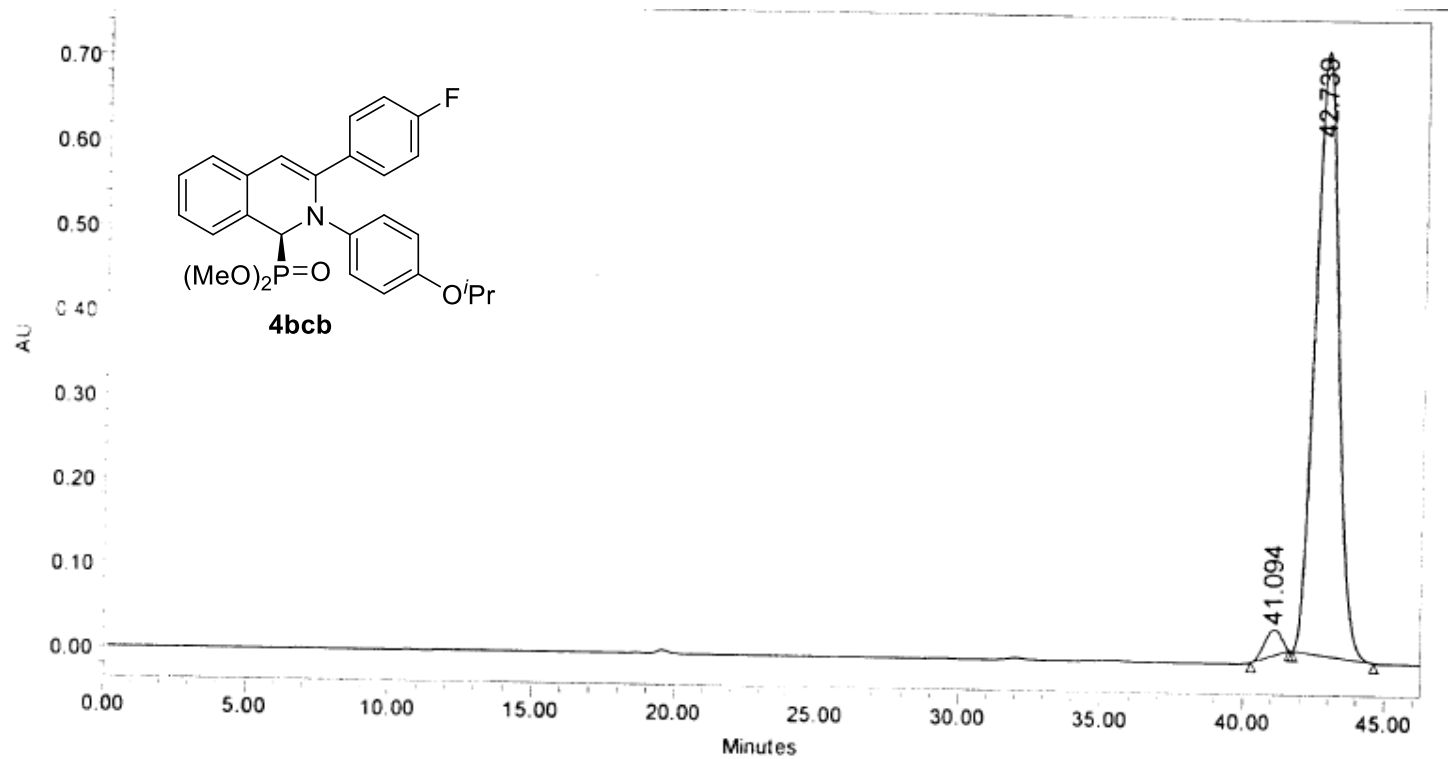

\begin{tabular}{|c|c|c|r|r|r|}
\hline & $\begin{array}{c}\text { RT } \\
(\mathrm{min})\end{array}$ & $\begin{array}{c}\text { Area } \\
(\mu \mathrm{V} * \mathrm{sec})\end{array}$ & \% Area & $\begin{array}{c}\text { Height } \\
(\mu \mathrm{V})\end{array}$ & $\begin{array}{c}\% \\
\text { Height }\end{array}$ \\
\hline 1 & 41.094 & 1259860 & 3.00 & 30393 & 4.08 \\
\hline 2 & 42.739 & 40747417 & 97.00 & 714295 & 95.92 \\
\hline
\end{tabular}




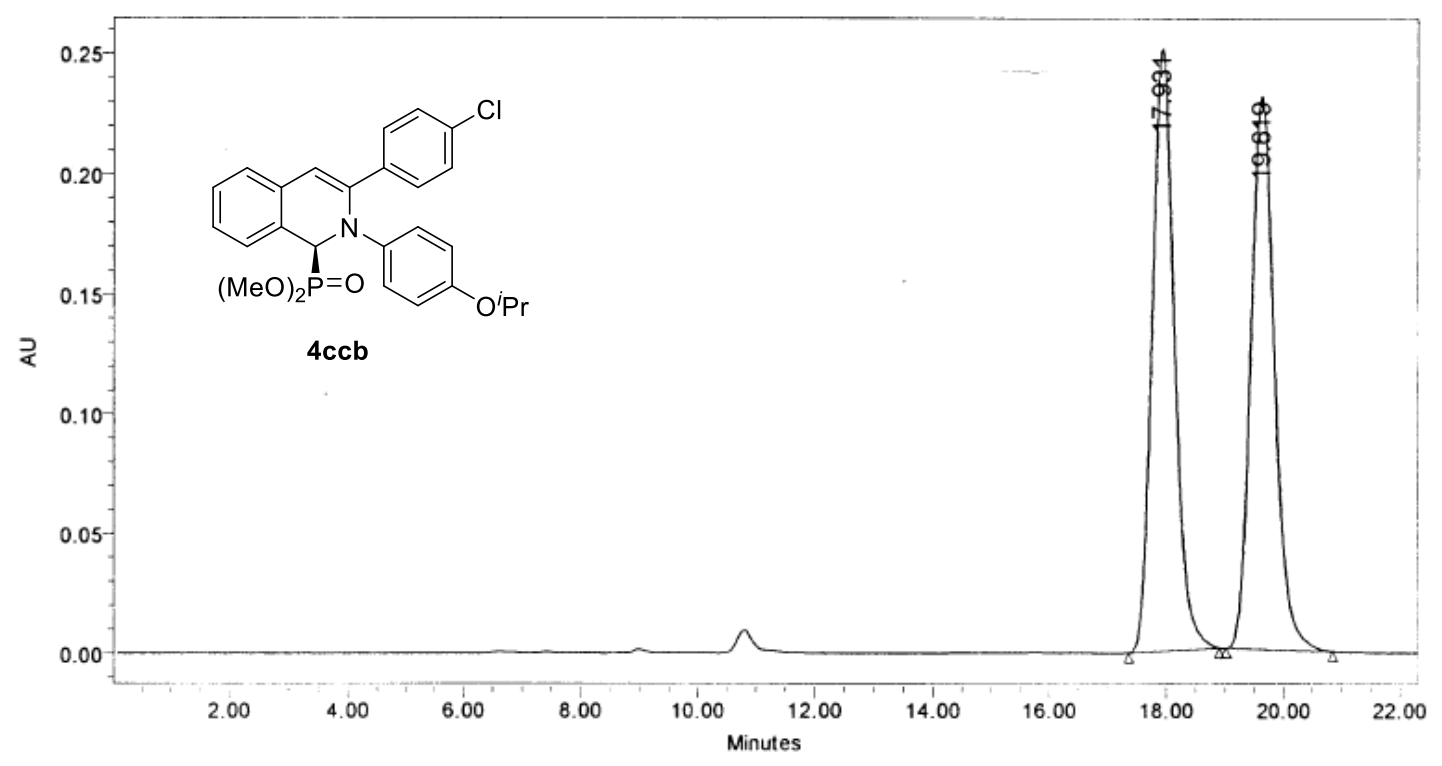

\begin{tabular}{|c|c|c|c|c|c|}
\hline & $\begin{array}{c}\text { RT } \\
(\mathrm{min})\end{array}$ & $\begin{array}{c}\text { Area } \\
(\mu \mathrm{V} * \mathrm{sec})\end{array}$ & $\%$ Area & $\begin{array}{c}\text { Height } \\
(\mu \mathrm{V})\end{array}$ & $\begin{array}{c}\% \\
\text { Height }\end{array}$ \\
\hline 1 & 17.931 & 6555523 & 50.06 & 251026 & 52.11 \\
\hline 2 & 19.619 & 6540166 & 49.94 & 230669 & 47.89 \\
\hline
\end{tabular}

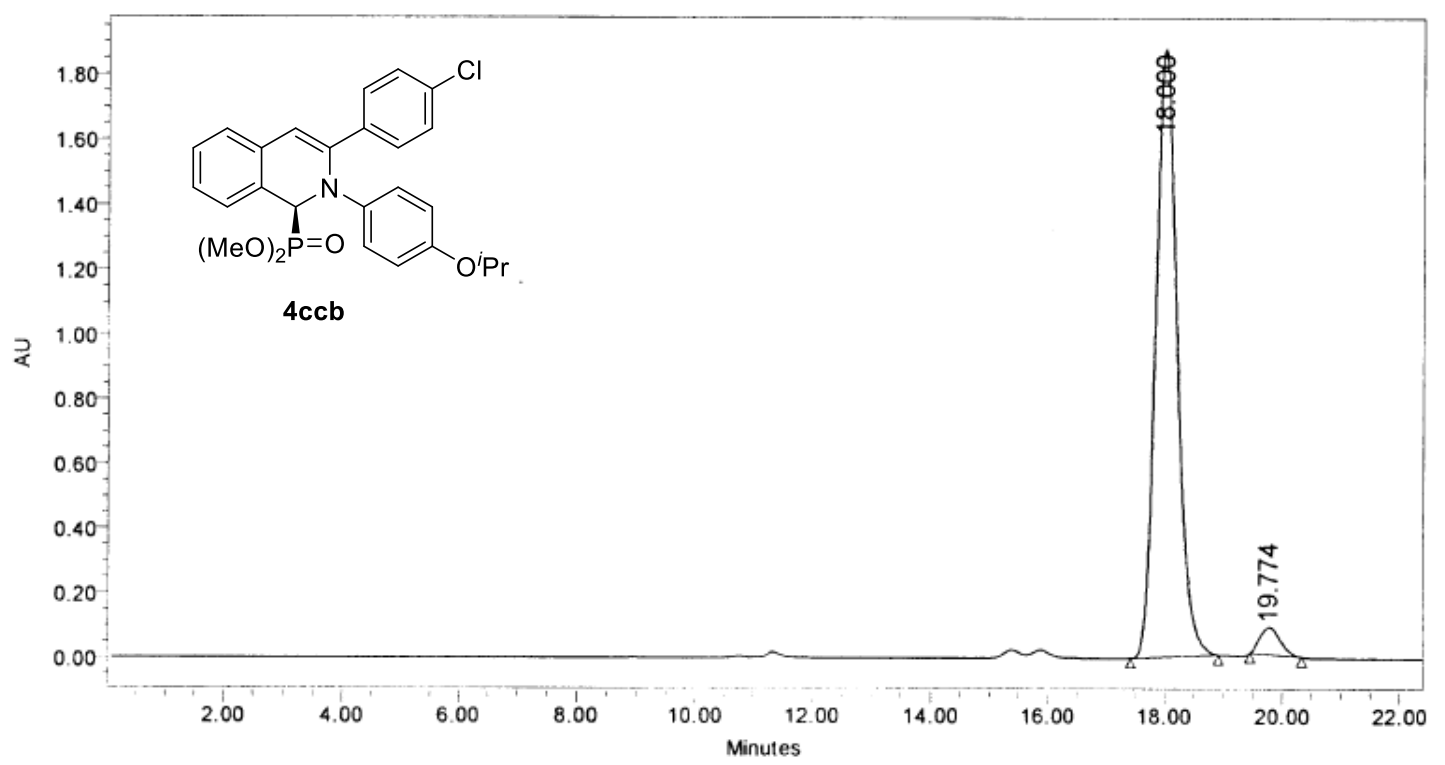

\begin{tabular}{|c|c|c|r|r|r|}
\hline & $\begin{array}{c}\text { RT } \\
(\mathrm{min})\end{array}$ & $\begin{array}{c}\text { Area } \\
\left(\mu \mathrm{V}^{*} \mathrm{sec}\right)\end{array}$ & $\%$ Area & $\begin{array}{c}\text { Height } \\
(\mu \mathrm{V})\end{array}$ & $\begin{array}{c}\% \\
\text { Height }\end{array}$ \\
\hline 1 & 18.000 & 47759977 & 95.91 & 1875972 & 95.69 \\
\hline 2 & 19.774 & 2037656 & 4.09 & 84455 & 4.31 \\
\hline
\end{tabular}




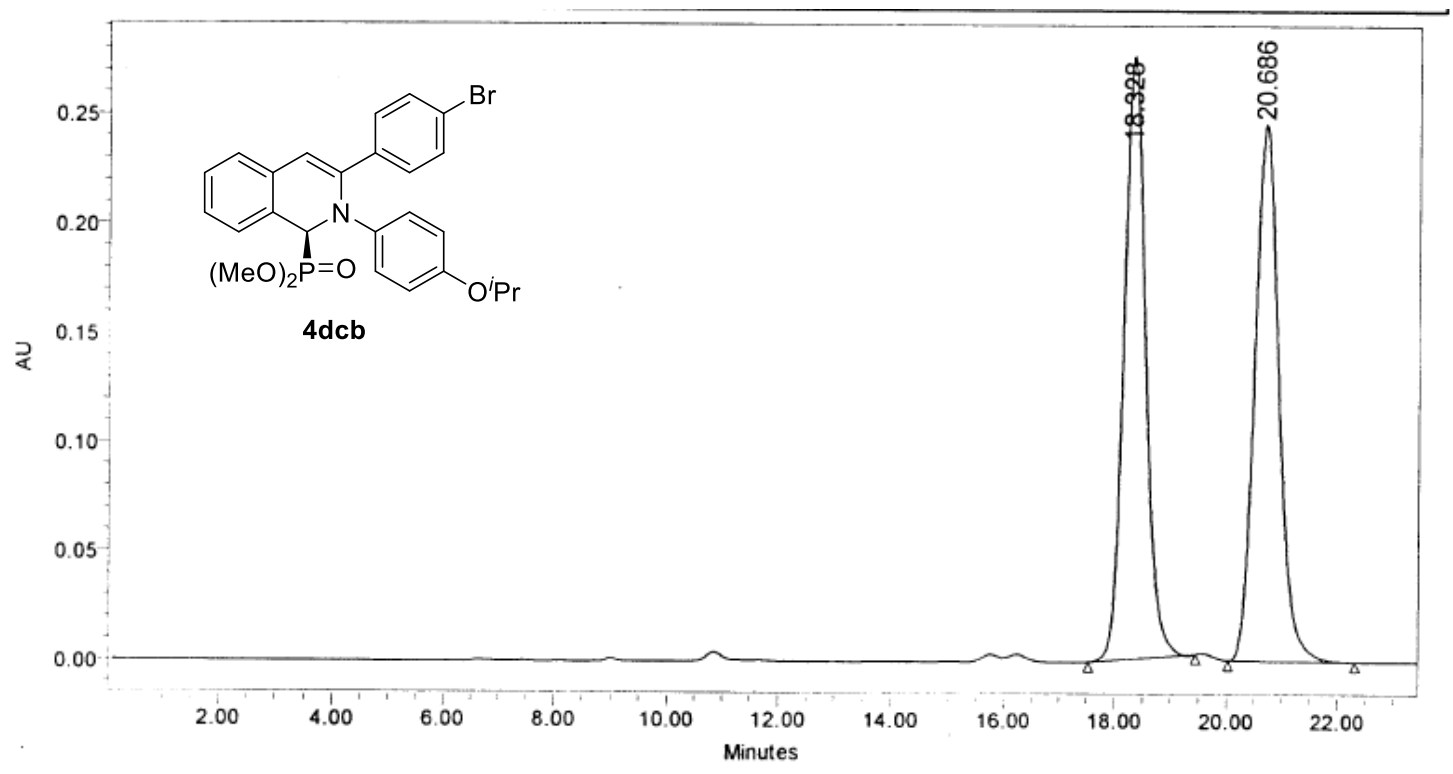

\begin{tabular}{|c|c|c|c|c|c|}
\hline & $\begin{array}{c}\mathrm{RT} \\
(\mathrm{min})\end{array}$ & $\begin{array}{c}\text { Area } \\
\left(\mu \mathrm{V}^{*} \mathrm{sec}\right)\end{array}$ & $\%$ Area & $\begin{array}{c}\text { Height } \\
(\mu \mathrm{V})\end{array}$ & $\begin{array}{c}\% \\
\text { Height }\end{array}$ \\
\hline 1 & 18.328 & 7453644 & 49.92 & 275931 & 52.87 \\
\hline 2 & 20.686 & 7477058 & 50.08 & 245930 & 47.13 \\
\hline
\end{tabular}

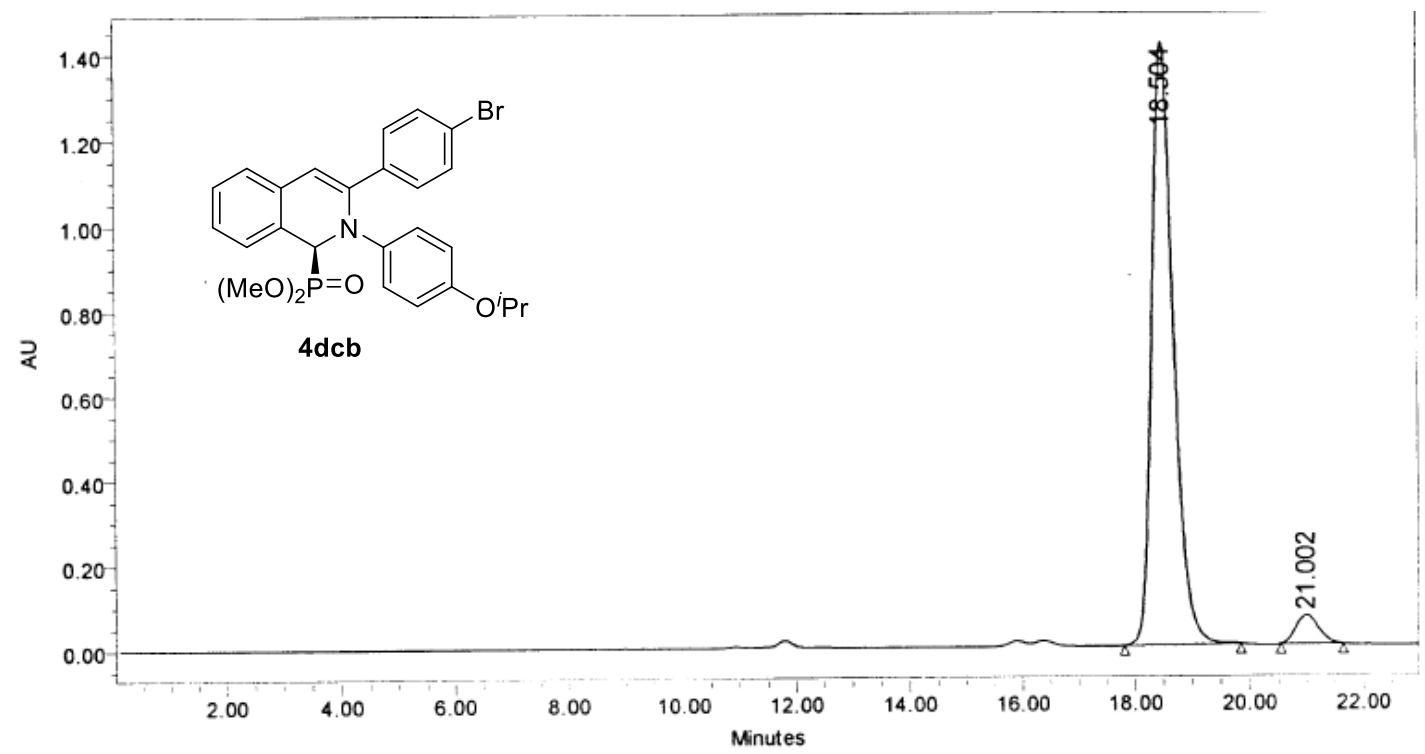

\begin{tabular}{|c|c|c|r|r|r|}
\hline & $\begin{array}{c}\mathrm{RT} \\
(\mathrm{min})\end{array}$ & $\begin{array}{c}\text { Area } \\
(\mu \mathrm{V} * \mathrm{sec})\end{array}$ & $\%$ Area & \multicolumn{1}{|c|}{$\begin{array}{c}\text { Height } \\
(\mu \mathrm{V})\end{array}$} & $\begin{array}{c}\% \\
\text { Height }\end{array}$ \\
\hline 1 & 18.504 & 37251790 & 95.25 & 1415561 & 95.49 \\
\hline 2 & 21.002 & 1859735 & 4.75 & 66858 & 4.51 \\
\hline
\end{tabular}




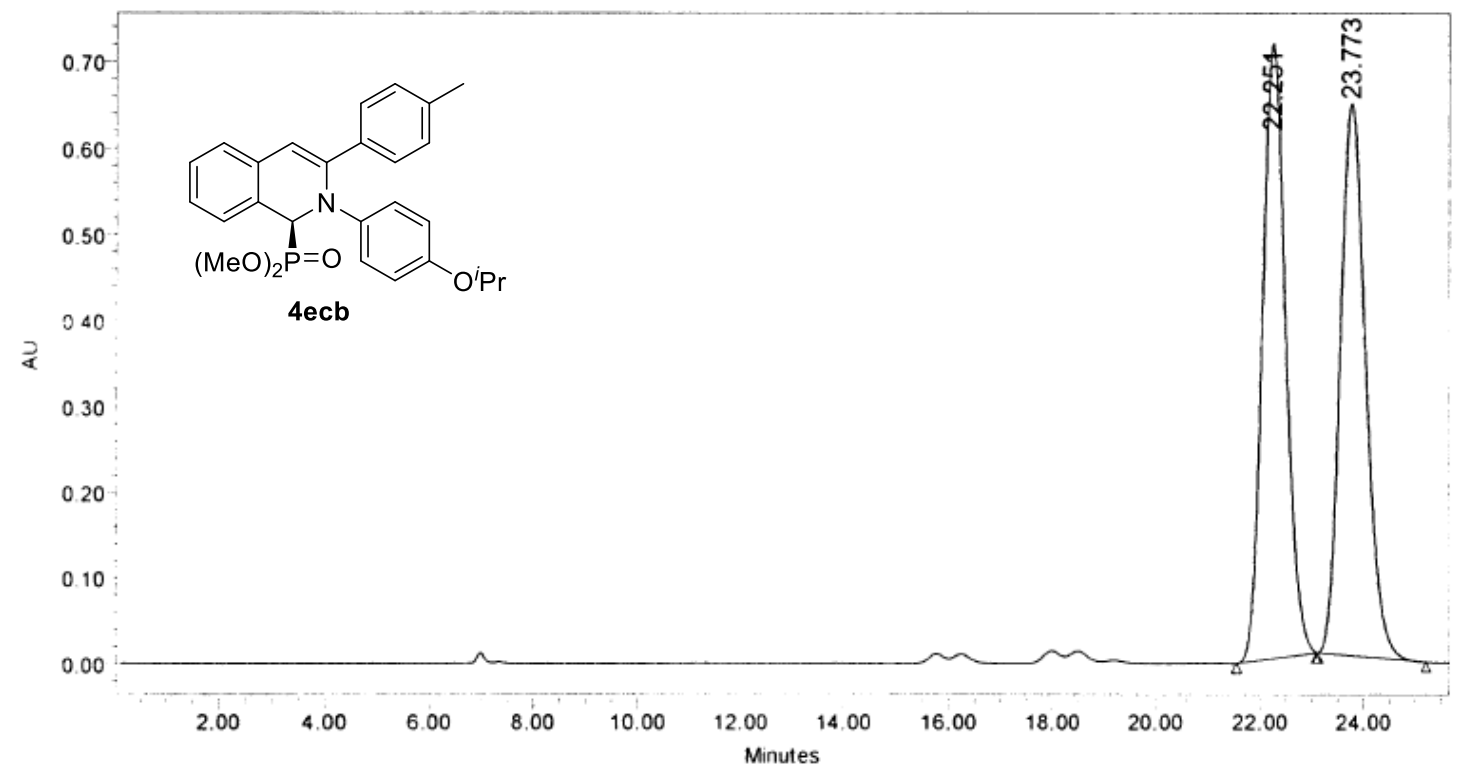

\begin{tabular}{|c|c|c|c|c|c|}
\hline & $\begin{array}{c}\text { RT } \\
(\mathrm{min})\end{array}$ & $\begin{array}{c}\text { Area } \\
(\mu \mathrm{V} * \mathrm{sec})\end{array}$ & $\%$ Area & $\begin{array}{c}\text { Height } \\
(\mu \mathrm{V})\end{array}$ & $\begin{array}{c}\% \\
\text { Height }\end{array}$ \\
\hline 1 & 22.251 & 22132803 & 50.05 & 714306 & 52.66 \\
\hline 2 & 23.773 & 22091581 & 49.95 & 642081 & 47.34 \\
\hline
\end{tabular}

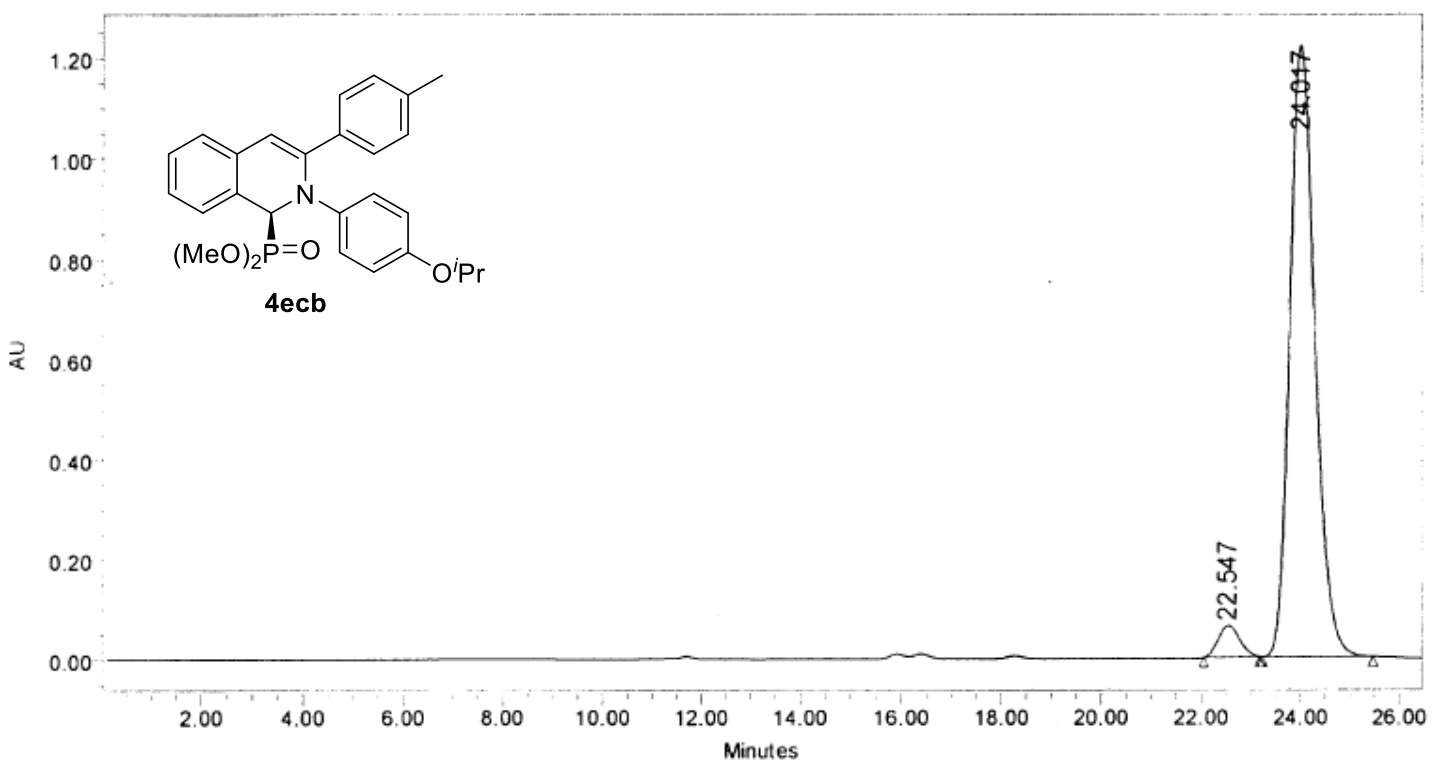

\begin{tabular}{|c|c|c|c|c|c|}
\hline & $\begin{array}{c}\text { RT } \\
(\mathrm{min})\end{array}$ & $\begin{array}{c}\text { Area } \\
(\mu \mathrm{V} * \mathrm{sec})\end{array}$ & $\%$ Area & $\begin{array}{c}\text { Height } \\
(\mu \mathrm{V})\end{array}$ & $\begin{array}{c}\% \\
\text { Height }\end{array}$ \\
\hline 1 & 22.547 & 1868798 & 4.17 & 62638 & 4.88 \\
\hline 2 & 24.017 & 42978676 & 95.83 & 1220641 & 95.12 \\
\hline
\end{tabular}




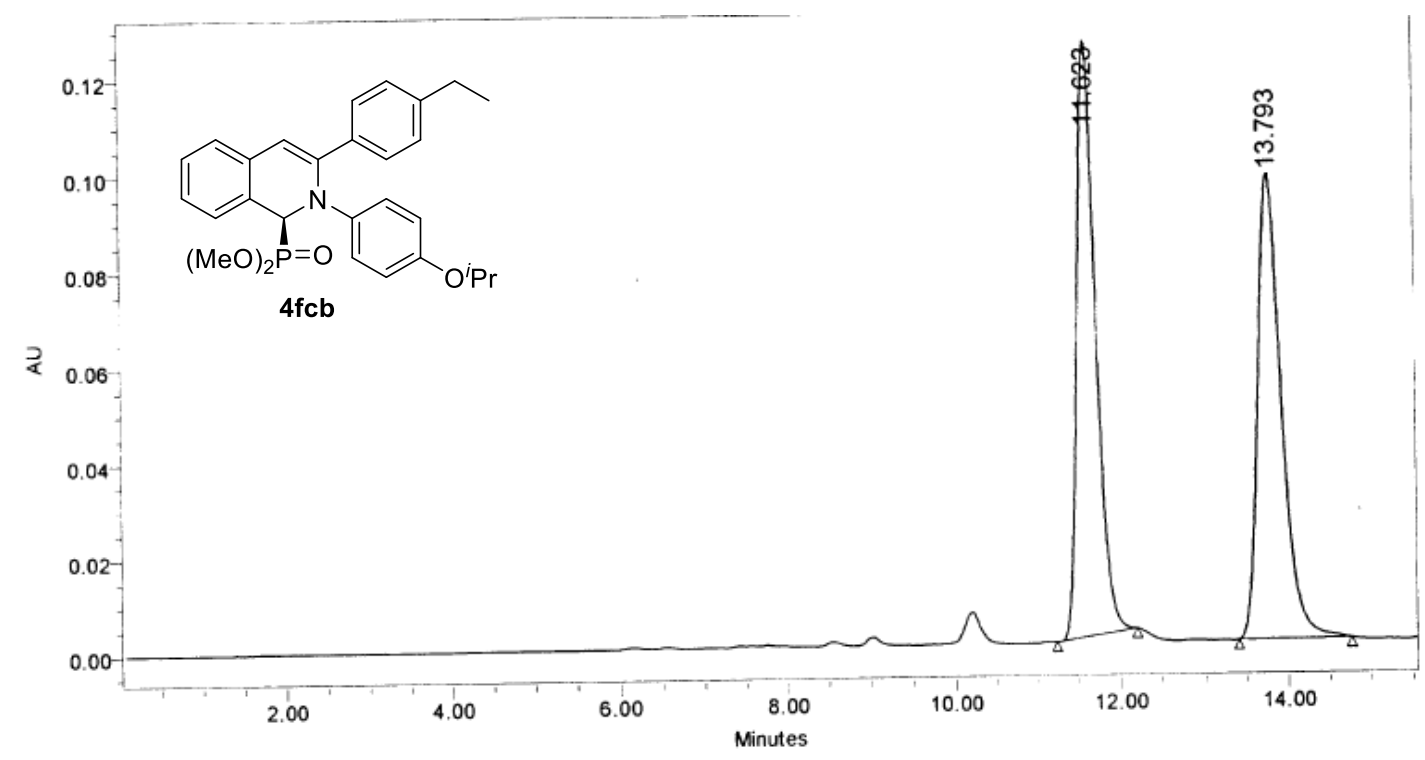

\begin{tabular}{|c|c|c|c|c|c|}
\hline & $\begin{array}{c}\text { RT } \\
(\mathrm{min})\end{array}$ & $\begin{array}{c}\text { Area } \\
(\mu \mathrm{V} * \mathrm{sec})\end{array}$ & $\%$ Area & $\begin{array}{c}\text { Height } \\
(\mu \mathrm{V})\end{array}$ & $\begin{array}{c}\% \\
\text { Height }\end{array}$ \\
\hline 1 & 11.623 & 1943401 & 50.33 & 124379 & 56.11 \\
\hline 2 & 13.793 & 1918048 & 49.67 & 97279 & 43.89 \\
\hline
\end{tabular}

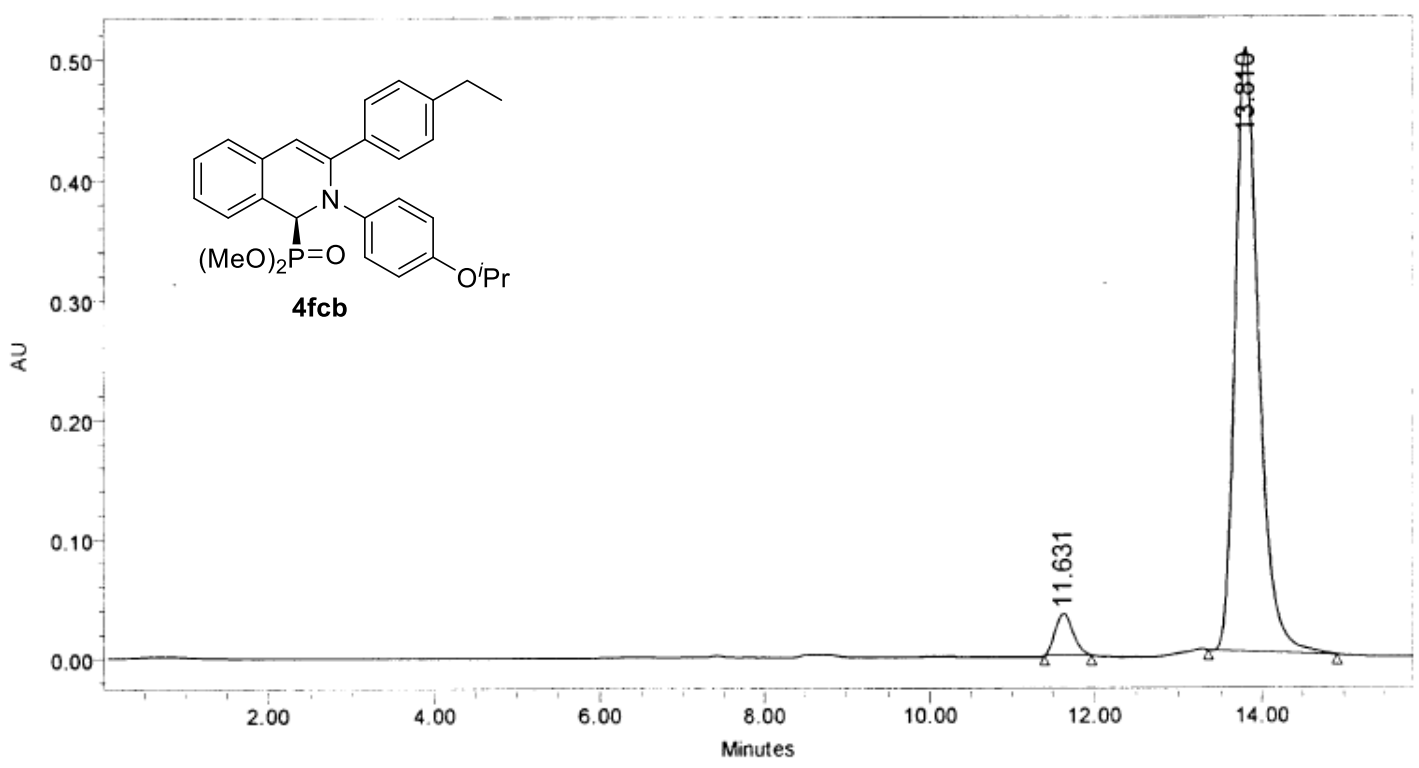

\begin{tabular}{|c|c|r|r|r|r|}
\hline & $\begin{array}{c}\text { RT } \\
(\min )\end{array}$ & $\begin{array}{c}\text { Area } \\
\left(\mu \mathrm{V}^{*} \mathrm{sec}\right)\end{array}$ & $\%$ Area & $\begin{array}{c}\text { Height } \\
(\mu \mathrm{V})\end{array}$ & $\begin{array}{c}\% \\
\text { Height }\end{array}$ \\
\hline 1 & 11.631 & 513841 & 4.89 & 34800 & 6.45 \\
\hline 2 & 13.810 & 9992246 & 95.11 & 504811 & 93.55 \\
\hline
\end{tabular}




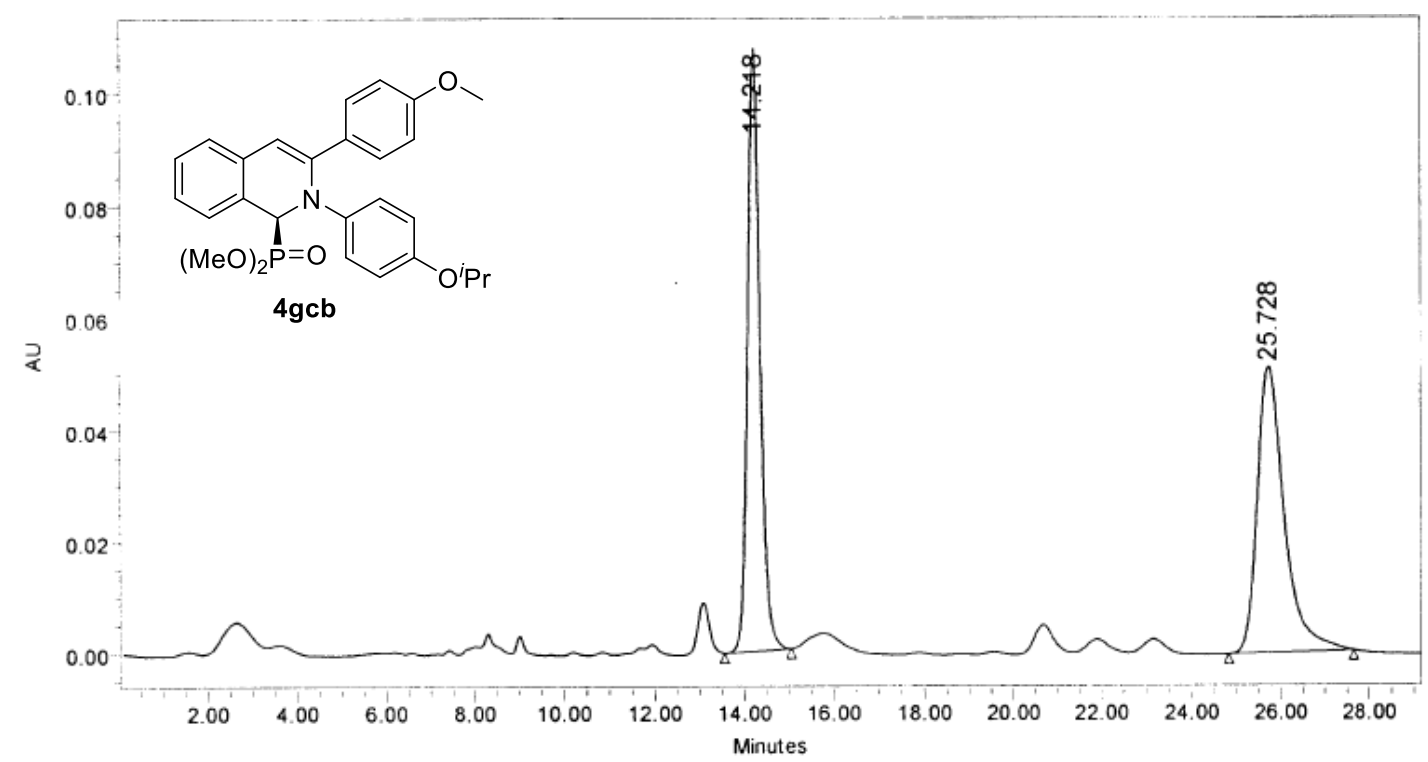

\begin{tabular}{|c|c|c|c|c|c|}
\hline & $\begin{array}{c}\text { RT } \\
(\mathrm{min})\end{array}$ & $\begin{array}{c}\text { Area } \\
(\mu \mathrm{V} * \mathrm{sec})\end{array}$ & $\%$ Area & $\begin{array}{c}\text { Height } \\
(\mu \mathrm{V})\end{array}$ & $\begin{array}{c}\% \\
\text { Height }\end{array}$ \\
\hline 1 & 14.218 & 2114799 & 49.30 & 107515 & 67.70 \\
\hline 2 & 25.728 & 2174996 & 50.70 & 51296 & 32.30 \\
\hline
\end{tabular}

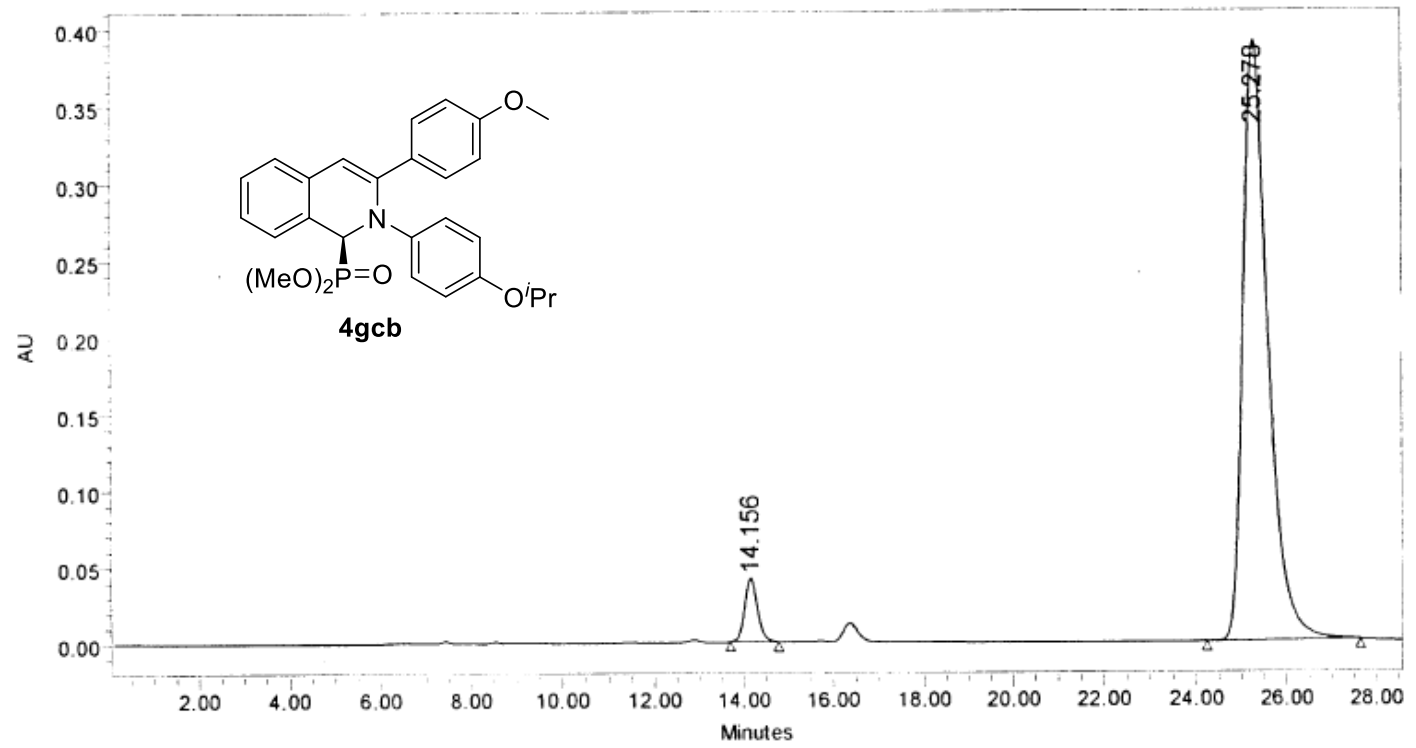

\begin{tabular}{|c|c|r|r|r|r|}
\hline & $\begin{array}{c}\text { RT } \\
(\mathrm{min})\end{array}$ & $\begin{array}{c}\text { Area } \\
(\mu \mathrm{V} * \mathrm{sec})\end{array}$ & $\%$ Area & $\begin{array}{c}\text { Height } \\
(\mu \mathrm{V})\end{array}$ & $\begin{array}{c}\% \\
\text { Height }\end{array}$ \\
\hline 1 & 14.156 & 821273 & 5.14 & 41483 & 9.59 \\
\hline 2 & 25.278 & 15159034 & 94.86 & 390900 & 90.41 \\
\hline
\end{tabular}



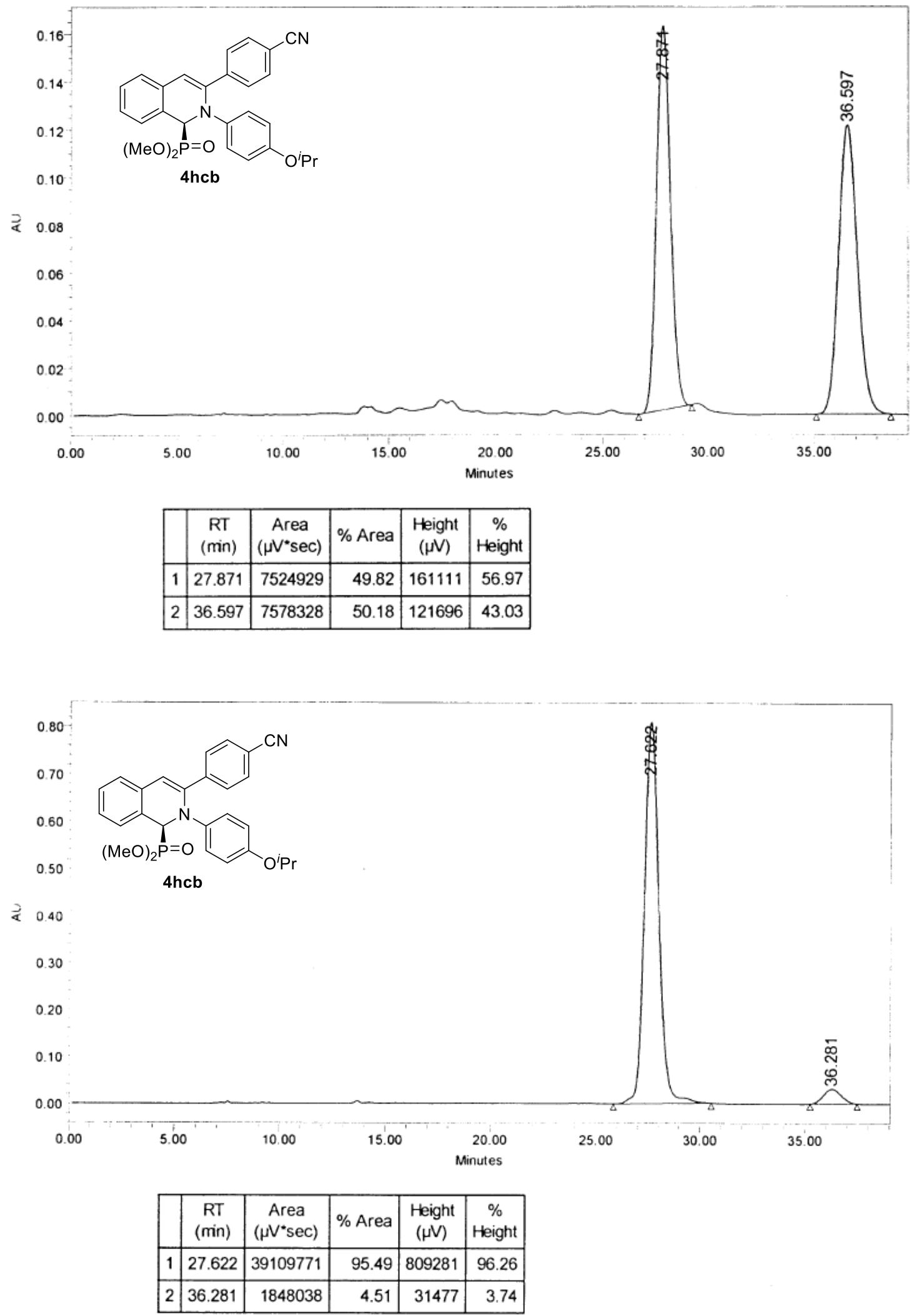


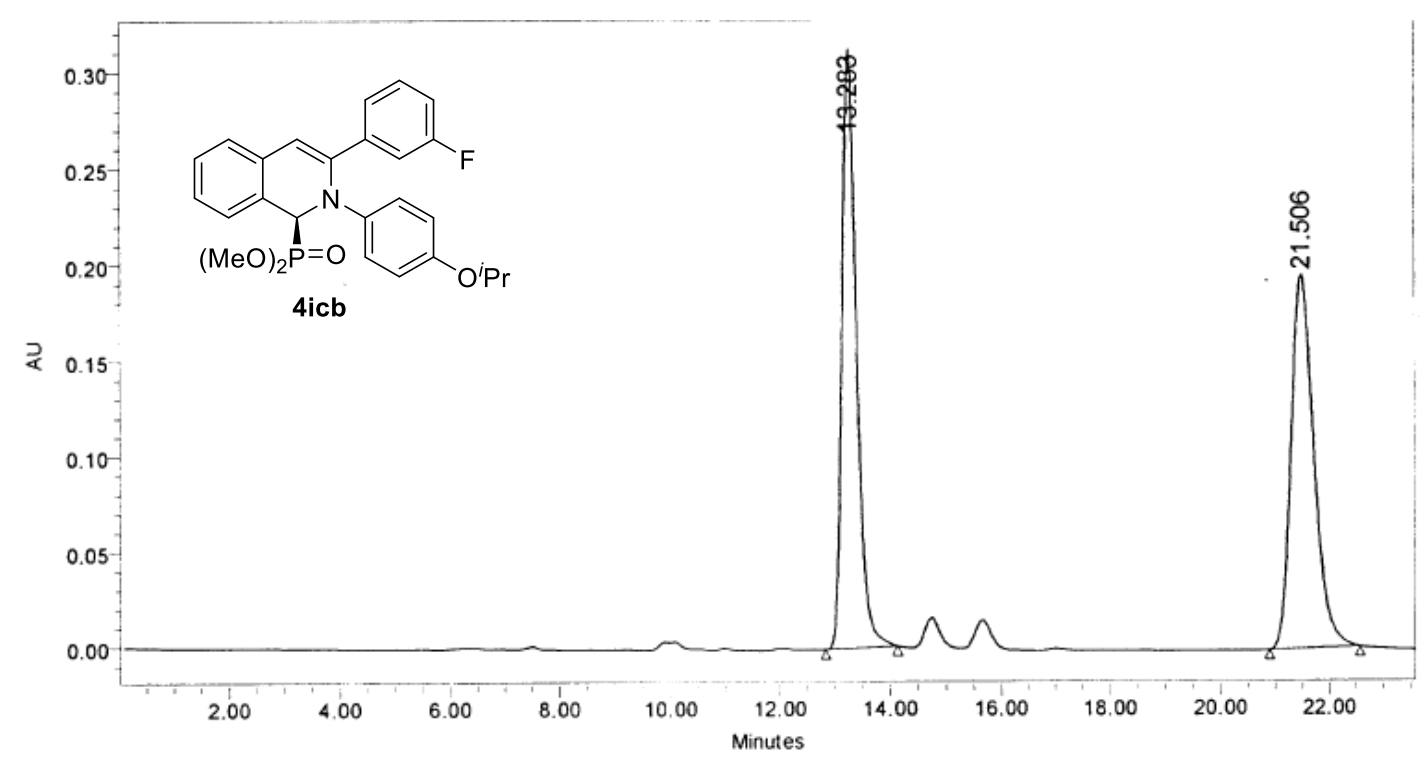

\begin{tabular}{|c|c|c|c|c|c|}
\hline & $\begin{array}{c}\mathrm{RT} \\
(\mathrm{min})\end{array}$ & $\begin{array}{c}\text { Area } \\
(\mu \mathrm{V} * \mathrm{sec})\end{array}$ & $\%$ Area & $\begin{array}{c}\text { Height } \\
(\mu \mathrm{V})\end{array}$ & $\begin{array}{c}\% \\
\text { Height }\end{array}$ \\
\hline 1 & 13.283 & 5648792 & 49.75 & 312344 & 61.50 \\
\hline 2 & 21.506 & 5706695 & 50.25 & 195492 & 38.50 \\
\hline
\end{tabular}

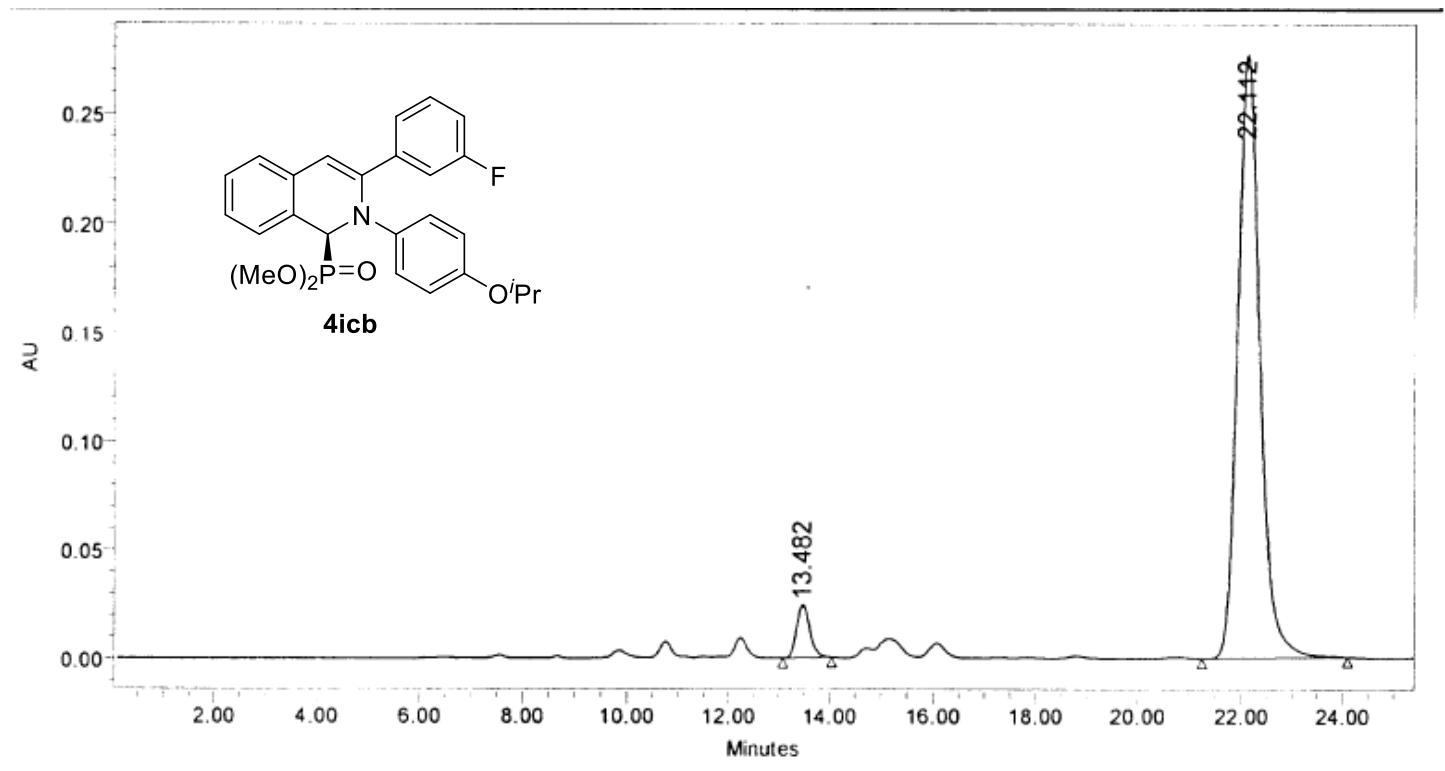

\begin{tabular}{|c|c|r|r|r|r|}
\hline & $\begin{array}{c}\text { RT } \\
(\mathrm{min})\end{array}$ & $\begin{array}{c}\text { Area } \\
\left(\mu \mathrm{V}^{*} \mathrm{sec}\right)\end{array}$ & $\%$ Area & $\begin{array}{c}\text { Height } \\
(\mu \mathrm{V})\end{array}$ & $\begin{array}{c}\% \\
\text { Height }\end{array}$ \\
\hline 1 & 13.482 & 440748 & 4.97 & 24408 & 8.10 \\
\hline 2 & 22.112 & 8423494 & 95.03 & 276894 & 91.90 \\
\hline
\end{tabular}




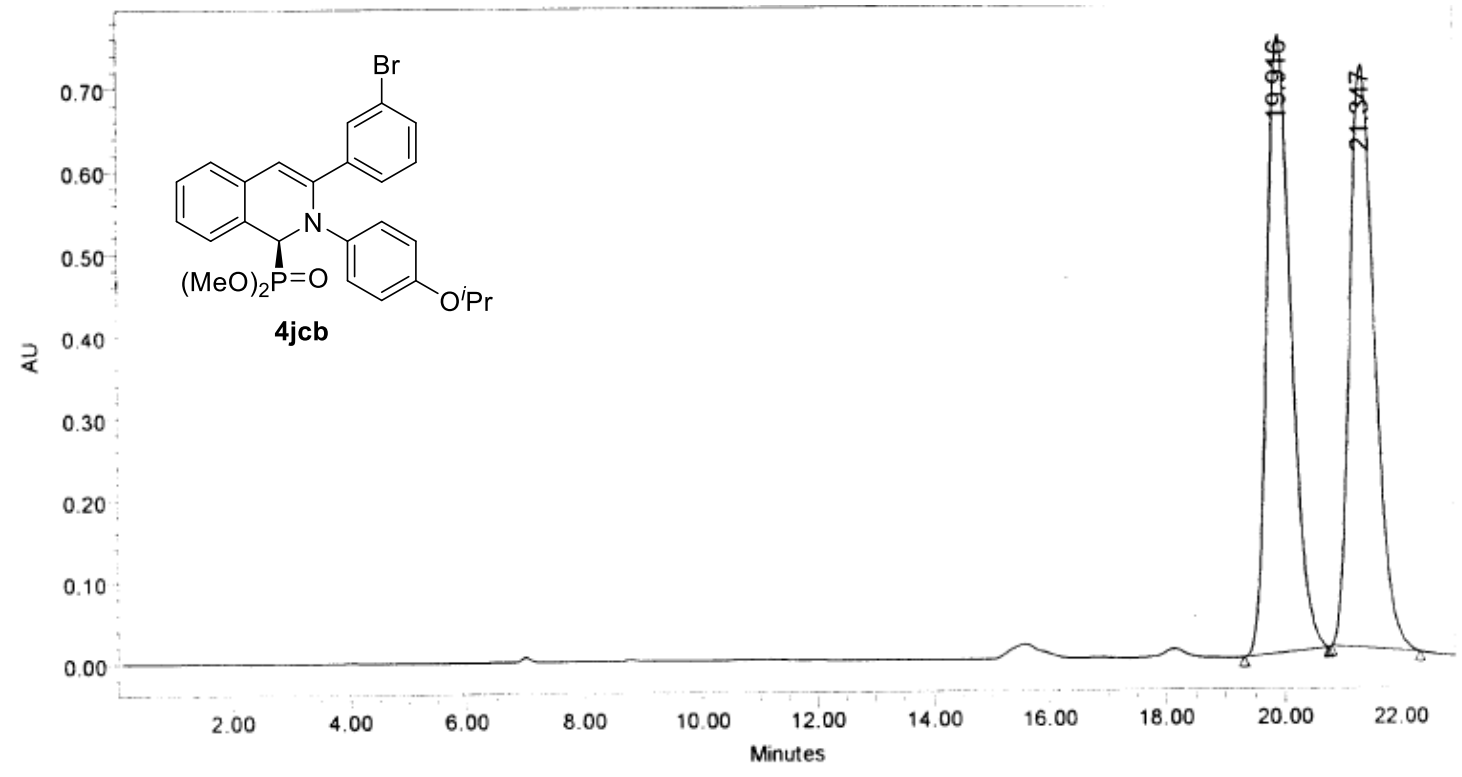

\begin{tabular}{|c|c|c|c|c|c|}
\hline & $\begin{array}{c}\mathrm{RT} \\
(\mathrm{min})\end{array}$ & $\begin{array}{c}\text { Area } \\
(\mu \mathrm{V} * \mathrm{sec})\end{array}$ & $\%$ Area & $\begin{array}{c}\text { Height } \\
(\mu \mathrm{V})\end{array}$ & $\begin{array}{c}\% \\
\text { Height }\end{array}$ \\
\hline 1 & 19.916 & 21490050 & 50.04 & 752003 & 51.46 \\
\hline 2 & 21.347 & 21458804 & 49.96 & 709357 & 48.54 \\
\hline
\end{tabular}

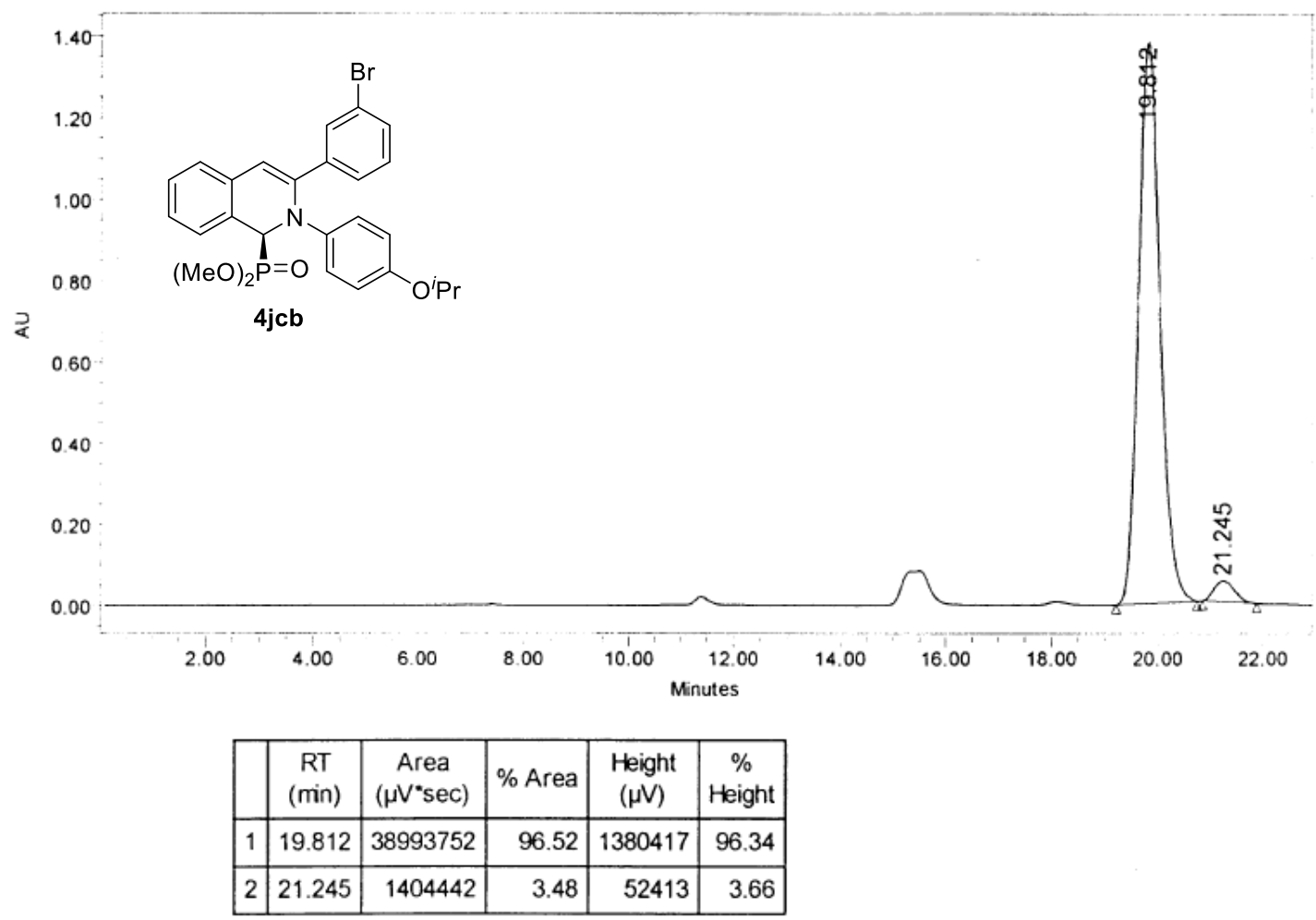




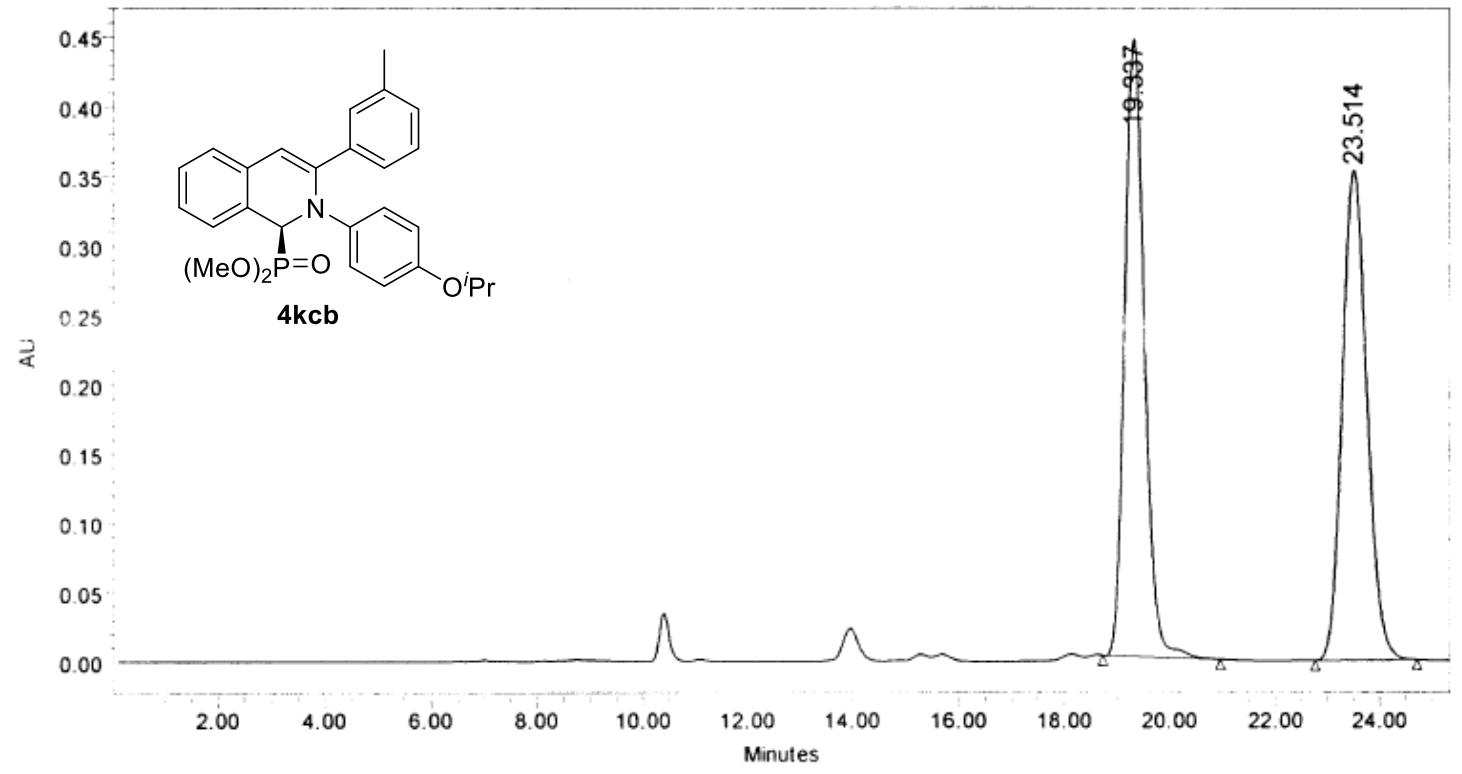

\begin{tabular}{|c|c|c|c|c|c|}
\hline & $\begin{array}{c}\mathrm{RT} \\
(\mathrm{min})\end{array}$ & $\begin{array}{c}\text { Area } \\
(\mu \mathrm{V} \text { *sec })\end{array}$ & $\%$ Area & $\begin{array}{c}\text { Height } \\
(\mu \mathrm{V})\end{array}$ & $\begin{array}{c}\% \\
\text { Height }\end{array}$ \\
\hline 1 & 19.337 & 11629421 & 50.04 & 444576 & 55.69 \\
\hline 2 & 23.514 & 11612852 & 49.96 & 353743 & 44.31 \\
\hline
\end{tabular}

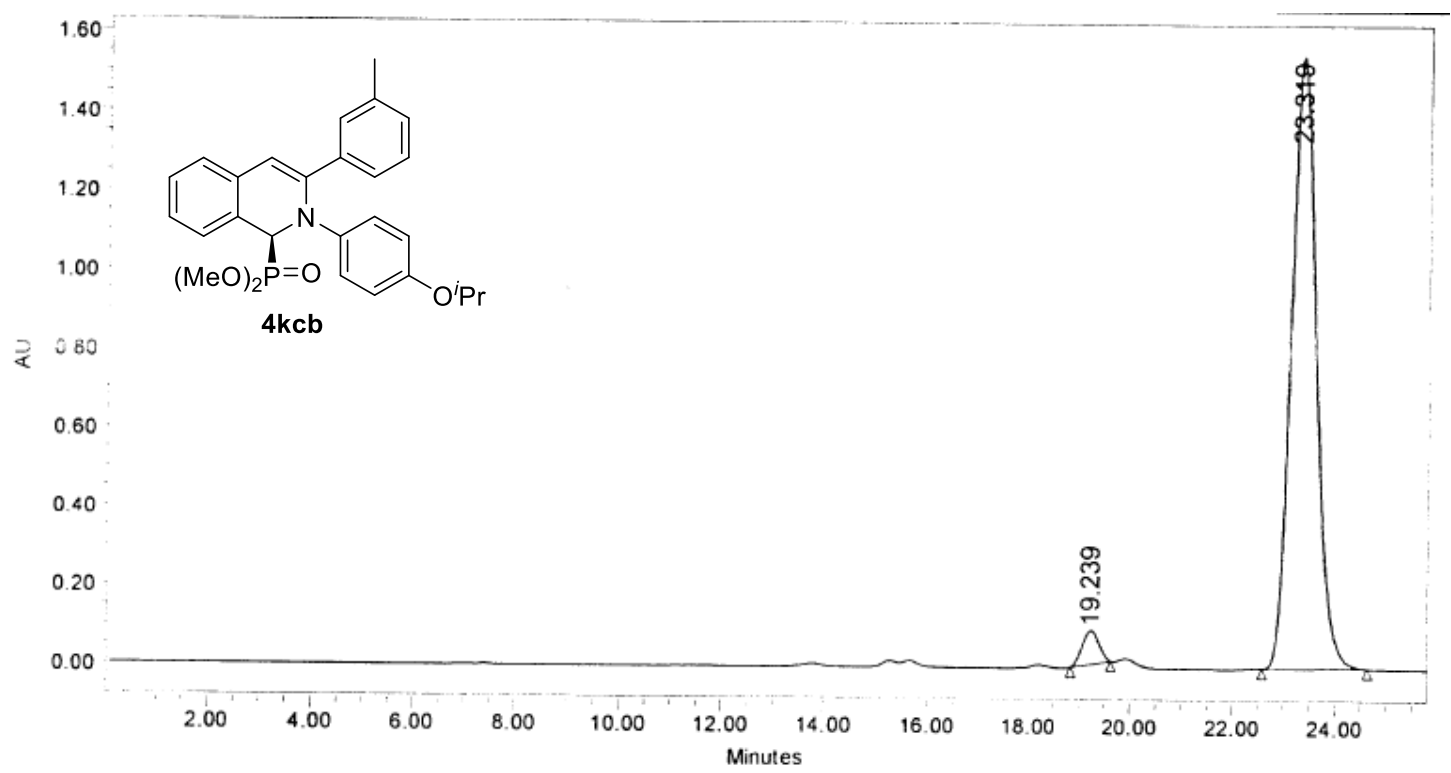

\begin{tabular}{|c|c|c|r|c|c|}
\hline & $\begin{array}{c}\mathrm{RT} \\
(\mathrm{min})\end{array}$ & $\begin{array}{c}\text { Area } \\
(\mu \mathrm{V} * \mathrm{sec})\end{array}$ & $\%$ Area & $\begin{array}{c}\text { Height } \\
(\mu \mathrm{V})\end{array}$ & $\begin{array}{c}\% \\
\text { Height }\end{array}$ \\
\hline 1 & 19.239 & 1943897 & 3.58 & 85185 & 5.21 \\
\hline 2 & 23.319 & 52288978 & 96.42 & 1548758 & 94.79 \\
\hline
\end{tabular}




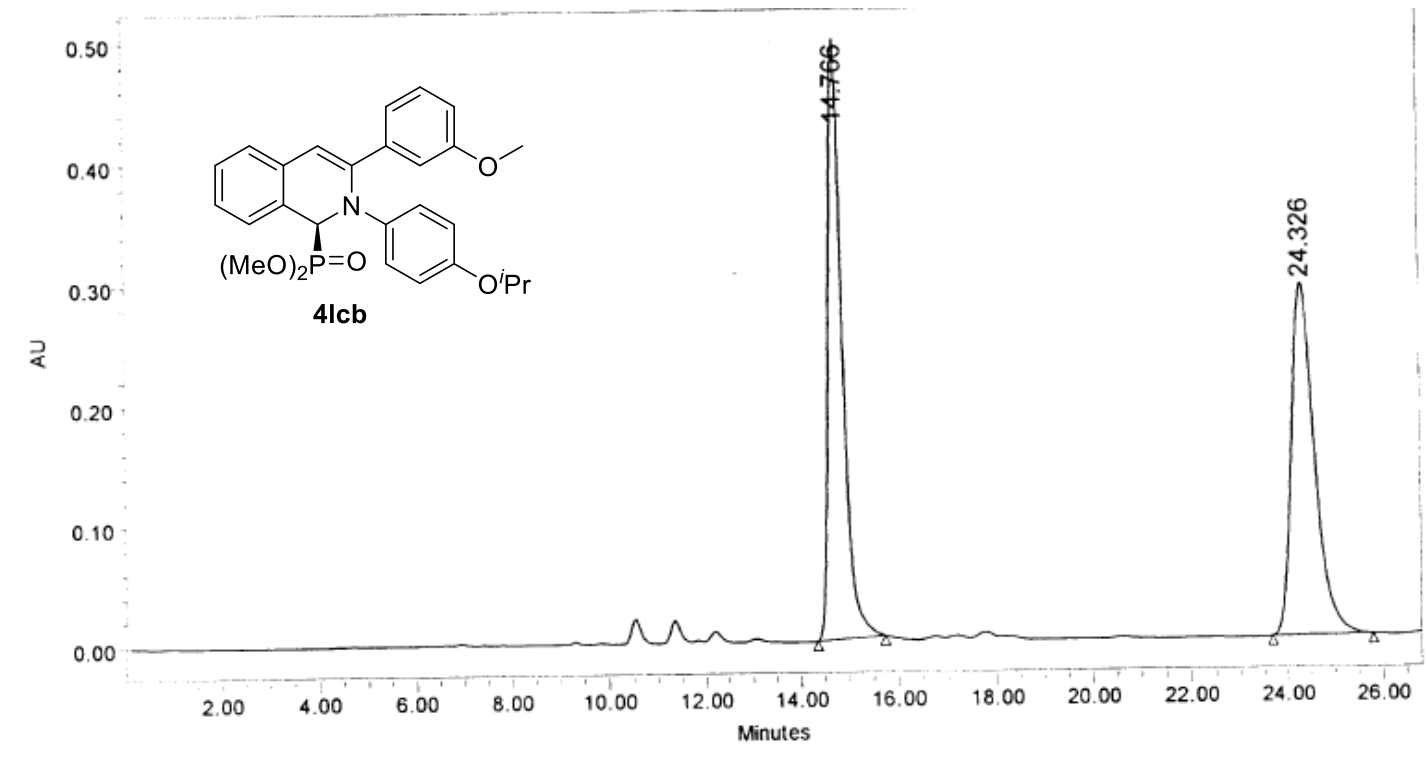

\begin{tabular}{|c|c|c|c|c|c|}
\hline & $\begin{array}{c}\text { RT } \\
(\mathrm{min})\end{array}$ & $\begin{array}{c}\text { Area } \\
\left(\mu \mathrm{V}^{*} \mathrm{sec}\right)\end{array}$ & $\%$ Area & $\begin{array}{c}\text { Height } \\
(\mu \mathrm{V})\end{array}$ & $\begin{array}{c}\% \\
\text { Height }\end{array}$ \\
\hline 1 & 14.766 & 9894381 & 50.10 & 496773 & 62.93 \\
\hline 2 & 24.326 & 9855078 & 49.90 & 292644 & 37.07 \\
\hline
\end{tabular}

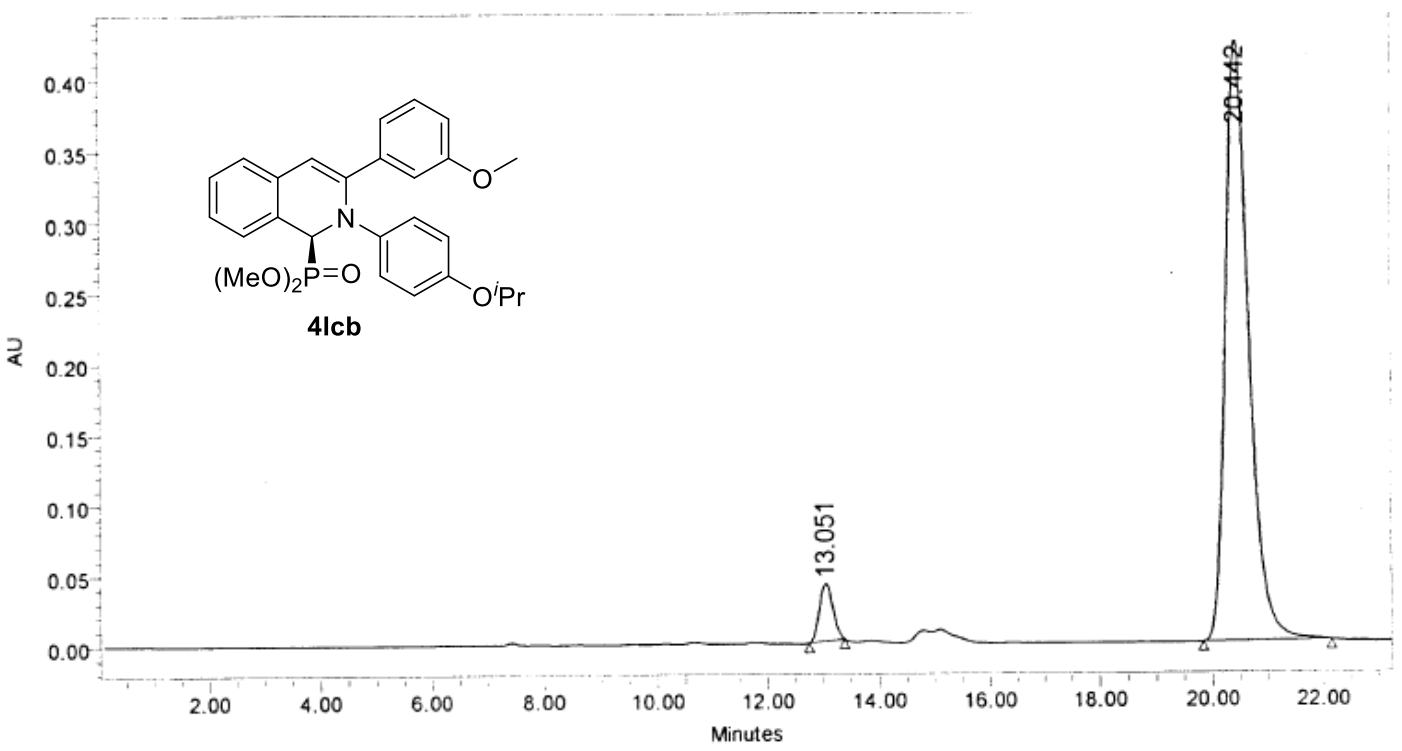

\begin{tabular}{|c|c|r|r|r|r|}
\hline & $\begin{array}{c}\mathrm{RT} \\
(\mathrm{min})\end{array}$ & $\begin{array}{c}\text { Area } \\
(\mu \mathrm{V} * \mathrm{sec})\end{array}$ & $\%$ Area & $\begin{array}{c}\text { Height } \\
(\mu \mathrm{V})\end{array}$ & $\begin{array}{c}\% \\
\text { Height }\end{array}$ \\
\hline 1 & 13.051 & 680269 & 5.16 & 40895 & 8.81 \\
\hline 2 & 20.442 & 12500298 & 94.84 & 423554 & 91.19 \\
\hline
\end{tabular}




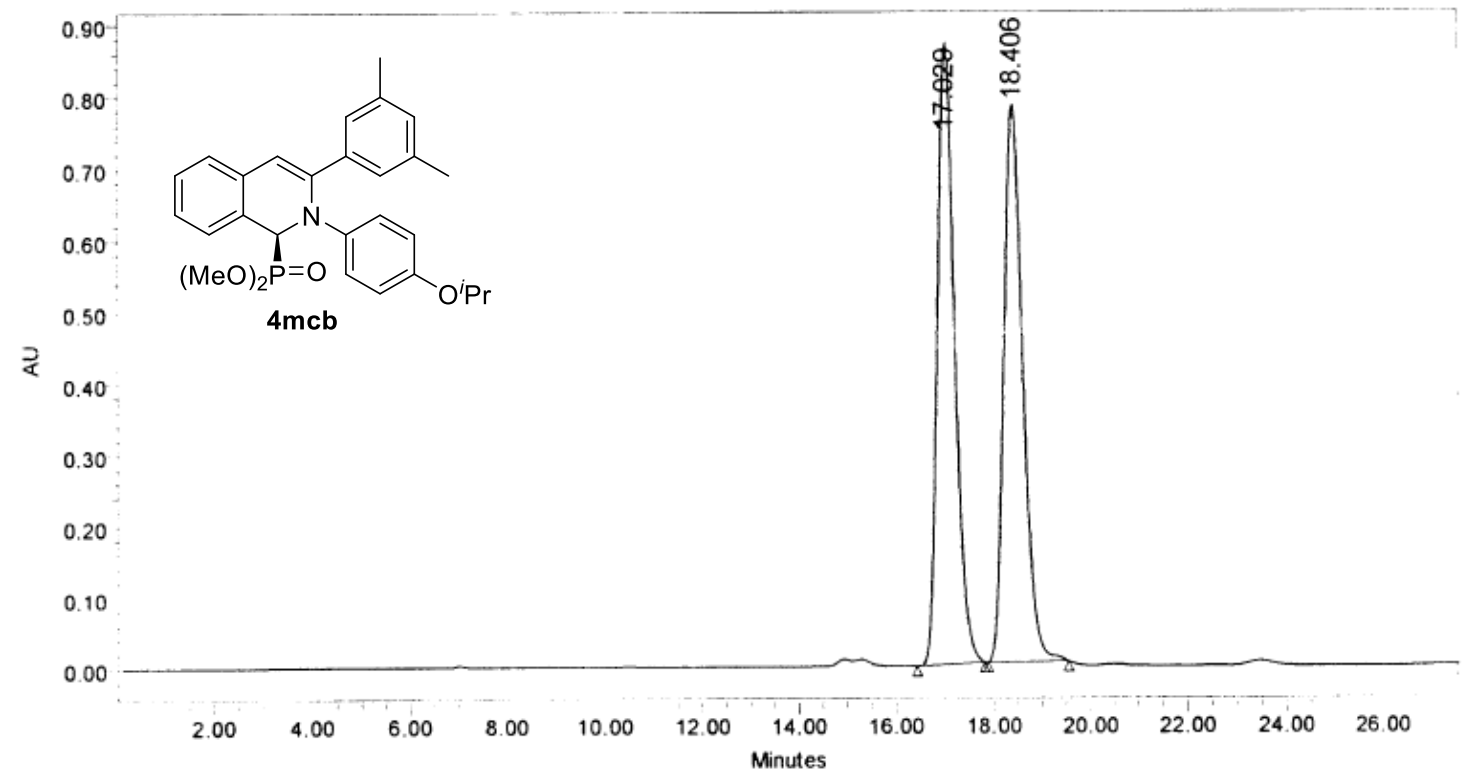

\begin{tabular}{|c|c|c|c|c|c|}
\hline & $\begin{array}{c}\mathrm{RT} \\
(\mathrm{min})\end{array}$ & $\begin{array}{c}\text { Area } \\
(\mu \mathrm{V} * \mathrm{sec})\end{array}$ & $\%$ Area & $\begin{array}{c}\text { Height } \\
(\mu \mathrm{V})\end{array}$ & $\begin{array}{c}\% \\
\text { Height }\end{array}$ \\
\hline 1 & 17.029 & 21125433 & 50.02 & 869374 & 52.70 \\
\hline 2 & 18.406 & 21112655 & 49.98 & 780256 & 47.30 \\
\hline
\end{tabular}

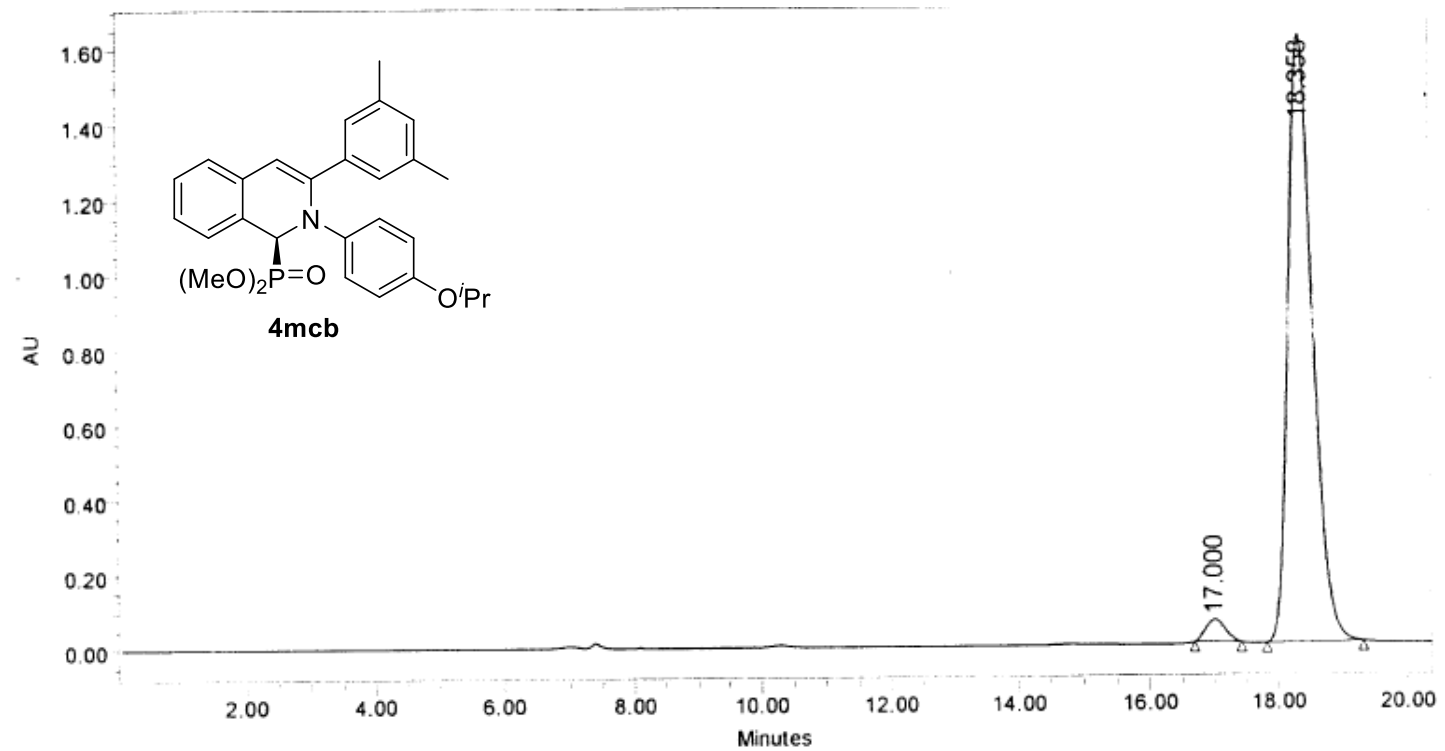

\begin{tabular}{|c|c|c|r|r|r|}
\hline & $\begin{array}{c}\mathrm{RT} \\
(\mathrm{min})\end{array}$ & $\begin{array}{c}\text { Area } \\
(\mu \mathrm{V} * \mathrm{sec})\end{array}$ & $\%$ Area & $\begin{array}{c}\text { Height } \\
(\mu \mathrm{V})\end{array}$ & $\begin{array}{c}\% \\
\text { Height }\end{array}$ \\
\hline 1 & 17.000 & 1257413 & 2.85 & 59568 & 3.54 \\
\hline 2 & 18.358 & 42867177 & 97.15 & 1621041 & 96.46 \\
\hline
\end{tabular}




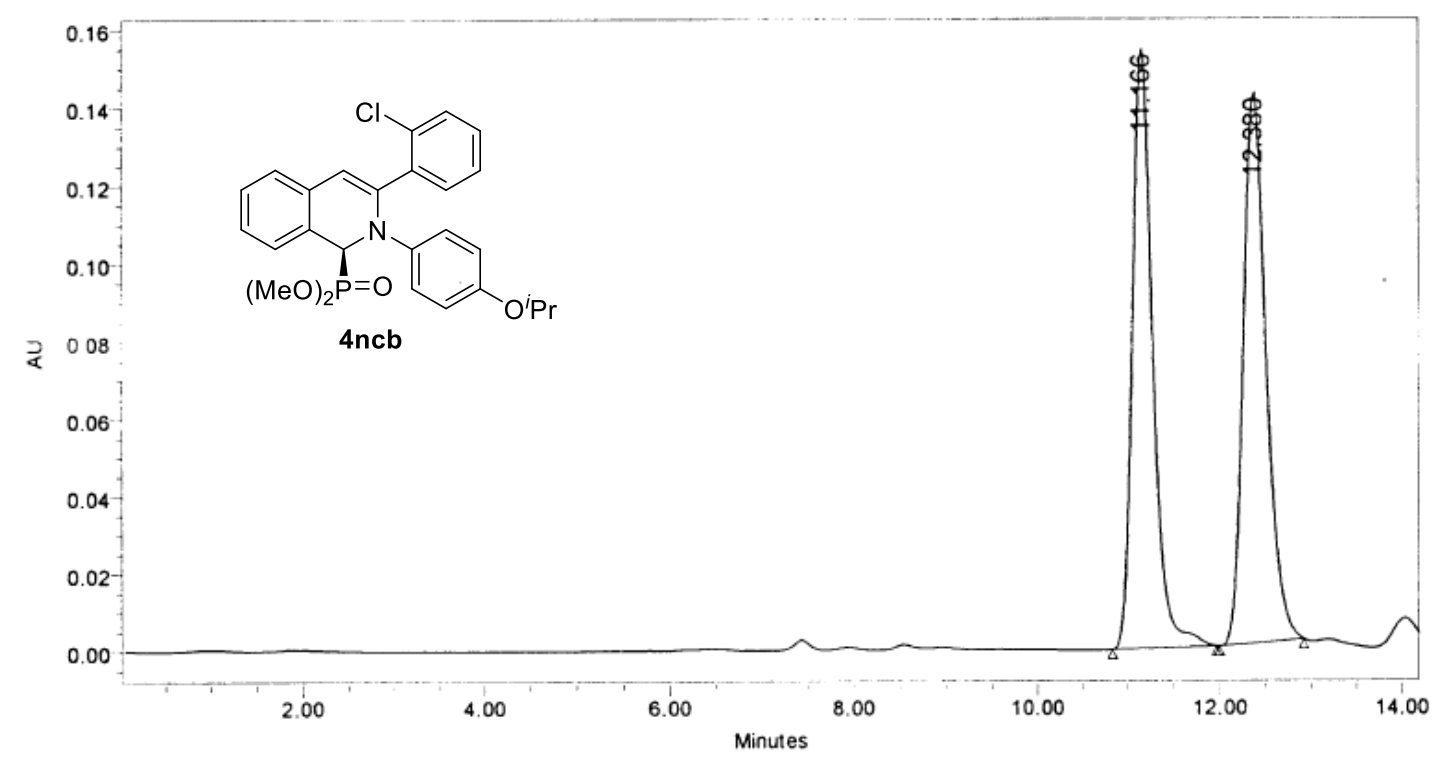

\begin{tabular}{|c|c|c|c|c|c|}
\hline & $\begin{array}{c}\text { RT } \\
(\mathrm{min})\end{array}$ & $\begin{array}{c}\text { Area } \\
(\mu \mathrm{V} * \mathrm{sec})\end{array}$ & $\%$ Area & $\begin{array}{c}\text { Height } \\
(\mu \mathrm{V})\end{array}$ & $\begin{array}{c}\% \\
\text { Height }\end{array}$ \\
\hline 1 & 11.166 & 2363313 & 49.18 & 154348 & 52.11 \\
\hline 2 & 12.380 & 2442021 & 50.82 & 141864 & 47.89 \\
\hline
\end{tabular}

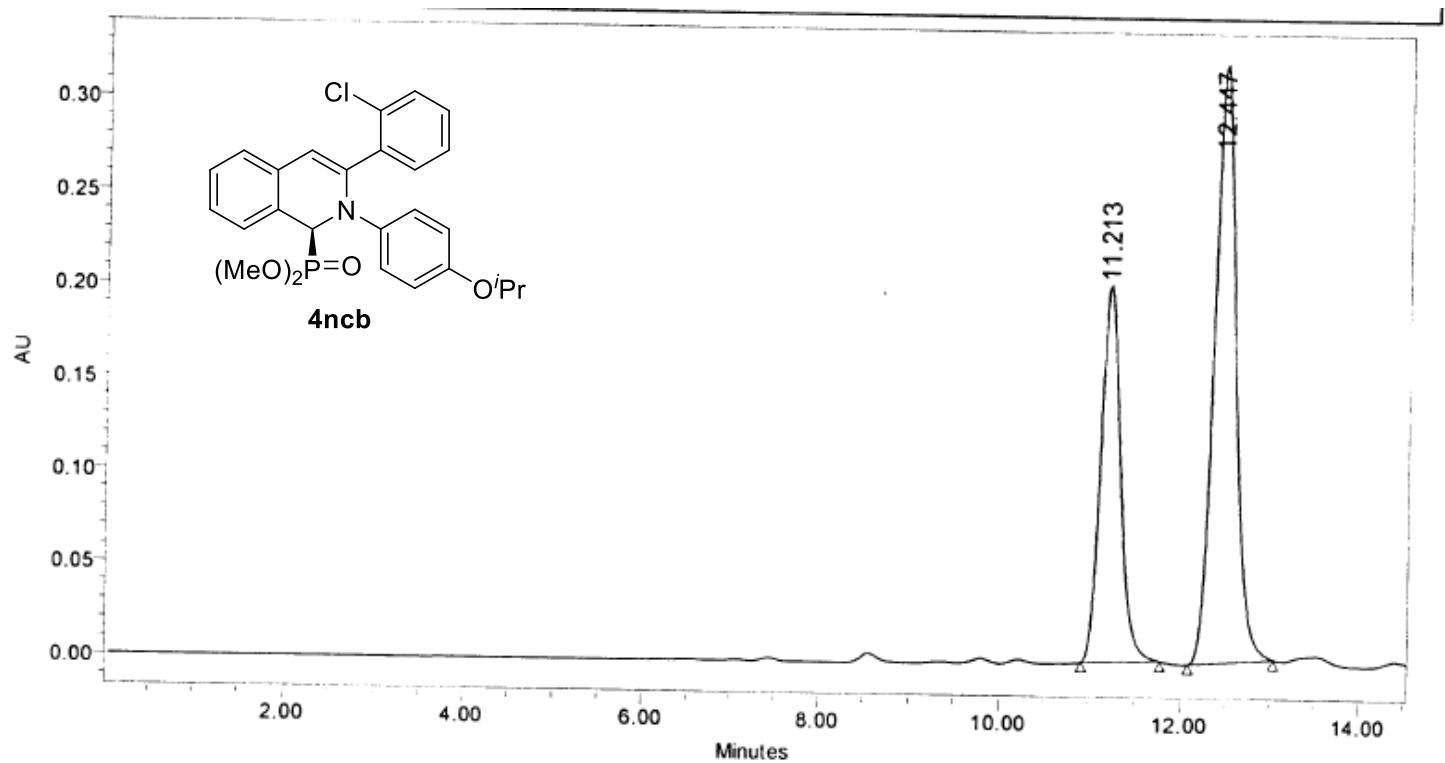

\begin{tabular}{|c|c|c|c|c|c|}
\hline & $\begin{array}{c}\text { RT } \\
(\min )\end{array}$ & $\begin{array}{c}\text { Area } \\
\left(\mu \mathrm{V}^{*} \mathrm{sec}\right)\end{array}$ & $\%$ Area & $\begin{array}{c}\text { Height } \\
(\mu \mathrm{V})\end{array}$ & $\begin{array}{c}\% \\
\text { Height }\end{array}$ \\
\hline 1 & 11.213 & 3062712 & 36.53 & 202799 & 38.75 \\
\hline 2 & 12.447 & 5321979 & 63.47 & 320588 & 61.25 \\
\hline
\end{tabular}




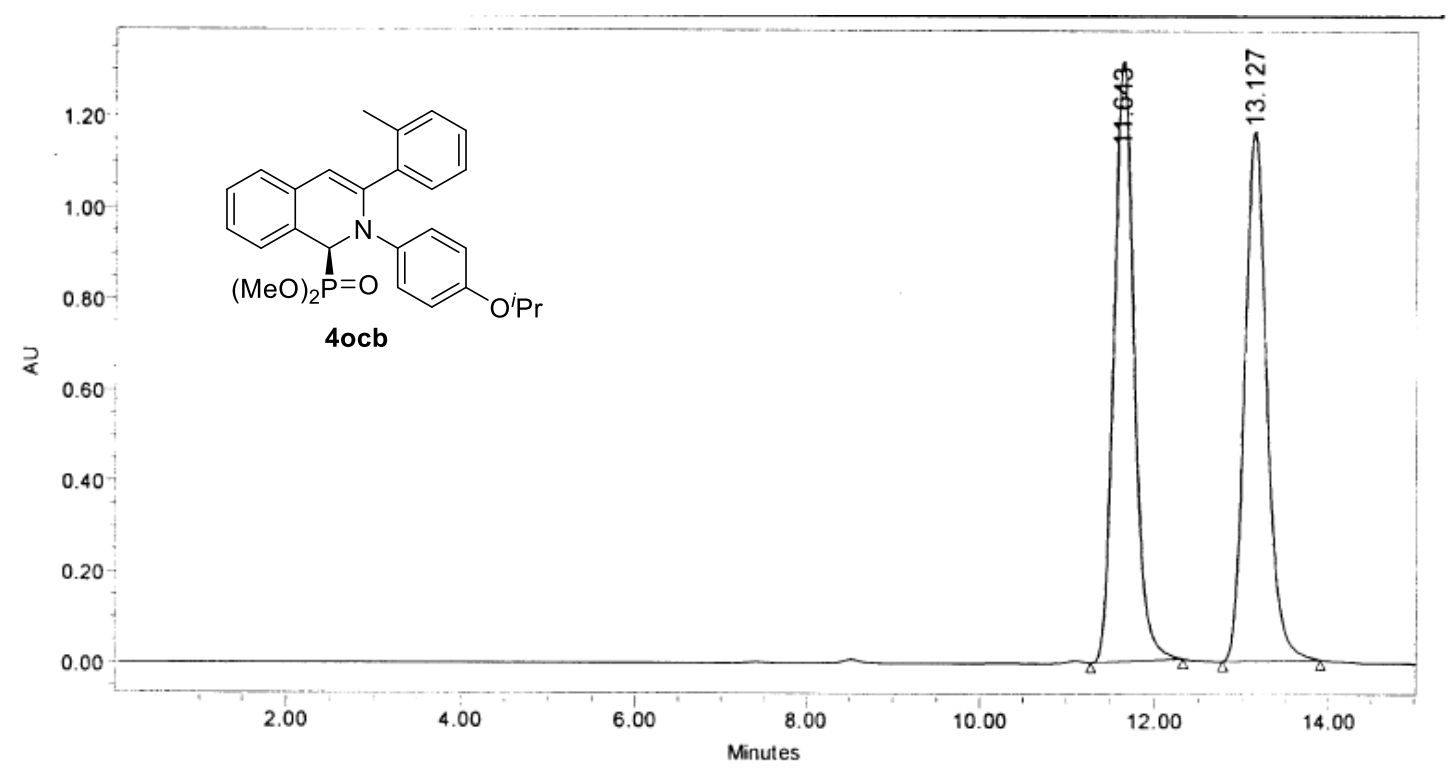

\begin{tabular}{|c|c|c|c|c|c|}
\hline & $\begin{array}{c}\text { RT } \\
(\mathrm{min})\end{array}$ & $\begin{array}{c}\text { Area } \\
(\mu \mathrm{V} * \mathrm{sec})\end{array}$ & $\%$ Area & $\begin{array}{c}\text { Height } \\
(\mu \mathrm{V})\end{array}$ & $\begin{array}{c}\% \\
\text { Height }\end{array}$ \\
\hline 1 & 11.643 & 20704089 & 50.02 & 1318831 & 53.12 \\
\hline 2 & 13.127 & 20687209 & 49.98 & 1163719 & 46.88 \\
\hline
\end{tabular}

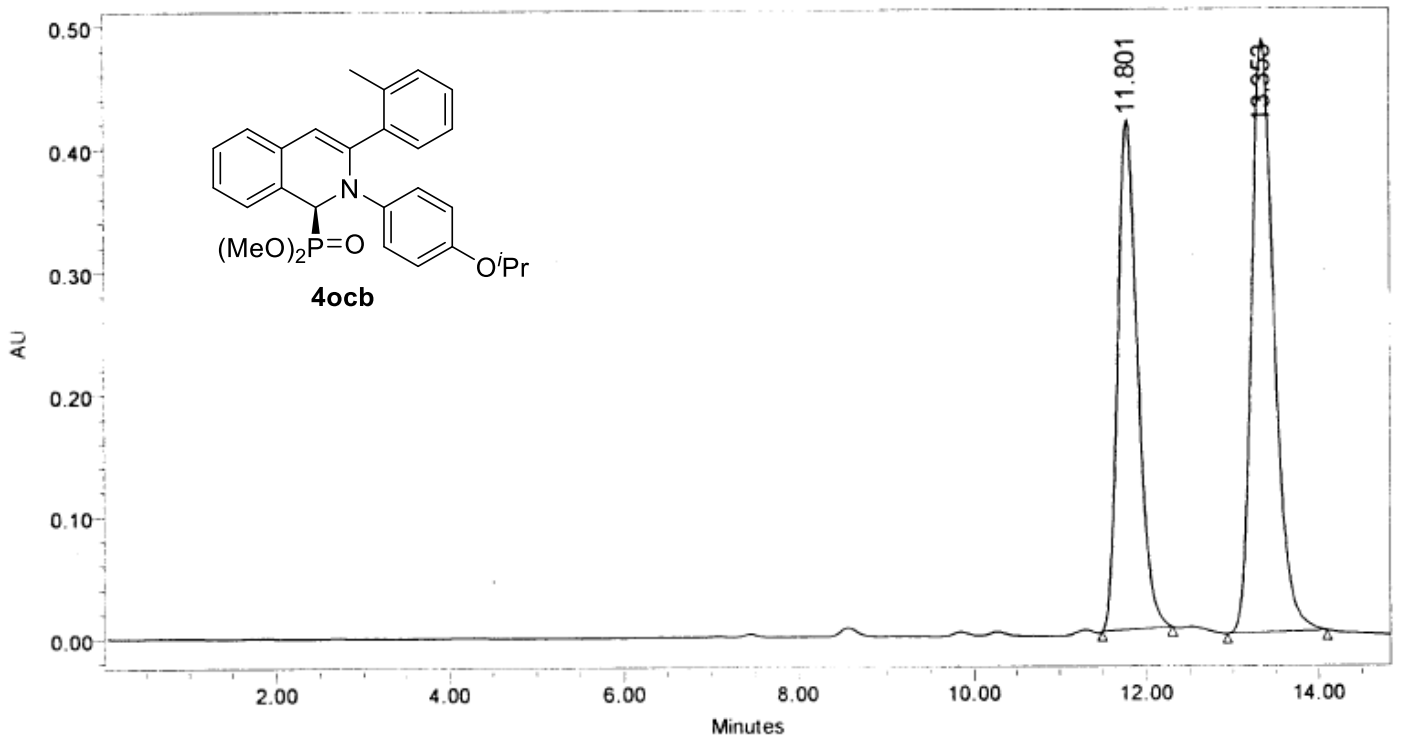

\begin{tabular}{|c|c|c|c|c|c|}
\hline & $\begin{array}{c}\text { RT } \\
(\mathrm{min})\end{array}$ & $\begin{array}{c}\text { Area } \\
(\mu \mathrm{V} * \mathrm{sec})\end{array}$ & $\%$ Area & $\begin{array}{c}\text { Height } \\
(\mu \mathrm{V})\end{array}$ & $\begin{array}{c}\% \\
\text { Height }\end{array}$ \\
\hline 1 & 11.801 & 6586812 & 42.57 & 416888 & 46.23 \\
\hline 2 & 13.353 & 8887680 & 57.43 & 484883 & 53.77 \\
\hline
\end{tabular}




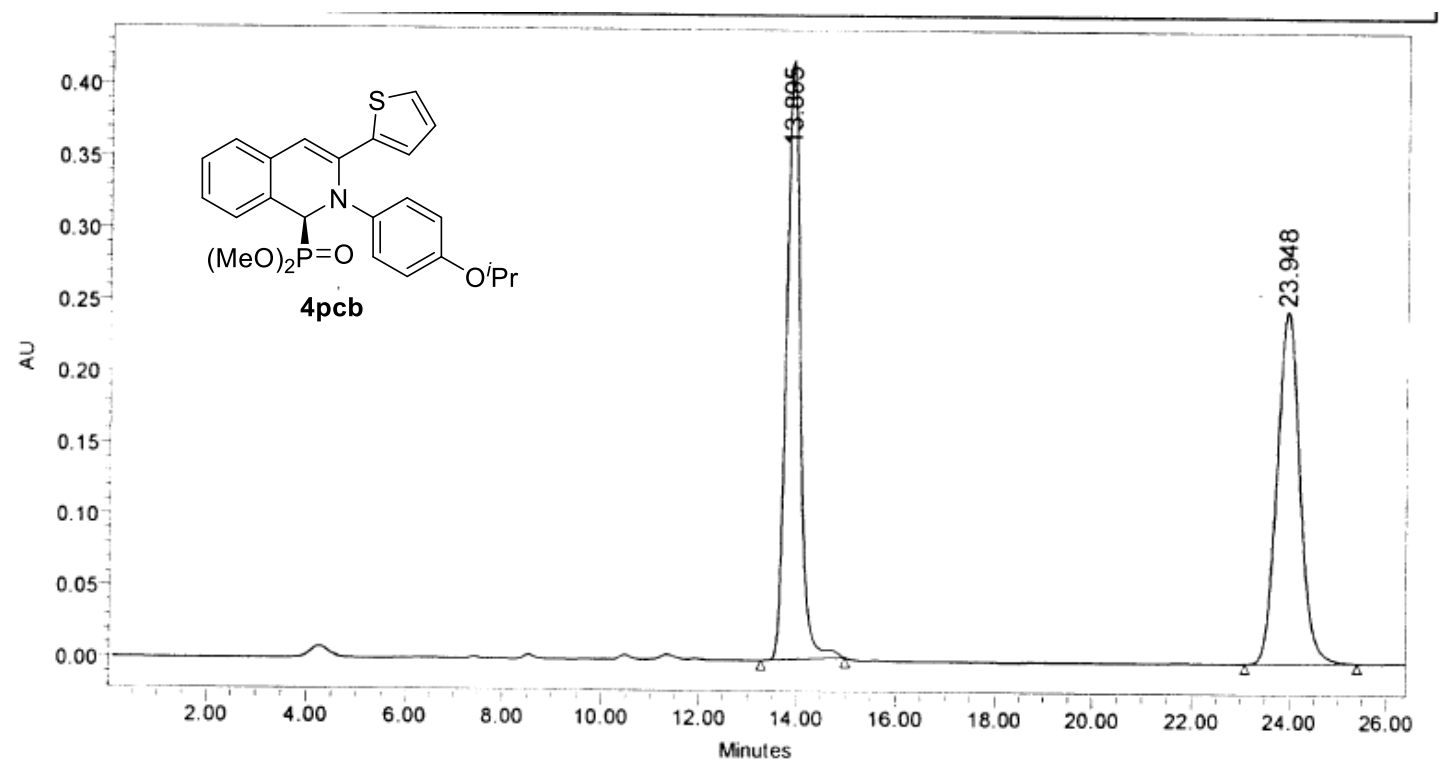

\begin{tabular}{|c|c|c|c|c|c|}
\hline & $\begin{array}{c}\text { RT } \\
(\mathrm{min})\end{array}$ & $\begin{array}{c}\text { Area } \\
\left(\mu \mathrm{V}^{*} \mathrm{sec}\right)\end{array}$ & $\%$ Area & $\begin{array}{c}\text { Height } \\
(\mu \mathrm{V})\end{array}$ & $\begin{array}{c}\% \\
\text { Height }\end{array}$ \\
\hline 1 & 13.895 & 8180641 & 50.20 & 418086 & 62.90 \\
\hline 2 & 23.948 & 8115275 & 49.80 & 246592 & 37.10 \\
\hline
\end{tabular}

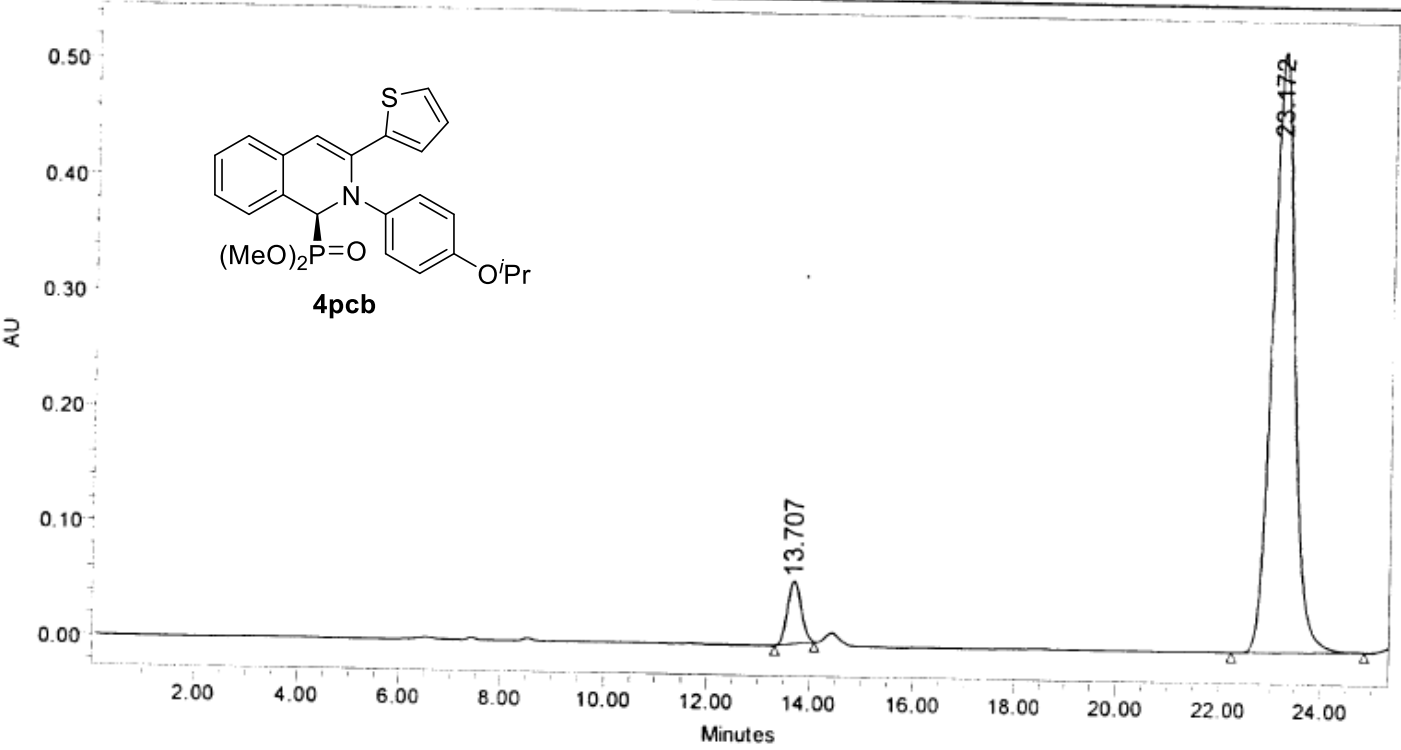

\begin{tabular}{|c|c|r|r|r|r|}
\hline & $\begin{array}{c}\text { RT } \\
(\mathrm{min})\end{array}$ & $\begin{array}{c}\text { Area } \\
(\mu \mathrm{V} * \mathrm{sec})\end{array}$ & $\%$ Area & $\begin{array}{c}\text { Height } \\
(\mu \mathrm{V})\end{array}$ & $\begin{array}{c}\% \\
\text { Height }\end{array}$ \\
\hline 1 & 13.707 & 1001858 & 5.98 & 54222 & 9.45 \\
\hline 2 & 23.172 & 15738069 & 94.02 & 519262 & 90.55 \\
\hline
\end{tabular}




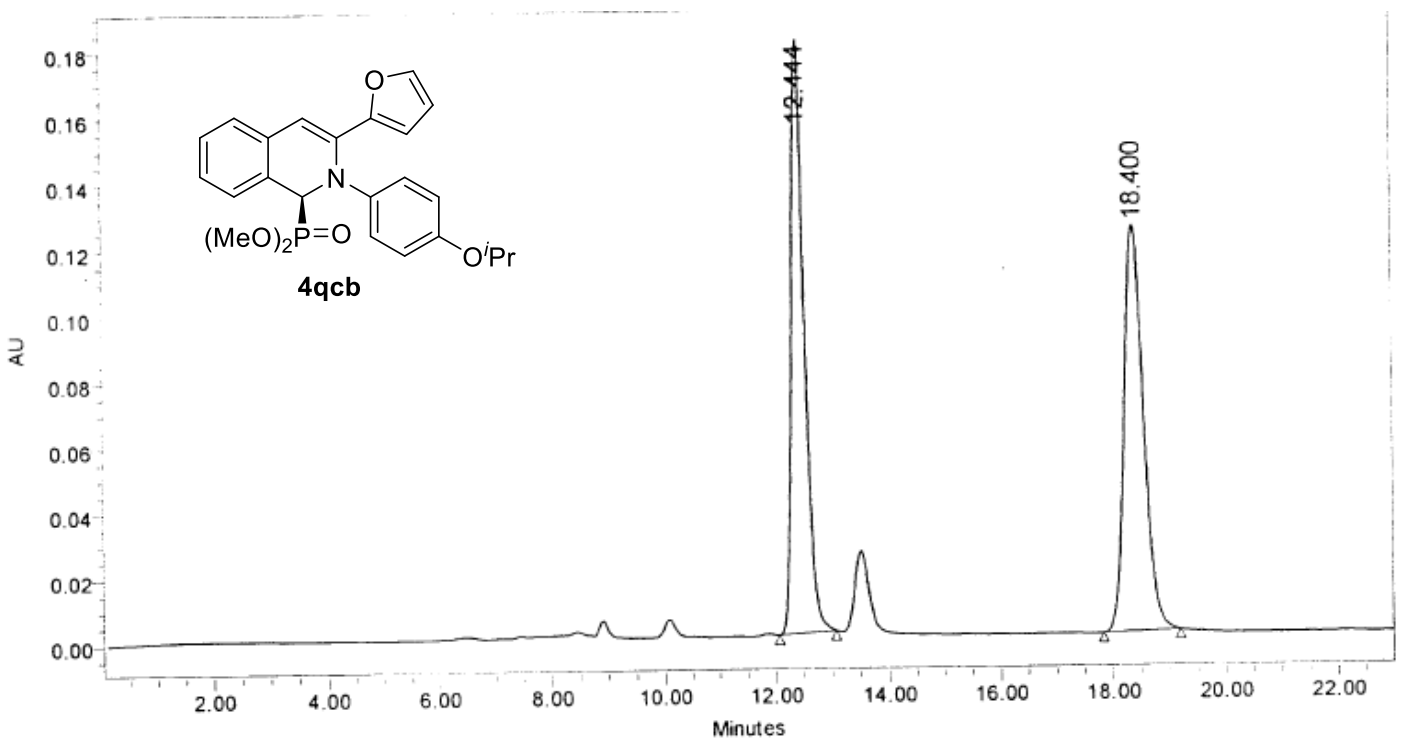

\begin{tabular}{|c|c|c|c|c|c|}
\hline & $\begin{array}{c}\text { RT } \\
(\mathrm{min})\end{array}$ & $\begin{array}{c}\text { Area } \\
(\mu \mathrm{V} * \mathrm{sec})\end{array}$ & $\%$ Area & $\begin{array}{c}\text { Height } \\
(\mu \mathrm{V})\end{array}$ & $\begin{array}{c}\% \\
\text { Height }\end{array}$ \\
\hline 1 & 12.444 & 2897773 & 49.91 & 180860 & 59.37 \\
\hline 2 & 18.400 & 2908653 & 50.09 & 123769 & 40.63 \\
\hline
\end{tabular}

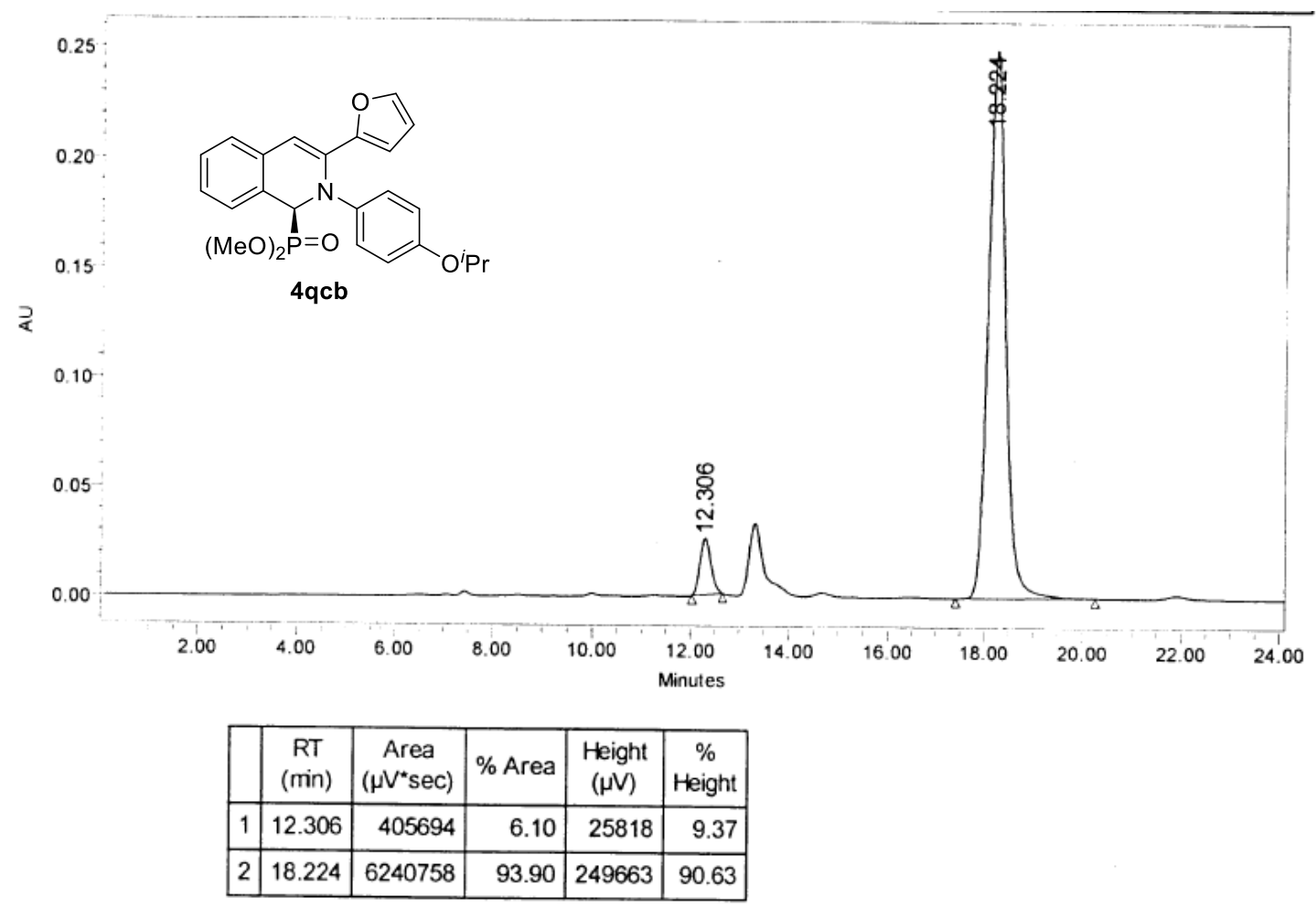



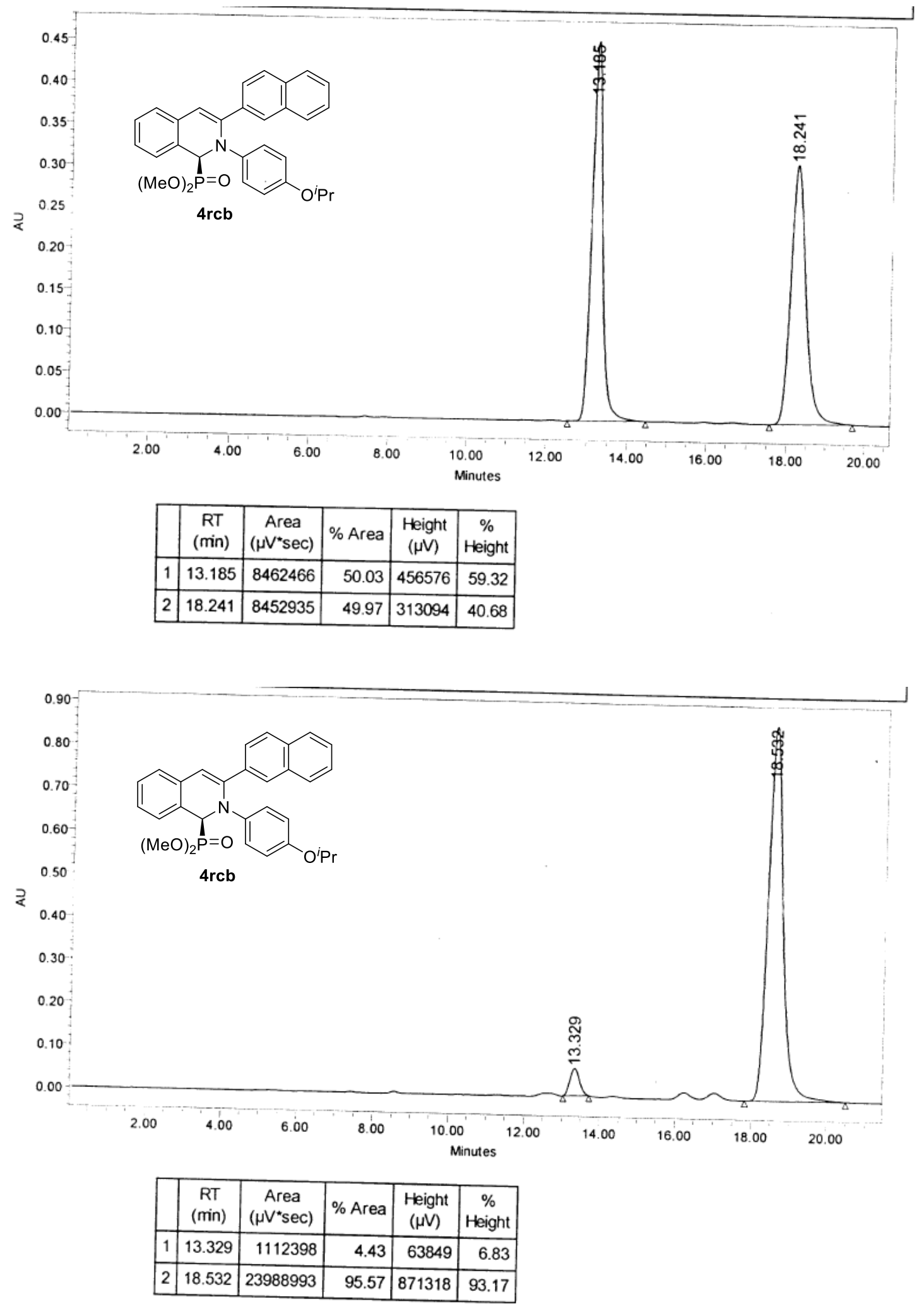

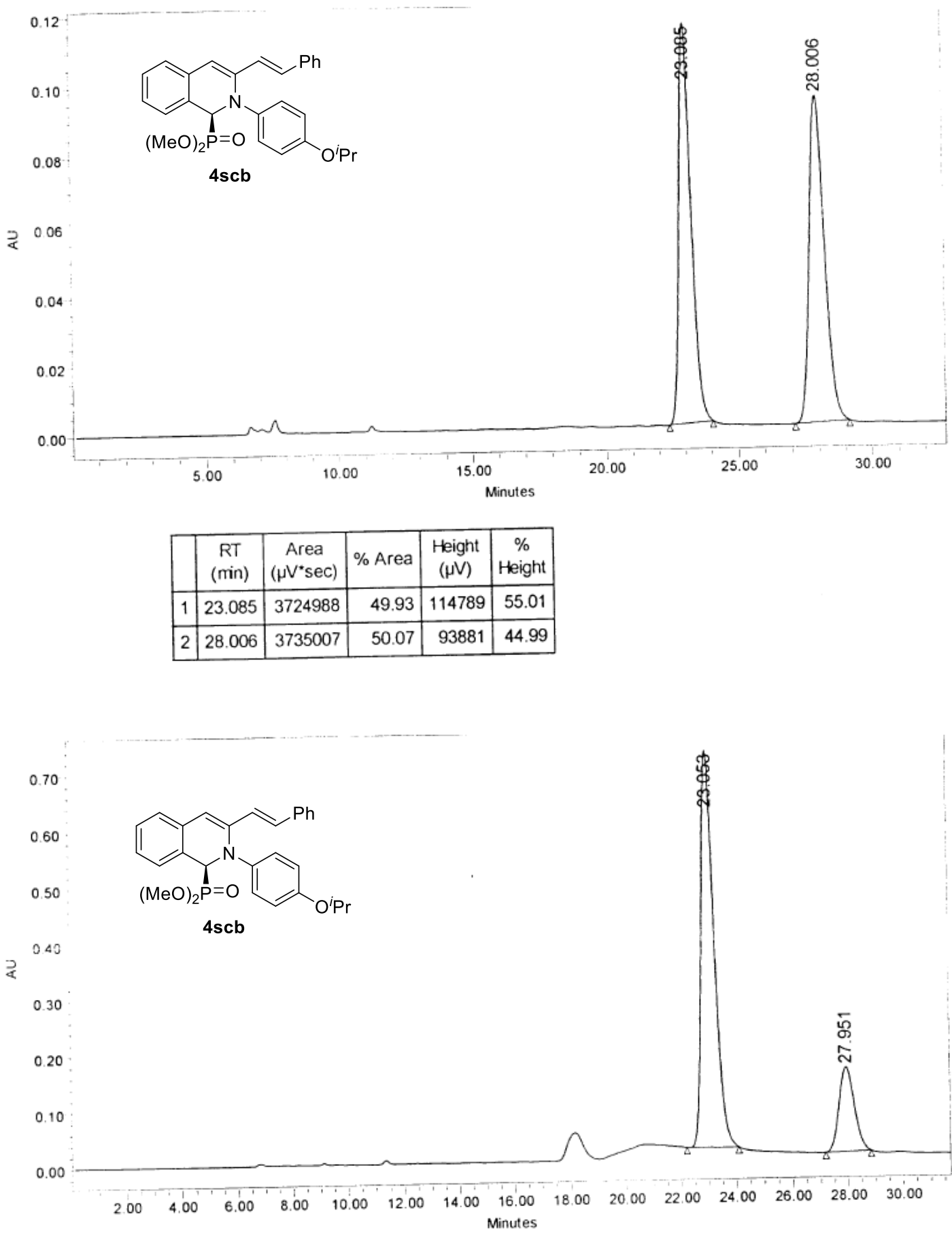

\begin{tabular}{|c|c|c|c|c|c|}
\hline & $\begin{array}{c}\text { RT } \\
(\mathrm{min})\end{array}$ & $\begin{array}{c}\text { Area } \\
(\mu \mathrm{V} * \mathrm{sec})\end{array}$ & $\%$ Area & $\begin{array}{c}\text { Height } \\
(\mu \mathrm{V})\end{array}$ & $\begin{array}{c}\% \\
\text { Height }\end{array}$ \\
\hline 1 & 23.053 & 22976034 & 79.75 & 707662 & 82.37 \\
\hline 2 & 27.951 & 5833656 & 20.25 & 151420 & 17.63 \\
\hline
\end{tabular}




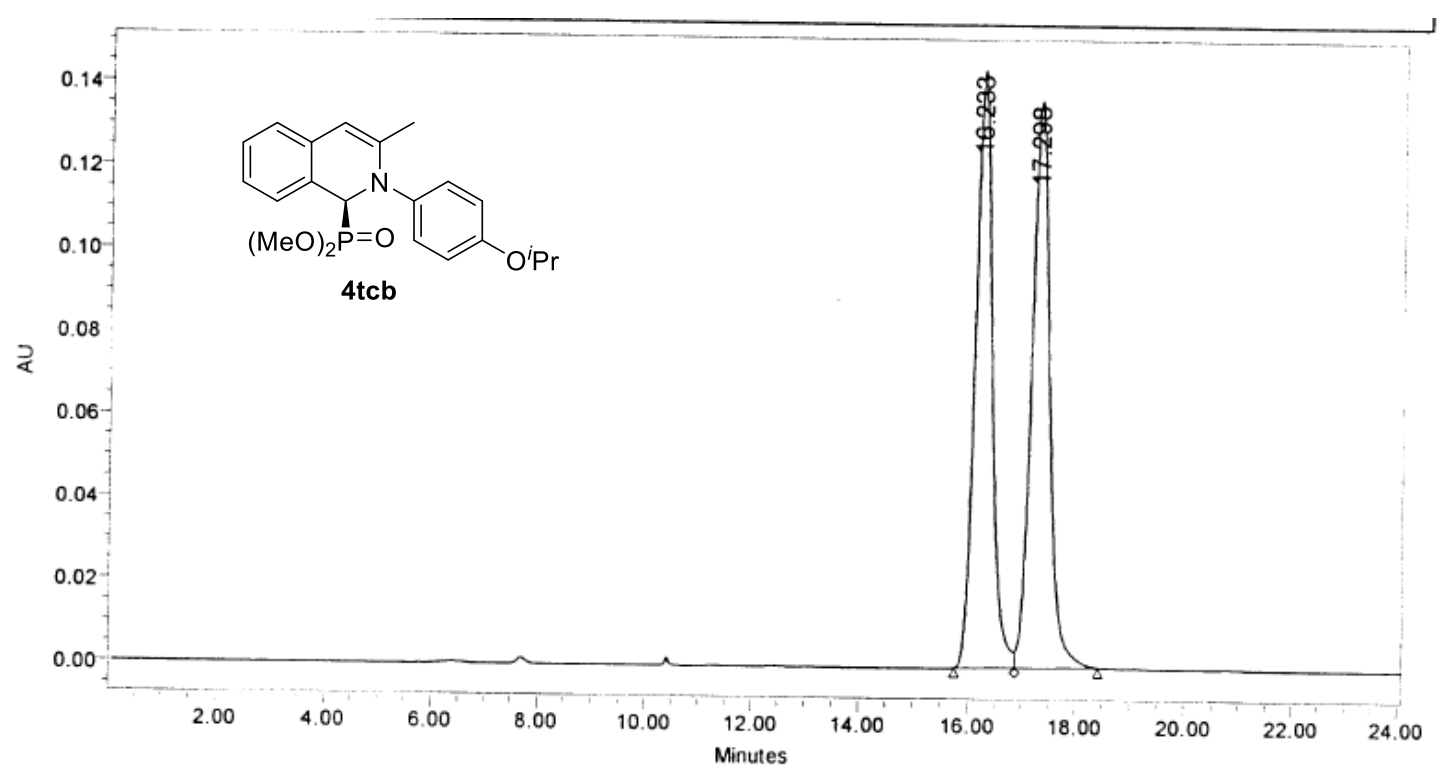

\begin{tabular}{|c|c|c|c|c|c|}
\hline & $\begin{array}{c}\text { RT } \\
(\mathrm{min})\end{array}$ & $\begin{array}{c}\text { Area } \\
(\mu \mathrm{V} * \mathrm{sec})\end{array}$ & $\%$ Area & $\begin{array}{c}\text { Height } \\
(\mu \mathrm{V})\end{array}$ & $\begin{array}{c}\% \\
\text { Height }\end{array}$ \\
\hline 1 & 16.233 & 3139568 & 49.47 & 144121 & 51.31 \\
\hline 2 & 17.298 & 3207458 & 50.53 & 136739 & 48.69 \\
\hline
\end{tabular}

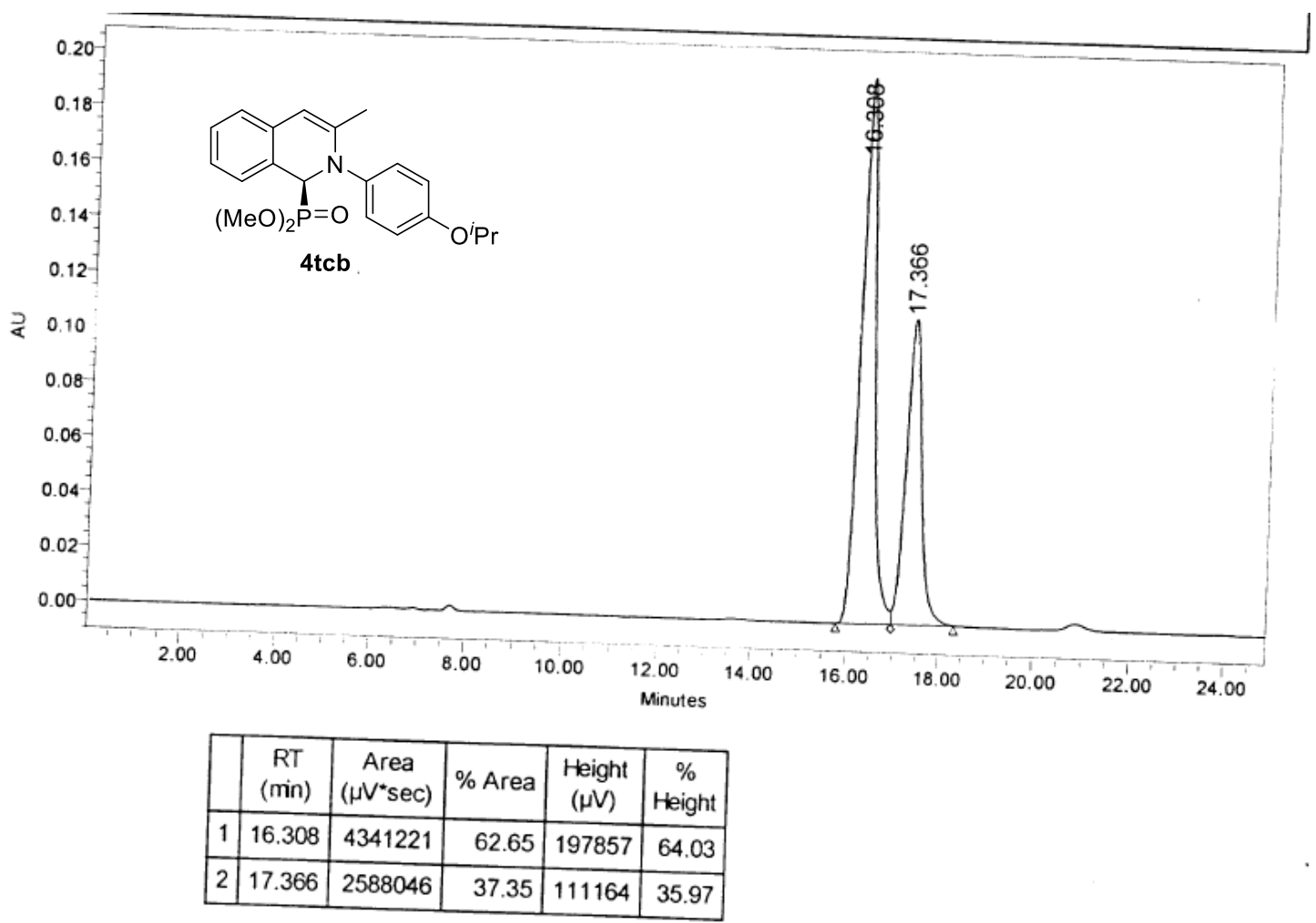




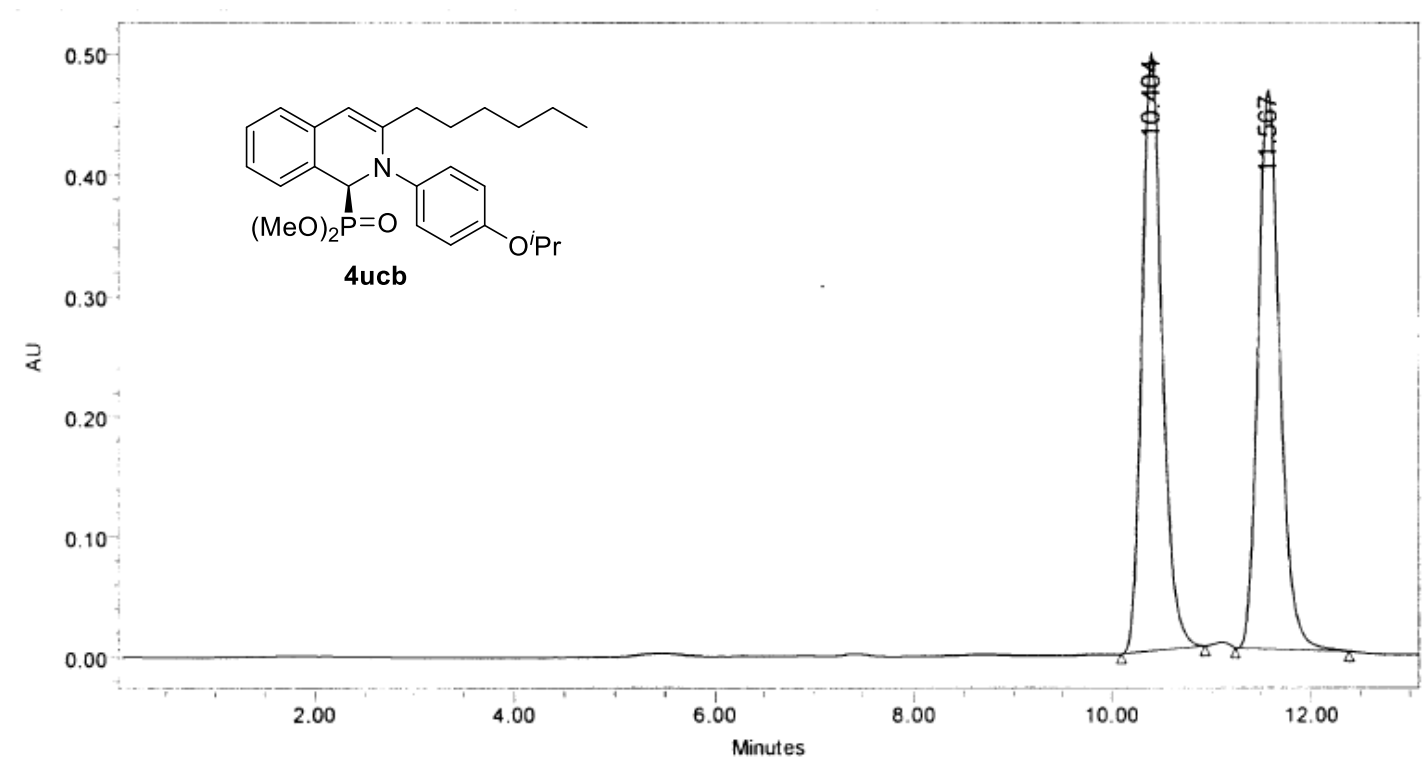

\begin{tabular}{|c|c|c|c|c|c|}
\hline & $\begin{array}{c}\text { RT } \\
(\mathrm{min})\end{array}$ & $\begin{array}{c}\text { Area } \\
\left(\mu \mathrm{V}^{*} \mathrm{sec}\right)\end{array}$ & $\%$ Area & $\begin{array}{c}\text { Height } \\
(\mu \mathrm{V})\end{array}$ & $\begin{array}{c}\% \\
\text { Height }\end{array}$ \\
\hline 1 & 10.404 & 6932859 & 49.50 & 495829 & 51.70 \\
\hline 2 & 11.567 & 7071928 & 50.50 & 463274 & 48.30 \\
\hline
\end{tabular}

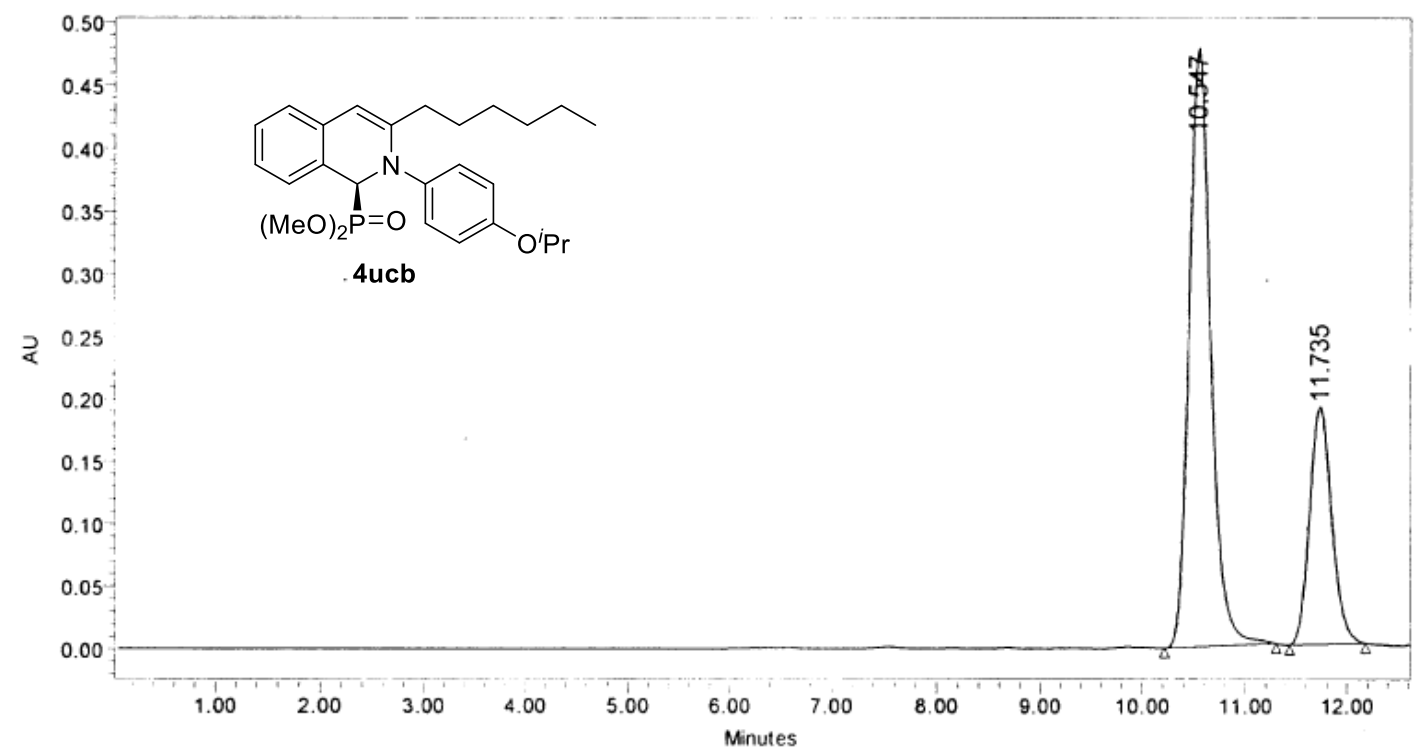

\begin{tabular}{|c|c|c|c|c|c|}
\hline & $\begin{array}{c}\text { RT } \\
(\mathrm{min})\end{array}$ & $\begin{array}{c}\text { Area } \\
(\mu \mathrm{V} * \mathrm{sec})\end{array}$ & $\%$ Area & $\begin{array}{c}\text { Height } \\
(\mu \mathrm{V})\end{array}$ & $\begin{array}{c}\% \\
\text { Height }\end{array}$ \\
\hline 1 & 10.547 & 6949611 & 70.66 & 477735 & 71.52 \\
\hline 2 & 11.735 & 2886126 & 29.34 & 190244 & 28.48 \\
\hline
\end{tabular}



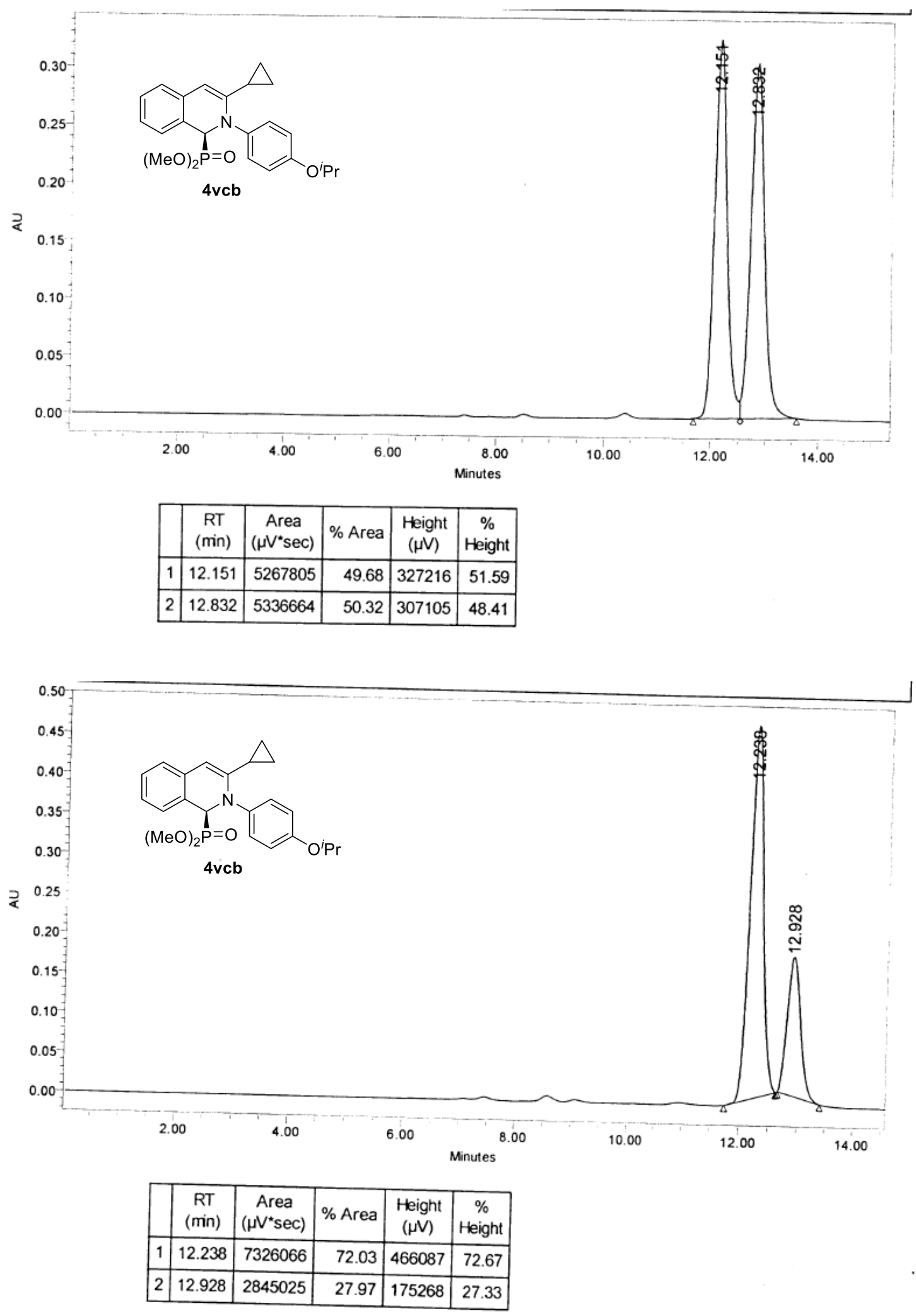


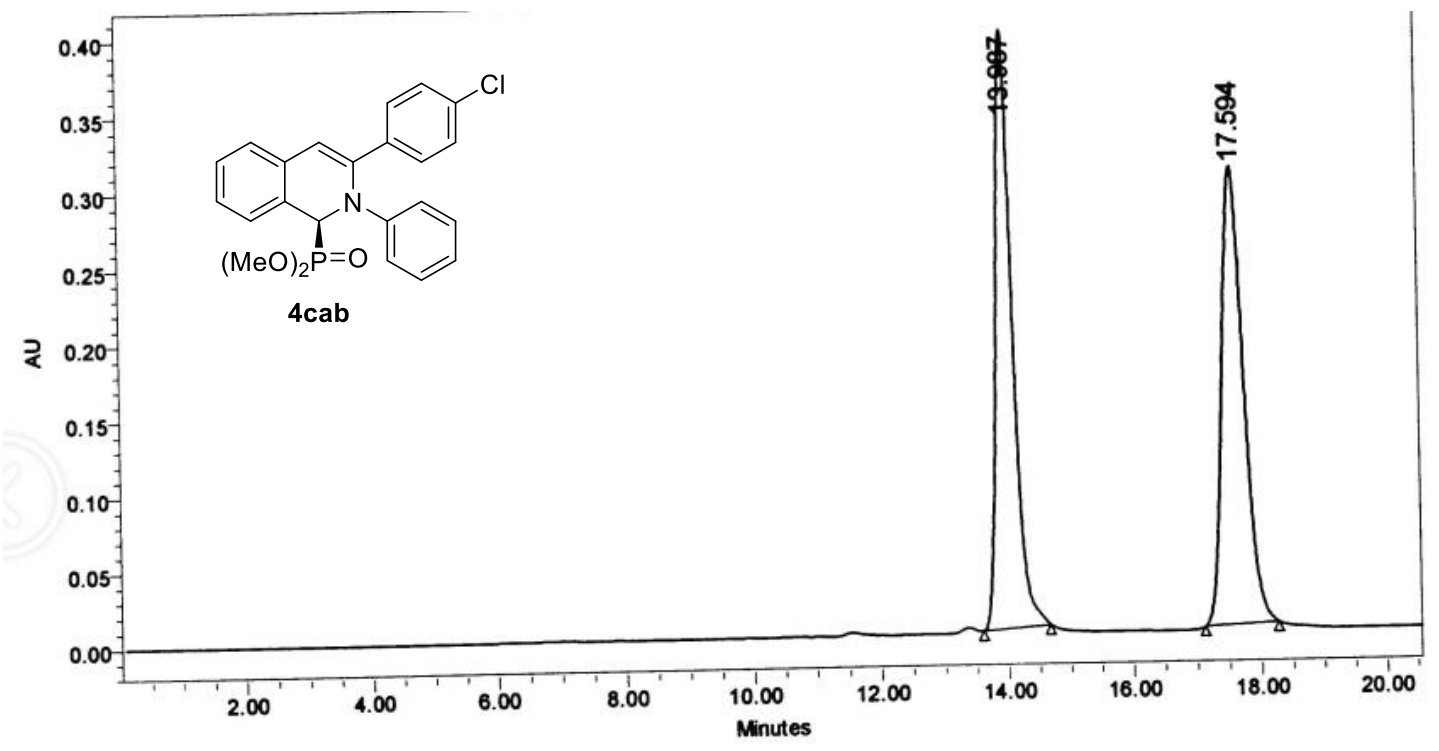

\begin{tabular}{|c|c|c|c|c|c|}
\hline & $\begin{array}{c}\mathrm{RT} \\
(\min )\end{array}$ & $\begin{array}{c}\text { Area } \\
\left(\mu \mathrm{V}^{*} \mathrm{sec}\right)\end{array}$ & $\%$ Area & $\begin{array}{c}\text { Height } \\
(\mu \mathrm{V})\end{array}$ & $\begin{array}{c}\% \\
\text { Height }\end{array}$ \\
\hline 1 & 13.987 & 7195918 & 50.45 & 394782 & 56.60 \\
\hline 2 & 17.594 & 7068707 & 49.55 & 302742 & 43.40 \\
\hline
\end{tabular}

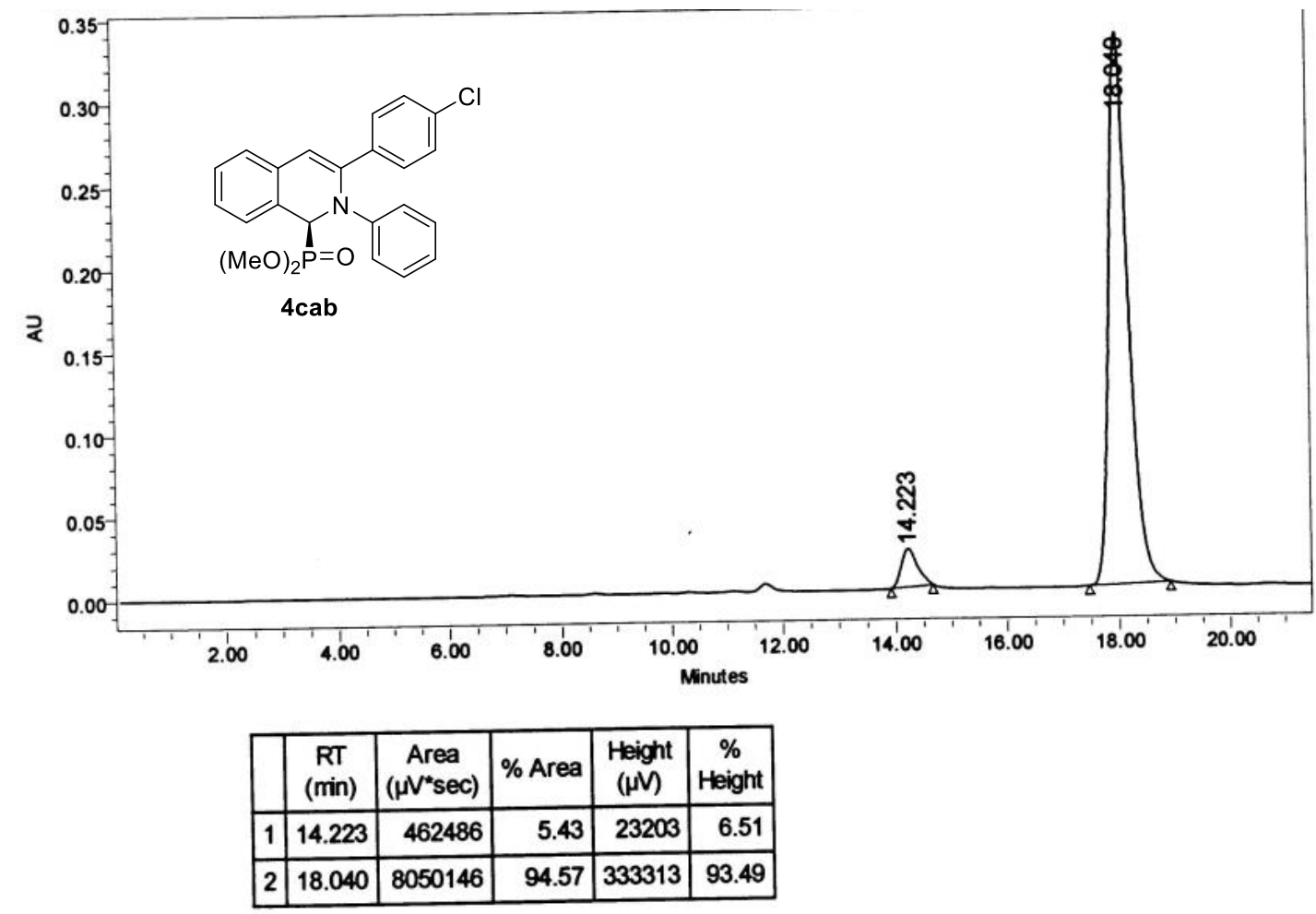




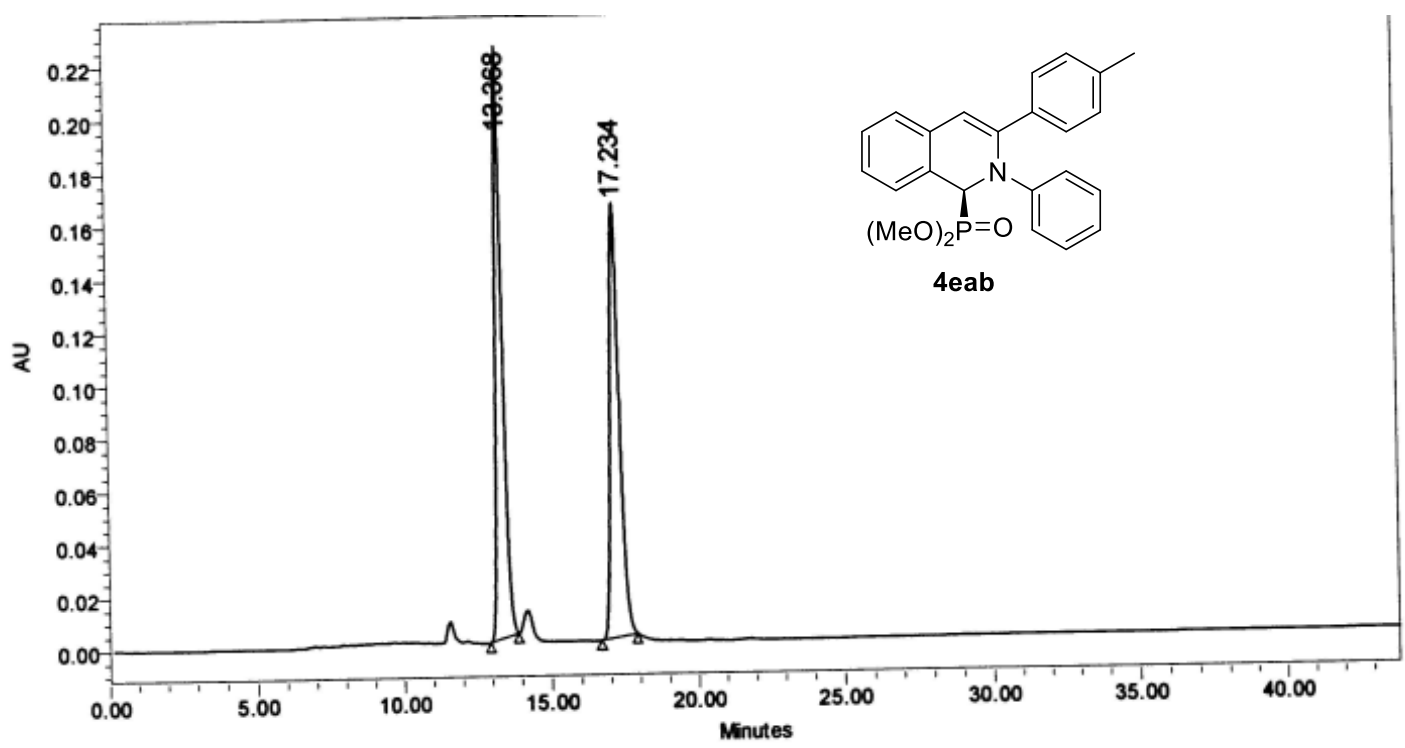

\begin{tabular}{|c|c|c|c|c|c|}
\hline & $\begin{array}{c}\mathrm{RT} \\
(\mathrm{min})\end{array}$ & $\begin{array}{c}\text { Area } \\
(\mu \mathrm{N} \text { sec })\end{array}$ & $\%$ Area & $\begin{array}{c}\text { Height } \\
(\mu \mathrm{V})\end{array}$ & $\begin{array}{c}\% \\
\text { Height }\end{array}$ \\
\hline 1 & 13.368 & 3718141 & 50.53 & 223191 & 57.62 \\
\hline 2 & 17.234 & 3640718 & 49.47 & 164180 & 42.38 \\
\hline
\end{tabular}

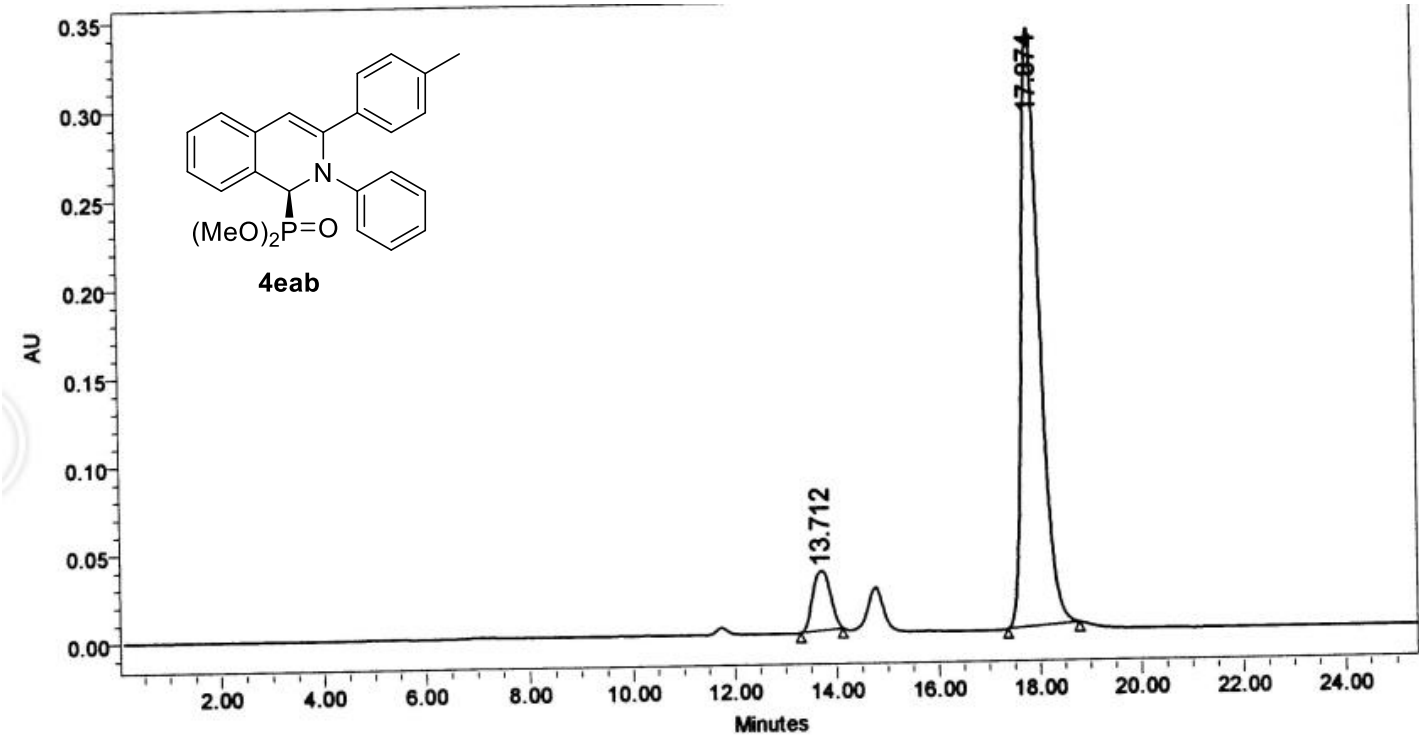

\begin{tabular}{|c|c|c|r|r|r|}
\hline & $\begin{array}{c}\text { RT } \\
(\mathrm{min})\end{array}$ & $\begin{array}{c}\text { Area } \\
\left(\mu \mathrm{N}^{*} \mathrm{sec}\right)\end{array}$ & $\%$ Area & $\begin{array}{c}\text { Height } \\
(\mu \mathrm{V})\end{array}$ & $\begin{array}{c}\% \\
\text { Height }\end{array}$ \\
\hline 1 & 13.712 & 804142 & 8.84 & 33666 & 9.07 \\
\hline 2 & 17.874 & 8296509 & 91.16 & 337617 & 90.93 \\
\hline
\end{tabular}




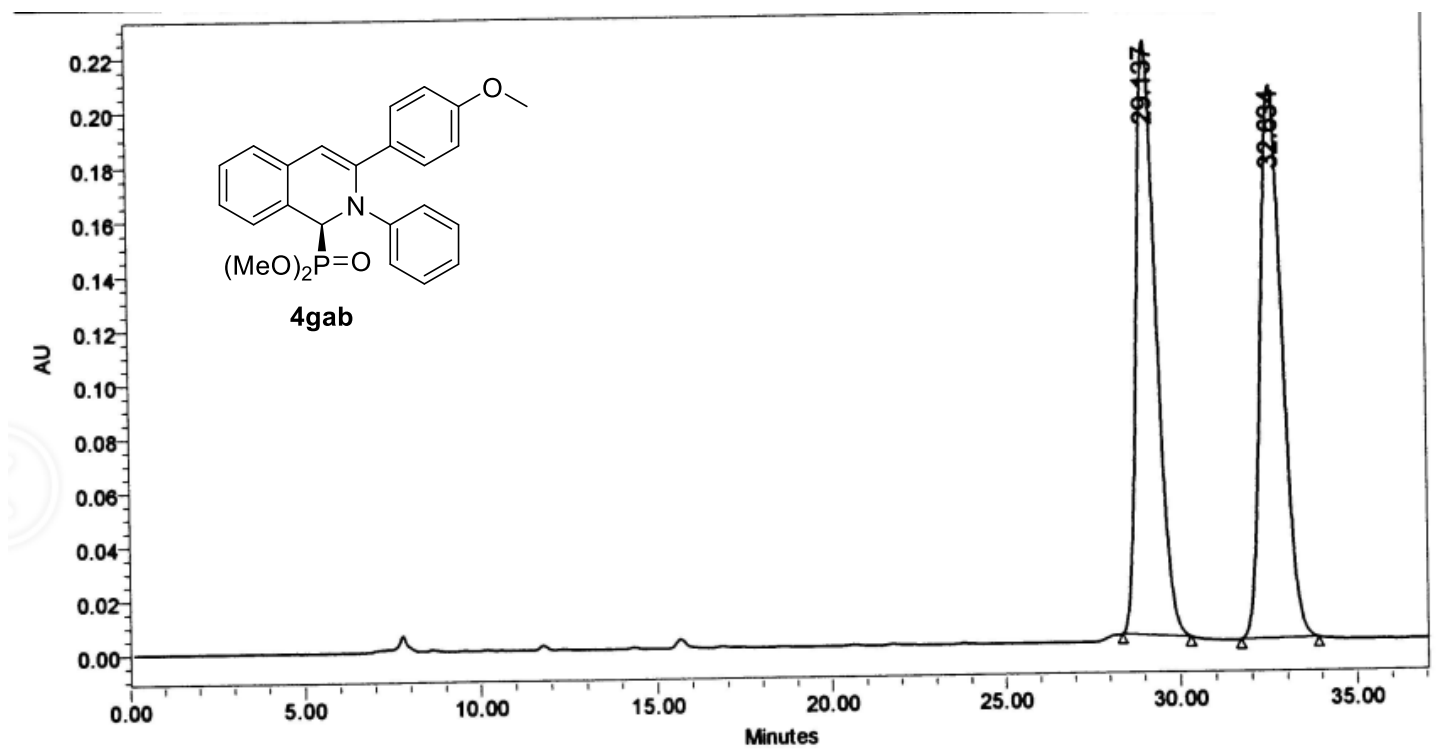

\begin{tabular}{|c|c|c|c|c|c|}
\hline & $\begin{array}{c}\text { RT } \\
(\min )\end{array}$ & $\begin{array}{c}\text { Area } \\
\left(\mu \mathrm{N}^{*} \mathrm{sec}\right)\end{array}$ & $\%$ Area & $\begin{array}{c}\text { Height } \\
(\mu \mathrm{V})\end{array}$ & $\begin{array}{c}\% \\
\text { Height }\end{array}$ \\
\hline 1 & 29.137 & 8587876 & 49.53 & 219515 & 51.78 \\
\hline 2 & 32.634 & 8750623 & 50.47 & 204412 & 48.22 \\
\hline
\end{tabular}

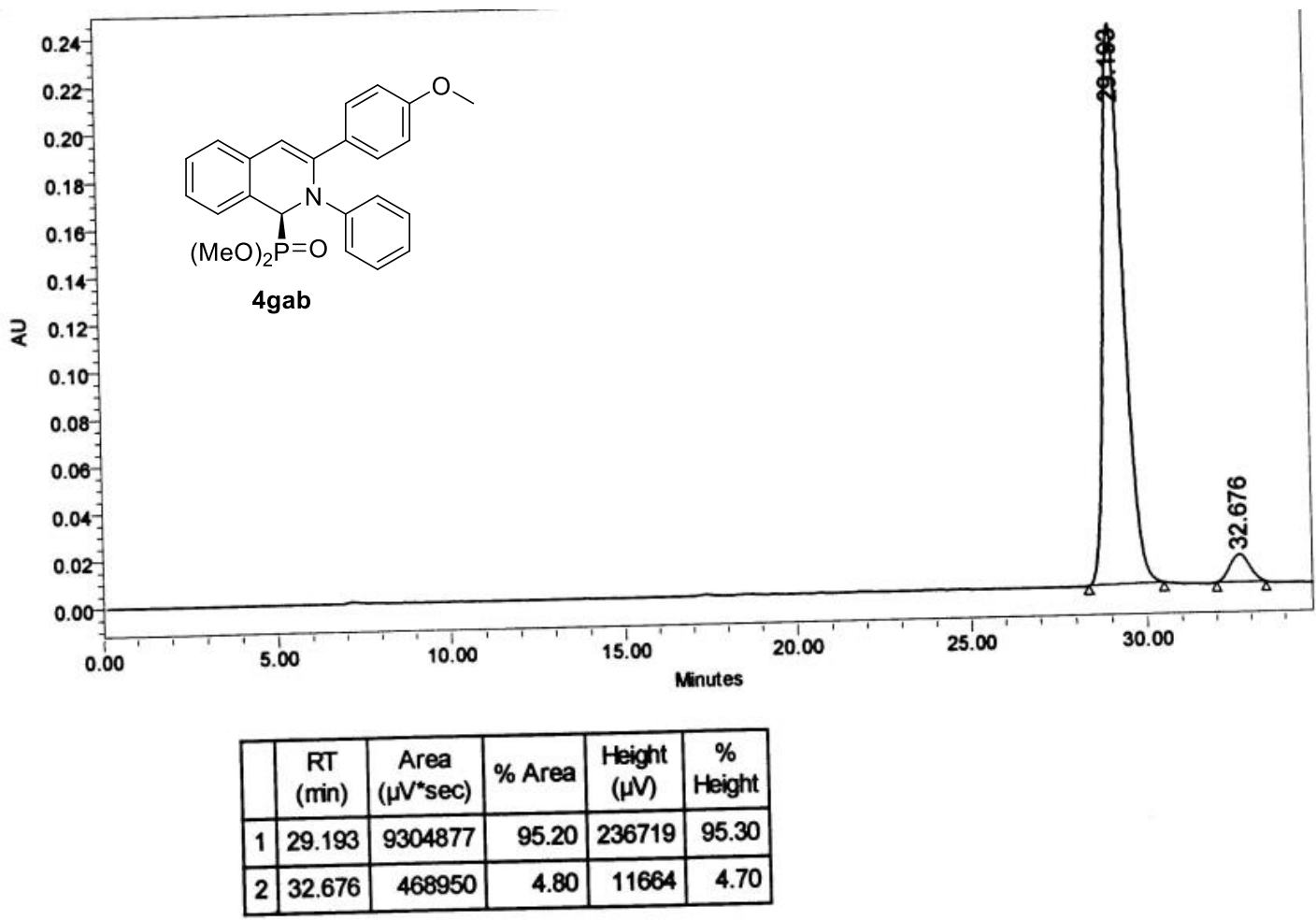




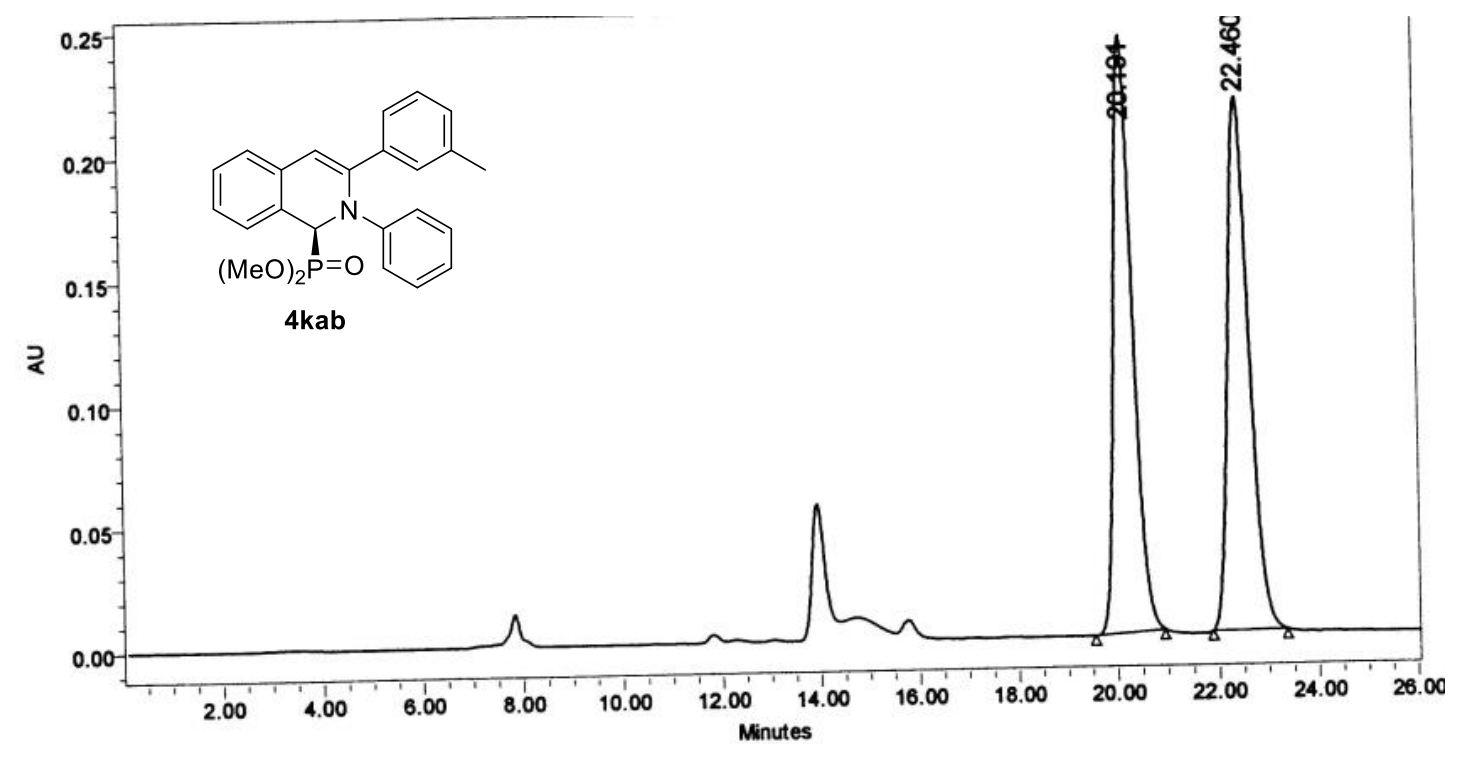

\begin{tabular}{|c|c|c|c|c|c|}
\hline & $\begin{array}{c}\text { RT } \\
(\mathrm{min})\end{array}$ & $\begin{array}{c}\text { Area } \\
(\mu \mathrm{N} \text { sec })\end{array}$ & $\%$ Area & $\begin{array}{c}\text { Height } \\
(\mu \mathrm{V})\end{array}$ & $\begin{array}{c}\% \\
\text { Height }\end{array}$ \\
\hline 1 & 20.191 & 6305643 & 49.76 & 241807 & 52.80 \\
\hline 2 & 22.460 & 6367692 & 50.24 & 216125 & 47.20 \\
\hline
\end{tabular}

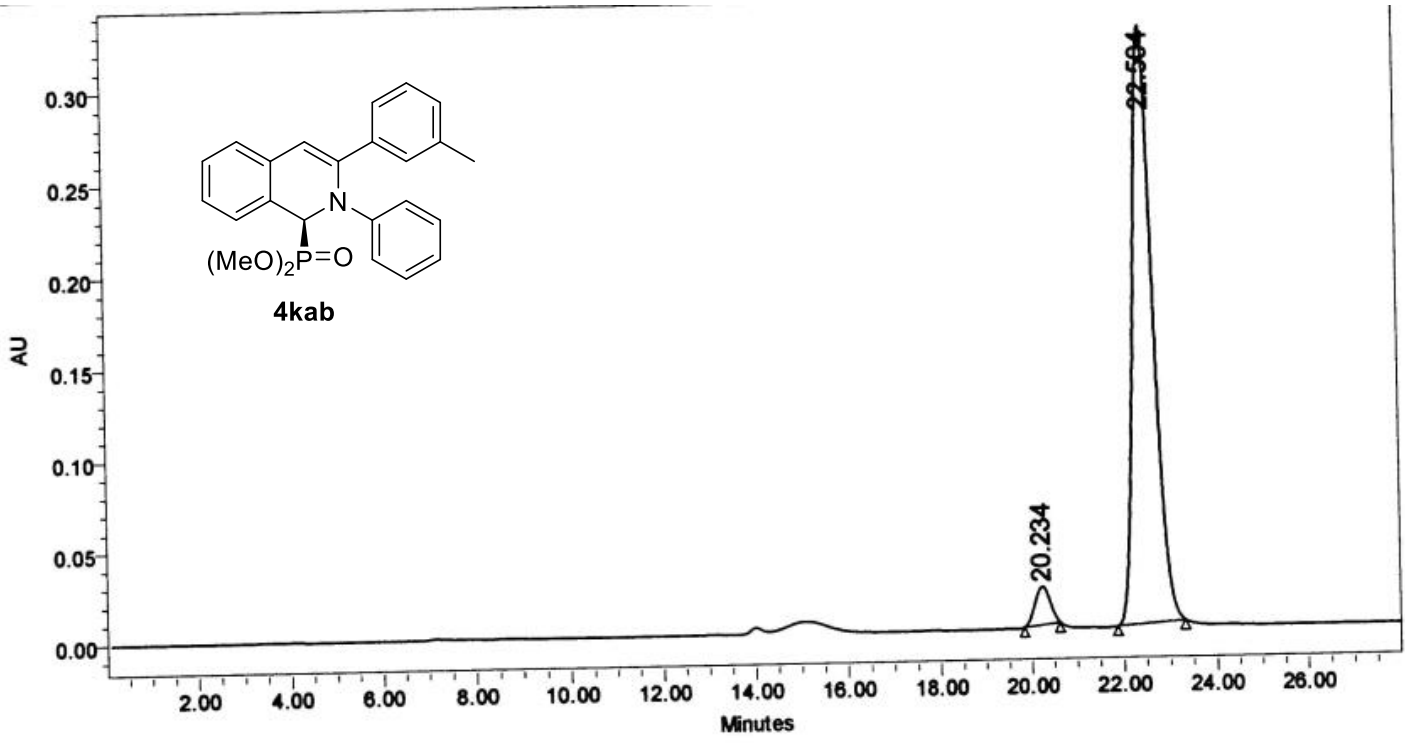

\begin{tabular}{|c|c|c|c|c|r|}
\hline & $\begin{array}{c}\text { RT } \\
(\mathrm{min})\end{array}$ & $\begin{array}{c}\text { Area } \\
(\mu \mathrm{V} * \mathrm{sec})\end{array}$ & $\%$ Area & $\begin{array}{l}\text { Height } \\
(\mu \mathrm{V})\end{array}$ & $\begin{array}{c}\% \\
\text { Height }\end{array}$ \\
\hline 1 & 20.234 & 481220 & 4.76 & 20799 & 6.01 \\
\hline 2 & 22.504 & 9622528 & 95.24 & 325357 & 93.99 \\
\hline
\end{tabular}




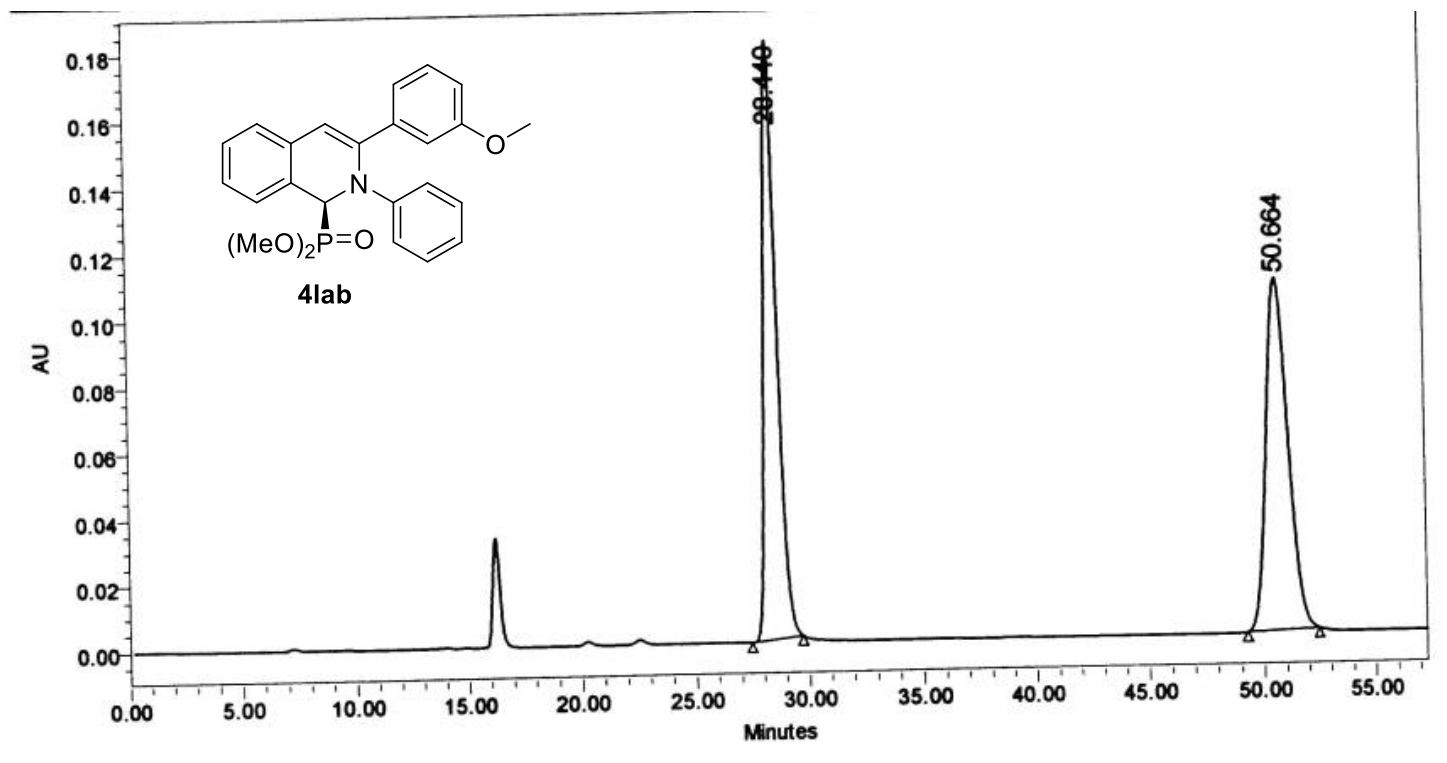

\begin{tabular}{|c|c|c|c|c|c|}
\hline & $\begin{array}{c}\text { RT } \\
(\min )\end{array}$ & $\begin{array}{c}\text { Area } \\
\left(\mu \mathrm{V}^{*} \mathrm{sec}\right)\end{array}$ & $\%$ Area & $\begin{array}{c}\text { Height } \\
(\mu \mathrm{V})\end{array}$ & $\begin{array}{c}\% \\
\text { Height }\end{array}$ \\
\hline 1 & 28.440 & 7323342 & 50.48 & 180976 & 62.89 \\
\hline 2 & 50.664 & 7185390 & 49.52 & 106799 & 37.11 \\
\hline
\end{tabular}

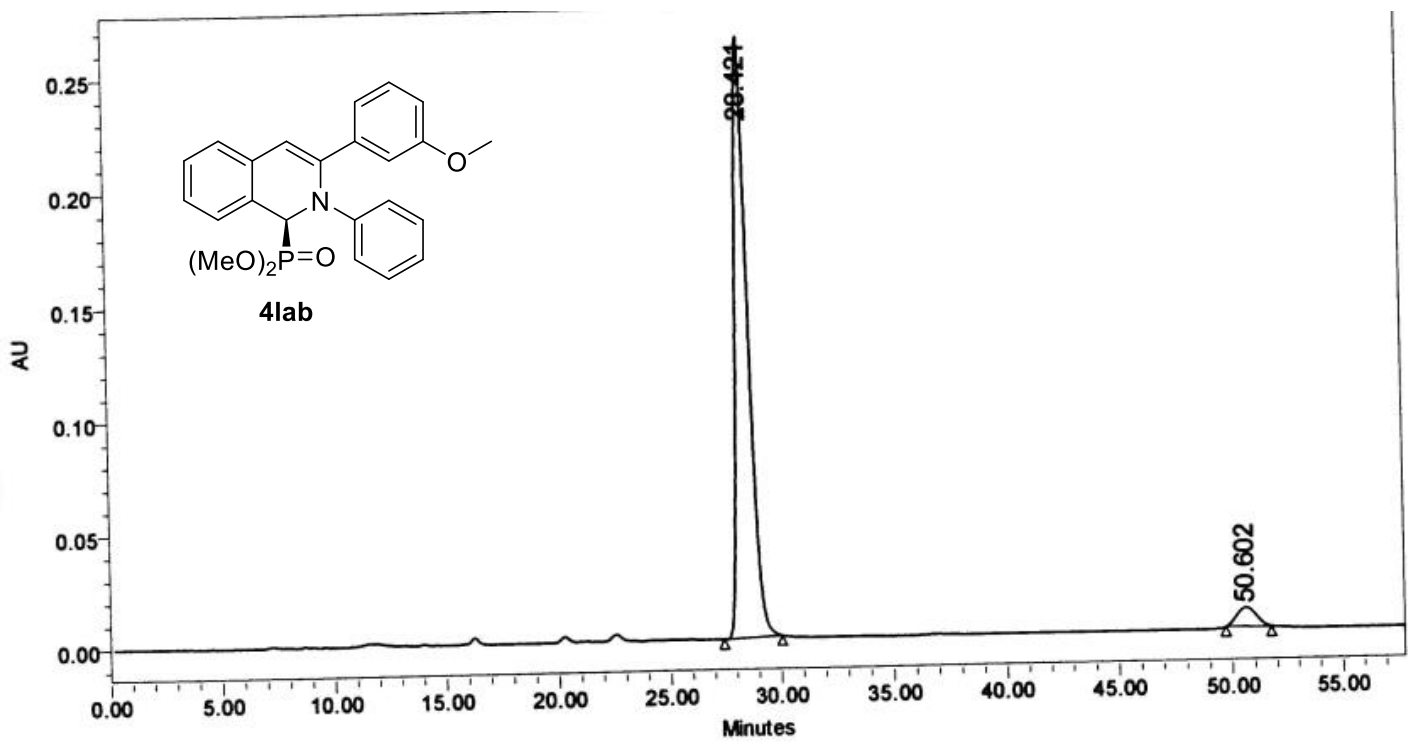

\begin{tabular}{|c|c|r|r|r|r|}
\hline & $\begin{array}{c}\mathrm{RT} \\
(\mathrm{min})\end{array}$ & $\begin{array}{c}\text { Area } \\
(\mu \mathrm{V} * \mathrm{sec})\end{array}$ & $\%$ Area & $\begin{array}{c}\text { Height } \\
(\mu \mathrm{V})\end{array}$ & $\begin{array}{c}\% \\
\text { Height }\end{array}$ \\
\hline 1 & 28.421 & 10687560 & 95.49 & 264015 & 96.92 \\
\hline 2 & 50.602 & 505285 & 4.51 & 8398 & 3.08 \\
\hline
\end{tabular}



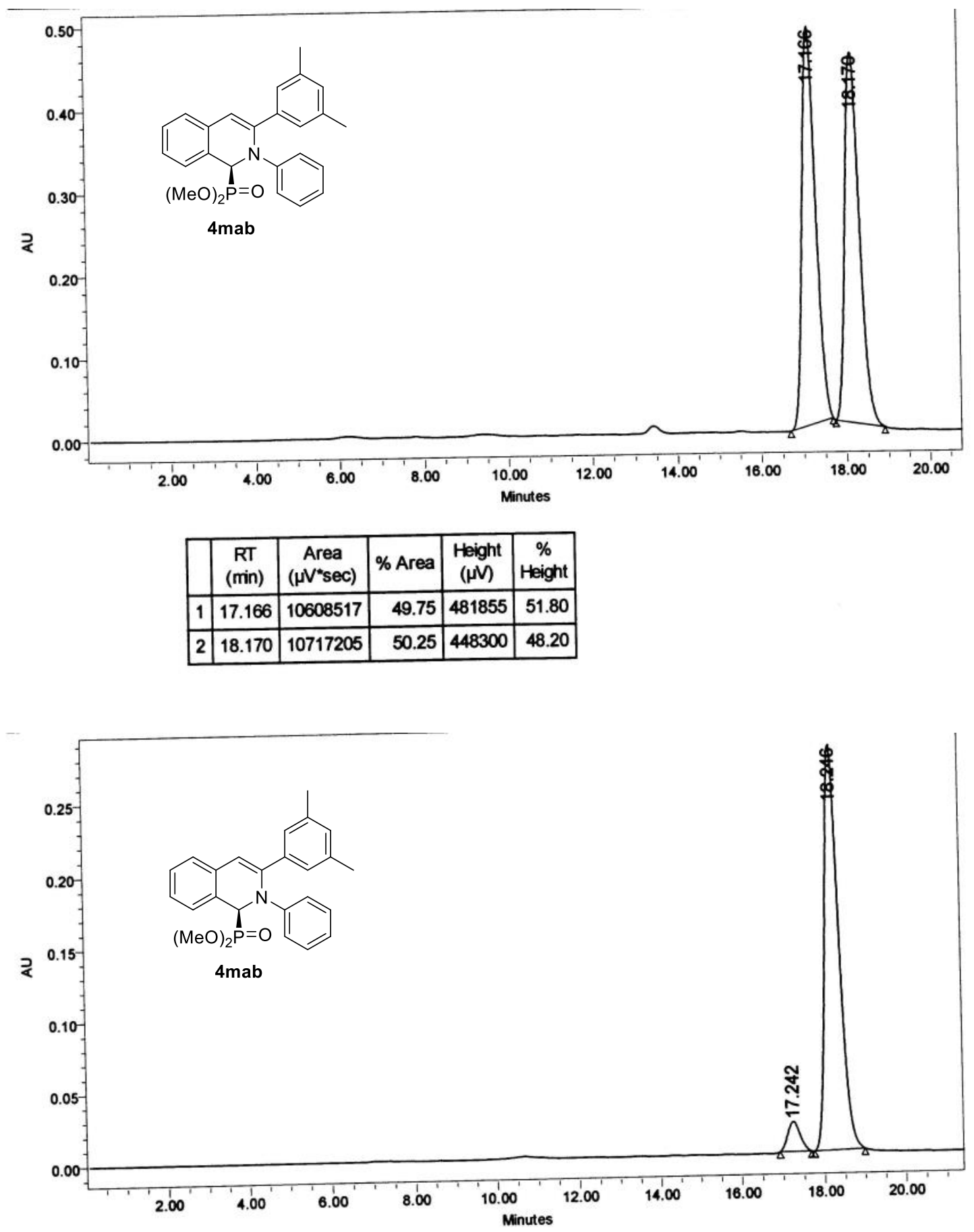

\begin{tabular}{|c|c|r|r|r|r|}
\hline & $\begin{array}{c}\text { RT } \\
(\min )\end{array}$ & $\begin{array}{c}\text { Area } \\
(\mu \mathrm{V} * \mathrm{sec})\end{array}$ & $\%$ Area & $\begin{array}{c}\text { Height } \\
(\mu \mathrm{V})\end{array}$ & $\begin{array}{c}\% \\
\text { Height }\end{array}$ \\
\hline 1 & 17.242 & 427706 & 5.91 & 20656 & 6.86 \\
\hline 2 & 18.246 & 6813519 & 94.09 & 280262 & 93.14 \\
\hline
\end{tabular}




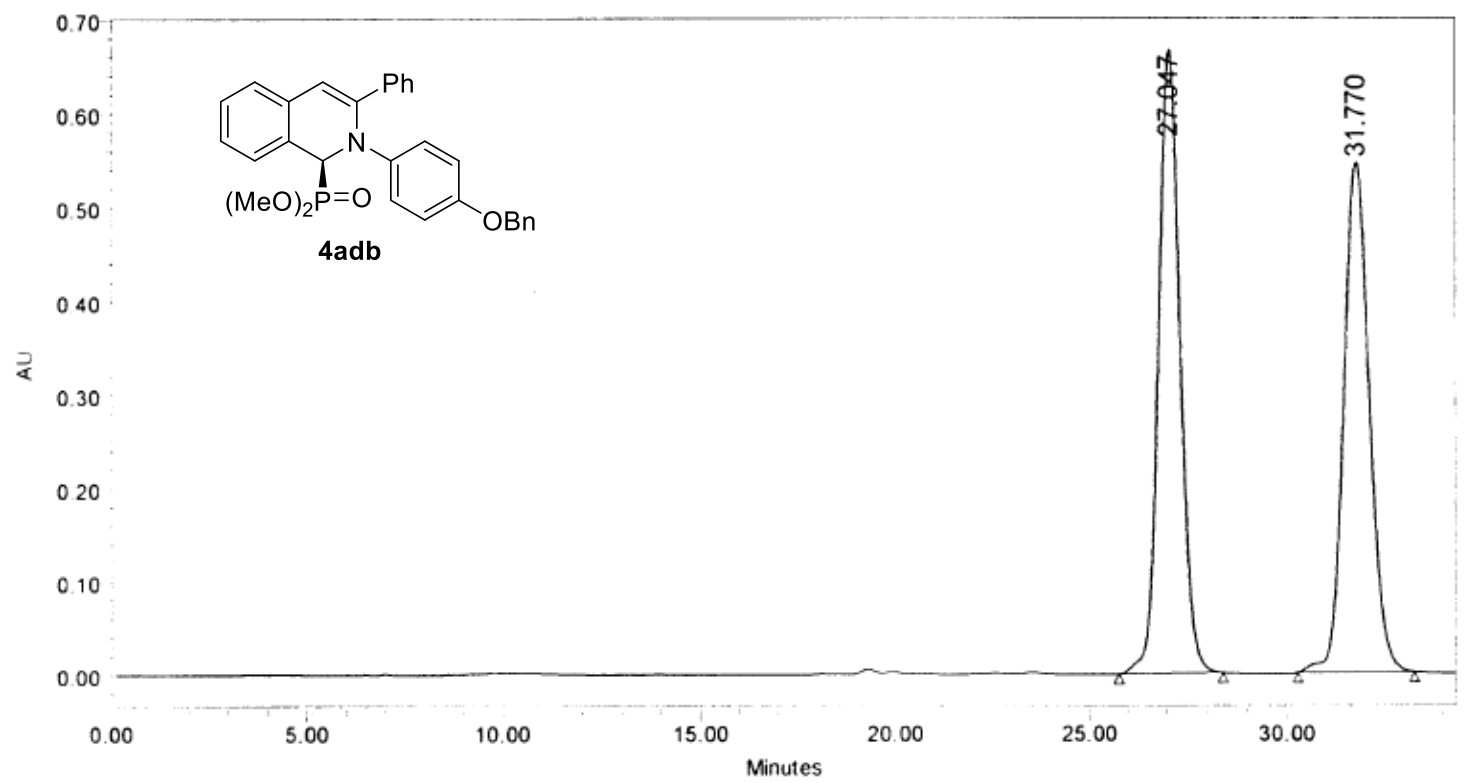

\begin{tabular}{|c|c|c|c|c|c|}
\hline & $\begin{array}{c}\text { RT } \\
(\mathrm{min})\end{array}$ & $\begin{array}{c}\text { Area } \\
(\mu \mathrm{V} * \mathrm{sec})\end{array}$ & $\%$ Area & $\begin{array}{c}\text { Height } \\
(\mu \mathrm{V})\end{array}$ & $\begin{array}{c}\% \\
\text { Height }\end{array}$ \\
\hline 1 & 27.047 & 25107719 & 50.25 & 666377 & 55.01 \\
\hline 2 & 31.770 & 24861778 & 49.75 & 545077 & 44.99 \\
\hline
\end{tabular}

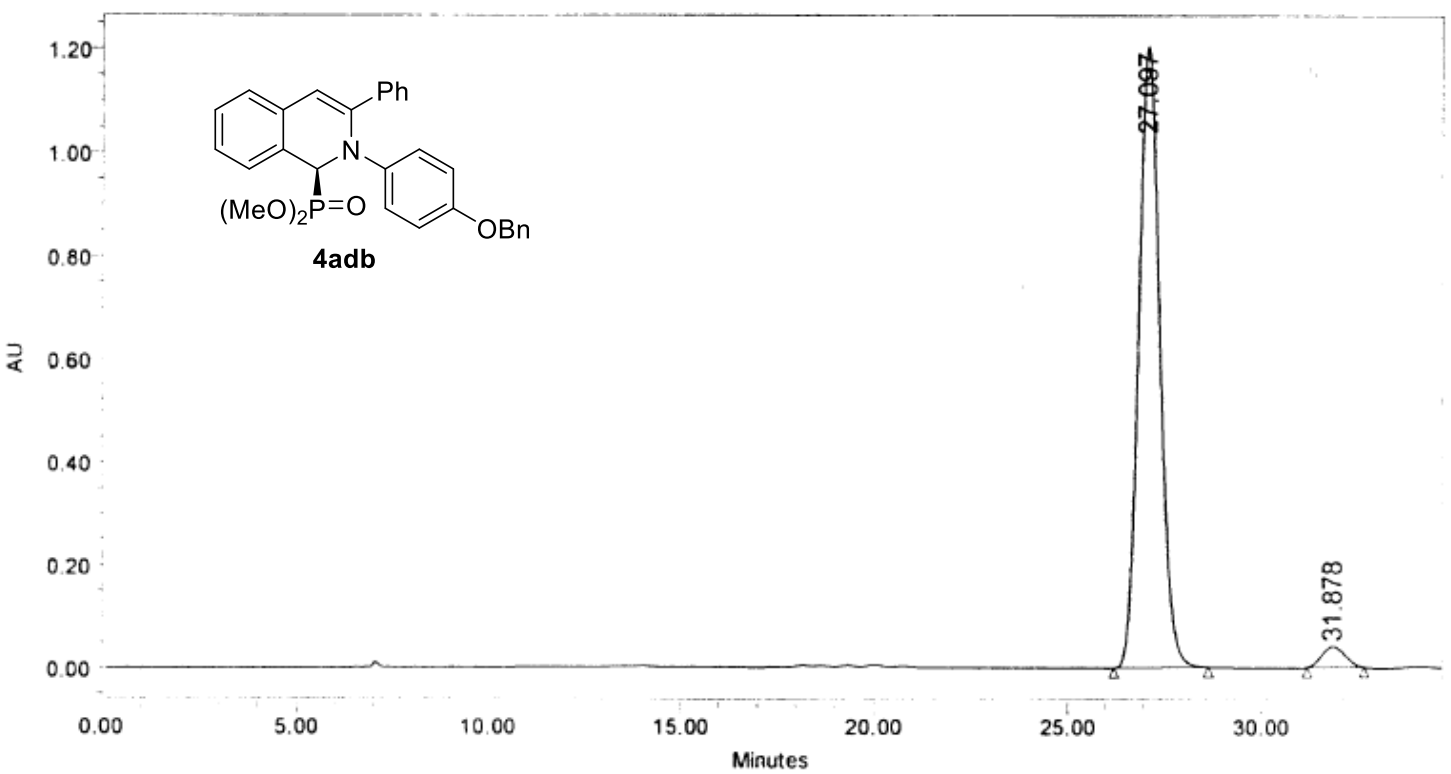

\begin{tabular}{|c|c|c|r|r|r|}
\hline & $\begin{array}{c}\mathrm{RT} \\
(\mathrm{min})\end{array}$ & $\begin{array}{c}\text { Area } \\
(\mu \mathrm{V} * \mathrm{sec})\end{array}$ & $\%$ Area & $\begin{array}{c}\text { Height } \\
(\mu \mathrm{V})\end{array}$ & $\begin{array}{c}\% \\
\text { Height }\end{array}$ \\
\hline 1 & 27.097 & 45716265 & 96.42 & 1202792 & 96.78 \\
\hline 2 & 31.878 & 1696994 & 3.58 & 40008 & 3.22 \\
\hline
\end{tabular}




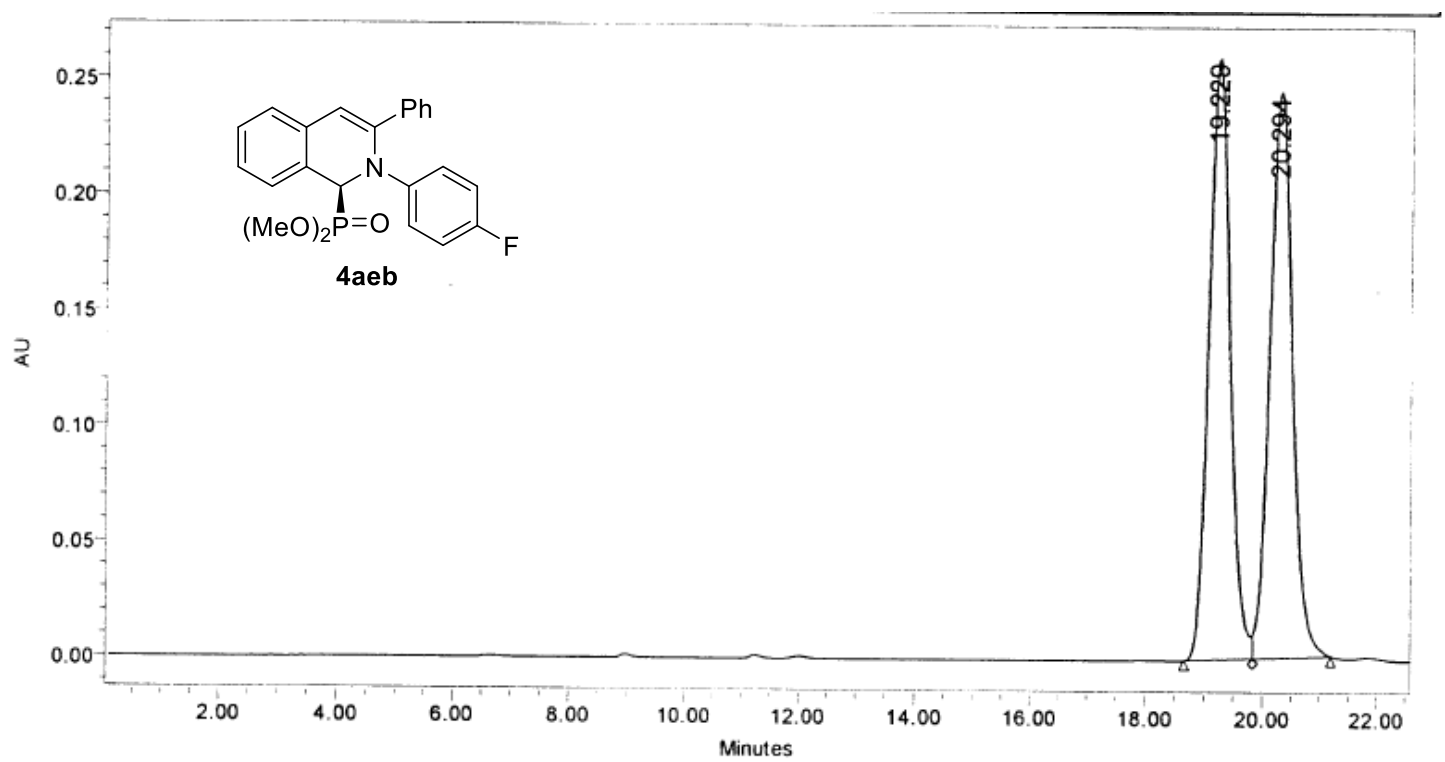

\begin{tabular}{|c|c|c|c|c|c|}
\hline & $\begin{array}{c}\text { RT } \\
(\mathrm{min})\end{array}$ & $\begin{array}{c}\text { Area } \\
(\mu \mathrm{V} \text { sec })\end{array}$ & $\%$ Area & $\begin{array}{c}\text { Height } \\
(\mu \mathrm{V})\end{array}$ & $\begin{array}{c}\% \\
\text { Height }\end{array}$ \\
\hline 1 & 19.229 & 6560903 & 49.59 & 259807 & 51.54 \\
\hline 2 & 20.294 & 6670506 & 50.41 & 244286 & 48.46 \\
\hline
\end{tabular}

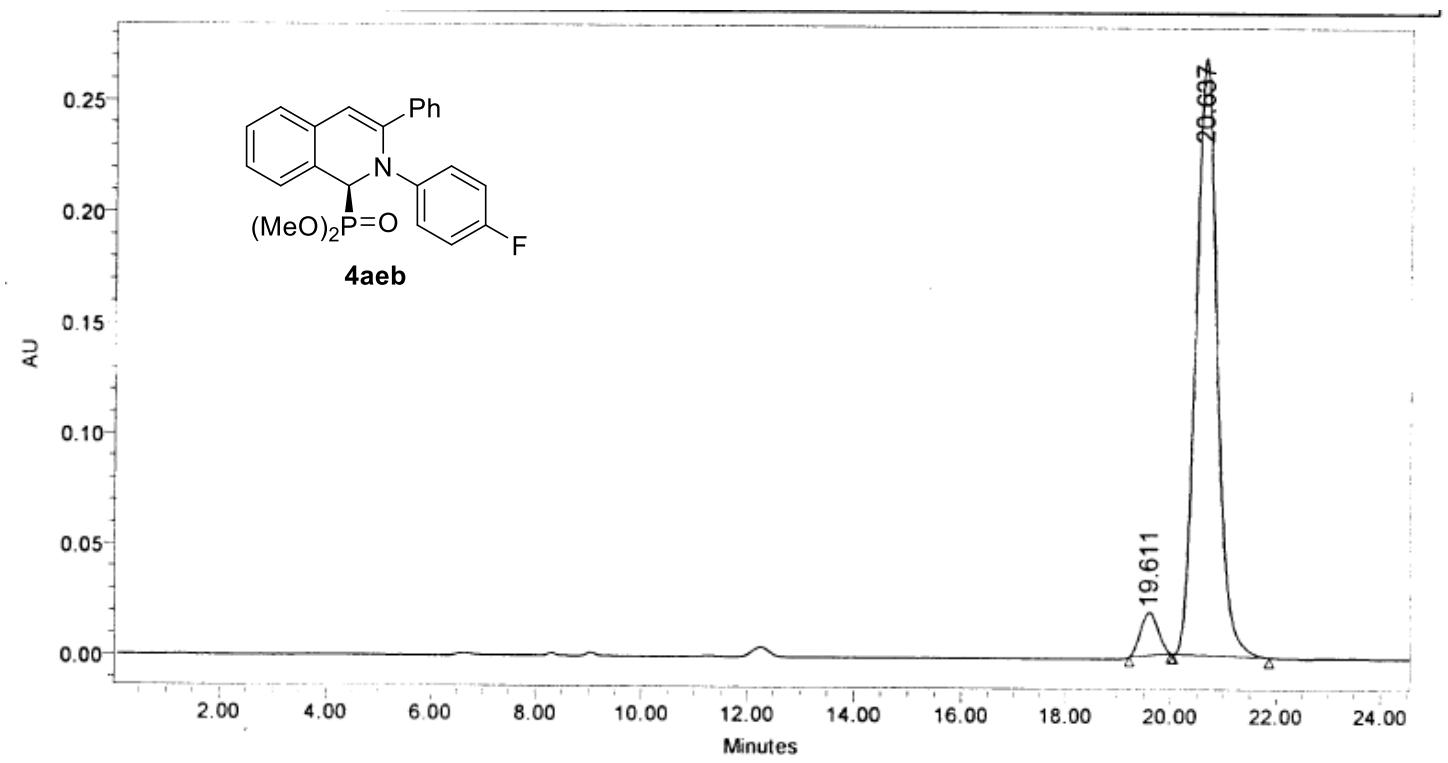

\begin{tabular}{|c|c|c|r|r|r|}
\hline & $\begin{array}{c}\text { RT } \\
(\mathrm{min})\end{array}$ & $\begin{array}{c}\text { Area } \\
(\mu \mathrm{V} * \mathrm{sec})\end{array}$ & $\%$ Area & $\begin{array}{c}\text { Height } \\
(\mu \mathrm{V})\end{array}$ & $\begin{array}{c}\% \\
\text { Height }\end{array}$ \\
\hline 1 & 19.611 & 442433 & 5.57 & 19326 & 6.70 \\
\hline 2 & 20.637 & 7503914 & 94.43 & 269103 & 93.30 \\
\hline
\end{tabular}




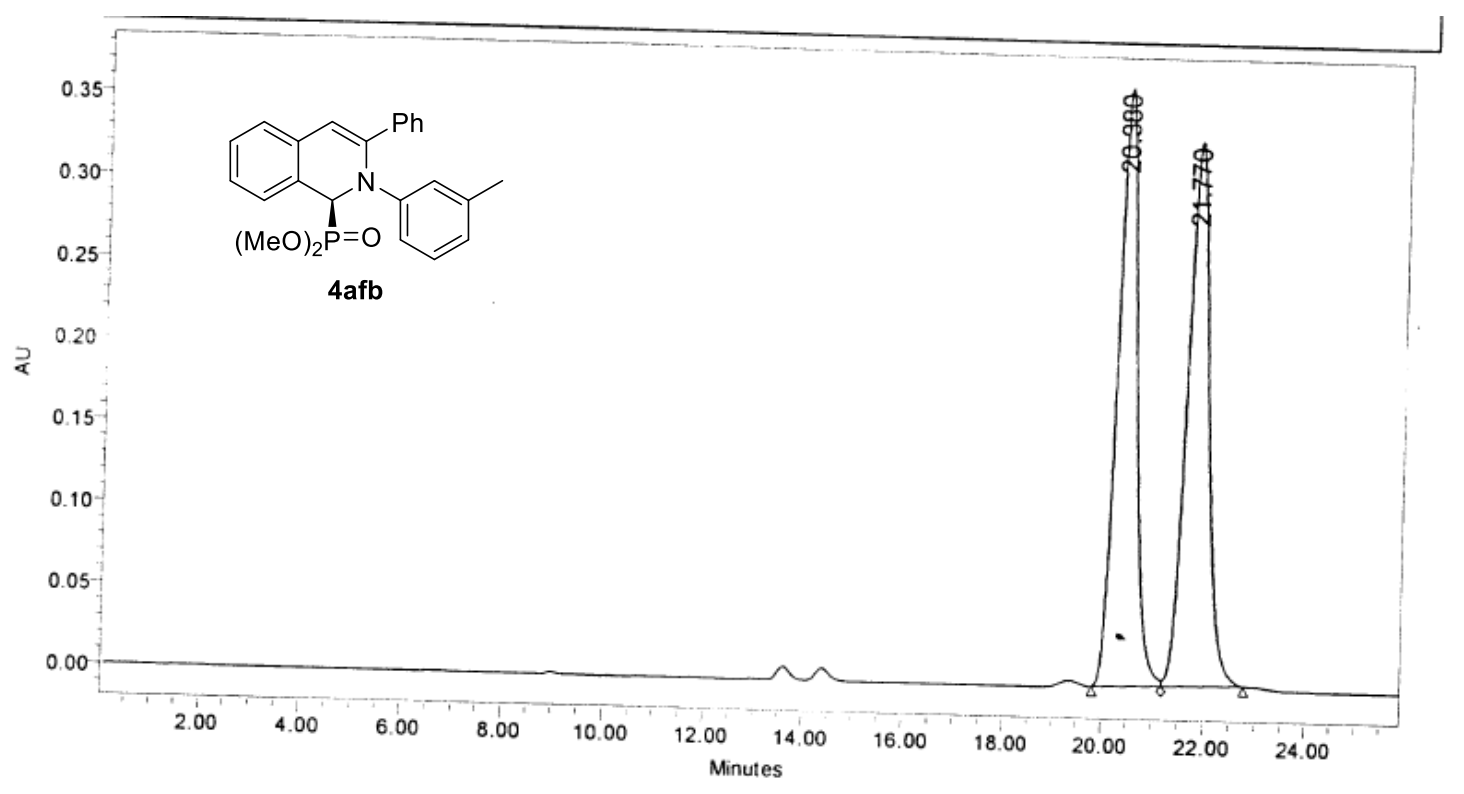

\begin{tabular}{|c|c|c|c|c|c|}
\hline & $\begin{array}{c}\text { RT } \\
(\min )\end{array}$ & $\begin{array}{c}\text { Area } \\
(\mu \mathrm{V} * \text { sec })\end{array}$ & $\%$ Area & $\begin{array}{c}\text { Height } \\
(\mu \mathrm{V})\end{array}$ & $\begin{array}{c}\% \\
\text { Height }\end{array}$ \\
\hline 1 & 20.380 & 9860061 & 50.16 & 365330 & 52.32 \\
\hline 2 & 21.770 & 9795845 & 49.84 & 332877 & 47.68 \\
\hline
\end{tabular}

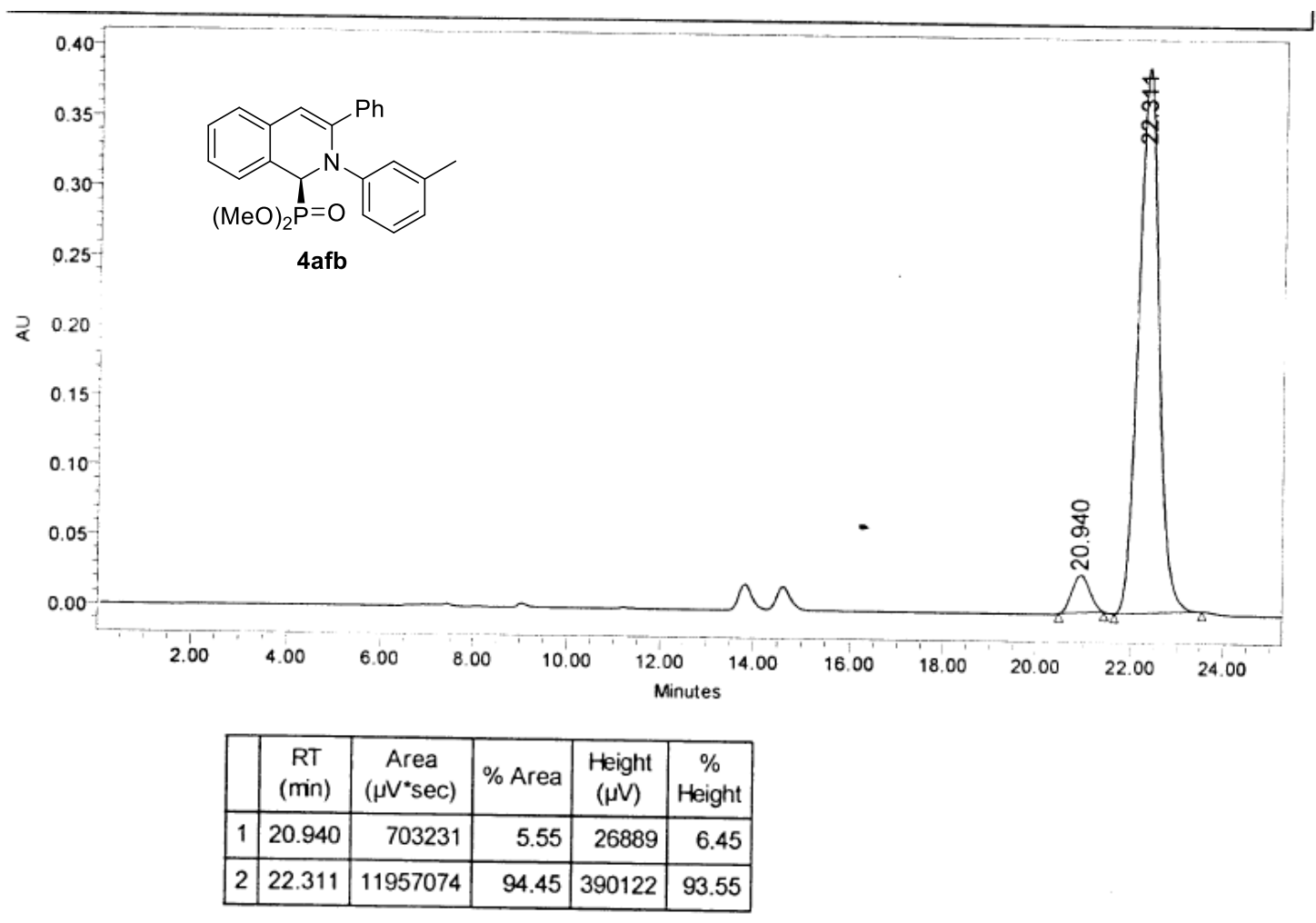




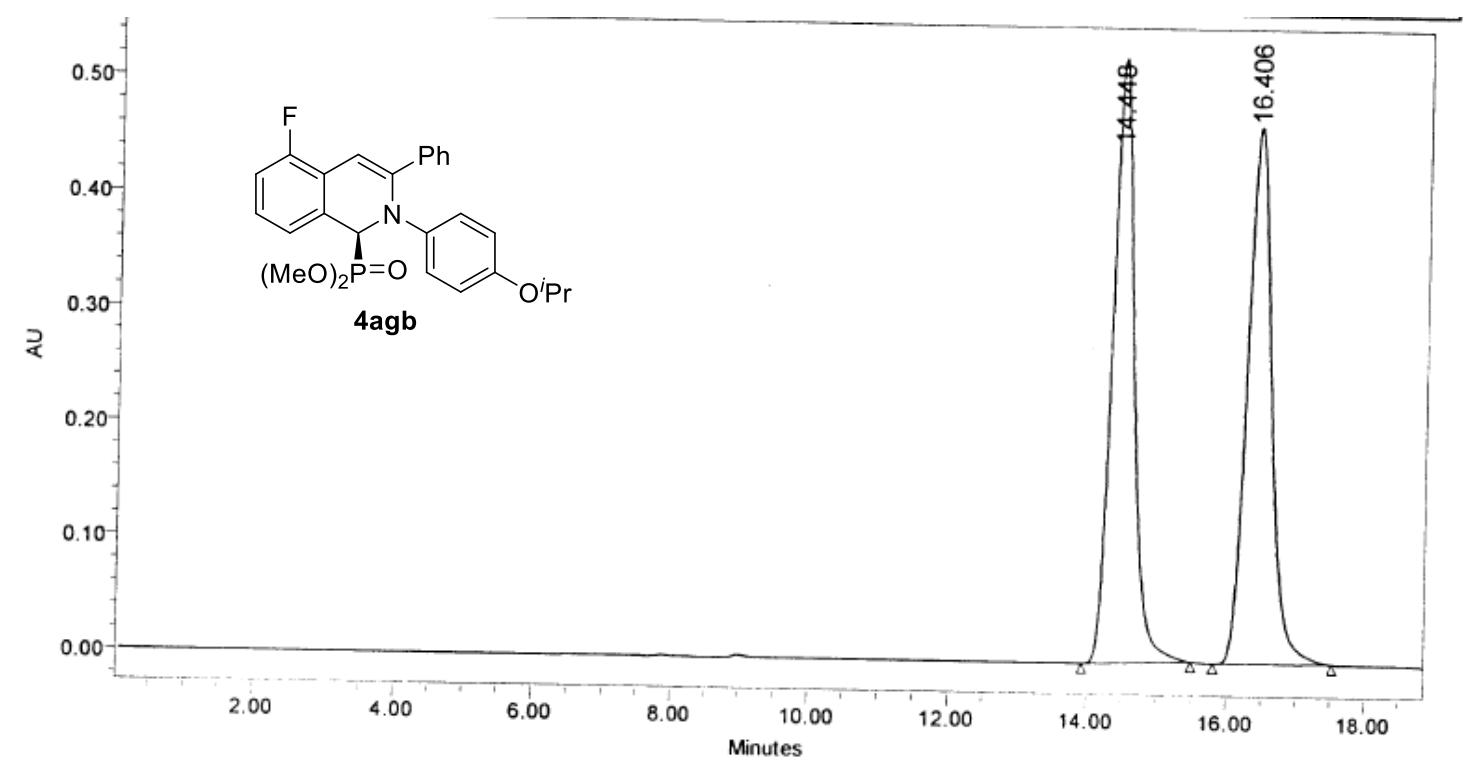

\begin{tabular}{|c|c|c|c|c|c|}
\hline & $\begin{array}{c}\text { RT } \\
(\mathrm{min})\end{array}$ & $\begin{array}{c}\text { Area } \\
(\mu \mathrm{V} * \mathrm{sec})\end{array}$ & $\%$ Area & $\begin{array}{c}\text { Height } \\
(\mu \mathrm{V})\end{array}$ & $\begin{array}{c}\% \\
\text { Height }\end{array}$ \\
\hline 1 & 14.448 & 11418725 & 50.03 & 525529 & 52.94 \\
\hline 2 & 16.406 & 11406491 & 49.97 & 467193 & 47.06 \\
\hline
\end{tabular}

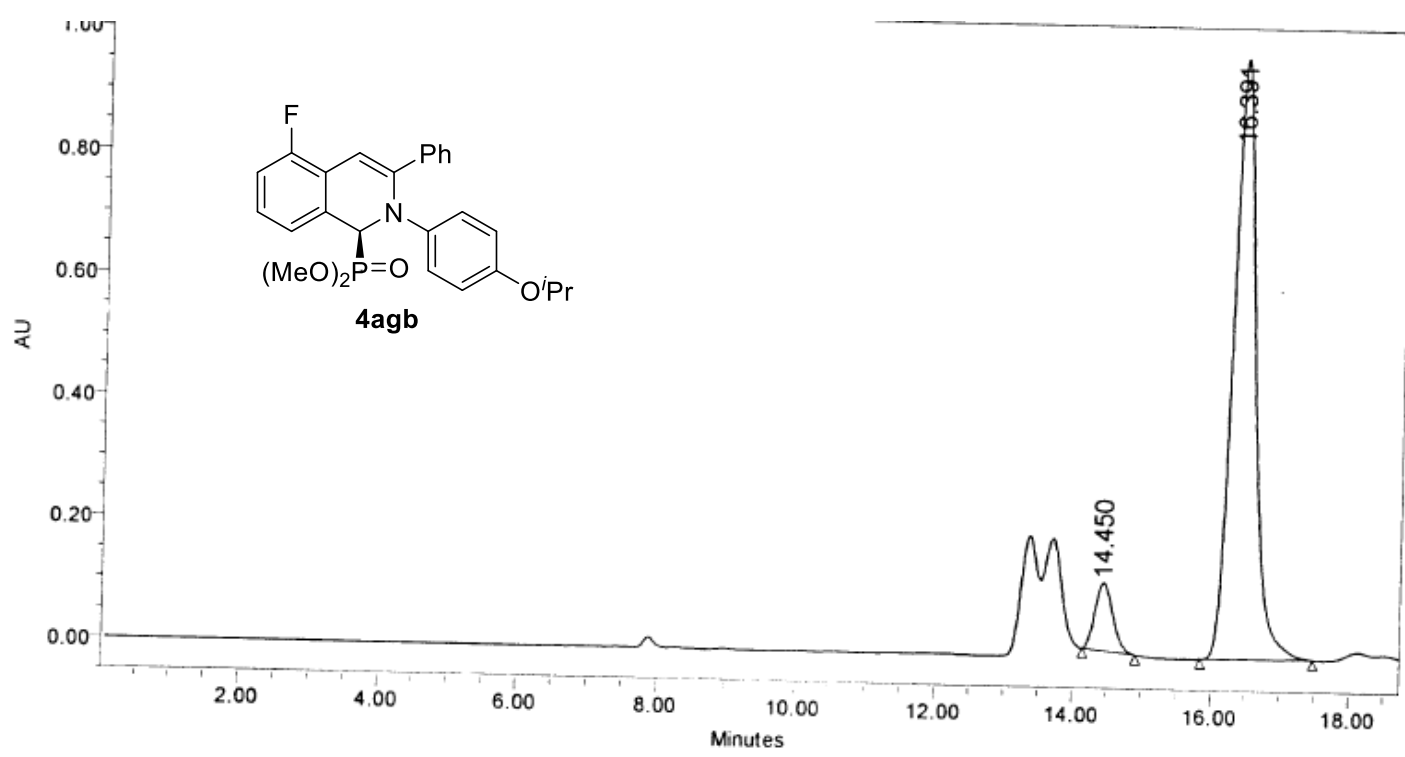

\begin{tabular}{|c|c|c|r|r|c|}
\hline & $\begin{array}{c}\text { RT } \\
(\min )\end{array}$ & $\begin{array}{c}\text { Area } \\
(\mu \mathrm{V} * \mathrm{sec})\end{array}$ & $\%$ Area & $\begin{array}{c}\text { Height } \\
(\mu \mathrm{V})\end{array}$ & $\begin{array}{c}\% \\
\text { Height }\end{array}$ \\
\hline 1 & 14.450 & 2013261 & 8.24 & 111539 & 10.20 \\
\hline 2 & 16.391 & 22410258 & 91.76 & 981826 & 89.80 \\
\hline
\end{tabular}




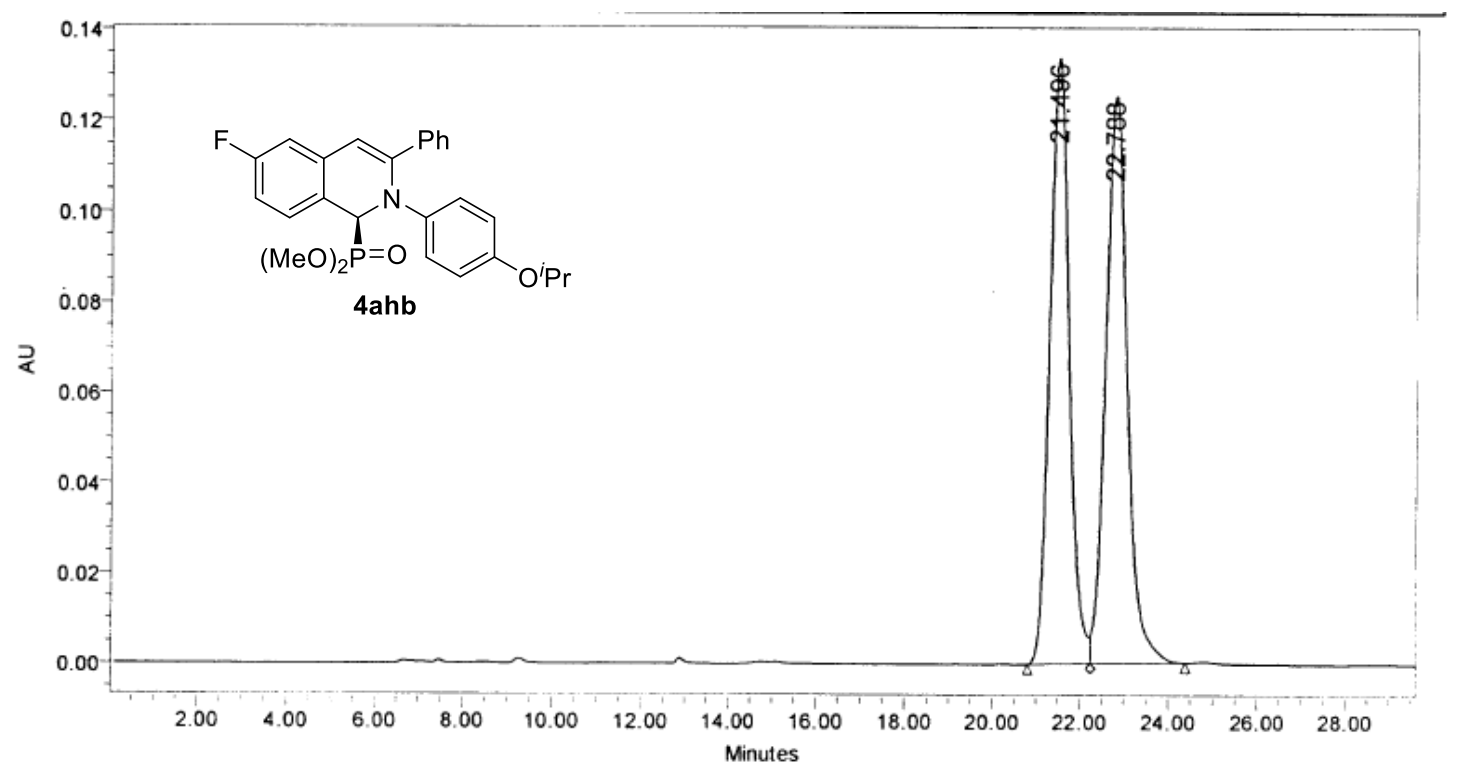

\begin{tabular}{|c|c|c|c|c|c|}
\hline & $\begin{array}{c}\mathrm{RT} \\
(\mathrm{min})\end{array}$ & $\begin{array}{c}\text { Area } \\
(\mu \mathrm{V} * \mathrm{sec})\end{array}$ & $\%$ Area & $\begin{array}{c}\text { Height } \\
(\mu \mathrm{V})\end{array}$ & $\begin{array}{c}\% \\
\text { Height }\end{array}$ \\
\hline 1 & 21.496 & 4137432 & 49.31 & 133873 & 51.63 \\
\hline 2 & 22.788 & 4253791 & 50.69 & 125426 & 48.37 \\
\hline
\end{tabular}

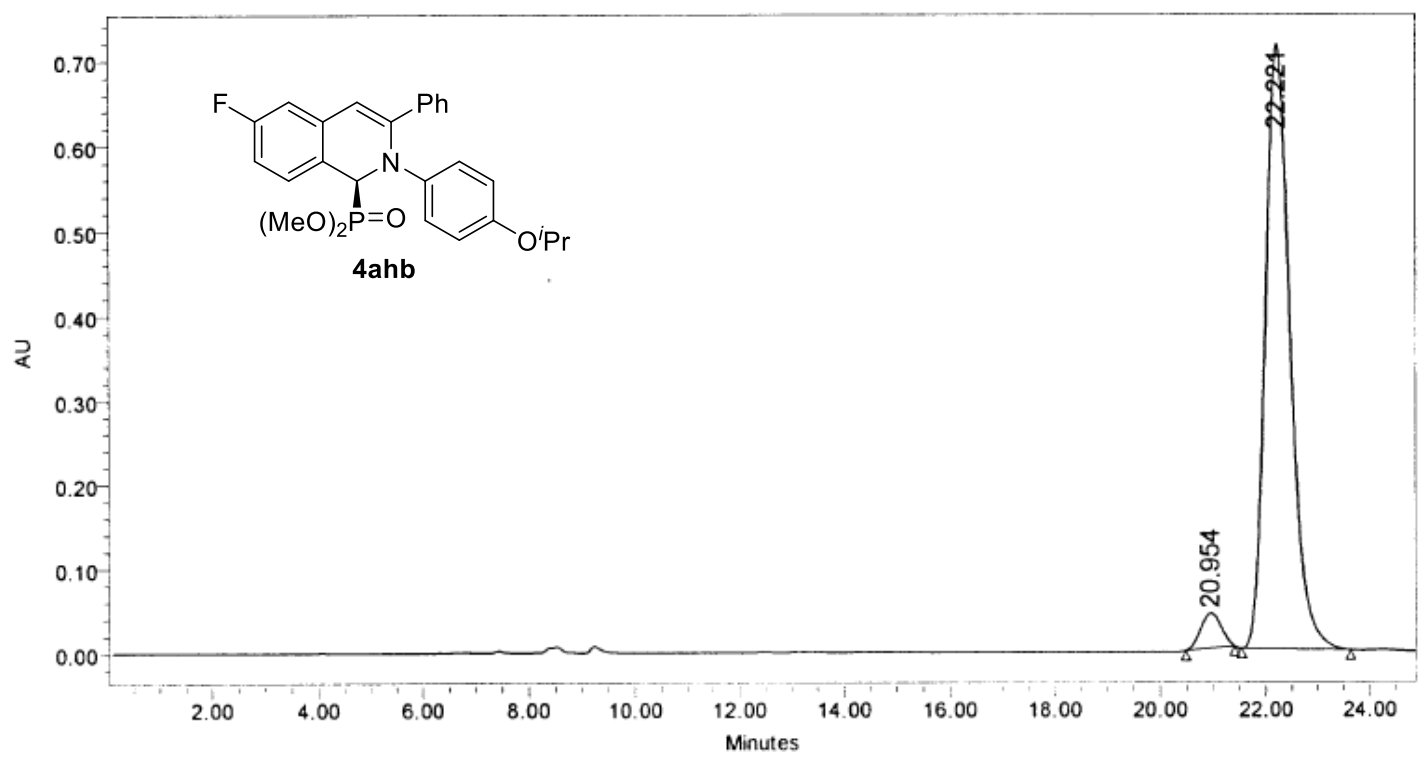

\begin{tabular}{|c|c|c|c|c|c|}
\hline & $\begin{array}{c}\mathrm{RT} \\
(\mathrm{min})\end{array}$ & $\begin{array}{c}\text { Area } \\
(\mu \mathrm{V} * \mathrm{sec})\end{array}$ & $\%$ Area & $\begin{array}{c}\text { Height } \\
(\mu \mathrm{V})\end{array}$ & $\begin{array}{c}\% \\
\text { Height }\end{array}$ \\
\hline 1 & 20.954 & 1114014 & 4.52 & 41649 & 5.51 \\
\hline 2 & 22.221 & 23556342 & 95.48 & 714562 & 94.49 \\
\hline
\end{tabular}



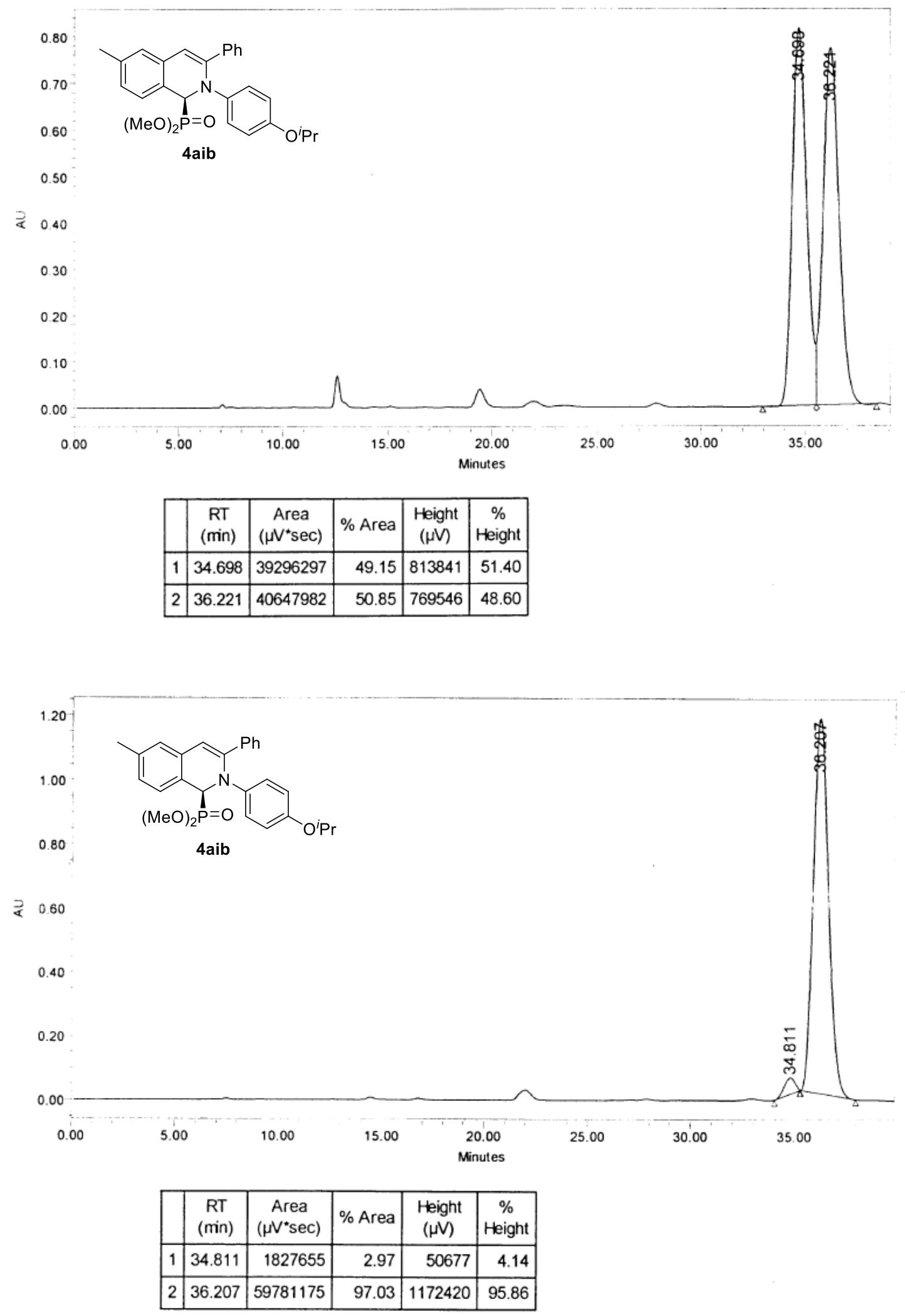


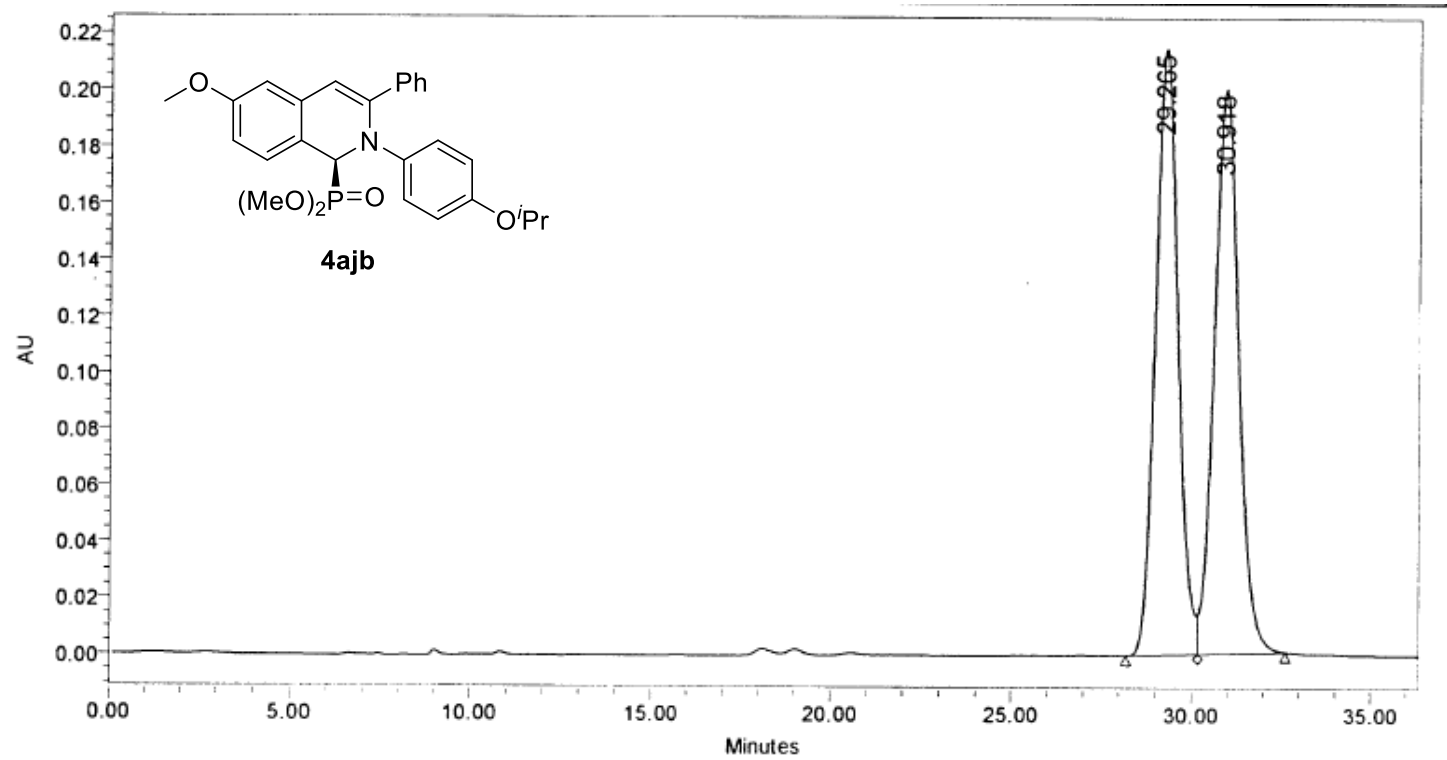

\begin{tabular}{|c|c|c|c|c|c|}
\hline & $\begin{array}{c}\mathrm{RT} \\
(\mathrm{min})\end{array}$ & $\begin{array}{c}\text { Area } \\
(\mu \mathrm{V} * \mathrm{sec})\end{array}$ & $\%$ Area & $\begin{array}{c}\text { Height } \\
(\mu \mathrm{V})\end{array}$ & $\begin{array}{c}\% \\
\text { Height }\end{array}$ \\
\hline 1 & 29.265 & 9319293 & 49.68 & 214737 & 51.77 \\
\hline 2 & 30.918 & 9438922 & 50.32 & 200060 & 48.23 \\
\hline
\end{tabular}

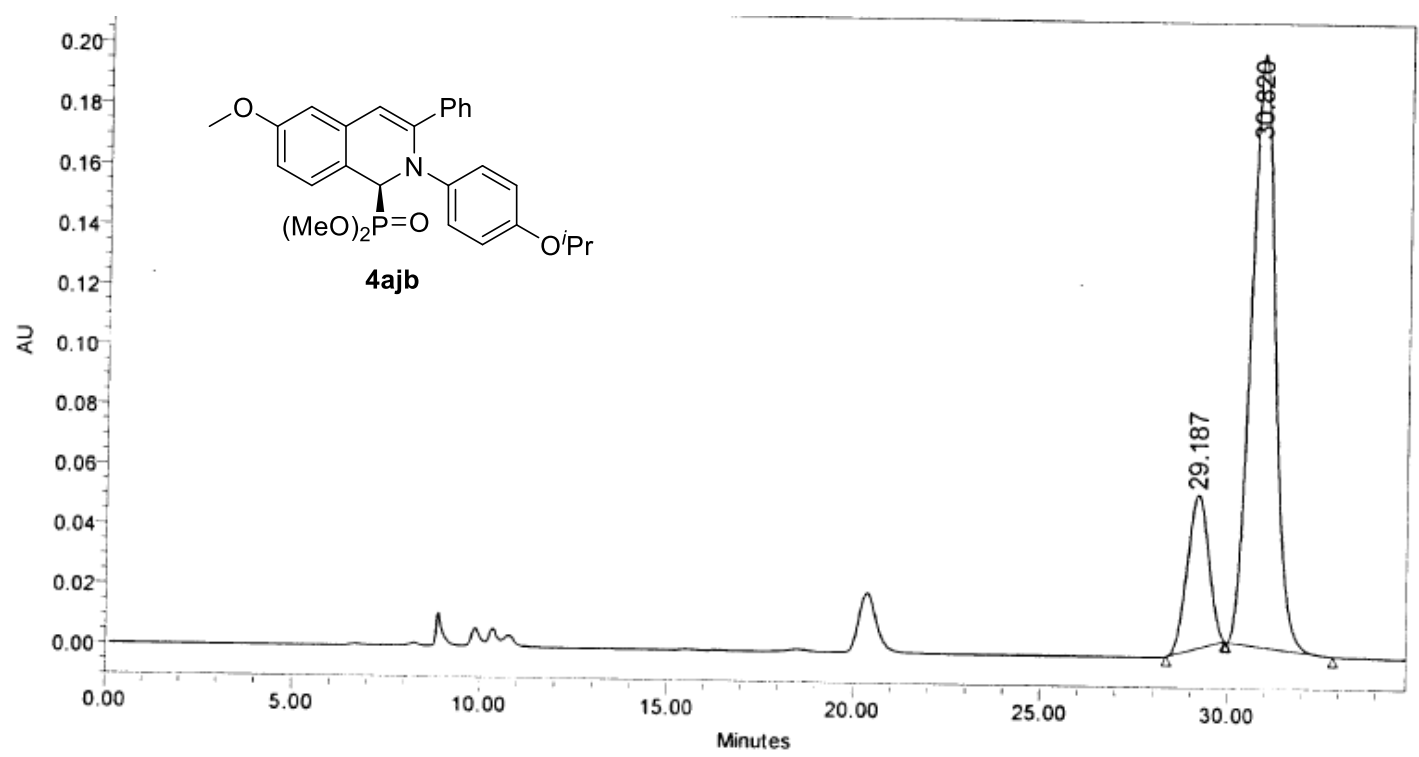

\begin{tabular}{|c|c|c|c|c|c|}
\hline & $\begin{array}{c}\text { RT } \\
(\mathrm{min})\end{array}$ & $\begin{array}{c}\text { Area } \\
(\mu \mathrm{V} \text { *sec })\end{array}$ & $\%$ Area & $\begin{array}{c}\text { Height } \\
(\mu \mathrm{V})\end{array}$ & $\begin{array}{c}\% \\
\text { Height }\end{array}$ \\
\hline 1 & 29.187 & 2035179 & 18.59 & 51360 & 20.66 \\
\hline 2 & 30.820 & 8913139 & 81.41 & 197215 & 79.34 \\
\hline
\end{tabular}




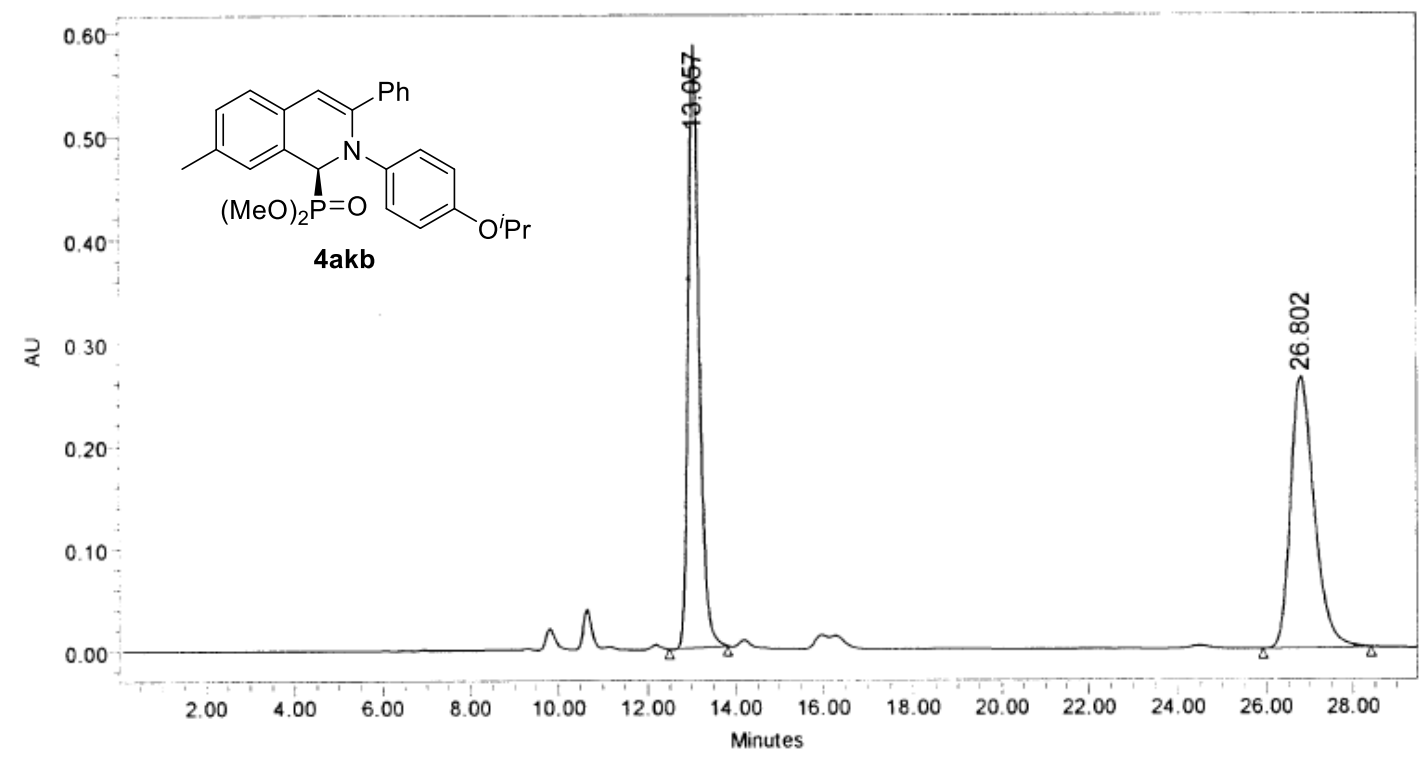

\begin{tabular}{|c|c|c|c|c|c|}
\hline & $\begin{array}{c}\text { RT } \\
(\mathrm{min})\end{array}$ & $\begin{array}{c}\text { Area } \\
\left(\mu \mathrm{V}^{*} \mathrm{sec}\right)\end{array}$ & $\%$ Area & $\begin{array}{c}\text { Height } \\
(\mu \mathrm{V})\end{array}$ & $\begin{array}{c}\% \\
\text { Height }\end{array}$ \\
\hline 1 & 13.057 & 10154269 & 50.30 & 585479 & 68.88 \\
\hline 2 & 26.802 & 10033569 & 49.70 & 264558 & 31.12 \\
\hline
\end{tabular}

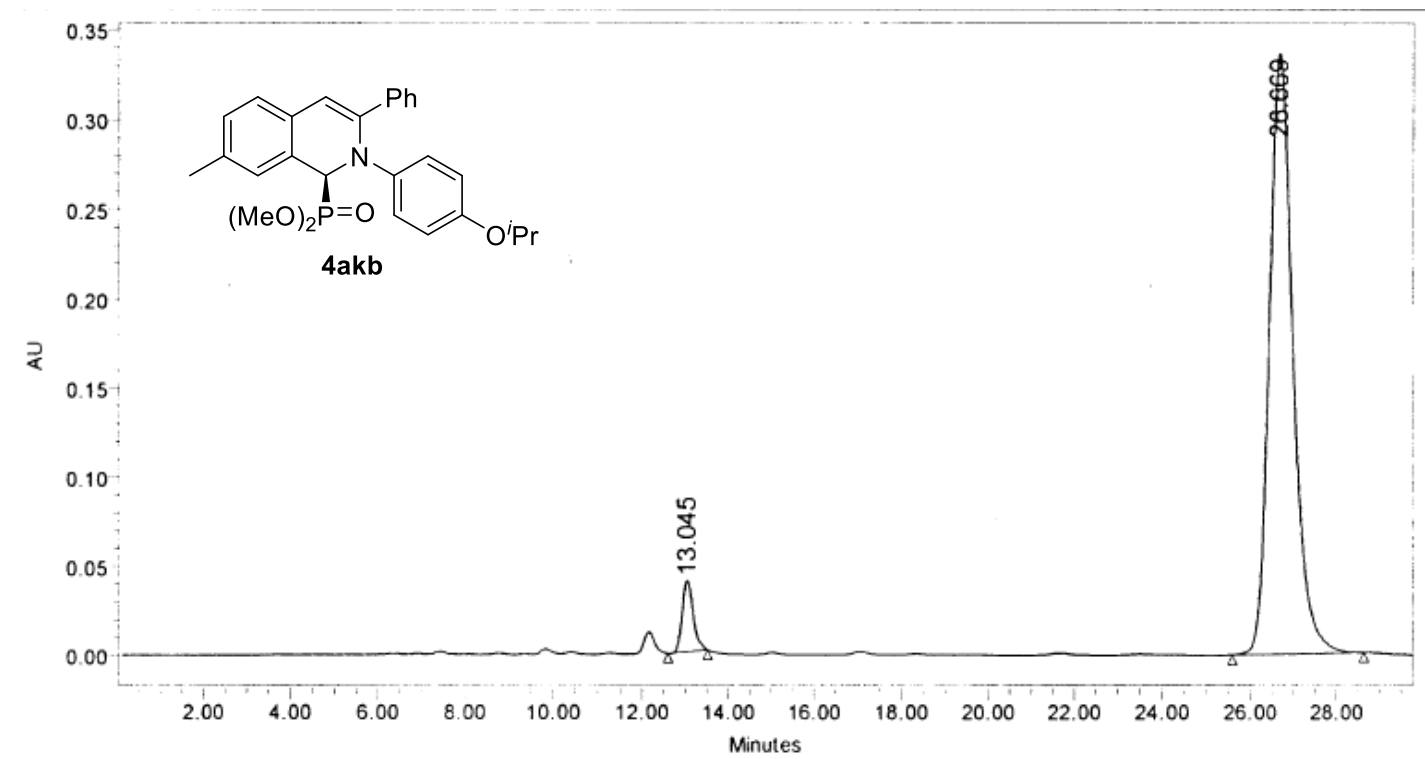

\begin{tabular}{|c|c|r|r|r|r|}
\hline & $\begin{array}{c}\text { RT } \\
(\mathrm{min})\end{array}$ & $\begin{array}{c}\text { Area } \\
(\mu \mathrm{V} * \mathrm{sec})\end{array}$ & $\%$ Area & $\begin{array}{c}\text { Height } \\
(\mu \mathrm{V})\end{array}$ & $\begin{array}{c}\% \\
\text { Height }\end{array}$ \\
\hline 1 & 13.045 & 709327 & 5.25 & 40176 & 10.68 \\
\hline 2 & 26.669 & 12792226 & 94.75 & 336081 & 89.32 \\
\hline
\end{tabular}




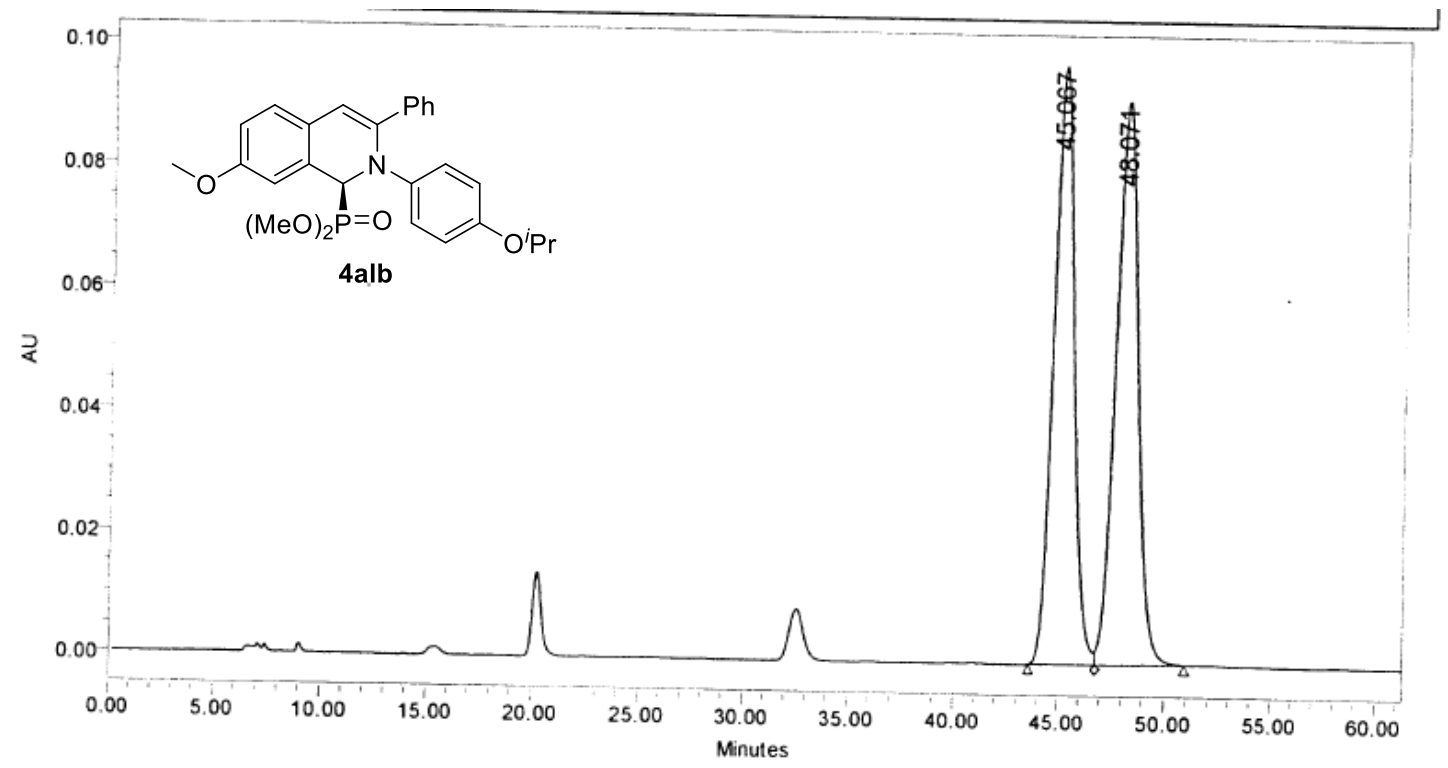

\begin{tabular}{|c|c|c|c|c|c|}
\hline & $\begin{array}{c}\mathrm{RT} \\
(\mathrm{min})\end{array}$ & $\begin{array}{c}\text { Area } \\
\left(\mu \mathrm{V}^{*} \mathrm{sec}\right)\end{array}$ & $\%$ Area & $\begin{array}{c}\text { Height } \\
(\mu \mathrm{V})\end{array}$ & $\begin{array}{c}\% \\
\text { Height }\end{array}$ \\
\hline 1 & 45.067 & 6577944 & 49.88 & 97647 & 51.51 \\
\hline 2 & 48.071 & 6609502 & 50.12 & 91940 & 48.49 \\
\hline
\end{tabular}

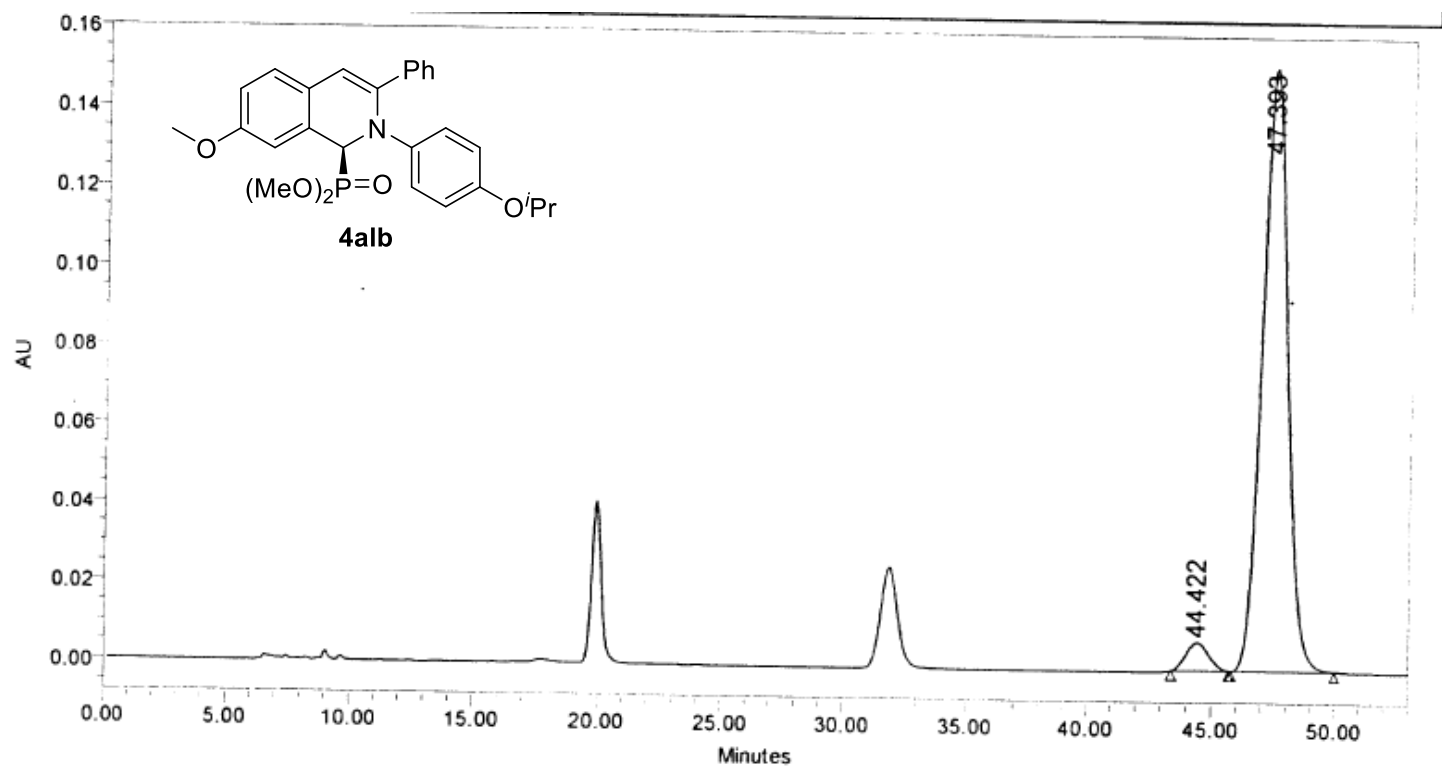

\begin{tabular}{|c|c|r|r|r|r|}
\hline & $\begin{array}{c}\mathrm{RT} \\
(\mathrm{min})\end{array}$ & $\begin{array}{c}\text { Area } \\
(\mu \mathrm{V} * \mathrm{sec})\end{array}$ & $\%$ Area & $\begin{array}{c}\text { Height } \\
(\mu \mathrm{V})\end{array}$ & $\begin{array}{c}\% \\
\text { Height }\end{array}$ \\
\hline 1 & 44.422 & 446401 & 3.93 & 7087 & 4.45 \\
\hline 2 & 47.393 & 10902857 & 96.07 & 152182 & 95.55 \\
\hline
\end{tabular}



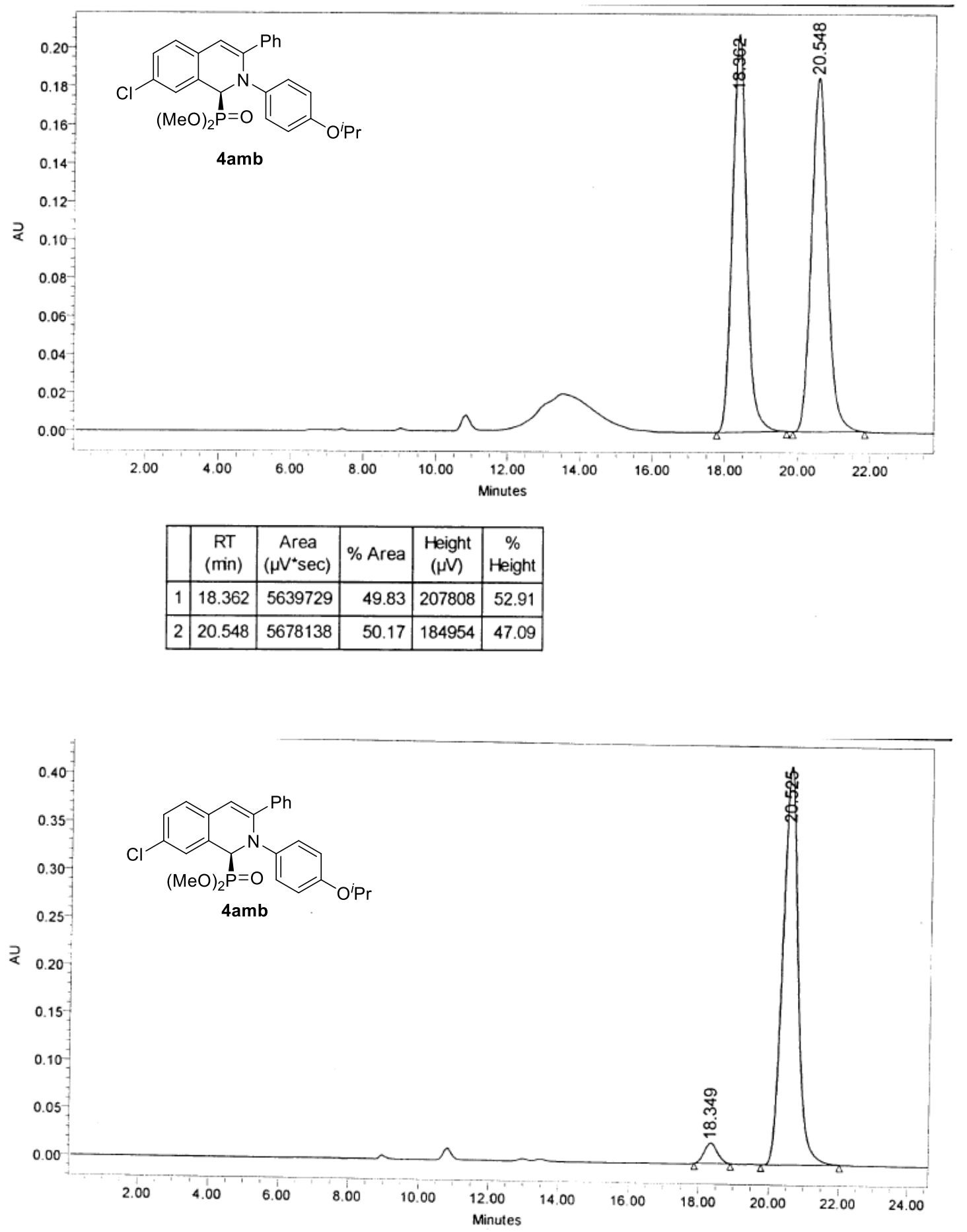

\begin{tabular}{|c|c|r|r|r|r|}
\hline & $\begin{array}{c}\text { RT } \\
(\mathrm{min})\end{array}$ & $\begin{array}{c}\text { Area } \\
(\mu \mathrm{V} * \mathrm{sec})\end{array}$ & $\%$ Area & $\begin{array}{c}\text { Height } \\
(\mu \mathrm{V})\end{array}$ & $\begin{array}{c}\% \\
\text { Height }\end{array}$ \\
\hline 1 & 18.349 & 555332 & 4.23 & 21386 & 4.88 \\
\hline 2 & 20.525 & 12582544 & 95.77 & 416544 & 95.12 \\
\hline
\end{tabular}



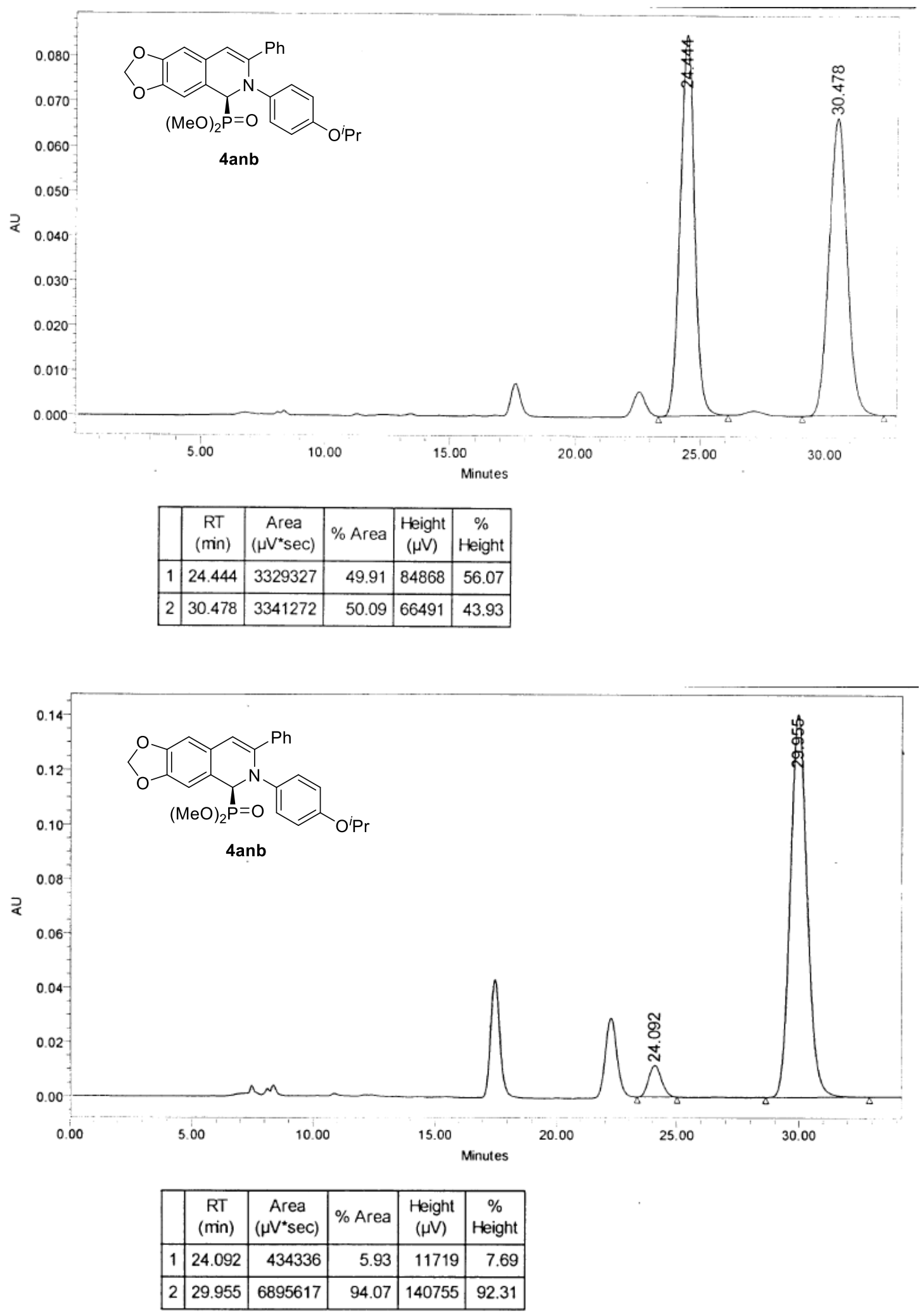


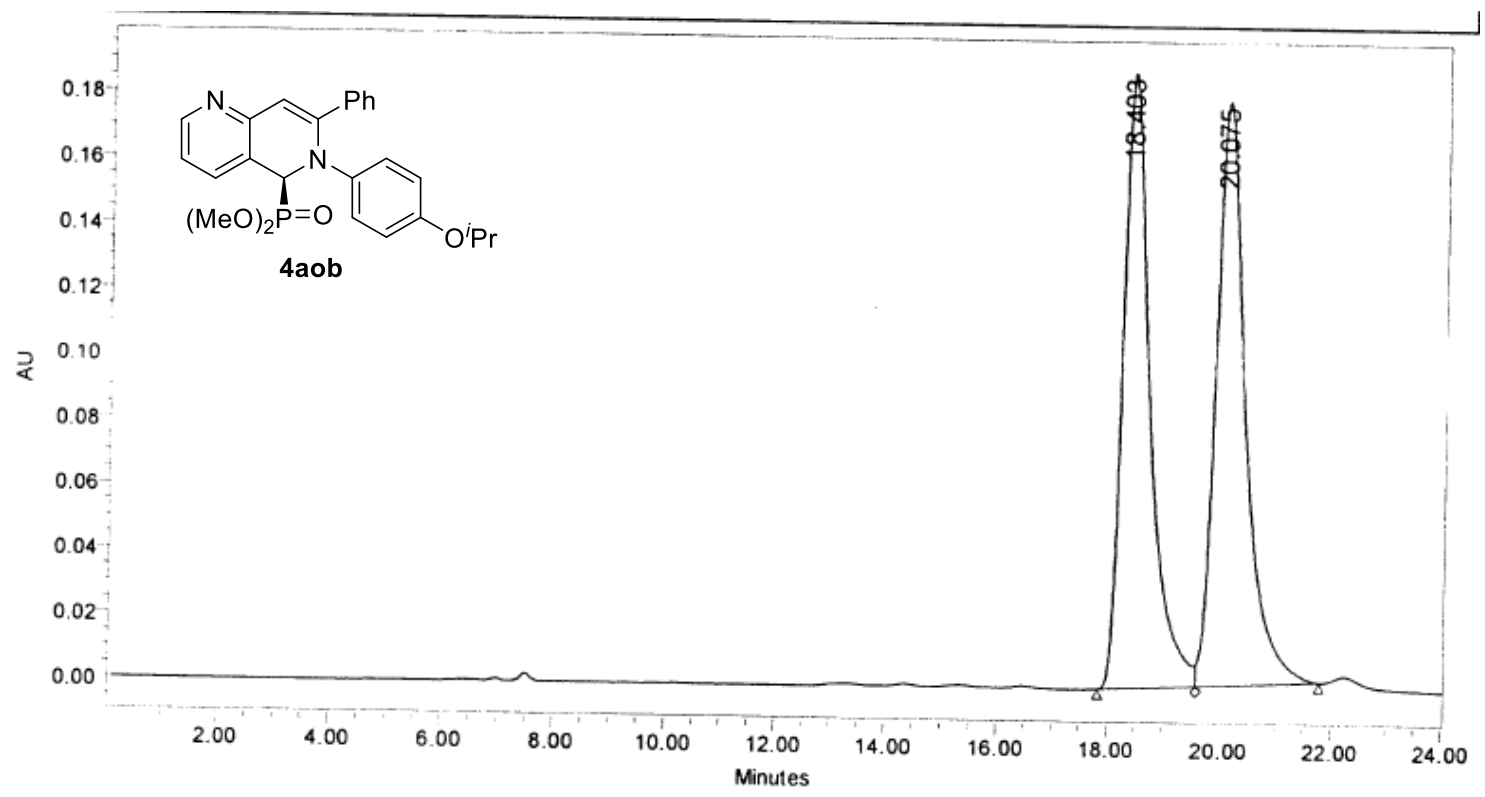

\begin{tabular}{|c|c|c|c|c|c|}
\hline & $\begin{array}{c}\text { RT } \\
(\mathrm{min})\end{array}$ & $\begin{array}{c}\text { Area } \\
\left(\mu \mathrm{V}^{*} \mathrm{sec}\right)\end{array}$ & $\%$ Area & $\begin{array}{c}\text { Height } \\
(\mu \mathrm{V})\end{array}$ & $\begin{array}{c}\% \\
\text { Height }\end{array}$ \\
\hline 1 & 18.403 & 6344527 & 49.48 & 188414 & 51.30 \\
\hline 2 & 20.075 & 6477850 & 50.52 & 178887 & 48.70 \\
\hline
\end{tabular}

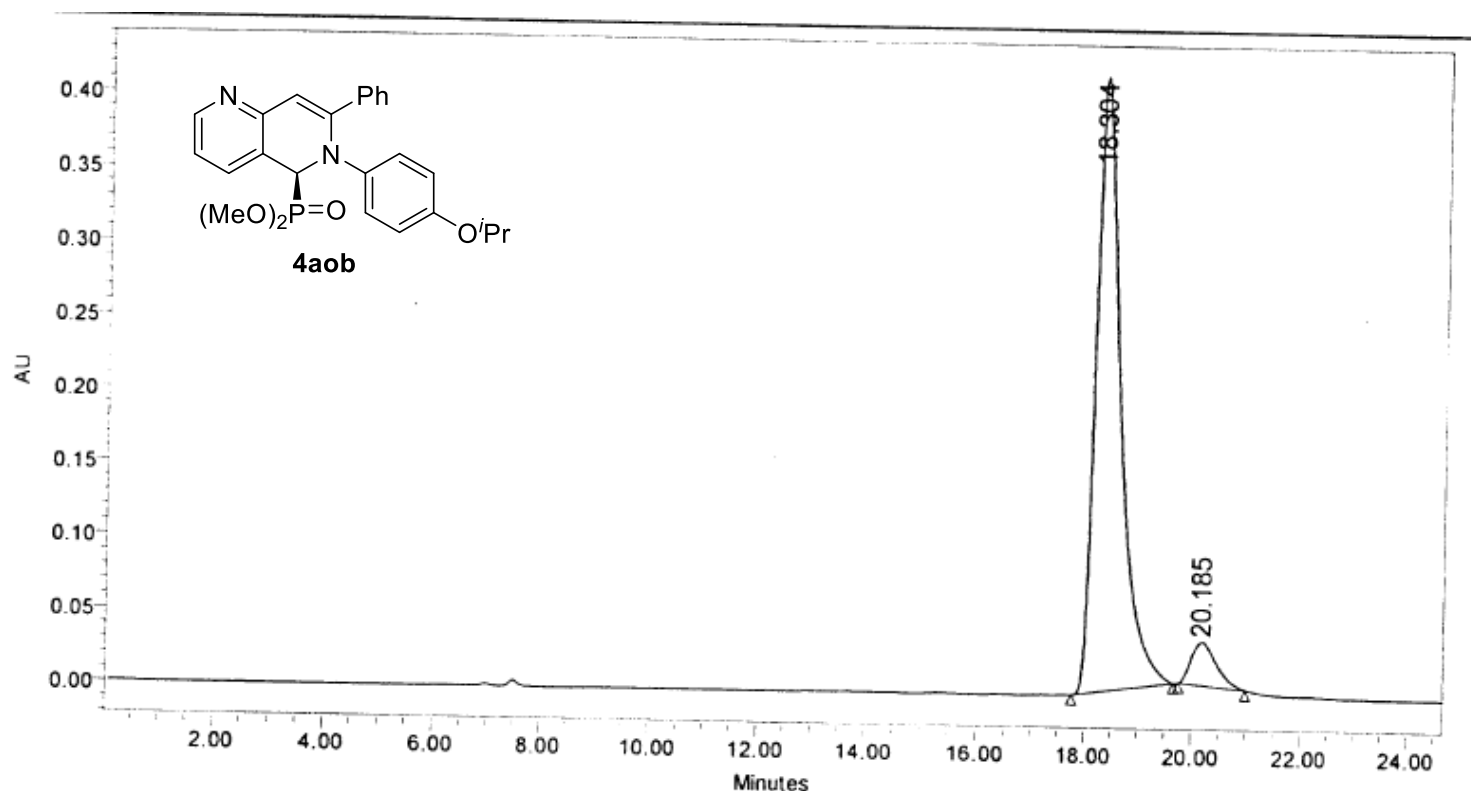

\begin{tabular}{|c|c|r|r|r|r|}
\hline & $\begin{array}{c}\text { RT } \\
(\mathrm{min})\end{array}$ & $\begin{array}{c}\text { Area } \\
(\mu \mathrm{V} * \mathrm{sec})\end{array}$ & $\%$ Area & $\begin{array}{c}\text { Height } \\
(\mu \mathrm{V})\end{array}$ & $\begin{array}{c}\% \\
\text { Height }\end{array}$ \\
\hline 1 & 18.304 & 13408102 & 93.51 & 416265 & 93.41 \\
\hline 2 & 20.185 & 930162 & 6.49 & 29362 & 6.59 \\
\hline
\end{tabular}




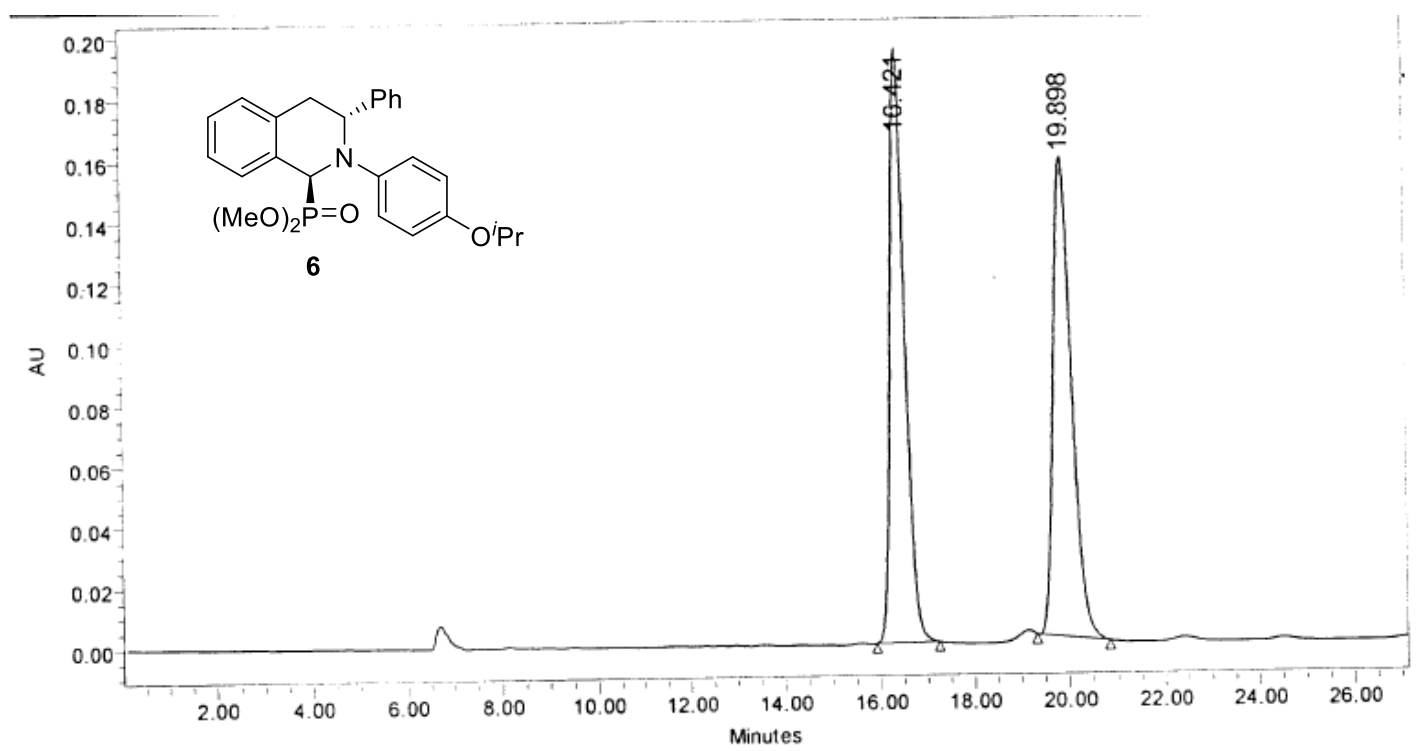

\begin{tabular}{|c|c|c|c|c|c|}
\hline & $\begin{array}{c}\text { RT } \\
(\mathrm{min})\end{array}$ & $\begin{array}{c}\text { Area } \\
\left(\mu \mathrm{V}{ }^{*} \mathrm{sec}\right)\end{array}$ & $\%$ Area & $\begin{array}{c}\text { Height } \\
(\mu \mathrm{V})\end{array}$ & $\begin{array}{c}\% \\
\text { Height }\end{array}$ \\
\hline 1 & 16.421 & 4225072 & 50.30 & 194962 & 55.29 \\
\hline 2 & 19.898 & 4174747 & 49.70 & 157637 & 44.71 \\
\hline
\end{tabular}

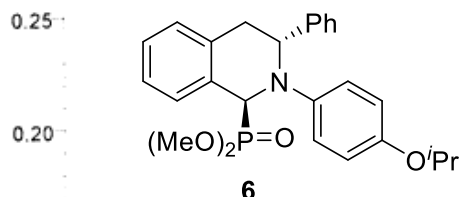

015

ج

0.10

0.05

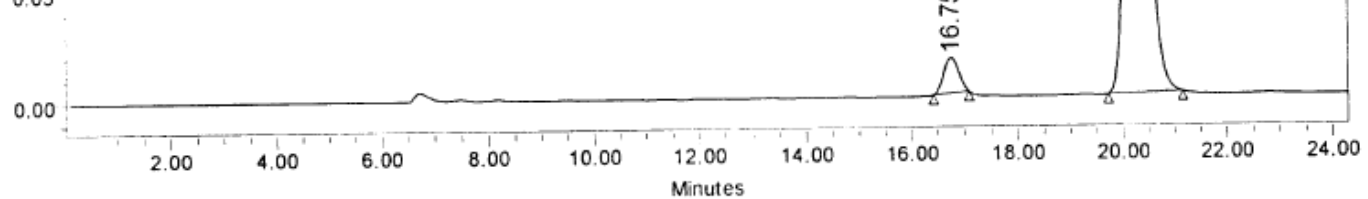

\begin{tabular}{|c|c|r|r|r|r|}
\hline & $\begin{array}{c}\text { RT } \\
(\mathrm{min})\end{array}$ & $\begin{array}{c}\text { Area } \\
(\mu \mathrm{V} * \mathrm{sec})\end{array}$ & \% Area & $\begin{array}{c}\text { Height } \\
(\mu \mathrm{V})\end{array}$ & $\begin{array}{c}\% \\
\text { Height }\end{array}$ \\
\hline 1 & 16.754 & 309497 & 4.15 & 15842 & 5.63 \\
\hline 2 & 20.284 & 7143926 & 95.85 & 265729 & 94.37 \\
\hline
\end{tabular}



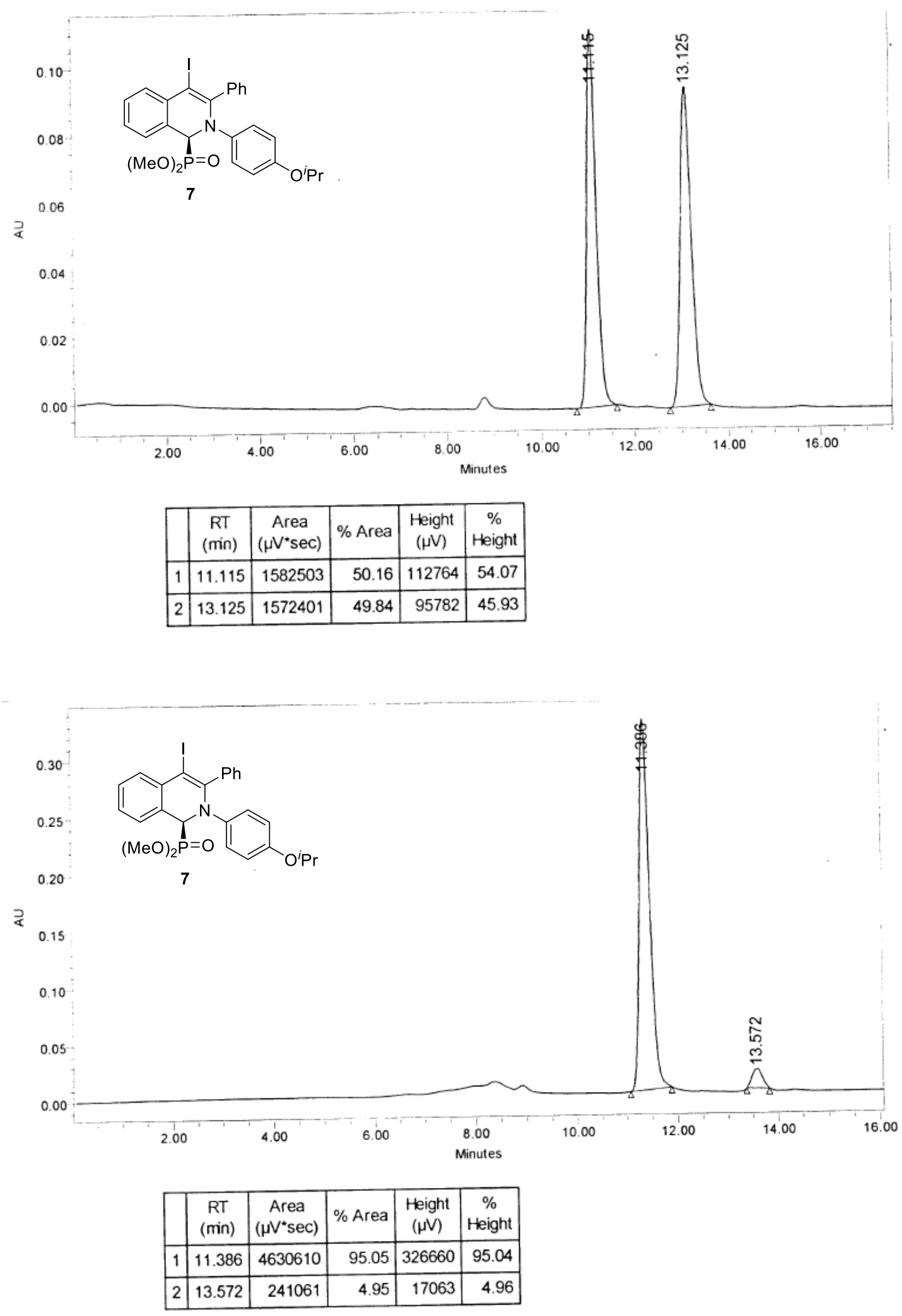

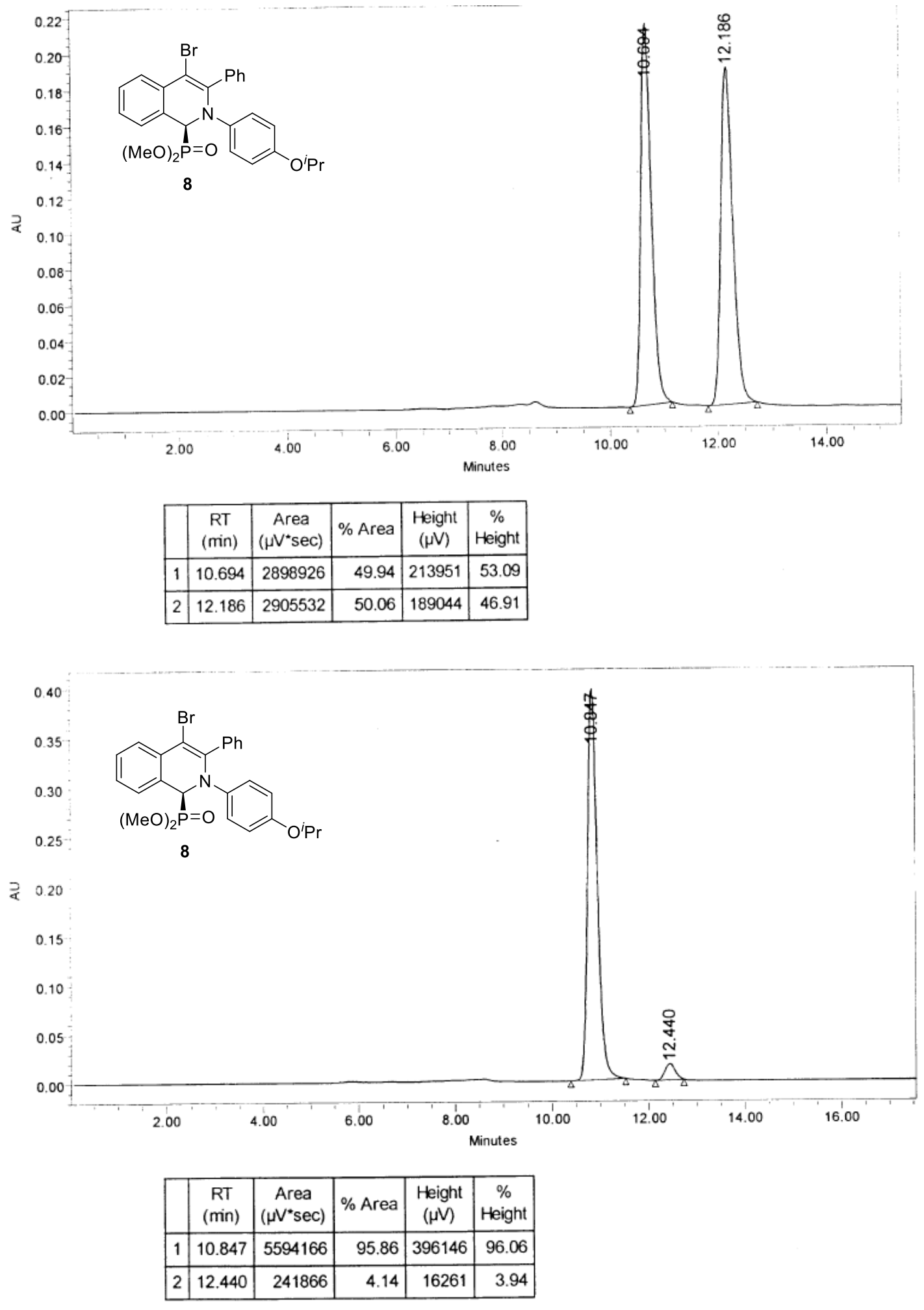